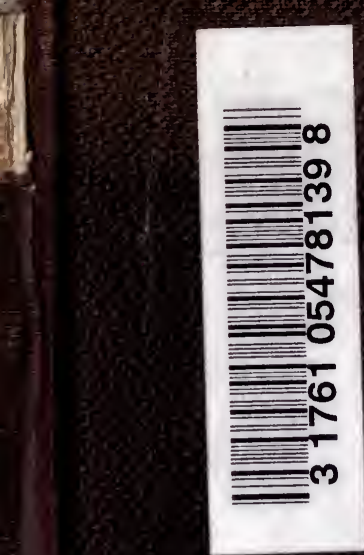




Digitized by the Internet Archive in 2007 with funding from Microsoft Corporation 






\section{A MANUAL OF}

\section{A N A T O M Y}

w

IRVING S. HAYNES, Pн.B., M.D.

ADJUNCT PROFESSOR AND DEMONSTRATOR OF ANATOMY IN THE MEDICAL DEPARTMENT OF THE NEW YORK UNIVERSITY; VISITING SURGEON TO THE HARLEM HOSPITAL; MEMBER OF THE SOCIETY OF THE ALUMNI OF BELlevUe hospital, OF THE AMERICAN ASSOCIATION OF ANATOMISTS, ETC., ETC.

\section{Wuitb 134 'balt=tone $\mathbb{1}$ llustrations and 42 Diagrams}

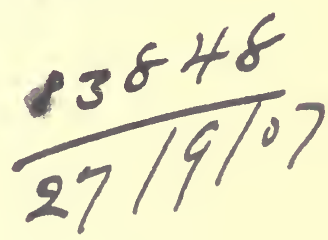

PHILADELPHIA

W. B. S A U N D E R S

925 Walnut Street

I 896 
Copyright, I896, BY

W. B. SAUNDERS 


\section{LEWIS A. STIMSON, B.A., M.D.}

PROFESSOR OF SURGERY IN THE MEDICAL DEPARTMENT OF THE NEW YORK UNIVERSITY

THIS SMALL VOLUME IS OFFERED AS A TRIBUTE

OF RESPECT AND AFFECTION

BY THE AUTHOR 



\section{PREFACE}

THE great practical importance of a thorough knowledge of the viscera and of their relations to the surface of the body has been recognized in preparing this MANUAL of Алатомy, by according to them the most prominent place in illustration and description. Further, to elucidate their formation and relations in the adult, a brief history of the development of the most important organs is introduced.

The anatomy of the extremities has been treated as fully as its requirements seem to demand.

Descriptions of the bones and the joints, and of those minute parts which require special preparation for their dissection, have intentionally been omitted.

Surgical references have been avoided, except in a few instances where it seemed advisable for the student's good to suggest the application of a process or a description.

The descriptions are given in the natural order-that is, the order in which the structures are discovered in the dissection; consequently some parts, being found in two or more regions, receive separate notices, but cross-references are freely used to harmonize such descriptions.

The writer's experience as a teacher in anatomy for several years is utilized in stating the "facts of anatomy" which have been gathered from the standard text-books on this and the allied subjects. Nothing new is presented in the text except a slight contribution touching the visceral 
(thoracic and abdominal) relations, obtained by means of "composite" photographs. Questions of theory are stated in conformity with the latest published opinions of recognized authorities; where they differ from previous writers (for example, see the development of the lamina terminalis) both opinions are expressed and the more rational one indicated.

All the illustrations are original, and excepting a few cases are from photographs by the author of his own dissections. Dissection for Fig. 72 was made by Mr. P. D. Shultz; for Figs. 80 and $8 \mathrm{I}$, by Mr. J. J. Moorhead. To these gentlemen, and also to Messrs. Asa Iglehart and G. $R$. Pisek, for their efficient assistance in the preparation of several dissections of the head and neck and the upper extremity, the writer gladly expresses his thanks.

The diagrams are mostly new; those copied from other text-books are duly credited to their proper sources.

Attention is invited to the Index, which has been written up from the beginning of the proof-reading, using the card system. Every reference has been verified from the completed book, and no pains have been spared to make this essential feature of any volume complete, comprehensive, and accurate. In conclusion, the publisher is to be congratulated for the clearness with which he has reproduced the photographs, and for the care and neatness displayed in the preparation of the text.

Irving S. Haynes.

NEW YoRk, May, 1896. 


\section{CONTENTS}

THE HEAD, ANTERIOR, ................ . I7

LANDMARKS, ............... . . I7

Craniocerebral Topography, . . . . . . . . . I I

Dissection, . . . . . . . . . . . . . . 24

FACE, Landmarks, . . . . . . . . . . . . 24

Dissection, . . . . . . . . . . . . 28

SkUllcap, Removal, . . . . . . . . . . . . 32

Meninges, Dissection, . . . . . . . . . . . . 32

Brain, Removal, . . . . . . . . . . . . 36

Fosse of SkUll, Dissection, . . . . . . . . . 36

Orbit And Contents, Dissection, . . . . . . . . . 48

Face, Dissection, . . . . . . . . . . . . 58

THE NECK, ANTERIOR, . . . . . . . . . . . . . . 68

LANDMARKS, . . . . . . . . . . . . . . 68

Dissection, . . . . . . . . . . . . . . . 69

Deep Face and Pharynx, Dissection, . . . . . . I I5

Pharynt, Dissection, . . . . . . . . . . . . . I 24

HEAD ANI NECK, Posterior,. . . . . . . . . . . I 28

LANDMARKS, . . . . . . . . . . . . . 128

Dissection, . . . . . . . . . . . . . . I 28

Pharynt, Interior, Dissection, ..... . . . . I4I

LARYNX, DISSECTION, . . . . . . . . . . 145

NASAl Fosse, Dissection, . . . . . . . . 148

THE BRAIN, ................. . . I52

Membranes, ................. . . 152

DURA, . . . . . . . . . . . . . . . 152

ARACHNOID, ................ . . . I54

PrA, . . . . . . . . . . . . . . . 156

Blood Supply, ................ . . . I58

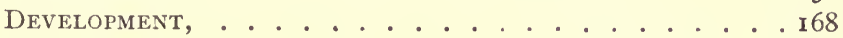

Cerebrum, . . . . . . . . . . . . . . . . . . I 78

BASE, . . . . . . . . . . . . . . . . . 192

Cranial Nerves, Superficial (and Deep), Origin, . . . 198

Cerfbellum, . . . . . . . . . . . . . . . . 242 
THE UPPER EXTREMITY AND THORAX, ANTERIOR, . . . 255 LANDMARKS, . . . . . . . . . . . . . . 255 Outlining Heart and Lungs on Chest Wall, . . . 258 Shoulder, Thorax, And Arm, Dissection, . . . . . 264 Axillary Space and Contents, Dissection, . . . . 274 Arm and Forearm, Dissection, . . . . . . . . 282 Palm of Hand, Dissection, . . . . . . . . . . . 300 The Brachial Plexus, . . . . . . . . . . . . 312 Thoracic Viscera, . . . . . . . . . . . 3I9 The Heart, . . . . . . . . . . . . . . . 330 Removal of Heart and Lungs from Thorax, . . . 343 Heart, Dissection, . . . . . . . . . . . . 352 Lungs, Dissection, . . . . . . . . . . . . . 358

THE UPPER EXTREMITY AND THORAX, POSTERIOR, . . . 360 Landmarks and Dissection, . . . . . . . . 360 BACK of ARM, Dissection, . . . . . . . . . 365 Back (of Trunk), Dissection, . . . . . . . . 373

SPINAL CORD, . . . . . . . . . . . . . . 389

Dissection, . . . . . . . . . . . . . . 389

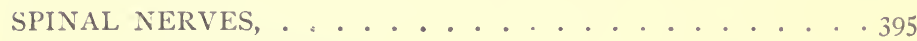

THE PERINEUM, Male, . . . . . . . . . . . . 397

LANDMARKS, . . . . . . . . . . . . . 397

Fascie of Pelvic Outlet, . . . . . . . . . . 398

Dissection, ........................ 404

THE PERineUh, Female, . . . . . . . . . . . . . . 422

External Genitals, .............. . . . 422

Dissection, . . . . . . . . . . . . . 4 424

THE ABDOMEN, Exterior, ............. . . 432

LANDMARKS, . . . . . . . . . . . . . 432

Reglons, . . . . . . . . . . . . . . . 4 435

Dissection, . . . . . . . . . . . . . 4 435

The Penis, Dissection, . . . . . . . . . . . 440

The Testicle, Dissection, . . . . . . . . . . . 443

Abdominal Rings, their formation, and the formation of the Coverings of the Cori and Testicle, . 457

THE ABDOMEN, INTERIOR, . . . . . . . . . . . . . . 462

The Peritoneum, Its Development, with that of the

Alimentary Canal, Liver, Spleen, and Pancreas, . 462

The Peritoneum, Its Reflections, . . . . . . . . 473 
The AbDominal Viscera, Page

Liver, .. . . . . . . . . . . 486

Stomach, . . . . . . . . . . . 4 494

Intestine, . . . . . . . . . . . . 496

Duodenum, ........... . . . 496

Small Intestine, . . . . . . . . . 499

Large Intestine, . . . . . . . . . 500

Spleen, . . . . . . . . . . . . 506

Pancreas, . . . . . . . . . . . . 508

Blood Supply for the Abdominal Viscera, . . . . . . 509

Kidneys, . . . . . . . . . . . . . 522

Suprarenal Bodies, ........... . . 524

Ureters,.............. . 524

The Posterior Abdominal Wall, . . . . . . 525

The Lumbar Plexus, . . . . . . . . . . . 529

The Peilic Viscera, Dissection, . . . . . . . 536

Bladder, . . . . . . . . . . . . 537

Ureter, . . . . . . . . . . . . 540

Vas Deferens, ............ . . 542

Uterus, . . . . . . . . . . . . 542

Ovary, ................. . . 544

The Pelvic Vessels, . . . . . . . . . . . . 546

The Interior of the Bladder and Urethra, . . . . 550

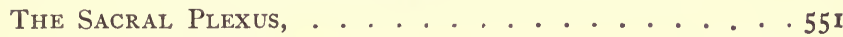

THE LOWER EXTREMITY, ANTERIOR, . . . . . . . 554

LANDMARKS, . . . . . . . . . . . . 554

Dissection of THE THIGH, ........... . 556

Dissection of THE Leg ANd Foot, . . . . . . . 584

THE LOWER Extremity, Posterior, . . . . . . . . 599

LANDMARKS, . . . . . . . . . . . . . . . . . 599

Gluteal Region and Thigh Posterior, Dissection, . . 599

Popliteal. Space and Back of the Leg, Dissection, . . 616

Sole of the Foot, Dissection, . . . . . . . . 634

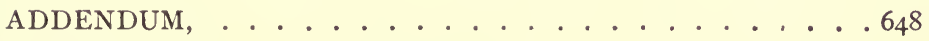

INDEX $\ldots \ldots \ldots \ldots \ldots . \ldots \ldots$ 



\section{MANUAL OF ANATOMY.}

\section{THE HEAD, Anterior.}

\section{The Landmarks.}

The student should fully investigate the landmarks of the head with especial reference to the subject of cerebral topography. A brief résumé of the more important guides is given below. See Fig. I.

All hair should be shaved or carefully clipped from the scalp and face.

In the Median Line.-The nasion is the centre of the nasofrontal suture and is marked by a groove at the root of the nose where it joins the forehead.

This is the more accurate point from which to measure for cerebral localizations than from the point usually given, namely the glabella, which is the smooth surface between the superciliary ridges and above the nasion.

Next the bregma, the junction of the coronal and sagittal sutures, and the lambda, the intersections of the sagittal and lambdoid sutures, are useful anatomical points to remember.

The former lies half an inch in front of a vertical line drawn upward from the external auditory meatus, the latter $23 / 4$ inches above the inion. 
The inion; this is another name for the external occipital protuberance.

Laterally.-The supra-orbital arch is the prominent margin bounding the orbital cavity above. It terminates internally at the extremity of the nasofrontal groove in the obscure internal angular process, and externally in the prominent external angular process (E. A. P.), which is one of the very useful cranial landmarks.

The zygomatic arch lies horizontally and limits the cranial from the facial regions. It also corresponds very nearly with the lower and external border of the temporal lobe of the brain.

The external auditory meatus is a conspicuous and useful landmark used in cranial measurements, as it is both easily felt and seen.

The parietal eminence is more of an area than a point, but it is useful in taking general measurements.

The mastoid process (its tip and posterior border), is a prominent and stable landmark in the adult, but in children cannot be of much use as it is not developed.

The superior curved line of the occipital bone forms usually a readily recognizable ridge arching forward from the inion to the base of the mastoid process. It determines the boundary between the back of the head and neck.

The "Sylvian point" is the point upon the cranium which indicates where the Sylvian fissure reaches the exterior of the cerebrum.

It is found at a distance of one and one-quarter inches directly behind the external angular process of the frontal bone.

Relations of the exterior of the brain to the surface of the cranium.

The margins of the cerebral hemispheres. 


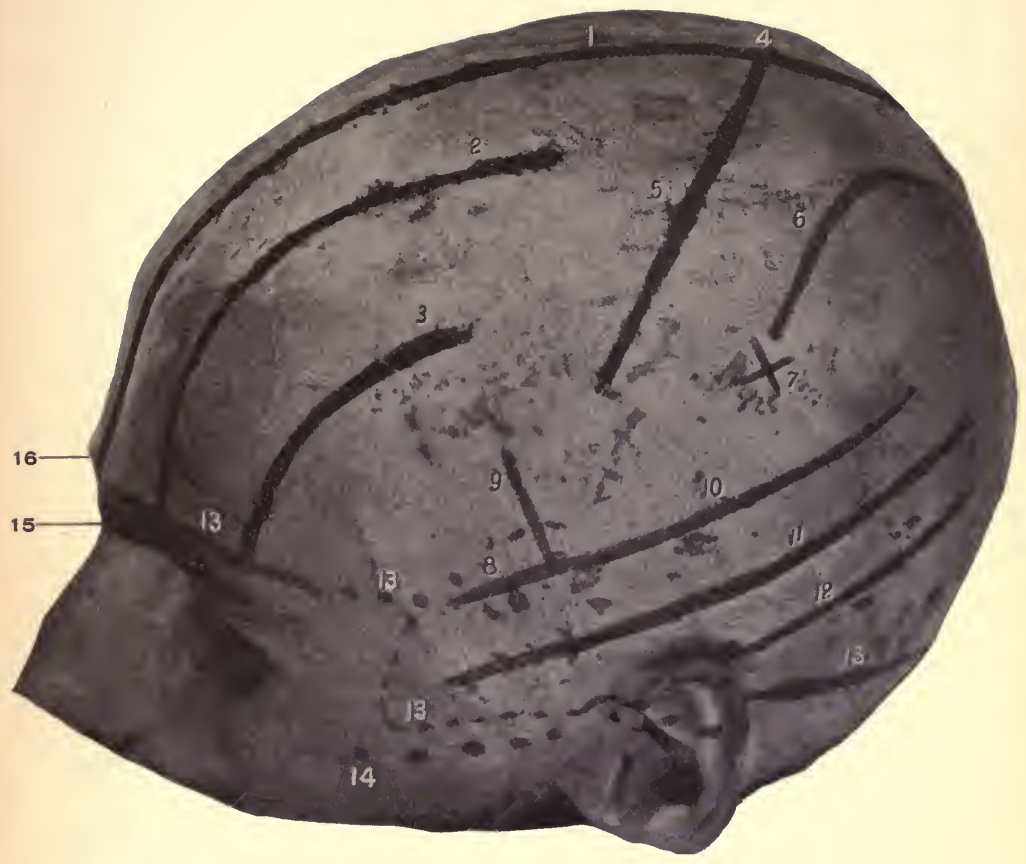

Fig. I. Cranio-Cerebral Topography.

I, Median line.

2, Superior frontal sulcus.

3 , Inferior frontal sulcus.

4, 5, Fissure of Rolando.

6, Intraparietal sulcus.

7, Parietal eminence.

8, Main part of fissure of Sylvius.

9, Vertical limb of fissure of Sylvius.

Io, Horizontal limb of fissure of Sylvius.

II, Superior temporal sulcus.

12, Middle temporal sulcus.

$13,13,13,13$, Lower border of the cerebrum.

I4, Zygomatic arch.

15, Nasion.

I6, Glabella. 
The junction of the vertical with the external surface of a hemisphere is indicated by a line drawn from the nasion over the top of the head to a point half an inch external to the inion.

This line when drawn on both sides will indicate the position of the superior longitudinal sinus.

The line of junction of the inferior and external surfaces of the cerebrum is indicated as follows :

Start the line at the nasofrontal groove, and carry it outward to a point five-eighths of an inch (one-half to threefourths of an inch) above the E. A. P.; from here pass backward to the Sylvian point, then curve a line downward and forward (with its concavity backward to correspond to the rounded extremity of the temporal lobe) to a point just above the junction of the zygoma with the frontal process of the malar bone. (This last point is situated one-fourth of an inch above the zygoma and the same distance from the posterior border of the frontal process of the malar bone. Or, if measured from the E. A. P., it is a vertical line dropped downward from a point five-eighths of an inch posterior to the E. A. P. to terminate one-fourth of an inch from the zygoma.) Now continue the line backward and slightly downward until the junction of the zygomatic arch with the skull is reached (just over the condyle of the inferior maxilla). The line then inclines upward and backward to reach a point one inch behind and half an inch above the level of the external auditory meatus. (This portion of the line passes from one-eighth to three-eighths of an inch above the upper margin of the external meatus and intersects a vertical line drawn upward from the posterior border of the root of the mastoid process, one inch behind and half an inch above the external auditory meatus.) The lower posterior border of the cerebrum is finished 
by continuing the line from the last point downward and backward to the inion, following for the posterior two-thirds of its extent the superior curved line of the occipital bone.

The lower border of the cerebellum is indicated by a line drawn forward and downward from the inion to the posterior border of the mastoid process at its base.

The Lateral Sinus.-This corresponds to a band onefourth of an inch wide extending from the inion, following the curved course of the superior nuchal line forward to a point one inch directly behind the external auditory meatus.

The Nasolambdoidal Line.-This is a line connecting the nasofrontal groove with a point five-twelfths to fiveeighths of an inch above the lambda.

\section{The Fissure of Sylvius.}

The Horizontal Portion.-This is indicated by drawing a line backward and slightly upward from the "Sylvian point" to a point three-fourths of an inch below the most prominent portion of the parietal eminence.

The posterior two inches of this line coincides with the nasolambdoidal line.

The vertical limb of the Sylvian fissure corresponds to a line one inch long drawn vertically upward from the horizontal limb at a point three-fourths of an inch from its beginning, or two inches distant from the E. A. P.

The Fissure of Rolando.-Take 57 per cent. of the distance from the glabella to the inion, or (preferably) fivesixths of an inch, behind the mid-point between the nasion and the inion,- - this will indicate the central extremity of the fissure of Rolando.

From this point draw a line three and three-eighths inches long downward and forward so as to make an angle with the median line of 7 I. 5 degrees. (Cunningham.) 
The External Portion of the Parieto-occipital Fissure.-This is five-twelfths to five-eighths of an inch in front of the lambda, or one and seven-eighths inches behind the central extremity of the fissure of Rolando, and extends about an inch outward and slightly forward on either side of the median line.

\section{The Principal Sulci.}

The Frontal Lobe.-The superior frontal sulcus is indicated by a line starting from the supra-orbital notch (at the inner and middle thirds of the supra-orbital arch), drawn backward parallel with the median line until within an inch of the fissure of Rolando.

The inferior frontal sulcus coincides with the frontal part of the temporal ridge (the bony not the muscular ridge). It begins at the E. A. P., and follows the curve of the ridge upward and backward until within an inch of the Rolandic fissure. This sulcus is parallel with and about an inch from the preceding one.

The superior, middle, and inferior convolutions are thus indicated, and the region in front of the fissure of Rolando and an inch in width will correspond to the ascending frontal convolution.

The Parietal Lobe.-The intraparietal sulcus is shown by drawing a line from a point one inch behind the fissure of Rolando and the same distance above the horizontal limb of the Sylvian fissure upward, one inch from and parallel with the first fissure, until at the junction of its middle and upper thirds, where the line turns backward to run parallel with the middle line, and about an inch and a quarter from it, until it terminates a little behind the parietooccipital fissure in the occipital region. The space behind the fissure of Rolando and in front of the first part of the 


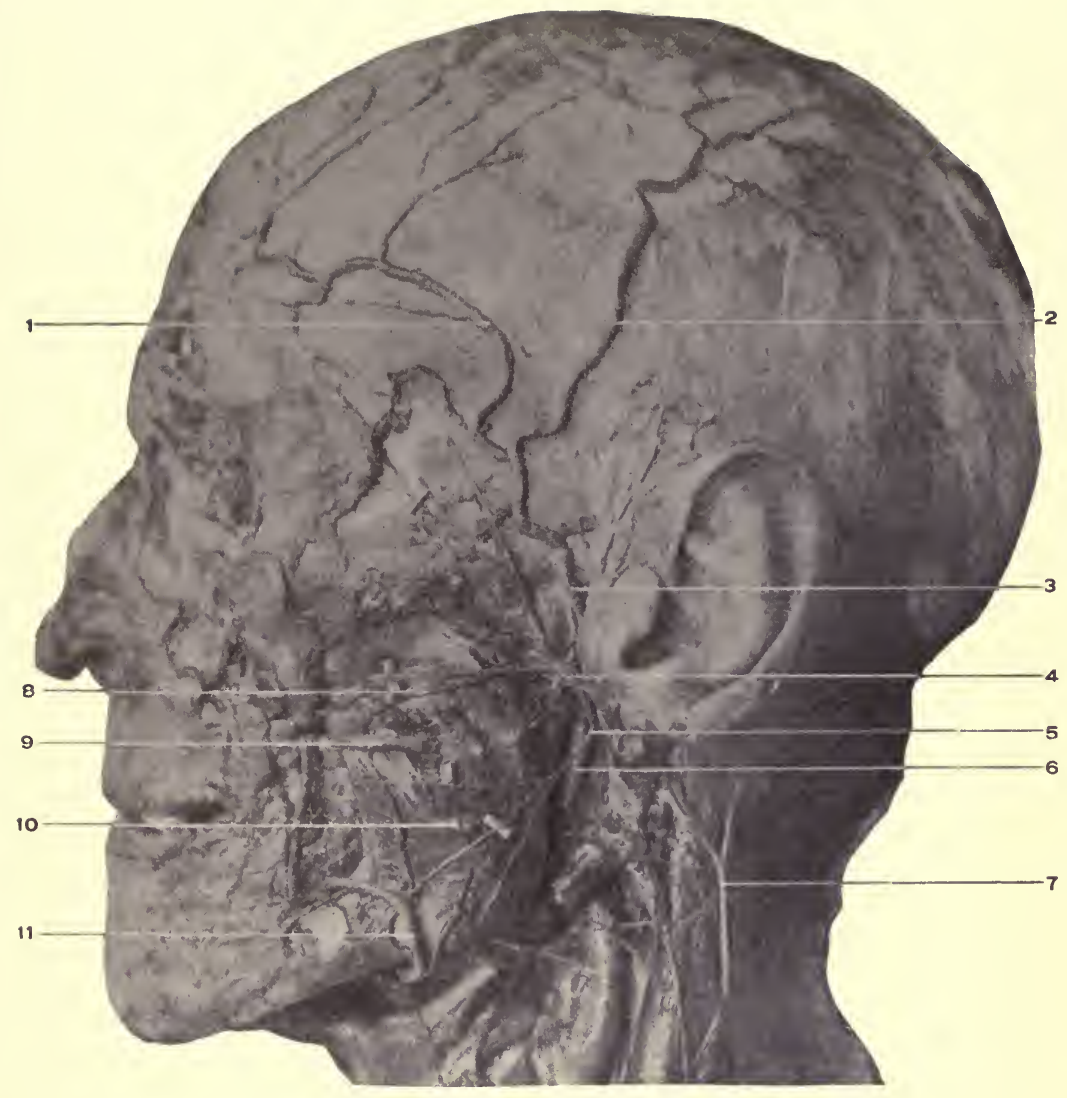

Fig. 2. Dissection of Head.-I, Anterior temporal artery. 2, Posterior temporal artery. 3, Temporal artery. Behind it is the auriculo-temporal nerve. 4, The temporo-facial division of the facial nerve and its three branches, the temporal, malar, and infra-orbital. 5, Cervico-facial division of the facial nerve, and its three branches, the buccal, supramaxillary, and inframaxillary. 6, The external carotid artery. 7, The great auricular nerve. 8, The transverse facial artery. 9, Anterior part of Stenson's duct. Io, Masseter muscle. II, Facial artery. 
intraparietal line is the ascending parietal convolution, the region between the second part of the sulcus and the median line is the superior parietal convolution, and the area within the arch of the intraparietal sulcus is the inferior parietal convolution.

The Temporal Lobe.-The sulci are indicated by two lines drawn backward and upward parallel with the horizontal limb of the fissure of Sylvius, - the first threefourths of an inch from the Sylvian fissure and the second one and one-half inches from the same.

The first temporal convolution corresponds to the region between the Sylvian fissure and the first sulcus, the middle convolution to the area between the first and second sulcus, and the inferior convolution to that below the last sulcus.

The Occipital Lobe.-This agrees with the area behind the parieto-occipital fissure. Its divisions into convolutions is by incomplete sulci, and both convolutions and sulci are unimportant.

The Region of the Face.-The infra-orbital arch limits the orbital cavity below. It leads outward to the malar bone, which varies in prominence with races and individuals. The margins of the lower jaw, posterior and lower, and the angle (formed by their junction) can easily be recognized. The lower border of the jaw, the tip of the mastoid process, a line connecting the angle of the jaw with the tip, and the superior curved line of the occipital bone form the artificial and natural boundary between the head and neck.

\section{DISSECTION.}

Support the head properly by suitable blocks for convenience of dissection. Incisions.-1. From the root of the nose (nasion) in the median line to the occipital protuberance (inion). 
2. Horizontally around the head just above the eyebrows and ears. Be very careful, in removing the skin, not to take with it the underlying muscles, which are closely united to it by a thin layer of dense fatty tissue.

Occipitofrontalis. Fig. 2.

Origin.-In two portions, the posterior from the mastoid process of the temporal and the outer two-thirds of the superior curved line of the occipital bones. The anterior portion from the corrugator supercilii, pyramidalis nasi, and orbicularis palpebrarum muscles with which it is continuous. Also from the overlying integument.

Insertion.-Both bellies are attached to the epicranial aponeurosis-galea capitis-which fills in the space left between the muscular portions. The epicranial aponeurosis also reaches over the side of the head, as a thin layer covering the temporal fascia, and gives attachment to the small superior and anterior auricular muscles.

Nerve Supply.-The facial or seventh; the posterior auricular branch supplying the occipital portion, and the temporal branches the frontal portion of the muscle.

Action.-To raise the eyebrows, and produce transverse wrinkling of the forehead.

\section{The Auricular Muscles.}

These are so insignificant as to scarcely repay the attention of the student, and their description is omitted here.

The Supratrochlear and Supra-orbital Nerves. Fig. 3.

These are both branches of the frontal branch of the ophthalmic nerve. See page 49.

The supratrochlear nerve leaves the orbit above the pulley of the superior oblique muscle and passes to the front of the forehead, supplying the skin over the inner third of the supra-orbital arch.

The supra-orbital nerve issues from the notch, or fora- 
men, of that name, and, ascending vertically over the forehead, is distributed to the integument of this region and the anterior portion of the scalp.

The Frontal and Supra-orbital Arteries. Fig. 3.

The first is one of the terminal branches of the ophthalmic, the second a branch also from the ophthalmic.

The frontal accompanies the supratrochlear nerve (see above); the supra-orbital the nerve of the same name through the supra-orbital foramen to the distribution of that nerve.

The former artery anastomoses with the opposite frontal and the supra-orbital arteries, the latter with the frontal and anterior branch of the temporal.

The Temporal Artery. Figs. 2 and 3.

This is the external branch of bifurcation of the external carotid. It starts under the parotid gland at the neck of the lower jaw, passes upward and over the zygoma, and divides into the anterior and posterior temporal branches. These are distributed to the anterior, lateral, and posterior portions of the head. The anterior anastomoses in front with the frontal and supra-orbital; the posterior, with the posterior auricular and occipital arteries; and both, over the top of the head, with the corresponding arteries of the other side, and in the lateral region with each other. For remaining branches see page 62 .

Temporal Branches of the Facial, and Auriculotemporal Branch of the Fifth Nerves. Figs. 2, 3.

For the former see the facial nerve, page 6 I.

The auriculotemporal branch of the fifth nerve is found (at a later stage of the dissection, see page I I 7 ) to arise by two roots trunks, which surround the middle meningeal 


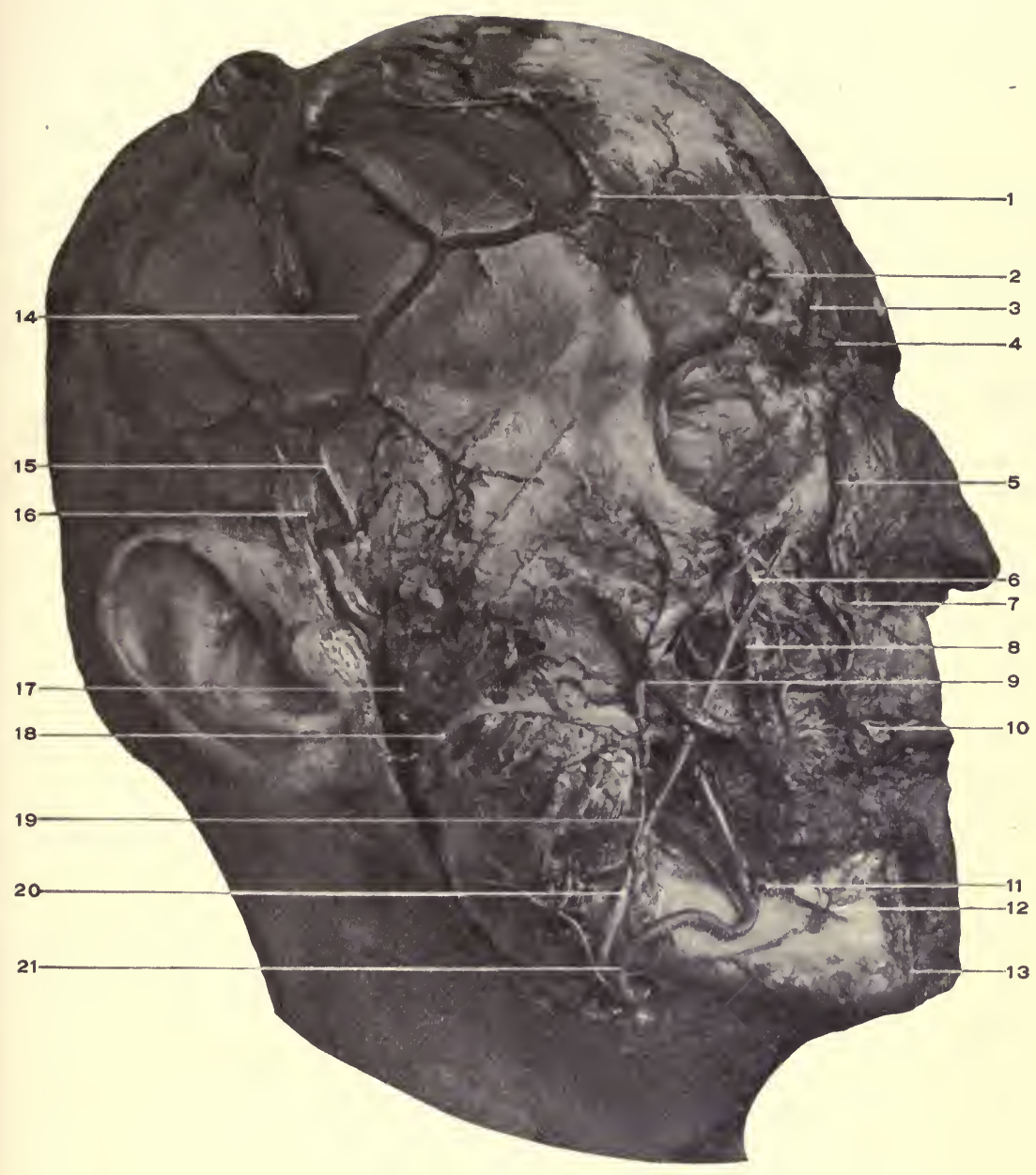

Fig. 3. Dissection of Head.-I, Anterior temporal artery. 2, Supra-orbital artery and nerve. 3, Frontal artery. 4, Supratrochlear nerve. 5, Angular artery. 6, Infraorbital artery and nerve. 7, Lateralis nasi artery. 8, Levator anguli oris muscle. 9, Zygomaticus major muscle. 10, Superior coronary artery. 11, Inferior labial artery. From this is given off the inferior coronary artery. 12, Mental nerve and artery. 13. Termination of the submental artery. 14, Temporal fascia. 15, Temporal artery. 16, Auriculo-temporal nerve. 17, Temporo-facial division of the facial nerve. 18, Stenson's duct crossing masseter and piercing buccinator muscles. The parotid gland is removed. 19, Masseteric artery anastomosing with the transverse facial and infraorbital. 20, Facial vein. 21, Facial artery. 
artery, from the inferior or posterior division of the fifth nerve.

Its course is between the internal lateral ligament and neck of the lower jaw, then between the temporomaxillary articulation and the parotid gland; issuing from under the gland, it turns upward along with the temporal artery, behind which it runs to supply the integument upon the side of the head.

Its branches are, also, distributed to the temporomaxillary articulation, the parotid gland, and the ear (meatus and upper part of the pinna).

\section{DISSECTION.}

Incision.-1. Continue the median incision to the point of the chin, carrying it around the wing of the nose and the angle of the mouth.

2. From the last point extend a cut backward along the lower border, to the angle of the jaw and upward to the lobe of the ear.

Reflect the flap from before backward. Much care will have to be exercised in removing the integument from the face, as it is very thin, especially so over the eyelids, and closely attached to the facial muscles, which are thin, pale, and indistinct.

Orbicularis Palpebrarum. Fig. 2.

Origin. - The palpebral portion, by means of the internal tarsal ligament (tendo-oculi) from the crest of the nasal process of the superior maxillary bone.

The orbital portion, from the internal tarsal ligament, the nasal process of the superior maxilla, and the inner portion of the infra-orbital arch.

Insertion.-The palpebral portion, into the external tarsal ligament and by it into the front of the frontal portion of the malar bone.

The orbital portion surrounds the palpebral or central portion and forms the sphincter muscle of the eyelids. Insertion same as the origin. 
Nerve Supply.-The temporofacial division of the seventh nerve.

Action.-To close the eyelids.

\section{The Tarsal Ligaments.}

The internal is a fibrous band less than a quarter of an inch long which is attached internally to the crest of the nasal portion of the superior maxilla and externally gives attachment to the orbicularis palpebrarum muscle, and divides into two portions, which are projected into the margins of the tarsal cartilages.

The external tarsal ligament passes from the outer extremities of the tarsal cartilages to the orbital process of the malar bone.

\section{Pyramidalis Nasi.}

That portion of the occipitofrontalis which is extended to the nasal bones where they are joined by their cartilages.

The compressor narium, depressor alæ nasi, dilator naris anterior, and dilator naris posterior are muscles of interest to the specialist in the field of anatomy and need not be dissected by the student (there are too many other structures of more importance than insignificant muscles of this sort, that demand his attention).

\section{DISSECTION.}

Divide the aponeurosis of the occipitofrontalis in the median line and also over the vertex of the head from ear to ear.

Reflect the anterior triangular portion forward and downward; when near the orbit the corrugator supercilii will be exposed. By turning the muscle still further downward the supra-orbital notch or foramen and its contents will be exposed.

Reflect the posterior half of the occipitofrontalis. The temporal fascia is now exposed. After learning its attachments cut it away from its upper attachment, beginning at the external angular process of the frontal bone, and turn it downward.

This discloses the temporal muscle. 


\section{Corrugator Supercilii.}

Origin.-From the inner surface of the superciliary ridge of the frontal bone.

Insertion. - Into the inner surface of the frontal portion of the occipitofrontalis and the skin above the middle of the eyebrow.

Nerve Supply.-The seventh, through its temporal branch.

Action.-To wrinkle the skin of the forehead vertically (frowning).

The Temporal Fascia. Fig. 3.

This is a dense shiny layer of fascia lying under the aponeurosis of the occipitofrontalis, and covering in the temporal muscle.

It is attached to the outermost ridge of the temporal fossa, from the external angular process of the frontal to the posterior root of the zygomatic arch behind, and below to the zygomatic arch in two layers, one to the inner and one to the outer border of its upper margin.

Between these layers is found a little fat, the orbital branch of the temporal artery, and the temporal branch of the temporomalar nerve (of the superior maxillary).

Temporal. Fig. 4 .

Origin.-From the whole of the temporal fossa of the skull and the inner surface of the temporal fascia.

Insertion.-Into the tip, anterior border, and inner surface of the coronoid process of the lower jaw.

Nerve Supply. - The fifth cranial nerve through the motor portion of its inferior maxillary branch.

Action.-To close the lower jaw, and, by the posterior portion, to retract it. 


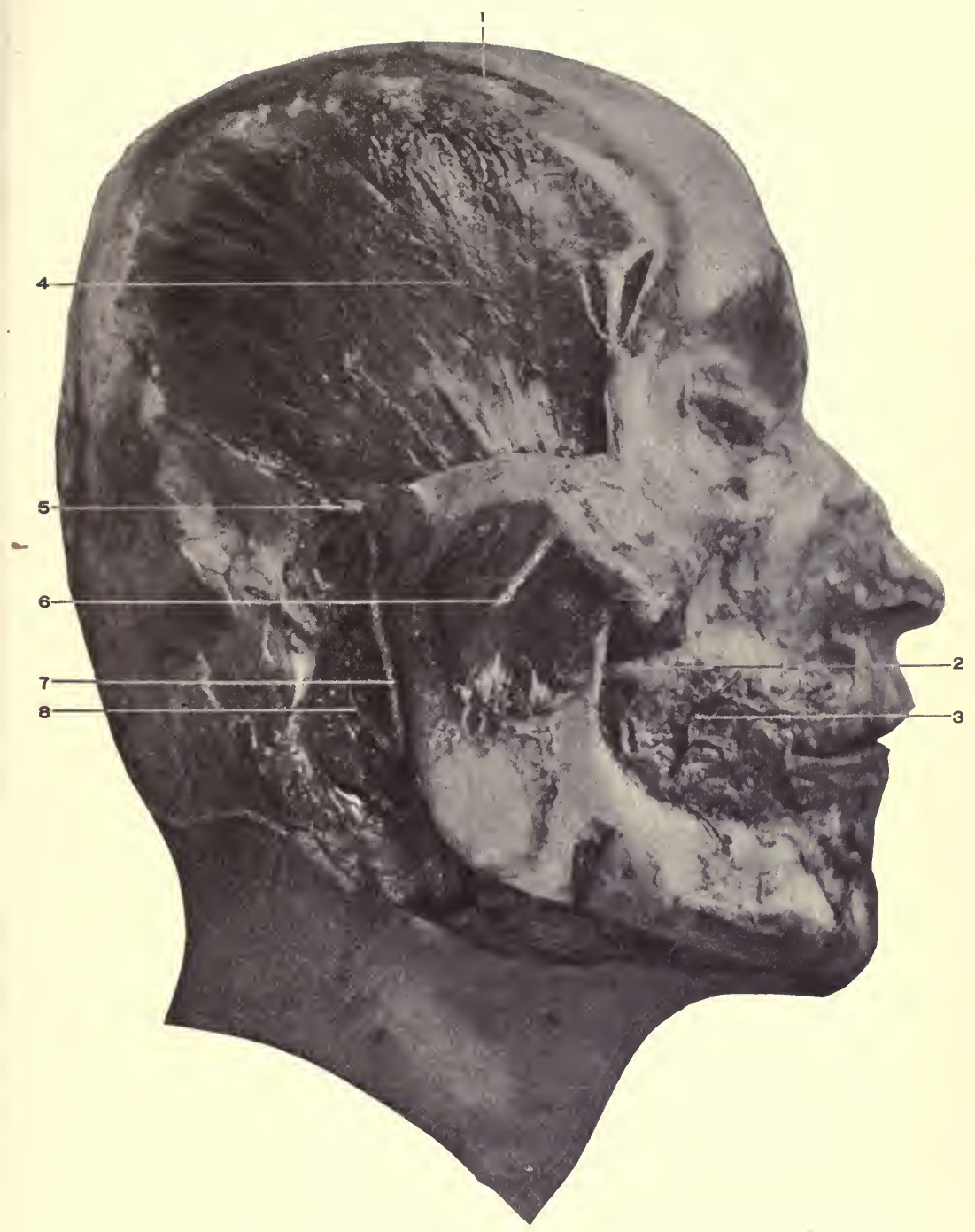

Fig. 4. Dissection of HEAD.-I, Ridge to which temporal fascia was attached. 2, Stub of Stenson's duct, and behind it the buccal branch of the inferior maxillary nerve. 3. Portion of the facial artery resting upon the buccinator muscle. 4, Temporal muscle. 5. Anterior auricular artery. From the side of the temporal artery opposite to the anterior auricular is seen the beginning of the orbital artery, and below this the transverse facial. 6, Deep portion of the masseter muscle. 7, External carotid artery. 8 , Posterior auricular artery. 


\section{DISSECTION.}

To Remove the Brain.-The anterior and posterior portions of the occipitofrontalis and the temporal fascia have been already turned down. The temporal muscle is now to be dissected from its attachment to the temporal fossa (the deep temporal vessels and nerves noted, see pp. I1 7, I 21) and turned over, or cut away entirely on a level with the zygoma. With a saw the outer table of the skull is to be cut through in a line from a point three-fourths of an inch above the supra-orbital arches to the occipital protuberance, completely encircling the head.

When the outer table has been cut through, as is shown by the bloody sawdust turned out, the saw is to be changed for a chisel. The ordinary "cold" chisel is suitable for this purpose. With a chisel break off the skull-cap, entering the chisel first at the external angular processes of the frontal bone, then over the mastoid processes. Be careful not to drive the chisel through into the brain. After breaking the skull at these four points, the entire top of the skull will probably be loosened.

Insert a strong hook at the forepart of the skull-cap, and with a sharp, quick pull tear it from the dura.

Before going farther study the middle meningeal artery and dura.

For the artery see page 44 .

The Dura. Figs. 6 and 8 .

The dura is the most external membrane which envelops the brain. It is closely applied to the inner surface of the cranium and forms the inner periosteum for the bones which compose it.

This attached surface is rough, as will be seen after the skull-cap has been removed. The inner surface of the dura is smooth, and is in close relation to the arachnoid (see page I 52), from which it is separated by the smallest interval, called the subdural space.

Offsets from the inner layer of the dura are found in certain places, as between the halves of the cerebrum-the falx cerebri, between the cerebrum and cerebellum-the tentorium cerebelli, and between the two lobes of the cerebellum-the falx cerebelli.

Along the lines where these offsets take place spaces are 


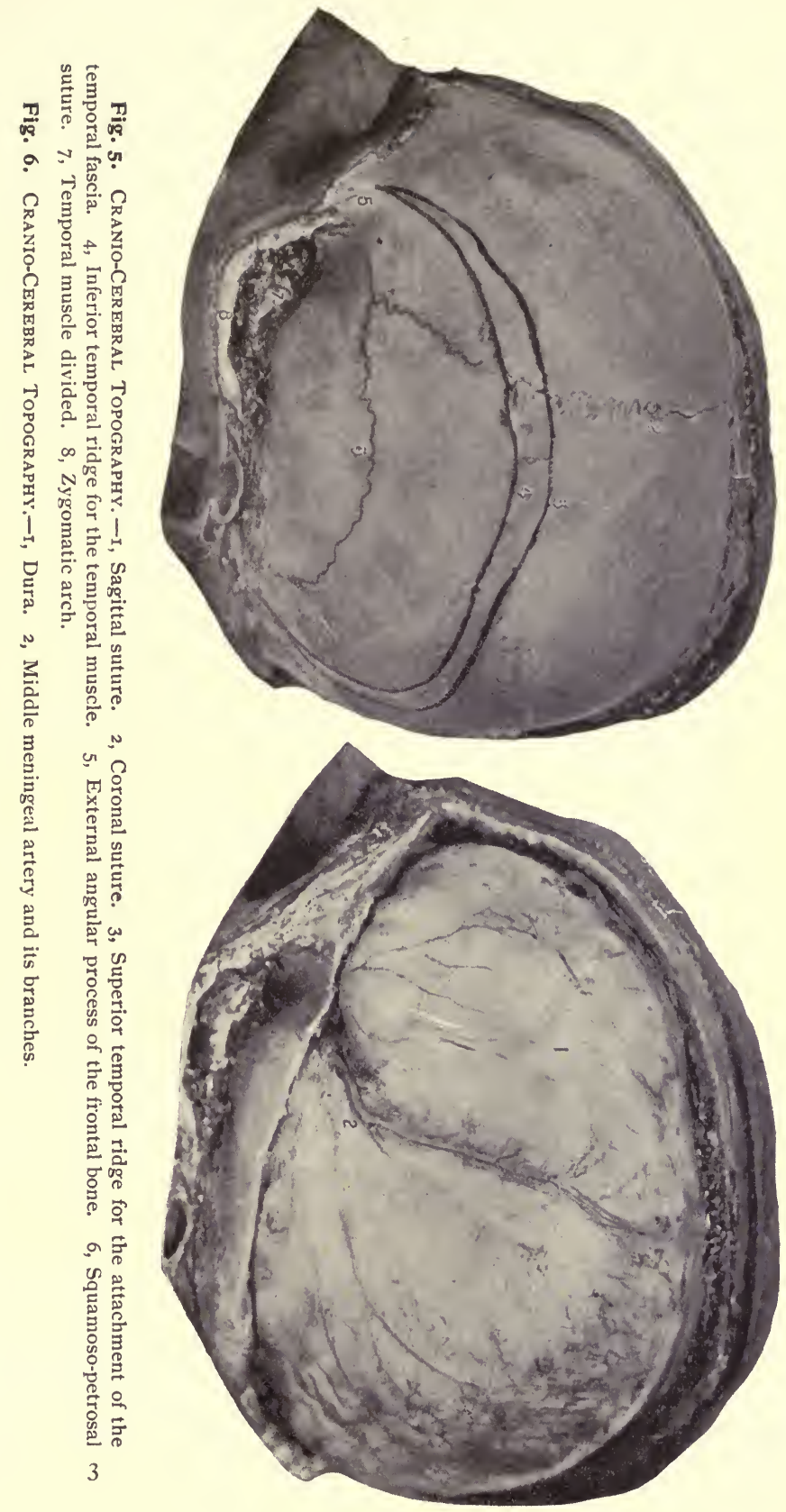


left between the two layers of the dura called the cranial sinuses. These sinuses are also found at other points than where these processes are given off, as at the base of the skull. The sinuses will follow later; see below.

\section{DISSECTION.}

Split open the superior longitudinal sinus from its beginning to its ending, and in doing so notice the cords which cross it. These are the chordx Willisii.

Divide the dura, just above the bone, entirely around the head. Raise the dura from the brain and turn it upward at the sides as far as possible; this will expose the attachment of the falx cerebri.

The falx is to be cut from its attachment, and with the dura turned backward. Divide the cerebral veins when encountered, and turn the dura off backward from the brain, exposing its upper surface. As a rule the dura is not removed far enough backward.

In the free margin of the falx will be seen a very small opening, looking like a slit. This is the inferior longitudinal sinus.

The Falx Cerebri. Figs. 8 and 9.

This is a sickle-shaped process of the dura which lies between the two cerebral hemispheres. It is attached all along its base to the dura (with which it is continuous) and at its attachment, by the divergence of its layers is formed the superior longitudinal sinus. See below. Its anterior extremity is fastened to the crista galli, and its posterior extremity to the upper surface of the tentorium cerebelli. At this rear attachment the straight sinus is formed by the separation of its layers, similar to the formation of the superior sinus.

The Superior Longitudinal Sinus. Diag. I. Fig. 8.

As stated above, this sinus is formed at the base of the falx cerebri, where its two layers pass off laterally into the inner layer of the dura. 
It will be found to extend from the foramen cæcum in the frontal bone over the convexity of the head in the middle line, and at the base of the falx cerebri (as will be apparent later), to terminate in a considerable cavity opposite the external occipital protuberance, called the torcular Herophili, or the confluence of sinuses; or it may pass into

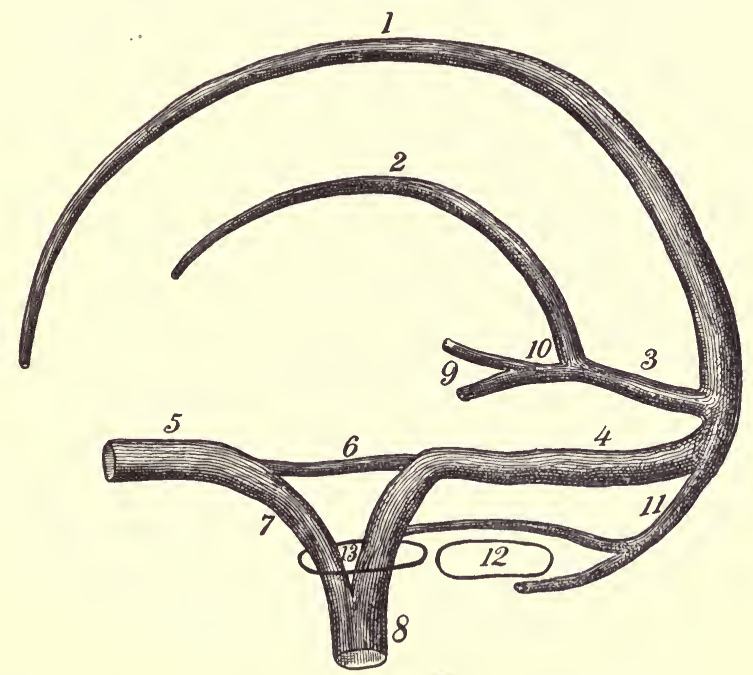

Diag. I. A Diagram of the Sinuses of the Skull, Vertical-Antero-posteRIOR. (I. S. $\left.H_{1}\right)-\mathrm{I}$, Superior longitudinal. 2, Inferior longitudinal. 3, Straight. 4, Lateral. 5, Cavernous. 6, Superior petrosal. 7, Inferior petrosal. 8, Internal jugular vein, formed below the skull by the junction of the inferior petrosal and lateral sinuses. 9, Right and left veins of Galen. Io, Common vein of Galen. II, Occipital. 12, Foramen magnum. 13, Jugular foramen.

the right lateral sinus (usual). By the scissors this sinus is to be laid open from its beginning to its ending. The bands crossing the sinus are the chordæ Willisii. Its tributaries are the nasal veins (sometimes) through the foramen cæcum, the parietal through the parietal foramen, the superior cere- 
bral. These will be found passing to the sinus over the top of the brain.

\section{The Inferior Longitudinal Sinus. Diag. I. Fig. 8.}

This is a small slit left between the two layers of the dura composing the falx cerebri at its free margin. It extends from the anterior and middle third of the falx to its attachment to the tentorium, where it opens into the straight sinus.

\section{DISSECTION.}

Raise the neck with a block, let the head (and with it the brain) drop backward, supporting the brain that it does not draw too forcibly upon its basal attachments.

Gently insert the fingers of one hand between the brain and the dura, separating the former from the latter, until the olfactory nerves are seen. Raise these from the groove in the bone with the handle of a knife; they will usually remain attached to the brain. Let the brain sag backward until the optic nerves come into sight; divide these close to the bone and the internal carotid artery, which will be seen at the same place.

The infundibulum is now seen extending to the pituitary gland, located within the sella turcica. To remove the gland cut through the dura covering the cavity containing it, and with the handle of the knife turn the gland out of its bed.

Still let the brain fall backward and cut through the third, fourth, fifth, and sixth nerves.

Cut the tentorium along the anterior attachment (to the superior border of the petrous portion of the temporal bone). This will free the brain so that all the remaining nerves can be divided close to the bone.

The last step is now the most important. Carefully cut through the vertebral arteries upon either side of the cord, and finally divide the cord across as low as possible.

The brain is now free, except its attachment by the vein of Galen to the straight sinus; divide this, after replacing the brain, by separating its hemispheres and cutting through the veins just in front of the tentorium.

Remove the brain and place it in a solution of alcohol (6o per cent.) and chloride of zinc (added to the alcohol until the brain floats midway between the top and bottom of the vessel; this will insure that when the brain is hardened it will preserve its normal outlines. With this solution the pia need not be removed until the brain is to be dissected, though it had better be removed in the course of the first week. For complete hardening of the brain in this solution about three weeks is needed). For the dissection of the brain see page 152. 


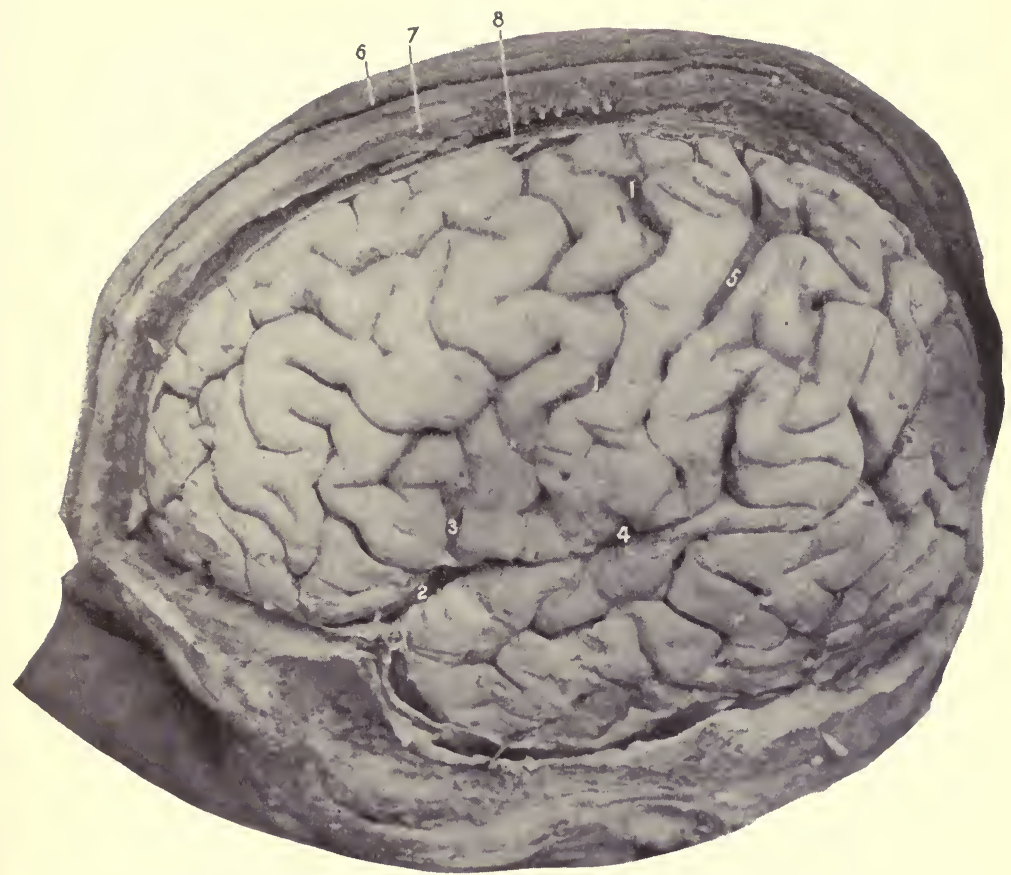

Fig. 7. Cranio-Cerebral Topography.

I, I, Fissure of Rolando.

2, Fissure of Sylvius, main portion.

3, Vertical limb of same.

4, Horizontal limb of same.

5, Intraparietal sulcus. For the remaining fissures, sulci, and convolutions consult Fig. 23.

6, Skin.

7 , Bone.

8, Dura. 
Now study the parts at the base of the skull. Fig. IO.

The base of the skull, internally, is divided into three fossæ-the anterior, middle, and posterior.

The anterior fossa has for its floor the orbital plate of the frontal, the cribriform plate of the ethmoid, and the lesser wings of the sphenoid bones.

The posterior margin of the last and the optic groove bounds the fossa posteriorly.

The Middle Fossa.- This is the central hollow, and its floor is composed of the greater wings and body of the sphenoid and the front surfaces of the petrous portions of the temporal bones. The fossa is limited behind by the superior margin of the petrous portions of the temporal bones and the dorsum ephippii. Anteriorly, by the lesser wings of the sphenoid and optic groove.

The posterior fossa of the skull is all that portion of the base internally which extends behind the superior margins of the petrosal bones and the dorsum ephippii.

The contents of these various fossæ will be apparent as the dissections of the floor of the cranial cavity proceeds.

\section{The Tentorium Cerebelli. Fig. 9.}

This is a portion of the dura which lies between the cerebrum and the cerebellum. It is attached externally to the dura (and so to the bone) along the line of the lateral sinuses (which it forms by the separation of its layers). Internally it is attached to the superior border of the petrous portion of the temporal bone (enclosing the superior petrosal sinuses at this point), and to the posterior and anterior clinoidal processes.

An opening is left between the petrosal attachments through which the crura cerebri, basilar artery, and third and fourth nerves pass. 


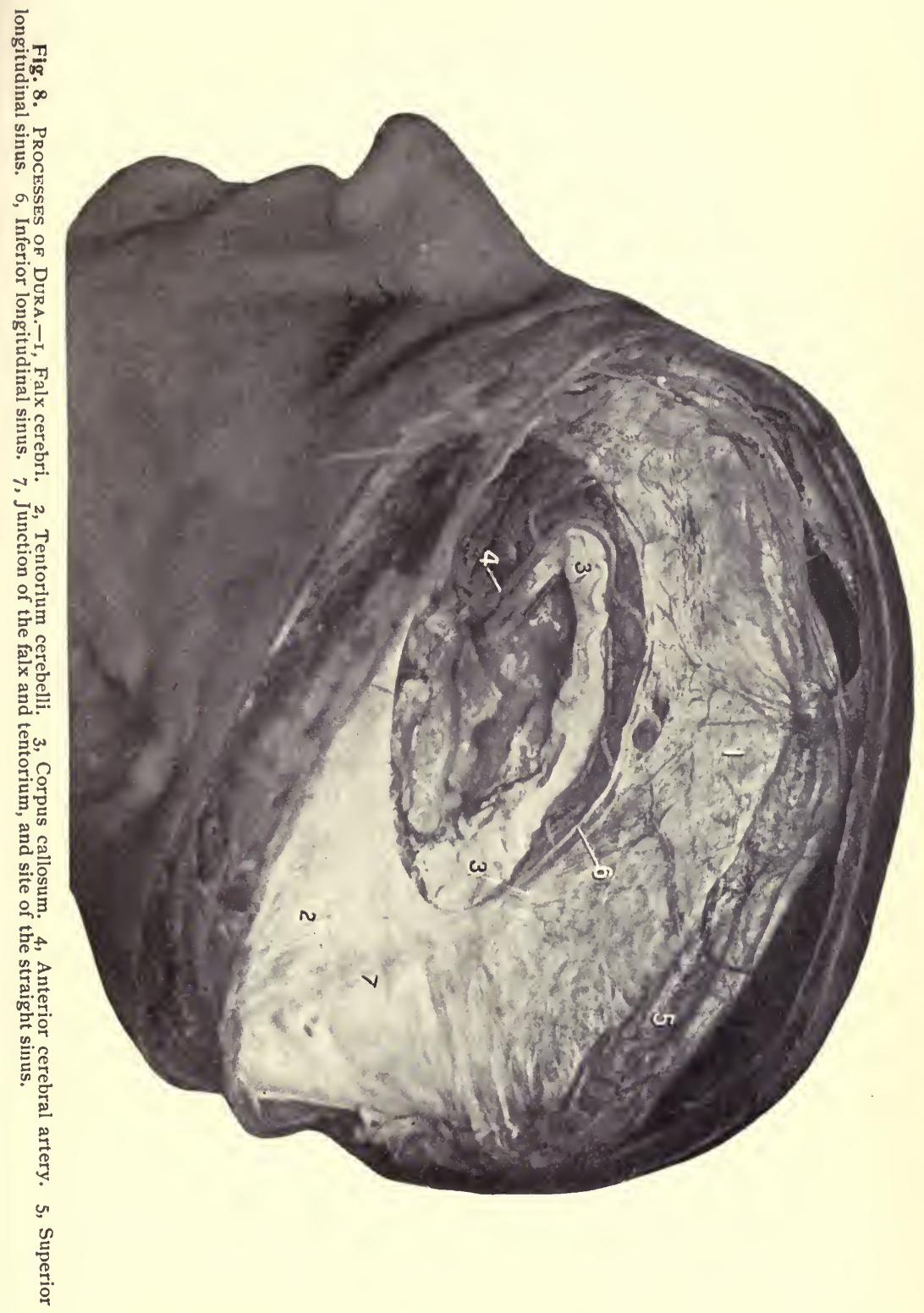


The Falx Cerebelli. Fig. Io.

This is the small ridge of dura placed between the two lobes of the cerebellum and extending from the under surface of the tentorium in the middle line to the posterior margin of the foramen magnum.

The Straight Sinus. Diags. I and 2.

This will be found by slitting up the attachment of the falx cerebri to the tentorium. It is contained or formed by the

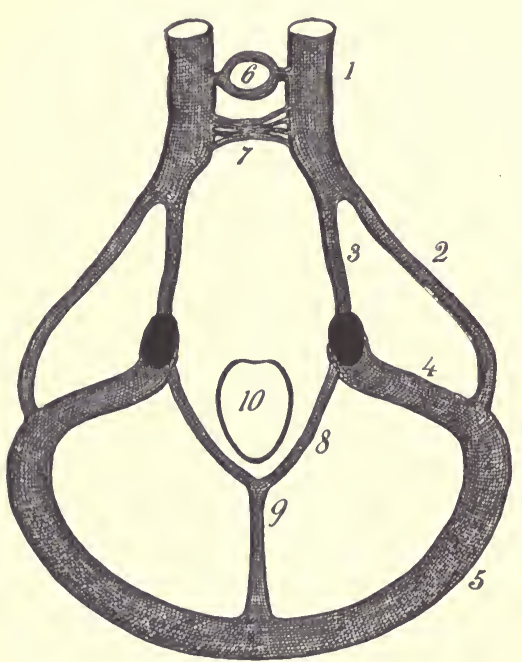

Diag. 2. A Diagram of the Sinuses at THE BASE OF THE SkUll. (I.S.H.)-1, Cavernous. 2, Superior petrosal. 3, Inferior petrosal. 4, and 5, Lateral. 6, Circular. 7, Transverse, (more of a plexus). 8 , Communicating between the lateral and 9, Occipital sinuses. 10, Foramen magnum. diverging halves of the falx at its tentorial attachment. It extends from the anterior (free) margin of the tentorium, where it receives the inferior longitudinal sinus and the vein of Galen, backward to end in the torcular Herophili, or be continued into one of the lateral (usually the left) sinuses.

The Lateral Sinuses. Diags. I and 2 . Fig. Io.

These run in the attached margins of the tentorium from the internal occipital protuberance behind to the petrous portion of the temporal bone, where they turn downward through the sigmoid groove of the temporal bone to pass through the posterior compartment of the jugular foramen and terminate in the internal jugular vein, $q . v$., page 92. 


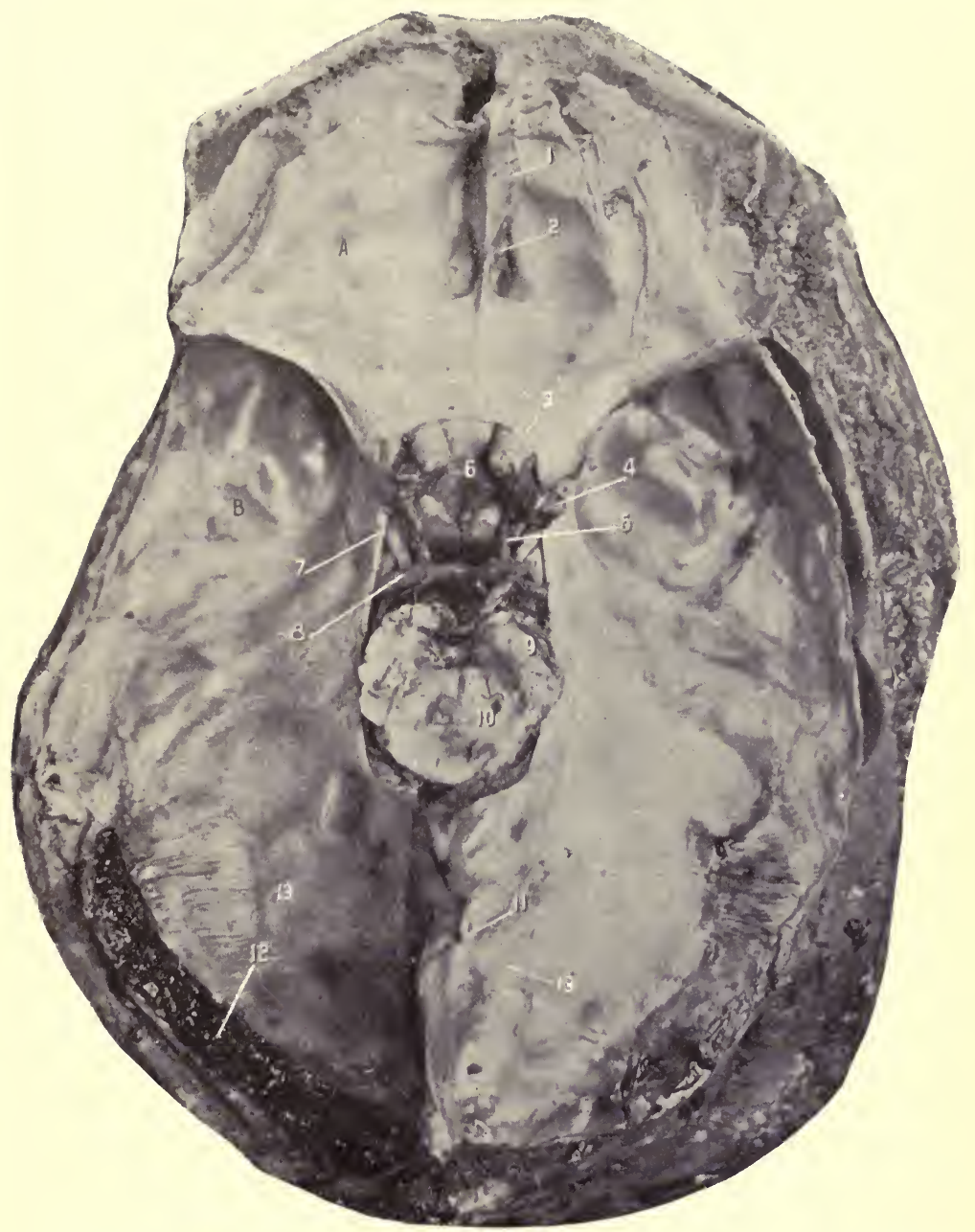

Fig. 9. Interior of the Base of the Skull, Tentorium in Place.-1, Crista galli and attachment of falx cerebri. 2, Olfactory bulb. 3, Optic nerve. 4, Middle cerebral artery. 5, Posterior communicating artery. 6, Infundibulum and pituitary gland. 7. Third nerve. 8, Posterior cerebral artery. 9, Crusta of crus cerebri. 10, Tegmentum of same. Between them is the locus niger. II, Attachment of the falx cerebri divided close to the tentorium cerebelli. 12, Lateral sinus. 13, Tentorium cerebelli. 
The right lateral sinus usually receives the superior longitudinal sinus; while the left receives the straight sinus, and the two are connected by a short sinus across the internal occipital protuberance.

In other cases the four sinuses open into a common cavity termed the torcular Herophili-or the confluence of sinuses. The lateral sinuses also receive the veins from the posterior part of the cerebrum, the upper and lower surfaces of the cerebellum, from the diploë, also the superior petrosal sinuses. They communicate with external veins through the mastoid and posterior condyloid foramina.

The Superior Petrosal Sinus. Diags. I and 2.

Two in number, one on either side. This sinus is located in a groove along the upper margin of the petrous portion of the temporal bone (the tentorium being attached to the margins of the groove). Externally it opens intb the lateral sinus, and internally into the cavernous sinus.

The Occipital Sinus. Diags. I and 2.

Is formed by the junction of the marginal sinuses at the posterior part of the foramen magnum. It extends upward in the median line to open into the confluence of sinuses over the internal occipital crest. The marginal sinuses communicate in front with the lateral sinuses.

The Inferior Petrosal Sinus. (A paired sinus.) Diags. I and 2 .

It extends backward from the cavernous sinus, at the margin of the basilar process of the occipital bone, to leave the skull through the anterior compartment in the jugular foramen, and below the margin of the foramen helps to form the internal jugular vein by joining with the lateral sinus. 
The Cavernous Sinus. Diags. I and 2. Fig. IO.

Two, one on either side of the body of the sphenoid bone.

It extends from the inner extremity of the sphenoidal fissure, where it receives (is really the continuation of) the ophthalmic vein from the orbit, to the apex of the petrous portion of the temporal bone, where it terminates in the superior and inferior petrosal sinuses.

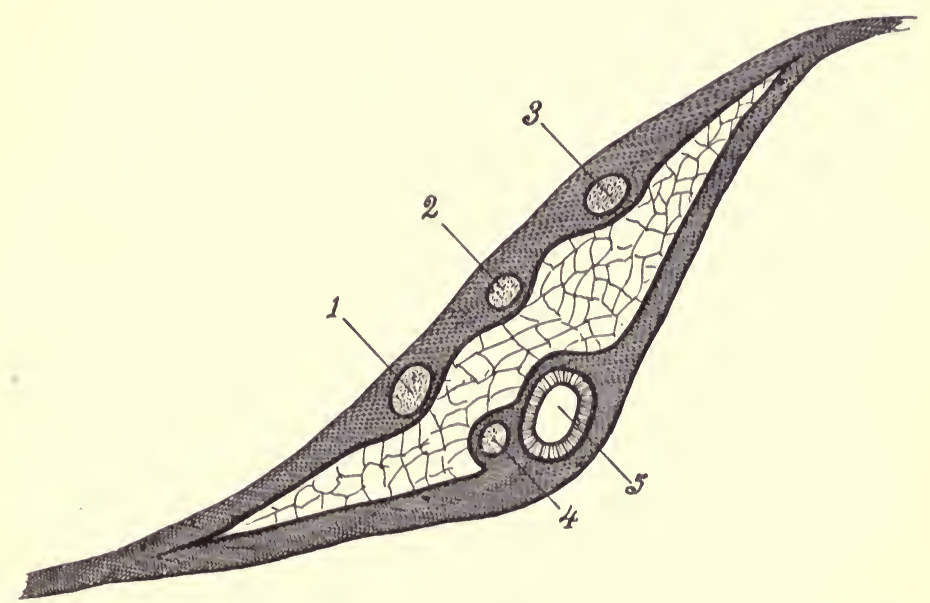

Diag. 3. A Diagram of the Cavernous Sinus. (I. S. H.)-The diagram shows the right sinus divided transversely and its cavity filled with fine interlacing trabeculæ. I, Ophthalmic division of the fifth cranial nerve. 2, Fourth cranial nerve. 3, Third cranial nerve. 4, Sixth cranial nerve. 5, Internal carotid artery.

The cavernous sinuses are connected across the sphenoid bone by the circular sinus.

Upon the inner wall of the cavernous sinus is the internal carotid artery and the sixth nerve; on the outer wall the third, fourth, and ophthalmic division of the fifth nerves ; all these structures are separated from the blood in the sinus by a covering of endothelium. 
The Circular Sinus. Diag. 2.

Connects the two cavernous sinuses in front and behind the pituitary body, which it encloses in a venous circuit.

The Transverse Sinus. Diag. 2.

This is really a venous plexus upon the basilar process of the occipital bone, joining the two inferior petrosal sinuses and communicating with the spinal veins below.

\section{DISSECTION.}

Trace out the middle meningeal artery, noting how deeply its trunk and the beginning of the anterior and posterior branches groove the bone, sometimes being bridged over with bone at the anterior inferior angle of the parietal bone.

Clean the stumps of the second to twelfth cranial nerves from before backward and learn their foramina of exit.

Trace the nerves and internal carotid artery, which pass through the cavernous sinus, and follow the carotid artery until it disappears through the middle lacerated foramen.

The Gasserian ganglion is to be carefully exposed, and the three branches of the fifth nerve shown.

The contents of the jugular foramen are to be separated and learned. They are the glosso-pharyngeal, pneumogastric, and spinal accessory nerves, occupying the central compartment; the inferior petrosal sinus, the anterior compartment; and the lateral sinus, the posterior compartment.

\section{The Middle Meningeal Artery. Figs. 6 and Io.}

This artery within the skull is found coming through the foramen spinosum and grooving the inner surface of the skull as it winds upward and backward.

It divides into two principal branches, the anterior and posterior meningeal. These between them supply the anterior, lateral, and posterior areas of the skull and dura. For the origin of the artery see page I 21 .

The anterior, small, and posterior meningeal arteries are very small, and will not repay any time expended upon their dissection. The anterior are furnished by the ethmoi- 


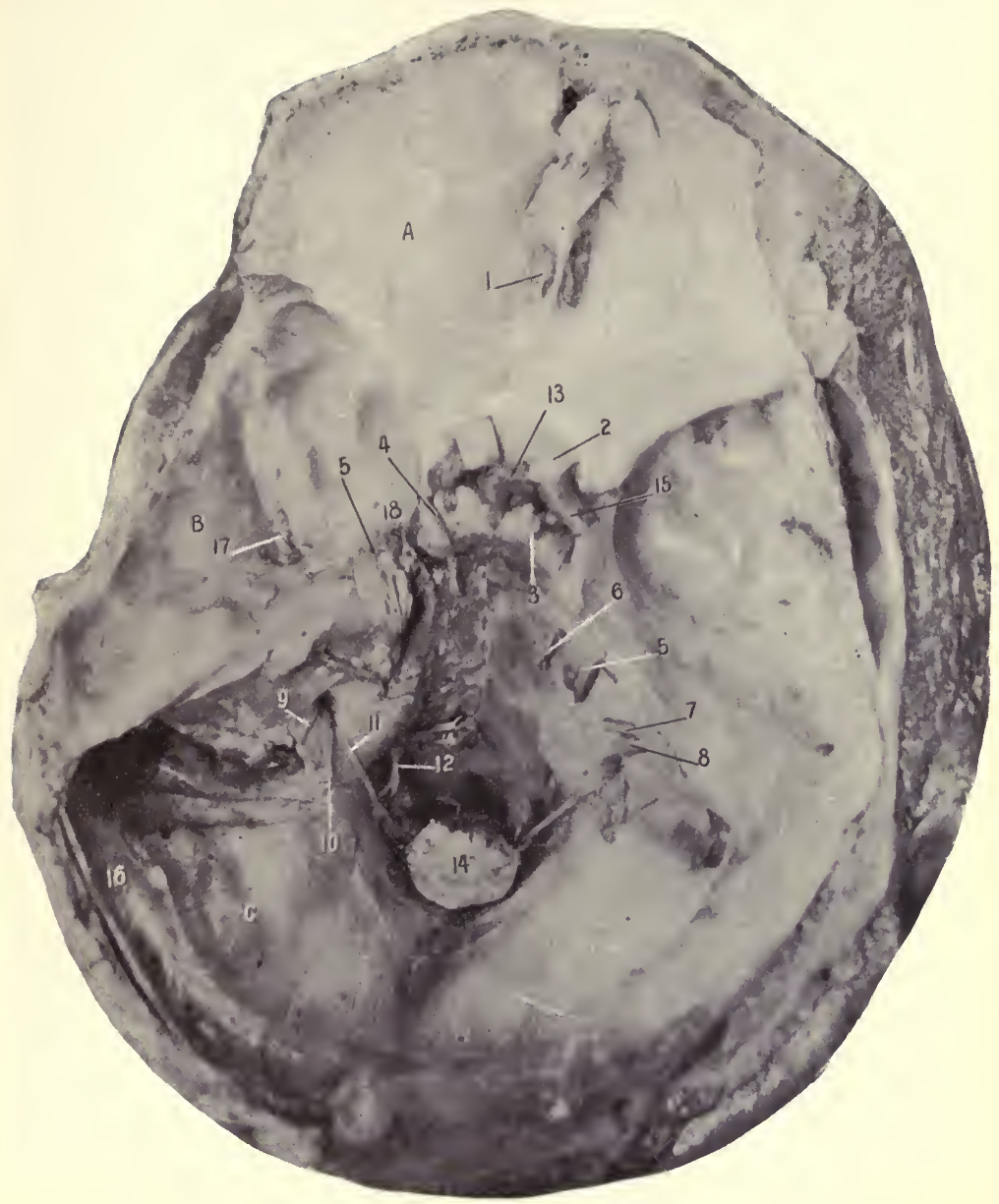

Fig. 10. Interior of the Base of the Skull, Tentorium Rfamoved.

I to 12 , The various cranial nerves at their foramina of exit.

I3, Pituitary gland and infundibulum.

I4, Spinal cord.

15, Internal carotid artery.

16, Lateral sinus.

17, Middle meningeal artery.

18, Cavernous sinus opened. Figure 5 points to the Gasserian ganglion on the fifth nerve. $A$, anterior, $B$, middle, $C$, posterior fossa of skull. 
dal and the internal carotid ; the posterior, by the ascending pharyngeal, occipital, and vertebral; the small meningeal is supplied to the Gasserian ganglion and parts of dura adjacent thereto; it enters the skull through the foramen ovale.

Structures Passing Througl the Sphenoidal Fissure.-The third, fourth, sixth, and ophthalmic branch of the fifth cranial nerves, the sympathetic nerve, the orbital branch of the middle meningeal artery and a recurrent branch from the lachrymal, and the ophthalmic vein.

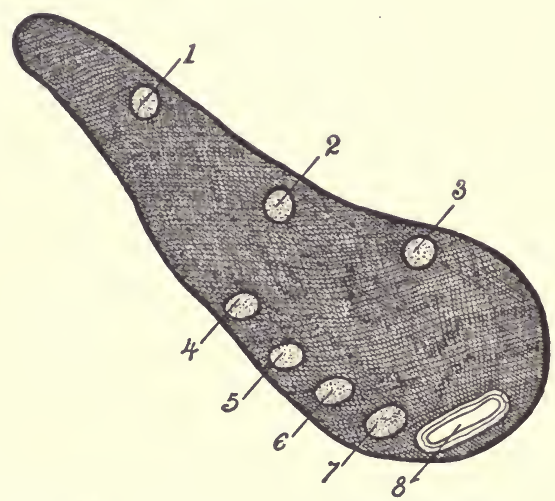

Diag. 4. A Diagram of the Sphenoidal Fissure. (I. S. H.)-I, Lachrymal nerve. 2, Frontal nerve. 3, Fourth cranial nerve. 4, Superior division of third cranial nerve. 5, Nasal nerve. 6, Inferior division of third cranial nerve. 7 , Sixth cranial nerve. 8 , Ophthalmic vein.

If great care is used the great petrosal nerve may be found extending from the hiatus Fallopii (see a dry skull) forward and inward, to disappear beneath the Gasserian ganglion. See Facial Nerve.

The Gasserian ganglion and beginning of the trunks of the three divisions of the fifth cranial nerve. Figs. Io and II. 


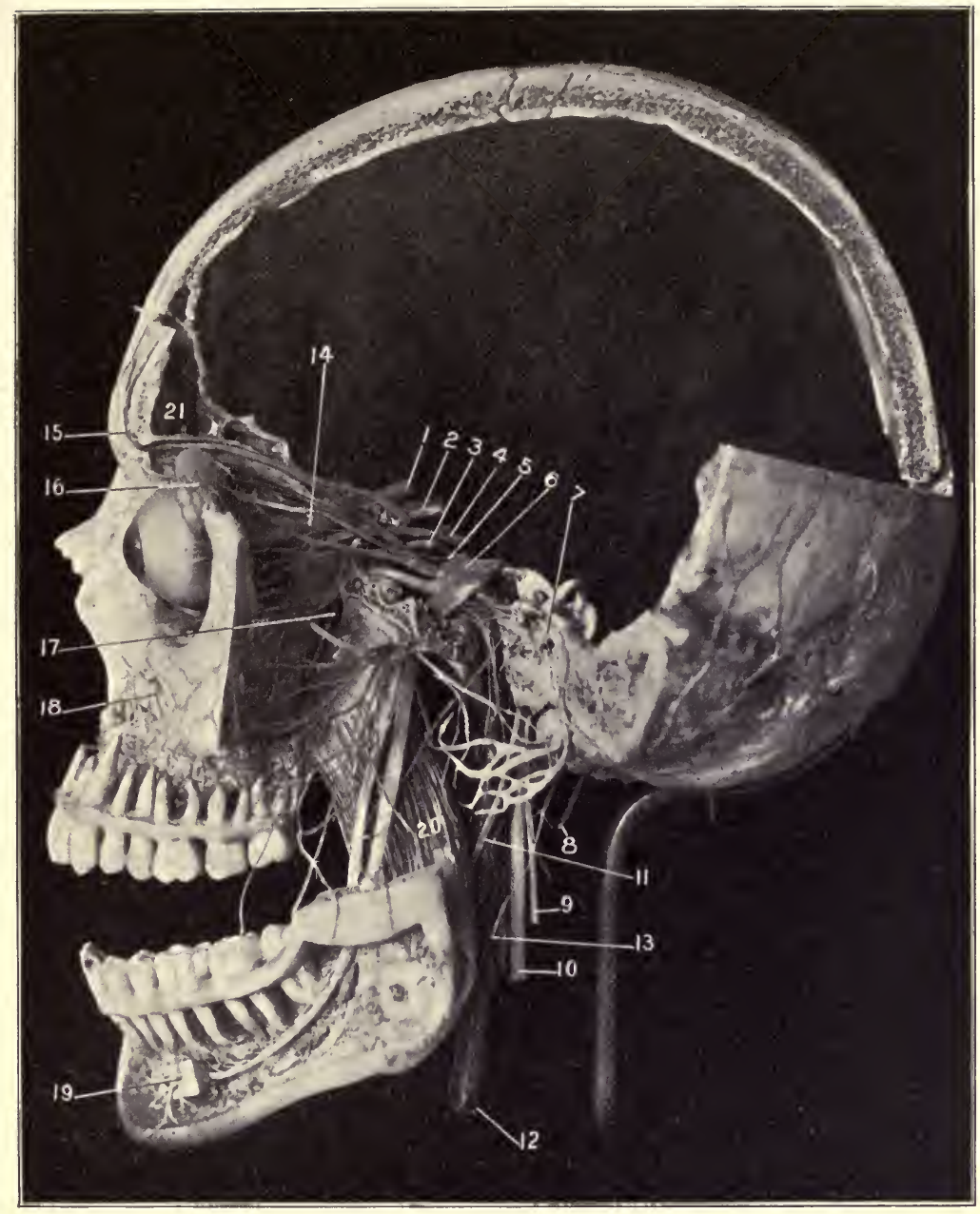

(From a preparation in the Museum of the University Medical College.)

Fig. II. The Course, Relations, and Distribution of the Cranial Nerves.I, Optic nerve. 2, The internal carotid artery. 3, The sixth cranial nerve. 4, Third cranial nerve. 5, Fourth cranial nerve. 6, Fifth cranial nerve, with the Gasserian ganglion. Its divisions into the ophthalmic, superior maxillary, and inferior maxillary branches is clearly shown. The course of these branches with their terminal divisions or distributions can easily be traced. To indicate each small branch on the photograph would ruin it. 7 , The facial nerve. The geniculate ganglion and branches, the cliorda tympani (passing downward and forward to join the gustatory nerve), and the terminal divisions and their branches are all shown. 8, Spinal accessory passing upward to the foramen magnum. 9, Same nerve in the neck. Io, Pneumogastric nerve. II, Glosso-pharyngeal nerve. I2, Internal carotid artery. Notice the various sympathetic plexuses upon it. 13, Superior cervical ganglion. 14, Ophthalmic ganglion. 15, Supra-orbital nerve. I6, Lachrymal gland. 17, Meckel's ganglion. I8, Infra-orbital nerve. 19, Mental nerve. 20, Internal pterygoid muscle. 2I, Frontal sinus. The student is urged to study the relations within the orbit and the three great trunks of the fifth nerve. 
The Gasserian ganglion is developed upon the sensory portion of the fifth nerve, and lies in a shallow depression upon the antero-superior surface of the apex of the petrous portion of the temporal bone. The ganglion receives sympathetic filaments from the carotid plexus.

From the anterior margin of the ganglion three trunks composed of sensory fibres are given off. The upper one is the ophthalmic branch, which traverses the cavernous sinus and takes its exit from the skull through the sphenoidal fissure, the middle is the superior maxillary, which leaves through the foramen rotundum, and the third branch is the inferior maxillary, which, with the motor root, descends through the foramen ovale; these two, being joined external to the skull, form the inferior maxillary nerve.

\section{DISSECTION OF THE ORBIT.}

If the skull is to be preserved intact the contents of the orbital cavity are to be removed as follows :-

Cut through the orbicularis palpebrarum around the margin of the orbit and open the socket of the eye.

Scratch in the fascia at the upper part until the levator palpebræ superioris is found. Also find the pulley of the superior oblique at the inner, superior, and anterior part of the cavity.

Below find the origin of the inferior oblique.

Now the pulley of the superior oblique and the origin of the inferior oblique must be divided, and then after separating the orbital fascia from the bony wall the arteries, nerves, veins, and muscles are to be divided as far posteriorly as possible, and the eye removed for further study.

If the brain has been removed as previously directed and the skull is not to be kept, the student should remove the upper wall of the orbit as follows :-

With a fine saw cut through the vertical plate of the frontal bone still left, over the outer and inner angles of the orbit. Break this piece of bone off. Separate the orbital fascia from the roof of the orbit, marking the location of the pulley of the superior oblique, as far posterior as the sphenoidal fissure. With a pair of bone cutters or a chisel cut the roof of the orbit away, leaving the pulley of the superior oblique in place.

Remove the presenting portion of the orbital fascia, and carefully pick away the fat which covers the several structures pertaining to the eye. 


\section{The Orbital Fascia, or Periosteum.}

This lines the interior of the bones composing the orbit, is continuous with the dura through the optic foramen and the sphenoidal fissure, forms the capsule of the lachrymal gland, the lining for the lachrymal canal, and the pulley for the superior oblique muscle, at the anterior margin of the orbit becomes continuous with the periosteum covering the exterior of the bones, and also sends to the eye-lids a fibrous expansion-the palpebral fascia.

\section{The Muscular Fasciæ.}

This consists of the connective tissue envelopes for the muscles and the eye-ball anterior to the insertion of the recti and oblique muscles, from where it is reflected on to the eyelids and orbital margin. This layer also forms the covering for the nerves and vessels.

\section{Tenon's Capsule.}

This is the connective sheath for the optic nerve and the posterior two-thirds of the eye-ball. Anteriorly it passes into the fasciæ for the muscles, the sclerotic coat, and ocular conjunctiva of the eye ; posteriorly it blends with the orbital fascia.

The Frontal Nerve. Diag. 4. Fig. I I.

This enters the orbit just external to the fourth nerve, crosses the levator palpebræ, and at the middle of the orbit divides into the supra-orbital and supratrochlear nerves.

The former leaves the orbit through the supra-orbital foramen or notch, and the latter, after passing above the pulley of the superior oblique, leaves at the inner angle of the orbit. For the termination distribution of these nerves see page 25 . 
The Lachrymal Gland, Nerve, and Artery. Fig. I I.

The lachrymal gland is a small gland lying at the anterior, outer, and upper part of the socket of the eye. Its capsule is formed by an extension from the orbital fascia (see above), by which the gland is retained in place.

The several small ducts open at the outer, upper part of the conjunctiva of the eye.

The lachrymal nerve enters the orbit through the external angle of the sphenoidal fissure. It is accompanied by the lachrymal artery from the ophthalmic, and both run along the upper border of the external rectus muscle to the lachrymal gland.

\section{Levator Palpebræ Superioris.}

Origin.-Above and in front of the optic foramen.

Insertion.-Into the tarsus of the upper eye-lid.

Nerie Supply.-Third cranial nerve.

Action.-To raise the upper eye-lid.

\section{Superior Oblique.}

Origin.-In front of the inner margin of the optic foramen. Insertion.-Into the outer surface of the eye-ball.

Nerie Supply.-The fourth cranial nerve.

Action.-Alone, to move the eye downward and outward. The pulley for the superior oblique is a ring of fibrous tissue attached to the anterior, inner, and superior portion of the orbit cavity through which passes the tendon of the superior oblique muscle.

The Fourth Cranial Nerve. Fig. I I. Diags. 3 and 4.

This comes through the sphenoidal fissure, lying at the inner and superior angle, and proceeds to the superior oblique, which it supplies. In its course it crosses above the origin of the levator palpebræ. 


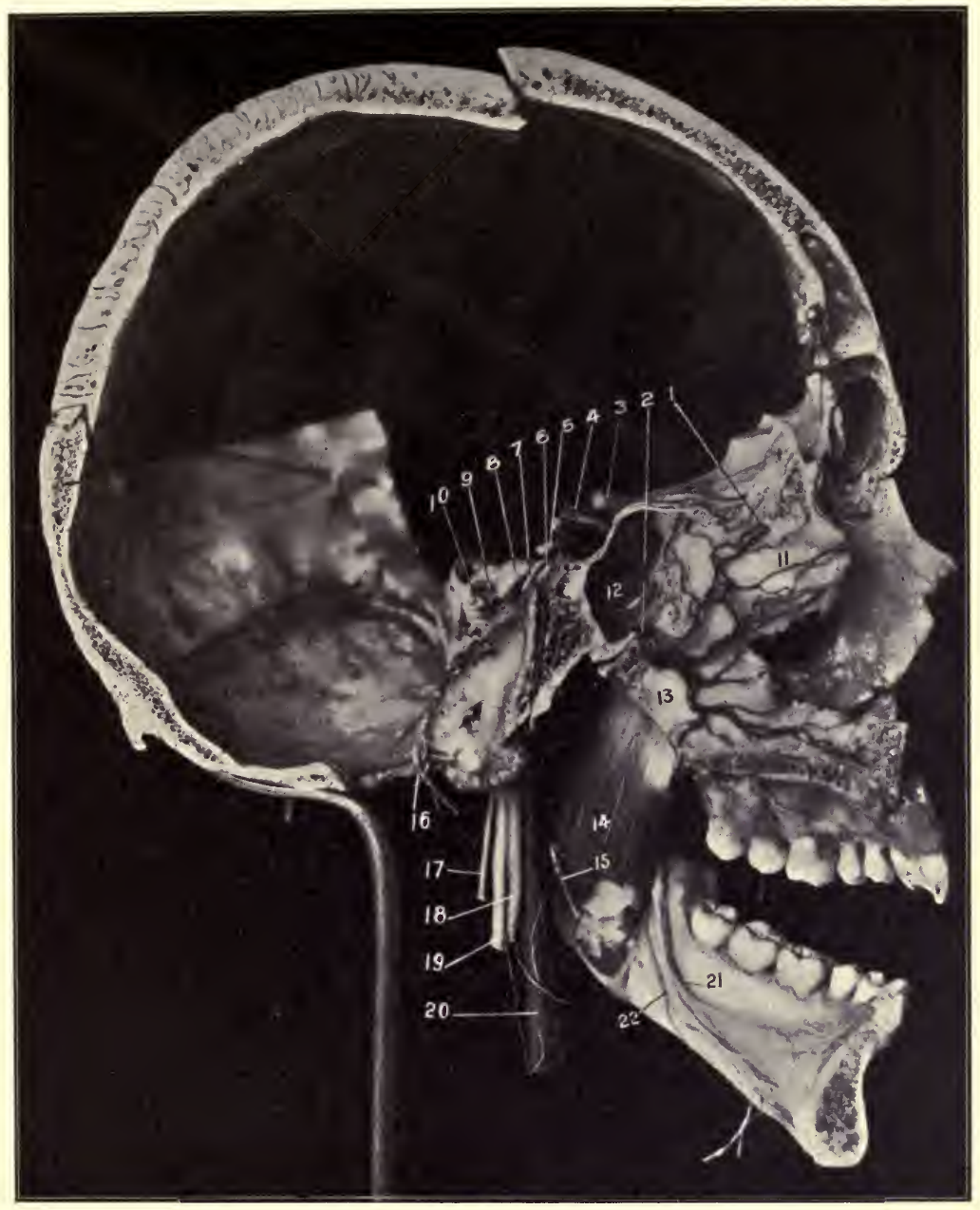

(From a preparation in the Museum of the University Medical College.)

Fig. 12.-I, Nasal branch of the anterior ethmoidal artery and vein. 2, Naso-palatine (septal) branch of the internal maxillary artery and vein. 3, Optic nerve. 4, Interilal carotid artery. 5, Third nerve. 6, Sixth nerve. 7, Fourth nerve. 8, Fifth nerve. 9, Seventh nerve. 10, Eighth nerve. I1, Vertical plate of ethmoid bone. 12, Sphenoidal sinus. 13, Vomer. 14, Internal pterygoid muscle. 15, Glosso-pharyngeal nerve. 16, Spinal portion of spinal accessory nerve. The nerve is seen passing up to the jugular foramen, into which are also passing the glosso-pharyngeal and pneumogastric nerves. 17, External portion of spinal accessory. 18, Cervical sympathetic and ganglion. 19, Pneumogastric nerve. 20, Internal carotid artery. 21, Gustatory nerve. 22, Mylo-hyoid nerve. 


\section{DISSECTION.}

Divide the levator palpebræ, superior oblique muscles, and frontal nerve.

\section{Superior Rectus.}

Origin.-From the upper margin of the optic foramen. Insertion.-Into the upper surface of the sclerotic coat just behind the cornea.

Nerve Supply.-The third cranial nerve.

Action.-To draw the cornea upward, also draw the cornea inward and slightly rotate it.

\section{DISSECTION.}

Remove the lachrymal gland, with its nerve and artery, and turn them outward. Divide the superior rectus. Clean the external and internal recti, the ophthalmic artery, vein, nasal nerve, and their branches. Be very careful to dissect out the ophthalmic ganglion which lies upon the outer and upper part of the optic nerve. Save the roots which pass to the ganglion and the ciliary nerves which pass forward to the eye. Clean the optic nerve from the foramen to the eye-ball.

\section{External and Internal Recti.}

The External.

Origin.-By two heads. The upper one from the outer margin of the optic foramen, the lower one from the lower margin of the sphenoidal fissure and the ligament of Zinn.

The Ligament of Zinn is a tendon attached around the margin of the optic foramen, except at its upper and outer part, and gives origin to the external, inferior, and internal recti.

Between the two heads of the external rectus pass the third, nasal branch of the fifth, the sixth cranial nerves, and the ophthalmic vein.

The Internal.

Origin.-From the inner side of the optic foramen. 
Insertion.-The two recti are inserted into the sclerotic of the eye-ball about a fourth of an inch behind the corneosclerotic junction.

Nerve Supply.-The external rectus by the sixth cranial nerve, the other by the third.

Action.-The external and internal recti muscles move the eye-ball outward or inward without any rotation.

\section{The Ophthalmic Artery and Branches.}

The ophthalmic is a large branch from the front of the internal carotid as it is turning upward to the brain through the cavernous sinus.

The artery enters the orbit through the optic foramen, with the optic nerve lying external and inferior to the nerve.

The artery runs forward in a tortuous course above the optic nerve and along the inner side of the orbit to the front of the same, where it divides into the nasal and frontal branches.

Its branches are the lachrymal, to the lachrymal gland; the supra-orbital, which accompanies the nerve of the same name through the supra-orbital notch or foramen to the front of the forehead (see page 26); the central artery of the retina, which penetrates the optic nerve one-fourth of an inch behind the eye-ball and runs within that nerve to the retina; the muscular, to the muscles of the eye; the ciliary arteries, grouped in three sets, the short posterior (five or six), the long posterior (two), and the anterior ciliary, from the surrounding muscular branches and lachrymal artery ; all these pass to the eye, the posterior to its fundus and the anterior along the tendons of the muscles to the middle zone of the eye ; the posterior ethmoidal, which leaves the orbit through the posterior ethmoidal 
foramen and breaks up into branches to the ethmoidal cells, the dura, and the interior of the nose; the anterior ethmoidal artery takes an identical course with the nasal nerve through the anterior ethmoidal foramen, cranial cavity, and nasal slit into the nasal cavity; its branches are ethmoidal, meningeal, nasal, and some small cutaneous twigs; the palpebral arteries, to supply the upper and lower eye-lids; lastly, the terminal branches, or the frontal and nasal. The frontal turns upward at the inner margin of the supraorbital arch to supply the front of the forehead between the median line and the supra-orbital artery. (See page 26.) The supratrochlear nerve and frontal artery run together. The nasal artery runs downward along the inner part of the eye to the side of the nose, where it anastomoses with the angular branch of the facial. It gives off a small transverse branch which, with a similar one from the other side, forms an arch over the front of the base of the nose.

\section{The Ophthalmic Veins.}

The superior is formed at the front of the orbit by the junction of the veins which accompany the frontal and supraorbital arteries. It passes backward above the optic nerve along with the ophthalmic artery to the sphenoidal fissure, through which it passes to empty into the cavernous sinus.

The inferior vein begins at the front of the orbit also, takes a course backward below the optic nerve, and at the back of the orbit empties into the superior ophthalmic vein or separately into the cavernous sinus. The inferior communicates with the pterygoid plexus through the sphenomaxillary fissure.

The Ophthalmic Nerve. Fig. I I. Diags. 3 and 4.

This is the first or superior division of the fifth cranial nerve. It is a sensory nerve solely. It enters the orbital 


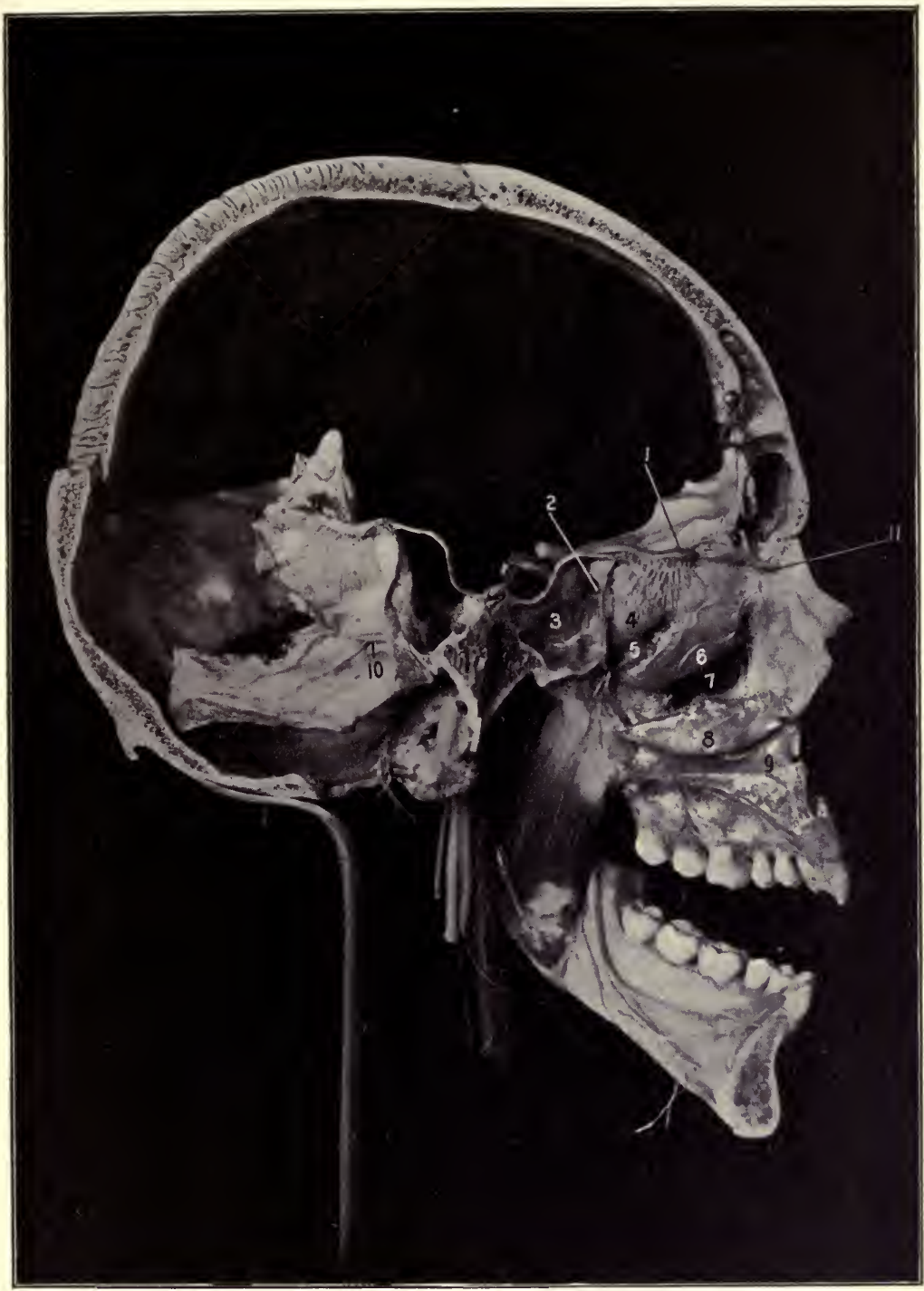

(From a preparation in the Museum of the University Medical College.)

Fig. 13. The Vertical Plate of the Ethmoid and the Vomer Turned Back so as to Show the Nasal Cavities and THEIR Nerve SuPPly.-I, Olfactory bulb, nerves, and tract. 2, Opening of sphenoidal sinus (No. 3) into the superior meatus. 4, Superior turbinated bone. 5, Superior meatus. 6, Middle turbinated bone. 7, Middle meatus. Shows the opening into the antrum of Highmore. 8, Inferior turbinated bone. 9. Inferior meatus. Io, Naso-palatine (septal) branch of Meckel's ganglion. II, Nasal nerve. 
cavity through the sphenoidal fissure in three divisions, the frontal, lachrymal, and the nasal.

The frontal and lachrymal nerves enter above the rectus externus, the nasal between the two heads of that muscle.

The frontal and lachrymal branches have already been given above. (Pages 49 and 50.)

The Nasal Nerve. Fig. I 3. Diag. 4.

This nerve runs forward and inward between the rectus superior and the optic nerve to the inner wall of the orbit, where it enters the anterior ethmoidal foramen, passes through it into the cranial cavity, runs forward on the outer border of the cribriform plate of the ethmoid bone, leaves the cranium by traversing the nasal slit at the side of the crista galli, and appears in the nasal fossa, where it terminates in three branches.

In the orbit the nerve gives off the following branches:-

Between the two heads of the rectus externus, the long root to the ophthalmic ganglion. A very slender filament about half an inch long.

Two long ciliary filaments to the eye. These run along the inner side of the optic nerve.

The infratrochlear nerve is the last branch given off just before the nerve leaves the orbit. It passes forward beneath the pulley for the superior oblique and supplies the integument about the inner part of the eye and upper part of the nose, also the mucous membrane of the inner part of the eye, lachrymal sac, and caruncle.

The remaining branches will be given when describing the nasal fossæ.

The Ophthalmic, or Lenticular Ganglion. Fig. I I.

This is a ganglion developed in connection with the ophthalmic division of the fifth nerve. It is about as large as 
the head of a small pin. Is situated at the outer and upper part of the optic nerve at about its middle, between the nerve and the external rectus and one-fourth of an inch in front of the sphenoidal fissure.

The ganglion receives its sensory root from the nasal nerve (the long root), the motor root, from the third cranial nerve (the short root), and its sympathetic root from the plexus on the carotid artery within the cavernous sinus.

Eight or ten minute filaments pass forward from the ganglion-the short ciliary nerves-to supply the eye-ball.

The Optic Nerve. Figs. IO, I I, I 2.

The optic nerve enters the orbit through the optic foramen with the ophthalmic artery, as already described, lying above and to the inside of the artery. It passes forward to enter the back of the eye-ball.

The optic nerve is the central structure passing to the eye. It is surrounded by the ocular nerves, arteries, veins, and muscles, and a packing of adipose tissue between them all to hold them in their proper places.

The Sixth Cranial Nerve. Figs. IO, I I, I 2.

It comes into the orbital cavity through the sphenoidal fissure and between the two heads of the external rectus muscle, to which it is distributed. Diags. 3 and 4 .

\section{DISSECTION.}

Complete the dissection of the eye cavity by dividing the internal and external oblique muscles and raising the eye-ball first to one side and then to the other.

The Third Cranial Nerve. Figs. IO, I I, I 2. Diags. 3 and 4 .

The motor oculi enters the orbit through the inner part of the sphenoidal fissure in the shape of two branches, 
which are separated by the nasal nerve. These branches run forward between the two heads of the external rectus.

The superior division of the third nerve. This is the smallest; it passes forward and inward over the optic nerve to the rectus superior and levator palpebræ superioris. The lower division is the larger and divides into three branches, one to the rectus internus, another to the rectus inferior, the third to the obliquus inferior. From this last branch the motor root of the ophthalmic ganglion arises.

Rectus Inferior.

Origin.-From the lower margin of the optic foramen.

Insertion. - Into the lower part of the sclerotic coat of the eye just behind the corneal margin.

Nerve Supply.-The inferior branch of the third nerve.

Action.-To depress and at the same time slightly rotate and draw the cornea inward.

\section{Obliquus Inferior.}

Origin.-From the front of the orbital cavity at the junction of its lower and inner surfaces, just outside the opening of the nasal duct.

Insertion.-Into the outer posterior part of the eye-ball under the external rectus.

Nerve Supply. - The third or motor-oculi through the inferior branch.

Action.-To rotate the cornea outward, and to turn the eye-ball slightly upward and outward.

\section{DISSECTION.}

If the skull has been opened, the brain removed, the interior of the base of the skull dissected, and the orbit finished, then take up the dissection of the face.

Nothing upon the face has been dissected except the orbicularis palpebrarum. The structures are covered by a layer of fascia and the upward projection of the platysma. 
The fasciæ and platysma and its facial portion, the risorius, are all to be studied and then removed, and the facial muscles above then below the mouth are to be cleaned. Along with the dissection of the muscles will proceed the cleaning of the nerves, veins (facial especially), and the arteries.

\section{Risorius.}

This is a thin sheet of loosely joined muscular fibres, an extension and derivation of the platysma, which extends from the masseteric and parotid fascix to the angle of the mouth.

\section{The Masseteric and Parotid Fasciæ.}

The upward continuation of the superficial layer of the deep cervical fascia from the neck over the masseter muscle and parotid gland is called the masseteric and parotid fascia respectively. See page 138 .

The former is attached, above, to the lower border of the zygomatic arch, and below, to the lower and posterior borders of the lower jaw. An extension of this fascia from the angle of the jaw to the styloid process is called the stylomaxillary ligament. It serves to separate the parotid from the submaxillary gland.

The latter (parotid) fascia covers in the parotid gland. In front it is continuous with the masseteric fascia; above, attached to the zygomatic arch ; behind, to the front of the mastoid process, and below, extends into the cervical fascia.

Zygomaticus Major. Fig. 3.

Origin.-From the outer surface of the malar bone.

Insertion.-Into the orbicularis oris, levator anguli oris, and skin at the angle of the mouth.

Nerve Supply. - The seventh or facial by its infra-orbital branch.

Action.-To raise and retract the angle of the mouth.

Zygomaticus Minor.

Origin.-The lower part of the anterior surface of the malar bone. 
Insertion.-Into the outer margin of the levator labii superioris.

Nerve Supply.-Same as above.

Action.-To raise the outer part of the upper lip.

Levator Labii Superioris.

Origin.-From the superior maxillary bone just below the orbit.

Insertion.-Into the orbicularis oris and skin of the upper lip.

Nerve Supply._-Infra-orbital branch of the facial.

Action. - To elevate the upper lip.

\section{Levator Labii Superioris Alæquæ Nasi.}

Origin.-From the nasal process of the superior maxilla.

Insertion.-An internal fasciculus into the wing of the nose, and by the outer portion into the orbicularis oris and the skin of the upper lip.

Nerve Supply. - The infra-orbital branch of the facial.

Action.-To raise the wing of the nose and the upper lip.

Levator Anguli Oris. Fig. 3.

Origin.-From the canine fossa of the superior maxillary bone, below the infra-orbital foramen.

Insertion.- Into the orbicularis oris at the angle of the mouth.

Nerve Supply.-The facial by the infra-orbital branch. Action.-To raise the angle of the mouth.

\section{DISSECTION.}

The parotid gland and masseter muscle are covered by a dense layer of fascia, the parotid and masseteric, a prolongation of the superficial layer of the cervical fascia upward to the zygomatic arch. Be very careful in removing this fascia to leave the branches of the facial nerve, the auriculo-temporal nerve, the transverse facial artery, Stenson's duct, and the detached portion of the parotid gland (glandula socia parotidis) uninjured upon the masseter muscle. 
The branches of the temporofacial division of the facial nerve are the temporal, malar, and infra-orbital. For these see below.

Two branches of the cervicofacial division are found upon the face; these are the buccal and the supramaxillary. These structures are to be carefully dissected out.

To find the branches of the facial nerve look for them in the region indicated by their names; it is usually best to begin with the infra-orbital, and after one has been found to trace it forward to its distribution and backward to its source. In tracing any nerve of the facial backward other branches will be found and the dissection carried on step by step until all the branches are cleaned.

The Facial Nerve. Figs. 2, 3, IO, I I, and I 4.

This leaves the skull by the stylomastoid foramen, gives off small branches (posterior auricular, nerve to the posterior belly of the digastric, and the nerve to the stylohyoid muscle), then divides into the temporofacial and the cervicofacial trunks.

The temporofacial gives off the temporal which passes upward through the parotid in front of the temporal artery, where it divides into several branches, to the temporal region; it communicates with the auriculo-temporal nerve behind the temporal artery; the malar branch runs upward and forward over the malar bone; the infra-orbital branch extends forward between the zygoma and Stenson's duct, then under the zygomaticus major and minor to supply the superior labial muscles and enter into a plexus with the infra-orbital of the fifth. See page $15 \mathrm{I}$.

The cervicofacial division gives off the buccal to the buccinator (the real motor supply); the supramaxillary above, and the inframaxillary below the lower jaw, the former supplying the inferior labial muscles and communicating with the mental nerve, the latter supplying the platysma and joining with the superficial cervical nerve.

Besides these communications mentioned the branches of both divisions communicate with the branch above and 
below itself, thus forming a plexus over the entire side of the face.

All these branches in the parotid gland lie anterior or superficial to the external carotid artery and the temporomaxillary vein.

The Temporal Artery. Figs. 2, 3.

Branches of the trunk of the temporal artery. The anterior auricular passes to the top and front of the ear; the orbital is a small branch which runs forward to the orbit between the two layers of the temporal fascia (see page 30); the middle temporal perforates the temporal fascia and muscle, just above the zygomatic arch, to reach the under surface of the temporal muscle and ramify in the fossa there; and the transverse facial, which is seen to run forward from the parotid gland, just below the zygomatic arch, between the arch and Stenson's duct, to anastomose with the infra-orbital branch of the internal maxillary in front, and the muscular branches of the facial below; besides these there are small twigs given to the parotid gland and temporomaxillary articulation.

The Facial Vein. Figs. 3, I 5.

This starts at the inner angle of the eye as the continuation of the angular vein. It takes a direct course downward and outward under the greater and lesser zygomatic muscles and platysma to the facial notch in the lower jaw, where it lies posterior to the artery. In its course it receives branches corresponding to the branches of the facial artery, and communicates with the infra-orbital vein. For continuation in neck, see page 74 .

Depressor Anguli Oris.

Origin.-From the external oblique line of the inferior maxillary bone. 
Insertion.-Into the angle of the mouth, blending with the other muscles at that point.

Nerve Supply. - The facial through its supramaxillary branch.

Action.-To depress and retract the angle of the mouth.

\section{Depressor Labii Inferioris.}

Origin.-From the upper border of the external oblique line of the inferior maxilla, from near the symphysis to beyond the mental foramen.

Insertion.-Into the orbicularis oris and the skin of the lower lip.

Nerve Supply.-The supramaxillary branch of the facial. Action.-To depress the lower lip.

\section{Levator Labii Inferioris.}

Origin.-From the incisive fossa of the lower jaw.

Insertion:-Into the skin of the chin.

Nerve Supply.-The supramaxillary branch of the facial. Action.-To raise the skin over the point of the chin and protrude the lower lip.

Orbicularis Oris. Fig. 3 .

This is the sphincter muscle of the mouth, and is arranged in two portions, an internal or labial, and an external or facial.

The labial portion has no bony attachments. The facial is attached by slender slips to the septum of the nose, and the incisive fossæ of the superior and inferior maxillary bones, besides blending with the muscles passing to the mouth.

Nerve Supply.-The facial through its buccal and supramaxillary branches.

Action.-To close the lips, vertically and transversely; 
the facial portion will pout the lips, the labial will press them against the teeth.

The Facial Artery. Figs. 2, 3, 14.

On the face. The facial artery comes into the face from the neck over the lower border of the inferior maxillary bone, lying in the facial notch just at the junction of the ramus and body, at the anterior border of the masseter muscle.

It takes a very tortuous course around the angle of the mouth and along the side of the nose to terminate at the inner angle of the eye.

In its course it passes under the platysma, zygomatic major and minor, the levator labii superioris, and levator labii superioris alæquæ nasi.

The facial vein lies behind the artery.

Branches of the Facial Artery. On the Face.

(I) Muscular, to the masseter and buccinator muscles. The masseteric branches anastomose with the transverse facial and muscular branches from the internal maxillary. The buccal, with the same, and also with the infra-orbital, from the internal maxillary. (2) The inferior labial. Its course is forward under the depressor anguli oris and midway between the border of the lip and the chin, to supply the muscles of the lower lip. It anastomoses with the facial branches above and below itself and across the median line.

All the anterior facial branches anastomose across the median line with corresponding arteries from the opposite facial, as well as with the arteries next above and below. Exception: the coronary arteries do not anastomose with each other, except at the angle of the mouth. (3) The inferior coronary. Extends forward beneath the depressor 
anguli oris to the lower lip, and runs inward between the mucous membrane and the orbicularis oris. It anastomoses with the opposite coronary artery and the arteries below. (4) The superior coronary. Takes a similar course along the upper lip. It supplies the upper lip and gives off the artery of the septum of the nose. (5) The lateral nasal artery. This is a small (sometimes a large) branch to the side of the nose. It anastomoses with the nasal from the ophthalmic, and with the facial branches adjacent. (6) The angular artery. The last portion of the facial along the side of the nose. It terminates at the inner angle of the eye by anastomosing with the nasal branch of the ophthalmic.

The facial anastomoses on the face.

As already stated, the facial branches toward the middle line freely communicate with each other. They also anastomose with the artery next above and below (excepting the coronary arteries do not anastomose with each other). The other connections are, with the temporal, by the masseteric and buccal branches uniting with the transverse facial; with the internal maxillary, through the inferior labial and coronary joining with the mental (of the inferior dental), by the muscular branches to the masseter and buccinator from both arteries, and through the infra-orbital; and with the ophthalmic by its nasal branch anastomosing with the artery of the septum, the lateral nasal, and angular branches of the facial.

\section{DISSECTION.}

Cut the parotid gland away piecemeal, finish the facial nerve; the upper portion of the external carotid artery and its terminal branches, the temporal and internal maxillary arteries; the transverse facial artery and posterior auricular arteries; and the temporomaxillary vein or sinus. 
The Parotid Gland. Figs. 3, I4.

This is a large salivary gland, which fills up the space below the zygoma, and between the ramus of the lower jaw and the auditory and mastoid processes, and extends downward as low as the level of the angle of the jaw.

Its lobes extend forward (pterygoid), upward (glenoid), and inward (carotid), between the surrounding structures. One portion usually detached and lying upon the masseter muscle is the socia parotidis, which connects with the parotid duct by a separate opening.

The duct of the parotid gland is Stenson's. It passes forward across the masseter half an inch below the zygoma, pierces the buccinator muscle, and opens into the mouth opposite the second upper molar tooth. Figs. 2, 3, 4.

The gland is covered by the extension upward of the cervical fascia called the parotid fascia. See page 59.

It is separated from the submaxillary gland by the stylomaxillary ligament. Page 59.

Within the parotid are found the facial nerve and its primary divisions and branches (forming the pes anserinus); the termination of the external carotid artery, its posterior auricular branch, the beginning of the internal maxillary and the temporal arteries; the transverse facial from the temporal, as also the glandular branches from these arteries to the parotid itself; and the temporomaxillary vein.

The arterial supply is from the arteries within the gland, the nerve supply from the seventh, great auricular, auriculotemporal, and sympathetic plexus on the carotid artery.

The veins open into the temporomaxillary sinus.

The lymphatics empty into the superficial and deep lymphatic glands of the neck. 
Masseter. Figs. 2, 3, 4.

Origin.-The deep portion, from the inner surface of the zygomatic arch and from the posterior third of the lower border of the same; the superficial portion, from the lower border of the anterior two-thirds of the zygomatic arch and malar bone.

Insertion.-The deep portion, into the upper half of the ramus of the lower jaw and coronoid process; the superficial portion, into the lower half of the ramus extending as far as the angle.

Nerve Supply. - The inferior maxillary branch of the fifth cranial nerve through its masseteric branch.

Action.-To close the jaw ; and, by the superficial portion, to slightly protract it.

Buccinator. Figs. 3, 4 .

Origin.-From the pterygomaxillary ligament (a ligament extending from the hamular process of the internal plate of the pterygoid bone to the posterior extremity of the mylohyoid ridge of the inferior maxilla) and from the outer surface of the alveolar process of the superior and inferior maxillary bones opposite the molar teeth.

Insertion.-Into the orbicularis oris, some of the lower fibres passing into the upper lip, and some of the upper fibres into the lower lip.

Nerve Supply.-The seventh cranial nerve through its buccal branch. The buccal branch of the inferior maxillary nerve passes to the muscle also. See page i i 7 .

Action.-To retract the angles of the mouth, to flatten the cheeks, and expel the contents of the mouth, also to force the food between the teeth. 
The Mental, Infra-orbital and Supra-orbital Foramina. Figs. 3, 4, I I.

The mental foramen is in the lower jaw, in a line with the supra-orbital and infra-orbital foramina. Through it come the mental nerve and artery (branches from the inferior dental nerve and artery, and these arise in turn from the inferior maxillary nerve and internal maxillary artery).

The supra-orbital foramen transmits the artery, vein, and nerve of this name. The artery is a branch of the ophthalmic, the nerve of the frontal, which arises from the ophthalmic. The vein empties into the ophthalmic, an important connection to remember.

The infra-orbital foramen transmits the artery, vein, and nerve of the same name. The artery is a branch from the internal maxillary, the vein empties into the internal maxillary vein, and the nerve is the termination of the superior maxillary nerve.

These three foramina are in the same line, and the nerves which come through them are sensory branches of the three trunks of the fifth cranial nerve.

\section{THE NECK, Anterior.}

The Landmarks. Figs. I4, I 5.

The region of the neck is limited above by the lower jaw, mastoid process, and a line joining them, and the superior curved line of the occipital bone; below, by the top of the sternum and clavicle; laterally, by the linear elevation formed by the anterior borders of the trapezius muscles.

This area is divided in front by the median line into two symmetrical portions.

The above boundaries should be identified. 
In the median line recognize from above downward the (1) hyoid bone. (2) The thyroid cartilage and its forward projection, called Adam's apple, and the angle left between the alæ of the cartilage. (3) The cricoid cartilage, just below (and connected to the thyroid cartilage by a stout membrane; see page I46); the cricoid cartilage is in front of the fifth intervertebral disc. (4) The trachea, (5) obscured by the thyroid gland, which lies upon either side of, and is connected by its isthmus across the front of the trachea. (6) Lastly, the depression just above the sternum-fonticulus gutturis, - and between the anterior ends of the clavicles-the suprasternal, or interclavicular notch.

Laterally, from above downward, identify superior curved line of the occipital bone and the mastoid process, its base and tip; the sternomastoid muscle, which forms a broad elevation, extending from the mastoid process to the sternum and inner end of the clavicle (when it is put upon the stretch).

As the arteries (subclavian, outer portion, and the carotids) are injected, their position can be recognized by touch, and their course sketched upon the skin by an aniline pencil. The superficial jugular veins usually show through the integument, and their course can be similarly indicated.

The position of the sternoclavicular articulation should be carefully noted, as it marks so many important structures.

Consult page 262 for the distance to which the lung extends into the base of the neck.

\section{DISSECTION.}

Incisions.-1. Carry the median incision to the top of the sternum.

2. Make a transverse incision outward along the clavicle to the point of the shoulder. (If the upper extremity is being dissected this incision has already been made. See page 264.) 
3. Continue the incision from the lobe of the ear along the line of its posterior attachment to the head.

Reflect the integument from behind the ear, front, and side of the neck, so as to expose the anterior border of the trapezius muscle.

Platysma Myoides. Fig. I4.

Origin.-From the deep fascia covering the upper part of the pectoralis major, deltoid, and trapezius muscles, and slightly from the front of the upper surface of the clavicle. Insertion.-Into the anterior three-fourths of the lower border of the inferior maxilla (the fibres of the muscle crossing the median line at the point of the chin), and into the angle of the mouth.

Nerve Supply. - The superficial cervical, from the second and third cervical nerves, and the inframaxillary branch of the cervico-facial division of the seventh. The latter is the main supply.

Action.-To depress the lower jaw. This being fixed, they will flex the head and retract the angle of the mouth. If one acts it will have the same action on one side of the mouth, depress the jaw; if this is resisted, the head will be flexed laterally and the chin rotated to the same side.

The upper part of the muscle being the fixed part, it raises the skin over its origin, and in the neck tends to draw it away from the deeper parts.

\section{DISSECTION.}

After completing the platysma, begin at its origin (below) and carefully separate it from the deeper structures beneath. The nerve supply to the muscle will be found, and, after being recognized, may be divided and the muscle rolled up on to the lower part of the face.

The first layer of the cervical fascia is now exposed; the other layers will follow in order and should be identified as far as possible. In order to do this, consult the technical description of the fascia as found on page 136 . Its description here now would not be so easily understood as after the dissection of the neck has been completed. 


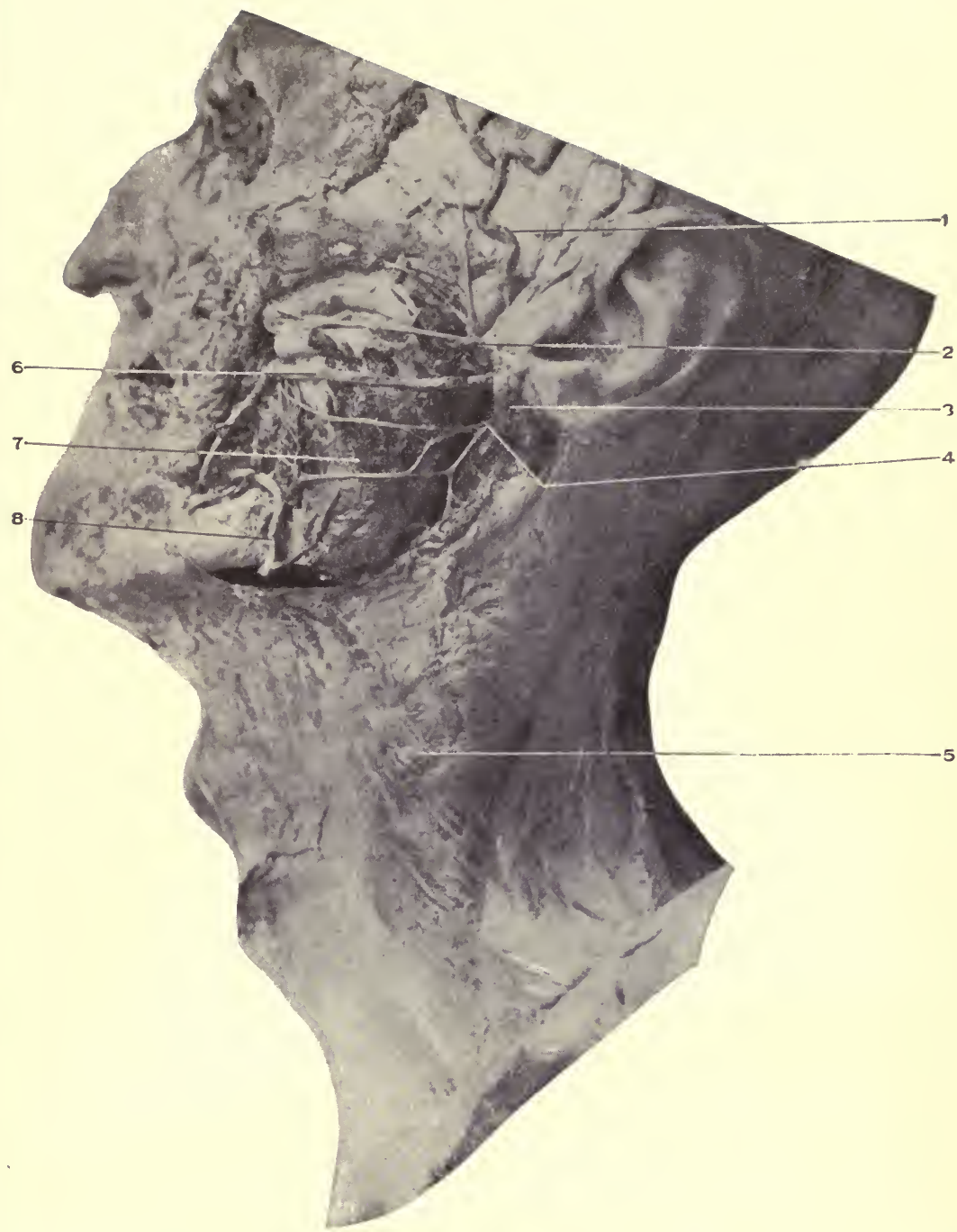

Fig. 14. Dissection OF Neck.

I, Temporal artery. Behind it is the auriculo-temporal nerve.

2, Temporo-facial division of the facial nerve and its three branches.

3 , Remains of the parotid gland.

4, Cervico-facial division of the facial nerve and its three branches.

5, Platysma myoides.

6. Stenson's duct.

7. Masseter muscle.

8, Facial artery and masseteric branch. 
The superficial veins (jugular) of the neck are to be dissected, their formation, course, and termination determined as far as possible.

The superficial nerves should be fixed in mind by consulting Fig. 16, before the dissection of the neck is attempted, and then traced out.

The Veins of the Neck. Fig. I 5. Diag. 5.

The temporal and internal maxillary veins unite opposite the neck of the lower jaw to form the temporomaxillary sinus (or vein).

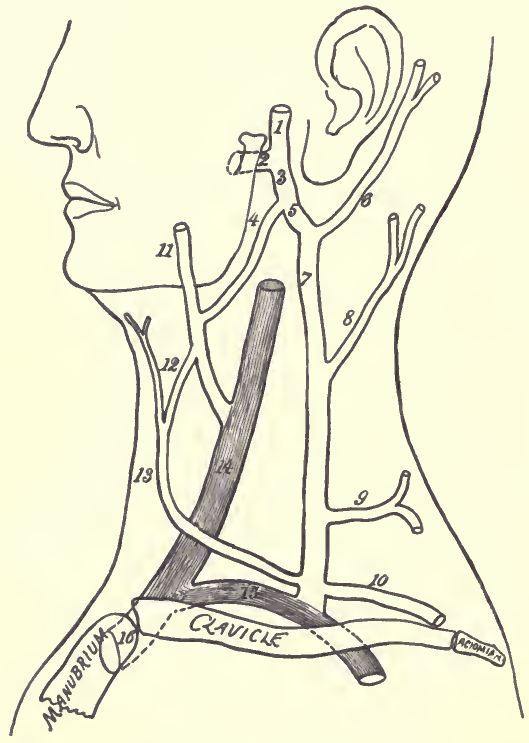

Diag. 5. The Veins of THE NECK.-I, Temporal. 2, Internal maxillary. 3, Temporomaxillary "sinus." 4, Anterior division of the temporomaxillary "sinus." 5, Posterior division of the temporomaxillary "sinus." 6, Posterior auricular. 7, External jugular. 8, Posterior external jugular. 9, Transverse cervical. Io, Suprascapular. II, Facial. I2, Communicating between facial and anterior jugular. I3, Anterior jugular. 14, Internal jugular. 15, Subclavian. 16, Innominate.

The temporomaxillary vein descends through the substance of the parotid gland, superficial to the external carotid, and at the angle of the jaw divides into an anterior and a posterior trunk. 


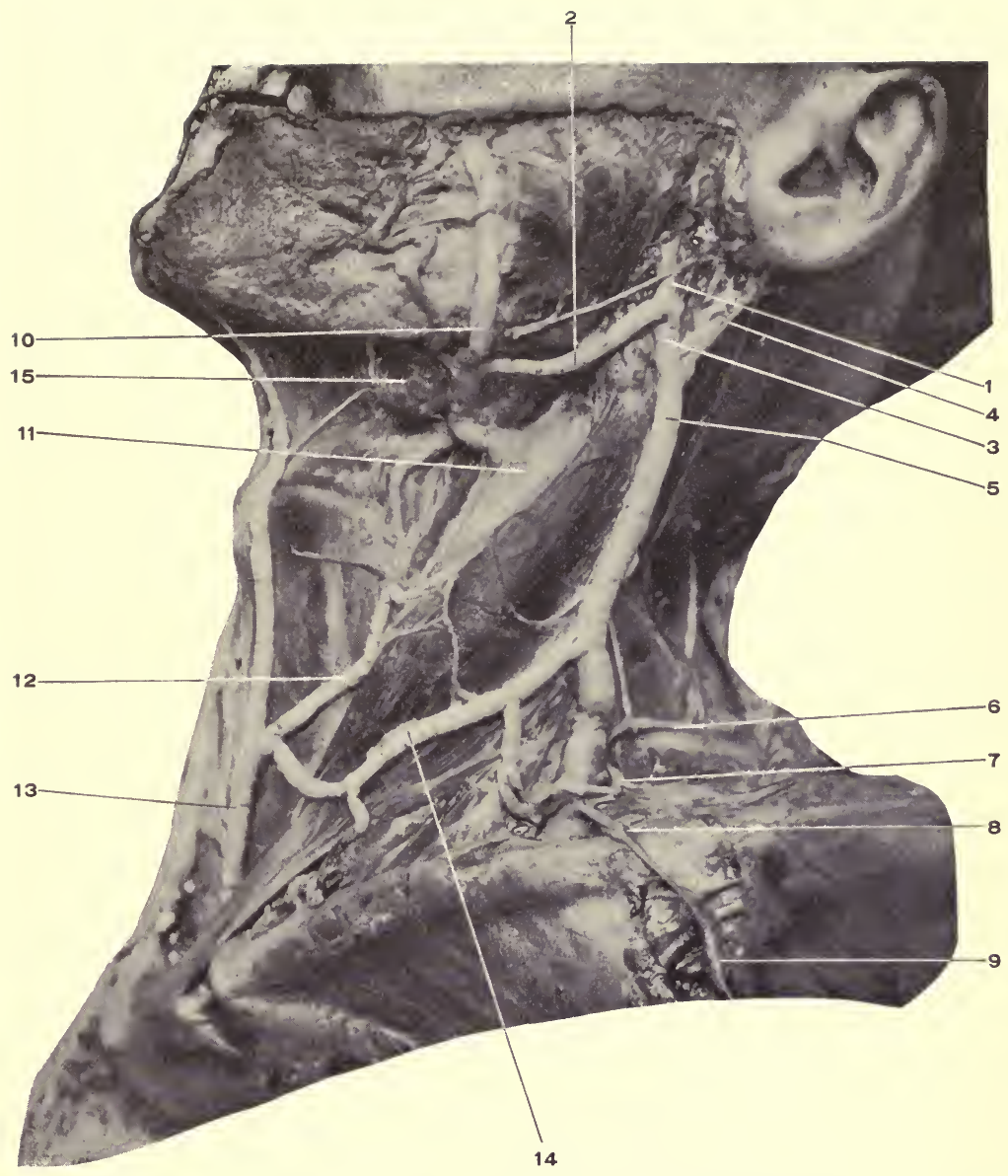

Fig. 15. Dissection of Veins of Neck.-I, Temporo-maxillary "sinus." 2, Anterior division of same. 3, Posterior division of same. 4, Posterior auricular vein. 5, External jugular. 6, Superficial cervical. 7, Suprascapular. 8, Connecting cephalic (9) with external jugular. 9. Cephalic. Io, Facial. II, Internal jugular. I2, Communicating between facial and, 13, The anterior jugular. 14, Communicating between external and anterior jugulars. 15, Submaxillary gland. 
The posterior branch is joined by the posterior auricular vein, and the trunk thus formed is the external jugular vein.

The external jugular descends vertically, crosses the sternomastoid (obliquely), pierces the deep cervical fascia behind the middle of the clavicle, and empties into the subclavian vein.

In its course it receives from the rear the posterior external jugular, the superficial transverse cervical, the suprascapular; from the front, a communicating vein from the facial, internal, and (sometimes) anterior jugular veins.

The Posterior External Jugular.-This vein drains the blood from the upper and back part of the neck. It opens into the external jugular at the posterior margin of the sternomastoid muscle.

The Anterior Jugular.-This is formed under the chin close to the median line by the union of several small veins. Its course is downward until near the sternal end of the clavicle, when it pierces the deep fascia, turns outward under the sternomastoid, and empties into the external jugular close to its termination, or into the subclavian vein.

It is connected by branches, varying in size, with the facial, external, and opposite anterior jugular veins.

The Facial Vein in the Neck.-Just below the neck this vein is joined by the anterior division of the temporomaxillary sinus; it gives off the anastomotic vein to the anterior jugular, and then empties into the internal jugular at the level of the hyoid bone.

The Superficial Nerves. Fig. I6. Diag. 6.

These are as follows :-

The inframaxillary branch from the cervicofacial division of the seventh; which passes downward and forward 
through the parotid gland (as already dissected), and then courses forward below the lower jaw to form under the platysma a loose plexus with the superficial cervical nerve.

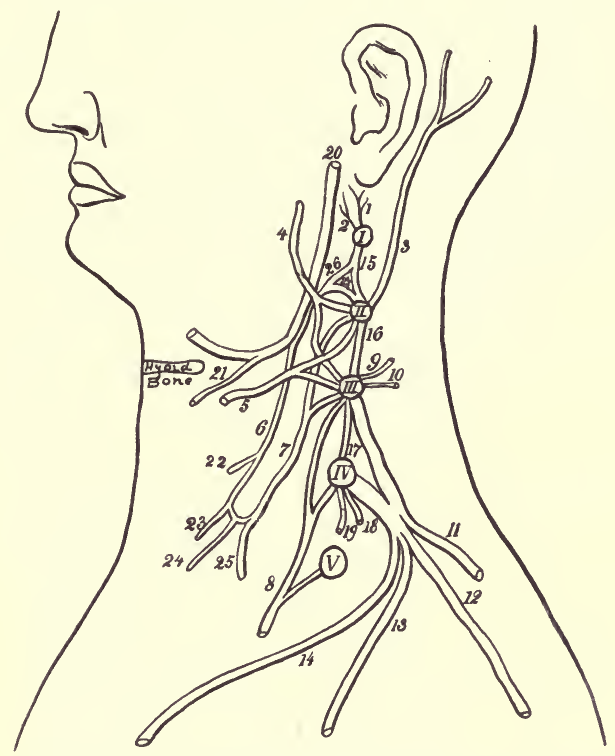

Diag. 6. Diagram of the Cervical Plexus. (I. $S . H)$.$-I , First cervical$ nerve. II, Second cervical nerve. III, Third cervical nerve. IV, Fourth cervical nerve. $V$. Fifth cervical nerve. I, Branch to rectus capitis lateralis. 2, Branch to rectus capitis anticus minor. 3, Lesser occipital. 4, Great auricular. 5, Superficial cervical. 6, Descendens hypoglossi. 7, Communicans hypoglossi. 8, Phrenic. 9, To scalenus medius. Io, To levator anguli scapulæ. II, To trapezius. I2, Acromial, 13, Clavicular, and 14, Sternal, cutaneous branches. 15, Cord joining first and second nerves. 16, Cord joining second and third nerves. 17, Cord joining third and fourth nerves. 18, To scalenus medius. 19, To levator anguli scapulæ. 20, Hypoglossal. 2I, Branch to thyrohyoid. 22, To anterior belly of the omohyoid. 23, To sternohyoid. 24 , To sternothyroid. 25, To posterior belly of the omohyoid. 26 , and 27 , The branches from the loop connecting the first and second cervical nerves which unite to form a cord that joins the hypoglossal nerve, being contained within its sheath, but leaves it again as the descendens hypoglossi.

The auricularis magnus, the small occipital, and the superficial cervical nerves are found at the posterior border 
of the sternomastoid muscle, just above its middle. The first passes upward diagonally across the sternomastoid to the region of the parotid gland, the second runs parallel with the posterior border of the sternomastoid to the side of the head, and the third crosses the muscle directly forward.

There are several cutaneous nerves which descend toward the chest.

They issue from the posterior margin of the sternomastoid also, and pass to the sternum (sternal), the clavicle (clavicular), and to the shoulder (acromial). For a full description of these several nerves, see the cervical plexus, page 89 .

Sternocleidomastoid. Fig. I6.

Origin.-The inner head, from the upper part of the manubrium; the outer head, from the upper surface of the inner third of the clavicle.

Insertion.-The mastoid process of the temporal, and the outer half of the superior curved line of the occipital bones.

Nerve Supply.-From the deeper branches of the second and third cervical nerves, and the spinal accessory, which passes through the muscle on its way to the trapezius.

Action.-Acting from below. Both muscles will flex the head and neck. One will flex the head laterally, and rotate the chin to the opposite side.

Acting from above. Will raise the clavicles and sternum, and so act in inspiration.

The spinal accessory and cervical branch to the trapezius muscle. These two nerves will be found as the fascia is cleaned out between the sternomastoid and the trapezius muscles. Figs. 16, I7.

The first issues from the sternomastoid muscle at its 


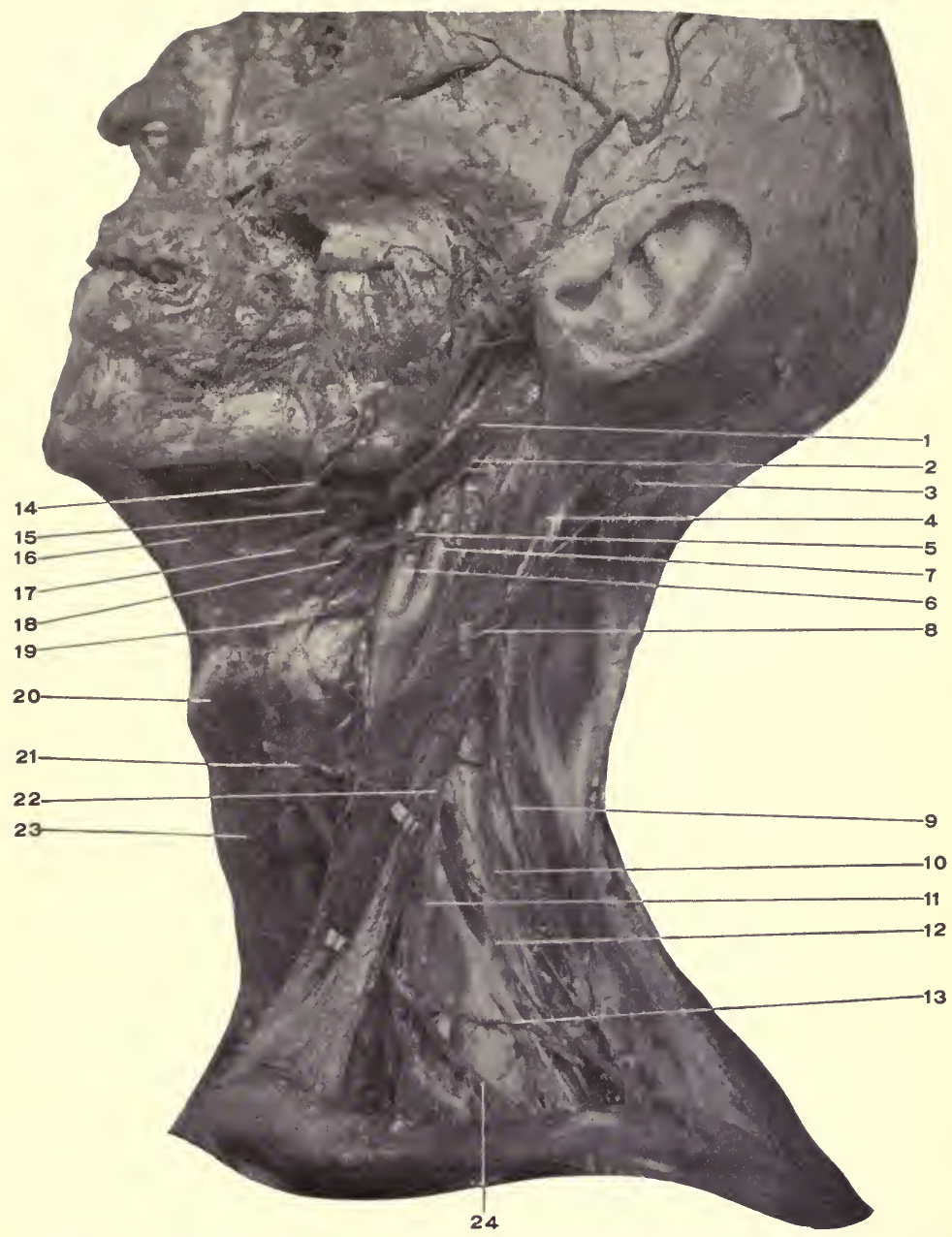

Fig. 16. Dissection of NEck.-[For the names of the structures shown on the face see preceding figures.] - I, Posterior belly of digastric. 2, Occipital artery. 3, Lesser occipital nerve. 4, Great auricular nerve. 5, Hypoglossal nerve. Note relation to occipital artery. 6, External carotid artery. 7, Internal carotid artery. Note their mutual relations and point of division of the common carotid. 8, Superficial cervical nerve. 9, Spinal accessory nerve. 10, Branch from cervical plexus to trapezius. II, Clavicular. 12, Acromial nerves. 13, Transverse cervical artery. 14, Facial artery and its submental branch. 15, Submaxillary gland. Most of it has been removed. 16, Anterior belly of the digastric, resting upon the mylo-hyoid muscle. I7, Tendon of the digastric. I8, Stylohyoid muscle. I9, Hyoid bone. 20, Thyroid cartilage. 21, Anterior belly of the omohyoid. 22, Sternal nerves. 23, Sterno-hyoid. 24, Posterior belly of the omo-hyoid. The triangles of the neck can all be easily recognized. In this connection consult Diagram No. VII. 
middle and upper thirds and the second from beneath the muscle at its middle. They run downward and backward to disappear in front of the trapezius muscle.

\section{Digastric. Figs. I6, I7.}

Origin.-Posterior belly, from the digastric groove in the temporal bone. Anterior belly, from the under surface of the lower jaw a little external to the symphysis.

Insertion.- The two bellies are joined by a central tendon, which is fastened to the body and greater cornu of the hyoid bone by a process of the deep cervical fascia. This fascial loop is lined by synovial membrane.

The tendon also passes through the stylohyoid muscle, divides the insertion of that muscle, and by it is aided in keeping its place.

Nerve Supply.-The posterior belly, by the facial. See facial nerve, page $6 \mathrm{I}$.

The anterior belly by the mylohyoid branch of the inferior dental, which is a branch of the inferior maxillary nerve.

Action.-(I) Posterior portion. Raises and retracts the hyoid bone (and the structures attached to it).

(2) The anterior portion. Depresses the lower jaw. Acting from above, it will raise and draw forward the hyoid bone.

(3) Both portions acting will elevate the hyoid bone (and the parts connected to it, especially the tongue). Hence the significance of raising the lower jaw upward and forward in anæsthesia, to prevent the tongue falling backward and suffocating the patient.

\section{DISSECTION.}

Divide the sternomastoid muscle and the external jugular vein and reflect their extremities. (Always divide veins between two cords tied firmly around 
them. The reason for this caution will grow more apparent as the dissection proceeds.)

Leave the spinal accessory nerve passing through the muscle and terminating in the trapezius.

Incise the fascia which holds the submaxillary gland in position along the lower margin of the gland and turn the gland upward.

Clean the suprahyoid and subhyoid muscles, being careful in all cases to save the nerve supply to them.

The internal jugular vein, carotid arteries, and the branches of the external carotid, the cervical plexus, and all the nerves of the deep region of the neck will have to be dissected as far as convenient.

Stylohyoid. Fig. I 7 .

Origin.-From the back and outer surface of the styloid process near its base.

Insertion.-Into the body of the hyoid bone near the greater cornu. The muscle splits to enclose the digastric tendon.

Nerve Supply. - The facial. See page 6r.

Action.-To raise and retract the hyoid bone (and the parts connected to it).

Omohyoid. Figs. I6, I 7 .

Origin.-From the superior border of the scapula near the suprascapular notch, and the transverse ligament crossing the notch.

Insertion.-Into the lower border of the hyoid bone, external to the sternohyoid. Behind the sternomastoid the muscle becomes tendinous and is held downward by a loop of the deep cervical fascia which is attached to the inner surface of the clavicle, first rib (its cartilage), and the manubrium.

Nerve Supply.-The anterior portion by a branch direct from the descendens hypoglossi. The posterior portion, by filaments from the loop of communication formed by the descendens and communicans hypoglossi. 
Action.-The hyoid bone being fixed, slightly to raise and draw forward the scapula, to tense the fascia of the neck below. The scapula being fixed, to depress (and retract) the hyoid bone.

Sternohyoid. Figs. I6, I7.

Origin.-From the inner surface of the first piece of the sternum, the posterior sternoclavicular ligament, and adjacent portion of the clavicle.

Insertion. - Into the lower border of the body of the hyoid bone.

Nerve Supply._By filaments from the descendens and communicans hypoglossi.

Action.-To depress and fix the hyoid bone.

Sternothyroid. Fig. I7.

Origin.-From the inner surface of the manubrium below and internal to the sternohyoid, from the cartilage of the first rib.

Insertion.-Into the oblique line on the outer surface of the thyroid cartilage.

Nerve Supply. - Same as for the sternohyoid.

Action.-To depress the thyroid cartilage and the hyoid bone above.

Thyrohyoid. Figs. I 7 and I8.

Origin.-From the oblique line on the outer surface of the thyroid cartilage.

Insertion.-Into the lower border of the body and greater cornu of the hyoid bone.

Nerve Supply. - A small branch directly from the hypoglossal nerve.

Action.-To approximate the hyoid bone and thyroid . cartilage, depressing the one or elevating the other according to its fixed point. 
The Mylohyoid Nerve. Figs. I 2, I 7.

This is a small branch from the inferior dental just before it enters the dental foramen in the lower jaw. It runs downward and forward, grooving the under surface of the lower jaw, and supplies the mylohyoid and anterior belly of the digastric muscles. In its course across the mylohyoid muscle it is accompanied by the submental branch of the facial artery.

Mylohyoid. Fig. I 7 .

Origin.-From the mylohyoid ridge on the inner surface of the inferior maxilla.

Insertion.-Into the body of the hyoid bone, and a median raphe extending from the hyoid bone to the lower jaw.

Nerve Supply.-The mylohyoid branch of the inferior dental (of inferior maxillary).

Action.-To raise the hyoid bone, and the parts connected to it, especially the tongue. To depress the lower jaw if the hyoid bone is fixed.

Having dissected out the preceding muscles, replace the sternomastoid and take up the study of the triangles of the neck, their formation or boundaries, the muscles or parts which form their floor, and most especially their various contents and their relations to each other.

The Triangles of the Neck. Figs. I6, I 7 .

The side of the neck forms an irregular parallelogram with the following boundaries.

Above, by the lower border of the inferior maxilla, the mastoid process of the temporal bone, and a line joining the angle of the jaw with the mastoid process.

In front, by the median line.

Below, by the top of the manubrium and the clavicle. 
Behind, by the anterior border of the trapezius muscle.

This quadrilateral space is divided obliquely from above downward and forward by the sternomastoid muscle into two triangles, the anterior and posterior cervical triangles.

The anterior triangle is further subdivided by the posterior belly of the digastric and the anterior belly of the omohyoid muscles into three triangles.

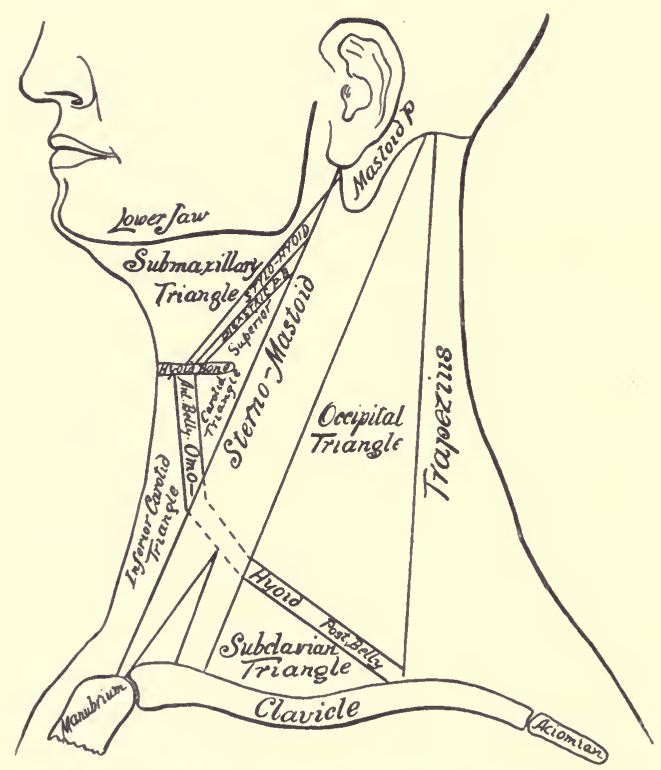

Diag. 7. A Diagram of the Triangles of the Neck. (I. S. H.)

These are from above downward, the submaxillary, superior and inferior carotid triangles.

The Submaxillary Triangle is bounded by the border of the lower jaw and the line from its angle to the mastoid process, by the posterior belly of the digastric (and the stylohyoid), and by the median line. (The student can 
locate the relative positions of these boundaries without further particulars.)

Its floor is formed by the anterior belly of the digastric, the mylohyoid, the hyoglossus, the styloglossus, superior constrictor, and the stylopharyngeus muscles.

Within it are found arteries, internal and external carotids, facial, and its cervical branches.

Veins: Internal jugular, facial, anterior and posterior divisions of the temporomaxillary vein or sinus.

Nerves: Inframaxillary branch of facial, mylohyoid, pneumogastric, and glossopharyngeal and hypoglossal.

Glands: Submaxillary, deep portion of parotid, and lymphatic glands.

The Superior Carotid Triangle. ("Triangle of Election.")

Boundaries.-By the posterior belly of the digastric, the anterior belly of the omohyoid, and the anterior border of the sternomastoid.

Floor is formed by the hyoglossus, thyrohyoid, middle and inferior constrictors; hyoid bone and thyroid cartilage.

Its Contents.-Arteries: Common, external and internal carotids; superior thyroid, lingual, facial, occipital, and ascending pharyngeal.

Veins: Internal jugular, veins corresponding to the above branches of the external carotid.

Nerves: Inframaxillary branch of facial, superficial cervical, descendens and communicans hypoglossi, hypoglossal, pneumogastric, spinal accessory, and superior laryngeal.

Parts of the larynx and pharynx and lymphatic glands. The Inferior Carotid Triangle. ("Triangle of Necessity.")

Boundaries.-By the median line, anterior border of the sternomastoid, and anterior belly of the omohyoid. 
Floor is formed by the scalenus anticus and longus colli muscles.

Contents.-Arteries: Common carotid, inferior thyroid, and vertebral.

Veins : Internal jugular, vertebral, middle thyroid.

Nerves: Superficial cervical, pneumogastric, recurrent laryngeal, descendens and communicans hypoglossi, sympathetic.

Parts of the larynx, trachea, thyroid gland, lymphatic glands, sternohyoid, and sternothyroid muscles.

The posterior triangle is divided by the posterior belly of the omohyoid into the occipital and subclavian triangles.

The Occipital Triangle.

Boundaries.-By the posterior border of the sternomastoid, the anterior border of the trapezius, and the posterior belly of the omohyoid muscles.

Floor is formed by the splenius capitis et colli, the levator anguli scapulæ, scalenus medius and posticus.

Contents.-Arteries : Occipital, muscular, superficial cervical, and transverse cervical.

Veins : Posterior external jugular, transverse cervical.

Nerves: The ascending and descending branches of the cervical plexus, and the spinal accessory.

Lymphatic glands.

The Subclavian Triangle (Molvenheim's Fossa).

Boundaries. - The posterior border of the sternomastoid, the posterior belly of the omohyoid, and the clavicle.

Floor is formed by the scalenus posticus and medius.

Contents.-Arteries: Second and third portions of the subclavian, and its suprascapular, transverse cervical, and superior intercostal (and usually the posterior scapular, see page I I 3) branches. 
Veins: External jugular, transverse cervical, suprascapular, and subclavian.

Nerves: Descending cervical branches, beginning of the brachial plexus, muscular branches from the lower four cervical nerves before the plexus is formed, the phrenic nerve.

Other parts: Scalenus anticus muscle, and lymphatic glands.

The Lingual Triangle.

This is a subdivision of the submaxillary triangle.

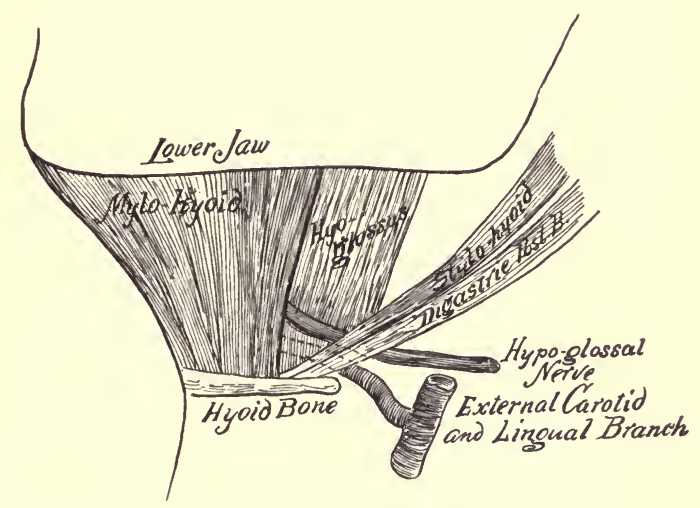

Diag. 8, A Diagram of the lingùal Triangle. (I. S. H.)

Boundaries.-In front, the posterior margin of the mylohyoid muscle. Behind, the posterior belly of the digastric and the stylohyoid muscle. Above, the hypoglossal nerve.

The floor is formed by the hyoglossus muscle, and the lingual artery is found under the muscle after dividing its fibres transversely.

The Submaxillary Gland. Figs. I 5 and 16.

The submaxillary gland is the second in size of the salivary glands. It lies under the lower jaw, and is covered 
by the platysma and deep cervical fascia which forms its capsule. Behind are the posterior belly of the digastric, the stylohyoid muscles, and the stylomaxillary ligament; in front, the anterior belly of the digastric; below, the tendon of the digastric, and the hyoid bone.

Internally the gland lies upon the mylohyoid, hyoglossus, and styloglossus muscles. The mylohyoid separates the submaxillary from the sublingual gland. Around the posterior border of the mylohyoid muscle a deep portion of the submaxillary gland extends forward, and from this deep portion the duct (Wharton's) passes forward and inward to open in the floor of the mouth at the summit of a small papilla at the side of the frænum of the tongue. The duct is about two inches long. Fig. I 8.

Relations about the Submaxillary Gland.-The facial vein and inframaxillary branch of the facial nerve cross the outer surface of the gland.

The facial artery deeply grooves the posterior and upper portion of the gland.

The submental branch of the facial artery and the mylohyoid nerve run together along its upper border.

Wharton's duct lies above the lingual nerve (which accompanies it to the tongue).

Between the lingual nerve and gland lies the submaxillary ganglion.

The arterial supply is from the facial (submaxillary branches) and lingual.

The nerves are derived from the submaxillary ganglion, mylohyoid, and sympathetic.

For the submaxillary ganglion, see page I I9.

DISSECTION.

Divide the tendon of the digastric on either side of the loop which binds it to the hyoid bone, and the stylohyoid at its insertion. 


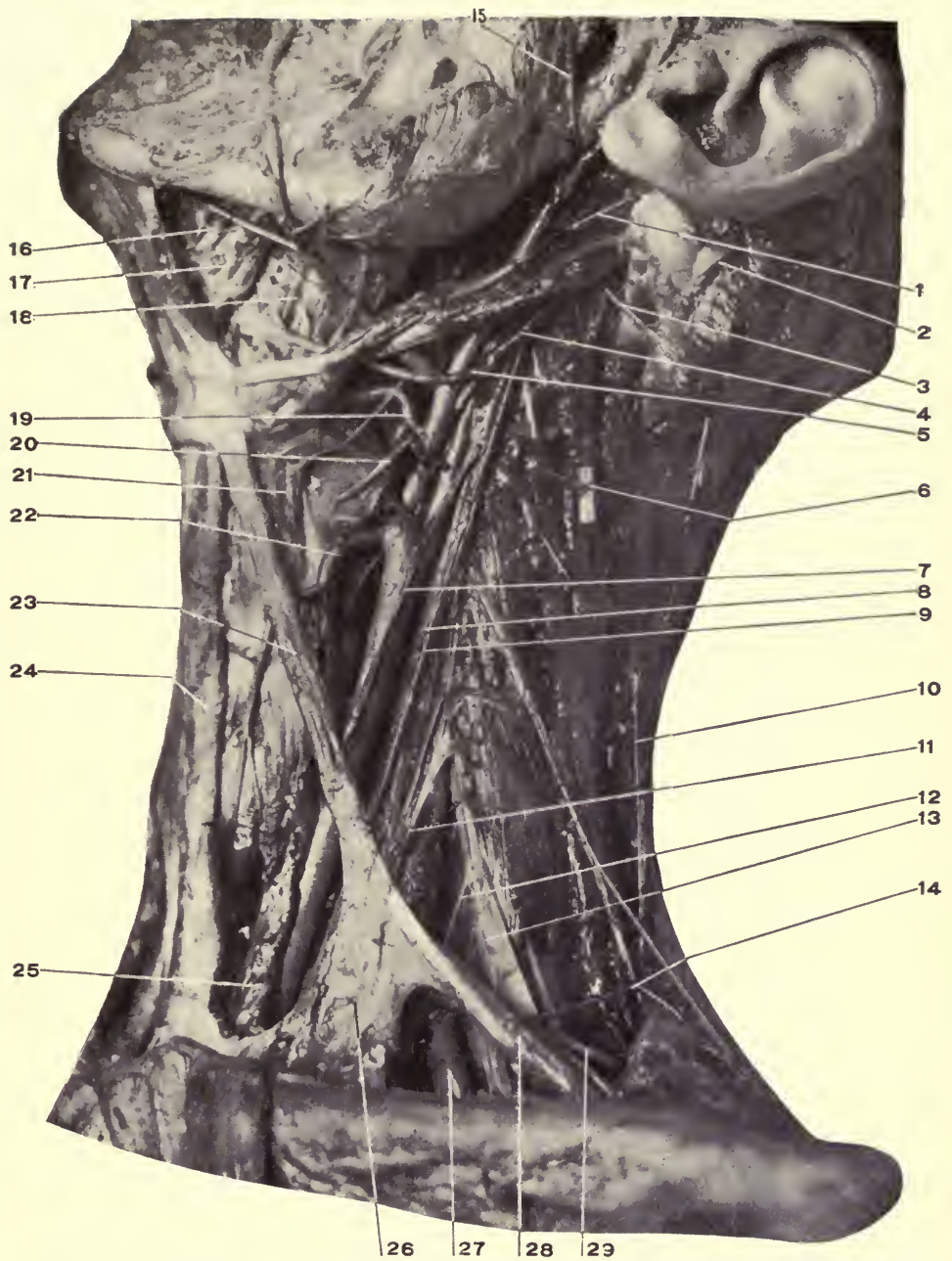

Fig. 17. Dissection of Neck.-I, Posterior auricular artery. 2, Sterno-mastoid muscle divided and drawn upward and backward. Shows the course of the spinal accessory nerve through it. 3. Spinal accessory nerve. 4, Occipital artery. 5, Hypoglossal nerve. 6, Lesser occipital nerve. 7, Descendens hypoglossi lying upon the common carotid. In this subject the nerve seems to be a branch (uses the sheath) of the pneumogastric nerve. 8, Communicans hypoglossi. In this connection consult Diagram No. VI. 9, Pneumogastric. 10, Spinal accessory. It is seen to be joined by a branch from the cervical plexus. II, Phrenic nerve. 12, Subclavian nerve. 13, Brachial plexus. 14, Transverse cervical artery. I5, Transverse facial artery. 16, Mylo-hyoid nerve, and behind it the submental branch of the facial artery. 17, Mylo-hyoid muscle. 18, Hyoglossus muscle. I9, Lingual artery. Consult Diagram No. VIII. 20, Superior laryngeal nerve. 21, Thyro-hyoid muscle. 22, Superior thyroid artery. 23, Anterior belly of the omo-hyoid. 24, Sterno-hyoid muscle. 25, Sterno-thyroid muscle. 26, Fascia binding the tendon of the omo-hyoid in place. 27, Subclavian artery. 28, Posterior belly of the omo-hyoid. 29, Posterior scapular artery. 
Divide the mylohyoid along the median raphé and at its insertion into the hyoid bone, and reflect the several parts of the muscles.

The Hypoglossal Nerve. Figs. I0, I6, I 7, I 8.

The hypoglossal nerve leaves the skull through the anterior condyloid foramen, winds outward around the ganglion of the trunk of the pneumogastric nerve from behind forward, passes between the internal jugular vein and the internal carotid artery, appears at the lower border of the digastric, turns forward around the occipital artery and across the internal and external carotids, and extends into the muscles of the tongue (resting upon the hyoglossus and under the mylohyoid), where it breaks up into its terminal branches.

For the part that the nerve takes in forming the lingual triangle, see page 85 .

As the hypoglossal nerve crosses the ganglion of the pneumogastric, it receives filaments from that nerve, the sympathetic, and a branch from the first and second cervical nerves. See Cervical Plexus, page 89.

Branches.-The descendens hypoglossi. This is composed of the fibres derived from the cervical plexus (first and second). It leaves the hypoglossal nerve as it is turning around the occipital artery (may come off much higher) and runs forward and downward upon the sheath of the carotid, where it is joined (in the middle of the neck) by the communicantes hypoglossi, forming the ansa hypoglossi (hypoglossal loop).

The descendens hypoglossi gives off a small branch to the anterior belly of the omohyoid muscle before the formation of the loop. From the loop branches are supplied to the posterior belly of the omohyoid, the sternohyoid, and sternothyroid muscles.

The hypoglossal also gives off a small branch just below 
the descendens hypoglossi, which supplies the thyrohyoid muscle, and a recurrent twig to the styloglossus.

Under the mylohyoid the hypoglossal gives branches to the hyoglossus, geniohyoid, geniohyoglossus, genioglossus, and the muscular substance of the tongue.

The Cervical Plexus. Figs. 16, 17, 18.

The cervical plexus is formed by the anterior branches of the four upper cervical nerves, which appear along the side of the neck between the prevertebral and lateral sets of muscles, and are connected in series by interlacing branches.

A diagrammatic representation of the plexus is given on page 75. Diag. 6.

Branches.-From the loop joining the first and second nerves is given off :-

(I) A branch which passes forward to join the hypoglossal nerve.

(2) From the second nerve is given off the small occipital.

(3) From the second and third nerves are given off branches to form the great auricular (4) the superficial cervical and (5) the communicantes hypoglossi. The second and third are joined by a loop.

From the third proceeds the (6) nerve to the trapezius.

From the third and fourth the (7) sternal, (8) clavicular, (9) and acromial branches.

From the fourth (and fifth, of brachial plexus) arises (IO) the phrenic.

(I I) Muscular branches are derived from the first for the rectus capitis lateralis and the rectus capitis anticus minor; from the first and second, for the rectus capitis anticus 
major; from the second, to the sternomastoid; from the third, to the scalenus medius and the levator anguli scapulæ; from the fourth, to the same.

The Branches of the Cervical Plexus.

(I) The branch to the hypoglossal: After joining the hypoglossal nerve this branch passes with it for a short distance. Some of the fibres continue onward to supply the geniohyoid, thyrohyoid, and anterior belly of the omohyoid. The greater part of the fibres leave the hypoglossal nerve as the descendens hypoglossi, form a loop below with the (5) communicantes hypoglossi, and supply the posterior belly of the omohyoid, sternohyoid, and sternothyroid muscles.

(2) The small occipital: This issues at the middle of the posterior border of the sternomastoid muscle along with the superficial cervical and the great auricular, turns upward parallel with this border of the sternomastoid to supply branches to the upper portion of the ear (auricular), to the skin over the mastoid process (mastoid), and to the scalp (occipital).

(3) The great auricular: This is found at the middle of the posterior border of the sternomastoid, passes vertically upward to supply the region of the parotid gland (facial), lower back part of the ear (auricular), and the mastoid region (mastoid).

(4) The superficial cervical : Comes from the middle of the posterior margin of the sternomastoid, passes forward transversely across the muscle and supplies the platysma, the skin of the antero-lateral region of the neck, and forms a loose plexiform interlacement with the inframaxillary branch of the facial.

(5) The communicantes hypoglossi: The branch from the second passes downward, joins that from the third, the 
united branch then passes downward and forward, usually over the internal jugular vein to unite upon the common carotid artery with the descendens hypoglossi. For the distribution see $(\mathrm{I})$.

(6) The muscular branch to the trapezius muscle appears on the posterior border of the sternomastoid at its middle, passes backward to the trapezius, and with the spinal accessory, which courses at a higher level, is distributed to this muscle.

(7), (8), (9) The branches to the integument over the sternum, clavicle, and acromial regions. They become superficial at the posterior border of the sternomastoid muscle and descend to their distribution. The suprasternal to the integument over the first piece of the sternum ; the supraclavicular, to the skin over the front of the clavicle (and chest as low as the fourth rib); and the supraacromial to the region of the shoulder.

(Io) The phrenic. For phrenic nerve, see page Ioo.

The Spinal Accessory Nerve. Figs. IO, I1, I2, 16, I 7 .

This will be found issuing under the posterior belly of the digastric muscle. It continues downward through the sternomastoid muscle, in the substance of which it forms a plexiform junction with a branch from the second cervical nerve for the supply of the muscle, then appears at the posterior border of the sternomastoid and extends downward and backward to the anterior surface of the trapezius, to which (with branches from the third and fourth cervical nerves) it is distributed. The deep course of the nerve after it comes through the central compartment of the jugular foramen (with the ninth and the tenth nerves) is downward and backward across the internal jugular vein and 
under the occipital artery, stylohyoid and posterior belly of the digastric muscles.

\section{The Internal Jugular Vein. Fig. I 5.}

This vein is formed just at the base of the skull by the junction of the inferior petrosal (anterior) and lateral (posterior) sinuses. Diags. I, 5 .

Its course is along the posterior surface of the internal and common carotid arteries to behind the sternoclavicular articulation, where it is joined by the subclavian, to form the innominate vein. See page 99.

In its course it receives veins corresponding to the branches of the external carotid artery.

It is also connected by communicating veins with the external and internal jugular veins.

\section{DISSECTION.}

The internal jugular vein should be tied off and divided about two inches from the clavicle, and as high toward the head as possible, and the intervening portion with all its tributaries removed.

Keep in mind that the thoracic duct opens into the angle at the junction of the internal jugular and the subclavian veins. The description of the duct is given with the dissection of the thorax.

The External Carotid. Figs. 16, I7, I8.

This is the outer branch of the common carotid. It extends from the bifurcation of the common carotid at the level of the upper border of the thyroid cartilage to behind the neck of the inferior maxilla, where it divides into the temporal and internal maxillary arteries.

At its beginning it lies anterior to the internal carotid, and at its termination superficial to the same.

The natural guide to the artery is the anterior border of the sternomastoid muscle.

Relations, External, or superficial._Skin, platysma, deep 
fascia, parotid gland; the sternomastoid overlaps the artery to a greater or less extent from behind forward.

It is crossed from above downward by the branches of the facial nerve, the temporomaxillary vein, stylohyoid, and posterior belly of the digastric muscles, the facial and lingual veins, and the hypoglossal nerve.

Internal, or centrally, the internal carotid, from which it is separated by the stylopharyngeus, styloglossus muscles, the glossopharyngeal, and pharyngeal branch of pneumogastric nerves, the posterior part of the parotid gland, and the stylomastoid ligament. The superior laryngeal nerve lies behind both internal and external carotids.

Anterior, or in front, the hyoid bone, pharynx, stylomaxillary ligament, ramus of jaw.

Posterior, or behind, the internal carotid for a short distance at its beginning, the hypoglossal nerve, the occipital artery, mastoid process.

Branches.-(I) The Superior Thyroid: This is the first branch from the anterior surface of the artery.

Its course is forward beneath the great cornu of the hyoid bone, then downward and forward to the upper part of the thyroid gland.

In its course it gives off branches to the larynx, pharynx, thyroid gland, trachea, and œesophagus; also, muscular branches to the sternomastoid, sternohyoid, sternothyroid, thyrohyoid, and inferior constrictor.

The named branches are (a) the hyoid, which passes transversely forward between the hyoid bone and the thyroid cartilage. (b) The sternomastoid, to that muscle. This branch crosses the carotid sheath. (c) The superior laryngeal, which accompanies the nerve of the same name into the interior of the larynx. (d) The cricothyroid. Of 
interest because it anastomoses with its fellow from the other side across the cricothyroid membrane, and must be divided in laryngotomy, or laryngotracheotomy.

(2) The Lingual Artery: The second branch from the front of the external carotid. Its course is forward and upward around the great cornu of the hyoid bone, then beneath the hyoglossus muscle, then under the tongue (as the ranine artery) to its tip.

The artery is reached for purposes of ligation through the lingual triangle. See page 85 .

Branches of the Lingual: (a) The hyoid; this runs forward over the hyoglossus muscle just above the hyoid bone. (b) The dorsal artery of the tongue is a branch from the lingual under the hyoglossus muscle; it passes upward to the back of the tongue. It supplies not only the tongue but small branches to the tonsil and fauces. (c) The sublingual is given off at the anterior border of the hyoglossus muscle, passes forward under the mylohyoid muscle to the sublingual gland, floor of the mouth, and in its course supplies the adjacent muscles. The artery of the frænum is a branch from the sublingual. (d) The ranine, or the last portion of the lingual along the under surface of the anterior portion of the tongue, to its tip. Supplies the tongue and the surrounding muscles.

(3) The Facial Artery (in the neck). Arises from the front of the external carotid as the third branch, or with the lingual by a branch common to both. It takes a tortuous course upward and forward, under the posterior belly of the diagastric and the stylohyoid muscles, through the upper and back part of the submaxillary gland, which it deeply grooves, then turns upward on to the face, over the lower jaw, just in front of the masseter muscle. For its course in the face, see page $6_{4}$. 
Between the facial artery and vein are found these structures, the posterior belly of the diagastric, the stylohyoid muscles, and the submaxillary gland.

Branches of the facial in the neck: (a) The ascending palatine. (b) The tonsillar. These branches pass upward to supply the palate, fauces, tonsils, and pharynx; the former is behind, the latter in front, of the styloglossus muscle, which separates them. (c) The glandular or submaxillary branches to that gland, as the facial passes through it. (d) The submental, a considerable branch which runs forward under the lower jaw and upon the mylohyoid muscle (being accompanied by the mylohyoid nerve), supplies the adjacent muscles, and turns upward over the chin to anastomose with the mental (of the inferior dental) and the inferior labial (of the facial).

Besides the chain of anastomoses which is formed by the branches of the inferior thyroid, lingual, and facial upon the same side of the neck and face, all these branches which pass forward to the median line inosculate across the median line with similar branches from the other side. This also holds for the nasal, frontal, and supra-orbital branches of the ophthalmic. See page 65 .

(4) The Occipital Artery is given off from the posterior aspect of the external carotid. It runs upward and backward under the posterior belly of the digastric to a point between the transverse process of the atlas and the mastoid process. Here it is covered by the sternomastoid, splenius capitis, and origin of the digastric. Then its course is backward in the occipital groove, where it will be found when dissecting the back. See page I 30 . The artery is crossed by the hypoglossal nerve, which winds downward and forward across the artery. In the anterior portion of the neck it gives off muscular branches to the adjacent muscles; the 
one to the sternomastoid is of some size and is the only one named.

The remaining branches of the occipital are given on page 130 .

(5) The Posterior Auricular Artery: See Dissection of the Face, page 65.

This is the second branch from the back of the external carotid. It is given off just above the occipital, runs upward and backward between the spinal accessory and facial nerves, and through the parotid gland to the groove between the external auditory meatus and the mastoid process, and supplies branches to the ear and the scalp behind it. From the posterior auricular is given off the stylomastoid branch which enters the foramen of that name.

(6) The Ascending Pharyngeal : This is the first branch of the external, and comes off in the angle between it and the internal carotid.

It is a long, slender artery, which runs upward between the pharynx, internal carotid, and spine to the base of the skull. It supplies fine branches to the pharynx, front of the spine, palate, and posterior meningeal, which reach the interior of the skull through the middle lacerated, jugular, and anterior condyloid foramina.

Geniohyoid. Fig. I 8.

Origin.-From the lower genial tubercle of the inferior maxilla.

Insertion.-Into the upper surface of the hyoid bone, close to the middle line.

Nerve Supply.-The hypoglossal.

Action.-To raise the hyoid bone or to depress the lower jaw, depending upon which is the fixed point. 
Geniohyoglossus. Fig. I8.

Origin.-From the superior genial tubercle.

Insertion.-Into the body of the hyoid bone, the tongue from its tip to its base just outside of the median plane, and into the side of the pharynx.

Nerve Supply. - The hypoglossal.

Action.-To depress the lower jaw, to raise the hyoid bone, to protrude the tongue (by its posterior fibres), to depress and protrude the middle of the tongue (by its middle fibres), and to retract the tip of the tongue (by its anterior fibres).

Hyoglossus. Figs. I 7 and I 8.

Origin.-From the body and great cornu of the hyoid bone.

Insertion.-Into the substance of the posterior half of the tongue external to the geniohyoglossus.

Nerve Supply. - The hypoglossal.

Action.-To depress the sides of, and retract the whole tongue.

Styloglossus. Fig. I 8.

Origin.-From the front of the lower portion of the styloid process.

Insertion.-Into the side of the tongue, interlacing with the hyoglossus.

Nerve Supply.-The hypoglossal.

Action.-To retract the tongue and elevate its sides.

Stylopharyngeus. Fig. I 8.

Origin.-From the inner surface of the base of the styloid process.

Insertion.-Passing between the superior and middle constrictor muscles, it is inserted into the posterior border of the thyroid cartilage and wall of the pharynx. 
Nerve Supply. - The glossopharyngeal.

Action.-To raise the pharynx and larynx.

The Glossopharyngeal Nerve. Figs. IO, I I, I 8.

This is found in the dissection of the neck, as it curves forward around the outer side of the stylopharyngeus muscle.

It leaves the skull through the jugular foramen, in the central compartment, with the tenth and eleventh nerves.

It runs forward between the internal jugular vein and the internal carotid artery, then winds around the outer border of the stylopharyngeus muscle to terminate in the under surface of the hyoglossus muscle.

Branches.-The glossopharyngeal nerve supplies the stylopharyngeus muscle, then gives off small filaments to help form the pharyngeal plexus, and to supply the tonsil and tongue.

The Internal Carotid Artery. Figs. I6, I7, I 8.

This begins at the same point as the external, viz. : at the bifurcation of the common carotid, at the level of the upper border of the thyroid cartilage.

It passes directly upward to the base of the skull, being internal to the external carotid, and separated from it by the structures mentioned on page 93 .

Relations.-See the relations of the external carotid, page 92.

In the beginning of its course the artery lies in the superior carotid triangle, and then within the submaxillary triangle. As it passes upward it rests upon the rectus capitis anticus major, the superior cervical sympathetic ganglion, and the pneumogastric nerve. Behind it is the internal jugular vein, which, near the base of the skull, is separated from the artery by the hypoglossal, pneumogastric, glossopharyngeal, and spinal accessory nerves. 
In front of the artery is the pharynx and the tonsil, from which it is separated by the superior constrictor muscle.

The course of the artery into the skull is through the carotid canal, within the petrous portion of the temporal bone, and then by the upper part of the foramen lacerum medium, beyond which it enters the cavernous sinus. See pages 43,53 , and 158 .

The artery gives off no branches in the cervical part of its course.

\section{DISSECTION.}

Leave the remaining structures beneath the lower jaw for dissection with the deep face and turn to the parts at the base of the neck.

Working carefully from the superficial to the deep structures, clean them in order, all the time noticing their mutual relations, which are many and complex, but which can be far better understood and remembered if in the dissection the student will only take the time to notice and repeat them to himself as his work proceeds.

Divide the omohyoid rear its middle, the sternohyoid and sternothyroid near the sternum, and reflect them.

The Subclavian Vein. Fig. I 8.

The subclavian vein extends from the lower border of the first rib, beyond which it is called the axillary, to behind the sternoclavicular articulation, where it ends by joining with the internal jugular to form the innominate vein.

It lies in front and slightly below the subclavian artery, from which it is separated by the scalenus anticus muscle and phrenic nerve.

Tributaries.-The external jugular, and sometimes the anterior jugular or the cephalic.

The Transverse Cervical and Suprascapular Veins. Fig. 15.

These veins are double (venæ comites) and return the blood from the region supplied by their respective arteries. Near their terminus each pair unites to form a single trunk, 
which opens into the lower part of the external jugular vein. See page 74 .

The Phrenic Nerve. Figs. 17, 18.

The phrenic nerve is formed by branches from the third, fourth, and fifth cervical nerves. The fourth is the constant branch, the fifth may be present, but the branch from the third is very inconstant.

The phrenic descends obliquely across the front of the scalenus anticus muscle, passes between the subclavian artery and vein, and enters the chest over the front of the internal mammary artery. See thorax for rest of course.

Scalenus Anticus. Fig. I8.

Origin.-From the anterior tubercles of the transverse processes of the third, fourth, fifth, and sixth cervical vertebræ.

Insertion.-Into the scalene tubercle upon the upper surface and inner border of the first rib.

Nerve Supply.-From the anterior divisions of the fourth, fifth, and sixth cervical nerves close to the intervertebral foramina.

Action.-Both muscles, acting from below, will flex the neck; one acting, will rotate the neck to the opposite side and flex the neck laterally. Acting from above, the muscles will raise and fix the first rib, so aiding in inspiration.

Scalenus Medius. Fig. I 8.

Origin.-From the posterior tubercles of the transverse processes of the lower six cervical vertebræ (sometimes the atlas also).

Insertion.-Into the upper surface of the first rib between the subclavian groove and the tuberosity.

Nerve Supply.-The branches from the posterior divisions of the cervical nerves. 
Action.-They flex the neck laterally if acting from below; if from above, they raise and fix the first rib, thus aiding inspiration.

Scalenus Posticus. Fig. 76.

Origin.-From the posterior tubercles of the transverse processes of the lower two or three cervical vertebræ.

Insertion.-Into the outer surface of the second rib behind the insertion of the serratus magnus.

Nerve Supply.-The three lower cervical nerves.

Action.-As slight lateral flexor of the lower cervical vertebræ, also as an accessory muscle of inspiration by raising and fixing the second rib.

The Common Carotid Artery. Figs. 18, 7 I.

On the right side the common carotid extends from the bifurcation of the innominate behind the sternoclavicular articulation, upward to the level of the upper border of the thyroid cartilage, where it divides into the external and internal carotids.

On the left side the artery arises as the middle branch from the arch of the aorta. Its course is upward to the same point as on the right side.

Relations.-The relations of the thoracic portion of the left common carotid are given on page 340 .

In the neck they are alike.

At the base of the neck the common carotids are about an inch apart, at their termination about two inches apart.

The course of the artery is indicated by the anterior border of the sternomastoid (natural landmark), or a line drawn from the sternoclavicular articulation to the front of the mastoid process (artificial).

The common carotid, internal jugular, and pneumogastric nerve are contained within the same sheath of the deep 
cervical fascia, yet each is separated from the other by a partition of the same. The artery is internal, the vein external, and the nerve between and behind them both.

Superficially or externally: Skin, platysma, anterior layer of deep cervical fascia.

It is overlapped by the sternomastoid behind, and by the thyroid gland in front, and below is covered by the sternomastoid, sternohyoid, and sternothyroid muscles. From above downward the artery is crossed by the superior and middle thyroid veins, the communicating vein from the facial to the anterior jugular, the sternomastoid artery (from the superior thyroid), the descendens hypoglossi (which courses downward and forward upon the sheath of the artery and is joined by the communicantes hypoglossi), the omohyoid muscle (dividing the artery into two portions, see page 79), and the anterior jugular vein (as it turns backward to empty into the external jugular, see page 74).

At the inner side: The trachea, œsophagus (and between them), the recurrent laryngeal nerve, the thyroid gland, cricoid and thyroid cartilages, the lower part of the pharynx, the superior laryngeal nerve, branches of the superior and inferior thyroid arteries, and the veins of the same.

At the outer side: The internal jugular vein, pneumogastric nerve (between and behind both artery and vein).

Centrally: Longus colli, rectus anticus major muscles, pneumogastric (see above), sympathetic and its cardiac branches, inferior thyroid artery, and recurrent laryngeal nerve.

Above: In the angle of bifurcation lies the carotid gland, a structure composed of fine capillaries invested with connective tissue. It is not a lymphatic gland. 
The Pneumogastric Nerve. Figs. IO, I I, I 7, I 8.

This leaves the interior of the skull through the jugular foramen, lying in the central compartment of the same with the ninth and eleventh nerves, passes straight downward behind and between the internal jugular vein and the internal carotid artery, then between the same vein and the common carotid, resting upon the fascia covering the rectus capitis anticus major, and longus colli muscles.

At the base of the neck the right nerve crosses over the front of the subclavian artery and lies between it and the innominate vein.

On the left side it lies between and behind the subclavian and common carotid arteries.

For its course and relations in the chest see page 342.

Just below the skull will be found the enlargement of the pneumogastric called the ganglion of the trunk. To this the hypoglossal nerve is firmly united by connective tissue.

Branches of the Pneumogastric.-The pneumogastric receives branches from the spinal accessory, hypoglossal, superior cervical sympathetic ganglion, and the loop between the two upper cervical nerves. These branches enter the ganglion of the trunk.

The pneumogastric gives off minute branches to the glossopharyngeal, spinal accessory, hypoglossal, and two upper cervical nerves.

The branches of distribution, in the neck.

(a) The pharyngeal: May be one or more ; come originally from the spinal accessory branch of communication. They form, with branches from the sympathetic and glossopharyngeal, the pharyngeal plexus, from which branches are given to the muscles and mucous membrane of the pharynx.

(b) The superior laryngeal: Arises from the lower part 
of the ganglion of the trunk, passes downward and forward under both external and internal carotids to the side of the larynx, where it divides into two branches.

The superior, larger, internal, or sensory branch enters the larynx through the thyrohyoid membrane with the laryngeal branch of the superior thyroid artery and supplies sensation to the mucous membrane of the interior of the larynx. It communicates with the inferior laryngeal nerve.

The inferior, smaller, external, or motor branch descends to terminate in the cricothyroid muscle, which it supplies.

From this branch are some fine filaments of communication to the sympathetic and pharyngeal plexus.

(c) The recurrent laryngeal or inferior laryngeal: The right one is given off as the pneumogastric turns over the subclavian artery, the left as it passes in front of the arch of the aorta. See page 342 .

In the neck they both pass behind the common carotids and inferior thyroid arteries, then in the groove between the trachea and the osophagus, where they are covered by the thyroid gland, then under the lower border of the inferior constrictor, where they enter the interior of the larynx and are distributed to all the muscles of that organ excepting the cricothyroid.

The recurrent nerve also furnishes small branches to the trachea, œsophagus, inferior constrictor, inferior cervical sympathetic ganglion, and the cardiac plexus.

(d) The cervical cardiac branches. For the Thoracic, see page 354 .

There are one or two upper cervical cardiac branches which join the cardiac branches of the sympathetic. See page 106.

There is one lower, which comes off the pneumogastric 


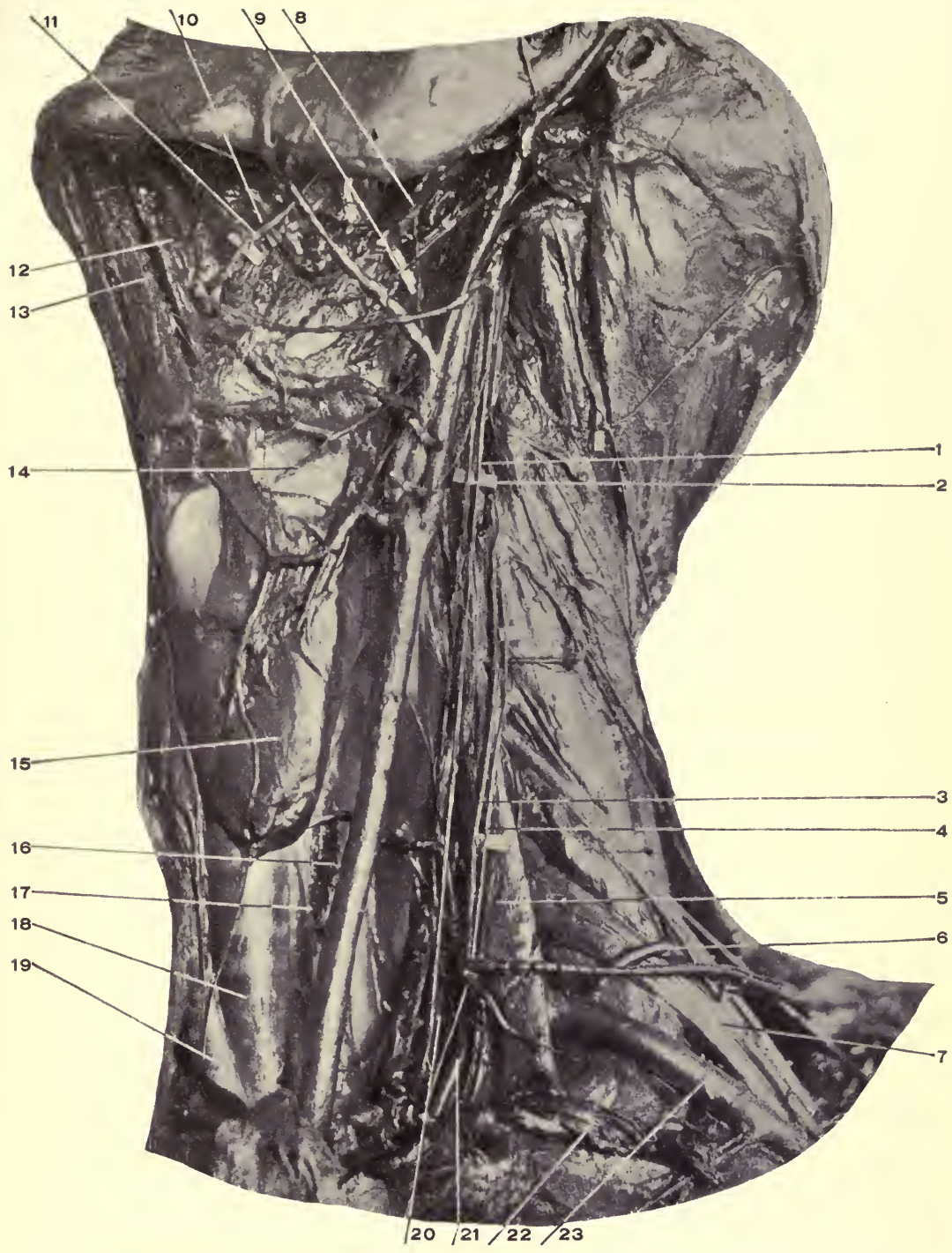

Fig. 18. Dissection of Neck.-[Structures named in preceding figures will not be re-named in this, except as necessary to clearness.]-i, Pneumogastric nerve. 2, Sympathetic cord. 3, Phrenic nerve. 4, Ascending cervical artery. 5, Scalenus anticus muscle. 6, Posterior scapular artery. See text, page II3. 7, Brachial plexus. 8, Stylo-glossus muscle. 9, Glosso-pharyngeal nerve. Io, Wharton's duct. II, Gustatory nerve and site of the submaxillary ganglion. 12, Genio-hyo-glossus muscle. 13, Genio-hyoid muscle. I4, Superior laryngeal nerve. I5, Thyroid gland. 16, Esophagus. I7, Inferior (recurrent) laryngeal nerve. 18, Trachea. 19, Innominate artery. 20, Vertebral artery. 21 , Internal mammary artery. 22, Subclavian vein. 23, Subclavian artery. 
as it is entering the chest at the root of the neck. The right nerve passes to the deep cardiac plexus and the left to the superficial cardiac plexus. See page 354 .

For the remaining branches of the pneumogastric nerve see page 342 .

The Cervical Sympathetic Cord and Ganglia. Figs. I 2, I 8.

There are three cervical ganglia, the superior, middle, and inferior, joined by the nerve cord which extends from the base of the skull into the thorax, where it is continued as the thoracic cord. The sympathetic cord lies upon the rectus capitis anticus major and the longus colli (separated from them by the prevertebral fascia), and behind the internal and common carotid arteries. The pneumogastric nerve lies external to the cord.

\section{The Superior Cervical Ganglion.}

This is about an inch in length; it lies in front of the transverse process of the second and third cervical vertebræ.

It gives off the ascending branch, which passes upward with the carotid artery and forms the carotid and cavernous plexuses; the arterial branches to the external carotid and its branches; the communicating filaments to the ninth, tenth, and twelfth cranial nerves; pharyngeal branches to the pharyngeal plexus ; the superior cardiac nerve, which on the right side passes to the deep cardiac plexus and on the left side to the superficial cardiac plexus (see page 354); and slender filaments to the anterior division of the first four spinal nerves.

The middle cervical ganglion is not always constant; when present is found in front of the inferior thyroid artery as a slight thickening of the cervical cord. 
It furnishes filaments to the thyroid gland and gives off the middle cardiac nerve, which passes into the thorax to end in the deep cardiac plexus, and is connected to the fifth and sixth cervical nerves.

\section{The Inferior Cervical Ganglion.}

This ganglion lies behind the vertebral artery, and between the neck of the first rib and the transverse process of the seventh cervical vertebra.

Its filaments extend to the seventh and eighth cervical nerves, to the vertebral artery, and by the inferior cardiac nerve to the deep cardiac plexus.

\section{DISSECTION.}

Divide the internal carotid near its bifurcation and retract the two portions.

Ligate the subclavian vein at the clavicle (if the axillary has already been tied, then draw the vein under the clavicle) and turn it inward. Keep track of the thoracic duct on the left side.

Divide the scalenus anticus over the subclavian artery and clean the entire course (in the neck) of the artery and its branches.

\section{The Right Lymphatic Duct.}

Receives the lymphatics of the right side of the head and neck, right upper extremity, right side of the thorax, and from the convex surface of the liver. It is about an inch long and empties into the angle between the internal jugular and subclavian as they form the right innominate vein.

The Thoracic Duct.-See Thorax, page 344.

The Subclavian Artery. Figs. I7, I 8.

The subclavian artery differs in its origin on the two sides of the body. On the right side it is the external branch of bifurcation of the innominate artery; on the left side, arises as the last branch from the transverse portion of the aorta. 
The artery terminates on both sides of the body at the lower border of the first rib, where the name for the arterial trunk becomes the axillary.

The artery is divided into three portions by the scalenus anticus muscle. The first portion, internal to the muscle; the second, behind, and the third portion external to the muscle.

The right artery is three inches, the left about four inches in length.

\section{Relations.-}

First Portion of the Left Subclavian Artery. (Inserted here to complete the description of the artery.)

In front: Sternohyoid, sternothyroid, and sternomastoid muscles. The left pleura and lung. The left innominate vein. (In the neck), internal jugular, vertebral, and subclavian veins. Phrenic nerve (in the neck), cardiac nerves (from sympathetic). Thoracic duct (root of neck).

Behind (and internal) : CEsophagus, thoracic duct, sympathetic and inferior ganglion, longus colli muscle, and spinal column. Behind (and externally): The lung and pleura.

At the right: Trachea, recurrent laryngeal nerve, œsophagus, and thoracic duct.

At the left: Left lung and pleura.

Right Subclavian Artery, First Portion.

In front: Skin, superficial fascia, platysma, deep fascia (two layers), the clavicular origins of the sternomastoid, sternothyroid, and sternohyoid muscles. The subclavian vein, the beginning of the innominate, end of the internal jugular and vertebral veins. Pneumogastric and phrenic nerves, the sympathetic cardiac branches. The artery is surrounded by a loop of the sympathetic, called the ring of Vieussens (annulus Vieussens).

Behind: Longus colli muscle, transverse process of the 
seventh cervical vertebra, sympathetic cord, cardiac nerves, recurrent laryngeal, lung, and pleura.

Below: Lung and pleura, loop of recurrent laryngeal, and sympathetic nerves.

The Second Portion of Right and Left Subclavian Arteries.

This lies behind the scalenus anticus muscle.

In addition to the fasciæ, skin, and muscles above given as being in front of the first portion of the right artery, there are the scalenus anticus muscle and the phrenic nerve (separated from the artery by the scalenus muscle).

Behind: Apex of the lung and pleura. Scalenus medius.

Below : Pleura and lung.

Above: The brachial plexus.

Third Portion of the Subclavian Artery, Right and Left.

This extends from the outer border of the scalenus anticus to the lower border of the first rib.

In front: The skin, layers of fascia, platysma, clavicular nerves, the suprascapular artery (if the arm be elevated); the external jugular and subclavian veins; the suprascapular and transverse cervical form a plexus in front of the artery before they empty into the external jugular vein.

The nerve to the subclavius muscle.

Behind: Scalenus medius, the lower or third trunk of the brachial plexus.

Below: The posterior of the two grooves upon the upper surface of the first rib.

Above: The cords and the first trunk (see page 312) of the brachial plexus. The posterior belly of the omohyoid muscle.

Branches of the Subclavian Artery.

From the First Portion.-Vertebral, thyroid axis, internal mammary. 
Second Portion.-Superior intercostal.

Third Portion.-Posterior scapular (more often than from the transverse cervical).

The Vertebral Artery. Figs. 10, 18, 20, 22.

This is the first branch of the subclavian. It arises from the upper and back part of the artery, near the inner edge of the scalenus anticus, passes vertically upward to enter the foramen in the transverse process of the sixth cervical vertebra.

The artery continues upward through all the foramina in the vertebræ above. On reaching the atlas it winds backward around the superior articular process, grooving the upper surface of the posterior arch, passes through a gap in the posterior occipito-atlantal ligament, winds forward and unites with the opposite vertebral at the anterior margin of the foramen magnum to form the basilar artery. See page 158.

For the relations of the artery in the suboccipital triangle, see page 132 .

The artery is divided into the cervical, vertebral, occipital (see page I 32), and intracranial portions.

The cervical portion is the part of the artery before it enters the vertebral foramen. It lies in the interval between the longus colli and scalenus anticus muscles, and upon the transverse process of the seventh cervical vertebra and sympathetic nerve. In front of it are the vertebral and internal jugular veins, the inferior thyroid artery, and, on the left side, the thoracic duct crosses it from within outward.

The vertebral portion is that part of the artery in transit through the vertebral foramina, where it is surrounded by a venous plexus and sympathetic filaments. 
The second portion gives off the lateral spinal and muscular branches to the contents of the spinal canal and external spinal muscles.

\section{The Vertebral Vein.}

Begins in a plexus of small veins in the suboccipital triangle, where it communicates with the intraspinal, deep cervical, and occipital veins. It retraces the course of the artery through the vertebral foramina, forming a venous plexus about it in its course, appears at the foramen in the sixth cervical vertebra, passes across the vertebral and subclavian arteries, and opens into the innominate vein.

Near its termination it is joined by the deep cervical vein. See page $\mathbf{I} 36$.

The Thyroid Axis. Fig. I 8.

This is a short trunk from the upper and front portion of the first portion of the subclavian, at the inner border of the scalenus anticus muscle.

It breaks up into the inferior thyroid, transverse cervical, and suprascapular arteries.

The Inferior Thyroid. Fig. I 8.

This is the largest branch of the axis. It takes a winding course upward and inward to the lower border of the thyroid gland.

In its course it passes in front of the vertebral artery, longus colli muscle, and the recurrent laryngeal nerve; and behind the internal jugular vein, pneumogastric nerve, common carotid artery, sympathetic nerve cord and its middle cervical ganglion (when that is present).

Its branches are $(a)$ muscular, to the adjacent muscles. (b) Ascending cervical, which runs upward between the vertebral attachments of the scalenus anticus and rectus capitis anticus major, following up the phrenic nerve, sup- 
plying the deep muscles of the neck, and some branches to the interior of the spinal canal. (c) Tracheal, to the trachea. (d) Esophageal, to the œsophagus. (e) The inferior laryngeal, which accompanies the inferior laryngeal nerve into the larynx.

The Transverse Cervical Artery. Figs. I 7 and 18.

It is the second (usually) in size of the branches of the thyroid axis.

Its course is as indicated by its name, transversely outward across the neck just above the clavicle, to disappear under the trapezius muscle, where it divides into its terminal branches.

In its outward course it crosses the phrenic nerve, scalenus anticus, brachial plexus, and scalenus medius; and is crossed by the omohyoid (posterior belly).

Its terminal branches are the (a) superficial cervical, which turns upward under the trapezius to anastomose with the superficial branch of the princeps cervicis of the occipital artery, in its course giving branches to the trapezius, levator anguli scapulæ and splenius muscles, between which it lies ; and (b) the posterior scapular, which is usually given off the third portion of the subclavian.

If given off the transverse cervical its course is downward and backward under the levator anguli scapulæ to the upper angle of the scapula.

For the continuation of its course, see page 370 .

The Suprascapular Artery. Fig. I 8.

The third and often smallest branch of the thyroid axis.

It takes a course outward behind the clavicle to the suprascapular notch, where it crosses above the ligament, which converts this notch into a foramen, the nerve of this name passing through the foramen. See page 37I. 
The artery crosses the phrenic nerve, scalenus anticus muscle, subclavian artery, and brachial plexus.

Its branches in this part of its course are $(a)$ muscular to the sternomastoid and subclavius; (b) nutrient to the clavicle; $(c)$ the suprasternal; $(d)$ supra-acromial to these regions ; and $(e)$ the articular to the acromioclavicular and shoulder joints. For the remaining branches, see page $37 \mathrm{I}$.

The Internal Mammary Artery. Fig. 18.

It is given off close to the scalenus anticus muscle from the under surface of the subclavian. It descends directly into the thorax behind the cartilage of the first rib. For its course, relations, branches, and veins, see pages 320 .

The Superior Intercostal Artery.-Second Portion of the Subclavian. Fig. 74 .

This branch arises from the back of the subclavian, behind the inner border of the scalenus anticus muscle.

It takes an arched course backward, upward, and then downward into the chest, passing in front of the neck of the first rib.

From the back of the arch it gives off the deep cervical (profunda cervicis) branch, which passes backward between the transverse process of the seventh cervical vertebra and the neck of the first rib to the back of the neck, where it will be found between the complexus and semispinalis colli muscles. See page 135 .

For the costal portion of the superior intercostal, see page 346 .

The Posterior Scapular.- - The Third Portion of the Subclavian. Figs. I7, I8.

The posterior scapular artery usually arises from this portion of the subclavian. When arising as a branch of the subclavian the posterior scapular takes a course backward 
between the lower trunks of the brachial plexus; to reach a position under the levator anguli scapulæ muscle; from here on its course is as described on page 370 .

The Thyroid Gland. Fig: I 8.

The thyroid gland is a ductless gland, composed of two symmetrical halves, which are joined by a narrow isthmus.

The halves, or lobes, of the gland rest upon the anteroexternal surface of the trachea, and the isthmus crosses its front.

Each lobe is about two inches long, and one and onehalf inches broad, and three-fourths of an inch thick. The lower end of the lobe is broader than its upper end. Its antero-external surface is convex, and its inner surface adapted to the trachea, against which it rests.

It reaches from the fifth or sixth tracheal ring upward to cover the lower fourth of the thyroid cartilage.

The isthmus is about one-half an inch wide and crosses the second and third tracheal rings.

The entire gland weighs from one to two ounces, and is usually heavier in females than males.

Relations.-In front: The integument, platysma, small part of the sternomastoid, deep fascia (two layers), omohyoid, sternohyoid, and sternothryoid.

Laterally: The sheath of the common carotid, internal jugular, and pneumogastric. The superior and inferior thyroid arteries.

Internally: The larynx and trachea, recurrent laryngeal nerves.

Posteriorly: Pharynx, and on the left side the œsophagus.

The arteries of the gland : The superior thyroid from the external carotid, the inferior thyroid from the thyroid axis, 
and sometimes the thyroidea ima from the arch of the aorta or the innominate artery.

Veins : Superior, middle, and inferior thyroid; the first two empty into the internal jugular, the last two into the innominate.

Nerves: From the middle and inferior cervical ganglia.

Lymphatics: Pass to the right lymphatic and the thoracic ducts.

Muscles: The thyroid gland is at times found to be connected to the hyoid bone by a detached slip of the thyrohyoid muscle, which is called the levator glandulæ thyroideæ.

Ligaments: The gland is retained in its position against the trachea and larynx by the third layer of cervical fascia (see page I39), which forms a capsule for the gland and encloses all these structures.

The student should prepare himself upon the trachea, thoracic duct, and brachial plexus. The description of these structures is omitted here, but is given, trachea, $\mathrm{p}$. 356 ; thoracic duct, p. 344, and brachial plexus, p. 3 I 2.

\section{DISSECTION OF THE DEEP FACE AND PHARYNX.}

The plan of dissection is determined by whether the skull is to be preserved for future use or not. If it is desirable to save the skull intact, the first plan can be adhered to, if otherwise, the second.

The first plan :-

Sever the attachment of the masseter muscle close to the zygomatic arch, draw it downward and carefully cut it away from its insertion. In doing this recognize and save the masseteric nerve and artery, which reach the muscle through the sigmoid notch in the lower jaw. Then remove the masseter entirely.

Cut the temporal muscle from its insertion into the coronoid process, keeping close to the bone. When the insertion is cut through, draw the tendon upward through the zygomatic arch, sever the muscular attachment to the temporal fossa, saving the deep temporal nerves and arteries, and remove the muscle entirely. (In the preceding plan the skull-cap has not been removed.) 
Expose the external lateral ligament of the lower jaw, then divide it, and continue the disarticulation of the lower jaw.

Sever the attachment of the internal pterygoid muscle and the stylomaxillary ligament close to the jaw. Work carefully forward until the dental foramen is reached, then sever the internal lateral ligament. This releases the lower jaw sufficiently to permit its being drawn forward and downward.

Expose the inferior dental nerve and artery and cut them close to the dental foramen. Save the mylohyoid nerve.

Trace the auriculo-temporal nerve, from where it was found posterior to the temporal artery, around the temporomaxillary articulation to its origin by two branches from the inferior maxillary nerve.

Clean the branches of the first portion of the internal maxillary artery, viz. : deep auricular, tympanic, middle meningeal, small meningeal, inferior dental ; and of the second portion, viz. : masseteric, deep temporal, buccal, and the buccal.

All of these will be found with care, excepting, perhaps, the first two.

Clean the pterygoid muscles as far as possible at this stage.

Divide the external pterygoid close to its insertion into the front of the neck of the jaw, cut away the buccinator from the lower jaw, and draw the jaw still farther downward and forward.

Trace the inferior maxillary nerve up to the foramen ovale, and its inferior dental (has been divided), mylohyoid, lingual, and buccal branches.

Cut away the external pterygoid, follow the internal maxillary artery to the sphenomaxillary fossa. Draw the jaw upward, complete the stylopharyngeus muscle, facial nerve, the occipital artery, to where it passes between the rectus lateralis and the origin of the posterior belly of the digastric, the spinal accessory, glossopharyngeal, pneumogastric, sympathetic, and hypoglossal nerves. Find the branch from the loop between the first and second cervical nerves to the last nerve.

Divide the stylopharyngeus, styloglossal muscles, the hypoglossal nerve.

Trace upward the ascending pharyngeal and internal carotid arteries to the carotid canal.

Cut the mylohyoid muscle, anterior belly of the diagastric, and the genial muscles close to the lower jaw, and remove it entirely. Fasten the tongue forward with a chain hook.

The second plan is intended to give more room for the dissection, and thus render it somewhat easier.

It contemplates the division of the zygomatic arch at both ends and its removal, the division of the lower jaw at the symphysis with a saw, and the separation of its halves.

The jaw can be divided in the first plan if the student so desires.

The dissection of the parts then takes place as outlined above.

The student should remember that the dissection of small spaces where im- 
portant organs are crowded together can be indicated in only the most general terms, and that he must rely upon his own judgment, guided by his previous study of the text-book, plates, and the directions given.

\section{The Inferior Maxillary Nerve. Figs. I I, I 2.}

This is the third or lowest branch of the fifth cranial nerve. It issues from the skull through the foramen ovale.

It is composed of the entire motor portion of the fifth and a part of the sensory fibres, which unite, after traversing the foramen ovale, to form a single trunk that at once divides into a smaller anterior and a larger posterior division. The anterior branch is mostly motor, and the posterior sensory.

From the trunk before its division a filament, the recurrent nerve, passes into the skull through the foramen spinosum with the middle meningeal artery; and a muscular branch to the internal pterygoid, which enters the deep surface of the muscle.

Branches of the Anterior Division.

(I) The deep temporal nerves. Usually two in number, anterior and posterior, enter the deep surface of the temporal muscle. (2) The masseteric. Passes through the sigmoid notch with the masseteric branch of the internal maxillary artery and terminates in the masseter muscle. (3) The external pterygoid nerve to that muscle. The buccal nerve comes forward between the two heads of the external pterygoid muscle, appears in the face issuing from under the anterior border of the ramus of the jaw and lying upon the buccinator muscle. The nerve is distributed to the skin and mucous membrane of the cheek.

Branches of the Posterior Division.

(I) The auriculo-temporal nerve. See page 26.

(2) The inferior dental nerve. This is the largest branch 
of the posterior division. It descends behind (usually internal to) the external pterygoid muscle, passes between the internal lateral ligament and ramus of the lower jaw, gives off its mylohyoid branch, and enters the inferior dental foramen. Its course is then through the inferior dental canal, supplying branches to the bone and teeth, until the mental foramen is reached, when it comes forward through it and terminates in the muscles and integument of the front of the chin and the mucous membrane and skin of the lower lip. The inferior dental nerve has the inferior dental branch of the internal maxillary artery accompanying it in its course.

The mylohyoid nerve passes downward and forward, grooving the inner surface of the lower jaw and lying upon (beneath) the mylohyoid muscle, to which it gives branches, and terminates in the anterior belly of the digastric muscle. See page 8I.

It is accompanied in the latter part of its course by the submental branch of the facial artery.

(3) The lingual or gustatory nerve. Next in size to the inferior dental. Its course is the same as the inferior dental, lying internal to and in front of it, until the inferior dental foramen is reached. Here the lingual nerve continues forward between the internal pterygoid muscle and the ramus of the lower jaw, then upon the mylohyoid muscle close to its origin, then between the mylohyoid and hyoglossus above the deep portion of the submaxillary gland (see page 86). It joins Wharton's duct on its lower side and passes with it to the tip of the tongue.

Near its origin the lingual nerve is joined by the chorda tympani, and below the external pterygoid muscle is connected to the inferior dental nerve by a small branch. 
It furnishes filaments to the submaxillary ganglion and hypoglossal nerve (at its terminus).

The lingual supplies the mucous membrane of the side of the tongue and the floor of the mouth, and the sublingual gland.

\section{The Otic Ganglion.}

This small ganglion (one-sixth of an inch in longest dimension) lies internal to the inferior dental nerve, in front of the middle meningeal artery, and close under the foramen ovale.

Its roots are sensory (and motor), from inferior maxillary (through the internal pterygoid) nerve; motor, from the small (lesser) petrosal nerve (which brings fibres from the seventh and ninth nerves); and sympathetic, from the plexus on the middle meningeal artery. Its branches of distribution are supplied to the tensor palati and tensor tympani muscles, to the parotid gland (by way of the auriculotemporal nerve with which these filaments pass), and to the chorda tympani.

The Submaxillary Ganglion. Fig. I 8.

The submaxillary ganglion lies between the lingual nerve, Wharton's duct, and the anterior portion of the submaxillary gland. It is about the size of the head of a common pin. It receives its motor root from the chorda tympani, the sensory root from the lingual, the sympathetic from the plexus upon the facial artery. Its filaments are distributed to the submaxillary gland, Wharton's duct, and filaments to the lingual nerve which pass on to the sublingual gland and the tongue.

\section{The Sublingual Gland.}

This is the smallest of the salivary glands. It lies immediately under the mucous membrane of the mouth, 
and in the sublingual fossa of the inferior maxilla. It rests upon the mylohyoid muscle, and has the geniohyoglossus muscle and the opposite gland at the inner side. Behind, it comes in contact with the deep lobe of the submaxillary gland.

Its main duct is called the duct of Rivinus. It accompanies Wharton's duct and opens with it upon the top of the same papilla in the mouth.

Numerous smaller ducts from the anterior portion of the gland open upon the floor of the mouth.

The blood supply is from the sublingual (from the lingual) and the submental (from the facial).

The nerve supply is from the gustatory (lingual) and sympathetic.

\section{The Internal Maxillary Artery.}

The internal maxillary artery is the larger branch of bifurcation of the external carotid. It is given off behind the neck of the lower jaw within the parotid gland.

It follows a tortuous course forward and inward, passing between the neck of the jaw and the internal lateral ligament, then upward and forward across the front (external surface) of the external pteryoid muscle, then turns into the sphenomaxillary fossa, where it divides into its terminal branches. The course of the artery divides it into three portions, the maxillary, behind the neck of the jaw; the pterygoid, in connection with the pterygoid muscle; and the sphenomaxillary, within this fossa.

Branches of the Internal Maxillary.-Maxillary portion or first portion.

(I) The deep auricular. A small branch to the anterior wall of the external meatus.

(2) The tympanic branch, a very slender artery which 
passes upward to the Glaserian fissure, and through this to the tympanum.

(3) The middle meningeal. The largest and most important branch of the internal maxillary artery. It arises from the upper surface of the artery, in its situation between the neck of the jaw and the internal lateral ligament, runs upward behind the external pterygoid muscle, then between the two roots of the auriculotemporal nerve, and enters the foramen spinosum in the sphenoid bone. For the course of the artery within the skull, see page 44 .

(4) The small meningeal. Often a branch from the middle. Follows upward the inferior dental nerve and disappears through the foramen ovale. It supplies the Gasserian ganglion, the cavernous sinus, and the adjacent dura.

(5) The inferior dental artery. This accompanies the nerve of the same name in its course through the inferior dental canal, and appears through the mental foramen as the mental artery.

Before it enters the inferior dental canal the artery gives off a branch (lingual) which follows the lingual nerve, and another (the mylohyoid) which runs forward with the mylohyoid nerve.

Pterygoid, or second portion :-

(I) The masseteric. Reaches the deep surface of the masseter muscle through the sigmoid notch of the jaw, and in company with the corresponding nerve to the muscle.

(2) The deep temporal, anterior and posterior, are distributed to the under surface of the temporal muscle. They and the deep temporal nerves will be found together.

(3) The buccal. Follows the buccal nerve downward and forward to supply the buccinator muscle and the skin and mucous membrane of the cheek. 
The sphenomaxillary, or third portion :-

(I) Branches which supply the molar and bicuspid teeth, mucous membrane of the antrum, and twigs to the gums, given off from the internal maxillary upon the back of the superior maxilla.

(2) The infra-orbital. This runs forward with the superior maxillary nerve through the infra-orbital canal, and appears upon the face through the infra-orbital foramen, where it breaks up into branches to the surrounding muscles and integument of the upper lip, lower eye-lid, and to the lachrymal sac.

Within the canal it supplies orbital branches to inferior oblique and rectus muscles; the anterior dental, to the canine and incisive teeth and mucous membrane of the antrum; and the nasal branch to the mucous membrane of the nose.

(3) The superior palatine artery. Its course is down through the posterior palatine canal, along the roof of the mouth, and up through Stenson's foramen into the nose to anastomose with the nasopalatine artery. It supplies the soft palate, tonsils, and mucous membrane of the mouth.

(4) The Vidian artery. Runs backward through the Vidian canal, supplying the Eustachian tube and roof of pharynx.

(5) The pterygopalatine. A twig through the pterygopalatine canal to the roof of the pharynx.

(6) The nasal, or nasopalatine. Passes through the sphenopalatine foramen to supply the ethmoidal cells, frontal sinus, and antrum. The continuation of the artery extends forward and downward upon the vomer and terminates by anastomosing with the anterior terminus of the superior palatine artery, through Stenson's foramen. 
The Stylohyoid Ligament.

This is the fibrous band or cord which connects the tip of the styloid process with the lesser cornu of the hyoid bone. It serves to hold the latter in position.

\section{The Pterygomaxillary Ligament.}

This consists of the fibrous interval between the buccinator muscle in front and the superior constrictor muscle behind, and extends from the tip of the hamular process of the internal pterygoid plate to the posterior extremity of the mylohyoid ridge.

\section{The Stylomaxillary Ligament.}

This is that portion of the deep cervical fascia which extends from the front of the styloid process to the posterior border of the ramus and angle of the inferior maxillary bone. It separates the masseter muscle and parotid gland externally from the internal pterygoid muscle and submaxillary gland internally.

\section{Pterygoideus Externus.}

Origin.-Upper head, from the under surface of the great wing of the sphenoid bone between the foramen ovale and spinosum and the pterygoid ridge.

The lower head, from the outer surface of the external pterygoid plate.

Insertion.-Into the front of the neck of the condyle of the lower jaw, and the interarticular fibrocartilage of the temporomaxillary articulation.

Nerve Supply._The inferior maxillary nerve.

Action.-To draw the interarticular cartilage and the condyle of the jaw forward (and inward). If both muscles act, the jaw is projected directly forward; when one acts, the jaw is turned to the opposite side ; if they act.alternately, the jaw is moved from side to side, as in the grinding movement. 
Pterygoideus Internus. Fig. I 2.

Origin.-From the pterygoid fossa, tuberosities of the superior maxillary, and palate bones.

Insertion.-Into the inner surface of the ramus and angle of the lower jaw below the inferior dental foramen.

Nerve Supply. - The inferior maxillary.

Action.-To close the jaw, to draw it forward, to assist in the grinding movements.

\section{DISSECTION.}

Clean the constrictor muscles, and remove the prevertebral fascia from the muscles it covers.

\section{The Pharynx.}

This is a fibromuscular cavity situated at the base of the skull, behind the nasal (above) and buccal (below) cavities and larynx, and in front of the spinal column.

Its length is four and one-half inches from the base of the skull to the level of the cricoid cartilage opposite the fifth cervical vertebra. Its cavity is flattened from before backward, and at its lower portion becomes obliterated by the contact of its anterior and posterior walls (except during swallowing).

Into it open the posterior nares (2), the Eustachian tubes (2), the mouth (I), the larynx (I), and the œsophagus (I), (total 7). The soft palate projects into it from the front and divides its cavity into the nasal (above) and the buccal (below) portions.

Its walls are composed of mucous membrane, fibrous tissue (pharyngeal aponeurosis), and muscles (the three constrictors, stylopharyngeus, and palatopharyngeus.)

The pharynx is attached above, to the base of the skull (base of sphenoid, petrous portion of temporal and Eustachian tube, and internal pterygoid plate); in front, to the 
sides of the posterior nares, mouth, and larynx; behind, it rests upon the prevertebral fascia; and below, is continued into the œsophagus. For the Esophagus, see Thorax.

\section{The Pharyngeal Plexus.}

This is a loose meshed plexus of fine nerve filaments formed upon the outer surface of the middle and inferior constrictor muscles by nerve fibres from the glossopharyngeal, pneumogastric, and superior cervical ganglion.

\section{Inferior Constrictor.}

Origin.-From the side of the cricoid cartilage, from the inferior cornu, the oblique line, and the upper border of the thyroid cartilage.

Insertion.-Into the fibrous raphe of the pharynx. Its lower fibres pass horizontally, the upper, obliquely upward, backward, and inward.

The recurrent laryngeal nerve passes upward under the lower border of the muscle, and the superior laryngeal perforates the thyrohyoid membrane above the upper border of the muscle.

\section{Middle Constrictor.}

Origin.-From both cornua of the hyoid bone and the stylohyoid ligament.

Insertion.-Into the median raphe of the pharynx. Its fibres run to the insertion; the lower, downward; the middle, horizontally; the upper, upward. It is overlapped below by the inferior constrictor and in turn overlaps the superior above.

\section{Superior Constrictor.}

Origin.-From the side of the tongue and mucous membrane, from the posterior portion of the mylohyoid ridge, the pterygomaxillary ligament, and the hamular process and lower portion of the internal pterygoid plate. 
Insertion.-Into the median raphe and the pharyngeal spine on the basilar process of the occipital bone.

The stylopharyngeus muscle passes to its insertion between the middle and superior constrictor muscles.

\section{The Sinus of Morgagni}

Is the semilunar space left between the upper border of the superior constrictor muscle and the base of the skull. It is covered by the pharyngeal aponeurosis, and through it passes the Eustachian tube and levator palati muscle.

Nerve Supply.-The three constrictors are supplied from the pharyngeal plexus, the inferior in addition by the recurrent laryngeal.

Action.-The constrictors act in rotation from above downward in the act of swallowing. Their action is inhibited, or reversed in vomiting.

Rectus Capitis Anticus Major.

Origin.-From the anterior tubercles of the transverse processes of the third to sixth cervical vertebræ, inclusive.

Insertion.-Into the under surface of the basilar process of the occipital bone.

Nerve Supply.-First and second cervical nerves.

Action.-To flex the head.

Rectus Capitis Anticus Minor.

Origin.-From the base of the transverse process of the atlas.

Insertion.-Into the basilar process of the occipital bone, behind the anticus.

Nerve Supply.-The first cervical nerve.

Action.-To flex the head.

Rectus Capitis Lateralis.

Origin.-From the upper surface of the transverse process (lateral mass) of the atlas. 
Insertion.-Into the jugular process of the occipital bone. Nerve Supply.-First cervical nerve. Action.-To flex the head laterally.

\section{Longus Colli.}

The vertical portion.

Origin.-From the sides of the bodies of the sixth and seventh cervical, and the first, second, and third dorsal vertebræ.

Insertion.-Into the sides of the bodies of the second, third, and fourth cervical vertebræ.

The upper oblique portion.

Origin.-From the anterior tubercles of the transverse processes of the third, fourth, and fifth cervical vertebræ.

Insertion.-Into the tubercle on the anterior arch of the atlas.

The lower oblique portion.

Origin.-From the sides of the bodies of the first, second, and third dorsal vertebræ.

Insertion.-Into the anterior tubercles of the transverse processes of the fifth and sixth cervical vertebræ.

Nerve Supply.-Filaments from the anterior divisions of the cervical nerves.

Action.-To flex the neck. If one acts alone, to flex the neck forward and laterally, and slightly rotate it.

The further dissection of the pharynx and the dissection of the larynx and nasal fossæ will be omitted until the back of the neck has been completed.

See page I4I for the directions and descriptions. 


\section{THE HEAD AND NECK, Posterior.}

LANDMARKS. Fig. 76 .

In the middle line is the inion-external occipital protuberance; outward from that extend the superior curved lines of the occipital bone to the posterior part of the mastoid processes.

Along the middle line of the neck is a depression between the two muscular masses, in which can be felt the spinous processes of the cervical vertebræ, especially the last, or vertebra prominens. The ligamentum nuchæ lies also in the central depression.

The spines of the three or four upper dorsal vertebræ can be felt.

The lower limit to the region of the neck is marked by the spine of the scapula and its acromial process.

\section{DISSECTION.}

Incisions.-(I) Continue the median incision (over the top of the head) down the back of the neck to midway between the shoulders.

(2) Make a transverse incision out along the spine of the scapula to the point of the shoulder to meet the similar anterior incision. This last incision is the same as number 2 , page 360 .

Remove the integument entirely, being careful not to take off too much of the dense fascia which underlies it.

The Superficial Fascia of the back of the neck and thorax.

This is a dense layer of brawny fascia, reaching as low down as the middle of the back, and upward into the base of the head.

Its fasciculi are very strong and the interstices filled with granular fat. Inflammation beneath this fascia produces very marked effects. Boils and carbuncles find their favorite seat here. 


\section{The Deep Cervical Fascia.}

This fascia is described at page 136.

\section{DISSECTION.}

Remove the superficial and deep fasciæ, exposing the trapezius muscle, the occipital artery, vein, and the cutaneous nerves, the larger of which are the internal cutaneous branch of the third cervical nerve, the great occipital, and the small occipital (already found on the dissection of the anterior of the neck, see page 75$)$.

\section{Trapezius Muscle, see page 362.}

\section{The Cutaneous Nerves.}

(I) The small occipital, described on page 90.

(2) The great occipital. Figs. 76 and 77.

The great occipital nerve is the internal branch of the posterior division of the second cervical nerve. The external branch of this division is a small nerve which supplies the inferior oblique, complexus, and trachelomastoid. The internal branch passes upward and inward and backward across the lower border of the inferior oblique, then through the complexus at its middle and inner thirds, and about one inch below the superior line of the occipital bone, then it pierces the trapezius close to this curved ridge and spreads out into branches, which accompany the branches of the occipital artery and supply the greater part of the back of the head. The great occipital communicates beneath the complexus muscle with the cervical nerves above and below, forming a loose plexus called the posterior cervical plexus of Cruveilheir, and upon the back of the head with the small and third occipital nerves.

(3) The third occipital nerve. This is the internal branch of the posterior division of the third cervical nerve. It is much smaller than the great occipital, and takes a course below and internal to that nerve, passing through the complexus (biventer), and, emerging at the inner 
margin of the trapezius, supplies the integument over the occipital protuberance.

The remaining branches of the posterior divisions of the occipital nerves are small. The internal branches supply the integument of the back of the neck near the middle line. The external branches are distributed to the muscles of this region.

The Occipital Artery. Figs. 76 and 77.

It is found at this stage of the dissection as it emerges from under the inner border of the splenius capitis muscle, between the sternomastoid and trapezius muscles close to the occipital bone. Its course is upward over the back of the head, breaking up into branches as it goes, to finally anastomose with the posterior temporal, posterior auricular, and the opposite occipital.

Its deeper course, from where its dissection stopped (page 95) as it lay between the rectus capitis lateralis and posterior belly of the digastric, is backward between those muscles to the occipital groove on the mastoid process of the temporal bone, then along the insertion of the superior oblique muscle and complexus, being covered by the sternomastoid, splenius capitis, trachelomastoid, and posterior belly of the digastric in this part of its course.

The artery gives off numerous small branches to the muscles, and one of considerable size, the princeps cervicis, which courses downward beneath the splenius and complexus muscles to form an anastomosis with the profunda cervicis. See latter, page I 35. The preceding is usually called the deep branch of the princeps cervicis. A small branch of the princeps cervicis perforates the splenius and forms an anastomosis with the superficial cervical branch of the transverse cervical. See page I I 2 . 
Besides the muscular branches, the artery supplies the integument of the back of the head; the cutaneous branches are accompanied by the cutaneous filaments from the occipital nerve.

\section{DISSECTION.}

Divide the trapezius half an inch from its attachment to the occipital bone, and along the median line (to meet the one dissecting it from below), and turn the muscle downward and outward over the shoulder.

Splenius Capitis et Colli. Figs. 76 and 77 .

Origin.-From the lower half of the ligamentum nuchæ, the spinous processes of the seventh cervical, and five or six upper dorsal vertebræ and the supraspinous ligament.

Insertion.-By two portions. The "capitis" portion, into the outer third of the superior curved line of the occipital bone and the mastoid process of the temporal bone. The "colli" portion, into the posterior tubercles of the three or four upper cervical vertebræ.

Nerve Supply.-The external branches of the posterior divisions of the cervical nerves.

Action.-When both act, they extend the head and neck; if one acts, it rotates and flexes the head and neck laterally, to the same side.

Levator Anguli Scapulæ. See Upper Extremity. Posterior Scapular Artery. See page I I 3 .

\section{DISSECTION.}

Sever the splenius along its cranial and spinal attachment and turn the muscle inward.

Trachelomastoid.

Origin.-From the articular processes of the three or four lower cervical and the transverse processes of the four or five upper dorsal vertebræ.

Insertion.-Into the mastoid process of the temporal bone. 
Nerve Supply. - The external branches of the posterior divisions of the cervical nerves.

Action.-To extend the head and neck, if both act; to flex the head and neck laterally and rotate them to the same side, if one acts.

Complexus. Fig. 76 .

Origin.-The transverse processes of the upper six dorsal and seventh cervical vertebræ, from the articular processes of the lower three or four cervical vertebræ.

Insertion.-Into the surface of the occipital bone between the inner halves of the superior and inferior curved lines.

The inner third of the complexus is also called the biventer cervicis, inasmuch as its muscular portions are separated by a median tendon.

Nerve Supply.-The upper three or four cervical nerves, through their posterior divisions.

Action.-To extend the head when both act. If one acts it flexes the head laterally.

\section{DISSECTION.}

Divide the complexus and the trachelomastoid at their cranial attachments, and the levator anguli scapulæ at its spinal attachments. The rhomboids and the serratus posticus superior should have been removed by this time by the one dissecting the upper extremity. Carefully remove the dense fascia which covers the muscles forming the suboccipital triangle and fills in the space between them. In dissecting off the fascia covering the suboccipital triangle and the deep muscles of the neck, save the princeps cervicis and the profunda cervicis arteries, and recognize their anastomosis with each other and with the vertebral artery through the triangle. Look for the suboccipital nerve and vertebral artery within the triangle.

The Suboccipital Triangle. Fig. 19.

This triangle is formed by the superior oblique externally, the inferior oblique below, and the rectus capitis posticus major internally. 


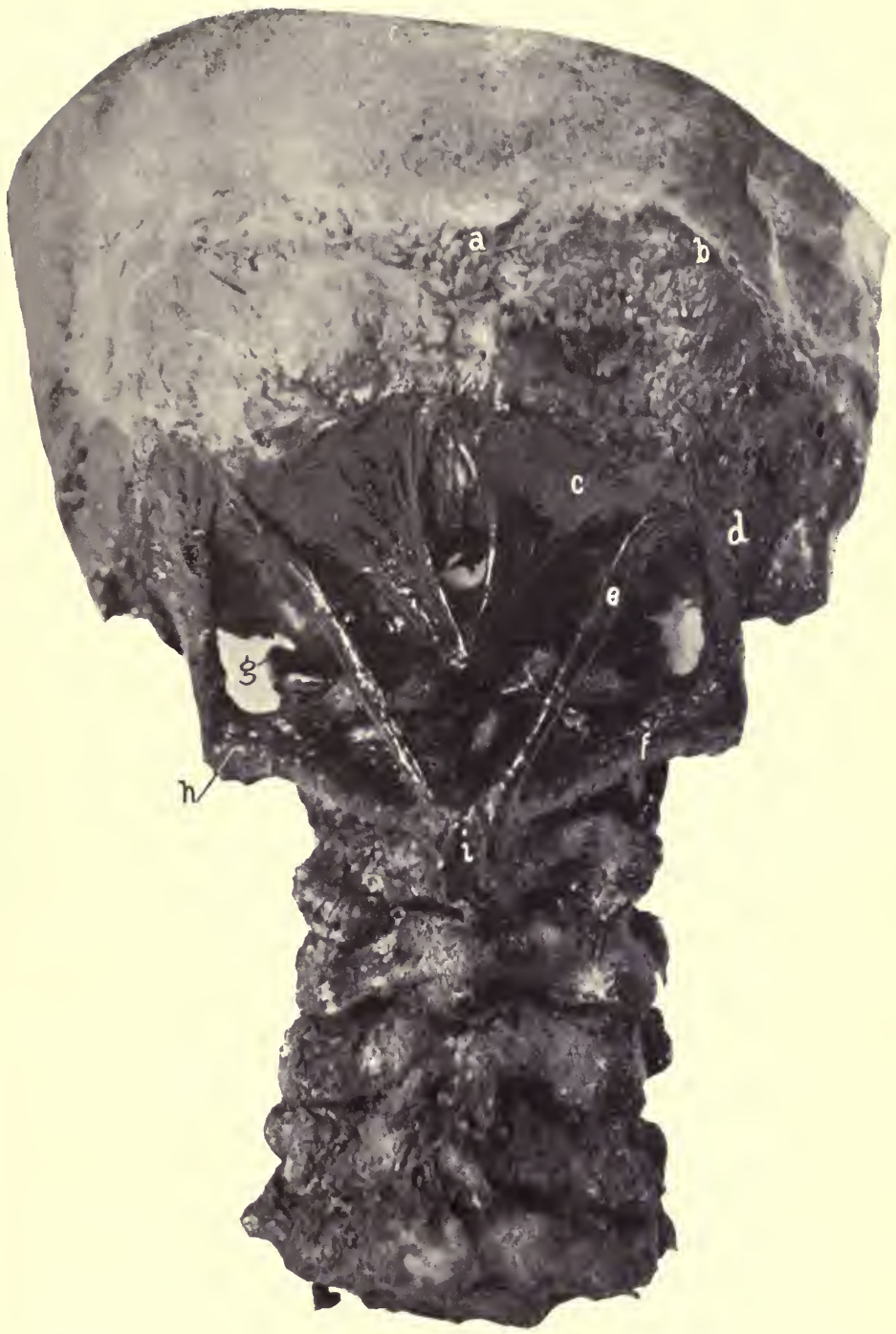

Fig. 19. The Suboccipital Triangle. [From a specimen in the museum of the University Medical College.]- $a$, External occipital protuberance. $b$, Superior curved line. $c$, Rectus capitis posticus minor. $d$, Obliquus superior. $e$, Rectus capitis posticus major. $f$, Obliquus inferior. $g$, Vertebral artery winding around the superior articular process of the atlas. $h$, Transverse process of the atlas. $i$, Spinous process of the axis. 
Within it are found the suboccipital nerve, the vertebral artery, and their branches. Its floor is formed by the posterior arch of the atlas and the occipito-atlantal ligament.

Obliquus Capitis Superior. Fig. 19.

Origin.-From the upper surface of the transverse process of the atlas.

Insertion.-Into the occipital bone between the outer halves of the curved lines.

Nerve Supply.-The suboccipital.

Action.-To extend and slightly flex the head laterally.

Obliquus Capitis Inferior. Fig. 19.

Origin.-From the side of the spine of the axis.

Insertion.-Into the lower, posterior surface of the transverse process of the atlas.

Nerve Supply.-The suboccipital.

Action.-To rotate the atlas and head upon the axis; to slightly flex the atlas upon the axis.

Rectus Capitis Posticus Major. Fig. 19.

Origin.-From the spinous process of the axis.

Insertion.-Into the middle third of the inferior curved line of the occipital bone.

Nerve Supply.-The suboccipital.

Action.-To extend the head upon the neck when both act; when one acts, in addition to the extension, it rotates the head to the same side.

Rectus Capitis Posticus Minor. Fig. I9.

Origin.-From the side of the tubercle upon the posterior arch of the atlas.

Insertion.-Into the inner third of the inferior curved line of the occipital bone, and the depression anterior to it.

Nerve Supply.-The suboccipital nerve.

Action.-To extend the head. 
Semispinalis Colli. Fig. 76 .

Origin.-From the transverse processes of the five or six upper dorsal vertebræ.

Insertion.-Into the spinous processes of the second to the sixth cervical vertebræ.

Nerve Supply.-The internal branches of the posterior divisions of the lower cervical nerves.

Action.-Both acting, they extend the cervical vertebræ. One acting, extends, and rotates the neck to the opposite side.

Transversalis Colli. Fig. 76 .

Origin.-From the transverse processes of the five or six upper dorsal vertebræ.

Insertion.-Into the posterior tubercles of the transverse processes of the cervical vertebræ from the second to the sixth.

Nerve Supply.-The external branches of the posterior divisions of the lower cervical and upper dorsal nerves.

Action.-Both, extend the neck; one, rotate the cervical vertebræ to the same side and flex them laterally.

Cervicalis Ascendens. Fig. 76.

Origin.-From the four or five upper ribs near their angles.

Insertion.-Into the transverse processes of the fourth, fifth, and sixth cervical vertebræ.

Nerve Supply.-The external branches of the posterior divisions of the lower cervical and upper dorsal nerves.

Action.-To extend the lower cervical vertebræ, to raise the ribs, and so aid inspiration; one acting, to flex the lower cervical vertebræ laterally.

The Deep Cervical Artery (Profunda Cervicis). See page I 3 for source. 
It appears in the dissection of the posterior region of the neck, under the complexus and upon the semispinalis; its course is vertically upward to anastomose at the suboccipital triangle with the deep branch (continuation) of the princeps cervicis of the occipital. The artery in its course enters into anastomoses with branches of the vertebral.

The Deep Cervical Vein (Profunda Cervicis Vein).

This begins in the suboccipital triangle by the confluence of smaller veins from the surrounding parts. It descends along the course of the deep cervical artery, and opens into the innominate or vertebral veins.

The Vertebral Vein. See page I I I.

In the suboccipital triangle the deep cervical and vertebral veins freely communicate.

The vertebral receives tributaries from within the spinal canal, the vertebræ, and muscles.

\section{The Suboccipital Nerve.}

The posterior division of the first cervical nerve passes backward between the vertebral artery and the posterior arch of the atlas, then through the suboccipital triangle to the deep surface of the complexus muscle.

It supplies the muscles forming the triangle, the rectus capitis posticus minor, the complexus, and sends a twig to the great occipital nerve.

The Deep Cervical Fascia.

The deep cervical fascia consists of the membranous sheaths of muscles, the covering of glands (capsules), the firm supporting encasement of vessels and the thinner ones of nerves, and the connecting processes (or ligaments) which join the detached to the fixed parts of the head and neck. 
All these separate portions belong to the same filling of connective tissue, and are grouped under the name of the deep cervical fascia.

These parts are continuous with one another, and are only divisions of the general connective tissue of the neck,

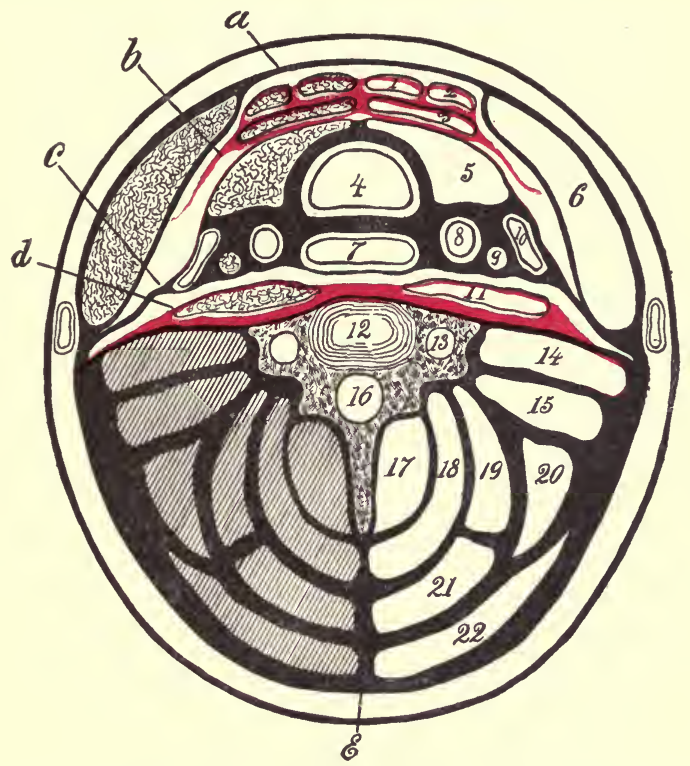

Diag. 9. A Diagram of the Cervical Fascia below the Hyoid Bone. (Modified from Gray.)-I, Sternohyoid. 2, Omohyoid. 3, Sternothyroid. 4, Trachea. 5, Thyroid gland. 6, Sternomastoid. 7, CEsophagus. 8. Common carotid. 9, Pneumogastric nerve. Io, Internal jugular. I1, Longus colli. I2, Body of the sixth cervical vertebra. 13, Vertebral foramen. 14, Scalenus anticus and rectus capitis anticus major. 15, Scalenus medius and posticus. 16, Vertebral canal. 17, Semispinalis colli. 18. Complexus (and biventer). 19, Splenius colli. 20, Levator anguli scapulæ. 21. Splenius capitis. 22, Trapezius. $a$, First layer enclosing sternomastoid. $b$, Second layer enclosing the subhyoid muscles. $c$, Third layer enclosing the vessels, thyroid gland, trachea, and œsophagus. $d$, Fourth (prevertebral) layer. $e$, Ligamentum nuchæ.

so in order to properly describe it, to have a starting point, its description must be taken up in parts and according to an arbitrary classification. 
The fascia is described in several layers.

(I) The first layer of the cervical fascia.

This starts behind from the spinous processes of the cervical vertebræ (through the ligamentum nuchæ), encloses the trapezius, receives a membranous expansion from the transverse processes of the cervical vertebræ at the side of the neck, passes forward to the posterior border of the sternomastoid muscle, splits into two layers which enclose the muscle and unite at its anterior margin, passes onward and becomes continuous at the middle line with the similar layer from the other side.

Above, this first layer is attached to the lower border of the jaw (from its angle being continued to the styloid process of the temporal bone forming the stylomaxillary ligament), and passing over the masseter muscle and parotid gland as the masseteric and parotid fascias, is attached to the lower border of the zygoma. Behind the ear this layer is attached to the superior curved line of the occipital bone and the mastoid process of the temporal bone (externally and internally). Below, to the front of the clavicle, outer margin of the acromion process, and the middle portion of the spine of the scapula.

In the middle line the first layer is also fastened to the hyoid bone.

(2) The second layer. This is given off from the inner surface of the first layer along the posterior border of the sternomastoid muscle.

It passes forward, enclosing the omohyoid, sternohyoid, and sternothyroid muscles, and unites in the median line with a similar layer from the other side, also with the first layer.

Above, it reaches to the hyoid bone. Below, it is attached to the inner margin of the manubrium and clavicle. 
It also extends downward to the cartilage of the first rib, this portion forming the loop by which the tendon and posterior belly of the omohyoid muscle is kept in position.

Between layers number one and two is left a triangular space-Burns' space-that has no width above, but below is represented by the space between the posterior margins of the sternomastoid. This space contains fat, lymphatic glands, the anterior jugular vein, and connective tissue to keep them in position.

It is also stated that the second layer is continued under the clavicle, splits to enclose the subclavius muscle, and reunites to form the costocoracoid membrane. That the sheath for the subclavian vein is derived from this second layer, while for the artery it comes, as stated below, from the third layer.

(3) The third layer of the cervical fascia. This invests the carotid vessels, arteries, and internal jugular vein, pneumogastric nerve, thyroid gland, trachea, œsophagus, and many other structures. This is also given off from the under surface of the first layer behind the sternomastoid muscle. It passes forward, splitting to enclose the internal jugular vein, pneumogastric nerve, carotid arteries (forming their sheaths), thyroid gland (forming its capsule), the trachea, and œsophagus, and finally unites in the middle line with corresponding layers from the other side.

Besides the structures above enumerated this layer ensheaths all the structures found between the under surface of the second layer and in front of the prevertebral (fourth) layer.

Above, this third layer passes up to the base of the skull and is attached to the basilar and jugular processes of the occipital bone, the petrous process of the temporal bone, the spinous and pterygoid processes of the sphenoid bone. 
Below, it passes along with the great vessels (trachea and œesophagus) into the thorax, forming their sheaths, and becomes continuous with the fibrous layer of the pericardium.

Laterally, this fascia passes outward under the clavicle with the subclavian artery and vein and brachial plexus, forming their sheath. In the axilla it receives the name of that region (axillary sheath).

(4) The fourth or prevertebral layer of fascia. The name indicates its position to be in front of the vertebræ (and their muscles). It lies immediately behind the third layer, and covers over the rectus capitis anticus major and minor, and longus colli muscles.

Above, it is attached to the basilar process of the occipital bone. Laterally, to the inner surface of the first layer along the side of the neck; below, it passes into the thorax behind the œsophagus, and extends into the posterior mediastinum.

While these various layers are described, it should be remembered that they are so described more for convenience and to indicate a general lamination, than to mean that these layers will be found as distinct and separated from each other as the description would indicate. On the contrary, all these layers blend wherever they come in contact, for the deep cervical fascia is only a part of the connectivetissue system of the body, whose function is to connect and support the various organs between which it is developed. Consequently, the student will obtain a more correct view of the cervical fascia (as well of any other) if he will imagine all the muscles, reins, arteries, and nerves of the neck removed, and the sheaths, capsules, and envelopes of these and the connective tissue which bind them all in position only remaining. This will then show that the 
separate parts usually described are only so separated by anatomists for convenience of description, and that such divisions do not exist as separate from the other parts or layers, but that all are united into a complicated whole.

The influence that the cervical fascia has upon determining the course of certain surgical affections, as abscesses and tumors, is a good deal more theoretical than real. It is found in practice that abscesses from cervical caries may descend into the thorax, but usually will point along the lateral region of the neck if above the sixth cervical vertebra. The postpharyngeal abscess will usually point in the pharynx. Abscesses in the lower part of the neck, unless behind the third layer of fascia, will usually point above the clavicle or sternum.

The Pharynx (concluded), Larynx, and Nasal Fossæ.

\section{DISSECTION.}

The dissection of the back of the neck having been completed, the remaining muscles which hold the head to the spine should be severed, and the skull separated from the atlas by the division of the connecting ligaments, or the skull should be sawn transversely across in front of the foramen magnum. The trachea should be separated from the larynx by dividing it just below the cricoid cartilage and the œsophagus cut across at the same level. Now remove the head and attached portions of the pharynx, larynx, and the arteries, veins, and nerves, which emerge from the base of the skull.

Identify all those structures which have been found already in the dissection of the deep neck.

Divide the pharynx along the median raphe and open its cavity.

The Structures seen on Opening the Pharynx.

Posterior nares, separated by the vomer, and within which can be seen the posterior extremities of the middle and inferior turbinated bones.

The opening for the Eustachian tube is behind the lower portion of the posterior nares; it leads into the middle ear. 
The cartilage which constitutes the anterior portion of the Eustachian tube forms a prominent ring around the opening orifice called the tubal eminence.

Behind the Eustachian tube opening is a blind pit, the fossa of Rosenmüller.

Below the posterior nares the soft palate hangs downward and terminates medianly in the uvula. The soft palate serves to shut off the nasal passages from the pharynx in deglutition.

Laterally the free margin of the soft palate is thrown into two prominent arches; the posterior, descending to the pharyngeal wall ; the anterior, to the side of the tongue, leaving a hollow between them. These arches are called the anterior and posterior arches of the fauces, or the anterior and posterior palatine arches. The depression between them is the tonsillar recess, and lodges the tonsil.

The posterior arch is produced by the palatopharyngeus, the anterior by the palatoglossus muscle.

The tonsils are two oval-shaped masses of spongy adenoid tissue, situated within the two palatine arches. Their size in the cadaver is never as large as in the living, consequently their relative size can best be estimated from an examination of the throats of friends.

The roof of the pharynx is occupied by an adenoid mass, the pharyngeal tonsil, which in children sometimes assumes very large proportions.

The base of the tongue is seen below the soft palate. The opening of the mouth posteriorly into the pharynx is called the isthmus faucium. It is bounded by the soft palate above, the faucial arches and tonsils laterally, and the base of the tongue below. Upon the base of the tongue will be seen in the middle line the foramen cæcum, 
in front of this blind depression the "V-shaped" group of circumvallate papillæ.

A third mass of adenoid tissue is seen at the base of the tongue and in front of the epiglottis; this is the lingual tonsil.

Below and behind the base of the tongue stands the epiglottis to protect the opening of the larynx, which presents posterior and inferior to it.

The epiglottis is connected to the base of the tongue by the general covering of mucous membrane which is thrown into three folds, one in the medial line and two laterally, which are called the median (frænum epiglottidis) and lateral glosso-epiglottic folds. On both sides of the frænum epiglottidis is a shallow depression-the glosso-epiglottic pouch.

From the sides of the epiglottis backward to the top of the larynx the mucous membrane is thrown into a prominent ridge, the aryteno-epiglottic fold. The upper margins and cornua of the thyroid cartilage can be easily appreciated.

\section{DISSECTION.}

Remove the mucous membrane which covers the interior of the pharynx, the posterior surface of the soft palate, and the posterior faucial arches.

In dissecting the muscles of the palate and the pharynx (interior), it must be remembered that these are very thin, and their outlines not at all as distinct as the illustrations in the text-books would lead one to think. The student should do the best he can with the aid of such illustrations and the descriptive anatomy to find the various parts.

\section{Palatopharyngeus.}

The thin muscular layer is attached to the soft palate near the median line and arches outward and downward, forming the posterior palatine arch, to be attached below to the posterior border of the thyroid cartilage and the lateral wall of the pharynx. At the soft palate the fibres of the muscle 
are separated by the insertion of the levator palati. The muscle acts to elevate the pharynx and larynx, or to depress the soft palate.

\section{Azygos Uvulæ.}

A few muscular fibres in the median line reaching from the posterior nasal spine to the tip of the uvula. Its function is to elevate the uvula.

\section{Levator Palati.}

This arises from the under surface of the petrous portion of the temporal bone in front of the carotid canal and from the cartilage of the Eustachian tube. It is inserted into the soft palate along the middle line.

The action is to raise the soft palate and close the posterior nares.

\section{DISSECTION.}

Remove the levator palati and the upper portion of the palatopharyngeus. This exposes the tensor palati muscle and the ascending palatine artery.

\section{Tensor Palati.}

Arises from the scaphoid fossa of the sphenoid bone, its tendon winds around the hamular process of the internal pterygoid plate and expands into a second muscular portion, which is attached along the posterior margin of the hard palate and into the median raphé of the soft palate.

For the ascending palatine artery, see Facial, page 95.

\section{DISSECTION.}

Remove the mucous membrane from the anterior pillar of the fauces and expose the palatoglossus muscle which forms this pillar.

\section{Palatoglossus.}

Extends from the anterior surface of the soft palate to the side of the base of the tongue, where it interlaces with the styloglossus muscle. Its action is to draw the sides 
of the soft palate downward and forward, or the tongue upward and backward.

\section{The Eustachian Tube.}

The cartilaginous portion of this tube will be exposed from its anterior part, lying between the levator and tensor palati muscles and giving origin to some fibres of these muscles. The palatopharyngeus also has a small bundle arising from the tube, the salpingopharyngeus.

The opening of the Eustachian tube is in the shape of a vertical slit, and the canal leads into the cavity of the middle ear.

\section{DISSECTION.}

Separate the tongue and larynx from the skull. Remove any remains of the œsophagus. Identify the epiglottis, hyoid bone, thyroid, cricoid, and (upon the top of the cricoid behind) the small arytenoid cartilages. Forward and upward from the arytenoid cartilages pass the aryteno-epiglottic folds to the side of the epiglottis. Between these folds is the upper opening of the larynx.

Looking into this opening the canal of the larynx will be seen to be constricted at two places by two anteroposterior bands. The upper pair are the false vocal cords and the lower pair the true vocal cords.

The slit between the vocal cords is the rima glottidis and varies in width according to the approximation of the vocal cords.

The true vocal cords may be made to approach or diverge from each other by depressing or elevating the thyroid upon the cricoid cartilage, or by rotating the arytenoid cartilages upon the cricoid cartilages.

The description of the cartilages of the larynx is omitted from this manual as being to the larynx what the bones are to the other parts of the body.

External Structures.

Muscles.

\section{Cricothyroid.}

Origin.-From the anterior part of the cricoid cartilage. Insertion.-Into the inferior border of the thyroid cartilage. 
Action.-To approximate the two cartilages and thus make tense the vocal cords.

Posterior Crico-arytenoid.

Arises from the quadrilateral posterior surface at the side of the median ridge of the cricoid cartilage.

It is inserted into the outer angle of the base of the arytenoid cartilage. The action is to slacken the vocal cords and widen the rima glottidis.

\section{Arytenoideus.}

Crosses from one arytenoid cartilage to the other. In action it draws together the arytenoid cartilages, thus narrowing the rima glottidis.

\section{Aryteno-epiglottidis.}

The name applied to a small bundle of fibres connecting the arytenoid cartilage with the aryteno-epiglottic fold. It is a part of the arytenoideus.

Arteries passing into the larynx.

The superior laryngeal branch of the superior thyroid and the inferior laryngeal branch of the inferior thyroid artery.

Nerves of the larynx.

The superior and inferior laryngeal branches of the pneumogastric.

\section{DISSECTION.}

Remove these external muscles, clean the fibrous membranes connecting the thyroid cartilage to the hyoid bone (thyrohyoid membrane), and that uniting the cricoid and thyroid cartilages (cricothyroid membrane). These membranes have to be divided in the operations of opening the larynx or the larynx and trachea.

Remove one-half of the larynx.

Above the false vocal cord is the fossa innominata ; between the false and true cords the ventricle.

Muscles of the Interior of the Larynx. 


\section{Lateral Crico-arytenoid.}

From the lateral upper border of the cricoid cartilage, into the muscular process of the arytenoid cartilage. The action is to approximate the vocal cords by drawing the arytenoid cartilages together.

\section{Thyro-arytenoid.}

By a vertical origin from the inner surface of the thyroid cartilage near the median line. It is attached posteriorly into the base and side of the arytenoid cartilage. The muscle consists of two portions, an upper and a lower. The lower portion lies parallel with the true vocal cord and some of its fibres pass into it.

ACTION OF THE MUSCIES MOVING THE VOCAL CORDS. (From Holden.)

Cricothyroidei, . . . . . To stretch the vocal cords.

Thyro-arytenoidei, . . . To relax the vocal cords and place them in the vocalizing position.

Crico-arytenoidei postici, . . Dilate the glottis.

Crico-arytenoidei laterales, . . Draw together the arytenoid cartilages.

Arytenoideus, . . . . . Draw together the arytenoid cartilages.

Aryteno-epiglottidei, . . . . Contract the upper opening of the larynx.

Nerve Supply for the Muscles of the Larynx.

The external branch of the superior laryngeal supplies the cricothyroid. The inferior laryngeal nerve supplies all the remaining muscles of the larynx.

The muscles connecting the epiglottis with the thyroid cartilage (thyro-epiglottideus, with the aryteno-epiglottideus superior and inferior) are thin bands of muscular fibres which need not detain the general dissector. 
The Nasal Fossæ. Figs. I 2, I 3.

\section{DISSECTION.}

Take the lower segment of the skull and divide it with a saw anteroposteriorly just at one side of the vomer.

Study the nasal fossæ. Each nasal fossa is divided by the superior, middle, and inferior turbinated bones into the superior, middle, and inferior meatus.

The nasal duct opens into the anterior part of an inferior meatus, the infundibulum opening of the frontal sinus and anterior ethmoidal cells is in the anterior part of a middle meatus, and at the middle portion is the opening of the maxillary sinus, or the antrum of Highmore.

In the anterior part of the superior meatus is the opening of the posterior ethmoidal cells, and in its posterior portion the opening of the sphenoidal sinus.

It will hardly repay the student to undertake the dissection of the small vessels and nerves which are found upon the walls of the nasal fossa.

Upon the Septum.-The septum is crossed from above downward and forward by the nasopalatine nerve and artery and the septal branch of the nasal nerve.

Each spongy turbinated mass is provided with an artery, which comes from the nasopalatine branches of the internal maxillary artery.

\section{DISSECTION.}

On a dry skull (sawn through anteroposteriorly) locate the sphenopalatine foramen. In the specimen dissect off the mucous membrane over a corresponding area and expose the sphenopalatine foramen and the small artery and nerves it transmits.

Remove the mucous membrane carefully from the nasal fossa under the cribriform plate of the ethmoid and find the nerves and arteries entering at this point.

Through the sphenopalatine foramen the nasopalatine or sphenopalatine artery and nerve enter the nasal fossa. They divide into two branches, an external and internal, to the outer wall and the septum (inner wall) of the nose.

The sphenopalatine artery is the terminal branch of the internal maxillary artery and enters the sphenopalatine 
foramen with the sphenopalatine branch of Meckel's ganglion. The artery and nerve are distributed to the outer wall and the septum of the nose. The septal branch of the artery anastomoses in front with the anterior palatine artery, which runs along the roof of the mouth and turns up through the transversely paired foramen (Stenson's).

The nasal nerve (see page $5^{6}$ ) enters the nose through the nasal slit with the anterior ethmoidal artery. The nerve supplies branches to the outer wall and the septum of the nose. The posterior ethmoidal artery enters the nasal cavity posterior to the nasal slit, and with the anterior is distributed to the upper part of the internal and external nasal wall.

\section{DISSECTION.}

With the bone cutters or a chisel carefully remove the bone surrounding the sphenopalatine foramen, the orbital process of the palate, and the body of the sphenoid until the foramen rotundum is reached and the superior maxillary nerve is exposed. Beneath the nerve as it is crossing the sphenomaxillary fossa is the small ganglion of Meckel, or the sphenomaxillary ganglion.

In the sphenomaxillary fossa the internal maxillary artery divides into numerous branches. See page 122 .

After finding the ganglion, its large posterior branch (the Vidian nerve) should be followed back to and through the Vidian canal (by cutting away the bone as necessary).

The various branches of the ganglion will have to be followed in the same way by removing piecemeal the bone covering them.

Finish this specimen by tracing the superior maxillary nerve through the infra-orbital groove and canal and out through the foramen to the exterior of the face, also its dental branches. In this dissection the antrum of Highmore is opened and should be studied from the descriptions of the bones in a dry state.

\section{The Superior Maxillary Nerve. Figs. IO, I I.}

This is the second branch from the front of the Gasserian ganglion of the fifth cranial nerve.

It leaves the skull through the foramen rotundum and enters the sphenomaxillary fossa. Continuing its course 
forward, it lies in the infra-orbital groove, then in the infra-orbital canal, through which it passes, to emerge upon the face at the infra-orbital foramen and supply the mid region of the face with sensory branches.

Branches of the Superior Maxillary Nerve.

(I) Within the skull, one or two recurrent filaments supplying the dura.

(2) In the sphenomaxillary fossa. (a) The orbital or temporomalar nerve. Enters the orbit through the sphenomaxillary fissure, and divides into the temporal and malar branch. The temporal branch passes through the temporal foramen in the malar bone, enters the temporal fossa, passes through it to pierce the temporal fascia above the zygoma and be distributed to the integument of the anterior part of the temporal region. The malar branch passes through the malar foramen of the malar bone to supply the integument over that bone. The posterior superior dental nerve divides into two branches, which descend along the posterior surface of the superior maxillary bone, enter the posterior dental foramina, and supply the molar teeth and mucous membrane adjacent to them. (c) The middle superior dental nerve is given off the superior maxillary in the posterior part of the infraorbital canal, runs downward and forward in a special canal on the outer wall of the antrum to supply the bicuspid teeth. (d) The anterior superior dental nerve is given off at the anterior part of the infra-orbital canal, and descends in a canal on the anterior wall of the antrum to supply the incisive and the canine teeth and gives off the nasal branch to the mucous membrane of the floor and wall of the anterior part of the inferior meatus. The three dental nerves have loops of communication with each other, thus forming the superior dental plexus. (e) The terminal 
branches on the face are four labial (usually), three or four nasal, and two small palpebral branches, supplying in turn the upper lip, side of the nose, and the lower eyelid.

The communications of these branches with the infraorbital branch of the facial nerve forms the infra-orbital plexus. See page 6 I.

Meckel's, the Sphenopalatine, or the Nasal Ganglion. Fig. I I.

This is a small ganglion placed in the sphenomaxillary fossa close to the sphenopalatine foramen and under the superior maxillary nerve, to which it is suspended by two sensory branches forming its sensory root. The motor and sympathetic roots enter the posterior part of the ganglion as the Vidian nerve.

The Vidian nerve is formed by the junction of the great superficial petrosal nerve from the facial with the great deep petrosal branch from the sympathetic plexus on the carotid artery in the carotid canal. The united nerves pass forward through the Vidian canal to terminate in Meckel's ganglion. Branches of the ganglion.

(I) Ascending or orbital branches. Three or more very small filaments which pass to the orbit through the sphenomaxillary fissure and supply the periosteum and mucous membrane of the posterior ethmoidal and sphenoidal sinuses.

(2) Descending branches. (a) The large or anterior palatine nerve. Passes through the posterior palatine canal to the roof of the mouth, which it supplies as far forward as the incisor teeth. This nerve gives off two inferior nasal filaments while in the posterior palatine canal, which pass forward through minute openings to supply the 
mucous membrane of the middle and inferior meatuses and inferior turbinated bone of the nose. (b) The small or posterior palatine nerve traverses the lesser or accessory palatine canal to reach and supply the soft palate, the tonsil and the uvula; also the levator palati and the azygos uvulæ muscles. (c) The external palatine nerve. A very small filament extends through the external palatine canal to supply the tonsil and the soft palate.

(3) The internal branches. (a) The superior nasal branches, six or seven filaments, passing through the sphenopalatine foramen to supply the mucous membrane covering the posterior part of the middle and superior turbinated bones and the posterior ethmoidal cells. (b) The septal branch-nasopalatine, or nerve of Cotunniuscrosses the roof of the nose to the septum, runs downward and forward buried in the mucous membrane of the septum, which it supplies, to terminate after passing through the anteroposterior pair of foramina (Scarpa's) in the mucous membrane of the roof of the mouth. (The left nerve takes the anterior, the right the posterior foramen.)

(4) The posterior branch has already been described as the Vidian nerve. See above.

\section{THE BRAIN.}

\section{The Membranes of the Brain.}

The membranes of the brain are the dura, arachnoid, and the pia.

The dura has already been described. See page 32.

The Subdural Space.-The space between the dura and the following membrane-the arachnoid-is called the subdural space. 


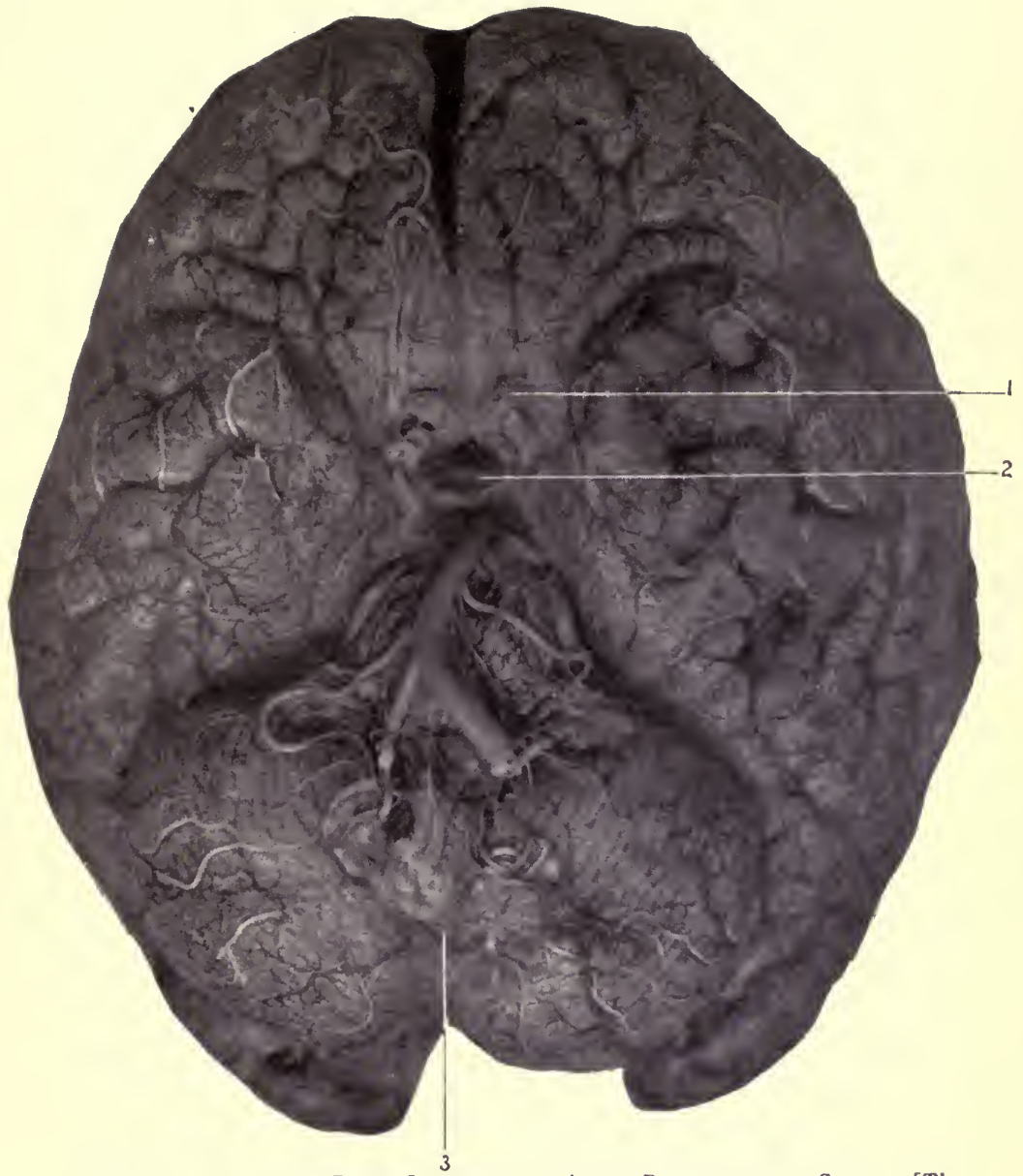

Fig. 20. Base of the Brain Immediately After Removal from Skull. [The slightly hazy appearance is due to the presence of the arachnoid membrane.]

I, Optic nerve.

2, Pituitary gland, which rests upon (as the brain lies base upward) the anterior subarachnoidean space.

3, The posterior subarachnoidean space. For the arteries and nerves consult the following figures. 
The Arachnoid Membrane. Fig. 20.

This is a very thin but distinct membrane which lies within the dura and in turn covers the pia. It is connected to the latter by delicate filaments, especially over the convexity of the brain, called the subarachnoid tissue, hence it is sometimes described as a part of that membrane (pia).

The arachnoid passes smoothly over the surface of the pia and does not follow it into the sulci and fissures, except into the great longitudinal fissure, in order to get from one side to the other beneath the falx cerebri; and into the great transverse fissure, where it has to turn around the tentorium cerebelli.

Over the convexity of the brain the arachnoid lies closely applied to the pia so that it can with difficulty be separated from it and demonstrated, but on the lower surface (base) of the brain it is much thickened and is separated from the pia in two well-marked localities.

These spaces within the arachnoid, between the arachnoid and the pia, are called the subarachnoidean spaces, anterior and posterior.

(I) The anterior subarachnoid space lies at the base of the brain. It reaches forward to the beginning of the great longitudinal fissure, laterally to the inner margins of the temporal lobes of the brain, and posteriorly it covers the pons and medulla, and communicates along the sides of both with the posterior subarachnoid space, and below with the similar cavity surrounding the spinal cord.

Within the anterior subarachnoid space are contained the medulla, the pons and its peduncles, the cerebral crura, the structures at the base of the brain which form the floor of the third ventricle (see page 222), the basilar, internal carotid arteries, and their branches, especially that arterial anastomosis which forms the circle of Willis. 
(2) The posterior subarachnoid space is larger than the anterior. It is formed at the base of the cerebellum by the arachnoid membrane which extends from the posterior part of the cerebellum to the upper surface of the medulla. It communicates with the spinal arachnoid space below, with the anterior subarachnoid space around the sides of the medulla, and with the interior of the brain through the foramina of Majendie, Key, and, Retzius.

The foramen of Majendie is centrally located in the pia which reaches from the under surface of the cerebellum to the upper surface of the medulla, and assists in forming the roof of the posterior part of the fourth ventricle. The opening of Majendie is found close to the medulla and in the middle line. The foramina of Key and Retzius are minute fissures at the extension of the pia into the lateral angles of the fourth ventricle. Other smaller and less important spaces are found (3) Below the falx cerebri and above the corpus callosum, in the great longitudinal fissure. (4) In the fissure of Sylvius. (5) Between the corpora quadrigemina and the anterior extremity of the cerebellum.

The Pacchionian Glands, Arachnoidal Villi.-These are up-growths from the arachnoid along the margins, and even into the cavity, of the superior longitudinal sinus. They look like small masses of granulation tissue. They penetrate the dura and produce corresponding depressions in the inner table of the skull.

The subarachnoid spaces are filled with a lymphatic fluid called the cerebrospinal fluid. This fluid is able to circulate freely from the cord to the exterior of the brain, and even to the interior of the latter through the foramina of Majendie, Key and Retzius. Its usual quantity is about two ounces. 


\section{The Function of the Cerebrospinal Fluid.}

It forms a "water-bed" upon which the brain rests, and is thus protected from injury by coming in contact with the projections of the bones forming the base of the skull. It equalizes the intracerebral pressure, by flowing away from the brain when the blood supply is too great, and toward the brain when it is insufficient.

The nerves to the arachnoid are derived from the fifth, seventh, and eleventh cranial nerves (Bochdaleck). The lymphatics from the subarachnoid spaces (which are really lymph channels) communicate with those of the internal ear, mucous membrane of the nose, and the superior longitudinal sinus; but are not in direct communication with the general lymphatic system of the head.

The subarachnoid spaces receive the lymphatics from the brain (and the spinal portion from) the cord:

\section{DISSECTION.}

Carefully remove the arachnoid covering in the anterior and posterior subarachnoid spaces. It will be useless to attempt to remove it from any other part of the brain.

The dissection of the pia consists in tracing out its vessels. This should be done with great patience; haste means destruction of important parts.

The Pia. Figs. 20 and $2 \mathrm{I}$.

This is the most internal membrane enveloping the brain. It is really a layer of blood-vessels held together by sufficient connective tissue to preserve their relations, which is closely applied to all parts of the outer surface of the brain, reaching into all the fissures and sulci, and even passing into the interior of the brain as the velum interpositum and choroid plexuses of the lateral, third, and fourth ventricles. It is to be noted that these extensions from the general pia, though apparently within the brain, are still really ex- 


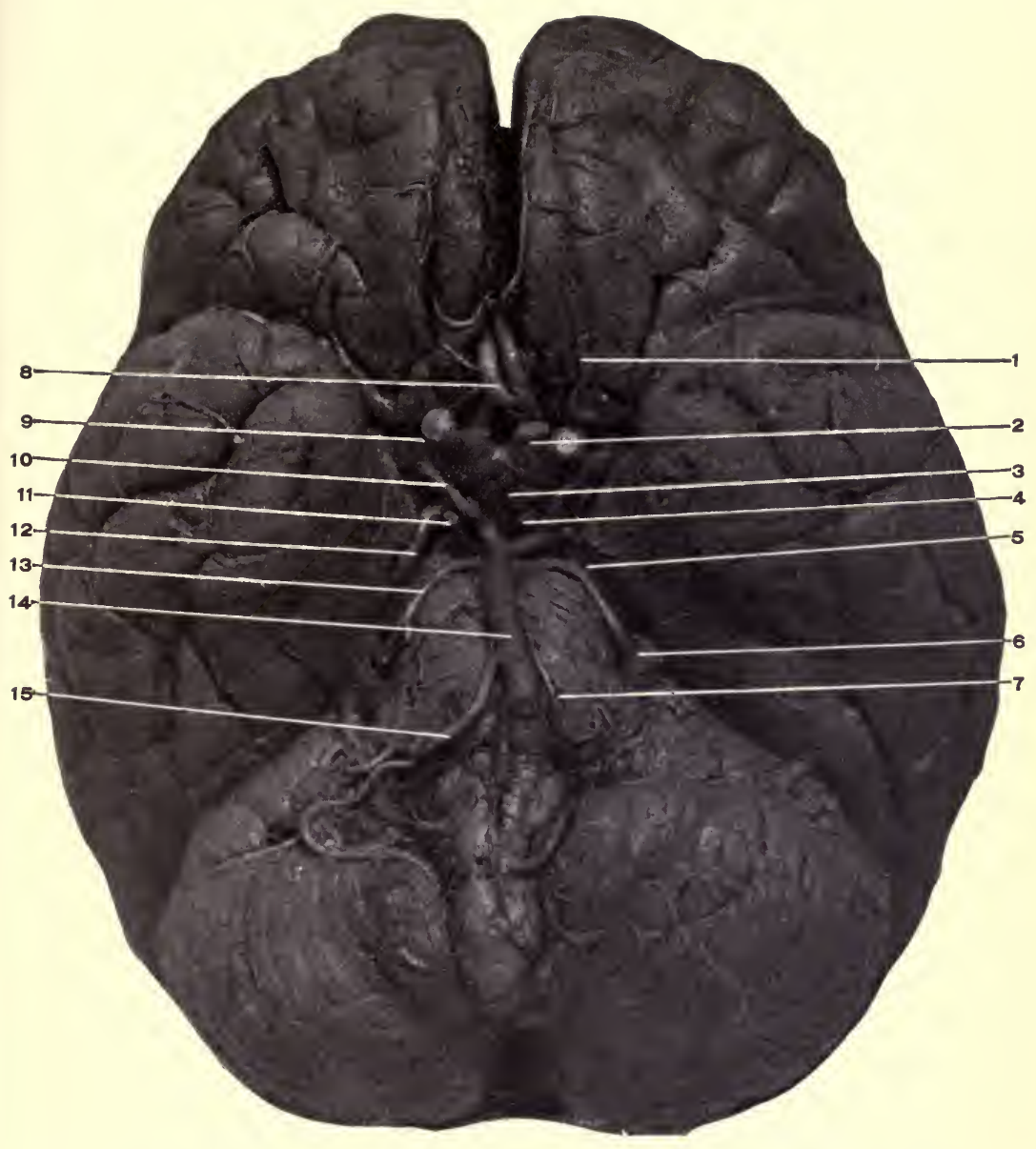

Fig. 21. The Arterial Supply at the Base of the Brain (Circle of Willis).-I, Olfactory nerve. 2, Optic nerve and chiasm. 3, Infundibulum torn off the tuber cinereum. 4, Corpora albicantia. 5, Fourth nerve. 6, Fifth nerve. 7, The sixth nerve. 8 , Anterior cerebral, united by the anterior communicating. 9, Internal carotid. Io, Posterior communicating. II, Third nerve. I2, Posterior cerebral artery. 13, Superior (anterior) cerebellar artery. 14, Basilar artery. I5, Inferior (posterior) cerebellar artery. 
cluded from the central cavities of the brain, as will appear later.

The Blood Supply of the Brain. Figs. 10 and 21 .

The brain receives its supply of blood through the internal carotids and vertebral arteries.

After entering the skull through the foramen lacerum medium the internal carotid artery passes forward through the cavernous sinus as given on page 43. At the anterior part of the sinus the artery turns upward through the dura, behind and internal to the anterior clinoidal process, and reaches the base of the brain at the beginning of the fissure of Sylvius, where it divides into the anterior and middle cerebral vessels.

The vertebrals, after entering the skull through the foramen magnum, are found at the side of the medulla. They converge as they pass forward and unite in the middle line at the junction of the pons and medulla to form the basilar artery.

The basilar artery runs forward in a median depression in the pons to its anterior margin, where it divides into the two posterior cerebral vessels.

The Circle of Willis. Figs. $2 \mathrm{I}$ and 22.

The anterior cerebral arteries pass forward and inward to the beginning of the great longitudinal fissure, which they enter. They continue upward and backward over the corpus callosum, distributing branches to the inner surface of the hemisphere as far as the parieto-occipital fissure (where the terminal branches anastomose with similar branches from the posterior cerebral arteries).

At the beginning of the great longitudinal fissure the anterior cerebral vessels are united by a very short artery 


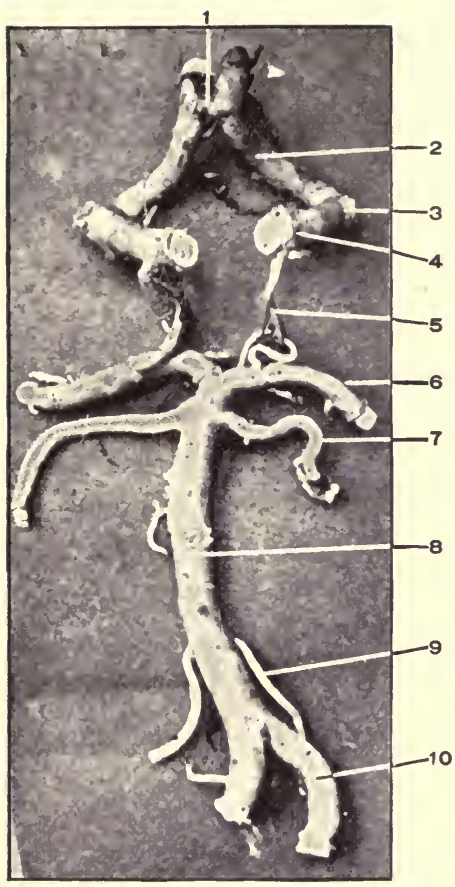

Fig. 22. The Circle of Willis, Natural SizE.-1, Anterior communicating. 2, Anterior cerebral. 3, Middle cerebral. 4, Internal carotid. 5, Posterior communicating. 6, Posterior cerebral. 7, Superior (anterior) cerebellar. 8, Basilar. 9, Inferior (posterior) cerebellar. Io, Vertebral. 
(shortest in the body) termed the anterior communicating.

For the branches of the anterior cerebral, see under the ganglionic and cortical systems.

The middle cerebral arteries follow, outward, upward, and then backward in the fissure of Sylvius, and near its middle part divide into four terminal branches; for their course and distribution see Cortical System.

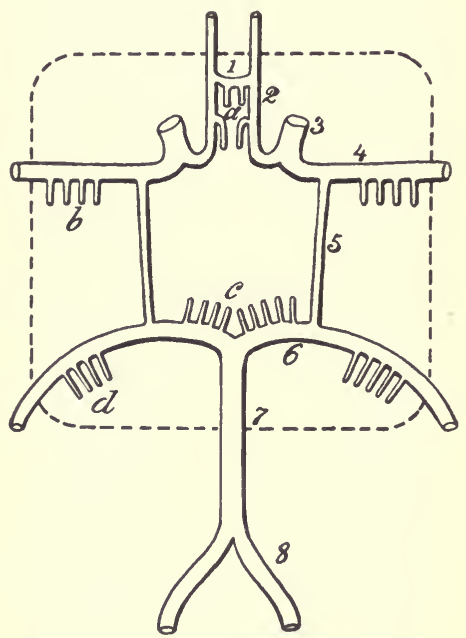

Diag. 10. A Diagram of the Ganglionic Arterial Supply of the Brain. (Charcot, from Gray.)-I, Anterior communicating. 2, Anterior cerebral. 3, Internal carotid. 4, Middle cerebral. 5, Posterior communicating. 6, Posterior cerebral. 7, Basilar. 8, Vertebral. $a$, Anteromedian group. $b$, Anterolateral group. $c$, Posteromedian group. $d$, Posterolateral group.

The posterior cerebral arteries pass outward and then backward around the crura cerebri and supply the inner and under surface of the occipital and temporal lobes.

The middle and posterior cerebrals are joined together in front of the crura cerebri by the posterior communicating arteries. These are usually small vessels; one may be 
very small or wanting, and the other quite large to make up for the deficiency. When both are present the arterial circuit called the circle of Willis is formed. The arteries which form it are the two anterior cerebrals, which are united by the anterior communicating, the two middle cerebrals (or internal carotids), which are joined by the two posterior communicating vessels to the two posterior cerebrals. Within this circle are found the following structures at the base of the brain. The posterior perforated space, third and fourth nerves, the corpora albicantia or mammillaria, the tuber cinereum, infundibulum and pituitary body, the optic chiasm tracts and nerves, and the lamina cinerea.

The function of the circle of Willis is to maintain an even supply of blood to all parts of the brain. The anastomoses of the opposite carotids through the anterior cerebrals and anterior communicating, and of the posterior cerebrals with each other, is so free that no trouble would arise from interference with one carotid or one vertebral.

But the anastomosis of these two systems through the posterior communicating is not so free and constant, owing to the smallness of these arteries, or even the absence of one. As a rule, we find both of these arteries small, rarely find both large, often one may be quite good sized and the other very small or wanting entirely. In cases where there is not a free communication between the anterior and posterior parts of the circle of Willis it would evidently be dangerous to ligate both carotids at one operation.

The cerebral blood-supply is divided into the vessels distributed to the base of the brain-ganglionic system,and those to the outer surface of the cortex-cortical system.

The blood supply to the brain differs from the blood 
distribution in other parts of the body in the following points :-

(I) The main arteries do not divide and subdivide and continue to do so until the arterial capillaries are reached, but each trunk gives off along its course branches which are of the same size, and the trunk ends in terminals which are of the same size as the branches. (2) The branches from the ganglionic system do not anastomose with each other, nor do the branches of the cortical system, nor do the branches of both systems anastomose with each other. (3) The arteries enter the brain vertically to its surface, and proceed inward along approximally straight lines.

The Ganglionic System. Diag. IO. Figs. 2 I, 22.

This system embraces all the branches arising from the circle of Willis and an inch beyond it. The branches are arranged in four groups (two being double, making six really).

(I) The anteromedian group. These are from the anterior cerebral and supply the anterior part of the corpus striatum. (2) (3) The anterolateral. Branches from the middle cerebral to the corpus striatum and anterior part of the optic thalamus. These branches entering the base of the brain produce that sieve-like appearance to which the name of "perforated" space (anterior) is given.

The lenticulostriate artery is the largest and most important branch of this group, because it is most frequently the seat of hemorrhage or embolism. Its course is upward between the lenticular nucleus and the external capsule, it then turns inward through the internal capsule, and terminates in the caudate nucleus.

(4) (5) The posterolateral groups. From the posterior 
cerebrals external to where the posterior communicating enter, supply the posterior part of the optic thalamus, crura cerebri, and the corpora quadrigemina. (6) The posteromedian group. Arise from the posterior communicating and posterior cerebrals, enter the brain (producing the posterior perforated space), and supply the optic thalami.

Other branches not supplying the basal ganglia :-

(I) The anterior choroid, a small branch from the back part of the internal carotid or the middle cerebral, runs backward, enters the lower part of the middle horn of the lateral ventricle through the transverse fissure, and supplies the choroid plexuses of the lateral ventricle, hippocampus major, and corpus fimbriatum. (2) The posterior choroid, from the posterior cerebral, supplies the upper part of the choroid plexuses of the lateral ventricles.

\section{The Cortical System.}

(I) The anterior cerebral takes a course forward and inward from the internal carotid, enters the great longitudinal fissure, through which it passes backward, lying close to the corpus callosum, as far as the parieto-occipital fissure. The anterior cerebral gives off three sets of cortical branches, which supply the under, inner, and outer surface (first and part of the second frontal convolutions) of the frontal lobe and the quadrate convolution of the parietal lobe.

(2) The middle cerebral. This is the larger artery, into which the internal carotid bifurcates at the inner end of the fissure of Sylvius. The artery passes outward, backward, and upward through the Sylvian fissure, until opposite the lower end of the fissure of Rolando, where it divides into four branches, which supply the greater part of the outer surface of the cerebrum.

The ganglionic branches of the middle cerebral are of the 
utmost importance, especially the lenticulostriate artery. For these see above.

The cortical arteries are distributed as follows :-

(a) The first supplies the inferior frontal convolution. This is called Broca's artery, supplying that convolution. Its obstruction in the left side produces degeneration of the inferior frontal convolution, which is manifested by disorders of speech. (b) The second supplies the lower two-thirds of the ascending frontal convolution. (c) The third is distributed to the ascending parietal convolution. (d) The fourth, to the supramarginal, angular, and first temporal convolutions.

(3) The posterior cerebral. After giving off the posterior lateral ganglionic arteries the posterior cerebral divides into three sets of branches, which are distributed to the under and lower part of the temporal lobe and the lower, inner, and outer surfaces of the occipital lobe.

The Branches of the Basilar Artery.-The formation, course, and ending of the basilar are given on page 158 .

(a) The transverse branches supply the pons. (b) One of these transverse branches follows the auditory nerve into the internal auditory meatus and is termed the auditory artery. (c) Another, a large branch, supplies the anterior part of the under surface of the cerebellum, and is called the inferior cerebellar artery. (d) The superior cerebellar arises near the end of the basilar, courses backward and upward around the crus cerebri to the upper surface of the cerebellum, which it supplies, also small branches are given to the pineal gland, velum interpositum, and the valve of Vieussens. 


\section{The Cerebral Veins.}

These are grouped into cortical, basilar, and ganglionic (deep or central).

The superior cortical veins gather the blood from the outer and inner surfaces of the cerebral hemispheres and terminate in the superior longitudinal sinus. They are eight to ten in number, and open into the sinus forward, contrary to the direction of the blood stream, which is backward.

The basilar or inferior cortical veins collect the blood from the lower and under surface of the cerebrum and empty into the cavernous, superior petrosal, and lateral sinuses. The more important of these are the $(a)$ middle cerebral vein, which accompanies the middle cerebral artery and empties into the cavernous sinus. (b) The great anastomotic vein of Trolard (which extends from the parietal lobe to open into the superior petrosal sinus. It communicates above with the superior cerebral veins and thus forms a communication between the superior longitudinal sinus and those at the base of the skull), and (c) the posterior anastomosing vein of Labbé (which passes from the middle cerebral vein to the lateral sinus over the outer part of the temporal lobe).

The central, ganglionic, deep cerebral veins empty into the veins of Galen, which will appear when dissecting the interior of the brain.

\section{DISSECTION.}

The pia and its numerous vessels are to be removed from the brain. This must be undertaken very slowly. It is best to begin the removal from the cortical surface, then from the inner surface, and finally from the base. During this dissection it is well to place the brain in a basin of water, which partially floats it and preserves its contour.

When working upon the inner surface of a hemisphere do not separate them too widely, or the corpus callosum will be torn through. Do not draw the vessels directly upward, but at right angles to the surface. 
At the base exercise the greatest caution, so as to leave behind the cranial nerves and the other important structures there. About the nerves it may be well to leave the pia, as they usually are torn off with it.

Anteriorly the lamina cinerea will be torn through if any force is attempted in taking up the pia. Do not attempt in any place to remove the blood-vessels entire, but with the scissors clip them wherever there is any resistance and turn to another place close by to continue their removal.

The Brain. Figs. 23, 29.

The brain is all that part of the cerebrospinal system contained within the cranial cavity. It varies in weight according to sex, age, race, and intelligence (see qualifications later). At birth the brain bears a larger proportion to the body weight than at any later time; it is for males as I : 5.85, and for females as $\mathbf{I}: 6.5$. The brain grows very fast up to the seventh year, but not so fast as the body weight increases, the ratio being brain to body as I : I 3 or 14. The increase is more slowly up to the twentieth year, when the ratio to the body is as $\mathrm{I}: 30$. From thirty to forty the brain grows very slowly, reaching its maximum weight between forty and fifty years of age. After the maximum is reached the brain begins to lose weight very slowly, at the rate of about an ounce for every ten years of life.

The ratio of the cerebrum to the cerebellum is $8 \frac{4}{7}: \mathrm{I}$ for males, and $8 \frac{1}{4}$ : I for females.

Race.-The largest brains belong to the civilized races, Europeans (English, Germans, Scotch, etc.) and the Chinese. The smallest to the savage races, as the native Africans and Australians.

Intelligence. - While there is some ratio between the degree of intelligence and the brain weight, it is at most very unreliable for comparison between individuals of the same race. As Dana states ("Text Book of Nervous Diseases "), among Ioo intelligent persons the proportion of 

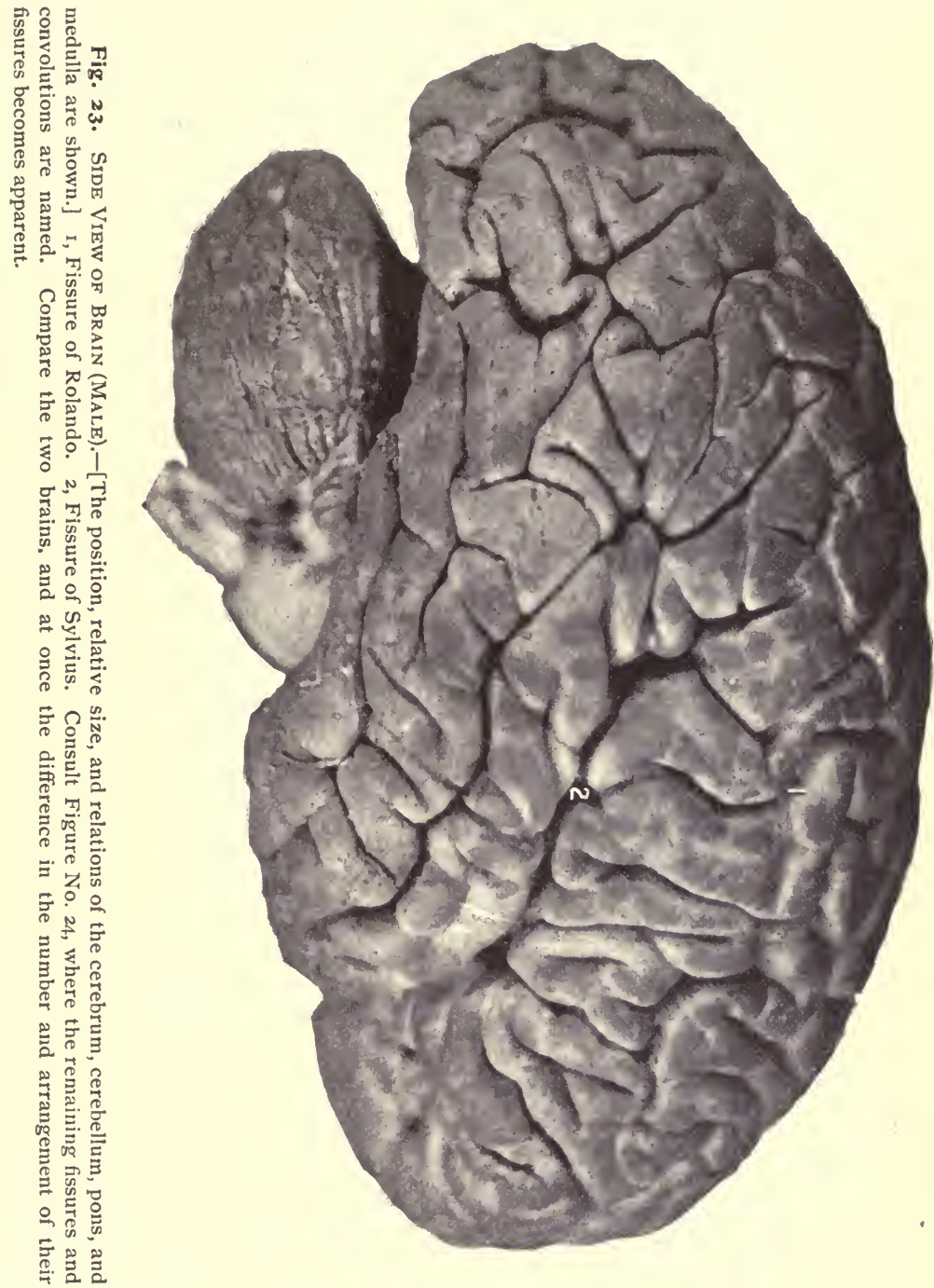
large brains would be as I : 4, while among ignorant people the ratio of large brains to the rest would be as $\mathrm{I}: 20$.

The Development of the Brain.-In order to understand the relations of the several parts of the brain to each other it is very necessary that the changes which take place during the process of development should be rehearsed. The following is only the briefest summary of the import-

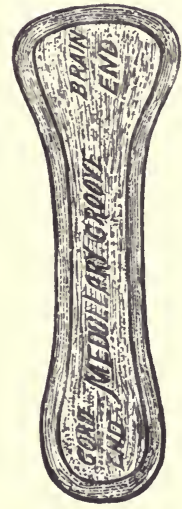

Diag. 11. A Diagram OF THE Primitive Medullary Groove. - $(I$. S. $H$.)

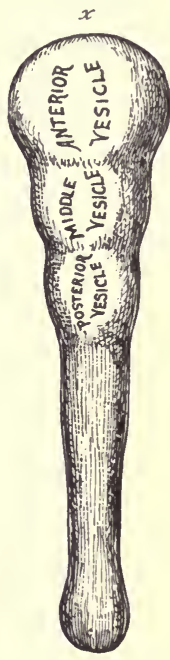

Diag. 12. The Primitive MeDULLARY "TUBE" AND ITS DIVISIONS into Cerebral Vesicles.- $(I . S$. $H$.) $x$, The lamina terminalis.

ant changes; many steps have to be omitted for lack of space. The student is referred for a complete treatise on the subject to Minot's “ Embryology."

The cerebrospinal system is developed from the epiblastic (ectodermal) layer of the blastoderm, and its first appearance is in the nature of a gutter along the blastoderm formed by the heaping up of the cells. 
This heaping up of cells goes on; they rise higher and higher and finally coalesce over the gutter, converting it now into a tube. During this time the anterior half of the tube spreads laterally and so becomes larger than the posterior portion. The dilated portion is the forerunner of the brain (cerebrum, cerebellum, pons, and medulla), while the long, slender hinder portion eventually forms the spinal cord.

We will confine these remarks to the anterior portion of the primitive cerebrospinal tube.

The next change is the production of three distinct swellings in the brain end of the tube, these swellings being produced by the dilatation of the central canal (internally) and the heaping up of cells (externally) opposite the dilatations. These dilatations are called vesicles, and are distinguished as anterior, middle, and posterior.

These three original vesicles are next increased to five by an outgrowth from the anterior vesicle and a subdivision of the posterior.

The anterior extension enlarges forward, outward, upward, and backward ; the outgrowth from the posterior vesicle takes place upward, laterally, and backward.

These five vesicles are now named the forebrain, interbrain (original anterior vesicle), the midbrain (middle vesicle), hindbrain, and the afterbrain (both from the posterior vesicle). From these vesicles all the complex structures of the fully formed brain are developed by a process of thickening here and there, thinning in other places, the pushing out of portions here, the invagination there, and the flexions or bending of the axis of the vesicles until the completed brain is the result.

The central canal of the primitive vesicles in the adult brain constitutes the various ventricles. 
The five vesicles furnish the following parts of the adult brain :-

The forebrain gives origin to the cerebrum, corpora striata, olfactory lobes and the lamina terminalis, (the extreme anterior part of the interbrain, which lies between

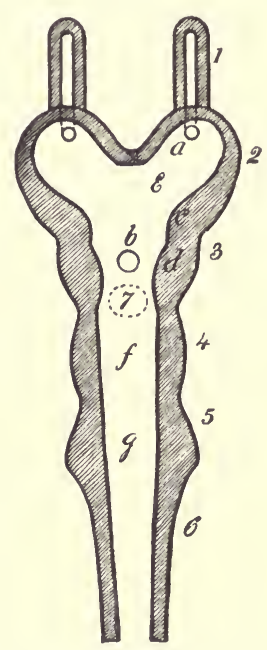

Diag. 13. A Third Stage in the Development of the Cerebral Vesicles. (Altered from Huxley.) Horizontal Anteroposterior Section, "roof" of Cerebral Vesicles Removed.-I, Olfactory bulb, opening into 2, The forebrain. (Cerebrum.) 3, Interbrain. (Optic thalamus.) 4, Midbrain. (Corpora quadrigemina.) 5 , Hindbrain. (Cerebellum.) 6, Afterbrain. (Medulla.) 7, Pineal gland removed. $a$, Opening from olfactory bulb into forebrain cavity. The letter lies in the future lateral ventricle. $b$, Blind pit of the infundibulum. $c$, Beginning of corpus striatum. $d$, Beginning of optic thalamus. $e$, Site of the future foramen of Monro, connecting the cavity of the forebrain (lateral ventricle) with the cavity of the interbrain (the third ventricle). $f$, Aqueduct of Sylvius. (Cavity of the midbrain.) $g$, Fourth ventricle. (Cavity of the hind and afterbrain.) $x$, Lamina terminalis.

and connects the forebrain), from which are developed the corpus callosum, septum lucidum, fornix, and anterior commissure. The cavity within the forebrain is the lateral ventricles. 
The interbrain gives origin to the optic thalami, pineal gland, infundibulum, (part of the) pituitary body, the optic nerve (primarily). The cavity within the interbrain remains as the third ventricle. It is joined to the lateral ventricles by the foramina of Monro, which are the remains of the comparatively wide openings in the embryo, that connects the fore and interbrain cavities.

The midbrain furnishes the crura cerebri, corpora quadrigemina, secondary portion of the optic nerve. The narrow canal which runs through it is the aqueduct of Sylvius.

The hindbrain forms the cerebellum, valve of Vieussens, pons, and the anterior part of the fourth ventricle.

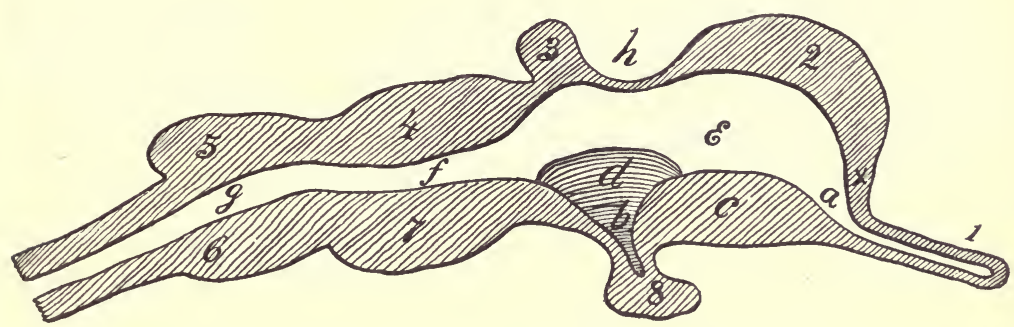

Diag. 14. Same stage as the preceding diagram. Vertical AnteroposteRior Section of the Cerebral Vesicles.-(Modified from Huxley.) 1, Olfactory bulb. 2, Forebrain roof, or pallium. 3, Pineal gland. 4, Corpora quadrigemina. 5, Cerebellum. 6, Pons, and behind it the medulla. 7, Crus cerebri. 8, Pituitary gland. $a$, Opening of olfactory bulb into forebrain cavity. $b$, Infundibular pit. $c$, Corpus striatum. $d$, Optic thalamus. $e$, Site of the foramen of Monro. $f$, Aqueduct of Sylvius. $g$, Fourth ventricle. $h$, Portion of the roof that will be involuted in front of the velum interpositum and choroid plexuses. $x$, Lamina terminalis.

The afterbrain expands into the medulla. From it arises the auditory nerve, and it completes the posterior part of the fourth ventricle.

To follow the changes a little more in detail that result in producing the above results: The forebrain enlarges rapidly forward, outward, upward, and backward until it 
covers in the rest of the brain. From the anterior inferior part of the forebrain extends forward a narrow prolongation, whose cavity is at first in direct communication with the cavity of the forebrain. This prolongation is the olfactory lobe. Its cavity becomes filled up during development and is no longer found in the adult. From the floor

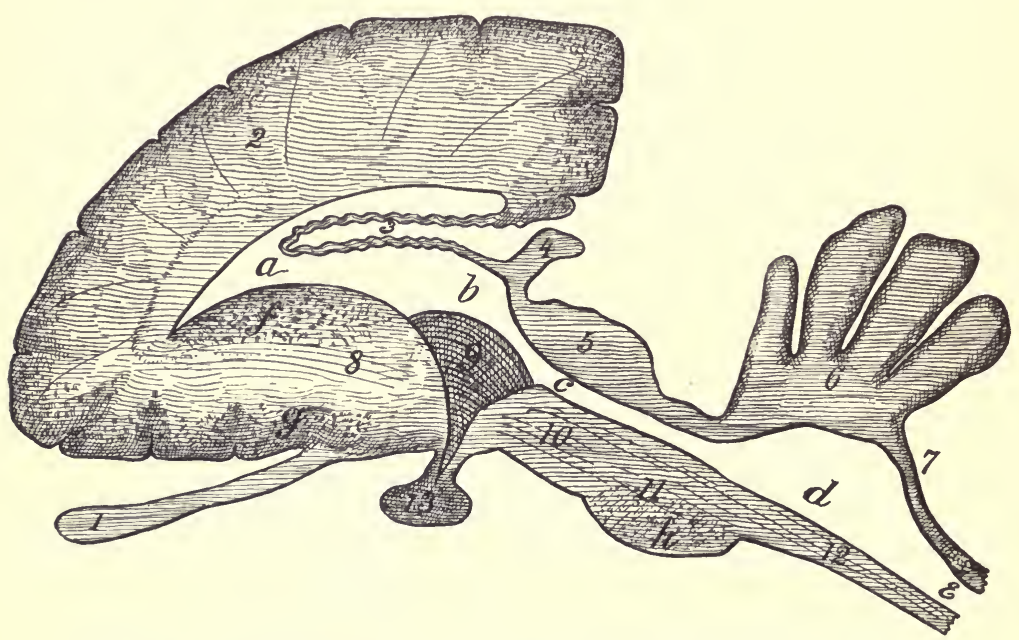

Diag. 15. A Fourth Stage in the Development of the Brain. Vertical Anteroposterior Section at one side of the Median Line.-(Modified from Edinger.) I, Olfactory bulb, central cavity obliterated. 2, Forebrain. (Cerebrum.) 3 . Attenuated roof of midbrain involuted into the cavity of the cerebral vesicles in front of the velum interpositum. 4, Pineal gland. 5, Corpora quadrigemina. 6, Cerebellum. 7, Attenuated roof of afterbrain which becomes involuted into the cavity of the fourth ventricle in front of its choroid plexus. 8 , Corpus striatum. The figure is placed on the fibres of the internal capsule which are beginning to be developed. 9, Optic thalamus. Io, Crus cerebri. I1, Pons. 12, Medulla. $a$, Lateral ventricle. $b$, Third ventricle. $c$, Aqueduct of Sylvius. $d$. Fourth ventricle. $e$, Central canal of spinal cord. $f$, Caudate, and $g$, Lenticular nuclei of the corpus striatum beginning to appear. $h$, Transverse fibres of pons developing.

of the vesicles opposite to and behind the site of the foramen of Monro there takes place an upgrowth of cells, in two elongated masses, which overlap each other. The long 
axis of these masses lies anteroposteriorly. The most anterior (and external) mass of cells becomes the adult corpora striata, the posterior (and internal) mass, the optic thalami. These masses are at first separated from each other, but as growth goes on they become united along their opposing surfaces. Furthermore, the primitive corpus striatum is separated from the lateral wall of the brain vesicle, but as development proceeds the two approach and finally fuse, but this line of junction is always a weak spot in the brain. Along this line of junction the lenticulostriate artery passes, and that this line of junction is a weak spot is proven by the fact that cerebral hemorrhage takes place here from this artery more often than at any other point. The effused blood separates the apparently united corpus striatum from the vesicular wall.

The fore part of the interbrain which unites the forebrains of opposite sides is called the lamina terminalis. By the extension of this backward along with the growth of the forebrain and by the development in it of fibres which connect the forebrains, the great median commissurecorpus callosum-is formed.

The portion of the lamina beneath the corpus callosum is projected backward in the shape of cylindrical bundles of fibres which are called the fornix.

Between the fornix and the corpus callosum the lamina terminalis persists as a triangular shaped, very thin layer of brain matter, which helps to separate the two lateral ventricles and is called the septum lucidum (pellucidum).

Within the septum lucidum by a process of vacuolation a cavity is formed which in the adult is called the fifth ventricle. It has no connection with the other ventricular cavities of the brain which are developed from the original cerebrospinal canal. 
(The above formation of the fifth ventricle is taken from Minot's "Embryology," and is undoubtedly the correct view, though contrary to the usual teachings.)

Within the lamina terminalis, below the septum lucidum, and in front of the anterior part of the fornix, is developed

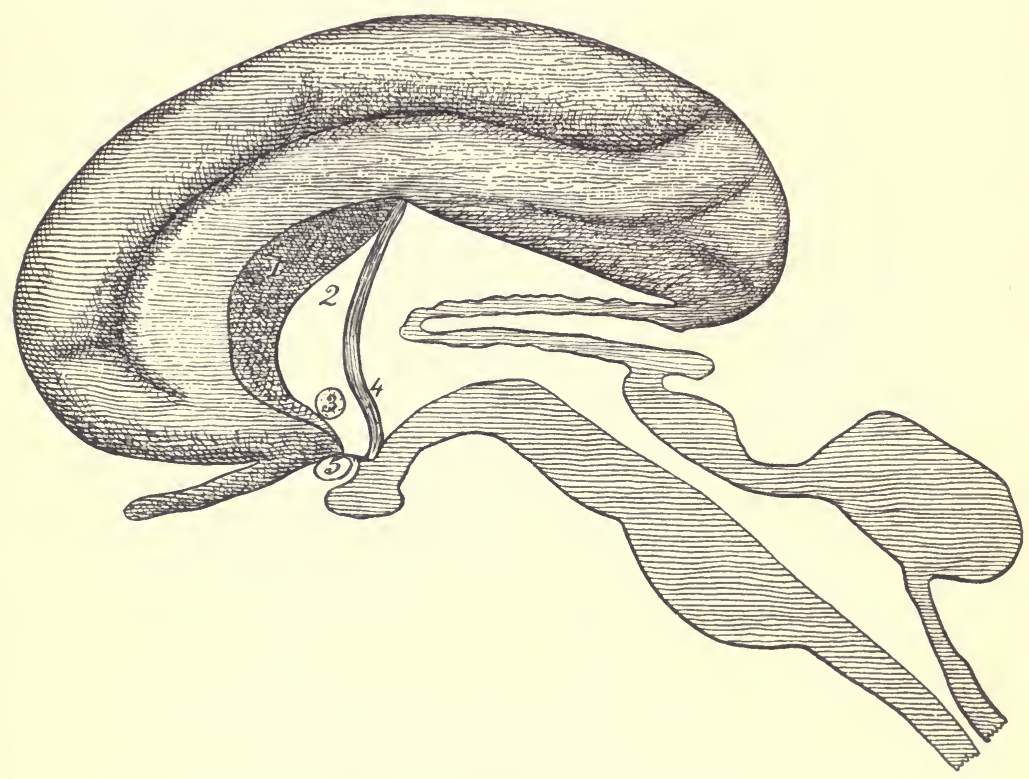

Diag. 16. Median Vertical Anteroposterior Section, at same stage as preceding diagram, to illustrate the development of the lamina terminalis into the corpus callosum, septum lucidum, fornix, and the anterior commissure.-(I.S. H.) I, Corpus callosum. 2, Septum lucidum. 3, Anterior commissure. 4, Fornix. 5, Optic chiasm. Remainder of parts as in preceding diagram.

a well-defined band of fibres which serves to connect the opposite forebrains and is called the anterior commissure.

The flexures of the cerebral vesicles take place early and consist of a "head-bend" at the midbrain, and later a "neck-bend" at the junction of medulla and spinal cord. 
The first bend is the most extensive, the axis of the vesicles being bent to a right angle. These bends have been and will be disregarded in the descriptions, as they would serve to confuse the reader.

The changes in the interbrain: The development of the optic thalami has been given above. The floor of the vesicle drops downward to form a blind tube-the infundibulum; the end of the infundibulum becomes enlarged, and meets an upward pouch from the pharynx of the fotus. This pharyngeal pouch becomes narrowed at its neck and finally cut off from the pharyngeal cavity and remains attached to the infundibulum. The rounded mass thus formed from the brain vesicle and the primitive pharynx is the hypophysis or the pituitary body. The cerebral portion constitutes a small central mass ensconced within the horseshoe shaped mass from the pharynx. Fig. 20.

The elevation (depression, really) at the junction of the infundibulum and floor of the vesicle is the tuber cinereum.

On each side of the median line two elevations appear, which are the corpora albicantia. Forward from the tuber cinereum the lamina terminalis is continuous under the name of the lamina cinerea.

The midbrain: The floor becomes thickened by the downward extension of fibres from the greatly enlarged forebrain (cerebrum), and these masses of fibres are called the crura cerebri. The roof of the midbrain becomes bunched to form the corpora quadrigemina. Its central canal is the aqueduct of Sylvius.

The hindbrain : It expands upward, outward, and backward until the result is the cerebellum, while its thickening in front and below by numerous transverse fibres forms the pons.

The afterbrain: In like manner forms the medulla, by 
the development of a great mass of connecting fibres, which unite the cord below with the rest of the cerebrospinal system above.

The cavity within the hind and the afterbrains dilates to form the fourth ventricle.

The Formation of Fissures.-As the forebrain increases in size and enlarges upward and backward there is left between the two halves a deep fissure, which is the great longitudinal fissure of the adult. As the forebrain enlarges it extends backward, so as to overhang the rest of the brain, and the interval thus left between the forebrain and the other parts of the brain becomes the transverse fissure (of Bichat) in the adult.

The above constitutes one method of formation of the fissures of the brain.

Another method is by the involution of the entire thickness of the cerebral surface itself. To this second classification belong the fissures of Sylvius, parieto-occipital, calcarine, and hippocampal.

A third class of fissures is formed by the unequal growth of the outer layers of the cerebral surface. The most important one of these is called the fissure of Rolando. To these depressions the name of sulci is applied in distinction to those grooves formed between parts of the brain, or by the involution of the entire thickness of the brain wall, which are called fissures.

The formation of the fissure of Sylvius is taken as an example of the method by which the second class of fissures is formed.

At the second month the fotal brain (forebrain) presents a smooth surface, but beginning with this period there appears upon the middle of its outer lower surface a depression. 
By the growth of the brain at a much faster rate above and below this depression its depth is increased, and as the brain becomes larger the fissure becomes deeper and longer, extending backward (the horizontal limb) with the backward prolongation of the forebrain. Thus the fissure is formed by the excessive growth of parts of the cerebrum about a very slowly growing portion, so at the last the slowly growing portion is covered in by the more rapidly growing portions, and there is left between them a fissure which leads to the former part of the brain (in this case to the insula). As an example of the third class of depressions-sulci -we will consider the formation of the fissure or sulcus of Rolando.

The first appearance of the fissure of Rolando is between the fifth and sixth months, and it is produced by the growth of the cortex of the cerebrum on either side of a linear depression at a faster rate than the cortex grows along the line of the depression itself. The result is to form a deep fissure. All the rest of the third class of sulci are formed in this manner-by the upgrowth of the cortex at a faster rate along certain lines than along other lines, these upgrowths being called convolutions and the depressions sulci.

The Formation of the Velum Interpositum and the Choroid Plexuses.-(Diags. I 3 to I6.) Go back to the time when there are the three primary vesicles; cover them with the vascular membrane, which is to become the adult pia. Now remember how the forebrain grows backward, taking with it the lamina terminalis (corpus callosum), so as to cover in the middle vesicle. The forebrain does cover in the middle vesicle or that portion of it which we call its roof, but upon this roof rests the vascular membrane (which later we designate as the velum interpositum), so at the end of the process the velum interpositum is found within the brain, 
but is excluded from the ventricular cavity by the remains of the roof of the middle vesicle, which persists as a thin layer of epithelial cells lining its under surface. Further, the lateral margins of the velum become converted into a plexus of capillary vessels, to which the name of choroid plexus is given. These lie within the hollow of the lateral ventricles, but are separated from them by the continuation of the layer of epithelial cells which lines the velum. These cells cover in all the extensions of the capillary loops.

In an exactly similar manner is the choroid plexus of the fourth ventricle developed from the portion of the primitive vascular membrane which covers in the roof of the hinder portion of this ventricle. At the adult stage, after the cerebellum has been formed, this vascular layer reaches from the under surface of the cerebellum to the upper surface of the medulla. It is lined throughout its extent by the attenuated remains of the original roof of this third cerebral vesicle. The layer of cells is applied to the membrane so as to cover in completely (exceptions below) all the festoons of capillaries which have been formed from it.

Remember that this portion of the roof of the fourth ventricle is perforated by the foramen of Majendie close to the medulla and in the middle line, and by the foramina of Key and Retzius at its lateral extensions.

The Cerebrum. Figs. 24 to 28 .

The cerebrum consists of the great mass of the brain situated at its anterior and superior part. It is the greatly overgrown forebrain.

Its shape is ovoid, flattened below, and partially divided in two symmetrical parts by the great longitudinal fissure. These halves are called hemispheres, right and left. 


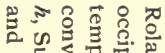

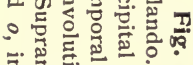

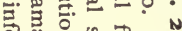

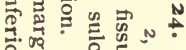

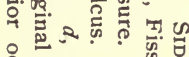

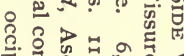
च. है

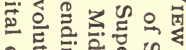

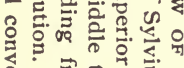

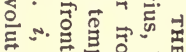

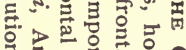
品

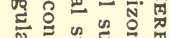

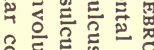

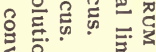
ํํㅇำ

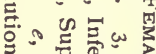
. นิ웡

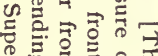

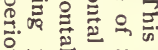

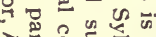
그.

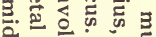
율

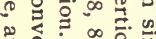

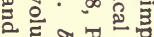
$\sim \vec{E}=\overrightarrow{0}$ 콜유유. 可以言要

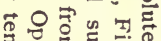

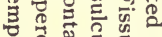
류유.

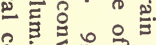
ᄋ 造

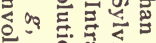
的。

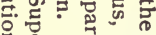

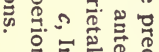
*

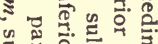
긍 응

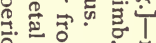
‡ उ

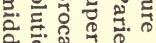
产

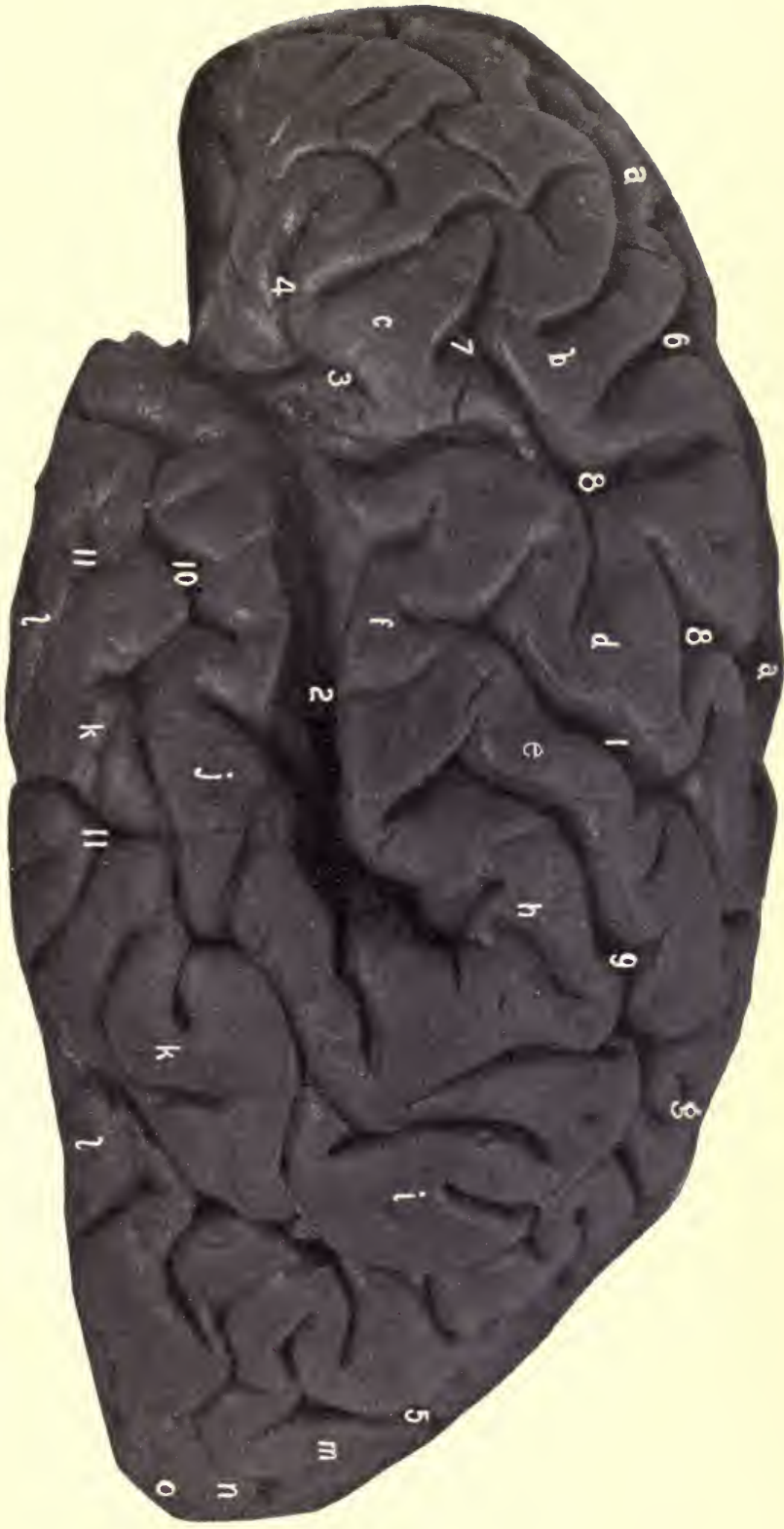


Each hemisphere is further subdivided into lobes, for convenience of reference, by the fissures.

The fissures of the brain may be divided into three classes:-

(I) The fissure between the halves of the cerebrum, and that between the cerebrum as a whole and the rest of the brain. To this class belong the great longitudinal and transverse fissures. (2) Fissures entirely within the cerebrum itself, which are formed by the involution of the entire thickness of the cerebral wall. These are the primary fissures and comprise the Sylvian, dentate or hippocampal, parieto-occipital, calcarine, and collateral fissures. Corresponding to the external depressions of the primary fissures are internal elevations within the lateral ventricles of the cerebrum. (3) Fissures within the cerebrum, which are formed by the upgrowth of the outer layer (cortex) of the cerebrum along certain lines, depressions being left between these ridges. The ridges are called convolutions, the depressions fissures and sulci. (The former term being restricted to the deeper and earlier appearing depressions of this class). These constitute the secondary fissures (and sulci) and are the Rolandic and callosomarginal fissures and all the remaining sulci of the cerebrum.

The Fissure of Sylvius. Figs. I, 7, 23, 24, 25, 28.

The Sylvian fissure, though strictly a primary fissure, belongs to the class of fissures formed by the outgrowth of the cortical wall rather than by an involution of the entire thickness of the cerebral wall.

It appears during the third month of development, opposite the floor of the interbrain, where the mass of cells which is to form the corpus striatum is found.

Its shape is first triangular, and at the bottom of the tri- 


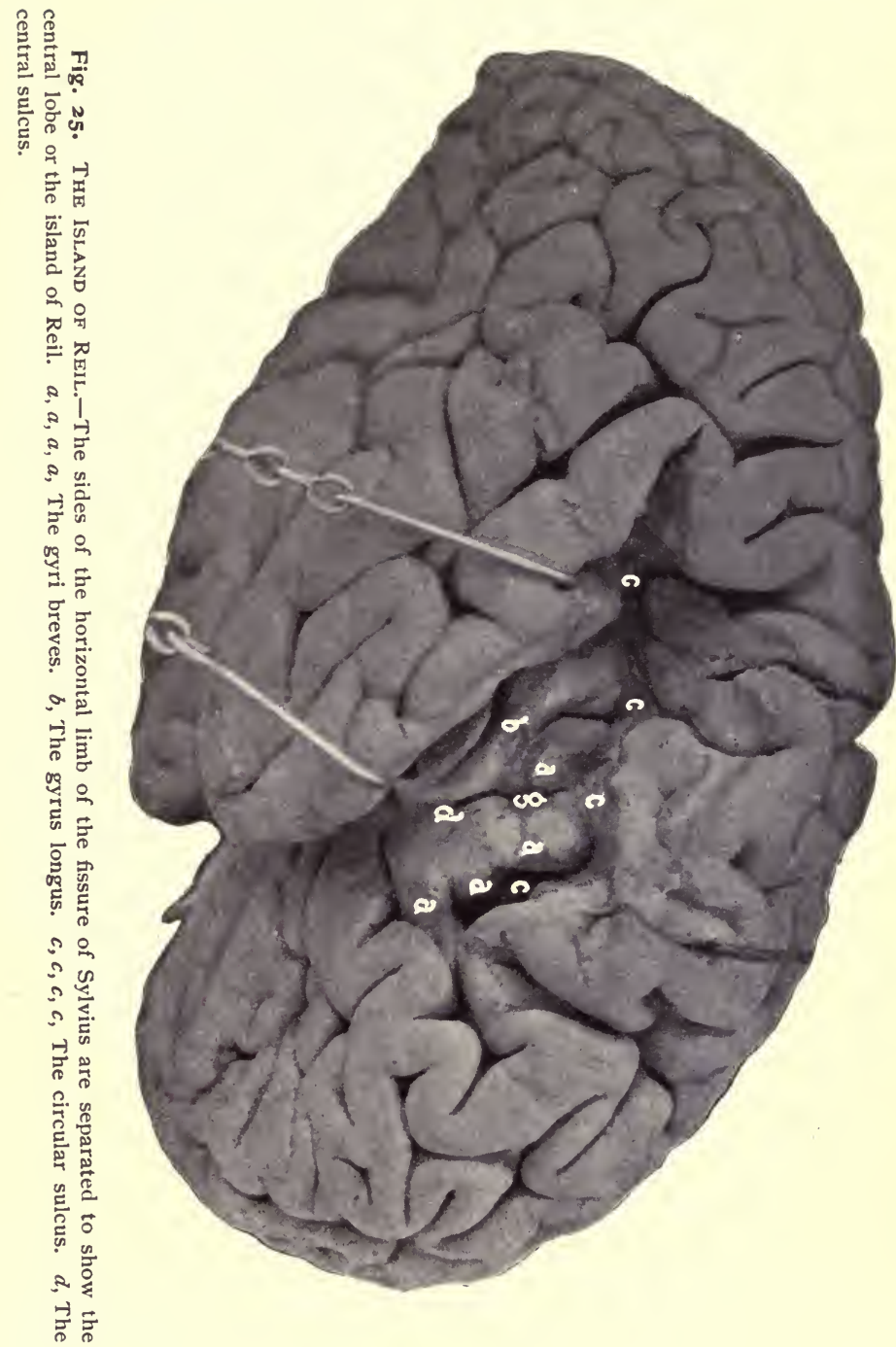


angle lies the portion of the cortex which is external to the corpus striatum. As growth proceeds and the cortex above and below overlaps more and more the central depressed portion, the fissure assumes a linear appearance.

This is the horizontal limb of the adult fissure. About the fifth month an offset from the main fissure appears; this ultimately becomes the vertical limb of the adult fissure.

In the adult the fissure of Sylvius begins at the base of the brain at the outer part of a perforated layer (anterior perforated space), passes outward, backward, and slightly upward until near its terminus, when it turns abruptly upward to end in the parietal lobe. This is the main, or horizontal limb of the fissure. Fig. 24.

From the anterior part of the horizontal limb a short (one inch long) fissure extends forward and upward into the frontal lobe; this is the ascending or vertical limb of the fissure of Sylvius. Sometimes this fissure is bifid and one part extends directly forward for a short distance into the frontal lobe, forming the anterior limb of the fissure of Sylvius.

The fissure of Sylvius serves to separate the frontal and parietal lobes above from the temporal lobe below.

The Fissure of Rolando. Figs. I, 7, 23, 24, 26.

This fissure appears between the fifth and sixth months of foetal development. It belongs to the class of fissures which are formed by the heaping up of the cortical portion of the cerebrum itself. It is found grooving the outer surface of the cerebrum about at its middle. Its course is downward and forward from about the middle of the great longitudinal fissure, into which it frequently opens, to near the horizontal limb of the fissure of Sylvius. It forms an 


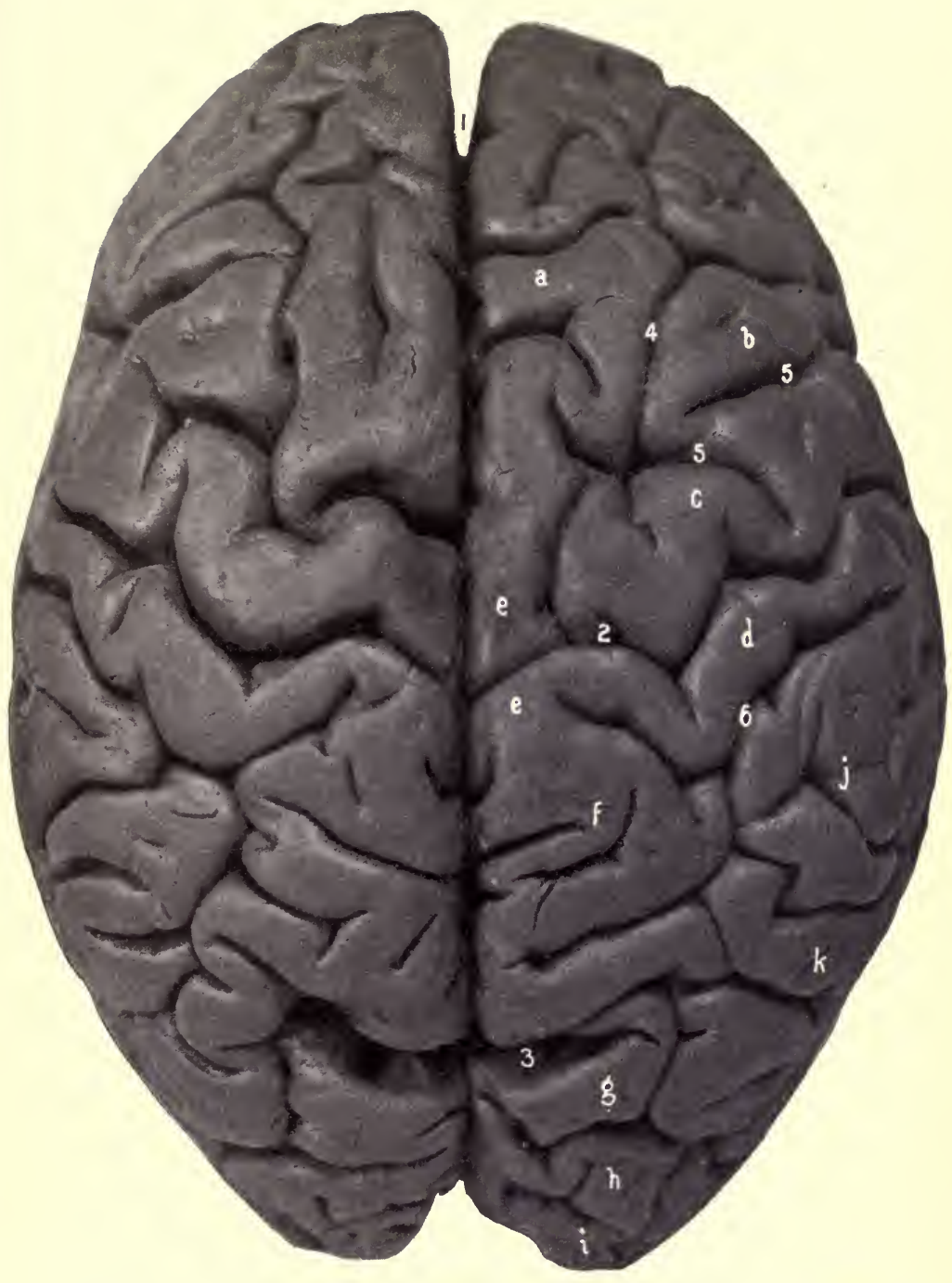

Fig. 26. The Upper Surface of the Cerebrum (same brain as No. 24).-1, Great longitudinal fissure. Divides the cerebrum into its two hemispheres. 2, Fissure of Rolando. The genua are very distinct. In this subject the fissure extends through into the great longitudinal fissure. Cunningham states this is the more usual condition. 3, Parietooccipital. The external portion of the fissure is unusually long. 4, Superior frontal sulcus. 5, 5, The præcentral sulcus. 6, Intraparietal sulcus. $a$, Superior, $b$, middle and $c$, ascending frontal convolutions. $d$, Ascending parietal convolution. $e, e$, Paracentral convolution. Consult the following figure. $f$, Superior parietal convolution. $g$, Superior, $h$, middle, and $i$, inferior occipital convolutions. $j$, Supramarginal convoIution. $k$, Ángular convolution. 
angle, opening forward, of about 7I degrees, with the longitudinal fissure.

The course of the fissure is not perfectly straight but winding, and may be divided into three segments or thirds; these segments make with each other angles or bends (genua); the angle formed between the upper and middle thirds points backward, that formed by the middle and lower thirds points forward (superior and inferior genu). Fig. 26.

The fissure of Rolando separates the frontal (anterior) from the parietal (posterior) lobe.

The Parieto-occipital Fissure. Figs. 26, 27.

As this shows only slightly upon the outer surface of the cerebrum its description will be omitted until the inner surface of a hemisphere is described. Suffice to say that its external portion is very short (one-half of an inch) and is found midway between the fissure of Rolando and the apex of the hemisphere behind, and partially divides the parietal from the occipital lobe.

The Frontal Lobe. Figs. 23, 24, 26.

The frontal lobe is situated at the anterior part of the cerebrum. It was thought to correspond to the limits of the frontal bone-hence its name-but such is not the case, as the lobe reaches backward beneath the anterior third of the parietal bone.

The frontal lobe presents three surfaces, the external, internal, and inferior; these are also called the frontal, mesial, and orbital. The external surface of the lobe is cut off from the rest of the cerebrum by the fissure of Rolando behind and the fissure of Sylvius below. The inferior surface rests upon the orbital plate of the frontal bone. The internal surface is separated from the corres- 
ponding portion of the opposite lobe by the falx cerebri, and is marked off from the rest of the internal surface by the callosomarginal fissure.

The frontal lobe presents three well-marked tertiary fissures: the superior and inferior-or first and second frontal sulci-which run anteroposteriorly, and the precentral, which is at right angles to the above, and irregularly parallel to the fissure of Rolando. The last fissure is usually interrupted in its course, and the anteroposterior sulci may terminate in it ; the superior usually does.

These sulci divide the frontal lobe into four convolutions: The ascending frontal, which lies parallel with and in front of the fissure of Rolando, and is limited in front by the precentral fissure. The superior frontal convolution, which becomes continuous on the inner surface with the marginal convolution, on the inferior surface with the internal convolution, and behind with the ascending frontal convolution. The middle frontal convolution, between the superior and inferior frontal sulci, is extended around to the inferior surface of the frontal lobe, and backward to the precentral sulcus, by which it is separated from the ascending frontal convolution. On the under surface of the lobe this convolution merges into the middle or the anterior convolution. The inferior frontal or Broca's convolution is below the inferior sulcus, arches over the vertical limb of the Sylvian fissure, and becomes continuous behind with the ascending frontal, and on the under surface of the frontal lobe with the posterior orbital convolutions. This convolution is deeply indented on its under surface by the vertical limb of the fissure of Sylvius, and above by the precentral sulcus.

The internal and inferior surfaces will be described on pages 188 and 192 . 
The Parietal Lobe. Figs. 23, 24, 26.

Anteriorly the parietal lobe is limited by the fissure of Rolando, posteriorly by the external portion of the parietooccipital fissure and a line extending in the direction of that fissure, below by the horizontal limb of the fissure of Sylvius and a line continuing its horizontal direction to the posterior boundary. Internally, the parietal lobe merges into the quadrate lobe. For its boundaries see page I9I.

The parietal lobe is furrowed by a single (named) fissure or sulcus, the intraparietal.

The intraparietal sulcus is usually found in the shape of a horizontal $\mathrm{T}$-shaped groove, the cross-bar of the $\mathrm{T}$ being parallel with the fissure of Rolando, and the upright arm with the great longitudinal fissure. This arm is usually prolonged into the occipital lobe, where it serves to mark off the superior from the middle occipital convolutions.

Between the fissure of Rolando and the transverse arm of the $T$ is the ascending parietal convolution. This convolution is usually continuous around the lower end of the Rolandic fissure with the ascending frontal convolution. The lower ends of these two convolutions together with the adjacent portion of the inferior frontal convolution are called the operculum-or roof-because they cover in the central lobe or island of Reil.

The ascending frontal and parietal convolutions are also often united around the upper end of the fissure of Rolando by the paracentral convolution.

Above the upright arm of the $T$ lies the superior parietal convolution, which is merged into the quadrate lobe on the inner surface of the hemisphere, and below this arm is the inferior parietal convolution, which is further subdivided into two convolutions-the anterior one, the supramarginal, which arches over the upturned extremity of the hori- 
zontal limb of the fissure of Sylvius and is continuous with the ascending parietal convolution, and a posterior portion, which similarly embraces the extreme posterior part of the first temporal sulcus, and is called the angular convolution.

The supramarginal convolution connects the ascending parietal with the first temporal and the angular convolutions.

The angular convolution connects the first and second (superior and middle) temporal convolutions with each other and also with the middle occipital convolution.

The Temporal Lobe. Figs. 23, 24, 25.

This is composed of all the cerebrum lying below the fissure of Sylvius. It rests upon the middle fossa of the skull, and posteriorly becomes insensibly continuous with the occipital lobe.

The external surface of the temporal lobe is divided by the superior or parallel and middle sulci into three convolutions, the superior (first), middle (second), and inferior (third) temporal convolutions. These run parallel with the fissure of Sylvius and behind are continuous with the convolutions of the parietal and occipital lobes, viz.: The superior convolution with the supramarginal and angular convolutions, the middle temporal with the middle occipital, and the inferior temporal with the inferior occipital convolution.

The parallel or superior temporal sulcus follows the direction of the Sylvian fissure and, like that fissure, turns upward at its terminus. Curving over the upturned extremity of this sulcus is the angular convolution of the parietal lobe.

The Central Lobe, or the Island of Reil. Fig. 25.

This lobe is seen only after separating the sides of the fissure of Sylvius. It is the portion of the cerebrum which 
is opposite and external to the corpus striatum, and which, not keeping up in growth with the adjoining portions of the cortex cerebri, becomes covered in by those portions. The island is traversed by three or four shallow sulci which radiate from the anterior portion of the lobe in a direction upward and backward. One of these fissures, the central sulcus, is deeper than the others, and divides the lobe into a large anterior portion and a smaller posterior part. The island is surrounded by a well-marked depression, the circular sulcus.

The Occipital Lobe. Figs. 23, 24, 26.

This constitutes that portion of the cerebrum posterior to the parieto-occipital fissure, and the prolongation of the course of this fissure across the external surface of the cerebrum.

The sulci and fissures are usually very poorly marked off.

The sulci may be determined as follows: The superior occipital sulcus is the posterior extremity of the intraparietal sulcus, or at least of a sulcus continuing the direction of this fissure backward ; similarly the inferior occipital sulcus is part of, or a backward extension of, the middle temporal sulcus. By these two irregular and indistinct grooves three convolutions are determined, the superior, middle, and inferior, or the first, second, and third occipital convolutions.

The superior convolution is continued into the cuneate convolution on the internal surface of the occipital lobe.

The Inner or Mesial Surface of the hemisphere taken as a whole. Figs. 27, 38 .

Upon the internal surface of the hemisphere are found four of the primary fissures of the cerebrum, viz.: The 


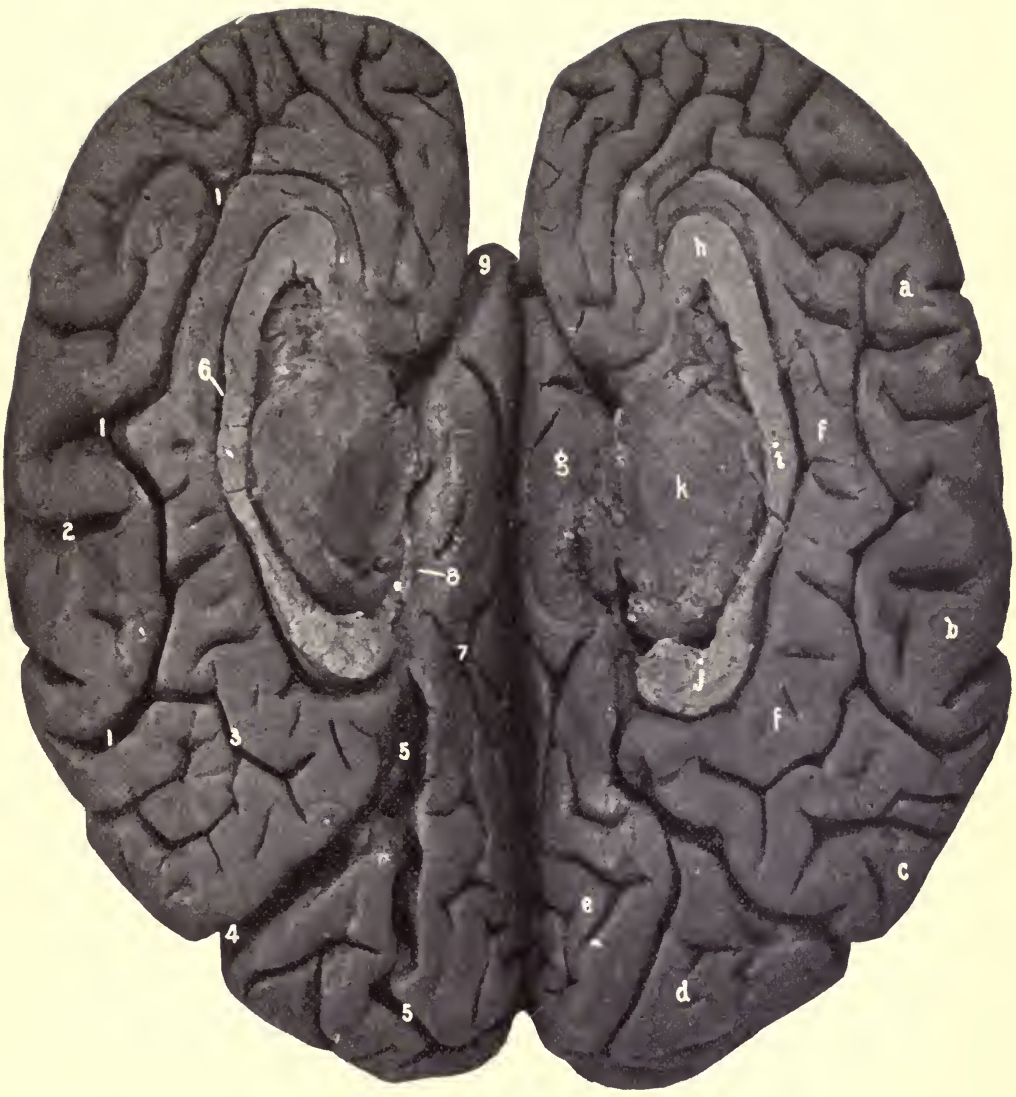

Fig. 27. The Inner Surfaces of the Cerebral Hemispheres. (Same brain as No. 23) - I, Callosomarginal fissure. 2, Fissure of Rolando terminating in the longitudinal fissure. 3, Subparietal sulcus. 4, Parieto-occipital fissure. 5, 5, Calcarine fissure. 6, Fissure of the corpus callosum. 7, Collateral sulcus. 8, Dentate fissure. 9, Fissure of Sylvius. Convolutions $-a$, Marginal. $b$, Paracentral. $c$, Quadrate. $d$, Cuneate. $e$, Lingulate $f, f, g$, Falciform lobe divided into two (some make three) divisions. $f, f$, Gyrus fornicatus. (Under the splenium of the corpus callosum is the isthmus or the gyrus hippocampi). $g$, Gyrus uncinatus. $h$, Genu. $i$, Body, and $j$, Splenium of the corpus callosum. $k$, Optic thalamus. The crura cerebri are divided close under the optic thalami. 
parieto-occipital, calcarine, hippocampal or dentate, and the collateral.

The parieto-occipital fissure deeply grooves the inner surface of the cerebrum in a direction downward and forward, starting at the junction of the posterior one-fifth with the anterior two-fifths of the margin (inner) of the hemisphere, and terminating opposite a point directly below the splenium of the corpus callosum.

The calcarine fissure begins in the posterior part of the occipital lobe and passes directly forward to merge into the parieto-occipital fissure at its middle point.

The corresponding elevation within the lateral ventricle produced by the calcarine fissure is called the calcar, or hippocampus minor.

\section{The Hippocampal or Dentate Fissure. Figs. 27, 28.}

This is a groove which begins just below the hinder end of the corpus callosum and runs forward in the internal margin of the temporal lobe. The elevation which it produces in the middle horn of the lateral ventricle is the hippocampus major. By separating the lips of the fissure the floor is seen to have a transversely striated appearance, hence the name of dentate fissure.

The Collateral Fissure. Fig. 28.

This fissure extends anteroposteriorly from the temporal into the occipital lobes.

The raised portion of brain matter produced by this fissure in the floor of the (posterior and descending horns of the) lateral ventricle is called the eminentia collateralis.

The Callosomarginal Fissure. Figs. 27, 38.

This is a well-marked sulcus which begins beneath the anterior extremity of the corpus callosum, turns backward, conforming to the curve of this structure until over its 
posterior extremity (the splenium), where it turns upward to the margin of the hemisphere.

In front of the fissure of Rolando there is an offset from the callosomarginal sulcus which serves to bound anteriorly the paracentral convolution. Also, the direction of the main part of the sulcus is continued posteriorly nearly to the parieto-occipital fissure under the name of the subparietal sulcus. This sulcus is often not distinctly marked.

The curved portion of cerebral matter external to the callosomarginal sulcus belongs to the frontal lobe and is called the marginal convolution. As above stated, the portion of the marginal lobe which connects the ascending frontal and parietal convolutions is called the paracentral convolution.

The square-shaped mass between the upturned extremity of the callosomarginal sulcus and the parieto-occipital fissure is the quadrate lobe, or convolution. It is the internal portion of the parietal lobe and is limited inferiorly by the subparietal sulcus.

Between the posterior portion of the parieto-occipital and the calcarine fissures is the triangular or wedge-shaped convolution - the cuneus-which is the internal part of the occipital lobe. The marginal, quadrate, and cuneate convolutions form the outer arch of the internal surface of the hemisphere.

Internal to this outer arch of convolutions is a second arch, which starts beneath the anterior extremity of the corpus callosum, curves backward over that structure, being separated from it by the callosal sulcus, then turns around the hinder extremity of the corpus callosum, and under the name of the uncinate convolution extends forward to the end of the temporal lobe. 
The first part of this arch is called the gyrus fornicatus, the second part the uncinate gyrus; they are connected by a narrowed portion-the isthmus-just under the posterior part of the corpus callosum. Together these convolutions, with some internal parts, as the septum lucidum, fascia dentata, and fornix, constitute the falciform or limbic lobe.

The uncinate convolution is the anterior part of the superior occipitotemporal convolution, which lies between the calcarine and the collateral fissures.

The posterior part of this convolution is also called the lingulate convolution.

External to the collateral fissure, between it and the inferior temporal sulcus, is the inferior, or simply the occipitotemporal, convolution. Some writers call the portion of the falciform lobe which lies behind and below the hinder end of the corpus callosum the gyrus hippocampi, restricting the uncinate gyrus to the anterior part of the hook.

The dentate convolution will be seen best during the dissection. This is the thin fold which passes from the end of the corpus callosum to the apex of the temporal lobe. It is found within the arch of the uncinate gyrus.

The Callosal Sulcus. Figs. 27, 38 .

This is the groove which is found between the corpus callosum and the gyrus fornicatus.

The Base of the Brain. Figs. 28, 29.

The Sulci and Convolutions. - The under surface of the frontal lobe: Here are found two shallow sulci, an external one shaped something like the letter $\mathrm{H}$ or $\mathrm{X}$. This is the orbital sulcus, and the convolutions formed by it are the internal, anterior, and the posterior or external. 


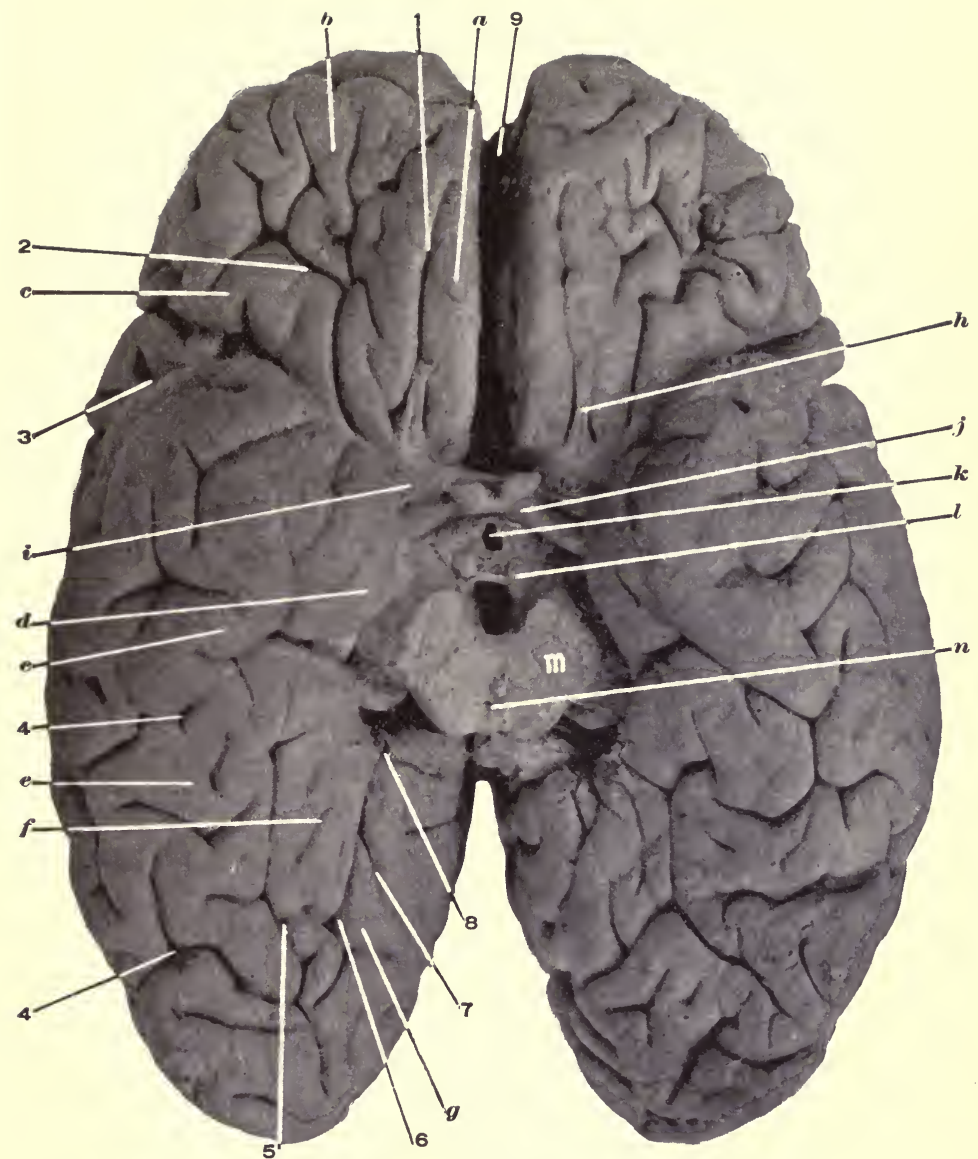

Fig. 28. Base of Cerebrum.-I, Olfactory sulcus. 2, Orbital sulcus. 3, Fissure of Sylvius. 4, 4, Inferior temporal sulcus. 5, Collateral fissure. 6, Calcarine fissure. 7, Parieto-occipital fissure. 8, Dentate fissure. 9, 9, Great longitudinal fissure. $a$, Internal frontal convolution. $b$, Anterior frontal convolution. $c$, Posterior frontal convolution. $d$, Uncinate convolution. e, e, Inferior temporal (occipitotemporal) convolution. $f$, Lingulate convolution. $g$, Cuneate convolution. $h$, Olfactory nerve (tract). $i$, Anterior perforated space. $j$, Optic commissure, nerves and tracts. $k$, Infundibulum torn away from the tuber cinereum. $l$, Corpora albicantia. $m$, Crura cerebri divided close to pons. $n$, Aqueduct of Sylvius. 
The internal is continuous with the superior frontal convolution, the anterior with the middle frontal, and the posterior or external with the inferior frontal convolution.

The internal convolution is grooved by the olfactory sulcus, within which rests the olfactory nerve.

The under surfaces of the temporal and occipital lobes should be taken together, as there is no line separating them.

The fissures seen here are portions of the hippocampal or dentate, the parieto-occipital, the calcarine, the collateral, and the inferior temporal. This last is more often composed of several short, indistinct grooves, which extend anteroposteriorly along near the external borders of the temporal and occipital lobes. External to the inferior temporal sulcus is the inferior temporal convolution (anteriorly) and the inferior occipital (posteriorly). Between the above sulcus and the collateral fissure lies the inferior occipitotemporal, or simply the occipitotemporal convolution. Then between the collateral and the calcarine, fissures is the superior occipitotemporal convolution, which, as explained before, is described under the names of the uncinate gyrus for the anterior part, and the lingulate convolution for the posterior portion. To see these convolutions and sulci the cerebellum must be raised.

The cerebral hemispheres will be found separated anteriorly and posteriorly by the great longitudinal fissure.

By raising the temporal lobe the beginning of the Sylvian fissure is found : it starts behind the frontal lobe in a depressed portion of the base of the brain (anterior perforated space).

Projecting backward from the centre of the base of the cerebrum, between the temporal lobes, are seen the two rounded masses of fibres, which are called the legs of the 


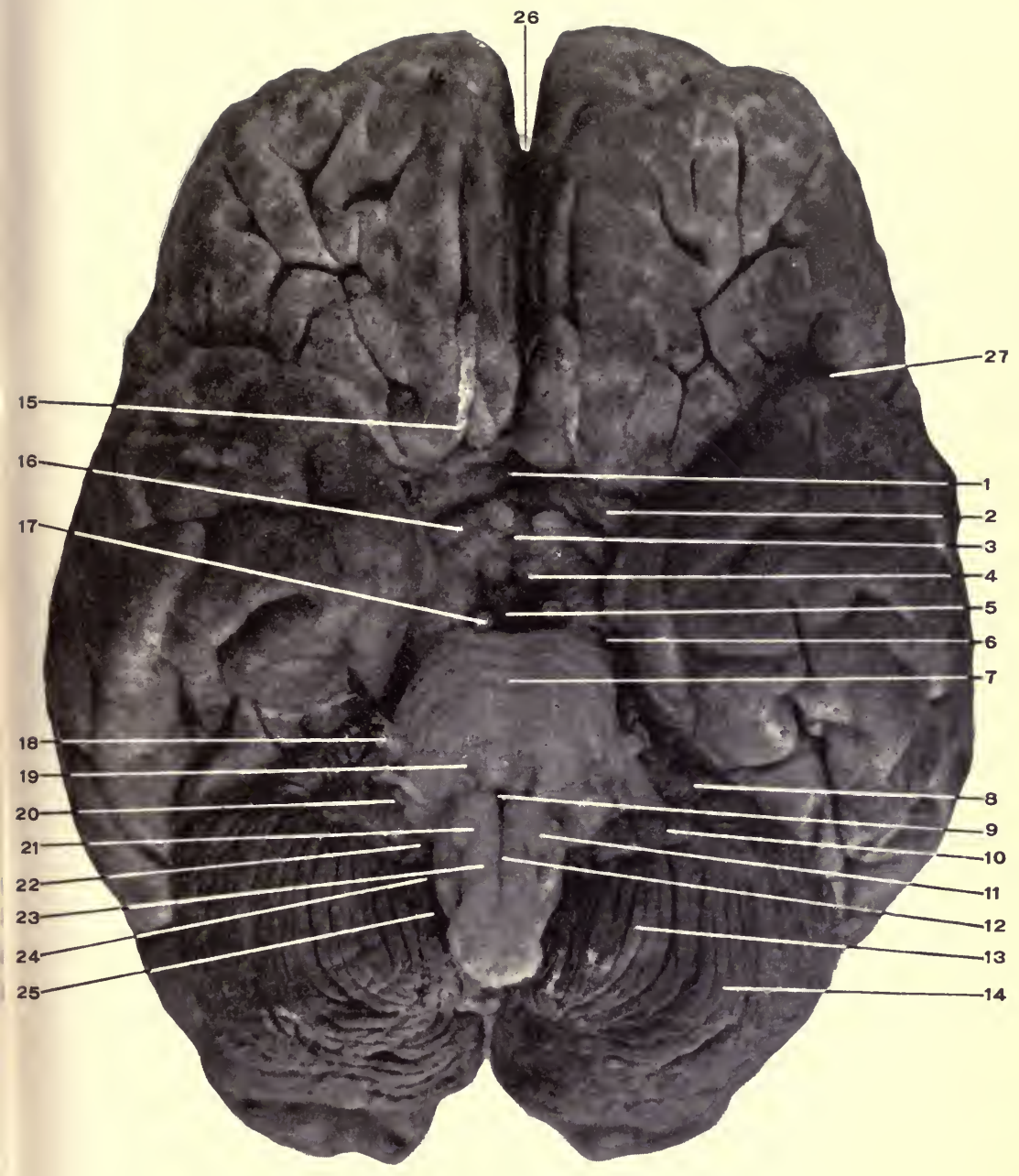

Fig. 29. BASE of THE BRain.-I, Lamina cinerea. 2, Anterior perforated space. 3. Tuber cinereum and point where the infundibulum was torn off. 4, Corpus albicans. 5, Posterior perforated space. 6, Fourth nerve crossing the crus cerebri. 7 , Pons, line points to the median groove. 8, Under surface of the quadrate convolution of cerebellum. 9, Foramen cæcum. 10, Flocculus. I1, Olivary body. 12, Anterior median fissure of medulla. 13, Amygdala. 14, Biventral, or cuneate lobe. 15, Olfactory nerve (tract really). 16, Optic tract. The optic commissure and nerves are shown. I7, Third nerve 18, Fifth nerve. I9, Sixth nerve. 20, Seventh and eighth nerves. The former internal to the latter. 21, Hypoglossal nerve. 22, Glosso-pharyngeal nerve. 23, Anterior pyramid of medulla. 24, Pneumogastric nerve. 25, Spinal accessory nerve. 26, Great longitudinal fissure. 27, Fissure of Sylvius. 
brain, or the crura cerebri. They are joined together at their line of contact along the middle line.

A short distance posteriorly the crura are crossed by the transverse fibres of the pons, which pass across the crura to connect the two halves of the cerebellum and form its middle peduncles.

Along the middle line the pons is marked by a shallow groove in which rests the basilar artery. (The pons really does not bear upon the artery at all, but upon the cerebrospinal fluid within the subarachnoid spaces, which form, as stated, a "water-bed" for its support.)

The longitudinal fibres issue from the posterior part of the pons, and from this point to the margin of the foramen magnum constitute the medulla.

A full description of these structures will appear later.

The Central Parts at the Base of the Cerebrum.-Beginning in front, in the anterior part of the great longitudinal fissure which separates the frontal lobes the under surface of the anterior part of the corpus callosum will be seen (this is the rostrum).

The extension of the floor of the cerebrum backward from the corpus callosum to the crossing of the optic nerves is in the shape of a thin lamella of grey matter called the lamina cinerea. The lamina cinerea becomes continuous laterally with the anterior perforated spaces, and posteriorly with the tuber cinereum. On either sides of the lamina cinerea and in the angle between the temporal and frontal lobes are the anterior perforated spaces, small areas of the base, where the small arteries of the anterolateral ganglionic systems enter the corpus striatum. The anterior perforated space is really the under surface of the lenticular nucleus of the corpus striatum.

From the sides of the corpus callosum a narrow band of 
white fibres extend backward and outward to the beginning of the fissure of Sylvius. These bands are called the crura, or peduncles of the corpus callosum.

The anterior perforated spaces are limited internally by these peduncles.

The beginning of the fissure of Sylvius is at the outer margin of the anterior perforated space, and separates the temporal from the frontal and central lobes of the cerebrum.

The junction of the optic nerves is called the optic chiasm or commissure. This junction takes place in the middle line and in front of the terminus of the crura cerebri. Outward and forward from the commissure projects the optic nerves, while backward and outward extend the optic tracts, which curve around the outer side of the crura cerebri and disappear between them and the inner margin of the temporal lobes.

Behind the optic chiasm is an elevation (really depression) of the floor of the cerebrum called the tuber cinereum. The tuber cinereum is prolonged (downward) in the shape of a funnel-shaped stalk, the infundibulum, upon which or attached to which is the pituitary gland (providing the brain has been carefully removed). The two lobes of the pituitary body can be determined-a small central posterior one situated within the horse-shoe shaped anterior and larger one. See Development, page I 75 .

Immediately posterior to the tuber cinereum and close to the middle line are two small but well-marked, rounded nodules, the corpora albicantia (from their white color) or mammillaria (from their resemblance to the mammæ).

Between the corpora mammillaria and the pons, and within the depression between the crura is the posterior perforated space formed by the arteries of the posterior median ganglionic system. 
All these structures are located in the area contained between the temporal lobes laterally, the pons posteriorly, and the frontal lobes anteriorly. This space is called the interpeduncular space. Within it the Circle of Willis has already been found, page i6 $\mathrm{I}$.

The lamina cinerea, optic chiasm, tuber cinereum, infundibulum, and posterior perforated space all take part in forming the floor of the third ventricle of the brain.

The Superficial Origin of the Cranial Nerves.

I. The Olfactory. Figs. 2 I, 28, 29, 9, I 3.

The olfactory nerve, as described, is really not a nerve but an aborted offset from the foetal forebrain.

Its central cavity, which is at first in communication with the cavity of the forebrain, becomes obliterated as development proceeds. Diag. I 4.

The nerve consists of four portions: the roots (of which there are three), the tract, the bulb, and the olfactory nerves proper. The three roots may be traced, one externally (which passes to the amygdaloid nucleus in the anterior part of the roof of the descending horn of the lateral ventricle), a middle (which terminates by connecting with the fibres of the anterior commissure within the substance of the corpus striatum), and the internal (which passes to the anterior part of the gyrus fornicatus).

The tract is a slender, triangular-shaped bundle which passes forward from the roots and terminates in an expanded extremity-the bulb. Both lie within the olfactory sulcus of the frontal lobe.

The olfactory bulb rests upon the cribriform plate of the ethmoid bone, and the olfactory nerves proper pass downward through the foramina in that plate to the mucous membrane of the nose. 


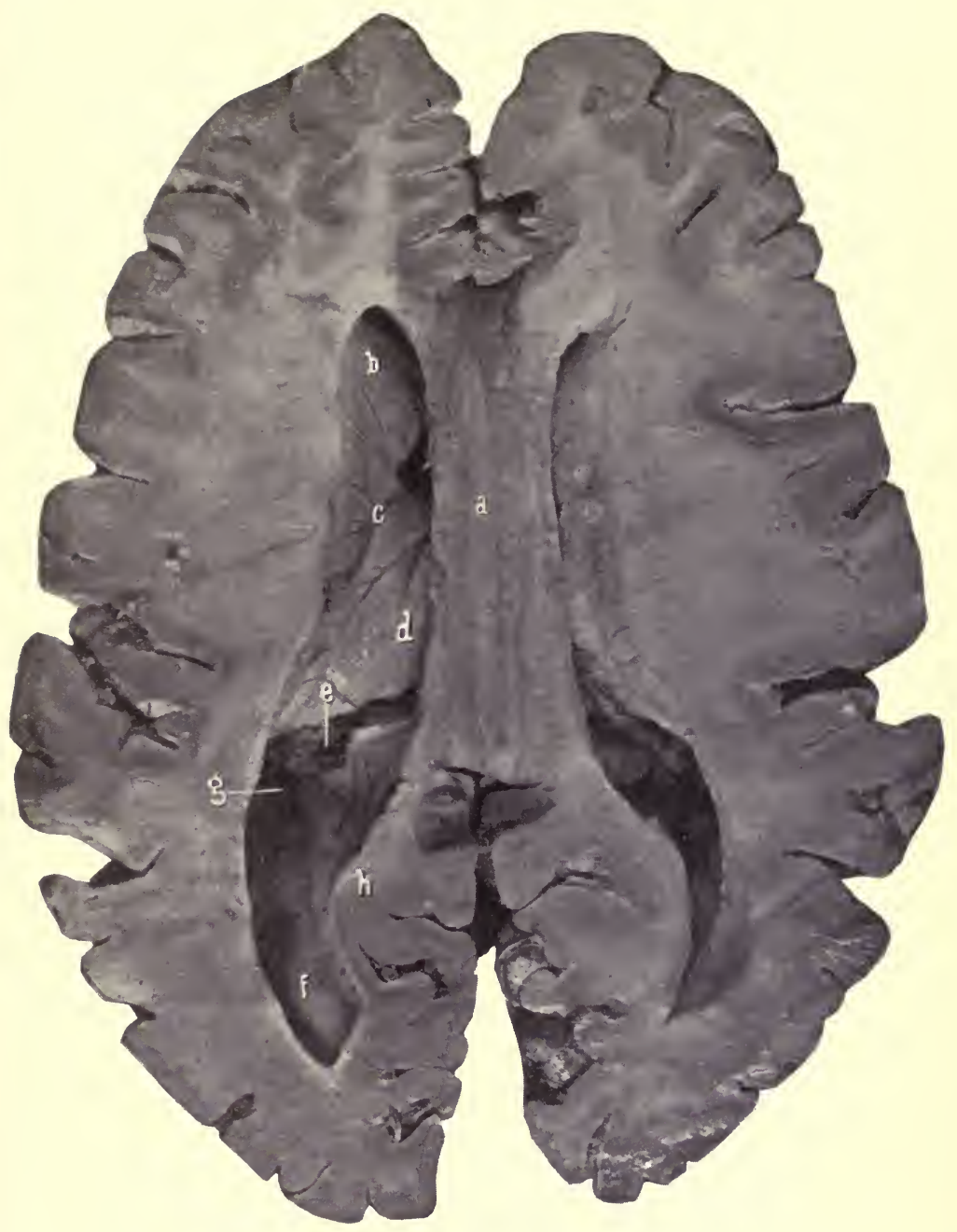

Fig. 30. Horizontal Section of Cerfebrum. Corpus Callosum and lat. ERAL VENTRICLES.

$a$, Corpus callosum, showing the longitudinal and transverse striations.

$b$, Caudate nucleus and anterior horn of the lateral ventricle.

$c$, Tænia semicircularis; groove between the caudate nucleus and the optic thalamus.

$d$, Optic thalamus.

$e$, Choroid plexus disappearing into the middle horn $(g)$ of the lateral ventricle.

$f$, Posterior horn.

h, Hippocampus minor. 
The cortical centre of smell is located in the uncinate convolution, hippocampus major, and the pes hippocampi.

2. The Optic Nerve. Figs. 2 I, 28, 29, 38, 53, 54, IO, I I, I 2.

This passes forward from the optic commissure or chiasm to enter the optic foramen in company with the ophthalmic artery, the latter being external and inferior to the nerve. See page 57 .

The optic chiasm is the junction of the two optic tracts at the middle of the base of the brain; it lies in front of the tuber cinereum and rests upon the optic groove of the sphenoid bone.

The optic tracts extend backward and outward from the optic chiasm, winding around the crura cerebri and under cover of the temporal lobes. At a later stage of the dissection the optic tracts will be found to arise from the external geniculate body, the pulvinar of the optic thalamus, and the anterior of the corpora quadrigemina (the external arm); and from the internal geniculate body and the posterior of the corpora quadrigemina (the internal root). The cortical centre of sight is in the cuneate convolution of the occipital lobe.

3. The Motor Oculi. Figs. 2 I, 29, 38, IO, I I.

This nerve issues from the inner side of a crus cerebri, close to the pons, passes forward through the cavernous sinus, and leaves the skull through the sphenoidal fissure. See page 57 .

(Its deep origin is from a nucleus in the floor of the aqueduct of Sylvius, and its cortical representation is in the convolutions about the lower extremity of the fissure of Rolando.) 
4. The Trochlear. Figs. 21, 29, 34, IO, I I.

This is found curving forward around the outer side of the crura cerebri. It enters the cavernous sinus and leaves the skull through the sphenoidal fissure. See page 50. (The deep origin of the nerve is from a nucleus in the floor of the aqueduct of Sylvius behind the third nucleus. The fibres of the fourth nerve decussate across the valve of Vieussens, then proceed on their way around the crura cerebri.)

5. The Trigeminus. Figs. 2 I, 29, 53, IO, I I, I 2.

The superficial origin of this nerve is from the side of the pons in two bundles, a larger anterior (sensory) and a smaller posterior (motor). These bundles pass forward over the inner end of the petrous portion of the temporal bone, and upon the front surface of the petrous bone the sensory root terminates in the Gasserian ganglion while the motor root passes beneath the ganglion to the foramen ovale. See page 46 :

(The deep origin of the fifth nerve is from three sets of nuclei; one in the floor of the aqueduct of Sylvius, from which comes the descending root; a middle from the floor of the fourth ventricle, from which arises the middle root; and a third nucleus from the upper part of the cervical division of the spinal cord, from which arises the ascending root. The descending root is sensory and trophic, the middle motor and sensory, and the ascending sensory.)

Ophthalmic division see page 54. Superior maxillary division see page I 49. Inferior maxillary division see page I I 7 .

6. The Abducens. Figs. 29, IO, I I.

The superficial origin is in the groove between the pons and medulla, and from between the olivary body and the anterior pyramids of the latter. The nerve continues for- 
ward through the cavernous sinus to the sphenoidal fissure, through which it passes. See pages 43, 46, 57 .

(The deep origin of the nerve is from a nucleus in the floor of the fourth ventricle.)

\section{The Facial Nerve. Figs. 29, 53, IO, I I, I 2.}

The facial arises from the groove between the pons and the medulla at the outer side of the olivary body. The nerve enters the internal auditory meatus with the eighth nerve, traverses the Fallopian canal, and emerges at the base of the skull through the stylomastoid foramen. See page $6 \mathrm{I}$.

Shortly after entering the canal the nerve is slightly thickened; this is called the geniculate ganglion. From this ganglion are given off the greater, lesser, and external superficial petrosal nerves.

The great superficial petrosal nerve passes through the hiatus Fallopii and then runs forward and inward to the foramen lacerum medium, pierces the cartilage which fills in this opening, joins the deep petrosal nerve, and forms the Vidian nerve. See page I $5 \mathrm{I}$.

The lesser superficial petrosal nerve passes through a small opening external to the hiatus Fallopii, runs forward and inward, and leaves the skull through the foramen ovale to terminate in the otic ganglion. See page I I9.

The external superficial petrosal nerve traverses a minute hole external to the above-mentioned foramina and terminates in the sympathetic plexus on the middle meningeal artery.

Below the geniculate ganglion the facial nerve gives off the following minute twigs: one to the stapedius muscle, another communicating with the pneumogastric, and a larger branch-the chorda tympani-which courses 


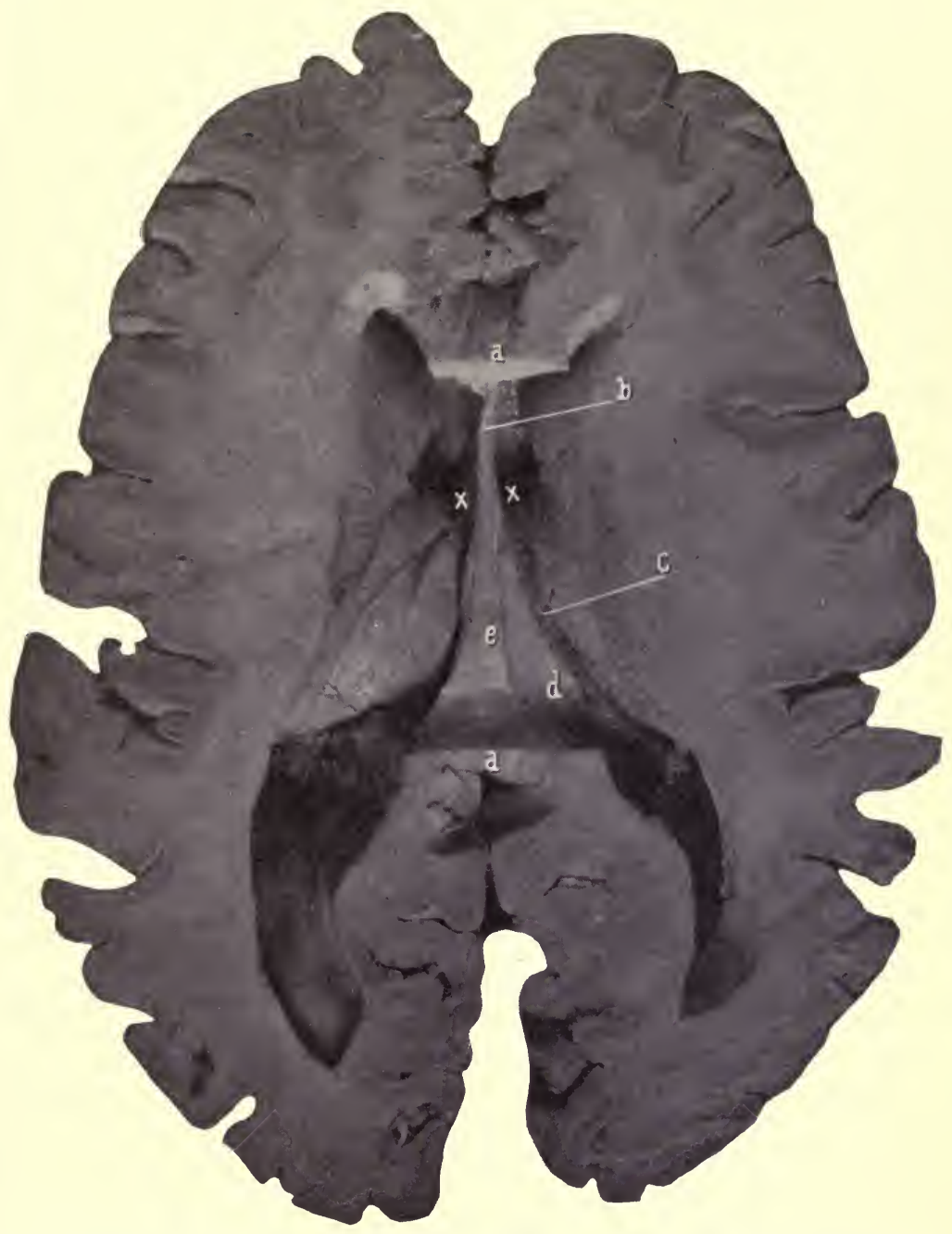

Fig. 3i. Horizontal Section of Cerebrum. Corpus Callosum Removed $a, a$, Divided extremities of corpus callosum.

$b$, Septum lucidum.

$c$, Choroid plexus.

$d$, Posterior pillar of fornix. The free margin of this is called the fimbria.

$e$, Body of fornix divided along its attachment to the under surface of the corpus callosum.

$x, x$, Foramina of Monro. Remaining parts same as in preceding figure. 
through the middle ear and reaches the base of the skull by way of the canal of Huguier. See page I I 8 .

(The deep origin of the facial nerve is from a nucleus in the floor of the fourth ventricle opposite the lower part of the pons ; its cortical area is in the lower part of the precentral convolution of the frontal lobe.)

\section{The Auditory Nerve. Figs. 29, 53, 10.}

This arises just external to the facial at the posterior margin of the pons. Its course is outward to the internal auditory meatus along with that nerve. Besides these two nerves the auditory vessels traverse this foramen.

The distribution of the auditory nerve is in two portions : one to the cochlea, utricle, and saccule; the other to the semicircular canals. (The deep origin of the nerve is from three nuclei in the floor of the fourth ventricle, and the cortical location for hearing is in the first or superior temporal convolution.)

\section{The Glossopharyngeal Nerve. Figs. 53, IO, I I.}

The ninth, tenth, and accessory portion of the eleventh nerves arise from the side of the medulla, in the groove between the restiform tract behind and the olivary body and the lateral tract of the medulla in front.

The deep origin of these nerves is from a continuous nucleus in the floor of the posterior part of the fourth ventricle and adjacent part of the cord.

The ninth, tenth, and the eleventh nerves leave the skull through the central compartment of the jugular foramen. See page 98 .

There are two ganglia developed upon the glossopharyngeal nerve in its course through the jugular foramenthe jugular and the petrous. 


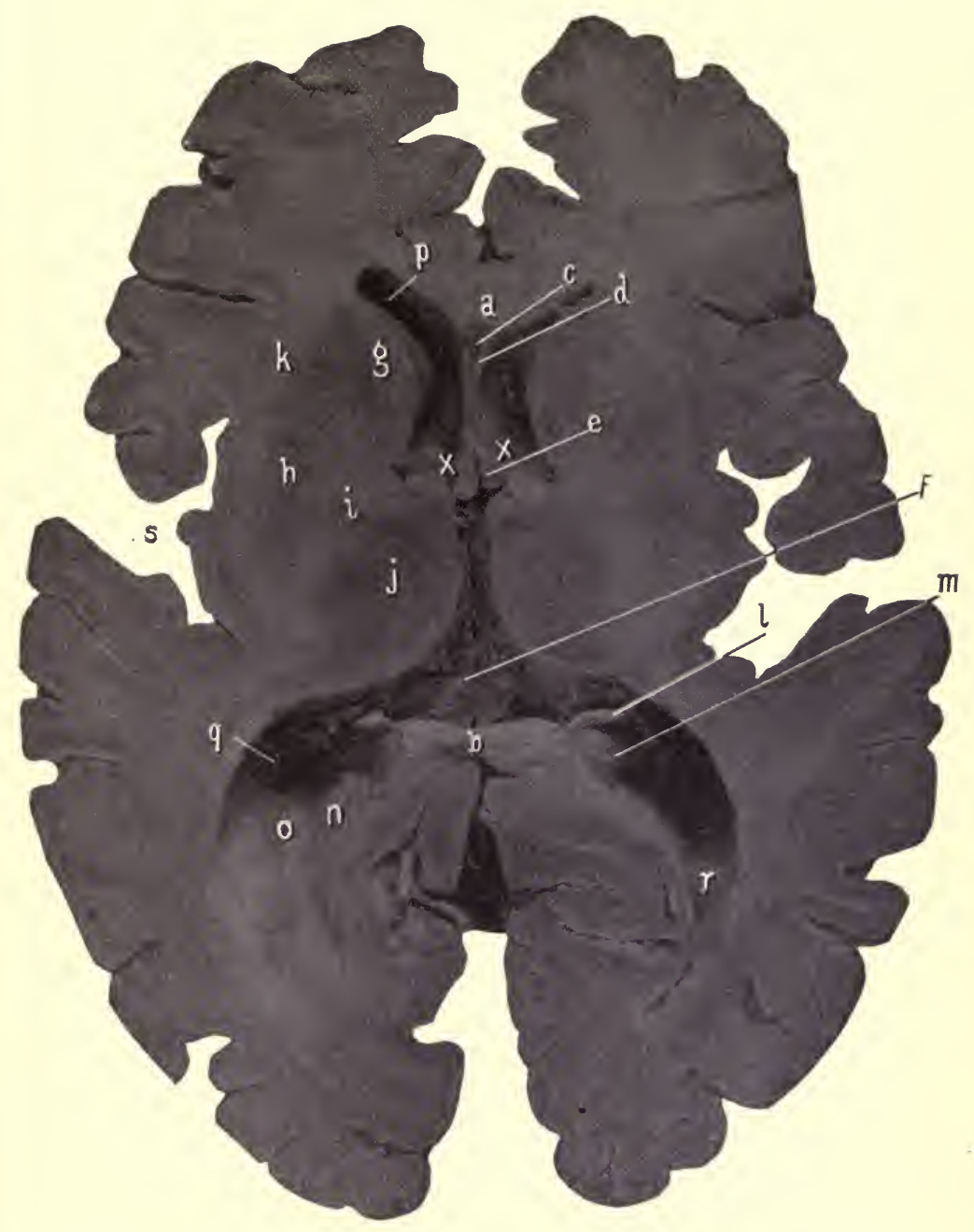

Fig. 32. Horizontal Section of Cerebrum. Fornix Removed.- $a, b$, Divided corpus callosum. $c$, Fifth ventricle. $d$, Right side of the septum lucidum. $e$, Anterior pillar of fornix descending to base of cerebrum. $f$, Velum interpositum. The choroid plexuses have been removed with the section of the cerebrum. Their divided extremities show in the descending horns of the ventricle. $g$, Caudate nucleus. $h$, Lenticular nucleus. $i$, Internal capsule. Note that the caudate and lenticular nuclei are continuous with each other around the anterior portion of the internal capsule. $j$, Optic thalamus. $k$, External capsule. $l$, Posterior pillar of fornix and fimbria. $m$, Hippocampus major. $\boldsymbol{n}$, Hippocampus minor. $o$, Eminentia collateralis. $p$, Anterior horn. $q$, Middle horn. $r$, Posterior horn of lateral ventricle. $s$, Within the fissure of Sylvius. The island of Reil and its convolutions. $x, x$, Foramina of Monro. 
Within the skull the ninth nerve gives off minute meningeal twigs to the dura and arachnoid.

Within the jugular foramen arises from the petrous ganglion the nerve of Jacobson, (which passes to the tympanum), communicating branches to the superior cervical ganglion of the sympathetic, to the pneumogastric, and to the lingual branch of the facial.

IO. The Pneumogastric Nerve. Figs. 53, IO, I I

As given above and described on page Io3.

\section{I. The Spinal Accessory. Figs. 53, IO, I I .}

The accessory portion has been given with the glossopharyngeal; the spinal part arises from the anterolateral surface of the cord as low as the fifth or sixth cervical vertebra. These filaments pass upward, uniting to form a single trunk, which enters the skull through the foramen magnum; then, being joined by the accessory portion, the nerve passes out from the skull as above given along with the ninth and tenth nerves through the central division of the jugular foramen. See page $9 \mathrm{I}$.

\section{2. The Hypoglossal Nerve. Figs. 53, IO, I I.}

The superficial origin of this nerve is from the groove between the olivary borly and the anterior pyramids of the medulla. The nerve is in the shape of two portions, which do not become joined until they enter the anterior condyloid foramen, through which the nerve leaves the skull. See page 88 .

(Within the foramen a recurrent meningeal twig is given off to the dura.)

(The deep origin of the nerve is from a long nucleus internal to that for the ninth, tenth, and eleventh nerves, close to and parallel with the median line in the floor of 
the fourth ventricle. The cortical area for the nerve is in the lower part of the convolutions about the fissure of Rolando.)

\section{DISSECTION.}

Separate the halves of the cerebrum and examine the upper surface of the corpus callosum.

With a long, sharp knife slice off the top of the hemispheres, about half an inch above the corpus callosum. The oval section presenting is called the centrum ovale minus. Its surface will show the divided vessels, and its margin the arrangement of the white and gray matter. The formation of the convolutions and sulci should be examined.

Remove a slice of the brain on a level with the corpus callosum, opening into the lateral ventricles. This section is called the centrum ovale majus. Or the brain matter can be torn from the fibres of the corpus callosum on one side, showing its arched formation and the forceps major and minor. While on the other side the brain can be cut away, opening into the lateral ventricle.

Consult the section, Fig. 30, which shows the horns of that ventricle, and expose them in the specimen in a similar manner on this one side.

\section{The Corpus Callosum.}

The form of the great central commissure which joins the two halves of the cerebrum must be learned from transverse and anteroposterior sections of the brain. See Figs. $27,30,38,45$.

The corpus callosum is composed of fibres which connect symmetrical parts of the opposite hemispheres, but these fibres being restricted to the central portion of the opposing surfaces of the hemispheres, they become crowded together, more at the anterior and posterior extremities.

On cross section of the corpus callosum, this bunching together of the fibres is best seen-and the result is to produce a thickening at these points. The posterior thickening is called the splenium and the anterior the genu or knee, because here the fibres of the corpus turn downward to the base of the brain. This bend constitutes the genu (knee), and the lower descending limb of the corpus gradu- 
ally becomes thinner and is termed the rostrum, where it passes backward into the lamina cinerea. The part of the corpus callosum between the genu and splenium is the body, and is thinner than those parts.

There is a small extension of the corpus callosum folded beneath the splenium, called the reduplicated fold of the corpus callosum. Fig. 39 .

The corpus callosum has a bow-shape on transverse section. The concave extremities of the bow arch over the lateral ventricles (whose roofs they form), and the central depressed portion is the portion of the corpus callosum which is seen on separating the hemispheres.

To the under surface of the corpus callosum is found attached (anteriorly) the septum lucidum and (posteriorly) the fornix. Figs. 4I to 48 inclusive.

Remember that these three structures are all developed from the fœetal lamina terminalis. Page I73. Diag. I6.

The anterior part of the corpus callosum covers in the anterior horn and body of the lateral ventricle, while the posterior part (the tapetum) forms, in a like manner, the covering for the descending and posterior horns of the same ventricle.

The bundles of fibres which radiate to the frontal lobe from the genu are called the forceps minor, while the bundles of fibres which pass from the splenium and the reduplicated fold of the corpus callosum radiate to the occipital lobe and constitute the forceps major.

As stated, the corpus callosum is made up of transverse fibres, with the exception of a few thin bundles of fibres which extend from the anterior part of the base of the brain to the forceps major, and use the corpus callosum as a bridge for their course. Fig. 30. 


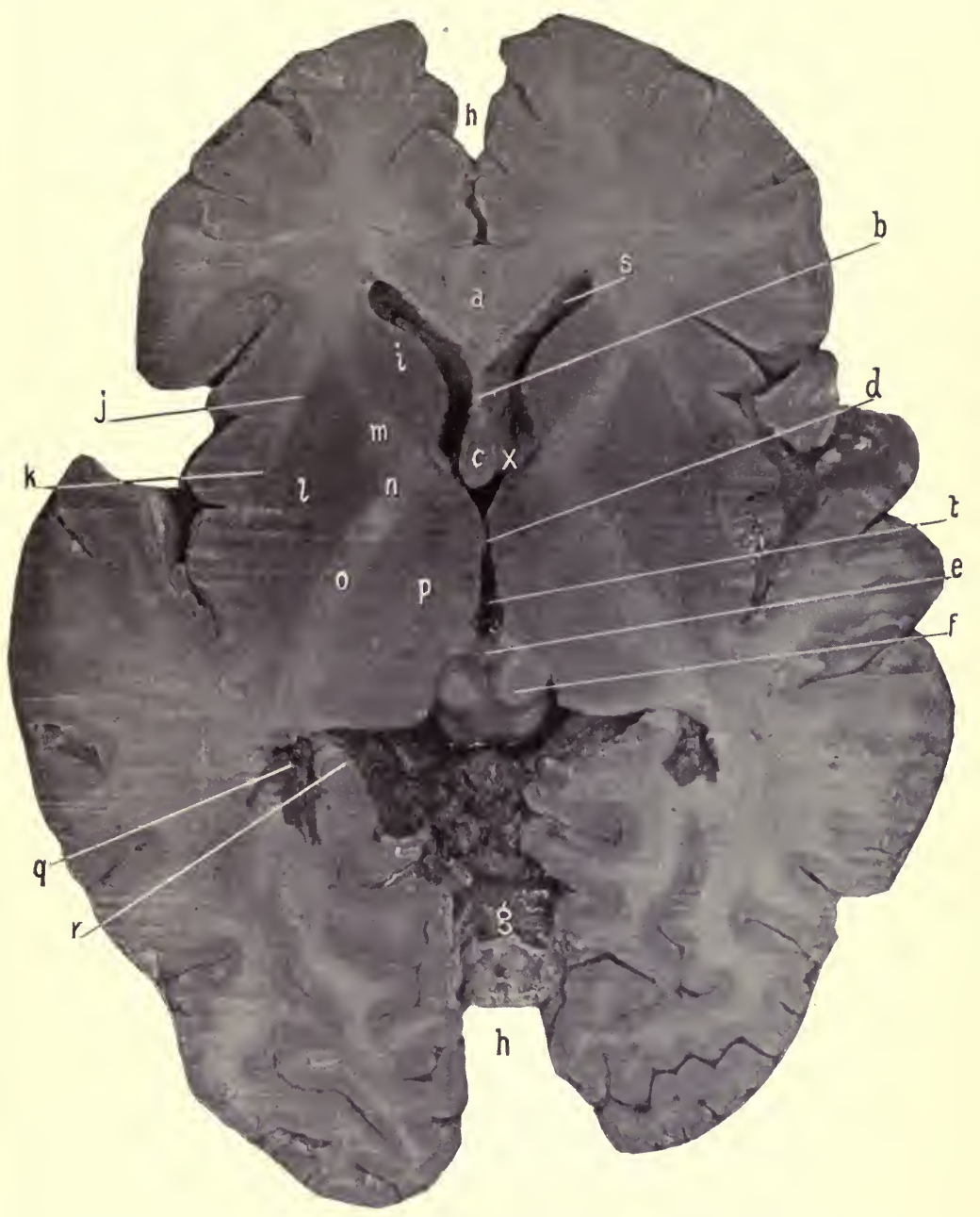

Fig. 33. Horizontal Section of Cerebrum. The Internal Capsule and AdJoINING NUClei.- $a$, Corpus callosum. $b$, Septum lucidum. $c$, Anterior pillars of fornix. $d$, Middle commissure. $e$, Pineal gland and posterior commissure. $f$, Corpora quadrigemina. $g$, Upper surface of cerebellum. $h, h$, Longitudinal fissure. $i$, Caudate nucleus. $j$, External capsule. $k$, Claustrum. $l$, Lenticular nucleus. Note that the claustrum and the lenticular nucleus are closely approaching each other at their posterior portions. Deeper down they become continuous. $m$, Anterior limb. $n$, Knee, and $o$, Posterior limb of the internal capsule. $p$, Optic thalamus. $q$, Middle horn, containing the choroid plexus. $r$, Hippocampus major. $s$, Anterior horn. $t$, Third ventricle. $x$, Foramen of Monro. The base of it. 
These fibres are grouped in four bundles, one beneath each gyrus fornicatus-tenice tecte-and one on either side of the median line-the strice longitudinales; between the latter a thin groove is left called the raphé.

The longitudinal fibres start from the base of the brain, at the beginning of the fissure of Sylvius, and pass forward to the corpus callosum (as its peduncles), over which they continue to the forceps major.

The tæniæ tectæ are stray fibres belonging to the gyrus fornicatus.

The Lateral Ventricles. Figs. 30, 3I, 32, 35, 4I to 49 inclusive.

These are the irregular cavities which are contained within the cerebral hemispheres.

Each lateral ventricle communicates with the third ventricle and with each other through the foramina of Monro. A lateral ventricle presents, in horizontal section, a central portion, an anterior, a posterior, and a middle prolongation or horn. The anterior horn curves forward, downward, and outward, the posterior horn curves backward and inward, and the middle horn takes a course backward, outward, downward, forward, and inward (the descending horn).

All these prolongations open into the body of the ventricle, which is that portion of the ventricle extending from the foramen of Monro to the beginning of the descending and posterior horns, and corresponds to the intraventricular portion of the optic thalamus.

The Boundaries of the Ventricle.-The anterior horn, body, and posterior horn are roofed over by the arched corpus callosum as previously described. The internal wall of the anterior horn is formed by the septum lucidum; of 


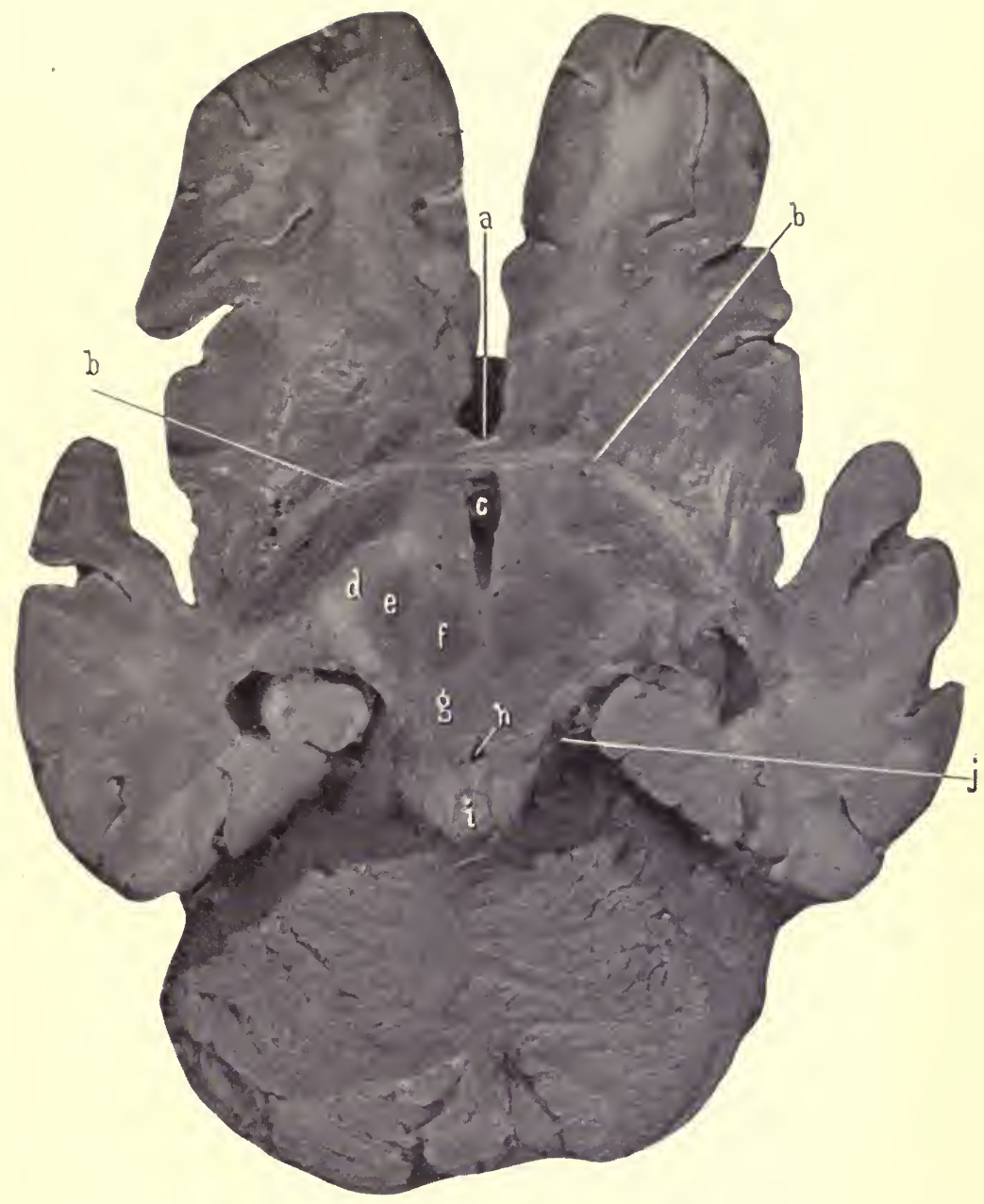

Fig. 34. Dissection to Show the Course of the Anterior Commissure.$a$, The rostrum of corpus callosum. (See Fig. 38.) The letter lies in the longitudinal fissure. $b, b$, Fibres of anterior commissure. Their course is outward from the middle line, then downward and backward underneath the lenticular nuclei (which have been removed) to terminate in the roof of the middle horn of the lateral ventricle. $c$, The third ventricle. $d$, Internal capsule, now the crusta of crus cerebri. $e$, Locus niger. $f$, Red nucleus. $g$, Tegmentum. $h$, Aqueduct of Sylvius. $i$, Lingula resting upon the valve of Vieussens. $j$, Fourth nerve. 
the body, by the posterior part of the septum lucidum and the fornix; of the posterior horn, by the hippocampus minor and the bulb of the posterior horn.

The hippocampus minor is an elevation along the inner wall of the posterior horn, formed by the involution of the brain corresponding to the calcarine fissure.

The bulb of the posterior horn is a bulging in the posterior horn above and parallel with the hippocampus minor produced by the bundle of fibres-the forceps major-which is proceeding from the splenium of the corpus callosum to the occipital lobe. The floor of the anterior horn and body of the ventricle present from before backward the caudate nucleus (the intraventricular portion of the corpus striatum), the tænia semicircularis, the optic thalamus, choroid plexus, and posterior pillar of the fornix.

The floor of the posterior horn is a flat surface behind but becomes gradually elevated as the floor passes into the descending horn ; this raise is the eminentia collateralis, and is produced by the upheaval of the brain over the collateral fissure.

The middle or descending horn of the lateral ventricle: The course of this extension of the cavity of the lateral ventricle is backward, outward, and downward, then forward, downward, and inward to the anterior extremity of the temporal lobe.

The roof of the horn is formed by that portion of the body of the corpus callosum passing to the temporal lobe and is called the tapetum, also by the tail of the caudate nucleus and the tænia semicircularis. At the extreme anterior portion the roof is formed by the amygdaloid nucleus in which the tail of the caudate nucleus and the tænia semicircularis terminate. Figs. 36, 37.

The inner wall presents a long, rounded eminence-the 


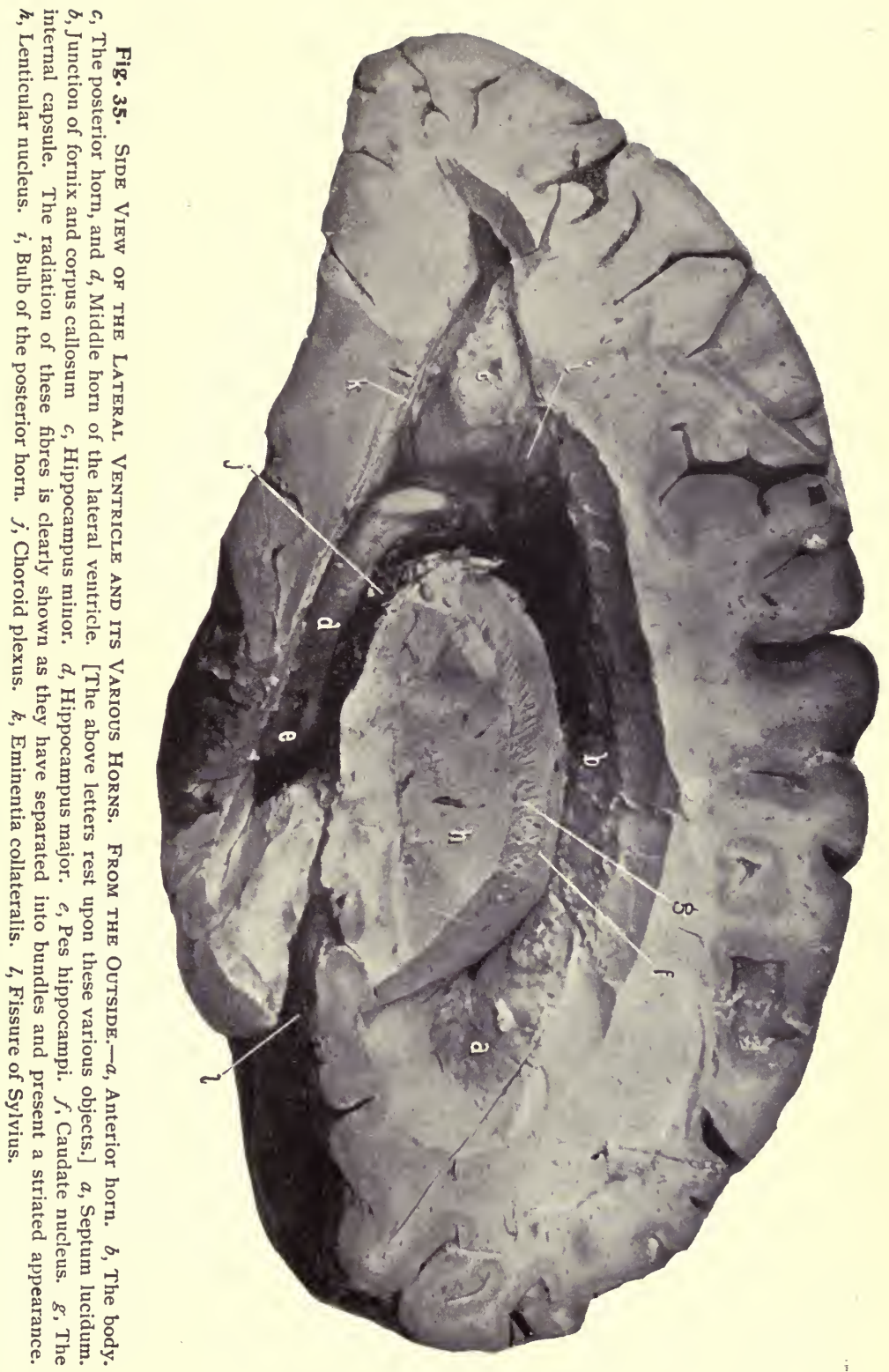


hippocampus major-the posterior pillar of the fornix, the fimbria (the free edge of this portion of the fornix), and the choroid plexus.

The floor is taken up by the eminentia collateralis.

The hippocampus major is an elongated roll of brain matter along the inner wall of the descending horn of the lateral ventricle, which is produced by the outward involution of the cerebral cortex of the temporal lobe to accommodate the hippocampal or dentate fissure. Figs. 35, 45, 46, 47 .

The hippocampus major terminates at the anterior extremity of the descending horn in a broadened indented end which resembles (slightly) the paw of an animalhence the name of pes hippocampi.

\section{DISSECTION.}

Remove the corpus callosum, exposing both lateral ventricles. Trace the descending horn on one side by cutting the brain away from the outside. Save the septum lucidum (between the halves of which find the fifth ventricle) and the fornix.

The Septum Lucidum. Figs. 3 I, 32, 33, 38, 39, 4 I to 44 inclusive.

This will be seen on transverse sections to be a thin, double layer, and on anteroposterior sections to have a triangular shape and to be attached in front and above to the inner surface of the corpus callosum, and below and behind to the fornix.

It is described as the attenuated wall of the hemispheres, which was imprisoned within the brain by the development of the fibres of the corpus callosum above it. But such is not its derivation, for it is a thinned and broadened lamination of the central portion of the lamina terminalis, from which it comes, the outer portion of the lamina terminalis being developed into the corpus callosum and the inner 


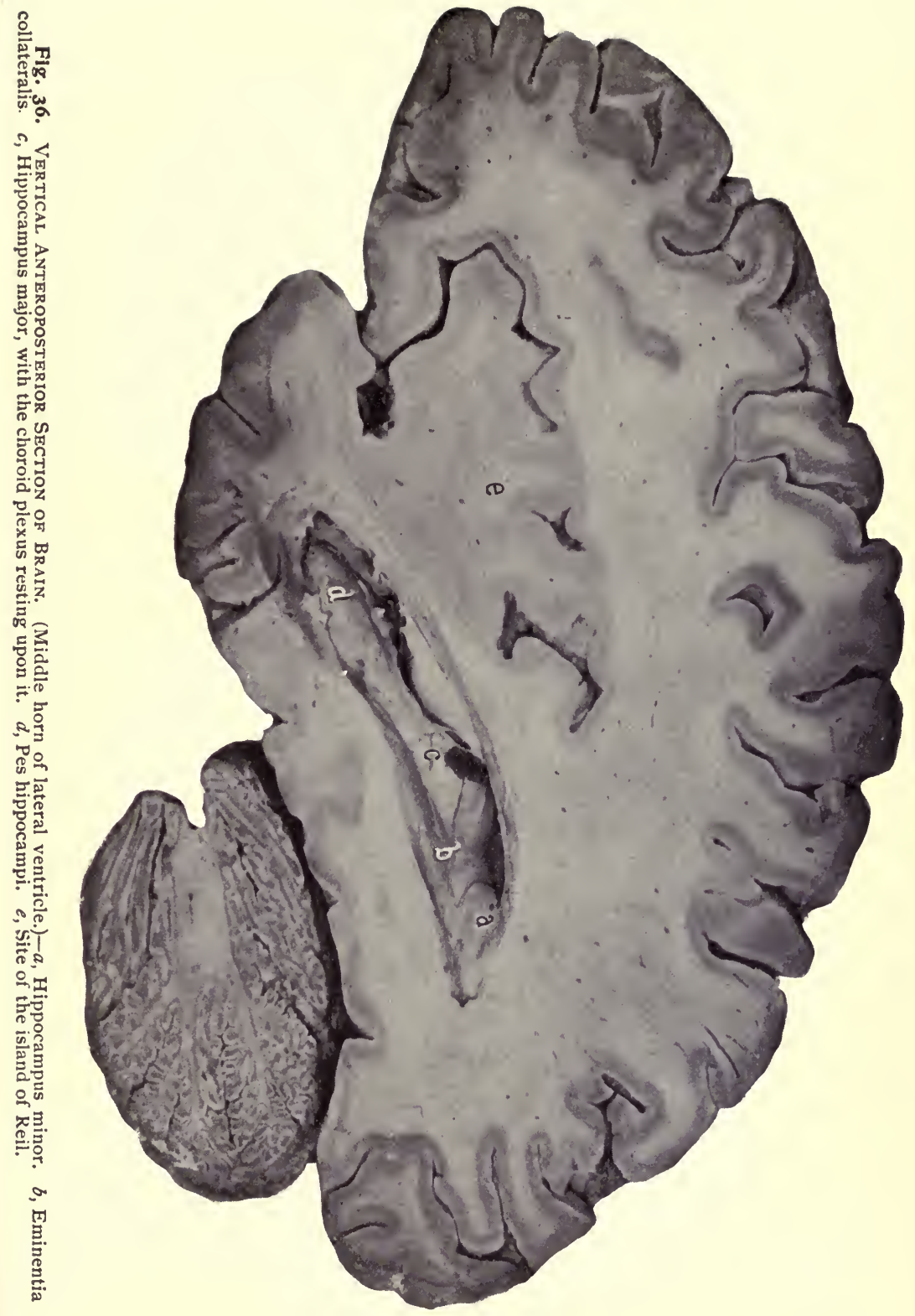


portion into the fornix. See Development, page I73 and Diag. 16.

The septum lucidum consists of a double layer for some extent ; the small, oval, slit-like cavity between the two layers is the fifth ventricle. This ventricle is not, in all probability, a portion of the fotal longitudinal fissure which has become included beneath the corpus callosum, but is formed within the originally solid septum lucidum by a process of vacuolation.

The fifth ventricle has no communication with, nor is it formed in any like manner to, the rest of the ventricular cavities of the brain.

The Fornix. Figs. 29, 31, 32, 35, 38, 39, 40, 43 to 47 inclusive.

The fornix is a double band of fibres, one on each side of the median line, separated in front and behind but coming together in the middle, where they fuse for a short distance and form the body. The anterior and posterior separated portions are called the anterior and posterior pillars respectively.

The fornix begins at the base of the brain, as the anterior pillars, in the corpora albicantia. The pillars ascend nearly vertically and at the anterior extremity of the third ventricle until they nearly reach the under surface of the corpus callosum, then they turn suddenly backward (the genua) (leaving an aperture extending into the third ventricle, which is the foramen of Monro), become united to each other, forming the body of the fornix, and to the under surface of the corpus callosum. After a short distance they again diverge, and, as the posterior pillars, pass outward and downward to the outer surface of the hippocampus major, with which they become blended. 


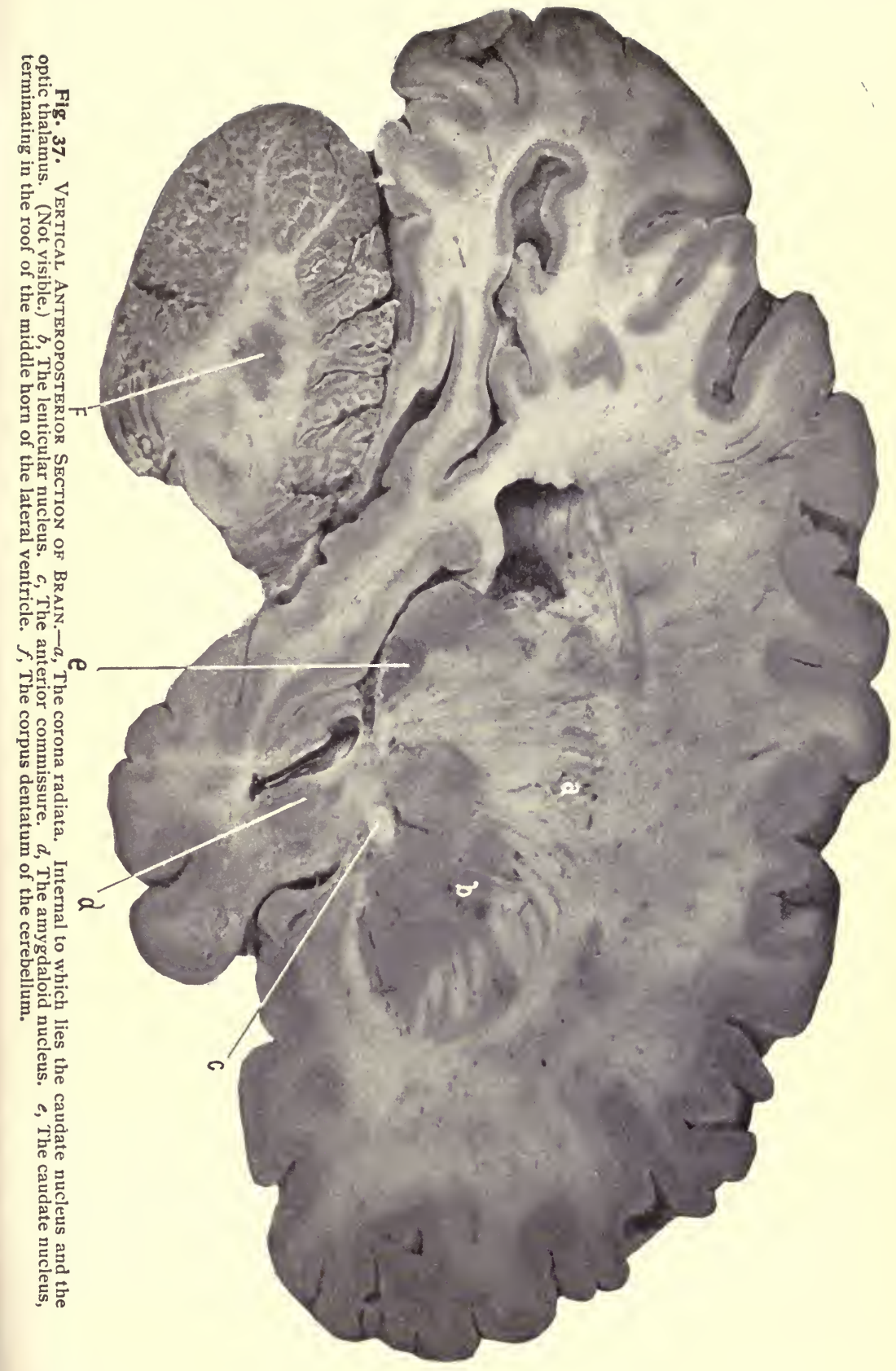




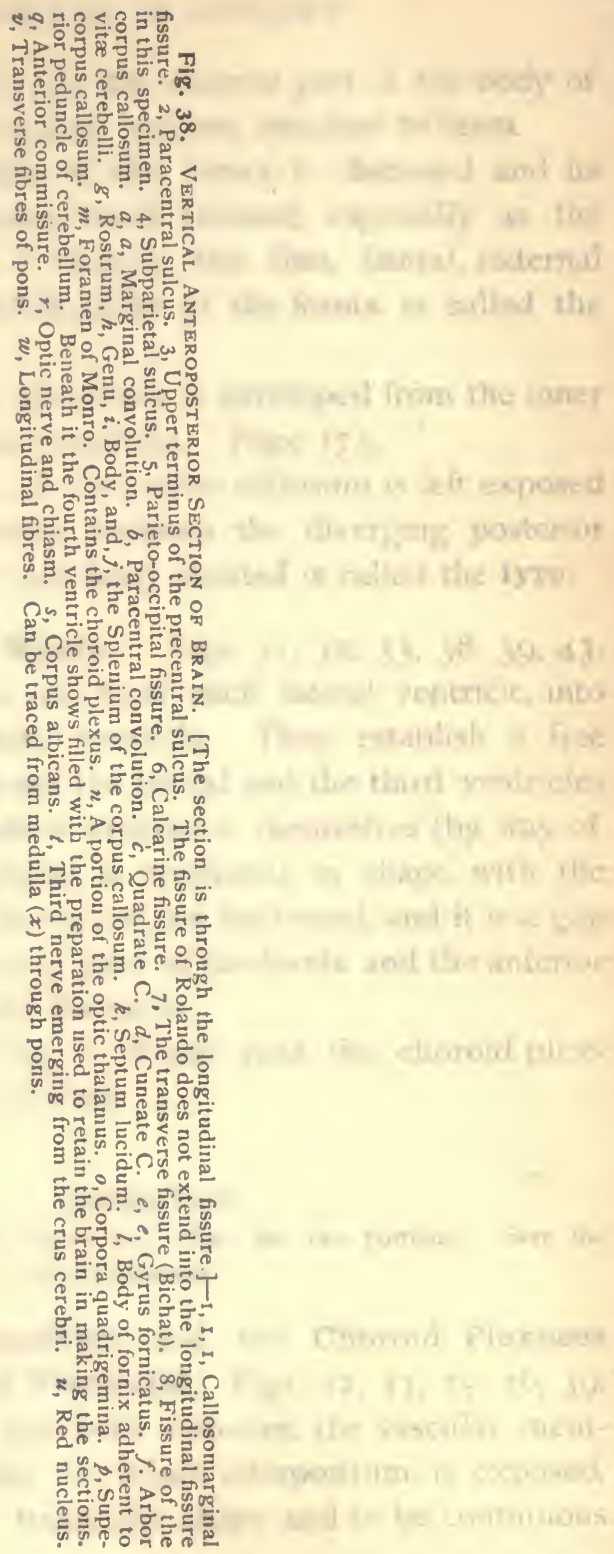




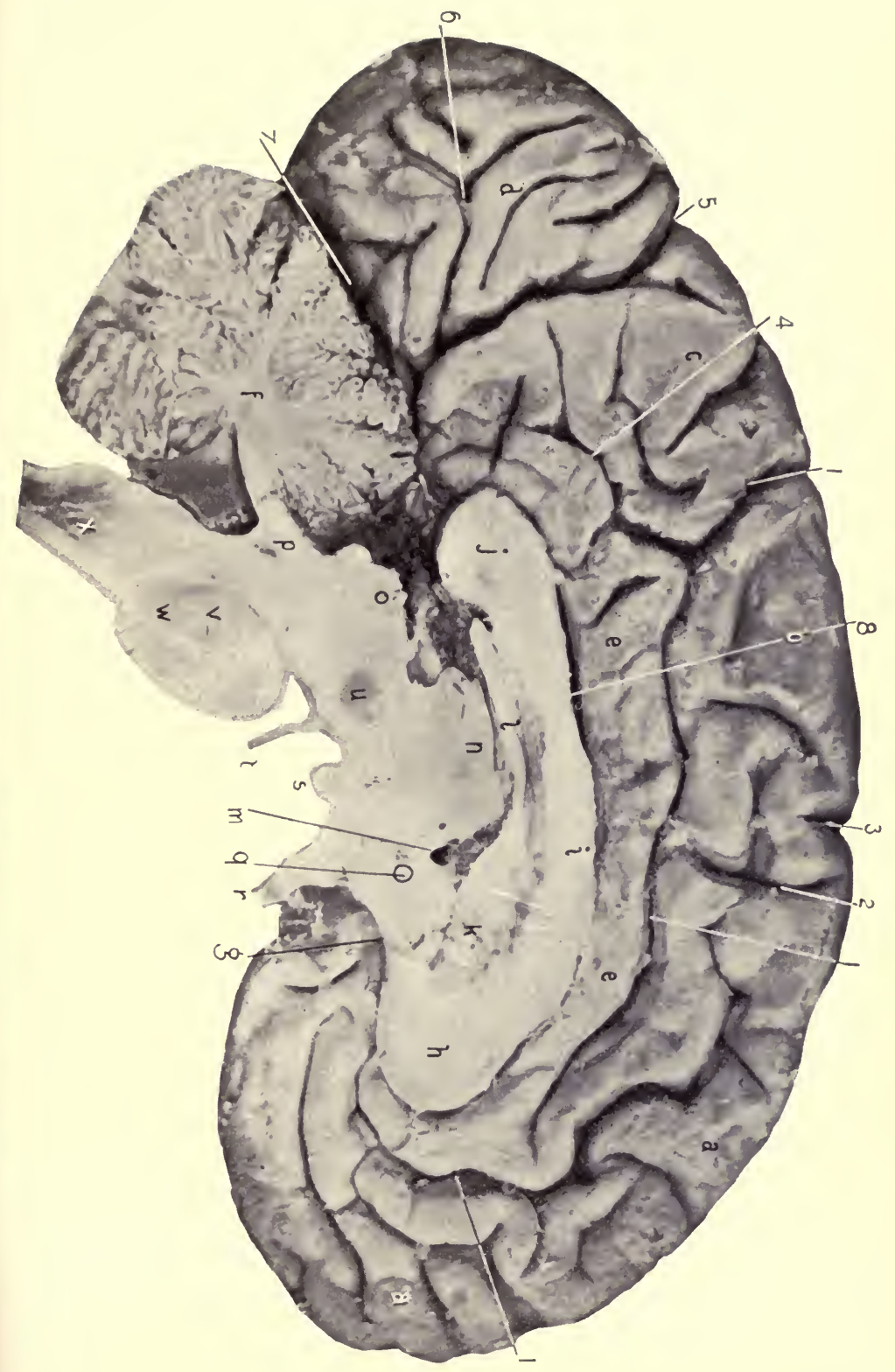


posteriorly with the pia, from which it originally springs. The velum interpositum forms the covering or roof for the third ventricle. Its margins project into the cavity of the lateral ventricles and are composed of masses of capillaries called the choroid plexuses of these ventricles.

In a similar manner from the under surface of the velum festoons of capillaries hang downward into the cavity of the third ventricle and furnish the choroid plexuses for that ventricle.

As stated above, the velum is a portion of the pia which seems to be contained within the ventricular cavity of the brain. But such is not really the case, for though it projects into the ventricles of the brain it carries in front of it a covering of epithelium which is the attenuated remains of the roof of the primitive vesicles of the brain. This layer covers the velum and the choroid plexuses and becomes continuous with the walls forming the boundaries of the ventricles and so shuts the velum and choroid plexuses out of the cerebral ventricles. Diag. I 5 .

The velum interpositum with its lining layer of epithelial cells (which represents the primitive roof of the brain) is called the tela choroidea superior, and the portion of the pia which reaches from the under surface of the cerebellum to the upper surface of the medulla, and forms the covering for the posterior portion of the fourth ventricle, is called the tela choroidea inferior.

\section{The Deep Cerebral Veins.}

(a) The vein of the corpus striatum collects the blood from the corpus striatum, optic thalamus, fornix, septum lucidum, courses forward and inward in the groove between the corpus striatum and the optic thalamus, and terminates at the entrance of the foramen of Monro. 


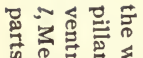

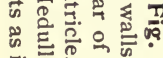

푸 웅요

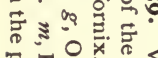

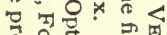

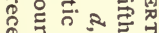
春者》的

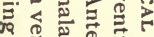

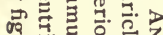

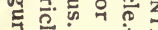
$?>01$ * $3 \Omega$ ○율 $\circ$ क क

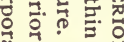

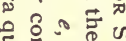

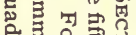
윽릉워

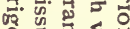
일 ह. ? $\cong$

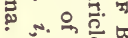

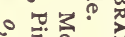
<苋 군 웅

哭证司.

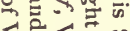
पद $<\leqslant$

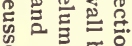

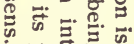
范

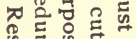

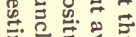
可 $\bar{\square}$ 든.

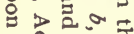
플 때유

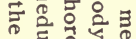

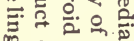

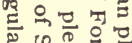

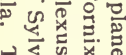
시 कै प्र 뭉융율

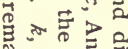
可可要这

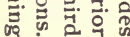

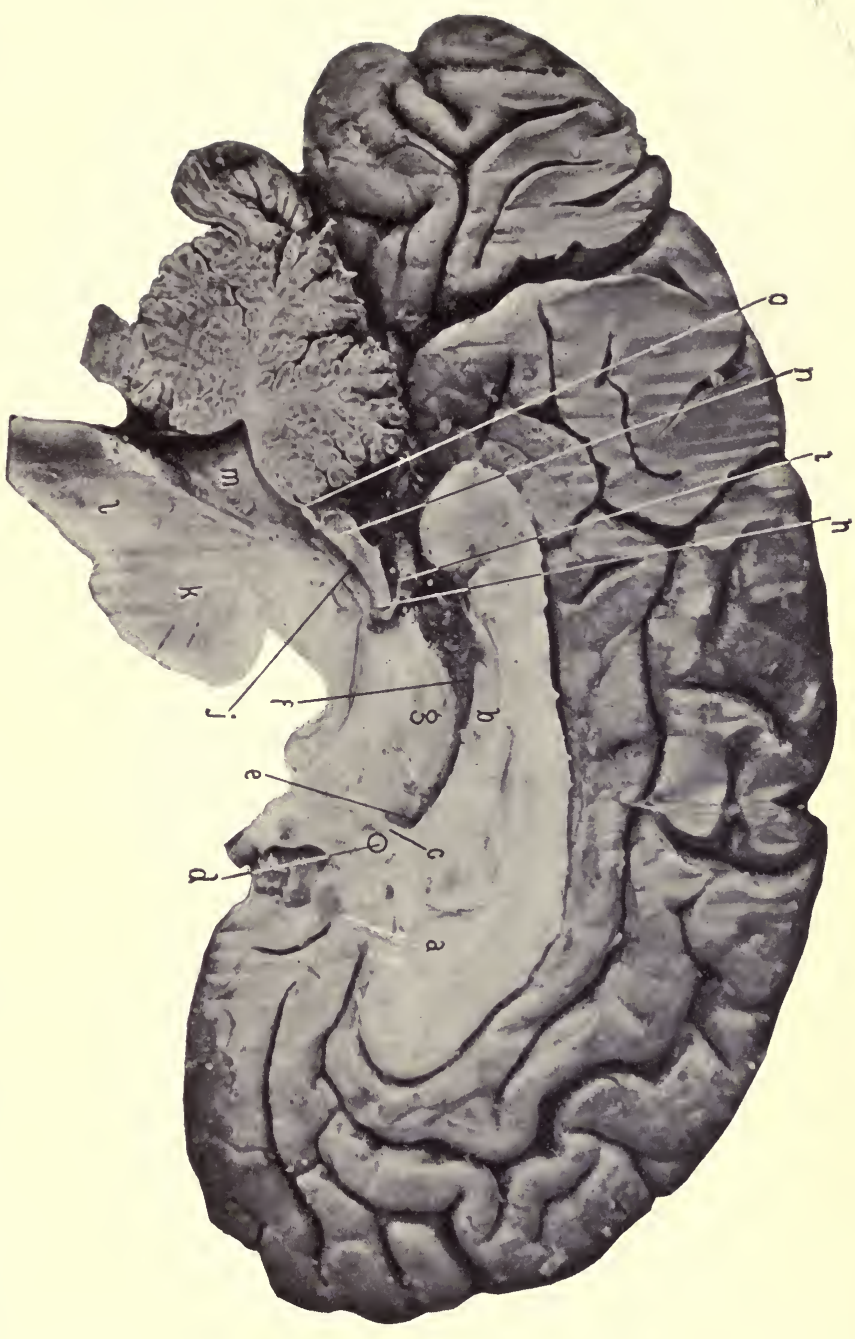


(b) The choroid vein collects the blood from the structures of the descending horn of the lateral ventricle, follows along the margin of the choroid plexus to the foramen of Monro, where it joins the vein above to form the vein of Galen.

(c) The vein of Galen is formed by the union of the veins of the corpus striatum and the choroid plexus at the foramen of Monro. It passes backward in the velum interpositum, close to the middle line, and at the posterior margin of the splenium unites with a corresponding vein of the other side to form the common vein of Galen, which is about half an inch long and terminates in the straight sinus. Besides the veins which unite to form it, the vein of Galen drains the third ventricle, and the posterior horn of the lateral ventricle, and the common or great vein of Galen the superior surface of the cerebellum.

\section{DISSECTION.}

Remove the velum interpositum and the choroid plexuses, being careful in doing so to leave the pineal gland behind, as the gland is firmly adherent to the under surface of the posterior part of the velum.

The Third Ventricle. Figs. 33, 34, 39, 43 to $46,50,5$ I, 54.

The third ventricle is now exposed. Its boundaries will be seen to consist of the following parts :-

Laterally, the optic thalami and the crura of the pineal gland; in front, by the anterior pillars of the fornix, the anterior commissure, and the septum lucidum which fills in the gap between the pillars of the fornix ; behind, by the posterior commissure, the pineal gland and its diverging crura; the floor is composed of the following structures already described in connection with the base of the brain,(see Fig. 29) the lamina cinerea, optic chiasm, tuber cine- 


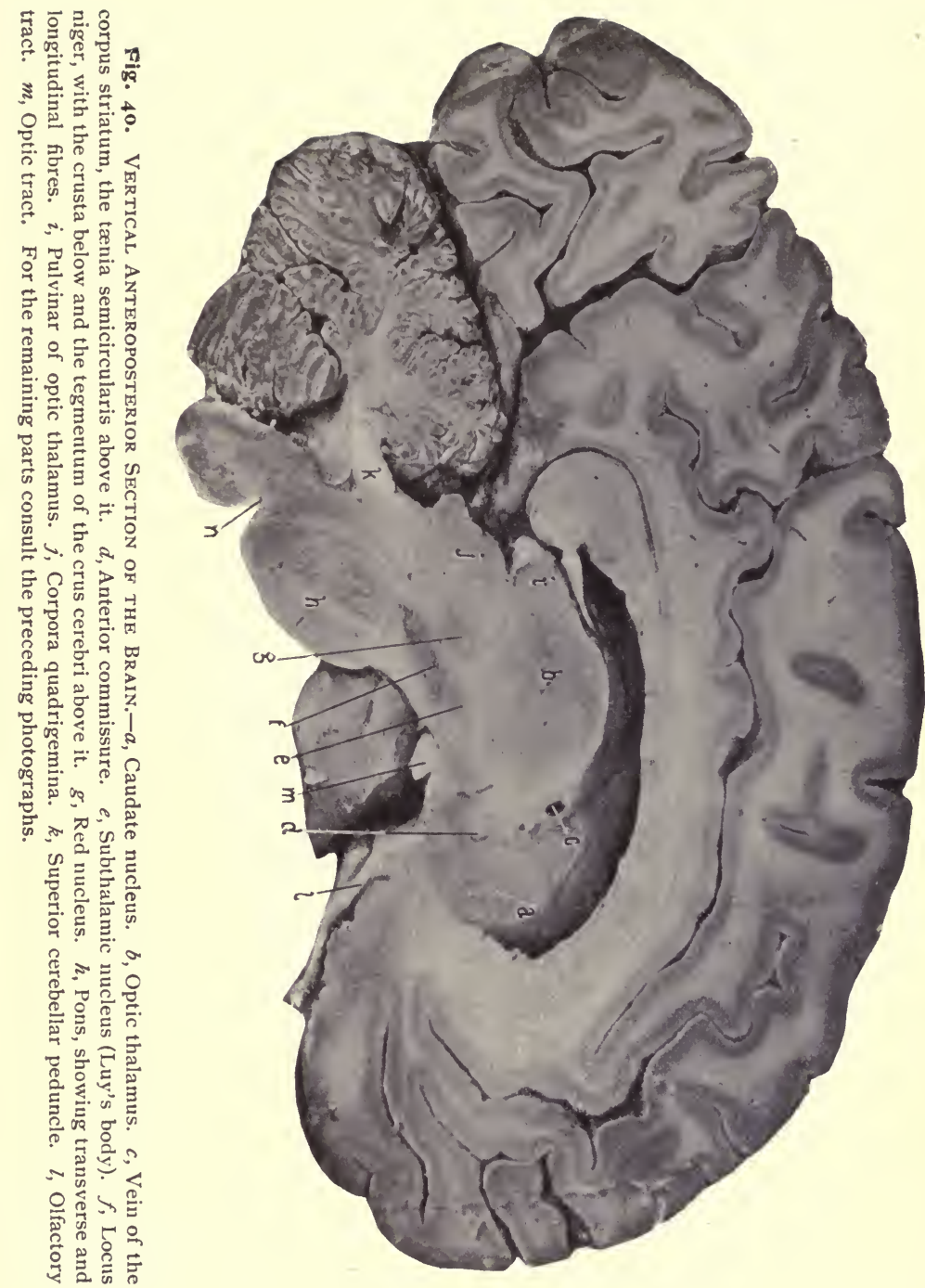


reum, infundibulum (and pituitary gland), the corpora albicantia, and the posterior perforated space. The third ventricle communicates by means of the foramina of Monro with the lateral ventricles anteriorly, and through the aqueduct of Sylvius with the fourth ventricle posteriorly. The opening into the infundibulum is only a blind one.

The Pineal Gland. Figs. 33, 39, 46, 47, 54 .

This is a small gland located between the posterior extremities of the optic thalami and resting upon the groove between the anterior pair of the quadrigeminal bodies. It is about as large as a small pea, and is connected to the rest of the brain by a divided stalk, the lower fibres of which connect it with the posterior commissure, the upper fibres, under the name of the peduncles of the pineal gland, running forward along the optic thalami, marking the junction of the superior and internal surfaces, to cross the depression at the floor of the foramen of Monro and join the anterior pillars of the fornix.

The Corpora Quadrigemina. Figs. 33, 38, 39, 40, 47, $48,53,54$.

These consist of four rounded elevations arranged in pairs, situated on either side of the middle line, between the optic thalami and the cerebellum, and upon the quadrigeminal lamina which forms the roof of the aqueduct of Sylvius. These pairs are separated from each other by vertical and horizontal intersecting grooves, forming a crucial sulcus. The anterior or superior pair of elevations is called the nates, the posterior pair the testes.

The quadrigeminal bodies have a narrowing prolongation forward which is called their brachia, or arms. Between these brachia and close to the groove under the pulvinar of the optic thalamus is the internal geniculate body, which 


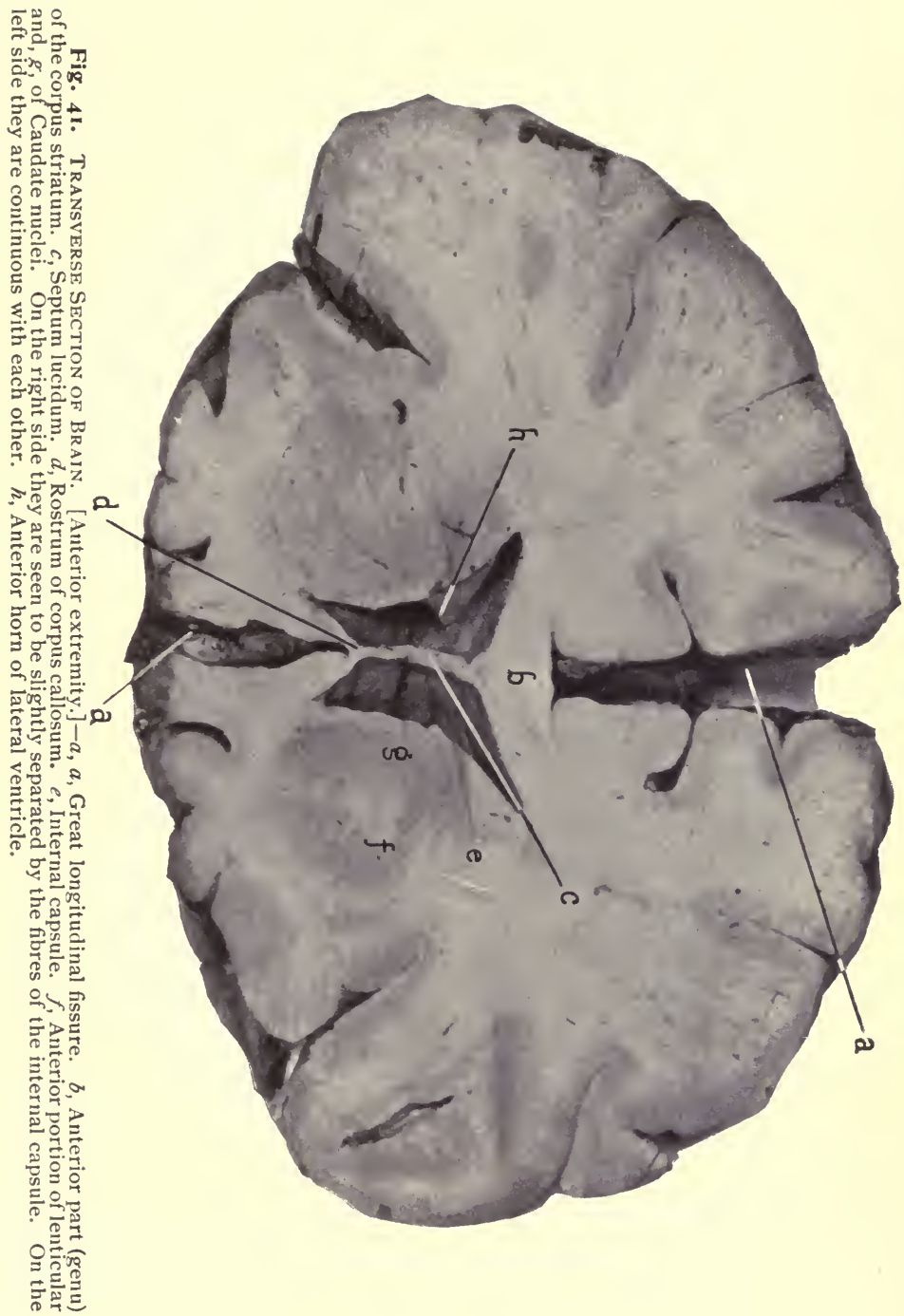


is a small oval nucleus with its long axis placed transversely. Figs. 53, 54.

The external geniculate body is described as an enlargement at the outer portion of the pulvinar, with which it is continuous. See Optic thalami, page 230.

\section{DISSECTION.}

One half of the cerebrum should now be carefully sectioned horizontally, and the other half transverse-vertically as far as the beginning of the aqueduct of Sylvius. From these sections study the situation, shape, and relations of the basal ganglia and nerve pathways.

The Corpora Striata. (Study carefully all the various sections, especially Figs. 30, 33, 35, 37, 43, 44, 5 I.)

These are symmetrical ovoid elevations of gray matter, which project into the floor of the lateral ventricles.

A corpus striatum, by consulting the various sections, will be seen to consist of an oval form with a long axis placed anteroposteriorly. It is located internal to and opposite the central lobe of the cerebrum. It reaches to and forms the base of the brain corresponding to the anterior perforated space, and extends into the cavity of the lateral ventricle as the caudate nucleus. It will be seen to consist of two masses of gray matter separated from each other by a sheet of white fibres, except at their extreme anterior inferior portion.

The two gray masses are the caudate and lenticular nuclei, the white fibres which separate them the internal capsule.

The Caudate Nucleus.-This is the inner and superior (also anterior and posterior) band of gray matter which lies within the lateral ventricle, and hence is the intraventricular portion of the corpus striatum. It is wider in front, where it forms a prominent elevation in the anterior horn, 


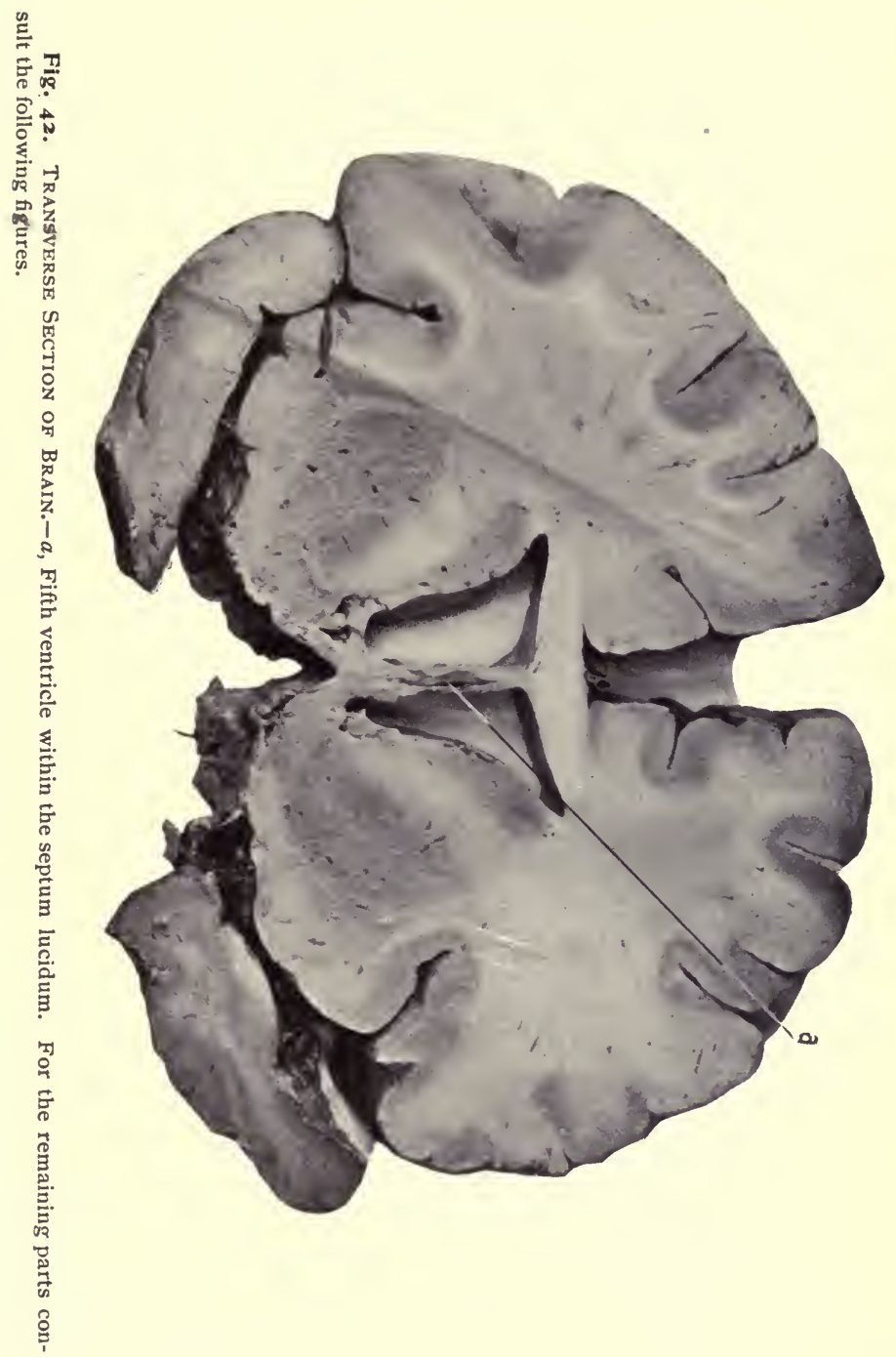


but narrows as it extends posteriorly, forming the outer portion of the floor of the body of the ventricle; then it turns downward into the descending horn, forming in this position the outer portion of its roof; finally it terminates in the amygdaloid nucleus, which is placed in the anterior part of the roof of the descending horn.

The large anterior portion in the anterior horn is the head of the caudate nucleus; its body corresponds to the body of the ventricle itself, while the tail of the nucleus is its slender prolongation into the descending horn.

The nucleus thus describes almost a complete circle and shows two cut surfaces in horizontal or vertical transverse sections.

The Lenticular Nucleus.-Is the lower and outerextraventricular-portion of the corpus striatum. In horizontal section it is biconvex in shape and faces inward and outward; in vertical-transverse section it is triangular or wedge-shaped, with the base of the wedge abutting on the central lobe; in vertical anteroposterior sections its outline is oval with its lower part reaching to the base of the brain (at the anterior perforated space).

The lenticular nucleus is separated from the caudate nucleus by a sheet of white fibres - the internal capsulewhich bounds it on the inside. There is a similar sheet of white fibres bounding the nucleus upon its external surface called the external capsule. The two nuclei are not entirely separated by the internal capsule, as will be seen on study of a series of anteroposterior sections, but they become continuous with each other around the anterior margin of the internal capsule; they are also connected by numerous fibres which pass through the anterior limb of the internal capsule.

A vertical transverse section of the nucleus in fresh speci- 


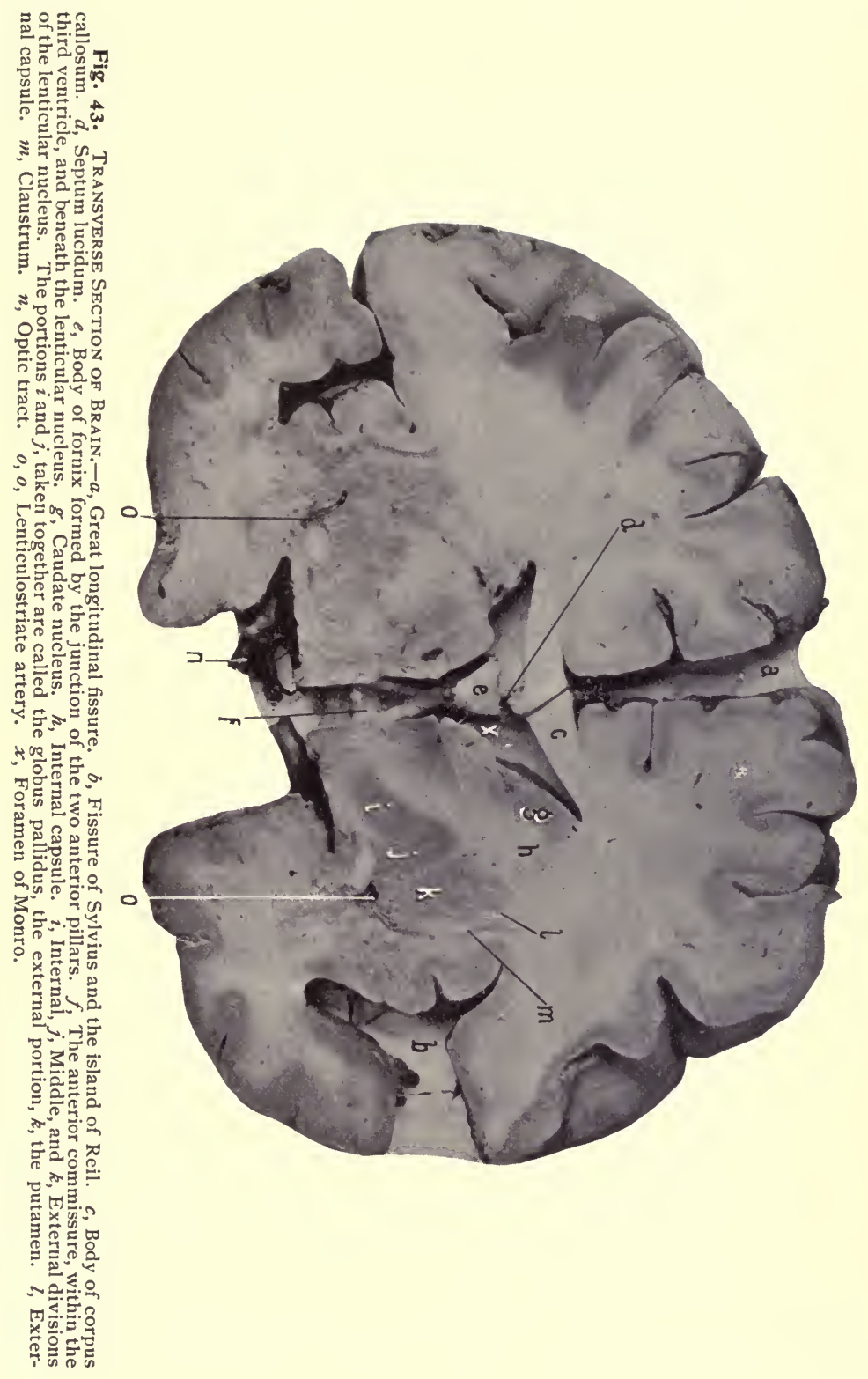


mens (and in the original photographs) shows the lenticular nucleus to consist of three portions vertically divided by thin striations of white fibres (the external and internal medullary laminæ). The two inner portions of the nucleus are lighter in color than the outer portion; the former constitute the globus pallidus, the latter the putamen.

The Optic Thalami. Figs. 30, 33, 39, 40, 46, 5 I, 53, 54. These are double structures, which form the lateral walls for the third ventricle and lie internal and posterior to the corpora striata. An optic thalamus is somewhat oval in shape with the long axis directed anteroposteriorly. It is slightly contracted in front and below, and expanded behind and above. The narrower anterior extremity is the anterior tubercle, which forms the posterior boundary for the foramen of Monro; the prominent posterior part is the posterior tubercle, or the pulvinar. The pulvinar terminates externally in a slight oval elevation, which is the external geniculate body.

The superior surface of the optic thalamus forms a part of the floor of the lateral ventricle, and furnishes support for the posterior pillar of the fornix and the choroid plexus. The course of the latter is indicated on the thalamus by a shallow groove, sulcus choroideus, which divides the upper surface into two portions, the external being within the lateral ventricle and the internal covered by the velum interpositum. The internal surface forms a nearly vertical wall and limits the third ventricle laterally. It is divided from the superior surface by the peduncle of the pineal gland which runs forward from the gland to join the anterior pillars of the fornix.

At the posterior internal part of the superior surface of the thalamus, in the angle between the peduncle of the 


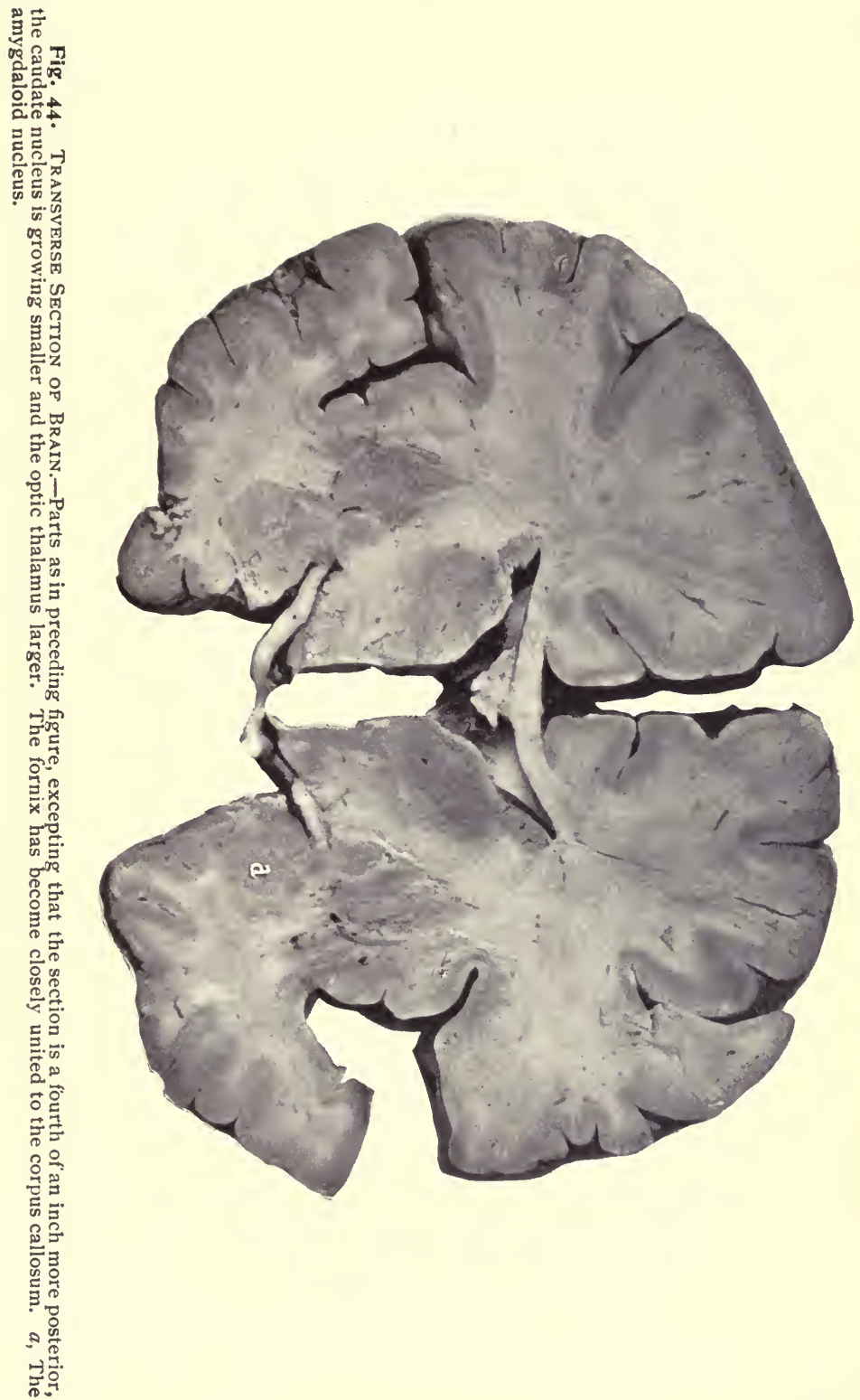


pineal gland and the pulvinar, is a shallow depression termed the trigonum habenulæ.

The optic thalami are joined by two connecting bundles of fibres, - the middle, gray or soft commissure, which unites them at the middle of their opposing surfaces, and the posterior commissure, which unites these surfaces beneath the pineal gland and over the beginning of the aqueduct of Sylvius.

The Development of the Corpus Striatum, Optic Thalamus, and Internal Capsule.

The optic thalamus and the corpus striatum are developed from the floor (the former) and the floor and side wall (the latter) of the fore and interbrains opposite and posterior to the narrowing orifice which connects the cavity of the fore and interbrains ( $i . e$., the future foramen of Monro). They first appear as separate swellings distinct from each other and from the wall of the vesicle, but later the corpus striatum becomes fused along its line of contact with the vesicular wall and also to the optic thalamus along their opposing surfaces. See page 173.

At this early stage the corpus striatum does not show the nuclei that distinguish it in the adult. They (the caudate and lenticular nuclei) are developed later as masses of gray cells which are bunched together in the superointernal and infero-external regions of the corpus striatum. From these cells and from the cortical wall (with which the corpus striatum has now become fused), white fibres develop which pass downward and inward, separating the two portions (caudate and lenticular) from each other (except at their extreme anterior and inferior parts), and also separating the corpus striatum from the optic thalamus. This white layer of fibres is the internal capsule. 


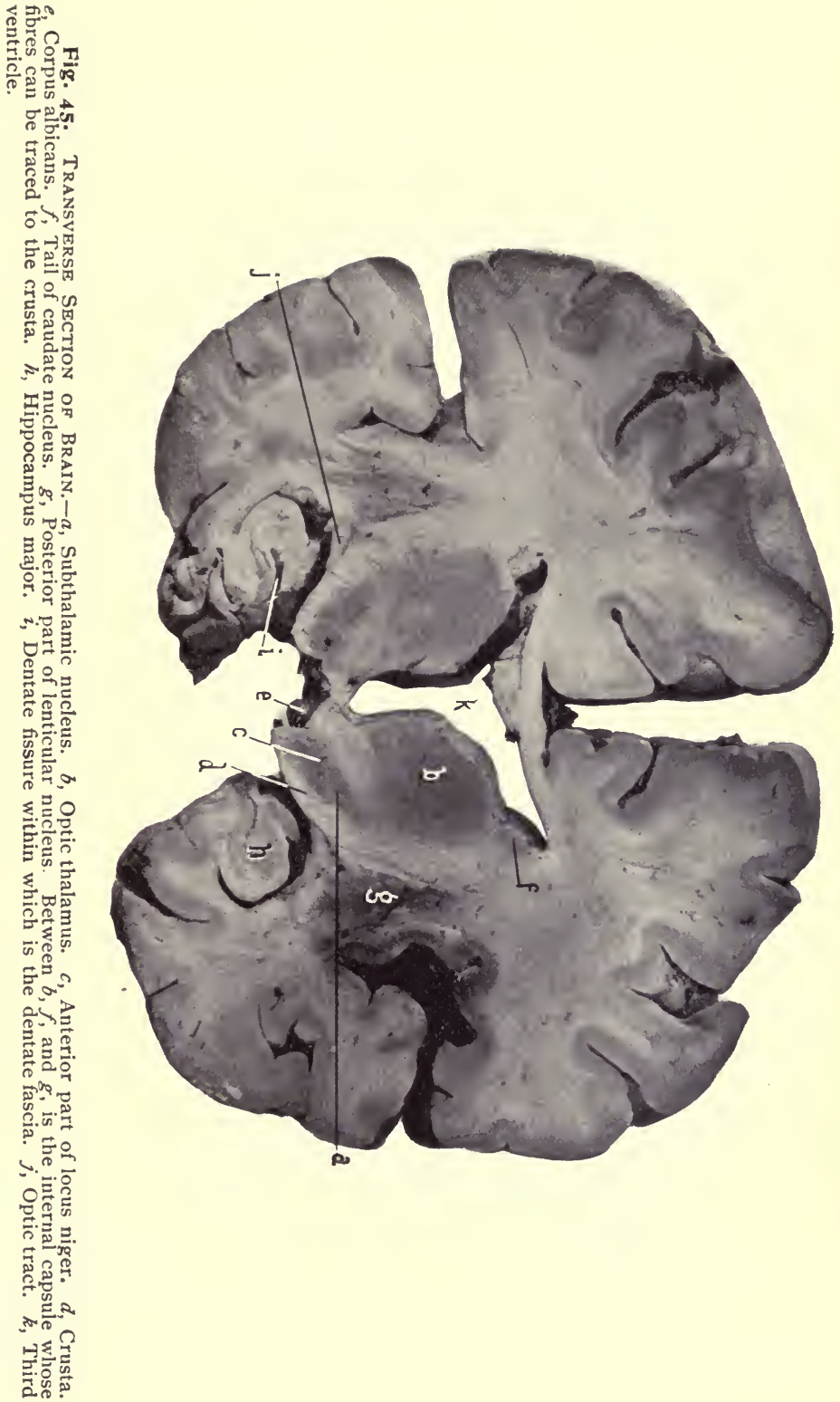


As the optic thalami grow upward they approach each other and become united along their opposing surfaces. This central bond of union later diminishes in extent, but persists as the middle commissure.

The fibres of the internal capsule turn downward and backward under the optic thalamus; thus the latter is said to rest upon the former.

\section{The Internal Capsule. Figs. 32, 35, 45.}

From what has been said it will be apparent that this part of the cerebrum is a mass of white fibres which pass from the cortex and ganglia to the base of the brain, where they appear as two rounded bundles of fibres under the name of the crura cerebri. Fig. 29.

From a vertical-transverse section the internal capsule will be seen to pass upward and outward between the optic thalamus on the inside and the lenticular nucleus on the outside, then between the caudate and lenticular nuclei, and then to radiate to the cortex of the brain. This radiation of the fibres of the internal capsule is called the corona radiata, and takes place to all parts of the cortex. Fig. 37.

On a horizontal section the internal capsule presents two portions or limbs, an anterior and posterior, united together at an angle of about I I 3 degrees. The angle is the knee, and is at the gap between the caudate nucleus and the optic thalamus.

The anterior limb lies between the caudate and lenticular nuclei, the posterior between the optic thalamus and the lenticular nucleus. As previously stated, the lenticular nucleus has a narrow sheet of white fibres covering its external surface ; this is the external capsule.

Again external to the external capsule is a plate of gray matter-the claustrum-which is placed vertically, and in 


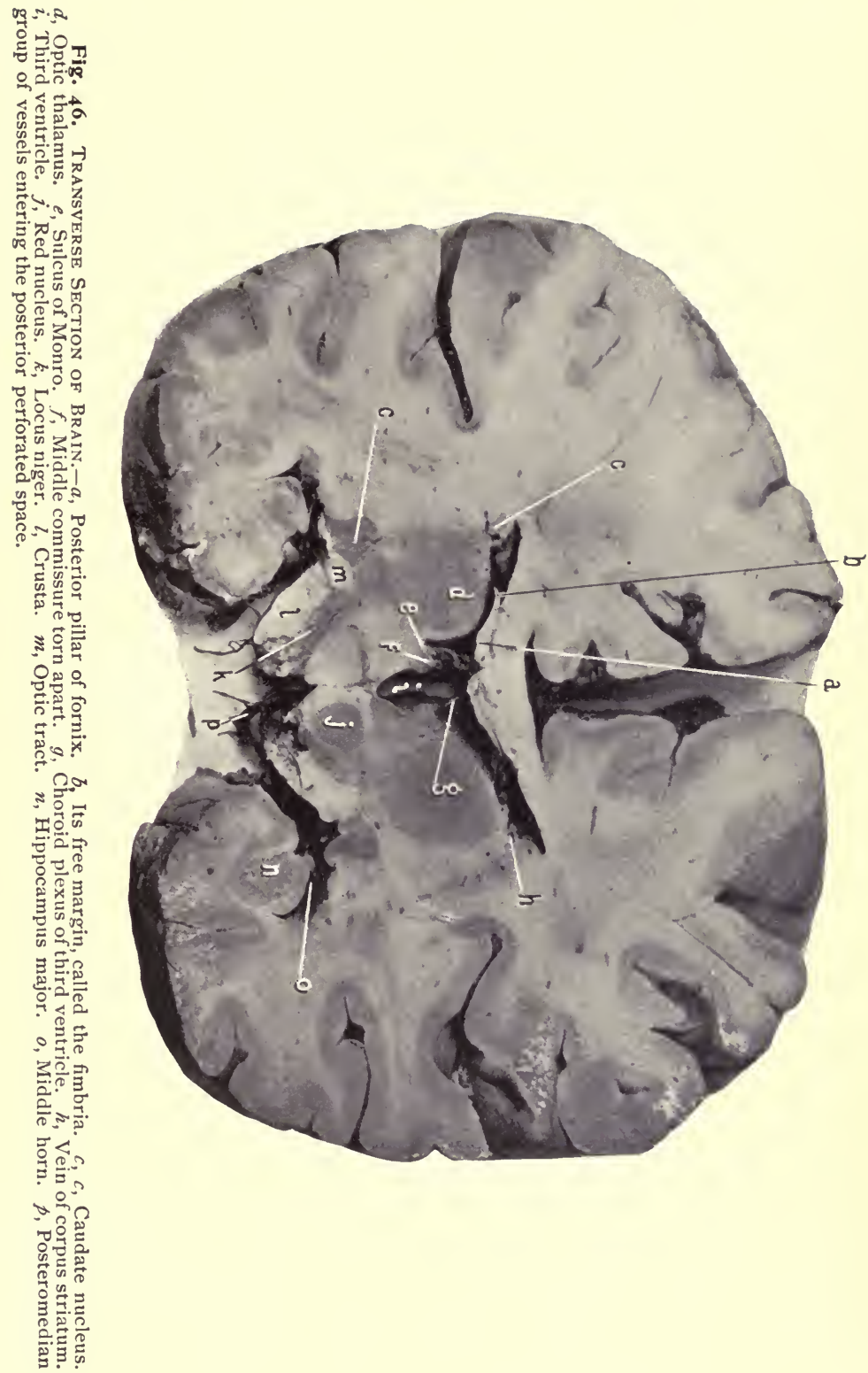


area corresponds to the base of the central lobe of the brain.

The claustrum is connected to the corpus striatum (lenticular nucleus) at its inferior margin, where the external capsule is wanting, also to the amygdaloid nucleus.

The amygdaloid nucleus (Figs. 37, 46, $5 \mathrm{I}$ ) is an oval, gray mass continuous with the cortex of the apex of the temporal lobe. It produces a bulging into the anterior part of the descending horn of the lateral ventricle. It is continuous with the claustrum, is immediately under the lenticular nucleus, and has terminating in it the tail of the caudate nucleus, the tænia semicircularis, also fibres from the external root of the olfactory nerve tract and from the anterior commissure.

The tænia semicircularis (one on each side, Figs. 30, 46 ), is a round bundle of white fibres which starts from the anterior pillar of the fornix, passes outward and backward along the inner border of the body and tail of the caudate nucleus, between this nucleus and the optic thalamus, then on into the descending horn of the ventricle, forming the inner part of its roof, to terminate in the amygdaloid nucleus with the tail of the caudate nucleus.

The anterior commissure can not be shown for its entire length in any one section, as it takes a curved course. The dissection in Fig. 34 (see also Figs. 37, 39, 43, 50) shows the commissure. It is a round bundle of white fibres (about an eighth of an inch in diameter) which crosses the anterior part of the third ventricle in front of the anterior pillars of the fornix, between which it can be seen from the third ventricle; its course is then downward and backward beneath the lenticular nucleus to terminate in the substance of the temporal lobe at the anterior part of the roof of the descending horn of the lateral ventricle, and 


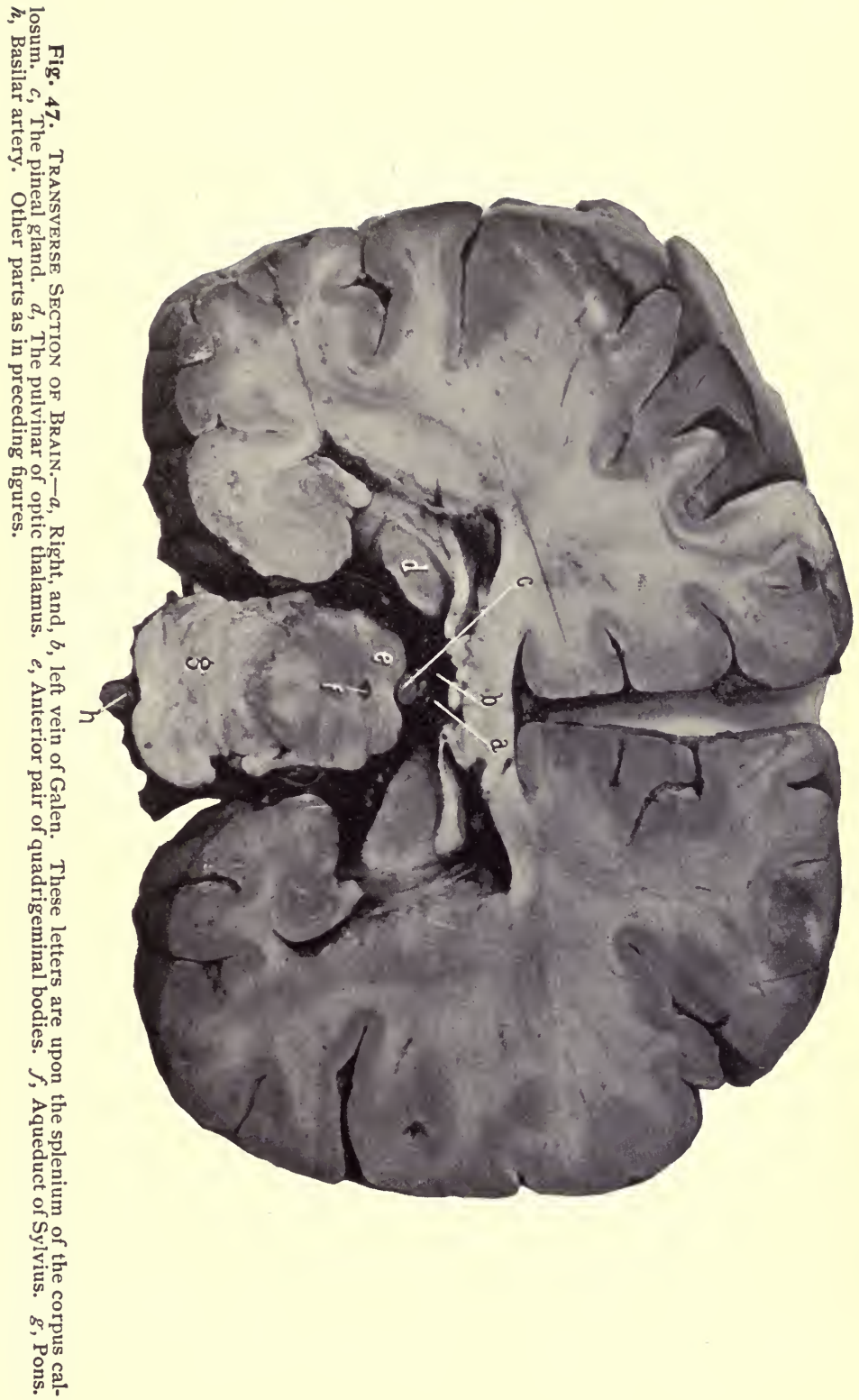


probably in the amygdaloid nucleus as well. Its fibres are connected with the fibres which constitute the middle root of the olfactory tract. The anterior commissure is developed from the lamina terminalis.

The basal gray commissure is that portion of the floor of the third ventricle which is formed by the lamina cinerea, tuber cinereum, and posterior perforated space.

The bundle of Vicq d' Azyr is a band of white fibres which passes from a corpus albicans vertically into the anterior extremity (anterior tubercle) of the optic thalamus. It was formerly described as a continuation of the anterior pillar of the fornix, which, after reaching the base of the brain, suddenly bent upward on itself (forming the corpus albicans) and passed to the optic thalamus. The optic thalami, pineal gland, pituitary body, and the upper arm of the optic nerve arise from the walls of the interbrain, while its central cavity remains as the third ventricle.

\section{DISSECTION.}

After studying the crura cerebri externally, transverse sections should be made of them as far back as the posterior quadrigeminal bodies.

The Crura Cerebri. Figs. 29, 38, 39, 40, 45, 46, 50, 53,

These are the rounded bundles of fibres which pass downward from the under surface of the cerebrum. They are oval in cross section and united along the posterior half of their opposing surfaces, while the anterior portions are separated, and in the interval between them is found the posterior perforated space and the third nerves. The crura cerebri are continuous posteriorly, around the aqueduct of Sylvius, with the quadrigeminal lamina.

Portions of the crura cerebri have been named for purposes of distinction. On a transverse section there will be seen a dark or black semilunar-shaped nucleus, which is 


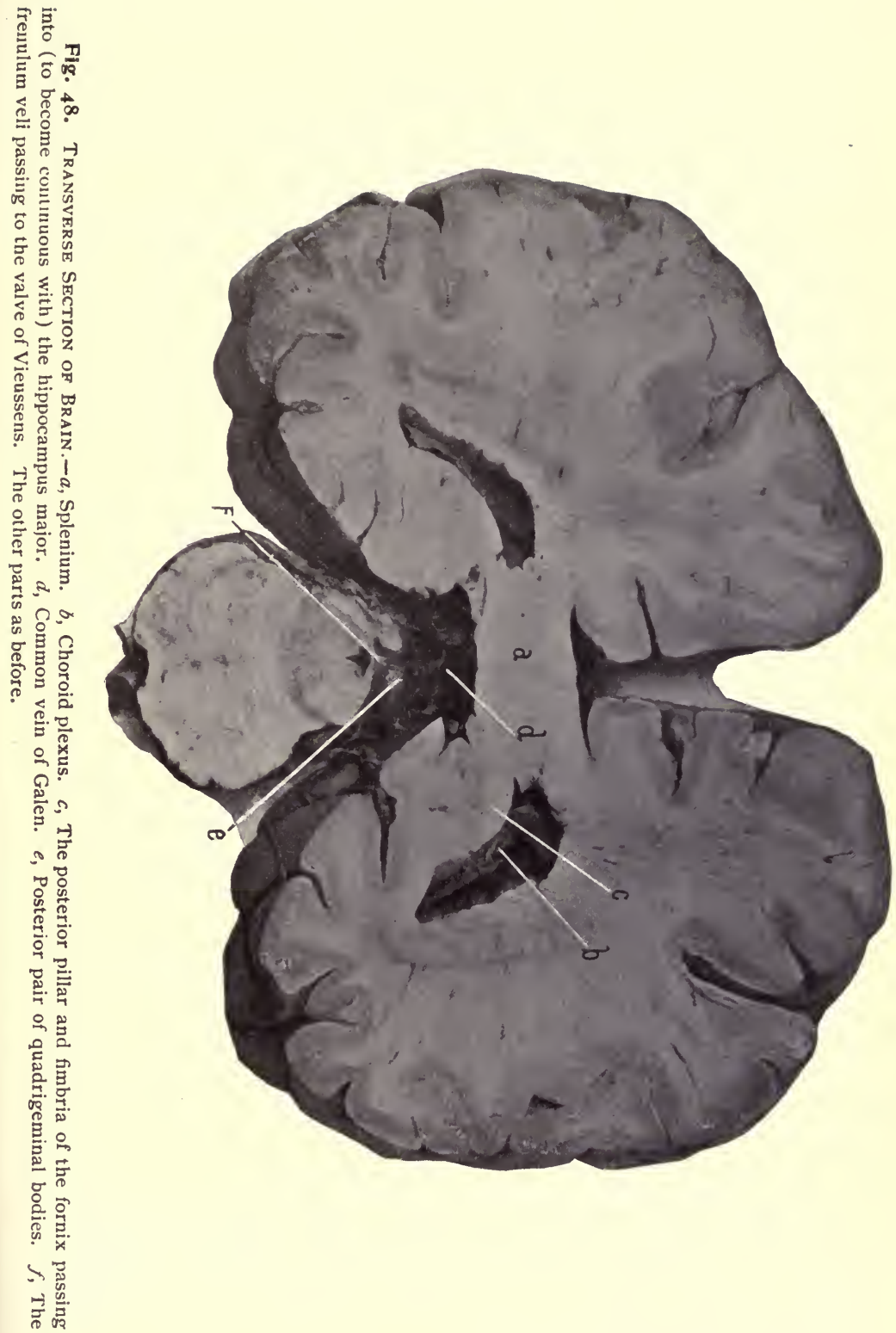


placed nearly transversely across the crus at about its middle; this is the locus niger and divides the crus into the crusta (anterior) and tegmentum (posterior).

The crusta and tegmentum are also separated from each other on their outer surface by two grooves which run parallel with each other. One groove is on the inner surface of the crus and is called the oculomotor groove, because out of this groove the third or oculomotor nerve emerges; the other groove is placed externally and called the lateral groove of the crus.

The crusta is composed of fibres which pass through the pons and appear as the anterior pyramids of the medulla, and of connecting fibres to the opposite cerebellar hemisphere. These fibres, continued upward, pass under the optic thalamus and take part in forming the internal capsule.

The tegmentum is composed of fibres passing from the internal capsule to the medulla and cerebellum.

The red nucleus (Figs. 34, 40, 46, 5I) is a round nucleus of brownish-gray matter, with a diameter of about three-eighths of an inch; it is situated in the tegmentum behind the middle of the locus niger and under the posterior part of the optic thalamus.

External to the red nucleus, on the same plane with it, but extending backward so as to appear behind it, is a second gray nucleus, the subthalamic ganglion, or the body of Luys. The plane of junction of the opposite tegmenta is called the median raphé.

The Aqueduct of Sylvius. Figs. 34, 39, 47.

This is the small canal which connects the third with the fourth ventricle. It passes beneath the posterior commissure and the lamina quadrigemina, and lies in the raphé between the tegmental tracts. 


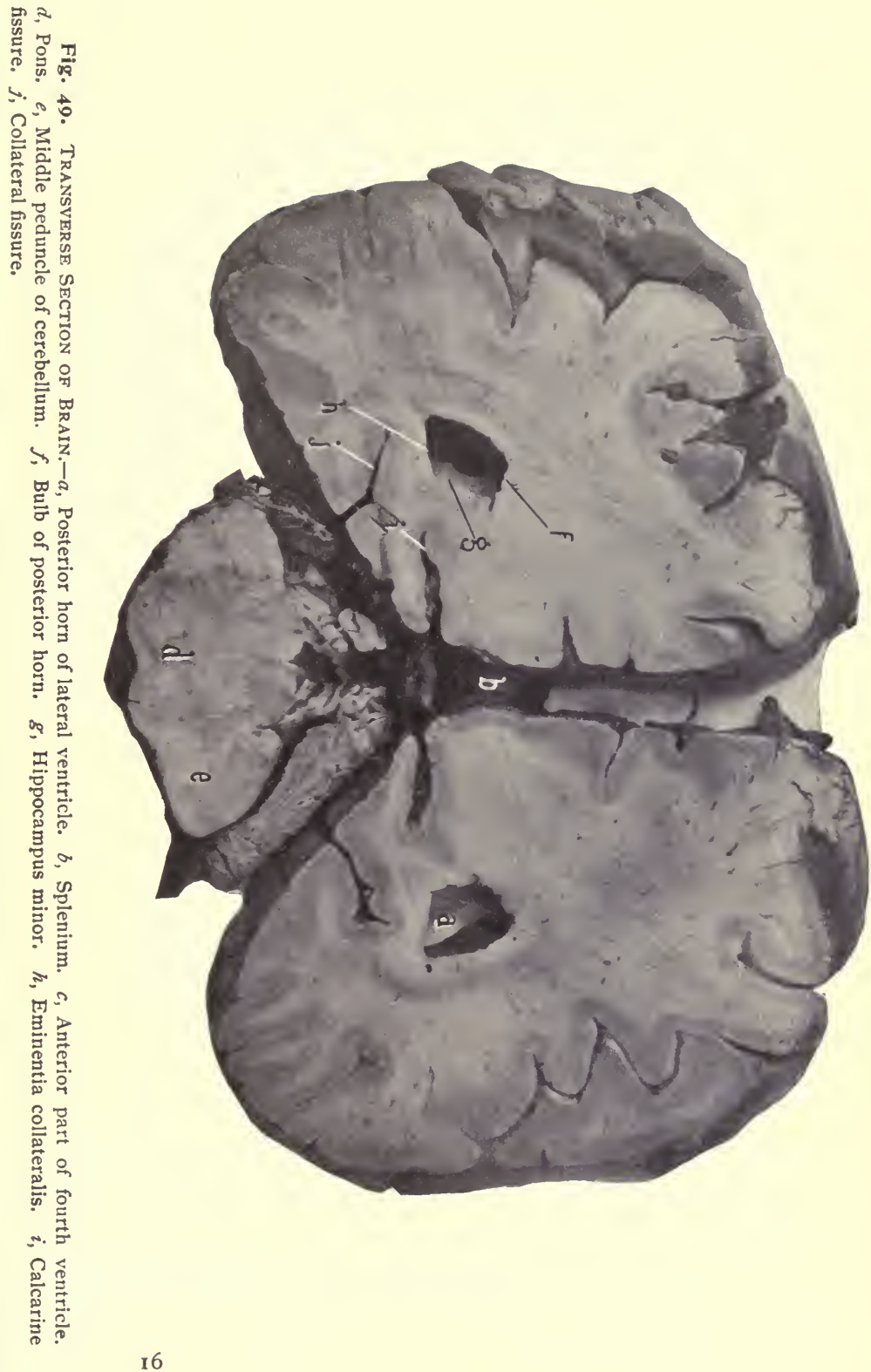


The crura cerebri, quadrigeminal lamina and bodies, and lower arm of the optic tract are developed from the primitive midbrain, and the aqueduct of Sylvius is the remains of the cavity within this vesicle.

The Cerebellum. Figs. 29, 34, 37 to 40,52 to 56 .

This consists of two symmetrical halves united at their opposing surfaces along a posterior median plane, and also, like the cerebral hemispheres, by a wide cross-band of commissural fibres which passes anterior to the backward prolongation of the crura cerebri, and is called the pons.

The Lobes of the Cerebellum.- "In descriptive anatomy an astounding variety of names are applied to the various parts of each lobe ; it would be an essential gain if at least three-fourths of these names could be discarded." (Minot's " Embryology.") The cerebellum is divided into five primary lobes by four sulci.

The superior surface of the cerebellum is divided into two lobes, the quadrate and the posterosuperior. The former is subdivided into the anterior and posterior crescentic portions.

The under surface of the cerebellum presents the flocculus, a small but prominent rounded lobe close to the posterior part of the pons. Behind this and close to the medulla, the tonsillar or amygdaloid lobe. Behind these are the cuneate, slender, and postero-inferior lobes.

Between the under surfaces of the cerebellar hemispheres is a deep notch-the vallecula-for the reception of the medulla.

The junction of the cerebellar lobes along the median plane is marked by a rounded elevation called the superior and the inferior vermis. These are further subdivided by 


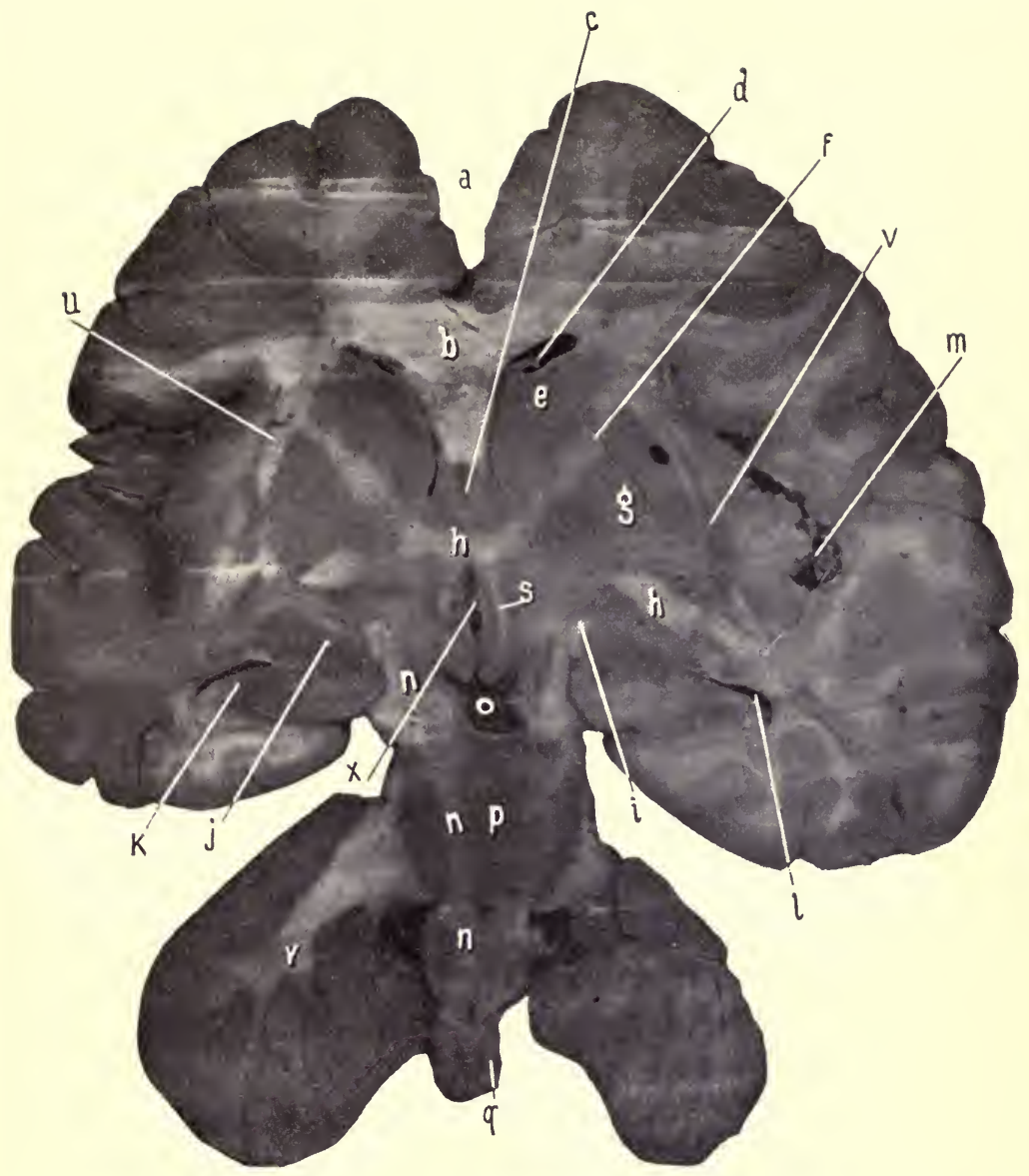

Fig. 50. Oblique - Transverse Section Through the Medulla, Pons, Crura, and Cerebrum. Viewed from in front.- $a$, Great longitudinal fissure. $b$, Genu of corpus callosum. $c$, Fifth ventricle. $d$, Anterior horn, lateral ventricle. $e$, Caudate nucleus. $f$, Internal capsule. $g$, Lenticular nucleus. $h, h$, Anterior commissure. $i$, Optic tract. $j$, Amygdaloid nucleus. $k$, Hippocampus major. $l$, Middle horn lateral ventricle. $m$, Fissure of Sylvius and island of Reil. $n, n, n$, Motor tract in crusta, pons, and medulla, $o$, Corpora albicantia. $p$, Pois. $q$, Medulla. $r$, Cerebellum showing arbor vitæ. $s$, Anterior pillar of fornix. $x$, Third ventricle. $u$, External capsule. $v$, Claustrum, becoming continuous with lenticular nucleus at lower part. 
an unnecessary refinement of description into seven other lobules.

The cerebellum is connected to the rest of the brain by three pairs of nerve-bundles, or peduncles: the superior, middle, and inferior.

The middle peduncles have already been referred to as the great transverse commissure or the pons, which unite the two hemispheres of the cerebellum in front of the fibres of the crura cerebri.

The superior peduncles are flattened bundles which project backward and outward from the testes of the corpora quadrigemina to reach the cerebellum internal to, and in front of, the middle peduncles.

The superior peduncles are united across the median line and from the posterior margin of the quadrigeminal lamina to the front of the under surface of the cerebellum by a thin sheet of white matter called the valve of Vieussens, or the superior medullary velum. A tongue-shaped projection-the lingula_from the superior vermiform process of the cerebellum rests upon the posterior part of the velum.

An extension of some fibres from the valve of Vieussens forward between the posterior pair of quadrigeminal bodies is called the frenulum veli.

The inferior peduncles of the cerebellum connect it with the lateral regions of the posterior part of the medulla. They are formed of the fibres from the direct cerebellar tract, the internal and the external arciform fibres, and go under the name of the restiform tracts. They enter the cerebellum internal to and behind the middle peduncles.

On sections of the cerebellum the peculiar arrangement of its white matter within the gray gives the appearance of a branching tree, hence the name of arbor vitæ which is applied to it. 


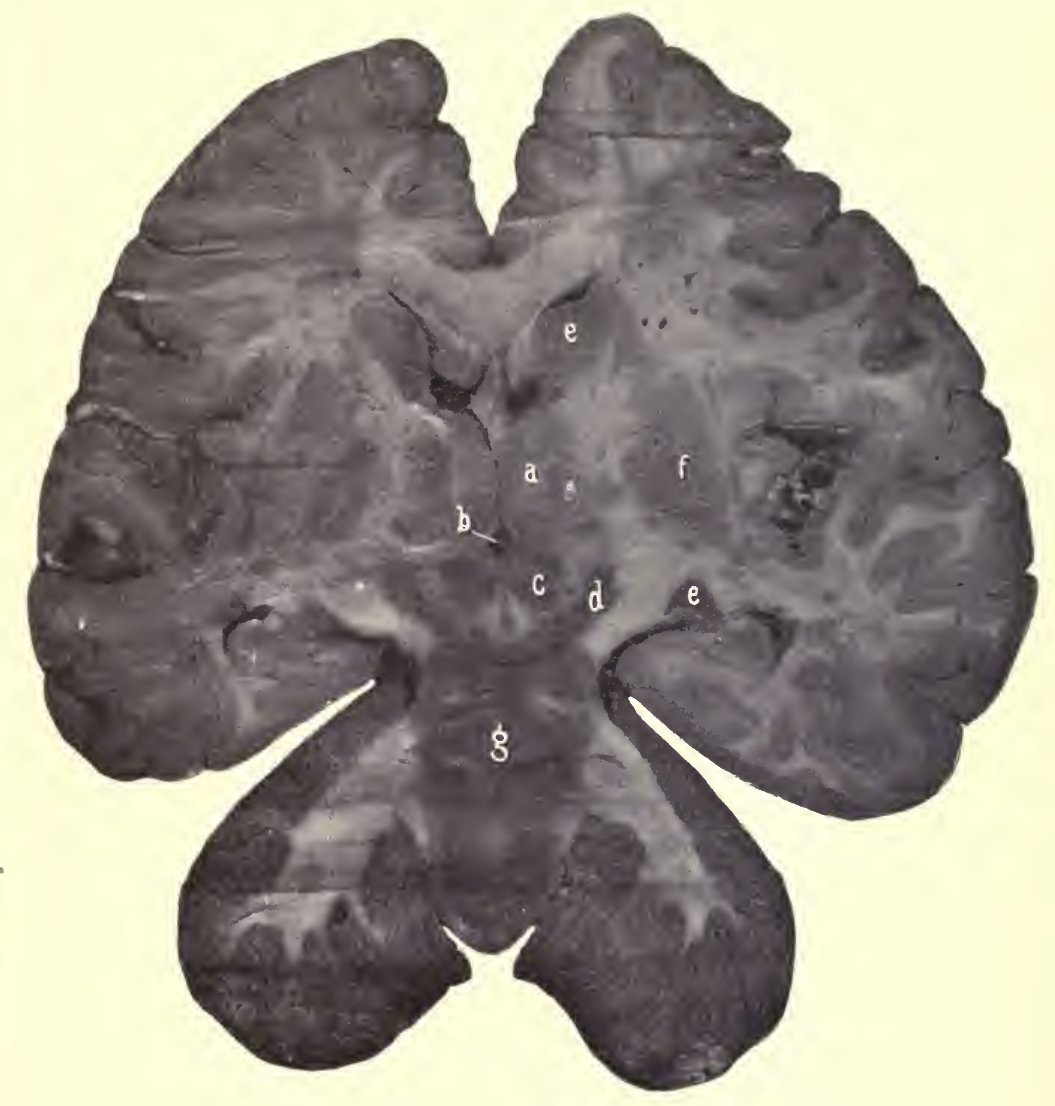

Fig. 51. Oblique-Transverse Section Through Medulla, Pons, Crura, and Cerebrum. Viewed from in front. $-a$, Optic thalamus. $b$, Third ventricle. $c$, Red nucleus. $d$, Locus niger. $e, e$, Caudate nucleus. $f$, Lenticular nucleus. $g$, Floor of the fourth ventricle. Remaining parts as in preceding figure. 
Within the stalk of white matter are situated four pairs of gray nuclei. The most conspicuous pair, situated within the centre of the white matter, is an oval nucleus with a dentated or serrated outline, and in consequence is called the corpus dentatum.

The Pons. Figs. 29, 38 to 40,47 to 49,53 .

This is the portion of brain which intervenes between the crura and the medulla, and consists of anteroposterior fibres crossed transversely by the fibres connecting the hemispheres of the cerebellum as stated above.

The pons is about an inch wide along the median line and narrows to half that width as it enters the cerebellum. It presents a shallow depression, in the middle line in which the basilar artery rests. See page i 58.

The upper and lower or anterior and posterior borders of the pons are well marked. At the upper border the crura cerebri pass backward out of sight, and at the lower border their continuation appears as the medulla.

The posterior surface of the pons will be described with the fourth ventricle, of which it forms the anterior portion.

On section, the pons presents two distinct areas. The ventral or anterior, and the tegmental or posterior (continuous with the tegmentum of the crura cerebri). The description of the subdivisions of these regions belongs to the histology of the brain.

The cerebellum and the pons are developed from the hindbrain, the former from its roof and the latter from its floor, while the cavity of the hindbrain remains as the anterior part of the fourth ventricle.

The Medulla. Figs. 29, 53, 54.

This portion of the brain extends from the pons backward for about an inch and a quarter to the margin of the 


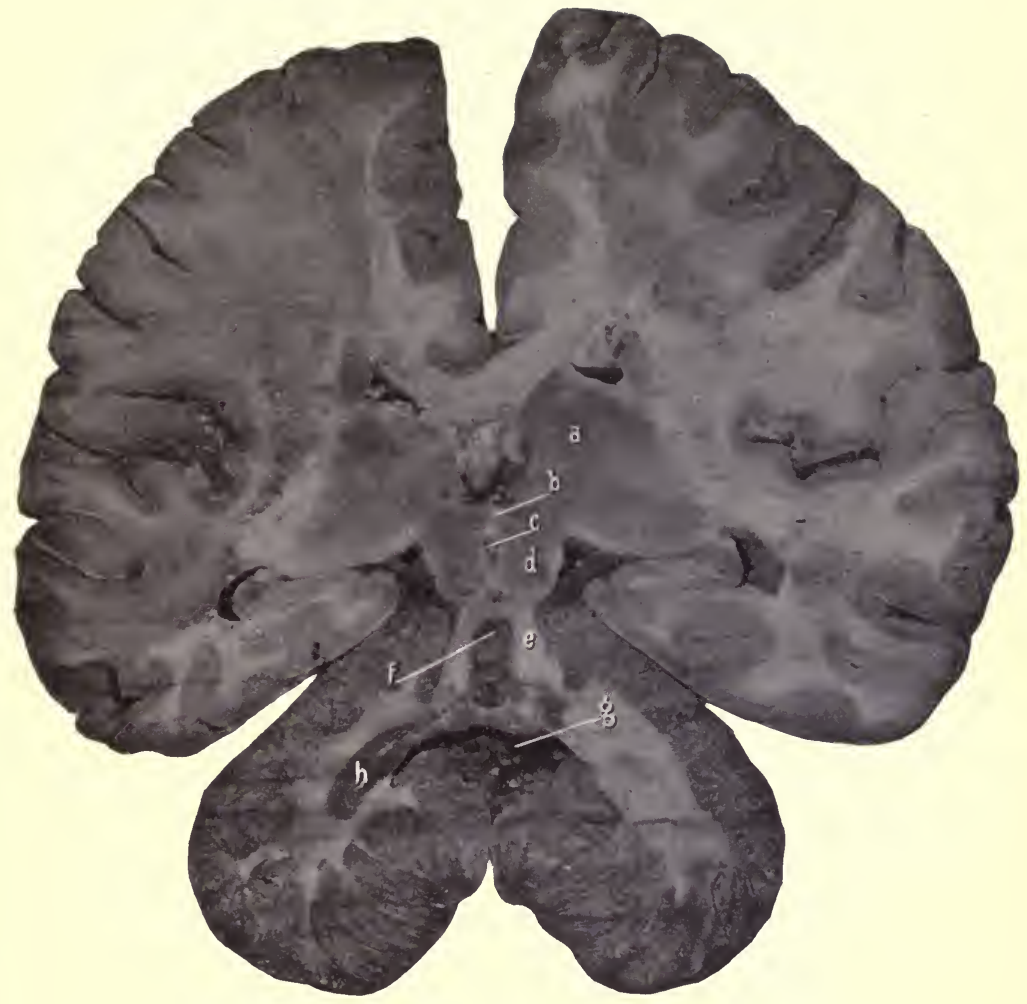

Fig. 52. Oblique-Transverse Section Through Cerfbellum, Corpora QuaDRIGEMiNA, AND CEREBRUM. Viewed from in front (the under side). $-a$, Optic thalamus. $b$, Posterior commissure. $c$, Roof of the aqueduct of Sylvius. $d$, Under surface of the corpora quadrigemina. $e$, Superior peduncle of cerebellum. $f$, Under surface of the valve of Vieussens with the lingula. $g$, Fourth ventricle. $h$, Corpus dentatum or cerebellum. 
foramen magnum, below which it is continued as the spinal cord. It is about three-quarters of an inch wide at the pons and three-eighths of an inch at its terminus.

The Under Surface of the Medulla.-Through the middle of the medulla from before backward extends a fissure-the anterior median fissure-which divides it into two symmetrical halves. The fissure terminates at the posterior margin of the pons in a slightly enlarged cavity named the foramen cæcum.

About an inch behind the pons the anterior fissure will be found crossed by fibres passing from one side of the medulla to the other. This is the decussation of the anterior pyramids of the medulla.

Along the side of the anterior median fissure lie two wellmarked ridges or bundles, which are the anterior pyramids of the medulla. They are limited externally by shallow grooves termed the anterolateral sulci; and external to these grooves are the lateral tracts or columns of the medulla.

At the outer side of the anterior pyramids and close to the pons is developed a small oval nucleus-the olivary body. The anterolateral groove is continued along the front of the olivary body and an offset from it around the back part called the posterior olivary sulcus.

Just at the olivary body the anterolateral groove is interrupted by a thin lamella of fibres which curve around from the anterior to the posterior parts of the nucleus and are called the external arciform fibres.

\section{DISSECTION.}

Divide the cerebellum along its median plane, open the halves, and expose the fourth ventricle. 


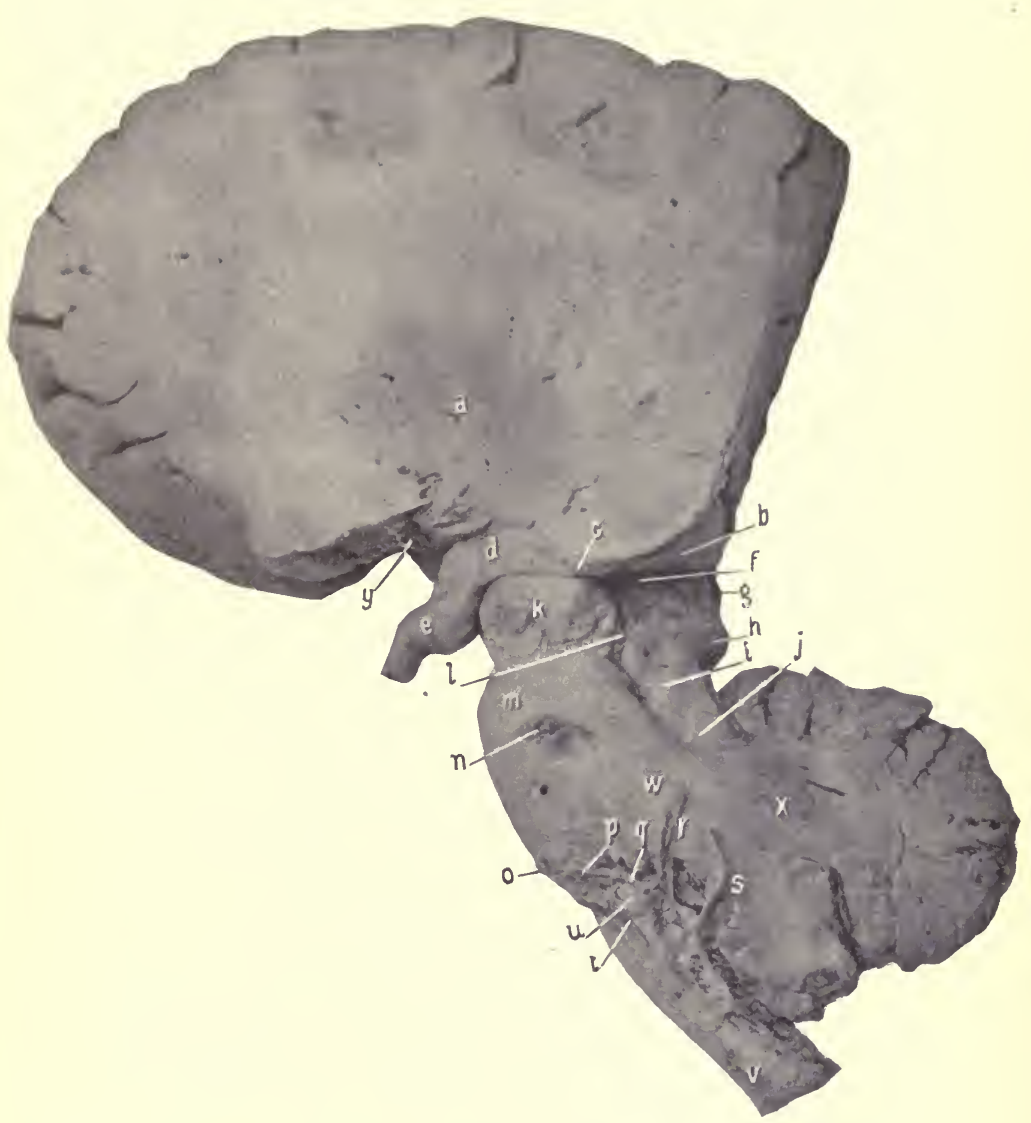

Fig. 53. Side View of Medulla, Pons, Crus Cerebri, with the Sectioned Cerebellum and Cerebrum.- $a$, Lenticular nucleus. $b$, Pulvinar of optic thalamus. $c$, External geniculate body. $d$, Optic tract. $e$, Optic nerve. $f$, Internal geniculate body. $g$, Nates. $h$, Testes, of corpora quadrigemina. $i$, Fillet. $j$, Superior cerebellar peduncle. $k$, Crusta. $l$, External lateral groove. $m$, Pons. $n$, Fifth nerve. $o$, Sixth nerve. $p$, Seventh nerve. $q$, Eighth nerve. $r$, Ninth and tenth nerves. $s$, Eleventh nerve. $t$, Twelfth nerve. $u$, Olivary body. $v$, Medulla. $w$, Middle peduncle of cerebellum. $x$, Cerebellum. $y$, Anterior perforated space with entering vessels. 
The Fourth Ventricle and the Posterior and Lateral Tracts of the Medulla. Figs, 39, 54.

The fourth ventricle is an elongated, diamond-shaped space communicating anteriorly by means of the aqueduct of Sylvius with the third ventricle and posteriorly with the minute central canal of the spinal cord. The lateral angles of the space are in the recesses left by the junction of the peduncles at the side of the cerebellum.

The roof of the ventricle is formed from before backward by the valve of Vieussens, the under surface of the cerebellum, and the portion of pia which forms the choroid plexus of the fourth ventricle and extends from the posteroinferior surface of the cerebellum to the upper surface of the lower part of the medulla. Remember that this portion of pia is composed of loops of capillaries, over which is laid the layer of cells which represents the attenuated roof of the posterior afterbrain, and consequently the plexus is shut out from the ventricular cavity. This layer of pia with its choroid plexus and lining membrane of epithelium is called the tela choroidea inferior. Also, remember that the tela inferior is perforated in the middle line and near the medulla by the foramen of Magendie, and in the lateral recesses by narrow slits of the foramina of Key and Retzius. Through these apertures a free communication is offered to the cerebrospinal fluid to pass from the subarachnoidean spaces into the cavity of the ventricles of the brain.

The Lateral Boundaries of the Fourth Ventricle.These are: In front, the superior peduncles; in the middle, the junction of the superior, middle, and inferior peduncles with each other and with the cerebellum; the posterior portion, by the inferior peduncles (restiform tracts), the fasciculi graciles, and their clavæ. 


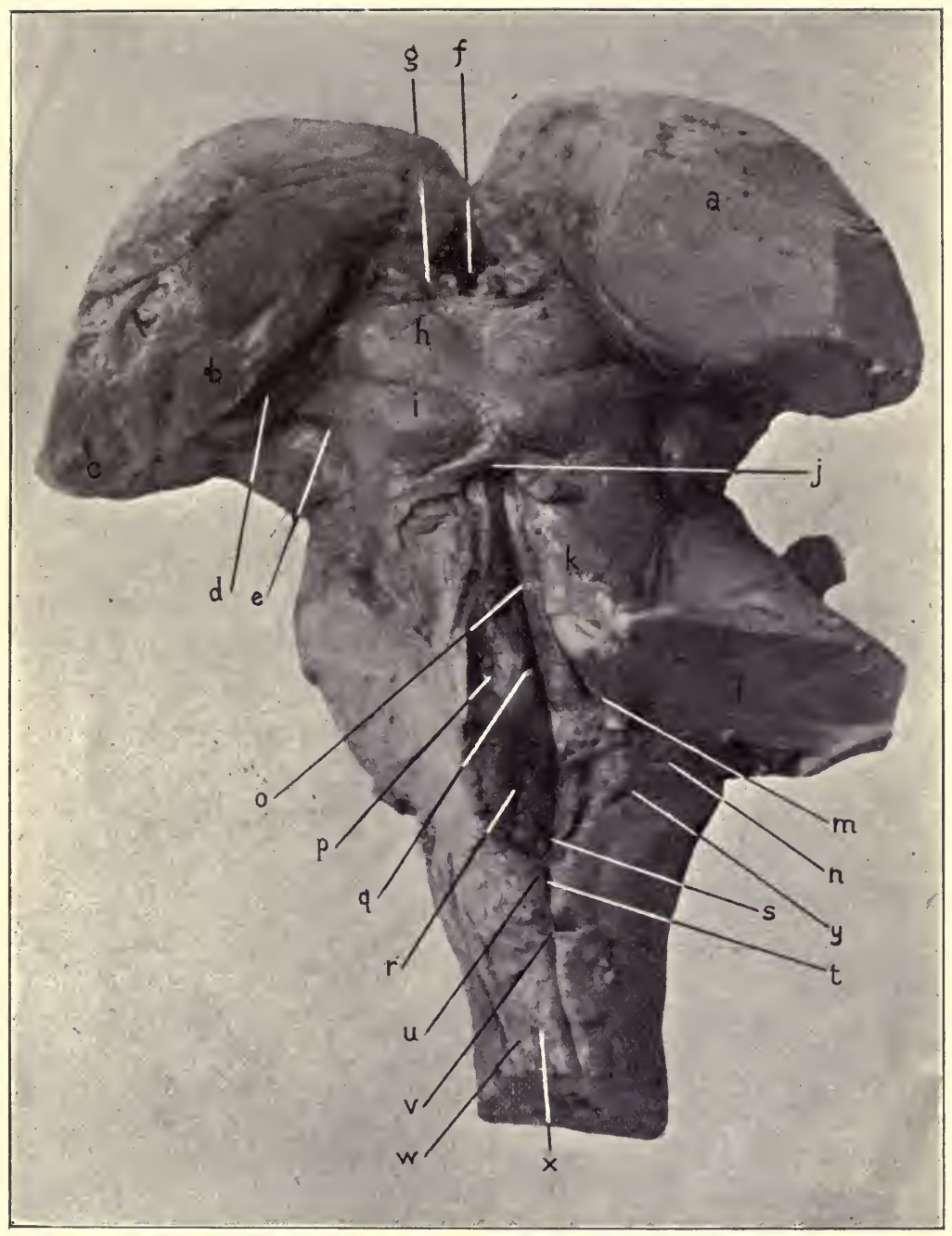

Fig. 54. Superior Surface of Medulla (cerebellum removed), Corpora QuadriGemina, AND OPTIC ThALAMI (cerebrum removed), $-a$, Optic thalamus. $b$, Pulvinar. $c$, External geniculate body. $d$, Internal geniculate body. $e$, External lateral groove. $f$, Third ventricle. $g$, Peduncle of pineal gland. $h$, Nates. $i$, Testes. Between them the crucial ridge. $j$, Frenulum veli, and the sectioned valve of Vieussens, $k$, Superinr cerebellar peduncle. $l$, Middle cerebellar peduncle. $m$, Inferior cerebellar peduncle. $n$, Striæ acusticæ. o, Posterior median fissure, leading anteriorly under the corpora quadrigemina into the aqueduct of Sylvius, and posteriorly by $(t)$ the calamus scriptorius, into the central canal of the spinal cord. $p$, Fovea anterior or superior. $r$. Fasciculus teres. $r$, Fovea posterior or inferior. $s$, Calamus scriptorius. $t$, Posterior medi 11 fissure of cord $u$, Clava. $v$, Funiculus gracilis. $w$, Funiculus cuneatus. $x$, Sulcus josterolateral. $y$, Restiform body. 
The floor of the fourth ventricle is the widely separated internal surface of the pons and medulla. It is traversed along the median line by a fissure-the posterior median sulcus.

Parallel with the anterior half, and on either side of the posterior median sulcus, is a rounded ridge-the fasciculus teres-which is produced by the fibres of the facial nerve.

A. small depression is in the lateral recess opposite the posterior part of the eminentia teres, the fovea superior, and in front of this depression is a small bluish spot, the locus cæruleus.

Some transverse fibres are seen crossing the floor of the ventricle just behind the lateral recesses; these are a part of the auditory nerve and are named the striæ acusticæ. They cross a little elevation, which is the tuberculum acusticum. Behind this tubercle is a second shallow depression, which is the fovea inferior. Extending backward and inward from the inferior fovea is the ala cinerea, a dark area over the nuclei of the ninth and tenth nerves.

The ventricle narrows to a pointed extremity - the calamus scriptorius-which is contained within the clavæ of the funiculi graciles.

Internal to the ala cinerea is another small elevation, the trigonum hypoglossi, which is over the nucleus for that nerve.

The Posterior and Lateral Surfaces of the Medulla.Back of the fourth ventricle the sides of the medulla come together, the space between them being called the posterior median fissure.

By the anterior and posterior median fissures the medulla is divided into two lateral halves; each half of the medulla is further subdivided by the antero- and posterolateral 


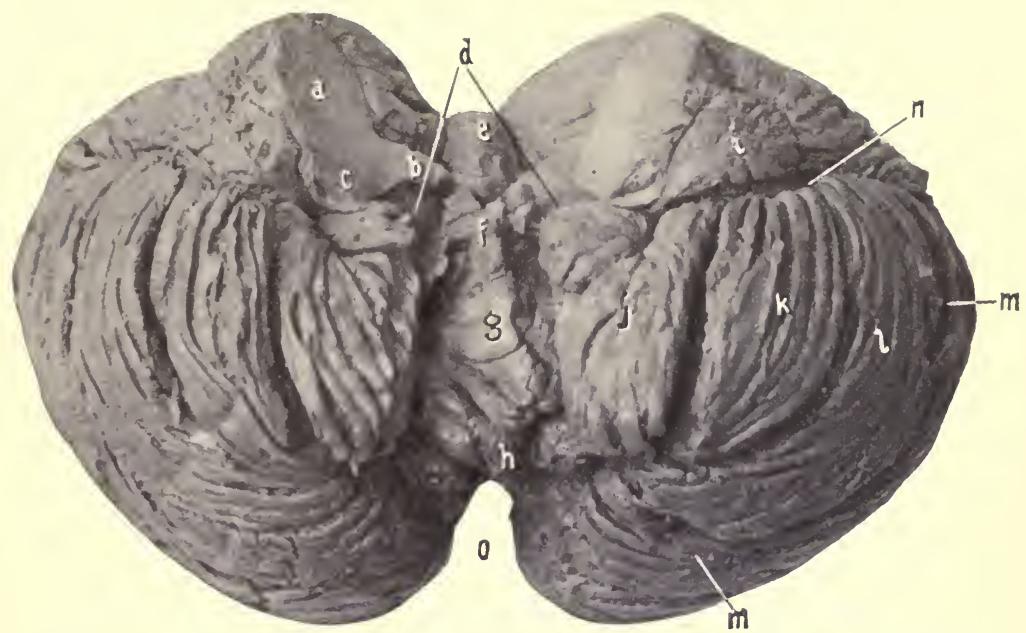

Fig. 55. The Under Surface of the Cerebellum Viewed from Behind.$a$, Middle, $b$, Superior, $c$, Inferior cerebellar peduncles. $d$, Roof of fourth ventricle. $e$, Central lobe. The lingula removed with the valve of Vieussens. $f, g, h$, Inferior vermiform process, called, respectively, the nodule, uvula, and the pyramid. $i$, Flocculus. $j$ Amygdala, or tonsil. $k$, Cuneate lobe. $l$, Slender lobe. $m$, $m$, Posteroinferior lobe. $n$, Beginning of the great horizontal fissure of the cerebellum. o, Incisura.

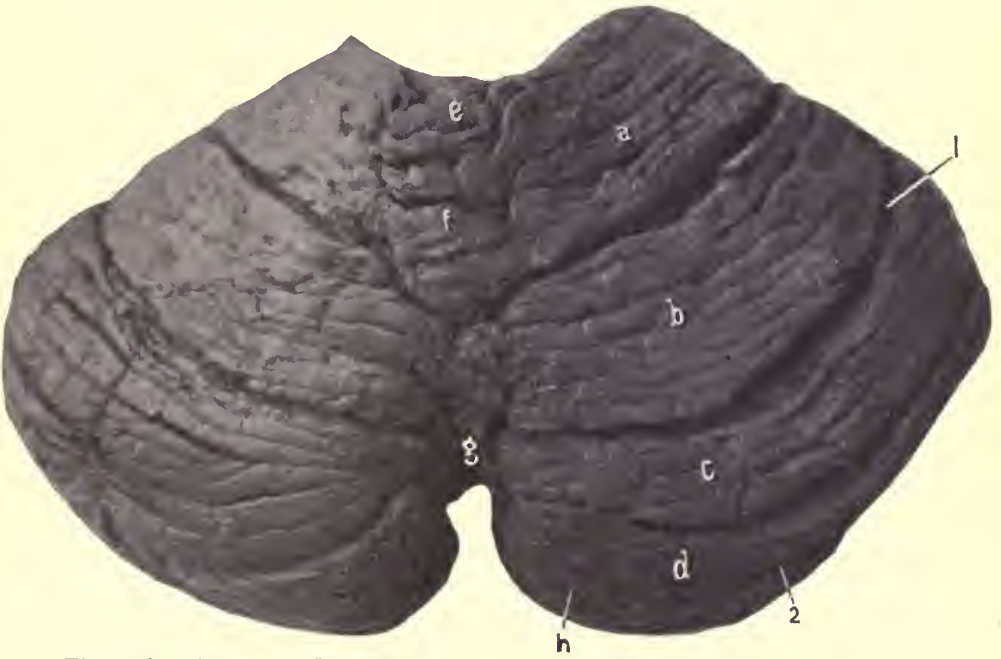

Fig. 56. Superior Surface of the Cerebellum Viewed from Behind.$a$, Anterior crescent, $b$, Posterior crescent, the two constituting the quadrate lobe. $c$ and $d$ form the posterosuperior lobe. 1, The superior cerebellar sulcus. 2, The great horizontal fissure of the cerebellum. $e, f$, and $g$, The superior vermiform process. $h$, Postero-inferior lohe. 
fissures, or sulci, into three areas-anterior, lateral, and posterior.

The anterior area is occupied, as already given, by the anterior pyramids, the lateral area by the lateral tract below and the olivary bodies above. The posterior tract is subdivided by a shallow fissure into two tracts: An internal one, the funiculus gracilis, and an external one, the funiculus cuneatus.

The funiculus gracilis borders upon the posterior median fissure, and as this opens to form the fourth ventricle, the funiculus forms its posterior lateral boundary.

At the beginning of the ventricle the funiculus is enlarged to form the clava.

The funiculus gracilis terminates, before the cerebellum is reached, in a fine point.

The funiculus cuneatus runs parallel with the funiculus gracilis and external to it. It terminates just beyond the lower part of the fourth ventricle.

What appears to be the direct continuation of the funiculus cuneatus and the lateral tract passes up to the cerebellum under the name of the restiform tract. But this tract is composed of fibres derived from the direct cerebellar tract, and the internal and external arciform tracts.

The external arciform tract is a thin lamina of fibres which issue from the anterior median fissure and pass outward across the pyramids, below the olivary body, to the restiform tract.

The medulla is developed from the afterbrain, and the posterior part of the fourth ventricle from the cavity of the same. 


\section{THE UPPER EXTREMITY AND THORAX, Anterior.}

\section{The Landmarks.}

In the median line there is the suprasternal or interclavicular notch between the large rounded ends of the clavicles. This notch is always easily felt even in very fleshy people, and is therefore a very valuable reference point. The notch is directly in front of the disk between the second and third dorsal vertebræ, and two and one-half inches from it. About two inches below the notch is a prominent transverse ridge marking the articulation of the first and second portions of the sternum. This ridge is also a guide to the second ribs and cartilages which join the sternum at this place. At the lower end of the sternum is the ensiform appendix, which can be felt on pressure, also the transverse ledge or jog which marks where the appendix unites with the second piece of the sternum. This place receives the cartilages of the seventh ribs, and is therefore a guide to them. The tip of the ensiform is in front of the body of the ninth dorsal vertebra.

For the remarks concerning the lower margin of the thorax, see page 432. The nipples are usually given as points from which to reckon distances and relations. Their position is so variable, especially in the female, as to scarcely deserve mention; however, it will be given here. As a rule, in both sexes they are found over the fourth interspace, in males about three and one-half inches, and in females four inches, from the median line, or about one inch external to the junction of the ribs and cartilages.

The clavicles are distinct, and extend outward and slightly upward from the lateral margin or angle of the manubrium 
to join the acromion process of the scapula, and thus hinge the upper extremity to the thorax.

The articulation between the clavicle and the acromion is usually easily found, and often is so prominent as to constitute almost a subluxation of the former upon the latter. The margin of the acromion can be traced, and the angle formed by the junction of the spine with the acromion is always easily located. This point is the one from which measurements are made in determining differences in the length of the upper extremity. The lower fixed points depend upon the part to be measured. If only the arm, the external condyle of the humerus or the tip of the olecranon with the forearm flexed to a right angle. For the arm and forearm, the tape is carried to the tip of the styloid process of the radius or ulna, and for the entire extremity, to the point of the middle finger (any finger or point will do provided the corresponding point is taken on the opposite side of the body).

In front of the middle and outer thirds of the clavicle is usually a depression corresponding to the interval between the pectoralis major and deltoid muscles; in this depression the tip of the coracoid process will be found by making a little pressure.

The cephalic vein lies in the groove between the deltoid and pectoralis major muscles and passes upward to empty into the axillary vein below the middle of the clavicle.

The deltoid tubercle may be prominent enough to be discerned by touch at the outer and middle thirds of the anterior border of the bone. Under the deltoid muscle the tuberosities of the humerus can be felt, especially on rotation of the bone. The greater is external, the lesser internal, and between them is the bicipital groove, which can be appreciated in thin people. 
By raising the arm half way to a right angle from the body, the anterior and posterior boundaries of the axillary space will appear, the pectoralis major in front and the latissimus dorsi behind. Between them is the cavity of the axilla. By carrying the fingers high up in this cavity the head of the humerus can be felt.

The line of the axillary artery: Extend the arm until it is at right angles with the trunk, then draw a line from the middle of the clavicle to the depression at the inner border of the coracobrachialis and biceps muscles.

The pectoral border leads down to the fifth rib.

To count the ribs, remember that the second rib joins the sternum (through its cartilage) at the articulation between the first and second pieces of the sternum; that the seventh at the junction of the second and third parts of the sternum; that the fourth rib is just above, and the fifth just below the nipple; that the tenth, eleventh, and twelfth can be located by touch along the lower border of the thorax.

The arm presents a prominence in front due to the biceps muscle, and a slight depression at its outer and inner margins; in the outer lies the cephalic vein and in the inner the brachial artery, and veins with the median nerve. The inner margin of the muscle is the guide to the artery. A similar prominence is upon the back of the arm, formed by the triceps muscle.

At the elbow recognize the two (epi) condyles of the humerus, the olecranon process of the ulna, and the head and tuberosity of the radius. The internal (epi) condyle, being more prominent than the external, is more often broken off than the external. It points in the direction to which the head of the humerus faces.

The prominent olecranon behind bears certain relations 
to the condyles of the humerus in the normal state that must be kept in mind :-

(I) When the forearm is extended on the arm, the top of the olecranon and the two condyles are all in the same straight line.

(2) If the forearm is flexed to a right angle with the arm, the condyles and the tip of the olecranon are in the same plane. The coronoid process of the ulna may be found in front of the inner part of the elbow on deep pressure. It is usually not easily distinguished. The head of the radius will be felt, on rotation of the shaft, threefourths of an inch below the external condyle. The "carrying angle:" This is the normal angle $\left(10^{\circ}\right)$ formed by the arm and the supinated forearm ; its opening is outward. It is produced by the trochlear surface of the humerus being prolonged lower on the inside than outside and thus throwing the lower end of the ulna outward. The angle may be lost in fractures involving the condyles of the humerus and should always be restored; if not maintained, a peculiar deformity—gun-stock-remains.

The shafts of the ulna and radius terminate below in their respective styloid processes. Demonstrate that the styloid process of the radius is about one-fourth of an inch lower than that of the ulna. If this relation is altered so as to bring the processes in the same line it shows that a fracture of one or the other bones has occurred. In the reduction of the usual (Colles's) fracture of the wrist see that the normal relation is reproduced.

Outlining the Heart and Lungs. Figs. 57, 58, 59.

The student now should take time to mark out the superficial outlines of the heart, lungs, and great vessels upon the exterior of the chest. The relations of the heart given below are the results of the writer's own investigations in 


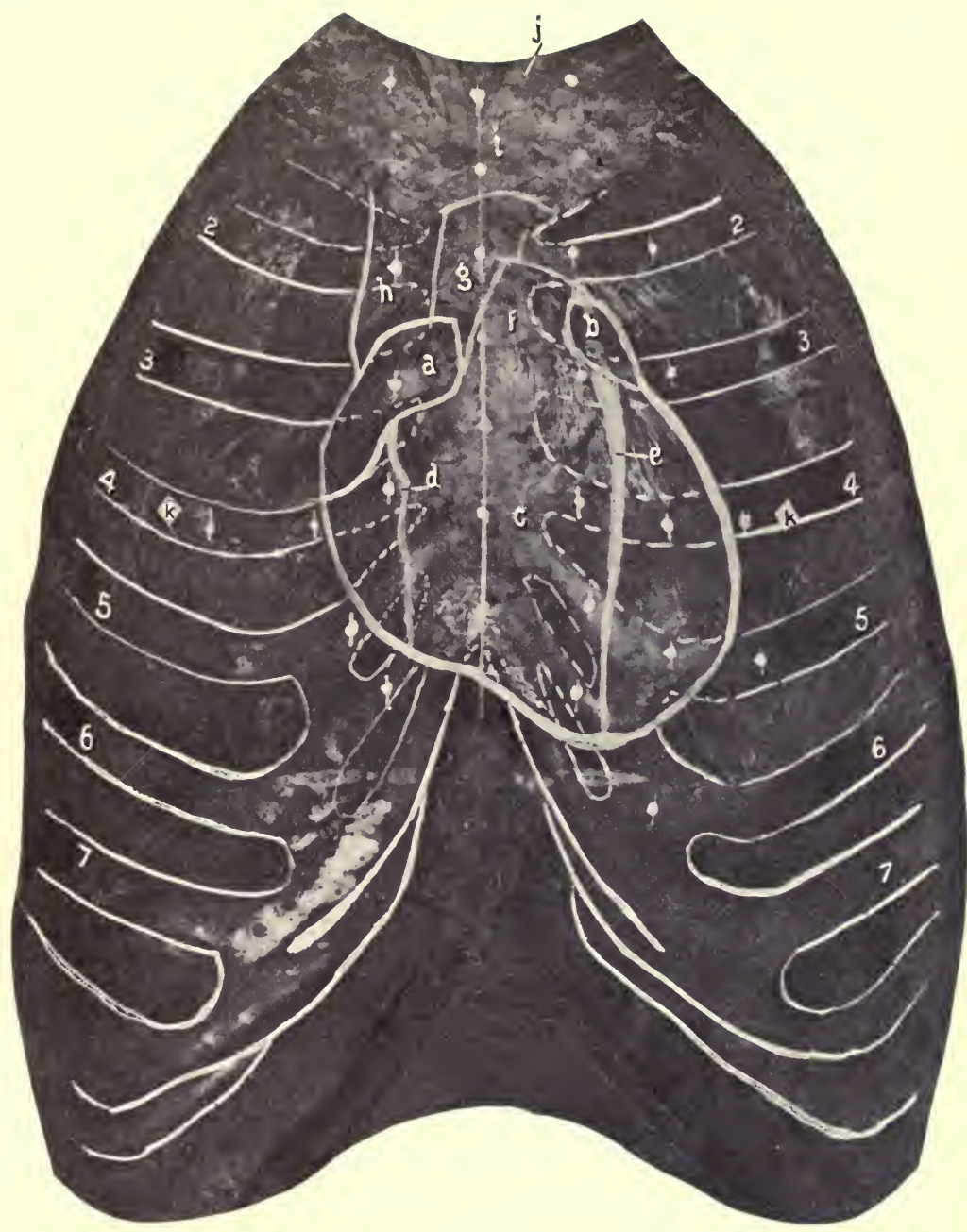

Fig. 57. Composite Chest-Heart Photograph. [From a paper by the author on "The Relations of the Heart and Lungs to the Anterior Chest-Wall, as Determined by Composite Photography." Abstract read before the Sections in Anatomy and General Surgery of the First Pan-American Medical Congress at Washington, September 9, 1893, and published in the New York Medical Journal, November II and December 9, 1893.]$a$, Right auricle. $b$, Left auricle. $c$, Ventricles of heart, in a moderately expanded condition. $d$, Anterior branch of right coronary artery. Right marginal. $e$. Left coronary artery. $f$, Pulmonary artery. $g$, Aorta. $h$, Superior vena cava. $i$, Manubrium. $j$, Sternomastoid. $k, k$, Nipples. 
I 893 with the camera (see "Relations of the Heart and Lungs to the Anterior Chest Wall," as determined by composite photographs, published in the Medical Journal of November I I and December 9, I 893) and by comparison of the conflicting statements of the various authors.

(I) Base of Heart.-A line crossing the sternum obliquely from the upper margin of the third right to the lower border of the second left costal cartilage, an inch and a half from the median line on each side.

(2) Apex.-In the fifth space near the upper margin of the sixth costal cartilage, two and a half inches to the left of the median line.

(3) Right Border.-From the right end of the base curved slightly outward to reach a point an inch and threequarters from the right of the middle line over the fourth cartilage and ending at the centre of the fifth cartilage an inch from the mid-sternal line.

(4) Left Border.-From the left end of the base with a convexity outward to the apex. It reaches its greatest distance (three inches) from the sternal centre over the fourth space.

(5) Lower Border.-A line curved downward at its beginning (at the lower extremity of the right border) and ending (at the apex), and slightly convex upward in its centre as it crosses the middle of the ensiform.

(6) The Area of Heart Dullness.-A quadrilateral area to the left of the median line and below the upper border of the fifth cartilage, nearly two inches in vertical and an inch and a half in extreme lateral measurements. See anterior border of left lung below.

(7) The Auricles._The Right: An "ear-shaped" area facing to the left, covering the first inch of the third right space and cartilage with the portion of the sternum adjacent to the latter. Its long axis measures about two inches and 


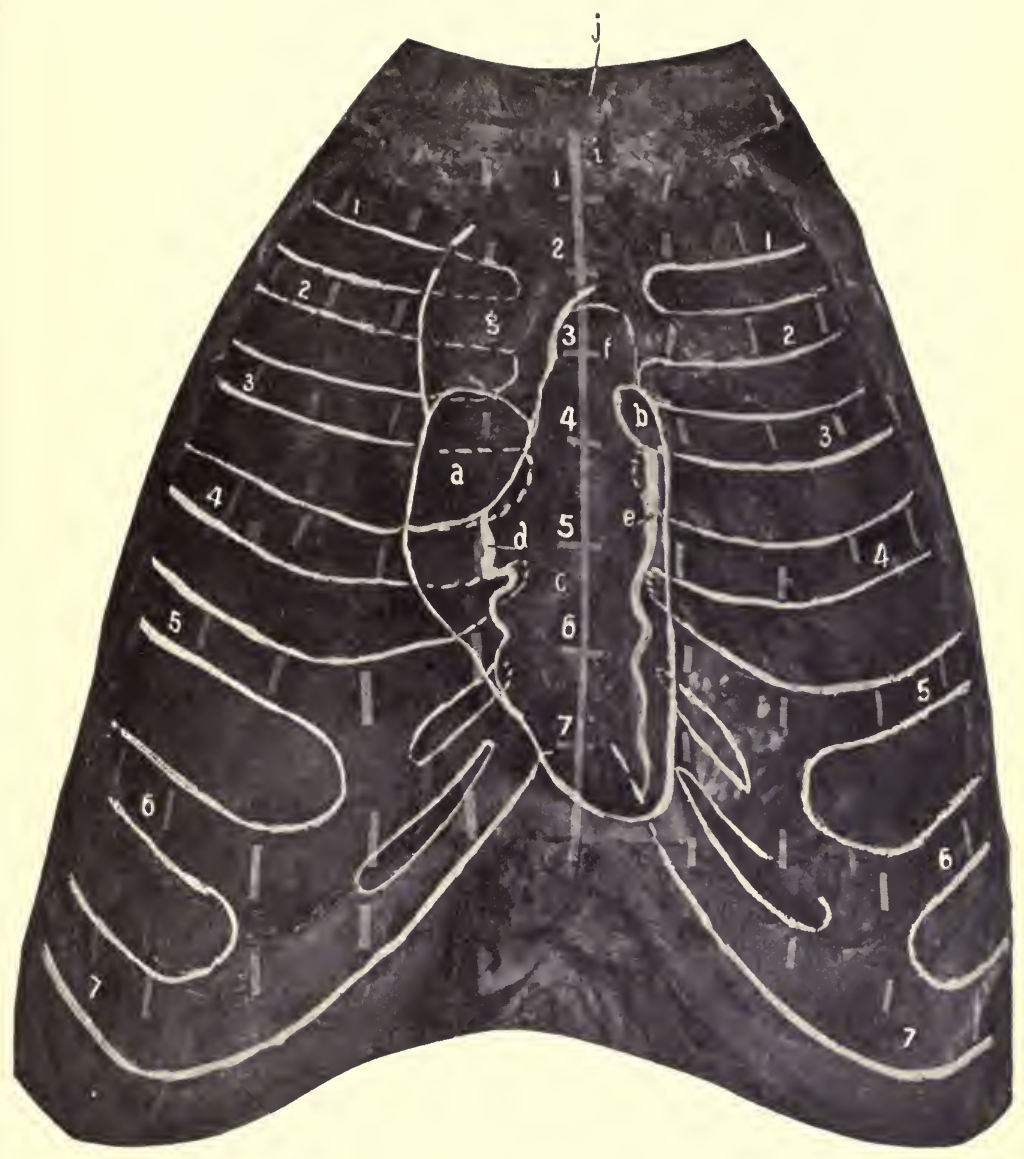

Fig. 58. Composite Chest-Heart Photograph.

See statement with Fig. 57.

The lettering is the same as in the preceding figure.

In this case the heart was in a firmly contracted condition. 
is inclined from above downward and outward. The Left: Is a small oval space half an inch by an inch, its centre an inch and a quarter to the left of the median line behind the second left space and third cartilage. Its long axis is directed from above downward and outward.

(8) The auriculoventricular groove is indicated by a line from the right to the left heart border, beginning on a level with the upper margin of the fourth right and ending on a level with the lower edge of the third left cartilage. This line is convex upward and crosses the middle of the sternum on a level with the lower border of the third cartilages.

(9) The aortic area is a little more than an inch wide and two inches in length. It extends from the upper border of the third to behind the middle of the first cartilages. At its beginning and ending its centre is behind the median line, but in the middle of its course the artery is convex toward the right.

(Io) The Area of the Pulmonary Artery.-About an inch and a quarter wide and an inch and a half long (in the photograph). Begins on a level with the lower border of the third, and ends behind the middle of the second left cartilages. Below, its centre is half an inch, and above, threequarters of an inch, to the left of the median line.

(I I) The superior vena cava is represented by a vertical area three-fourths of an inch wide at the right of the aortic area, reaching from the (right auricle) right extremity of the base line upward to the first intercostal space, where the innominate veins coalesce.

(I 2) The outline of the lungs (in inspiration) is indicated by the following lines:-

The apices of the lungs reach into the base of the neck a distance of from one and a half to two inches above the first ribs, consequently the lines representing the apices of 
the lungs would begin over the middle of the clavicles, pass upward and inward for a distance of three-fourths of an inch until over the sternal end of the clavicles, then drawn downward and inward, crossing the sternoclavicular articulations, toward the middle line of the body, to meet at the level of the upper border of the second cartilages (junction of first and second pieces of the sternum). The anterior borders of the two lungs lie in contact (separated by the pleuræ) from this point to the level of the middle of the sternum (the mid-point between the fourth cartilages). From here they separate, due to the presence of a notch in the anterior border of the left lung for the accommodation of the heart. The right continues directly downward to the sternal end of the sixth cartilage. The left turns outward and downward along the upper border of the fifth cartilage until it reaches a distance of one and one-half inches from the middle line, when it turns downward to the upper margin of the sixth rib three inches from the median line. Each lung is divided into two main lobes by the great fissure and the right has in addition a third lobe cut off by a secondary fissure.

The great fissure is indicated on the chest by drawing a line from the third dorsal spine obliquely downward and forward to the sixth costochondral articulation, for the left side; and from the fourth dorsal spine to the seventh costochondral joint on the right side. The secondary fissure, on the right side, is drawn from the angle of the sixth to the cartilage of the fourth rib. The lower border of the lungs is represented by a line drawn around the chest diagonally from the sixth cartilage at the sternum, to the tenth dorsal spine behind. On the left side this line represents the lower border also, but it should be started at a distance of three inches from the middle line. This entire border 
varies, of course, with the descent of the lungs during inspiration.

(13) The Line Indicating the Level of the Pleura.This is lower than that of the lungs. It is represented by a line starting at the sternum at the articulation of the seventh cartilage, and passes obliquely around the chest to the spine of the eleventh dorsal vertebræ. This line crosses the lower border of the ninth rib in the axillary line, and behind is as low as the upper border of the twelfth rib.

(14) The trachea bifurcates on a level with the articulation between the first and second pieces of the sternum.

(15) The internal mammary artery runs vertically downward behind the costal cartilages and intercostal muscles one-half inch external to the sternal border as low as the sixth cartilage, where it divides into its two terminal branches, the superior epigastric and musculophrenic.

\section{DISSECTION.}

Incisions.-1. In the median line from the top, to the bottom of the sternum.

2. From the beginning of $\mathbf{I}$, out along the clavicle to the point of the shoulder (same incision as 2, page 69).

3. From the lower end of $\mathrm{I}$, transversely around the body, as far as the posterior axillary line (same as 2 , page 435 ).

4. From the centre of the clavicle down the front of the arm to the elbow. Remove the integument from the chest and upper part of the arm.

\section{The Superficial Fascia.}

This sheet of subcutaneous tissue covers the entire area. It is continuous with the similar fascia of the abdomen, neck, back, shoulder, and arm. Its thickness depends upon the amount of adipose tissue present in its meshes. Within the fascia are found the subcutaneous vessels, nerves, and the mammary gland.

Superficial Vessels and Nerves and Origin of Platysma. At the upper part of the chest the origin of the platysma 


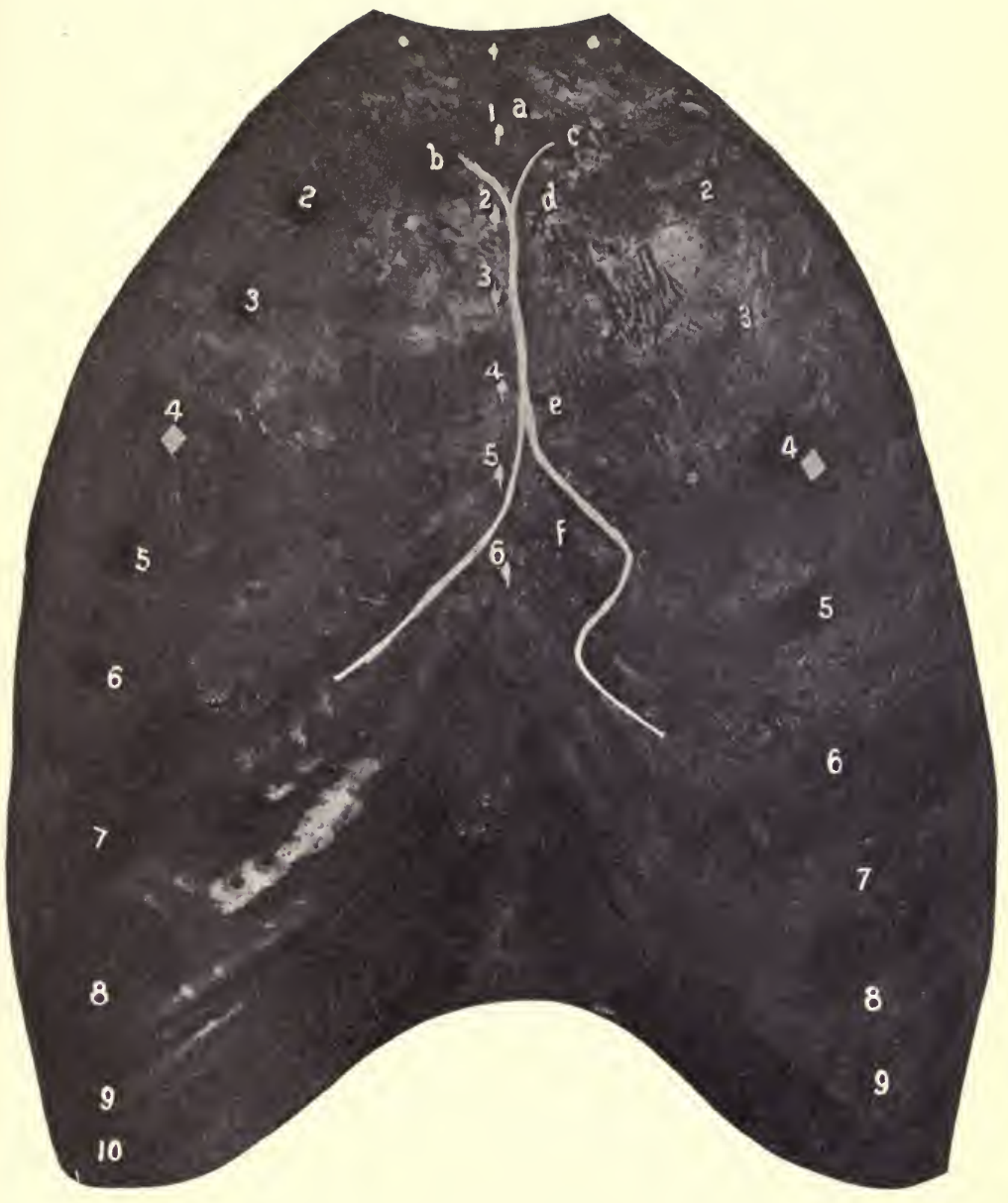

Fig. 59. Composite Chest-Lungs Photograph. [See Fig. 57 for explanatory remarks.] $-a$, Manubrium. $b$, Anterior border of right lung. $c$, Anterior border of left lung. $d, e$, Extent of contact of these borders. $f$, Notch in left lung for the heart. Corresponds to the area of heart dullness. 
will be seen extending about an inch below the border of the clavicle. In this region, too, will be found the terminal filaments of the sternal, clavicular, and (over the point of the shoulder) the acromial nerves from the cervical plexus. See page 9I.

Along the front of the chest are the terminal twigs of the intercostal nerves, second to sixth inclusive, and perforating branches of the internal mammary artery. At the side are the lateral cutaneous branches of the intercostal nerves, second to sixth inclusive. These perforate the fascia along the mid-axillary line. One large one, the intercostohumeral, must be saved.

\section{The Mammary Gland.}

The mammary gland is rudimentary in the male. In the female it varies greatly in size. Usually it covers a space represented by a circle whose centre is at the nipple (see below), and whose diameter reaches from the third to the sixth ribs inclusive. The nipple is usually over the fourth intercostal space, four inches from the median line. It is surrounded by a darker area-the areola-about one inch in diameter.

The arteries are the intercostal branches of the internal mammary, through the second, third, and fourth spaces, the long thoracic artery, and often an abnormal branch (external mammary) from the axillary.

The veins follow the arteries.

The nerves are derived from the fourth, fifth, and sixth intercostal nerves, and from the terminal filaments of the clavicular branch of the cervical plexus.

The Lymphatics are Important.

The main lymphatic trunks proceed to the pectoral and 


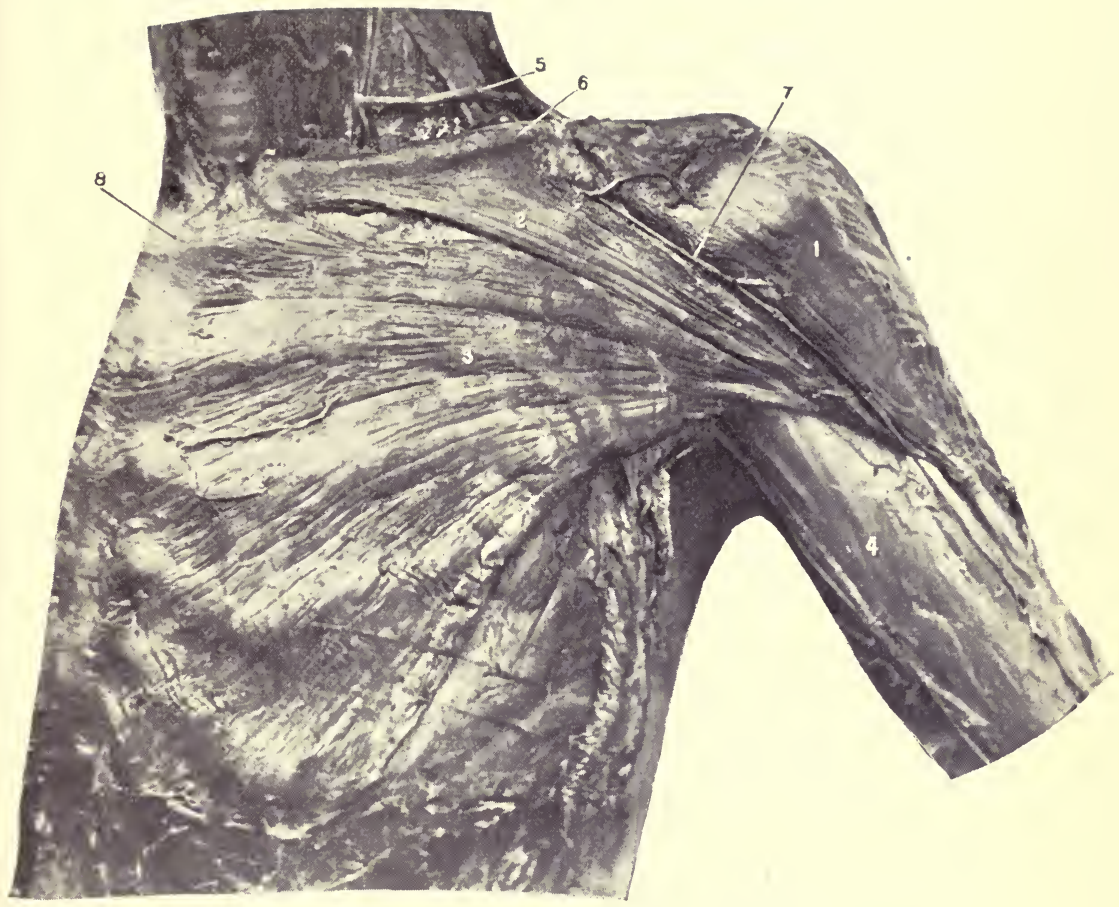

Fig. 6o. Superficial Shoulder, Anterior.-1, Deltoid. 2, Clavicular, and 3, Sternal portions of the pectoralis major. 4, Deep fascia of arm. 5, Transverse cervical artery. 6, Clavicle. 7, Cephalic vein. 8, Sternum. 
thence to the axillary glands. They follow along the long thoracic artery.

\section{DISSECTION.}

Remove the superficial fascia from the same area as the skin, leaving the deep fascia uninjured behind.

\section{The Deep Fascia.}

This is a very thin layer covering the pectoralis major. Anteriorly it is attached to the front of the sternum, above to the clavicle, externally it passes on to the deltoid muscle, and below turns over the lower border of the pectoralis major and stretches across the axillary space (axillary fascia) to the latissimus dorsi muscle, which it covers. Externally the axillary fascia passes into the deep fascia covering the arm.

The axillary fascia (and integument) is deeply hollowed when the arm is extended to an angle of 45 degrees with the trunk. This is due to the attachment of the fascia covering the pectoralis minor muscle. See page 270.

\section{DISSECTION.}

Remove the deep fascia, cutting parallel with the fibres of the muscle it covers. In turning down the axillary fascia be careful to save the nerves which will be found coming out from the axillary space.

Clean the cephalic vein from the middle of the arm to where it disappears through the costocoracoid membrane (see below), the branches of the acromiothoracic artery, and the external anterior thoracic nerve.

Pectoralis Major. Fig. 6o.

Origin.-The clavicular portion, from the anterior surface of the inner half of the clavicle. The sternal portion, from the anterior surface of the sternum, from the anterior surface of the second to sixth costal cartilages inclusive, also, sometimes, the first and seventh cartilages, and from the aponeurosis of the obliquus externus abdominis.

Insertion.- Into the anterior or external lip of the bicipi- 
tal ridge, for a distance of two inches below the greater tuberosity of the humerus. The tendon of insertion is in two layers, the anterior layer belonging to the clavicular portion of the muscle ; the posterior layer to the sternocostal portion. These layers are open above but joined below along the lower border of the tendon. The fibres of the muscle (sternocostal) inserted into the posterior layer of the tendon are twisted on themselves until the lower fibres of origin take the highest point of insertion, and the highest fibres of origin take the lowest point of insertion.

Nerve Supply.-From the anterior thoracics: The external thoracic nerve arises from the outer cord and enters the muscle above the pectoralis minor; the internal nerve comes from the inner cord and pierces the minor to end in the major muscle.

Action.-(I) On the humerus. The muscle adducts and flexes the humerus if previously extended; when the arm is at the side it will rotate it internally and draw it across the front of the chest (adduction and flexion). (2) On the shoulder. When the arm is fixed the muscle will draw the shoulder downward and forward. (3) On the chest. When the body is supported on the arms the pectoral muscles sling the chest between them. If the arms are fixed upward and forward the muscles will raise the body (as in climbing).

The sternocostal portion of the muscle may have some action in enlarging the chest in difficult respiration, and thus become an accessory muscle of inspiration.

The Cephalic Vein. Figs. 60, 6I.

(Now dissected from the middle of the arm; see below for its formation at the elbow.)

The cephalic vein starts just above the elbow-joint by the confluence of the median cephalic and the radial veins; 
it runs upward in the groove at the outer margin of the biceps muscle, then enters the interval between the deltoid and pectoralis major muscles, to finally pierce the costocoracoid membrane in front of the clavicle and empty into the axillary vein. In the course between the last muscles it is accompanied by the descending (humeral) branch of the acromiothoracic artery.

\section{DISSECTION.}

Section the pectoralis major at its middle and outer thirds, and cut it away from the clavicle. Raise the parts and reflect them. Notice the formation and method of insertion of the tendon, and the nerve and arterial supply to the inner part of the muscle. Carefully expose the deep fascia (costocoracoid membrane). The nerves and arteries exposed can now be completed.

\section{The Costocoracoid Membrane. Fig. 6I.}

This starts from the under surface of the clavicle in two layers, one in front, the other behind the subclavius muscle. These two layers become united along the front of the muscle and form a single plane of fascia that spreads downward to the pectoralis minor muscle, inward to the first rib and cartilage, and the aponeurosis of the first intercostal space, and outward to the coracoid process. The portion of the membrane between the coracoid process and the first costal cartilage is much thicker and stronger than any other part of the membrane, and is known as the costocoracoid ligament.

The costocoracoid membrane is perforated by the cephalic and acromiothoracic veins, the acromiothoracic artery, the external anterior thoracic nerve, and the axillary lymphatic trunk.

The outward continuation of this membrane is in the shape of two layers of thin fascia which enclose the pectoralis minor muscle, unite at the lower margin of the same, 


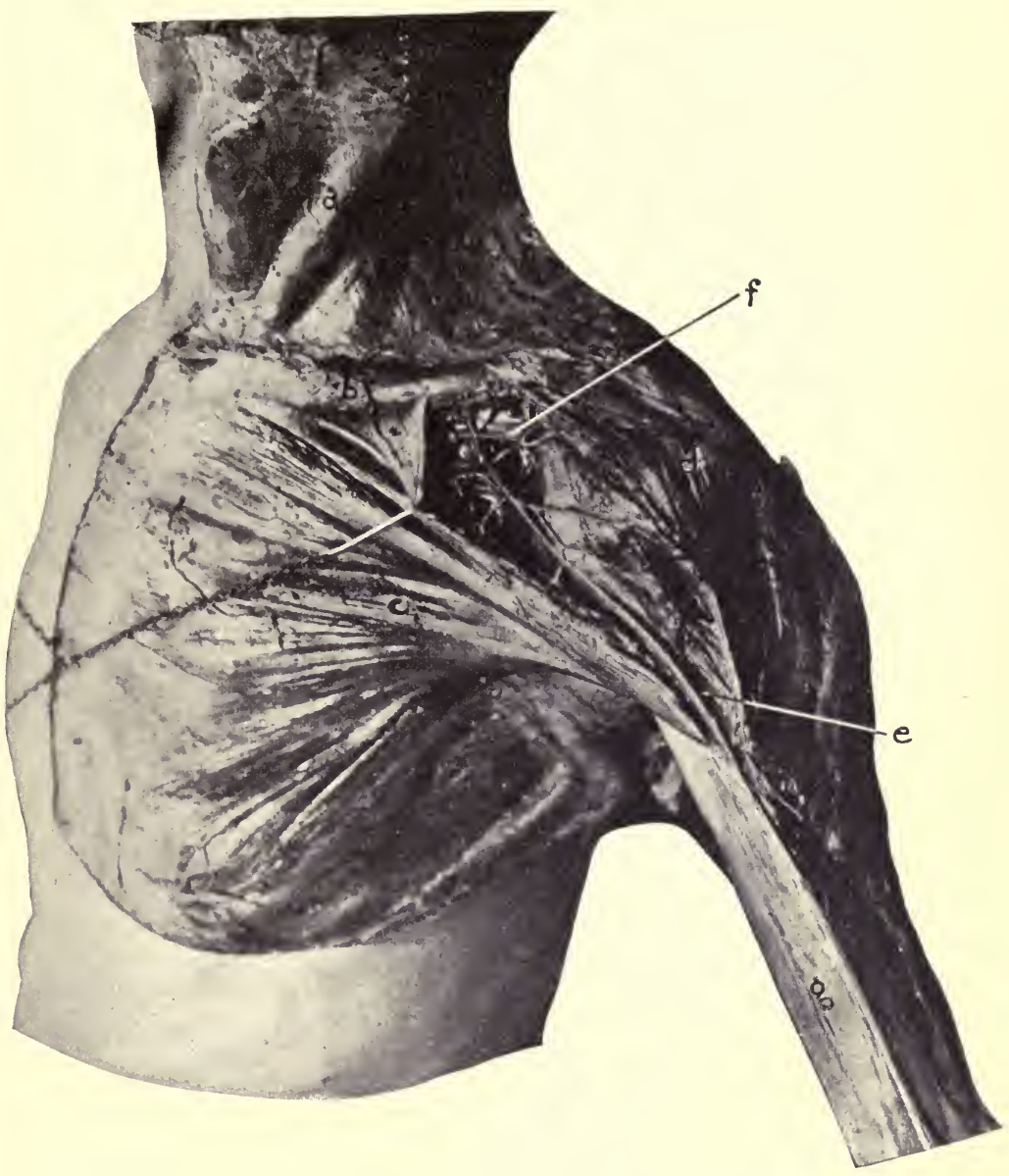

Fig. 6I. Superficial Dissection of Shoulder. Costocoracoid Membrane.$a$, Platysma myoides. $b$, Clavicle. $c$, Pectoralis major. $d$, Deltoid. The pectoralis major and deltoid muscles are separated so as to show the costocoracoid membrane and the structures which traverse it. $e$, The cephalic vein. $f$, The costocoracoid ligament. The branches of the acromiothoracic artery are seen along with the cephalic vein. 
and become continuous with the axillary fascia. The attachment of the lesser pectoral (clavipectoral) fascia to the axillary fascia draws upward the latter, and this explains why it is that the floor of the axilla is so hollowed out or concave externally (the pit of the arm). This concavity is greatest when the arm is midway between the side of the chest and right-angle abduction.

The branches of the acromiothoracic artery will be found in the interval between the pectoralis major, deltoid, and clavicle. They are the humeral, acromial, clavicular, and thoracic. Figs. 60, 61, 62.

The humeral (descending) is found along with the cephalic vein in the groove between the deltoid and pectoralis major muscles, supplying both.

The acromial may be a branch directly from the main trunk or often from the humeral. It supplies the region about the shoulder.

The clavicular branch is a constant branch to the subclavius, but may arise from the thoracic branch.

The thoracic distribution is usually, by two or more branches, to the pectoral muscles and side of the chest.

The External Anterior Thoracic Nerve.-This will be found coming through the costocoracoid membrane and passing to the pectoralis major. Fig. 62 .

\section{DISSECTION.}

Remove the costocoracoid membrane and expose the subclavius muscle, first portion of the axillary artery and its two branches, the accompanying vein, and cords of the brachial plexus.

Clean the pectoralis minor muscle and the floor of the axilla external to the muscle, saving the nerves and arteries found.

Subclavius. Fig, 62.

Origin.-From the cartilage and first rib at their point of junction. 

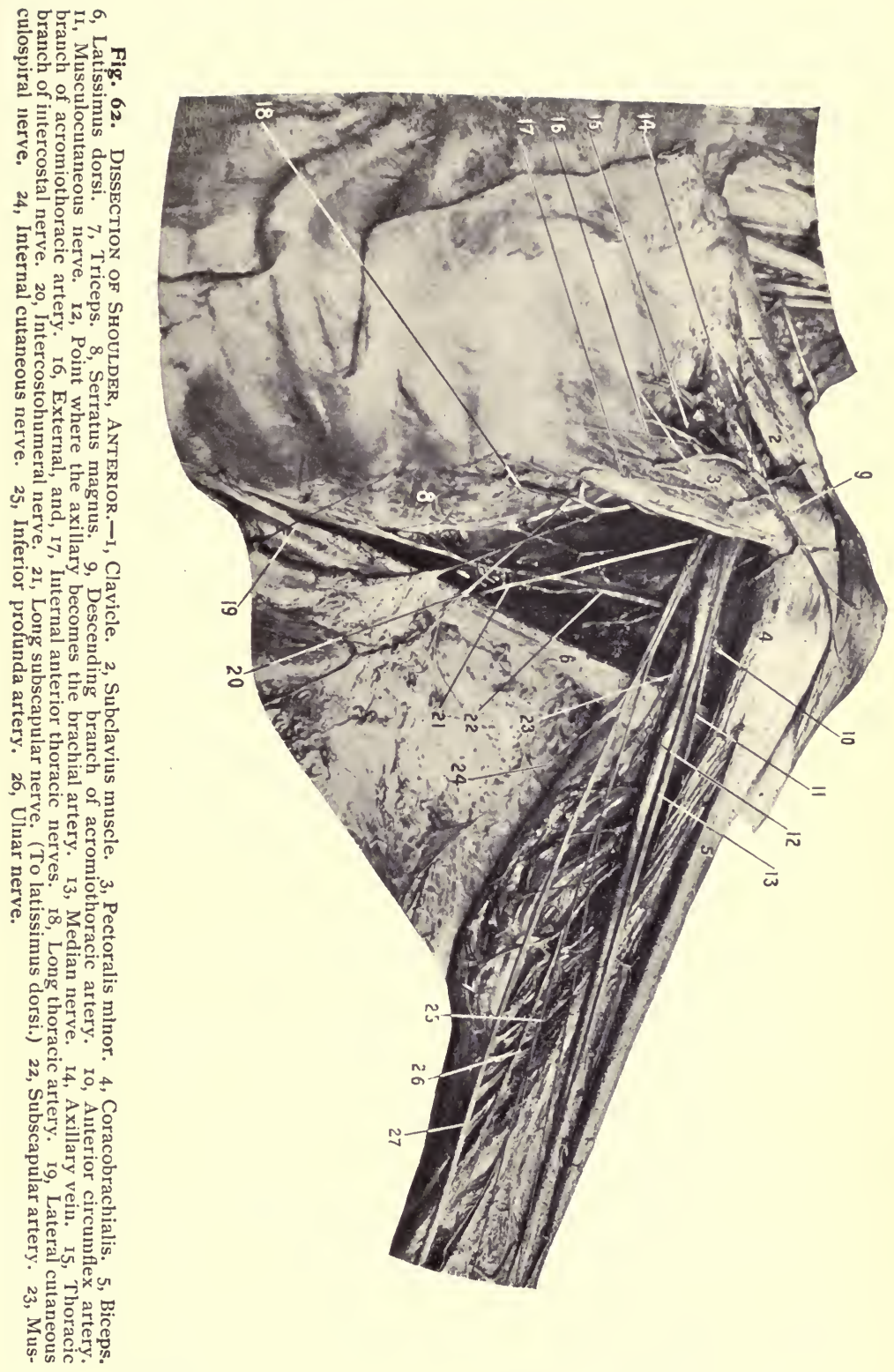
Insertion. - Into the middle third of the lower surface of the clavicle, between the conoid and rhomboid ligaments.

Nerve Supply._-Supraclavicular, from the first trunk of the brachial plexus (fifth and sixth nerves).

Action.-To depress and draw forward the clavicle, to prevent outward displacement of the clavicle.

Pectoralis Minor. Fig. 62.

Origin.-By three slips from the anterior surfaces and upper borders of the third, fourth, and fifth ribs near their cartilages. From the intercostal aponeurosis of the included spaces.

Insertion.-Into the inner side and tip of the coracoid process of the scapula, and inner side of the tendon of the coracobrachialis and biceps muscle.

Nerve Supply._The internal anterior thoracic from the inner cord (eighth and first dorsal nerves).

Action.-(I) On the scapula. To depress, adduct, and draw forward the shoulder. (2) On chest. If the scapula is fixed it will tend to spread the ribs and raise them, thus assisting in inspiration.

\section{DISSECTION}

Section the pectoralis minor near its insertion, turn the inner part inward and save the nerve and blood supply to it.

Complete the dissection of the axillary space, beginning with the axillary vein, which can be removed between two ligatures (one at its beginning and one at its ending), after studying it carefully and noting its relations.

Observe the location of the lymphatic glands, especially those along the long thoracic artery and axillary vein.

Trace the branches of the axillary artery, the nerves contained in the axilla, and clean the presenting surfaces of the subscapularis, serratus magnus, teres major, and latissimus dorsi muscles, being sure to remove all the tissue filling the space between the various muscles.

\section{The Lymphatics.}

The axillary lymphatics are from ten to twelve in num- 
ber. They are grouped in several sets, two of which are especially worthy of notice. (I) The pectoral set: four or five in number, below the lower border of the pectoralis major and with the long thoracic artery. These receive the drainage from the front and side of the chest (including the mammary gland); hence in inflammatory or malignant disease of the chest or mamma these lymphatics are first affected. (2) The axillary set: three or four, located along the axillary vein. These receive the lymph from the upper extremity and from the pectoral set of glands. They become enlarged in inflammatory affections of the extremity, or from the chest by extension through the first set. They are closely connected to the vein, especially in the inflamed condition. Their removal should proceed with caution.

\section{The Axillary Vein.}

The axillary vein is formed by the confluence of the venæ comites of the brachial artery and the basilic vein, at the lower border of the subscapularis muscle. It takes a course upward along the inner side and front of the artery (overlapping it in front), to become the subclavian vein at the lower border of the first rib. It receives, besides the veins which unite to form it, the veins corresponding to the branches of the axillary artery, and the cephalic vein, which passes through the costocoracoid membrane to empty into it.

\section{The Axillary Artery. Figs. 62, 63 .}

This is the continuation of the subclavian, from the lower border of the first rib to the lower border of the teres major muscle. It lies near the outer angle of the axillary space. It is divided into three portions by the pectoralis minor, that above, behind, and below the muscle.

The Relations of the Axillary Artery.-The axillary 
vein lies to the inside and a little in front of the artery for its entire extent. Besides the vein there is the internal anterior thoracic nerve to the inside of the first portion of the artery, the inner cord of the brachial plexus for the second, and the ulnar nerve for the third part. These nerves intervene between the artery and the vein. Also at the inner part of the third portion is the lesser, and the internal cutaneous nerves, and the inner head of the median. On the outside, for the first part, are the cords of the brachial plexus; for the second part, the outer cord of the same, and the coracoid process; for the third part, the median and musculocutaneous nerves, the coracobrachialis and biceps muscles.

Behind the first part is the first intercostal space and muscle, second rib, and second digitation of the serratus magnus muscle, and the posterior thoracic nerve. In the second part, posterior cord and first subscapular nerve, and the subscapularis muscle. Behind the third part, the musculospiral, second and third subscapular and the circumflex nerves, the subscapularis, teres major, and latissimus dorsi muscles. In front of all parts of the artery, the skin, superficial and deep fascia, and the pectoralis major muscle (does not cover the lower part of the artery). In front of the first part, origin of platysma, cephalic and acromiothoracic artery, veins and branches, external anterior thoracic nerve, axillary lymphatic trunk and costocoracoid membrane. Over the second part, the pectoralis minor muscle. In front of the third portion, inner head of median, outer brachial vena comes.

Branches of the Axillary Artery.

First Portion.-(a) Superior thoracic. This is a small artery from the inside of the axillary close below the subclavius muscle. It passes to the first intercostal space 


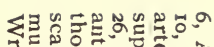

क. ใด 궁

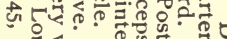
३ง

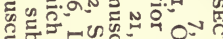

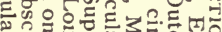
7 舟舟

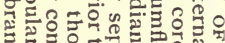

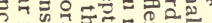

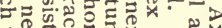
००

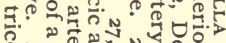
莡

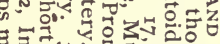

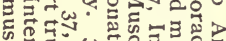
ผึ.

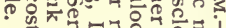

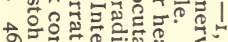
ลै

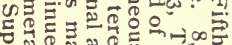

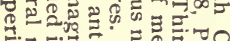
하쿵

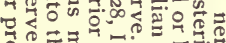
우욜

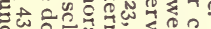
w क 7 क क

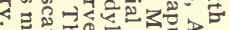

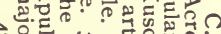
숭요웅

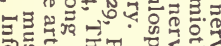

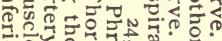

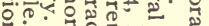

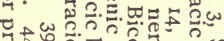

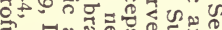

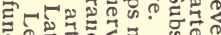

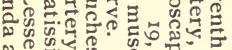
क क ज्य

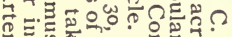
co

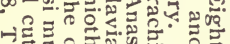

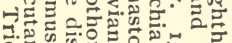
त

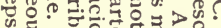
जै

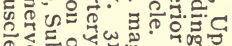
-

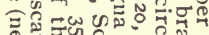

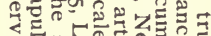

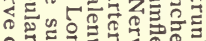

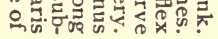

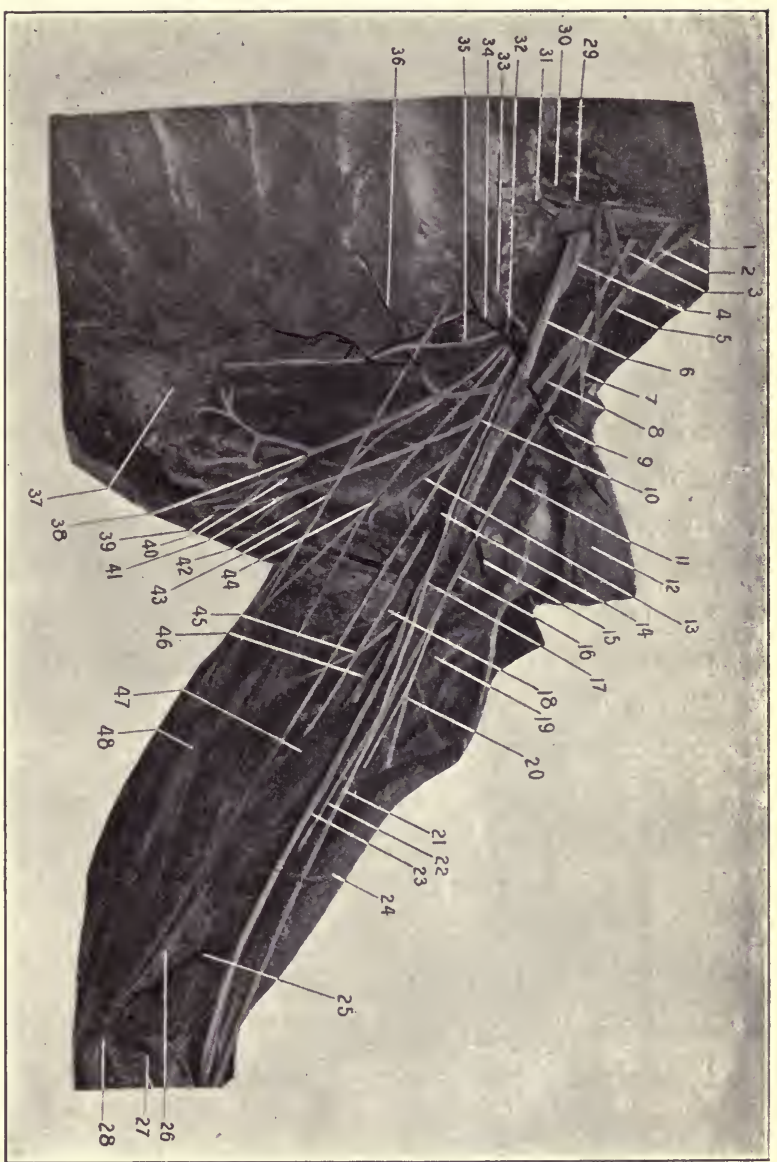


along the upper border of the pectoralis minor. (b) The acromiothoracic. This arises as a short trunk from the front of the axillary, just above the pectoralis minor, pierces the costocoracoid membrane, and breaks up into three or four sets of branches; the humeral (descending), which accompanies the cephalic vein into the groove between the deltoid and pectoralis major muscles; the acromial, usually a branch from the above, to the region of the point of the shoulder; the thoracic, two or three branches to the chest and pectoral muscles; the clavicular, often from the thoracic to the subclavius muscle.

Second Portion.-(a) The alar thoracic. There may be one or several alar arteries, and they may arise from the axillary or from the neighboring branches, usually from the long thoracic. They supply the glandular and adipose tissue in the axillary space. (b) The long thoracic is given off from the inner side of the axillary behind the pectoralis minor, passes along the lower border of the same, and is distributed to the pectoral muscles, side of chest, and mammary gland.

Third Portion.-(a) The subscapular, the largest branch of the axillary artery, arises from its inner side just above the lower border of the subscapularis muscle, passes downward and inward to the chest, lying in the angle between the subscapularis, teres major, and latissimus dorsi muscles, and terminating in the serratus magnus muscle. About one inch from its origin the artery gives off the dorsal artery of the scapula (dorsalis scapula), a large branch (really the continuation of the subscapular) which winds backward around the axillary (outer) border of the scapula to its posterior surface. It passes through the triangle formed by the subscapularis, teres major, and long head of the triceps muscles. For rest of course see page $37 \mathrm{I}$. (b) The anterior 
circumflex is a small branch from the outside of the axillary opposite the surgical neck of the humerus, around which it turns, under the coracobrachialis and biceps muscles, to end in the deltoid. At the bicipital groove it gives off a branch (articular) which passes upward in the groove to the shoulder-joint.

(c) The posterior circumflex, nearly as large as the subscapular, is given off the back of the axillary, continues through the quadrilateral space formed by the subscapularis, teres major, long head of triceps, and humerus, with the circumflex nerve. For the continuation of the artery see page 372 . (d) The external mammary. This is usually described as an abnormal branch, but is found so frequently that it deserves a place with the more constant branches of the axillary, from which it arises at the lower margin of the axillary space and takes a course downward, forward, and inward in the subcutaneous tissue to the mammary region.

Subscapularis. Fig. 63 .

Origin.-From the inner two-thirds of the subscapular fossa on the anterior surface of the scapula, from the lower two-thirds of the axillary border of the same and intermuscular septum between it and the teres minor.

Insertion.-Into the lesser tuberosity of the humerus, the surgical neck just below it, and into the capsule of the shoulder-joint. The nerve supply, from the first (upper) and third (lower) subscapular nerves.

Action.-To rotate the humerus inward; if the arm is extended, to slightly adduct the humerus. When the humerus is fixed it acts upon the scapula to turn it forward.

Serratus Magnus. Figs. 63, 76 .

Origin.-From the outer surfaces and upper margins of the eight (or nine) upper ribs at their anterior and middle 
thirds by nine (or ten) fleshy slips, two arising from the second rib.

Insertion.-Into the anterior aspect of the vertebral border and superior and inferior angles of the scapula.

Nerve Supply. - The posterior or long thoracic (external respiratory of Bell), which brings filaments from the fifth, sixth, and seventh cervical nerves.

Action.-(I) On the scapula. The muscle draws the scapula forward, and owing to the lower portion of the muscle being longer than the upper, the lower angle of the scapula will be advanced farther than its upper angle, thus producing a rotation of the scapula around a central axis perpendicular to its plane surface. This action is further developed in raising the arm to a vertical position, the deltoid carrying it to a right angle, the trapezius and serratus magnus completing the action. (2) On the ribs. If the scapula is fixed backward, the muscle would tend to draw the ribs outward, and so enlarge the chest laterally and aid inspiration.

The Posterior Thoracic Nerve. Figs. 63, 64.

For formation, see description of brachial plexus, page 3 I 5 .

Appears in the axilla posterior to the axillary artery and vein, continues downward across the middle of the serratus magnus muscle to the various digitations to which it is distributed.

The Intercostohumeral Nerve. Figs. 62, 63, 64 .

This comes through the second intercostal space. It is the lateral cutaneous branch of the second intercostal nerve. In the floor of the axilla some of its filaments interlace with others from the lesser internal cutaneous nerve to form a loose plexus. The nerve is then distributed to the integument of the inner, upper, and posterior part of the arm. 


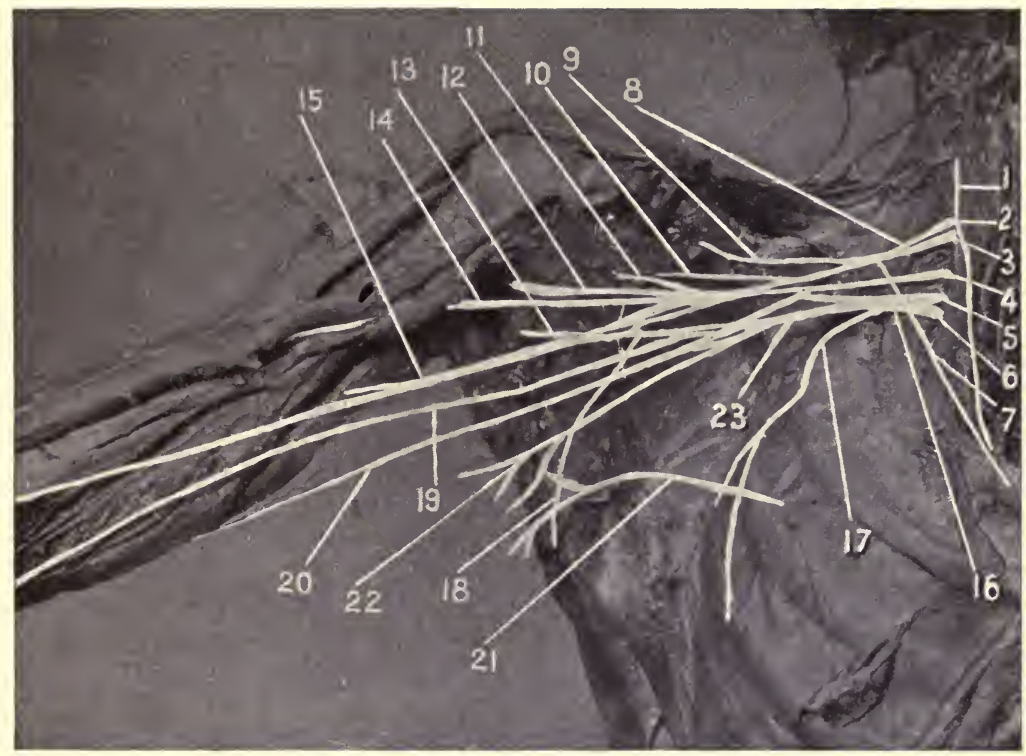

Fig. 64. Dissection of Brachial Plexus.-1, Phrenic nerve. 2, Fifth C. 3, Sixth C. 4, Seventh C. 5, Eighth C. 6, First D. 7, Subclavian nerve. 8, Upper trunk. 9, Suprascapular nerve. Io, First subscapular nerve. II, External anterior thoracic nerve. I2, Circumflex nerve. I3, Third subscapular nerve. I4, Musculocutaneous nerve. 15, Median nerve. I6, Lower trunk. The middle trunk is formed by the continuation of the seventh nerve (4). 17, Long thoracic nerve. 18, Long subscapular nerve. 19, Ulnar nerve. 20, Internal cutaneous. 21, Intercostohumeral. 22, Lesser internal cutaneous. 23, Internal anterior thoracic. 
The lateral cutaneous branches of the third, fourth, fifth, and sixth intercostal nerves may be found as they appear along the side of the chest after coming through the intercostal muscles. The first one or two usually help to form a loose plexus with the intercostohumeral nerve. These nerves decrease in size from above downward. The third is usually distributed to a small part of the integument of the inner side of the arm and axilla, the others to the integument of the side of the chest.

(The description of the brachial plexus will be delayed until the dissection of the neck has proceeded far enough to permit removal of the clavicle, when the entire extent of the plexus will be uncovered.)

\section{DISSECTION.}

Incisions.-(I) Continue the median incision to the wrist.

(2) Make two short transverse cuts at the lower end of (I).

Reflect the skin from the arm and forearm, saving the superficial nerves and veins.

\section{The Superficial Fascia.}

This is usually a thin layer, but may be thickened by the presence of adipose tissue over the lower part of the forearm and hand. In the hand the superficial fascia consists of fatty granules between the palmar fascia and the skin. In the superficial fascia ramify the subcutaneous vessels and nerves.

The Superficial Veins of the Arm and Forearm. Fig. 65.

On the outer side of the forearm is the radial vein; in front, the median ; at the inner side, two, the anterior and posterior ulnar, which unite to form the common ulnar at the elbow. These pass up to the elbow, where the anterior and posterior ulnar unite to form the common ulnar, 
and the median receives the deep median vein from the interior of the forearm and divides into the median cephalic and median basilic. The deep median vein is formed by the venæ comites of the ulnar, radial recurrent, and muscular arteries.

The median cephalic joins the radial vein to form the cephalic, which passes upward in the groove at the outer border of the biceps muscle.

The median basilic joins with the common ulnar to form the basilic, which ascends in the inner brachial groove, pierces the deep fascia in the middle and lower thirds of the arm, and terminates as one of the tributaries forming the axillary vein.

The Superficial Nerves. Figs. 62, 63, 65.

These are, on the inside of the arm, branches of the intercostohumeral, lesser internal cutaneous, internal cutaneous; on the outside, the cutaneous branches of the circumflex and the superior external cutaneous branch of the musculospiral.

In the forearm there are, on the inside, the anterior division of the internal cutaneous, which reaches to the wrist; on the outer side, the external cutaneous branch of the musculocutaneous, which is distributed as low as the ball of the thumb.

The Deep Fascia. Figs. 61, 65.

The deep fascia of the arm and forearm encases the muscles like a tight sleeve, forming their compartments and fixing them in position. Above it is continuous with the deep fascia covering the deltoid, pectoralis major, teres major, latissimus dorsi, and axillary space. Through these extensions it passes to the spine and acromion process of the scapula and the clavicle. Below, the fascia is attached 
about the wrist by means of thickened portions called annular ligaments.

At the elbow the fascia is united to all the bony points. Behind, to the olecranon, and here it receives the fibrous expansion of the tendon of the triceps muscle. At the sides, to the humeral condyles, and by means of the intermuscular septa to the condyloid ridges. In front, it receives the fascial prolongation of the bicipital tendon, and is pierced for the deep median vein.

The intermuscular septa in the arm separate the anterior from the posterior muscles. In the anterior compartment are the biceps, coracobrachialis, brachialis anticus, origin of the supinator longus, and the extensor carpi radialis longior. In the posterior is the triceps muscle.

In the forearm the intermuscular septa are especially well-marked and appear as "white lines," indicating the separations between the various muscles and forming guides to the arteries.

\section{DISSECTION.}

Remove the superficial veins and the deep fascia to just below the elbow. Leave the insertion of the biceps into the deep fascia.

Complete the dissection of the parts exposed.

Coracobrachialis. Fig. 62.

Origin-From the tip of the coracoid process of the scapula with the short head of the biceps.

Insertion.-Into the middle third of the inner border of the humerus for a distance of two inches.

Nerve Supply.-By the musculocutaneous, which usually passes through the muscle. The nerve filaments come from the seventh cervical nerve.

Action.-It is an adductor and flexor of the arm; helps to hold the humerus against the scapula. 
Biceps. Figs. 62, 63.

Origin.-By a long tendinous head from the top of the glenoid cavity of the scapula, by a shorter muscular head from the coracoid process of the same bone.

The former tendon passes over the top of the head of the humerus within the capsular ligament of the shoulder, and blends with the glenoid ligament, and the latter has a common origin with the coracobrachialis muscle.

Insertion.-By the broad expansion into the deep fascia of the forearm. By the tendon into the posterior margin of the bicipital tuberosity of the radius.

Nerve Supply.-From the musculocutaneous, which brings fibres from the sixth and seventh cervical nerves.

Action.-(I) It is a feeble flexor and adductor of the arm. Serves to retain the head of the humerus in the glenoid cavity. (2) On the forearm. A strong flexor of the forearm. If the forearm has been pronated the biceps acts as a strong supinator, the strength of its action of supination increasing until it is the strongest when the forearm is at right angles with the arm.

Brachialis Anticus. Figs. 63, 67.

Origin.-From the anterior surface of the shaft of the humerus below the insertion of the coracobrachialis and deltoid (the latter it embraces), and from the anterior surface of the internal intermuscular septum.

Insertion.-The rough surface at the front and inside of the base of the coronoid process of the ulna.

Nerve Supply.-By the musculocutaneous in front and the musculospiral behind. The sixth and seventh cervical nerves supply the filaments.

Action.-To flex the forearm upon the arm, or the reverse. 
The Brachial Artery. Figs. 62, 63, 67.

The brachial artery is the continuation of the axillary from the lower border of the teres major to half an inch below the bend of the elbow, where it divides into the radial and ulnar arteries.

Relations.-On the outside: The coracobrachialis, biceps (which slightly overlaps the artery in front), and the median nerve (at the upper part).

The inner border of these muscles is the sure guide to the artery.

The median nerve, though formed at the outside of the artery above, crosses in front (usual) or behind (unusual) the artery to its inside at the elbow. One of the accompanying veins is to the outside.

Behind: The brachial rests upon the triceps, tendon of the coracobrachialis and brachialis anticus muscles. It may have the median nerve behind its middle.

At the inside is the other accompanying vein. These venæ comites are joined by frequent cross branches. The basilic vein takes a course upward at the inside of the artery but separated from it by the deep fascia in the lower part of its course.

The ulnar and internal cutaneous nerves are at some little distance from the upper part of the artery, and in its lower two-thirds have no direct relation to it.

In front: The brachial is covered by the integument, two layers of fascia, and the inner border of the biceps muscle. The median nerve is in close relation to the artery and crosses its front about the middle (usual). At the elbow the brachial has the median basilic vein in front of it, and separated from it by the bicipital fascia. It lies between the tendon of the biceps at the outside and the median nerve at the inside. 
Branches of the Brachial.

(I) The superior profunda. This is the largest branch of the brachial. It arises from the posterior surface of the artery, takes a course downward; then with the musculospiral nerve, downward and outward through the musculospiral groove to terminate by anastomosing with the radial recurrent at the outer side of the arm in the space between the brachialis anticus and the supinator longus. Besides the muscular branches it gives off the ascending and articular, which will be found in the dissection of the posterior of the arm.

(2) The inferior profunda is given off the inside of the brachial opposite the insertion of the coracobrachialis. It often arises with the superior profunda as a common trunk from the brachial. It takes a course downward and inward with the ulnar nerve to get behind the internal condyle and terminates in an anastomosis with the posterior ulnar recurrent and anastomotica magna arteries.

(3) The nutrient. This small branch is given off at the lower part of the insertion of the coracobrachialis and enters the nutrient foramen of the humerus.

(4) Muscular branches are supplied to the adjacent muscles and vary from six to eight in number.

(5) The anastomotica magna. This is from the inside of the brachial from one to two inches above the bend of the elbow. Its course is inward, and at the inner border of the arm it divides into two branches: (a) the anterior which is smaller than the posterior, and descends between the brachialis anticus and pronator radii teres to anastomose at the inside of the elbow with the anterior recurrent ulnar; (b) the posterior, which continues backward around the inner side of the arm, anastomoses with the inferior profunda and posterior recurrent ulnar arteries, and terminates 
(usually) by uniting with the articular branch of the superior profunda to form an arch above the olecranon fossa.

The veins. These are venæ comites, accompanying the arteries above and opening into the brachial veins.

The Nerves. Figs. 63, 64, 65 .

(I) The musculocutaneous nerve. This is the direct continuation of the outer cord of the brachial plexus. It passes through the coracobrachialis muscle, then between the biceps and brachialis anticus; appearing at the outer border of the tendon of the biceps, it pierces the deep fascia over the elbow and becomes the cutaneous nerve to the outer side of the front of the arm. In its course through the arm the musculocutaneous supplies the coracobrachialis, biceps, and brachialis anticus.

(2) The median nerve is formed by a branch from the outer and inner cords of the brachial plexus, which unite at the outer side of the axillary artery ; the nerve passes down the arm to reach the inner side of the brachial artery in front of the elbow, having crossed the middle of the artery in front or behind it.

The median nerve has no branches in the arm, but just above the bend of the elbow it gives off the articular branch to the joint.

(3) The ulnar nerve continues the inner cord down the inside of the arm, is joined by the inferior profunda artery about its middle, and disappears behind the internal condyle. An articular branch is furnished to the elbow-joint behind the condyle.

(4) The internal cutaneous. This comes from the internal cord, descends along the inner side of the arm, pierces the deep fascia above the elbow, divides into an anterior and posterior branch. The anterior continues down the 


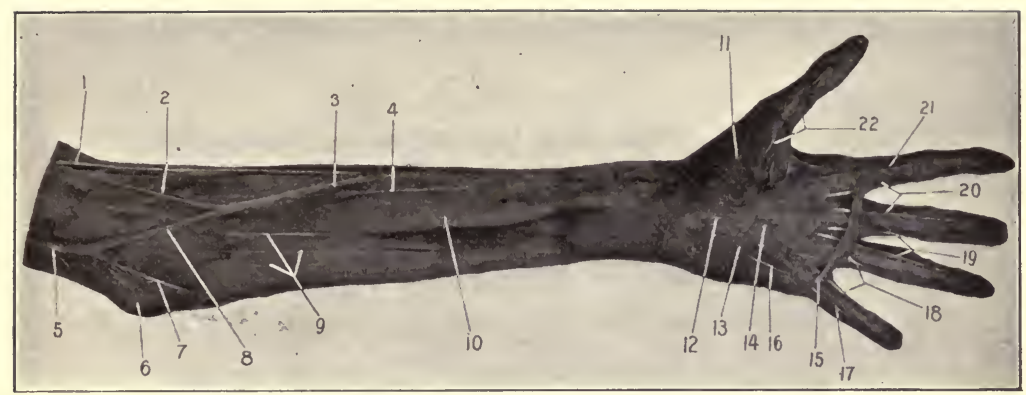

Fig. 65. Superficial Forearm and Hand.-1, Radial vein. 2, Median cephalic. These two unite to form the cephalic vein. 3, Median vein. 4, Cutaneous branch of musculocutaneous nerve (the external cutaneous). 5, Basilic vein. 6, Internal condyle. 7, Ulnar veins. 8, Median basilic vein. 9, Internal cutaneous nerves. Io, Deep fascia of forearm. I , Outer portion of palmar fascia. 12, Annular ligament (position of). 13, Inner portion of palmar fascia, and the palmaris brevis muscle. I4, Middle portion of palmar fascia. 15, The superficial transverse ligament of the fingers (Fibers of Gerdy). The division of the central portion of the palmar fascia into its fasciculi and the position of the digital vessels and nerves is shown. 16, Digital nerve to little finger. 17, 18, 19, 20, 21, 22, Collateral digital nerves and vessels.

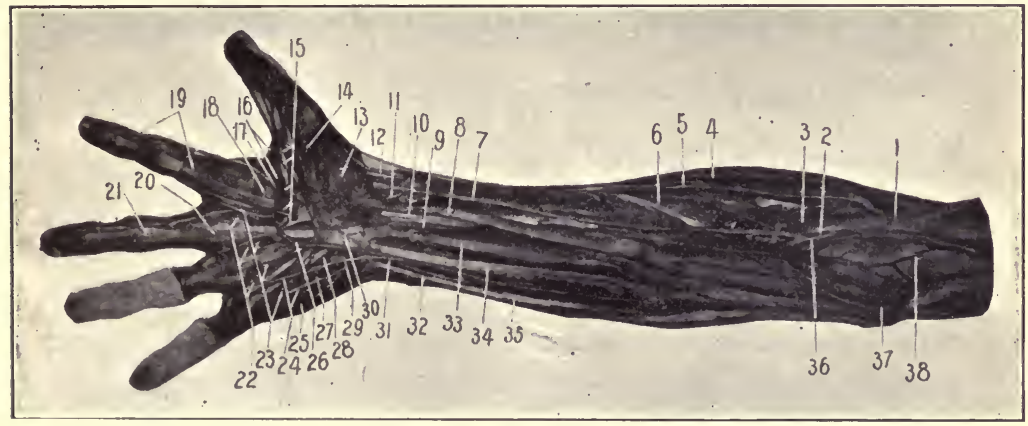

Fig. 66. Dissection of Forearm and Hand.- I, Tendon of biceps muscle. 2, Brachial artery at point of bifurcation. 3, Radial recurrent artery. 4, Brachioradialis or supinator longus. 5, Radial nerve. 6, Tendon of pronator radii teres. 7, Radial artery. 8, Flexor carpi radialis muscle. 9, Median nerve. Io, Flexor longus pollicis. II, Superficial volar artery. 12, Tendon of extensor ossis metacarpi pollicis. I3, Abductor pollicis. I4, Outer head of flexor brevis pollicis. 15, The digital branches of the median nerve. 16, Digital arteries to thumb and index finger. 17, External lumbrical muscle. 18, Adductor pollicis muscle. 19, Vaginal ligaments of the sheaths of the long flexor tendons. 20, Tendon of the flexor sublimis digitorum. 2I, Tendon of the flexor profundus digitorum. 22, The three inner lumbrical muscles. 23, Digital arteries. 24, Ulnar digital nerves. 25 , Abductor minimi digiti. 26, Superficial palmar arch. 27, Flexor brevis minimi digiti. 28, The slender nerve which passes from the ulnar to the median. 29, The deep communicating from the ulnar artery to the radial to form the deep palmar arch. 30, The anterior annular ligament. 3I, The ulnar nerve. 32, The ulnar artery. 33, Palmaris longus muscle. 34, Flexor sublimis digitorum. 35, Flexor carpi ulnaris. 36, Median nerve. 37. Internal condyle of humerus. 38, The anastomotica magna artery lying upon the brachialis anticus muscle and dividing into its two terminal branches. 
inner side of the front of the forearm to the wrist, the posterior passes backward to a similar distribution to the posterior surface of the forearm.

The triceps muscle shows at the inside of the arm and should be cleaned, and the musculospiral nerve and the superior profunda artery traced between its two lower heads.

(5) The musculospiral nerve. This nerve is the direct continuation of the posterior cord. It is found first behind the axillary, then to the inner side of it and the brachial artery, and disappears with the superior profunda artery by entering the musculospiral groove or canal between the two lower heads of the triceps muscle.

\section{DISSECTION.}

Remove the deep fascia from the forearm as low as the annular ligament of the wrist.

Carefully clean the following structures:-

Pronator Radii Teres. Fig. 66.

Origin.-By the common tendon from the lower halfinch of the internal condyloid ridge and the internal condyle, for the upper head; from the inner margin of the coronoid process of the ulna, by the lower and smaller head; also from the intermuscular septum between it and the flexor carpi radialis.

NotE.-It will be stated here for once, to save repetition, that all the muscles arising from the internal condyle by the common tendon also arise from the intermuscular septum between each other.

Insertion.-Into the rough oval impression on the outer surface of the radius at its middle.

Nerve Supply.-By the median, which passes between its two heads. These filaments come from the sixth cervical nerve. 
Action.-To pronate the forearm; to aid in flexion of the forearm. This muscle is a valuable accessory to the flexors of the elbow-joint.

Flexor Carpi Radialis. Fig. 66.

Origin.-By the common tendon, from the internal condyle of the humerus. (See note above.)

Insertion.-Into the base of the second metacarpal bone. The tendon grooves the trapezium upon its inner side and passes superficial and external to the central compartment of the annular ligament.

Nerve Supply.-The median, by filaments from the sixth cervical nerve.

Actions.-To flex the wrist and slightly abduct the wrist, to pronate and feebly flex the forearm.

Palmaris Longus. Fig. 66.

Origin.-Same as the flexor carpi radialis. (See note above.)

Insertion.-Into the anterior annular ligament and central portion of the palmar fascia.

Nerve Supply.-The median from the eighth cervical nerve.

Action.-Tensor of palmar fascia, feeble flexor of wrist and forearm.

The Flexor Carpi Ulnaris. Fig. 66.

Origin.-By the common tendon from the internal condyle (see note), by a second head from the internal surface of the olecranon and the upper two-thirds of the posterior ridge of the ulna.

Insertion.-Into the pisiform bone, hook of the unciform, and base of the fifth metacarpal bone. 
Nerve Supply.-Through the ulnar nerve by filaments from the eighth cervical and first thoracic nerves.

Action.-It is a flexor and adductor of the wrist and a weak flexor of the forearm.

Supinator longus. (Brachioradialis.) Fig. 67.

Be sure to carry the dissection of this muscle to its origin; save the nerves and arteries found.

Origin.-From the upper two-thirds of the external condyloid ridge, from the external intermuscular septum of the deep fascia of the arm.

Insertion.-Into the base of the styloid process of the radius.

Nerve Supply. - The musculospiral from the sixth cervical nerve.

Action.-Primarily it is a flexor of the forearm, secondarily a pronator in complete supination of the forearm, and has a slight action of supination only when the arm is fully pronated.

The Anterior Elbow Space. Fig. 66.

The hollow in front of the elbow is bounded on the outside by the supinator longus, on the inside by the pronator radii teres. Its floor is formed by the brachialis anticus.

From without inward is found the musculospiral nerve (dividing into its radial and posterior interosseous branches), radial recurrent artery, musculocutaneous nerve (under the outer margin of the biceps), the tendon of the biceps muscle, the brachial artery and its terminal branches, the ulnar and radial, and their accompanying veins, the median nerve and its muscular branches, and the anastomosis between the anterior branch of the anastomotica magna and anterior ulnar recurrent. 


\section{DISSECTION.}

Trace the radial nerve to the posterior margin of the tendon of the supinator longus (brachioradialis). The radial artery until it disappears under the extensor tendons of the forearm, and its branches in the forearm. The ulnar artery to the wrist, and its branches.

\section{The Radial Nerve. Fig. 66.}

The anterior branch of the musculospiral nerve. It descends behind the inner portion of the supinator longus muscle, until at the lower part of the muscle it passes backward to the posterior surface of the thumb and hand.

\section{The Posterior Interosseous Nerve.}

This is the posterior branch of the musculospiral nerve and disappears from the front of the elbow by passing into the substance of the supinator brevis muscle.

\section{The Radial Artery. Figs. 66, 67.}

This is the outer branch of bifurcation of the brachial, the direction of which it continues. The portion of the artery that lies in the forearm extends from below the elbow to the wrist, where the artery passes posteriorly under the extensor tendons of the thumb.

Its course is outward and downward, between the pronator radii teres and the supinator longus (brachioradialis) (the latter of which overlaps the artery in front), then between the tendons of the flexor carpi radialis and the supinator longus. The artery is indicated by a line drawn from just below the centre of the elbow to the inner side of the styloid process of the radius. The radial nerve is at the radial side of the artery for the upper two-thirds of its course, but not in close relation with it, and is not seen in ligation of the artery.

The artery rests upon the tendon of the biceps, supinator 
brevis, pronator radii teres, flexor sublimis digitorum, flexor longus pollicis, and pronator quadratus muscles, and the lower end of the radius.

The accompanying veins are on the outer and inner sides of the artery. While the artery is overlapped by muscles in the upper part of its course, it is only covered by the fascia and skin in the lower two-thirds.

Branches of the Radial Artery.

(I) Radial recurrent. This is a large branch from the outer side of the artery below its origin. It passes outward, supplying branches to the supinator longus, extensor carpi radialis longior and brevior, and supinator brevis, then turns upward between the supinator longus (brachioradialis) and brachialis anticus to terminate in branches to these muscles and by anastomizing with branches from the superior profunda artery. (2) Muscular. To the surrounding muscles. (3) Anterior carpal. A very small branch to the front of the carpus. (4) Superficial volar. Variable in size and occurrence. Usually a small branch to the base of the thumb muscles, and terminating in them; at other times a larger branch that crosses beyond the thumb to anastomose with the ulnar to form the superficial palmar arch. For the remaining branches see back of hand.

\section{DISSECTION.}

Divide the flexor carpi radialis, palmaris longus, and flexor carpi ulnaris where they become tendinous, and the pronator radii teres at its outer and middle thirds.

Clean the flexor sublimis digitorum, the anterior surface of the supinator brevis, the upper and lower thirds of the ulnar artery, and the ulnar nerve from the internal condyle to the wrist.

Flexor Sublimis Digitorum. Fig. 66.

Origin.-(I) From the internal condyle of the humerus and internal lateral ligament of the elbow-joint. (2) From 
the inner margin of the coronoid process of the ulna. From the oblique ridge on the front of the upper part of the radius.

See note, page 290.

Insertion.-By four tendons which perforate the tendons of the long flexor muscle (as described in the dissection of the hand, see page 3 I I), and are attached to the sides of the the second set of phalanges of the four fingers.

Nerve Supply.-The median (seventh and eighth cervical and first thoracic nerves).

Action.-Beginning from below, the muscle will flex the second, then the first set of phalanges, then the hand, and perhaps slightly the forearm.

\section{DISSECTION.}

Remove the flexor sublimis digitorum from its radial attachment and turn it inward, cleaning the median nerve, interosseous arteries, and nerve. Finish the ulnar artery; clean the last layer of muscles.

The Ulnar Artery. Fig. 67.

This is the other branch of brachial bifurcation just below the elbow. It passes inward and downward under all the superficial layer of muscles, and also between the flexor sublimis and profundus digitorum. At the middle and upper thirds of the forearm it bends directly downward and enters the wrist over the annular ligament at the outer side of the pisiform bone.

The course of the lower two-thirds of the artery is indicated by a line from the internal condyle to the outer side of the pisiform bone. The guide to the artery is the intermuscular septum between the flexor sublimis digitorum and the flexor carpi ulnaris. The artery has two accompanying veins.

Relations.-In front: Pronator radii teres, flexor carpi 
radialis, palmaris longus, flexor sublimis digitorum, for the upper half. In the lower half, and for the entire course, by the skin and fasciæ.

Internally: The flexor carpi ulnaris. The ulnar nerve is close to the ulnar side of the artery for the lower twothirds of its course in the forearm.

Outside: The tendons of the flexor sublimis digitorum for the lower two-thirds. Behind: Brachialis anticus, flexor profundus digitorum.

\section{Branches of the Ulnar Artery.}

(I) Recurrent: (a) The anterior. Usually a branch from the posterior recurrent may arise from the ulnar just after its origin. It ascends behind the pronator radii teres to anastomose upon the brachialis anticus with the anterior branch of the anastomotica magna and the inferior profunda. (b) The posterior recurrent. A larger artery than the anterior (which is usually derived from it) from the inner side of the ulnar. It runs upward upon the flexor profundus digitorum to behind the internal condyle, and between the two heads of the flexor carpi ulnaris muscle, and terminates by an anastomosis with the inferior profunda, posterior branch of the anastomotica magna, and interosseous recurrent. Behind the internal condyle it is close to the ulnar nerve.

(2) Common interosseous: This is a large branch from the outer part of the ulnar, about half an inch long, which passes backward and divides into the anterior and posterior interosseous arteries. (a) The anterior interosseous continues the direction of the main trunk down the front of the interosseous membrane, between the flexor longus pollicis and flexor profundus digitorum, to the upper margin of the pronator quadratus muscle, behind which it disappears. 
There are two veins with the artery, and the interosseous branch of the median nerve.

The anterior interosseous artery supplies numerous branches to the adjacent muscles, and one which follows the median nerve and receives its name from this fact. Sometimes this median artery is very large, passes into the palm, and forms the superficial arch with the ulnar.

The nutrient arteries for the radius and ulna are from the anterior interosseous artery.

(b) The posterior interosseous passes directly backward between the radius and ulna, and will be found in dissecting the back of the arm.

(3) The Muscular. These are numerous and supply the muscles near by.

(4) Posterior carpal. Is given off just above the wristjoint, and turns backward under the tendon of the flexor carpi ulnaris to the back of the hand.

(5) The anterior carpal. A small artery to the front of the carpus to supply this region along with the anterior carpal branch of the radial.

For the remaining branches in the hand see page 304 .

The Ulnar Nerve. Fig. 67.

Is found entering the forearm from behind the internal condyle of the humerus, then passes between the two heads of the flexor carpi ulnaris, and descends upon the flexor profundus digitorum. It joins the ulnar artery at the upper and middle thirds of the forearm, continues at the ulnar side of the artery, and with it passes over the anterior annular ligament at the outer side of the pisiform bone into the hand.

In the forearm the ulnar nerve supplies filaments to the flexor carpi ulnaris and the inner portion of the flexor profundus digitorum. 
The dorsal cutaneous branch of the ulnar nerve is: found about two inches above the wrist; it turns backward under the tendon of the flexor carpi ulnaris muscle to the back of the hand, where it will be found.

The Median Nerve. Fig. 67.

At the elbow the median nerve lies at the inner side of the brachial artery. It descends between the two heads of the pronator radii teres, then directly down the centre of the forearm between the flexor sublimis and flexor profundus digitorum muscles to enter the hand through the central compartment of the anterior annular ligament with the tendons of the above muscles.

It supplies directly the pronator radii teres, flexor carpi radialis, palmaris longus, flexor sublimis digitorum, and through the anterior interosseous branch the flexor longus pollicis, the outer portion of the flexor profundus digitorum, and the pronator quadratus.

The Palmar Cutaneous branches of the median and ulnar nerves.

These small nerves should be looked for just above the wrist, while removing the superficial fascia from the forearm; the median branch is in the middle and the ulnar along the inside of the wrist.

Flexor Longus Pollicis. Fig. 67.

Origin.-From the anterior surface of the radius between the oblique line above and the pronator quadratus below, also (often) by a slender head from the inner side of the coronoid process of the ulna, and from the outer part of the interosseous membrane.

Insertion.-Into the palmar surface of the base of the last phalanx of the thumb. 


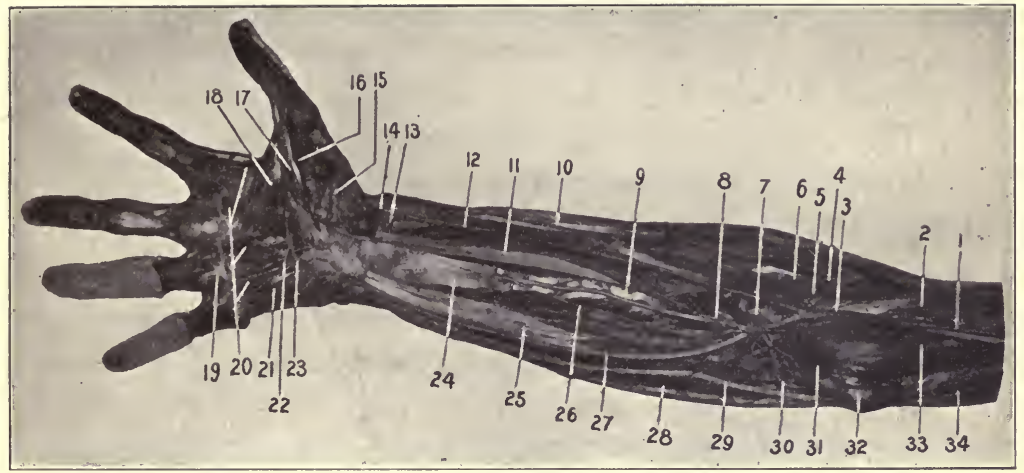

Fig. 67. Dissection of Forearm and Hand.- - , The median nerve crossing the brachial artery. 2, Brachialis anticus muscle. 3, Brachial artery. 4, Posterior interosseous nerve. Its branching from the musculospiral is seen also. 5, Radial recurrent artery. 6, Supinator brevis muscle. 7, Bicipital tuberosity of radius. 8, Posterior interosseous artery. 9, Anterior interosseous artery. Io, Radial nerve. If, Flexor longus pollicis. I2, Radial artery. 13, Superficial volar artery. 14, Tendon of the extensor ossis metacarpi pollicis muscle. 15, Opponens pollicis. 16, End of the tendon of the outer head of the flexor brevis pollicis. I7, Inner head of the flexor brevis pollicis (the adductor pollicis obliquus). 18, The adductor (transversus) pollicis. I9, The deep transverse ligament of the fingers. 20 , The interossei muscles. 21, Opponens minimi digiti. 22, Deep palmar arch. 23, Deep palmar nerve. 24, Pronator quadratus. 25, Flexor profundus digitorum. 26, Anterior interosseous nerve. 27, Ulnar artery. 28, Flexor carpi ulnaris. 29, Ulnar nerve. 30, Posterior ulnar recurrent artery. 3r, Anterior ulnar recurrent artery anastomosing with the anastomotica magna. 32, Internal condyle. 33, Anastomotica magna artery. 34, Inferior profunda artery. 
Nerve Supply.-From the median by the anterior interosseous (eighth cervical and first dorsal nerves).

Action.-To flex the thumb, then the wrist.

Flexor Profundus Digitorum. Fig. 67.

Origin.-From the anterior and inner surfaces of the ulna above the pronator quadratus, from the inner and upper two-thirds of the interosseous membrane, from the posterior ridge of the ulna in common with the origin of the flexor and extensor carpi ulnaris muscles.

Insertion.-Into the front of the bases of the last phalanges of the four fingers.

Nerve Supply.-Ulnar to the inner portion, median (anterior interosseous) to the outer portion. The filaments come from the eighth cervical and first dorsal nerves.

Action.-Flexor of fingers, wrist (all the joints between the lower end of the radius and the last set of interphalangeal articulations).

\section{DISSECTION.}

Incisions.-(I) Continue the median incision down the palm and along the front of the middle finger. (2) Make a transverse cut across the palm near the web of the fingers. (3) Incise the skin down the front of each finger. (4) Carry an incision from the front of the wrist along the palmar surface of the thumb.

Reflect the integument from the palm, thumb, and two or more fingers.

Be very careful not to injure the palmar fascia and the digital (cutaneous) vessels and nerves, which lie close under the integument.

The palmaris brevis muscle, the palmar cutaneous branches of the median and ulnar nerves, the palmar fascia, the digital and collateral digital nerves and arteries are to be carefully cleaned.

Palmaris Brevis. Fig. 65.

Origin.-From the front of the palmar fascia and anterior annular ligament.

Insertion.-Into the skin on the ulnar border of the palm. 
Nerve Supply.-Ulnar, from first thoracic nerve.

Action.-To draw the skin toward the middle of the palm.

The palmar cutaneous nerves have already been dissected; their distribution is seen in removing the integument. The ulnar branch supplies the inner, the median the middle portion of the skin of the palm.

The Palmar Fascia. Fig. 65.

This is the deep fascia covering the muscles (and vessels) of the palm. The outer and inner thirds resemble the deep fascia in other parts of the body in being thin and forming a covering to the muscles. The middle third is so strengthened by additional transverse and vertical fibres that it becomes a very dense fascia. It is narrow above, where it is attached to the annular ligament and receives the fibres from the palmaris longus muscle, spreads out fan-shaped below, where it divides into four fasciculi which pass to the fibrous structures about the metacarpophalangeal articulations and lateral margins of the bases of the first phalanges for their ultimate attachments.

These fasciculi are bound together by cross fibres that near the fingers exist as a separate band and are termed the superficial transverse ligament of the palm. Between these fasciculi the digital nerves and vessels are passing to the fingers, and the lumbrical and interossei muscles at a deeper level to their insertions.

Each fasciculus just over the finger splits just before its insertion to form a passageway for the tendons of the long flexor muscles to pass on to the fingers. Distally each fasciculus is continuous with the digital sheaths of the flexor tendons.

There is another fasciculus from the outer side of the 
palmar fascia to the thumb, which, though thin and not so easily demonstrated as the four internal ones, still can be shown to have a similar arrangement to them, and to be attached to the ligaments and fibrous structures about the metacarpophalangeal articulation. Between the divided fasciculus the tendon of the long flexor passes. The palmaris brevis muscle lies superficial to the inner portion of the inner or hypothenar fascia.

At the extreme web of the fingers exists a more or less distinct band of transverse fibres (fibres of Gerdy), which serve to bind together the fingers and strengthen the hand.

The digital vessels will be found between the fasciculi of the palmar fascia, and their position with reference to the bones of the fingers is to be noted.

\section{DISSECTION.}

Remove the lateral portions of the palmar fascia, with the inner the palmaris brevis muscle, and the central portion in the following way: Draw downward on the tendon of the palmaris longus muscle and cut it and the fascia carefully away from the annular ligament. When the lower border of the annular ligament is passed, raise all of the central portion of the palmar fascia and separate it from the vessels and nerves beneath. When the attachments of the fasciculi are reached, notice how they split for the transmission of the flexor tendons, while the lumbricales and the digital vessels and nerves occupy the space between the fasciculi themselves. Then divide the fasciculi and remove the fascia entirely.

Trace the superficial palmar arch, digital vessels, and nerves. Clean the sheath of one or more of the long flexor tendons of the fingers. Notice how the sheath is reinforced by oblique and transverse cross-bands of fibres, then open it.

Fxamine the attachments of the anterior annular ligament.

The muscles of the thumb and little finger are to be divested of their connective-tissue covering.

The Anterior Annular Ligament of the Wrist. Fig. 66.

This is a dense band of fibres reaching from the base of the thumb (scaphoid and trapezium) across the front of the 
wrist to the base of the little finger muscles (pisiform and hook of the unciform). Above it is continuous with the deep layer of the forearm fascia, tendon of the palmaris longus muscle, and thin fascia intervening between the superficial and deeper layer of flexor muscles of the forearm. Below the palmar fascia passes off from its lower border and anterior surface.

Behind the ligament the tendons of the long flexors of the fingers and thumb and the median nerve (and artery if present) pass into the hand. Over the ligament, yet contained in compartments of the deep fascia, are found the tendon of the ulnar flexor, ulnar vessels and nerve, the tendon of the palmaris longus and radial flexor of the carpus, then the radial vessels, and the tendons of the extensor muscles of the thumb. This order is from within outward.

Sheaths of the tendons of the long flexors of the fingers. These are formed of fibrous tissue passing from the sides of the fingers over the front of the tendons. In front of the joints the sheaths are very thin, but over the first and second phalanges they are reinforced by transverse fibres (vaginal ligaments). The sheaths are continuous above with the lower margins of the divided fasciculi of the palmar fascia. Internally they are lined with synovial membrane.

\section{The Superficial Palmar Arch. Fig. 66.}

This arterial arch is formed by the ulnar artery curving downward from the inside to the outside of the hand, where an anastomosis is established with the radialis indicis, princeps pollicis, superficialis volæ, separately; or with any two or all of them. It is sometimes formed by the median artery, see page 297. The position of the superficial arch is indicated by a line drawn across the palm on a level with the base of the extended thumb. 
Branches of the Ulnar in the Hand.

(I) The deep palmar or communicating branch, is given off the posterior surface of the ulnar, passes deeply into the palm between the abductor and flexor brevis minimi digiti muscles, and ends by inosculating with the radial to form the deep arch. See page 3 IO.

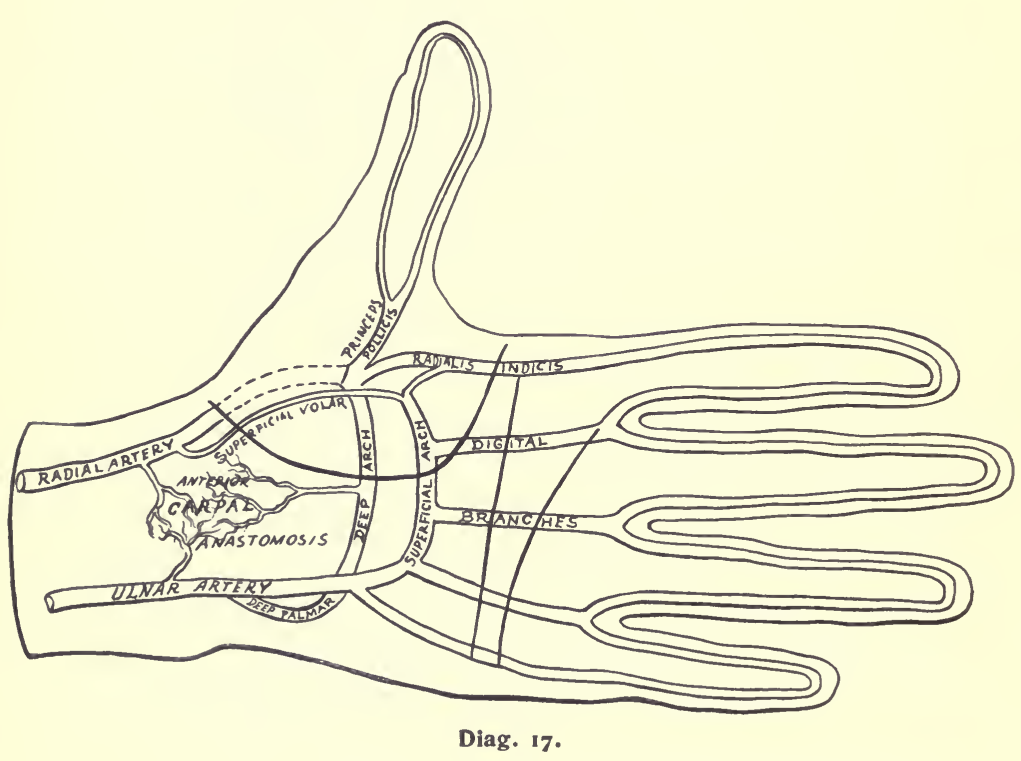

(2) Four digital arteries. These arise from the lower border of the superficial arch and are distributed to the fingers. The first crosses the muscles of the little finger to its inner side and then runs along the inner side of this finger.

The second descends in the interval between the fourth and fifth metacarpal bones to the web of the finger, where it divides into two collateral digital arteries, which pass to 
the adjacent sides of the ring and little fingers. The third takes a similar course and distribution to the middle and ring fingers. The fourth is distributed to the adjoining sides of the index and middle fingers in the same manner.

These digital arteries lie between the fasciculi of the palmar fascia, and long flexor tendons of the fingers, and upon the lumbrical muscles and digital nerves, and are covered by the palmar fascia.

Just before they bifurcate they are joined by the interosseous branches of the deep arch and the perforating from the dorsal interosseous arteries. The remaining side of the index finger is supplied by the radialis indicis, and the thumb by the princeps pollicis, both from the radial artery. See page 3 IO.

(3) The muscular and cutaneous branches from the superficial arch are few and small.

The Digital Nerves. Fig. 66.

The median nerve supplies two branches to the thumb, one on either side; a third to the outer side of the index finger; a fourth, to between the index and middle; and a fifth, between middle and ring fingers; each of which subdivide to supply the contiguous sides of these fingers.

The third and fourth nerves send a fine branch to the two outer lumbrical muscles, the fifth receives a communicating branch from the ulnar nerve. From the ulnar nerve the rest of the fingers are supplied, the first digital branch being given off to the inner side of the little finger, and the second to between the little and ring fingers, which then divides into collateral branches to the adjacent surfaces of these two fingers. From the outer side of the second branch is given a small filament to join the median. See above. From the median nerve just below the annular 
ligament will be found the muscular branches to the thumb muscles. From the ulnar there passes into the palm with the deep communicating artery the deep palmar branch of the ulnar nerve. It also sends branches to the palmaris brevis and little finger muscles.

\section{Superficial Layer of Palmar Muscles.}

Abductor Pollicis. Fig. 66.

Origin.-From the tuberosity of scaphoid, ridge of trapezium, and fibres of palmar fascia, annular ligament, and tendon of the extensor ossis metacarpi pollicis.

Insertion.-Into the anterior side of the base of the first phalanx of the thumb and the aponeurosis of the extensor longus pollicis.

Nerve Supply.-Median. (Filaments from the sixth cervical nerve.)

Action.-To abduct, flex thumb, and (through its attachment to the thumb extensor) it will slightly extend the iast phalanx of the thumb. (See action of lumbricales and interossei.)

Outer Head of the Flexor Brevis Pollicis. (For the inner see page 308.) Fig. 66.

Origin.-From the annular ligament and ridge of trapezium.

Insertion.-The outer side of the base of the first phalanx of the thumb.

Nerve Supply.-From the median (filaments from the sixth cervical).

Action.-Flexion of the carpometacarpal and metacarpophalangeal articulations of the thumb. Acts with abductor pollicis. For deeper muscles of thumb see page 308 .

Abductor Minimi Digiti. Fig. 66.

Origin.-From the pisiform bone. 
Insertion. - The inner side of the base of the first phalanx of the little finger, and by an extension to the extensor tendon behind.

Nerve Supply.-The ulnar, by filaments from the eighth cervical.

Action.-Abduction and flexion of the metacarpophalangeal joint, with extension of the last two phalanges of the little finger. (See action of lumbricales and interossei.)

Flexor Brevis Minimi Digiti. Fig. 66.

Origin.-From the annular ligament and hook of the unciform bone.

Insertion.-Into the inner side of the base of the first phalanx of the little finger.

Nerve Supply._Ulnar. Same as for the abductor.

Action.-Flexor of first joint of little finger.

NotE.-Between the abductor and flexor muscles pass the deep palmar communicating artery and nerve.

\section{The Lumbricales.}

Four in number.

Origin.-Begin at the lower border of the annular ligament from the anterior surface and radial border of all the tendons of the flexor profundus digitorum. In addition, the two inner muscles are attached to the ulnar sides of the adjacent tendons.

Insertion.-Into the radial sides of the aponeurosis of the extensor communis digitorum muscle opposite the first phalanx.

Nerve Supply.-The two outer by the median the two inner from the deep branch of the ulnar.

Action.-Flexion, abduction (from median line of the body) of the first set of phalanges of the fingers ; extension 
of the last two sets of phalanges through their connections with the common extensor aponeurosis.

\section{DISSECTION.}

Divide the abductor pollicis and reflect its portions; cut through the annular ligament, note the relations of the tendons of the long flexor muscles and the median nerve, also the extension up under the ligament of the synovial sac of these tendons.

Divide the ulnar artery and nerve just below their deep palmar branches; sever the connections of the superficial arch at the base of the thumb and turn the digital nerves and arteries and the tendons of the sublimis and profundus digitorum, with the lumbricales, below the web of the fingers.

Clean the rest of the thumb and little. finger muscles, the deep palmar arch and its branches.

The Inner Head of the Flexor Brevis Pollicis. (Adductor Obliquus.) Fig. 67.

Origin.-From the sheath of radial flexor of carpus, os magnum, and bases of second and third metacarpal bones.

Insertion.- - Into the inner side of the base of the first phalanx of the thumb with the adductor pollicis ; and by a separate fasciculus into the outer side of the base of the same bone with the outer head of the flexor brevis pollicis.

Norve Supply.-By the deep branch of the ulnar from the eighth cervical nerve.

Action.-To adduct and flex the carpometacarpal and metacarpophalangeal articulations acting with the adductor pollicis muscle.

Adductor Pollicis. (Adductor Transversus.) Fig. 67. Origin.-From the lower two-thirds of the front of the third metacarpal bone.

Insertion.-Into the inner side of the base of the first phalanx of the thumb, with the inner head of the flexor brevis muscle, and into the inner portion of the aponeurosis of the extensor longus pollicis. 
Nerve Supply.-The ulnar, through the deep branch ; the filaments coming from the eighth cervical nerve.

Action.-To adduct and flex the carpometacarpal joint, to flex this and the metacarpophalangeal joints. By its attachment to the extensor aponeurosis it will extend the last phalanx of the thumb.

Opponens Pollicis. Fig. 67 .

Origin.-From the annular ligament and the ridge of the trapezium.

Insertion.-Into the front of the metacarpal bone of the thumb.

Nerve Supply.-The median from the sixth cervical nerve. Action.-To flex and adduct the metacarpal bone of the thumb.

Opponens Minimi Digiti. Fig. 67.

Origin.-From the annular ligament and the hook of the unciform bone.

Insertion.-Into the entire length of the inner border of the fifth metacarpal bone.

Nerve Supply.-The ulnar nerve from the eighth cervical. Action.-Flexion and adduction (slight) of the fifth metacarpal bone.

\section{Palmar Interossei. (Three.)}

Origin.-From the side of the metacarpal bones of the index, ring, and little fingers. The first arises from the inner, the second and third from the outer side of the respective metacarpal bones.

Insertion.-Into the base of the first phalanges of the corresponding fingers, and by an extension into the aponeurosis of the extensor tendons. The first being inserted on the inner, the second and third into the outer sides of their respective fingers. 
Nerve Supply.-From the ulnar, by its deep palmar branch. The filaments arising from the eighth cervical nerve.

\section{Action.-See Dorsal interossei.}

The Deep Palmar Arch. Diag. I 8. Fig. 67.

After the radial artery has gained the front of the hand by coming through between the first and second metacarpal bones, it turns inward between the adductor and inner head of the flexor brevis pollicis, and crosses the bases of the second, third, and fourth metacarpal bones and interossei muscles, to form the deep arch by anastomosing with the deep communicating artery from the ulnar.

The line indicating the deep arch is half an inch nearer the wrist-joint than the superficial.

Branches of the Deep Arch. (Radial in the hand.)

(a) Princeps pollicis, is a branch from the radial just as it enters the palm. It passes outward and downward between the adductor pollicis and the abductor indicis muscles. Over the metacarpophalangeal joint it divides into two branches, one for each side of the thumb. Radialis indicis, issues between the adductor pollicis and abductor indicis muscles, descends along the outer anterior border of the index finger to its tip. (c) Palmar interosseous arteries, three in number; descend between the index and middle, middle and ring, and ring and little fingers, along the anterior surface of the interossei muscles, and terminate by joining the digital branches from the superficial arch. See page 305. They supply the adjoining muscles. (d) The recurrent, are two or more small twigs which pass upward to the front of the carpus and form an anastomosis with the anterior carpal branches of the radial, ulnar, and anterior interosseous arteries. (e) The perforat- 
ing, also usually three in number, pass to the back of the hand through the second, third, and fourth interosseous spaces, to end in the dorsal anastomoses.

The Tendons of the Long Flexor Muscles of the Fingers.

After opening the sheath of one or two fingers and having seen its formation, notice how the long flexor tendons are attached to the phalanges. It will be seen that the flexor sublimis tendon lies anterior to the profundus until the middle of the first phalanx is reached, where it divides into two parts, which pass around and reunite behind the deep tendon, over the base of the second phalanx. The tendons again divide and pass to their insertion into the sides of the second phalanx.

The tendons within their sheaths are surrounded by synovial membrane. These synovial sacs reach from the insertion of the long flexor tendons to the heads of the metacarpal bones. The synovial sheaths for the index, middle, and ring fingers are distinct from the great palmar synovial membrane. That for the little finger reaches up to it and may connect with it. See Diag. I 8.

The great palmar synovial membrane envelopes the tendons of the flexor sublimis and profundus digitorum from the middle of the palm to an inch above the annular ligament of the wrist. This is distinct from the synovial membranes for the fingers, excepting that the sheath for the little finger may open into it.

The flexor longus pollicis is surrounded by a similar synovial sheath that reaches from the insertion of the tendon to a like distance above the annular ligament. This sac lies in contact with, and often communicates with, the above sheath for the flexors of the fingers. 
Hence a suppuration starting in the little finger may point in the region of the thumb or extend above the wrist. Inflammations of the sheaths of the index, middle, and ring fingers are limited to those fingers and do not as a rule spread into the palm.

The foregoing completes the dissection of the anterior part of the upper extremity, excepting the study of the formation of the brachial plexus, which was purposely omitted until the neck had been dissected. Now, working

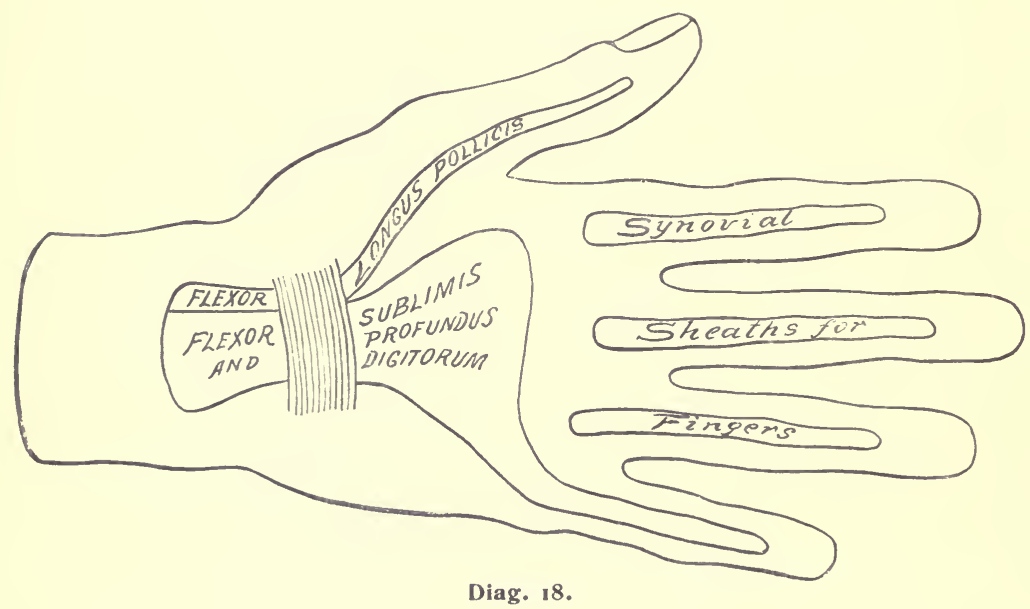

with the one dissecting the neck, disarticulate the clavicle at its sternal end (having previously studied the ligaments of this joint), divide the subclavius muscle near its costal attachment, and carry the clavicle outward. This will give sufficient room without entirely removing the clavicle.

For the third portion of the Subclavian Artery see page I o9. The Brachial Plexus. Figs. 63, 64.

The brachial plexus is formed by the anterior branches of the fifth, sixth, seventh, and eighth cervical and the first 


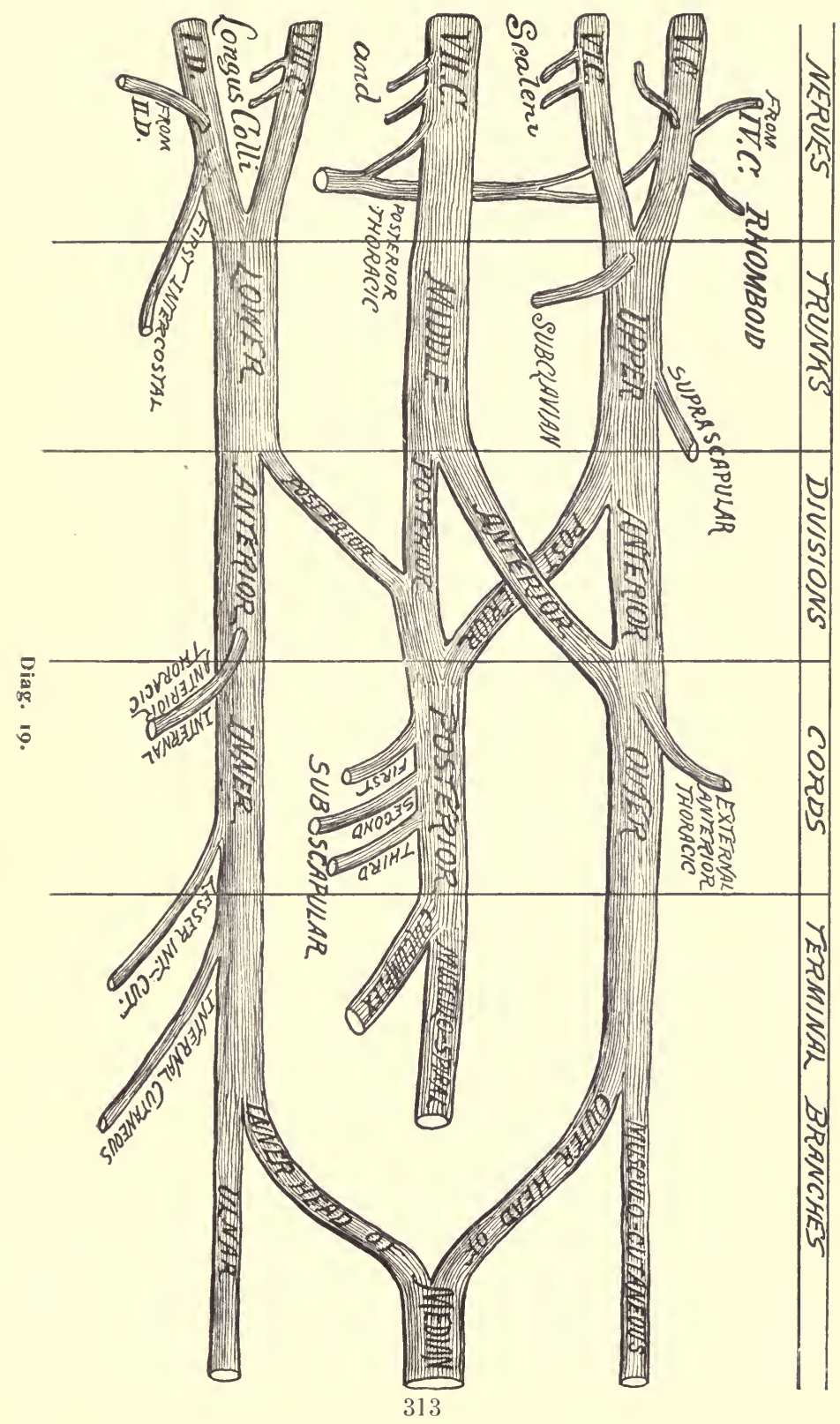


dorsal nerve, with branches from the fourth cervical and the second dorsal nerves.

The cervical nerves emerge along the side of the neck between the anterior and middle scaleni muscles. The first dorsal passes over the upper border of the first rib to join the last cervical nerve.

The plexus is conveniently divided into stages for description :-

(I) The nerve roots. (2) The junction of the nerves to form trunks, the fifth and sixth nerves forming the upper, the seventh the middle, and the eighth and first dorsal the lower trunks. (3) The division of these trunks into anterior and posterior branches. The division is about equal in the upper and middle trunk, but in the lower the anterior division is much larger than the posterior. The union of these divisions to form cords. The anterior divisions of the upper and middle trunks forming the outer cord. The anterior division of the lower trunk continuing as the inner cord, and the junction of the posterior divisions of all three trunks forming the posterior cord. The first and second portions of the plexus lie in the neck above and to the outside of the subclavian artery. The third portion is behind the clavicle and the subclavius muscle and at the outside of the axillary artery. The fourth portion surrounds the axillary artery, the outer cord being to the outside, the inner cord to the inside, and the posterior cord behind, the axillary artery.

Branches of the Plexus.

(I) From the first portion of the plexus.

(a) From the fifth nerve, the rhomboid branch. (b) The communicating to the phrenic. (c) The first part of the posterior or long thoracic. (d) From the sixth and the seventh, branches to the scaleni, longus colli muscles, and 
to the posterior thoracic nerve. (e) The posterior or long thoracic nerve is formed in the substance of the scalenus medius by branches from the fifth, sixth, and seventh cervical nerves. It passes downward behind the plexus and the beginning of the axillary artery into the axillary space, where it is found coursing along the middle of the serratus magnus muscle, to which it is distributed.

(2) From the second portion of the plexus. (a) Nerve to the subclavius. This small nerve comes from the upper trunk just after its formation, descends across the brachial plexus and subclavian vessels to supply the subclavius muscle. (b) Suprascapular nerve. This arises from the upper trunk, passes outward and downward through the suprascapular foramen in the scapula (notch converted into a foramen by the transverse ligament), supplies the supraspinatus muscle, the shoulder-joint, continues around through the great scapular notch, and terminates in the infraspinatus muscle. All the preceding nerves arise from the plexus above the clavicle.

(3) From the fourth portion of the plexus. Below the clavicle. From the outer cord. (a) The external anterior thoracic nerve receives filaments from the fifth, sixth, and seventh cervical nerves, arises from the outer cord, turns inward across the axillary artery and vein, perforates the costocoracoid membrane, and supplies the pectoralis major. (b) The musculocutaneous nerve. This nerve is the direct continuation of the outer cord. It continues to the outer side of the third portion of the axillary artery and the beginning of the brachial, perforates the coracobrachialis muscle (supplying it), descends between the biceps and brachialis anticus (supplying both), and as a cutaneous nerve is distributed to the outer portion of the 
anterior surface of the forearm as low as the ball of the thumb. (c) The outer head of the median.

From the inner cord. (a) The internal anterior thoracic. It passes upward between the axillary artery and vein from the inner cord to the under surface of the pectoralis minor muscle. Some filaments pass through the minor and terminate in the major. The external and internal nerves form a slight interlacement with each other between the two pectoral muscles. (b) The lesser internal cutaneous descends to the floor of the axillary space and enters into a loose plexus with the intercostohumeral nerve. The nerve itself is distributed to the skin of the inner and back part of the arm over and above the olecranon process. (c) The internal cutaneous nerve, another branch from the internal cord, passes through the axillary space, along the inner side of the arm above the elbow, divides into two branches, the anterior, which supplies the skin of the inner half of the front of the forearm as low as the wrist, and the posterior branch, which takes a similar supply upon the back of the inside of the forearm. (d) The ulnar nerve. This continues the direct course of the inner cord, descends along the inside of the arm with the inferior profunda artery, behind the internal condyle of the humerus with the recurrent branch of the ulnar, between the two heads of the flexor carpi ulnaris muscle, then between the flexor sublimis and profundus digitorum muscles; at the middle and upper thirds of the forearm joins the ulnar artery at its inner side, keeps this position with reference to the artery into the hand; here it divides into its terminal digital branches.

Branches of the Ulnar Nerie.-In the arm (behind the condyle) articular to the elbow-joint. In the forearm, 
muscular to the flexor carpi ulnaris and flexor profundus digitorum (inner portion). In the hand, digital to the inner side of the little finger, the adjacent sides of the little and ring fingers; communicating to the inner digital branch of the median; muscular to the palmaris brevis, flexor, abductor, and opponens minimi digiti, to the two inner lumbricals, all the interossei, the adductor and inner head of the flexor brevis pollicis (adductor obliquus). (e) The inner head of the median.

The median nerve. The median nerve is formed at the outer side of the third portion of the axillary artery by a branch from the outer and inner cords, the branch from the inner cord crossing in front of the artery to get to its outer side. The nerve then continues down the front of the arm in the same sheath with the brachial artery, crossing either in front or behind the artery to attain its inner side at the elbow. From the elbow the nerve descends through the forearm, passing between the two heads of the pronator radii teres, then between the flexor sublimis and the flexor profundus digitorum muscles. At the wrist the median traverses the central compartment of the annular ligament and enters the palm, where it breaks up into its terminal branches. At the elbow, the median gives off an articular branch to the joint and muscular to the superficial layer of muscles; these muscles are the pronator radii teres, flexor carpi radialis, palmaris longus, and flexor sublimis digitorum; then through its anterior interosseous branch it supplies the flexor longus pollicis, the outer side of the flexor profundus digitorum, and the pronator quadratus. In the hand, the median supplies the abductor, opponens, and outer head of the flexor brevis pollicis muscles, and the outer two lumbricals. The digital distribution is to the thumb, the index, middle, and outer side of the ring fingers. 
The digital branch to the ring finger receives a communicating branch from the ulnar. See Ulnar above.

From the posterior cord. (a) The subscapular nerves, three in number, arise from the posterior cord immediately after its formation. The first (upper) supplies the upper part of the subscapularis muscle. The second (the long) the latissimus dorsi muscle. The third (lower) the lower portion of the subscapularis and the teres major muscles. (b) The circumflex nerve. Turns backward with the posterior circumflex artery through the quadrilateral space formed by the humerus, long head of triceps, subscapularis (teres minor behind), and teres major muscles, to be distributed to the teres minor and deltoid muscles and the integument over the outer and posterior part of the shoulder. (c) The Musculospiral. Continues the posterior cord into the arm. It winds through the groove of the same name with the superior profunda artery to reach the interval at the outer side of the elbow between the supinator longus (brachioradialis) and the brachialis anticus, where it divides into its two terminal branches, the radial and posterior interosseous. The radial descends under cover of the supinator longus to the lower third of the wrist, turns backward to the posterior part of the hand, and supplies the integument on the back of the hand and two and one-half fingers (thumb, index, and outer side of the middle fingers). The posterior interosseous passes backward through the supinator brevis, and descends between the posterior muscles of the forearm as low as the back of the carpus, where it terminates in a gangliform enlargement. The musculospiral supplies the triceps, supinator longus, extensor carpi radialis longior, and anconeus muscles; and by its superior and inferior cutaneous nerves the integument of the lower part of the outer side of 
the arm, and the posterior, outer surface of the forearm nearly to the elbow. The posterior interosseous nerve supplies the extensor carpi radialis brevior, supinator brevis, extensor communis digitorum, extensor minimi digiti, extensor carpi ulnaris, extensor metacarpi pollicis, extensor indicis, extensor brevis, and longus pollicis muscles. The intercostohumeral nerve, after entering into a plexiform arrangement with the lesser internal cutaneous and cutaneous branch of the third intercostal nerve at the floor of the axilla, continues outward, downward, and sends some filaments posteriorly to supply the integument of the upper posterior part of the arm and axilla, over the latissimus dorsi muscle.

The nerves receive their filaments from the following sources:-

The suprascapular and subclavius- $5,6,7 . *$

The external anterior thoracic, 5, 6, 7 .

Musculocutaneous, 5, 6, 7 .

The internal anterior thoracic, 8, I.

Lesser internal cutaneous and internal cutaneous, 8, I. Ulnar, (7), 8, I.

Subscapular, circumflex, and musculospiral, 5, 6, 7, 8 .

The median, $5,6,7,8$, I.

\section{THE THORAX.}

For the landmarks see page 255 .

The dissection of the soft parts anterior to the ribs has been already performed, and their description given in the preceding pages.

Remove the sternum and costal cartilages by cutting through the latter as close to the ribs as possible (when the ribs are not needed, the division had

* Numerals refer to the number of the cervical and dorsal nerves forming the brachial plexus. 
better be made through them along the anterior axillary line, using a small saw or bone cutters for the purpose) until the seventh rib is reached; cut the muscles between it and the sixth up to the sternum, and saw through the latter just above the point where the seventh costal cartilage articulates with it.

Beginning above, raise the sternum, dividing the internal mammary arteries as close to it as possible, and remove the sternum and cartilages (and portions of ribs if they have been sawn through) from the thoracic contents by careful dissection, so as not to injure them.

\section{On the Sternum.}

Triangularis Sterni. (Continuation upward of the transversalis.)

Origin.-From the internal surface of the fifth, sixth, and seventh costal cartilages, the ensiform appendix, and the lateral margins of sternum for its lower third.

Insertion.-Into the inner surface and lower border of the second to the sixth cartilages, close to their costal junction.

Nerue Supply.-The upper intercostals.

Action.-Feeble muscle of expiration, by depressing the inner anterior portions of the ribs to which it is attached.

\section{DISSECTION.}

Remove the above muscle. Trace the internal mammary artery and its anterior and lateral branches as far as possible.

The Internal Mammary Artery. Figs. I 8, 68.

Arises from the under surface of the first portion of the subclavian artery, close to the inner border of the scalenus anticus muscle, and opposite the thyroid axis. See page I I3. It passes into the chest and descends behind the cartilages of the ribs about half an inch from the external margin of the sternum until the sixth space is reached, where it divides into the superior epigastric and the musculophrenic. 
The superior epigastric continues downward, enters the under surface of the rectus muscle (piercing its sheath), and finally terminates in anastomotic branches with the deep epigastric.

The musculophrenic passes downward and outward across the costal cartilages of the false ribs and along the origin of the diaphragm, which it perforates over the ninth rib, to the tenth or eleventh intercostal space. In its course it supplies the intercostal muscles and the diaphragm.

Relations. - In the neck, the internal mammary is crossed in front by the phrenic nerve, which passes from the outside to the inner side of the artery, and by the subclavian vein. The artery in the upper part of its course is in contact with the pleura, but in the lower is separated from it by the triangularis sterni muscle.

Branches.-A small artery to accompany the phrenic nerve, given off at the upper opening of the thorax.

The anterior intercostal arteries are a pair in each of the upper six intercostal spaces. These turn outward to anastomose with the aortic intercostals, supplying the surrounding parts.

The perforating. One for each of the six upper spaces. Pass forward and are distributed to the parts upon the front of the chest. The mammary gland receives blood from the second, third, and fourth.

Besides these there are small branches to the mediastinum, sternum, and pericardium.

The internal mammary artery is accompanied by two veins which empty into the innominate vein of that side.

Parts Within the Thorax.-If the pleuræ have been saved uninjured in removing the sternum and cartilages (and ribs), they will be seen to form a closed sac within which the lungs are contained. By inflating the lungs they will ex- 
pand and fill up the chest, their anterior borders will come into contact. The heart will be seen to be contained in a similar membranous sac, the pericardium, though it is much stronger than the pleuræ.

The space between the lungs (and pleuræ), sternum, and spine, and reaching from the root of the neck to the diaphragm, is the mediastinum.

\section{The Mediastinum. Figs. 68 to 72 .}

The mediastinal space is the central portion of the thoracic cavity between the pleural sacs laterally, the sternum and cartilages in front, the vertebral column behind, the diaphragm below, and the superior aperture of the thorax above.

The mediastinum is divided into several portions, as the superior, anterior, middle, and posterior.

The superior mediastinum lies behind the first piece of the sternum. In front are the sternal origins of the sternohyoid and sternothyroid muscles; behind, are the four upper dorsal vertebræ and the longus colli muscle. It contains the trachea, œsophagus, and thoracic duct; transverse aorta, innominate artery, the thoracic portions of the left carotid (common), and subclavian arteries ; the innominate veins, the upper portion of the superior vena cava; the pneumogastric, left recurrent laryngeal, the phrenic, sympathetic, and cardiac nerves ; lymphatic and thymus glands ; longus colli, sternohyoid, and sternothyroid muscles.

The anterior mediastinum is all that portion of the mediastinum anterior to the pericardium. It corresponds to the area of heart dullness. It contains a little areolar tissue and a few small lymphatic glands.

The middle mediastinum is the central portion of the space. It contains the heart, ascending aorta, pulmonary 
artery, and lower portion of the superior vena cava (all within the pericardium); the phrenic nerves and their accompanying arteries; the azygos vein (termination); the roots of the lungs and some bronchial lymphatic glands.

The posterior mediastinum is that portion of the space between the pleuræ laterally, the pericardium and roots of the lungs in front, and the spine (below the fourth dorsal vertebra) behind.

It contains the descending and thoracic aorta, the œsophagus, pneumogastric nerves, azygos veins, thoracic duct, and lymphatic glands, sympathetic cord, ganglia, and splanchnic nerves.

Structures which traverse the superior opening of the chest :-

Muscles.-Sternohyoid, sternothyroid, longus colli.

Arteries. - Innominate, left common carotid, left subclavian, internal mammary, superior intercostal.

Veins.-Left innominate, right internal jugular and subclavian, inferior thyroids, and superior intercostals.

Nerves.-Phrenics, pneumogastrics, cardiac, sympathetic cord, recurrent laryngeal.

Other Structures.-Thymus gland, thoracic duct, trachea, œsophagus, apices of lungs, pleuræ, cervical fascia.

\section{DISSECTION.}

If the pleuræ have been preserved whole, remove the presenting portion. Trace the phrenic nerves and save the arterial branch (very small) that accompanies them.

Carefully dissect away the areolar tissue in the superior and anterior mediastina, exposing the remains of the thymus gland, the great vessels, and pericardium.

\section{The Pleuræ.}

Each pleura is a closed sack containing a lung, over which it is reflected, passing into the fissures. This reflec- 
tion of the pleura over the lung is the visceral layer. The pleura also lines the interior of the thorax, diaphragm, and sides of pericardium, and extends upward into the root of the neck about an inch above the clavicle; this portion is the parietal pleura. These layers are continuous at the root of the lung, and between them is the pleural cavity (not a cavity unless the lungs have retracted into the back of the chest). From the root of the lungs a fold of the pleura descends to the diaphragm; this is the broad ligament of the lung.

The lower level of the pleural reflection from the diaphragm on to the chest wall has been given on page 264 .

\section{The Thymus Gland.}

The remains of this structure may be found lying below the left innominate vein and over the transverse arch of the aorta, reaching up into the root of the neck.

\section{The Phrenic Nerves. Figs. 18, 69, 70, 72.}

Each phrenic nerve is formed in the neck by branches from the anterior divisions of the third, fourth, and fifth cervical nerves. It descends obliquely downward and inward across the front of the scalenus anticus muscle, between the subclavian artery and vein, to enter the thorax, passing in front of the internal mammary artery.

It then descends to the diaphragm under cover of the pleura; on the right side passing along the outer side of the right innominate vein, superior vena cava, and the pericardium; on the left side, it is to the outside of the transverse aorta and behind the left innominate vein; on both sides they pass in front of the roots of the lungs, and when the diaphragm is reached pierce it and are distributed to the under side of the muscle. 


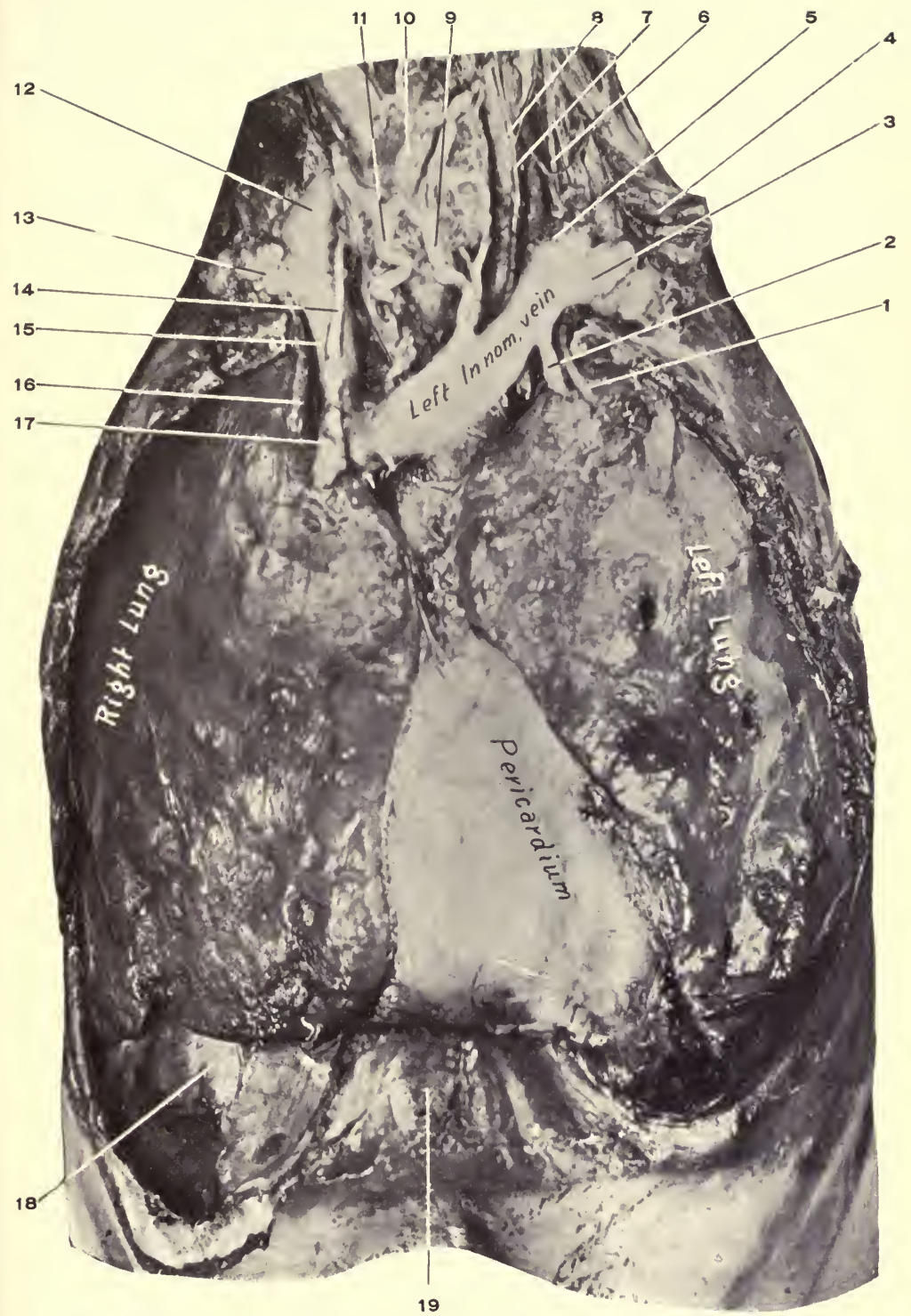

Fig. 68. Dissection OF THE ThORAX.-1, Left internal mammary artery. 2, Left internal mammary vein. 3, Left subclavian vein, resting upon first rib. 4 , Brachial plexus. 5, Left internal jugular. 6, Phrenic nerve, left. 7, Left pneumogastric nerve. 8, Left common carotid artery. 9 and 11, Inferior thyroid veins. 10, Trachea. 12, Right internal jugular vein. 13, Right subclavian vein, resting upon first rib. I4, Right inferior thyroid vein. 15, Right innominate vein. 16, Right internal mammary artery. 17, Right internal mammary vein. 18, Diaphragm. I9, Ensiform appendix. 
The Innominate Veins. Figs. 68, 69, 70.

Each is formed by the internal jugular and subclavian veins behind the sternoclavicular articulation. The right is only an inch long, and vertical in its course; the left,
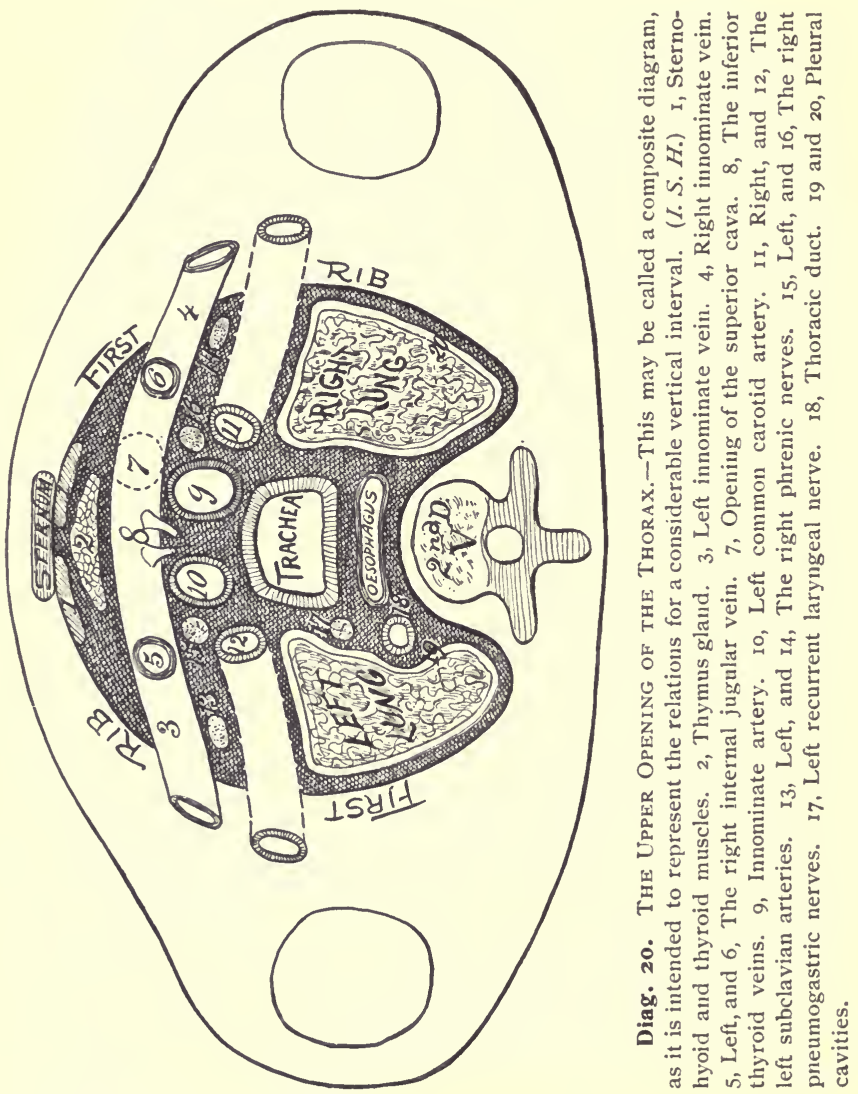

three inches long, and very oblique in its course; both veins unite in the first intercostal space of the right side close to the sternum and form the superior vena cava. 
Relations.-Right: In front, sternal origins of the sternohyoid and sternothyroid muscles, clavicle, first costal cartilage, and remains of the thymus gland; behind, pleura and lung; to the right, pleura, lung, and phrenic nerve (right); to the left, right subclavian artery and pneumogastric nerve, the innominate artery, and trachea.

The left: In front, sternohyoid and sternothyroid muscles, manubrium, clavicle, sternoclavicular articulation, remains of thymus gland; behind, innominate, left carotid and subclavian arteries, trachea, left phrenic nerve, left pneumogastric nerve; below, the transverse aorta; above, the cervical fascia and inferior thyroid veins.

Tributaries.-Both receive the internal jugular, subclavian, vertebral, deep cervical, and inferior thyroid veins above, and the internal mammary below. The left vein also receives the left superior intercostal, thymic, mediastinal, pericardiac veins, and the thoracic duct; the right, the right lymphatic duct. The ducts empty into the innominate veins at the junction of the internal jugular and subclavian.

The Superior Vena Cava. Fig. 70. Diags. 2 I, 22.

This is formed in the first intercostal space close to the right side of the sternum by the junction of the right and left innominate veins. Its course is downward to the right auricle of the heart, into which it opens.

Relations.-In front, first and second intercostal spaces and the second costal cartilage, remains of the thymus gland, pericardium, pleura, and right lung ; behind, azygos major, right bronchus, right pulmonary artery, and right superior pulmonary vein, the pericardium; to the right, phrenic nerve, lung, and pleura; to the left, ascending aorta, innominate artery. 
Tributaries. - The right and left innominate veins, azygos major, mediastinal and pericardial veins.

The arteria comes nervi phrenici is a very small artery from the internal mammary to the phrenic nerve just after the latter crosses the former. It accompanies the nerve to the diaphragm.

\section{DISSECTION.}

Make a vertical and a transverse incision in the pericardium.

Reflect the flaps; study the situation and relations of the heart and its great vessels.

The Pericardium. Figs. 68, 69.

The pericardium is a fibrous sack enclosing the heart and beginning of the great vessels. Below it is attached to the central tendon of the diaphragm, mostly at the left, of the median line; above it passes on to the great vessels and becomes continuous with the deep cervical fascia from the neck.

The fibrous pericardium is lined by a serous layer that is also reflected on to the heart and beginning of the great vessels. The portion of the serous membrane lining the sack is the parietal, that reflected over the vessels and heart is the visceral layer.

The serous layer extends upward on to the beginning of the great vessels for a distance of one or one and one-half inches from the base of the heart.

Relations.-In front, sternum and cartilages from third to the sixth inclusive, anterior intercostal muscles, triangularis sterni, to some extent by the lungs and pleuræ; laterally, pleuræ, lungs, phrenic nerves, and accompanying arteries; posteriorly, bronchi, œesophagus, pneumogastric nerves, descending aorta; below, the diaphragm, 


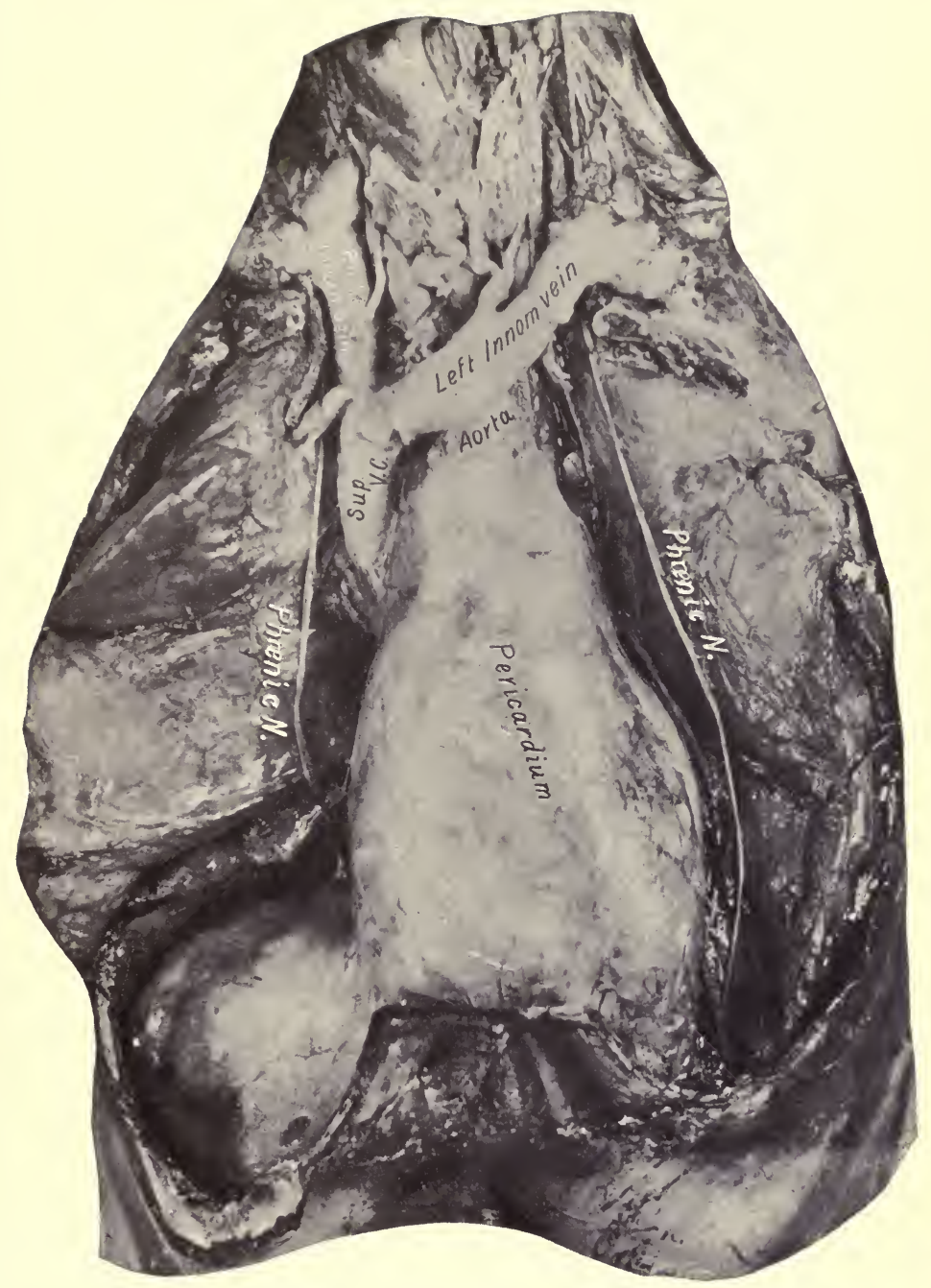

Fig. 69. DisSECTION OF THE THORAX. - The lungs are separated to show the pericardium, its extension upon the great vessels of the heart, and the course of the phrenic nerves. The structures are the same as in the preceding figure. 
to which it is attached. Above it is continuous with the deep cervical fascia on the great vessels.

Contents of the Pericardium.-Heart (its auricles, ventricles, arteries, veins, and nerves), ascending aorta, pulmonary artery, pulmonary veins, superior vena cava.

The Heart. Figs. 57, 58, 68 to 73 .

Is a hollow muscular cone, placed with its base upward, backward, and to the right, and its apex downward, forward, and to the left. For the surface relations consult description already given.

Its dimensions: Length, 5 inches; breadth, $3 \frac{1}{2}$ inches ; thickness, $21 / 2$ inches; weight, I I ounces in the male, 9 in the female.

The heart is attached by its base to the great vessels which proceed from it. Its apex is free and in life describes considerable movement.

It is divided into four cavities by two partitions, one between the ventricles, the interventricular, one between the auricles and ventricles, the auriculoventricular.

The location of the partitions is indicated on the outside of the heart by grooves, the auriculoventricular groove being deeper and better marked than the interventricular. The former is obliterated in front by the pulmonary artery. The heart (and vessels for their first one and a half inch) is covered by the visceral layer of the pericardium, and its cavities are lined by a similar smooth membrane-the endocardium-continuous with the lining of the blood vessels.

On opening the pericardium the greater part of the heart presenting is composed of the right ventricle and auricle; the smaller part, of the left ventricle and auricle. The ascending aorta, pulmonary artery, and superior vena cava are also in view. 


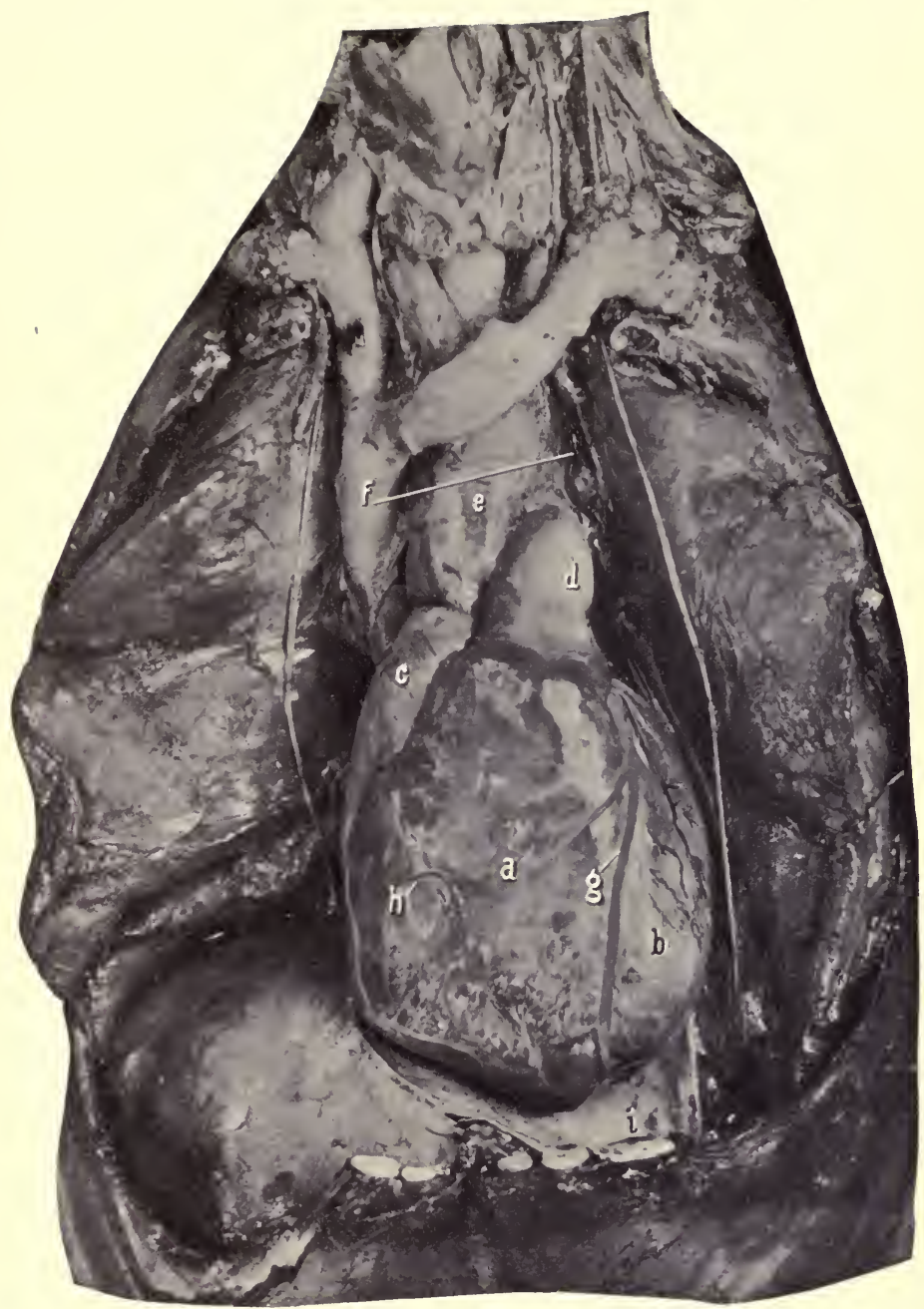

Fig. 7o. Dissection of Thorax.- $a$, Right ventricle. $b$, Left ventricle. $c$, Right auricle. $d$, Pulmonary artery. $e$, Ascending aorta. $f$, Superior vena cava. $g$, Left coronary artery and vein. $h$, Right marginal artery and vein; anterior branch of right coronary artery. $i$, Pericardinm; inner surface. Note the difference hetween the external and internal appearance of the pericardium. Also the notch at the apex of the heart where the ventricles meet. 
The dissection of the heart, see page 354 .

The Pulmonary Artery. Figs. 57, 58, 70 to 73. Diag. 22.

Conveys the venous blood from the right heart to the lungs. It is about two inches long, and is contained within the pericardium, and ends by dividing into two terminal branches, which pass through the pericardium.

It presents in the interval between the two auricles, curves upward, to the left, and backward beneath the aortic arch, to which it is attached by the obliterated ductus arteriosus (remains of the foetal communication between the pulmonary artery and the aorta), where it divides into its two terminal branches.

Relations.-At the beginning it is between the two auricles, and has the ascending aorta behind and to the right. The coronary arteries are on either side of it. It is covered by the pericardium. At its termination it lies beneath the aortic arch, to the left of the ascending aorta, and over the left auricle, and is covered by pericardium on the left.

(See page 352 for dissection.)

\section{The Ductus Arteriosus. Fig. 72.}

This is the fibrous cord in the adult which is found joining the under surface of the transverse aorta to the upper surface of the pulmonary artery. In the foetus it was relatively a large trunk and conducted the blood from the pulmonary artery into the aorta.

\section{The Right Pulmonary Artery.}

Is longer than the left, passes to the right lung, where it divides into three main branches to the three lobes.

Relations.-In front, ascending aorta, superior vena cava, phrenic nerve, and anterior pulmonary plexus of nerves; 


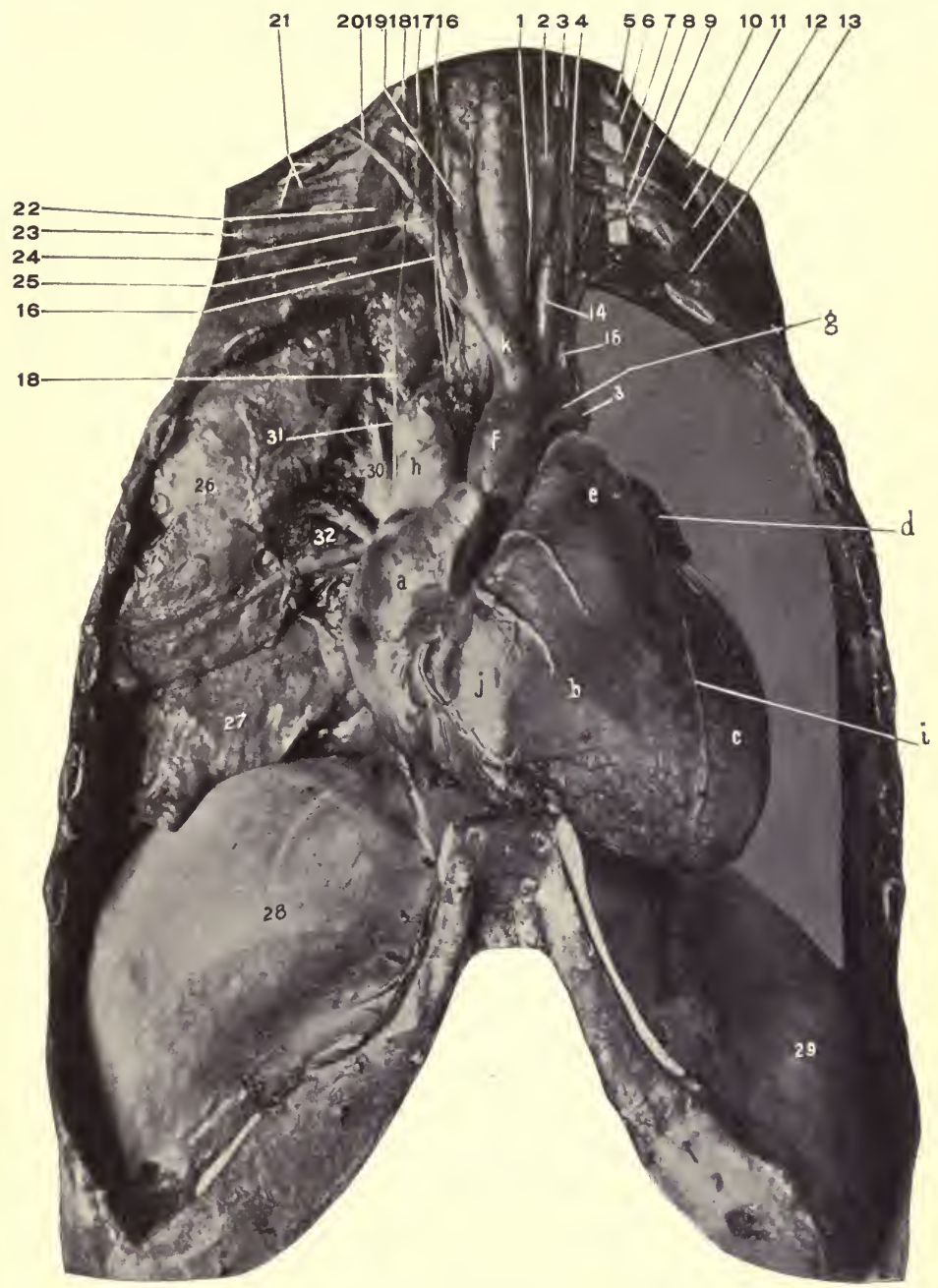

Fig. 7I. Dissection of Thorax.- $a$, Right auricle. $b$, Right ventricle. $c$, Left ventricle. $d$, Left auricle. $e$, Pulmonary artery. $f$, Ascending aorta. $g$, Transverse aorta. $h$, Superior vena cava. $i$, Left coronary artery. $j$, Right marginal artery from right coronary. $k$, Innominate artery. 1 , Left recurrent laryngeal nerve. The reference line crosses the trachea. 2, Esophagus. 3, Left pueumogastric nerve. 4, Vertebral artery. 5, 6, 7,8, and 9, Nerves going to form the brachial plexus. 10, i1, and 12, The outer, middle, and inner cords. 13, Scalenus medius muscle; also first rib. 14, Left common carotid artery. 15, Left subclavian artery. 16, 16, Right pneumogastric nerve. 17, The ascending cervical artery. 18, Right phrenic nerve. 19, Right common carotid 20, Transverse cervical artery. 21, Brachial plexus. 22, Scalenus anticus muscle. 23, Right axillary artery. 24, Right subclavian artery. 25, Internal mammary artery. 26, Upper lobe of right lung. 27, The lower lobe of the same. 28, 29, The diaphragm. 30, Pulmonary artery. 31, Bronchus. 32, Pulmonary veins. 
behind, right bronchus; above, transverse aorta; below, the left auricle.

In the root of the lung it is between the bronchus (above) and the pulmonary veins (below); behind is the azygos vein, pneumogastric nerve, and posterior pulmonary plexus.

\section{The Left Pulmonary Artery.}

Is shorter than the right, reaches to the left lung, and divides into two branches.

Relations.-In front, phrenic nerve, anterior pulmonary plexus, left pleura ; behind, descending aorta, left pneumogastric nerve, posterior pulmonary plexus; below (and in front), pulmonary veins, left bronchus.

The Aorta. (The description will be given here in full.

For dissection see page 352). Figs. 57, 58, 69 to 74 .

The outlet for the blood from the left ventricle.

In the subject is seen to be contained in the pericardium for its first two inches (or less). It presents to the right of and behind the pulmonary artery and right auricle. Its direction is upward, backward, and to the left, then downward along the spinal column to leave the thorax through the aortic opening. It is divided into the ascending, transverse, descending, and thoracic portions.

The Ascending Aorta. Diag. 22.

This is about two inches long. It begins at the left ventricle of the heart, passes upward, curving slightly to the right in its course; it ends where the artery turns to pass transversely into the left portion of the thorax. From its base on the right and left sides are given off the coronary arteries to the heart, the left coronary artery supplying the left ventricle mostly, and the right, the right ventricle. At the base of the aorta it is dilated into three 
pouches forming the sinuses of Valsalva, one in front and two posterior.
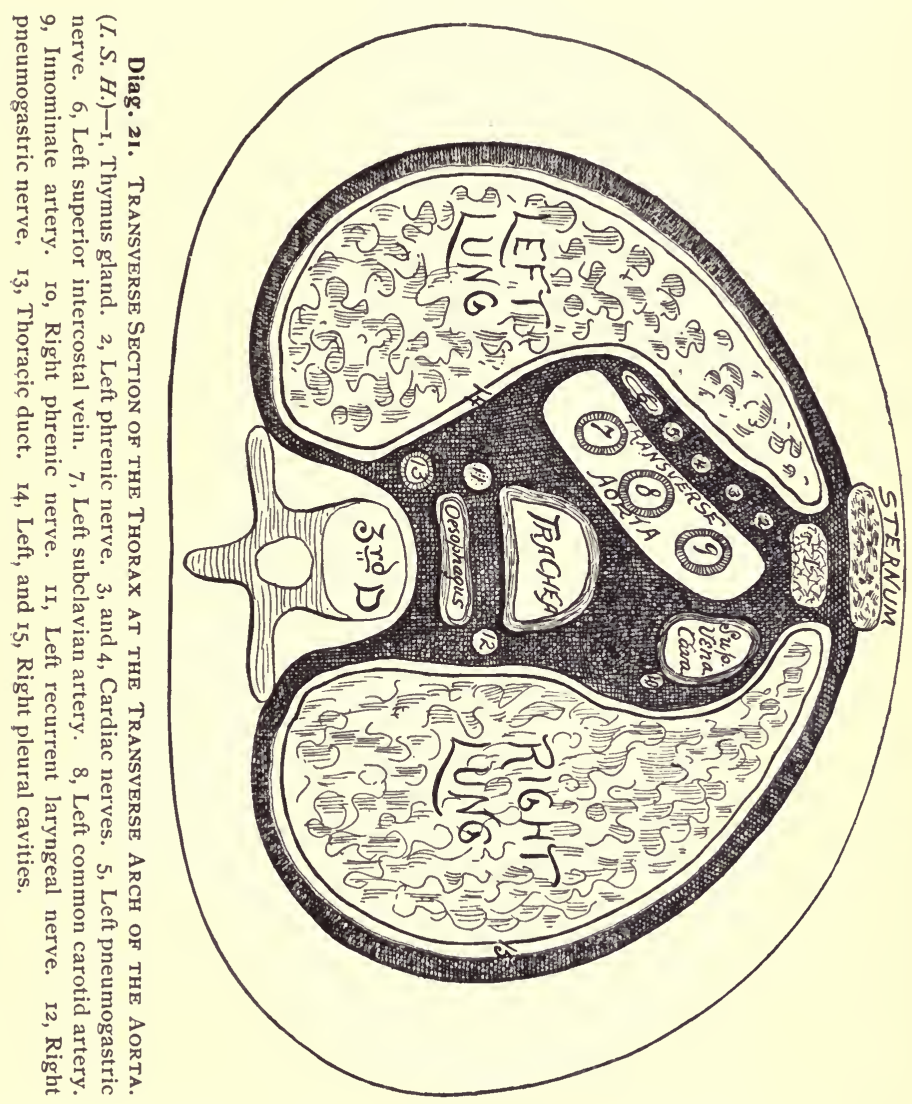

Relations. - In front is the pericardium (and the structures that overlie the pericardium in the middle line).

At its base the pulmonary artery: Behind, left auricle of heart, right pulmonary artery, right bronchus, anterior 
cardiac nerves; at the right, right auricle and superior vena cava; at the left, pulmonary artery and cardiac nerves.

The Transverse Aorta. Diag. 2 I.

This is nearly two inches long, also. Takes a course back-
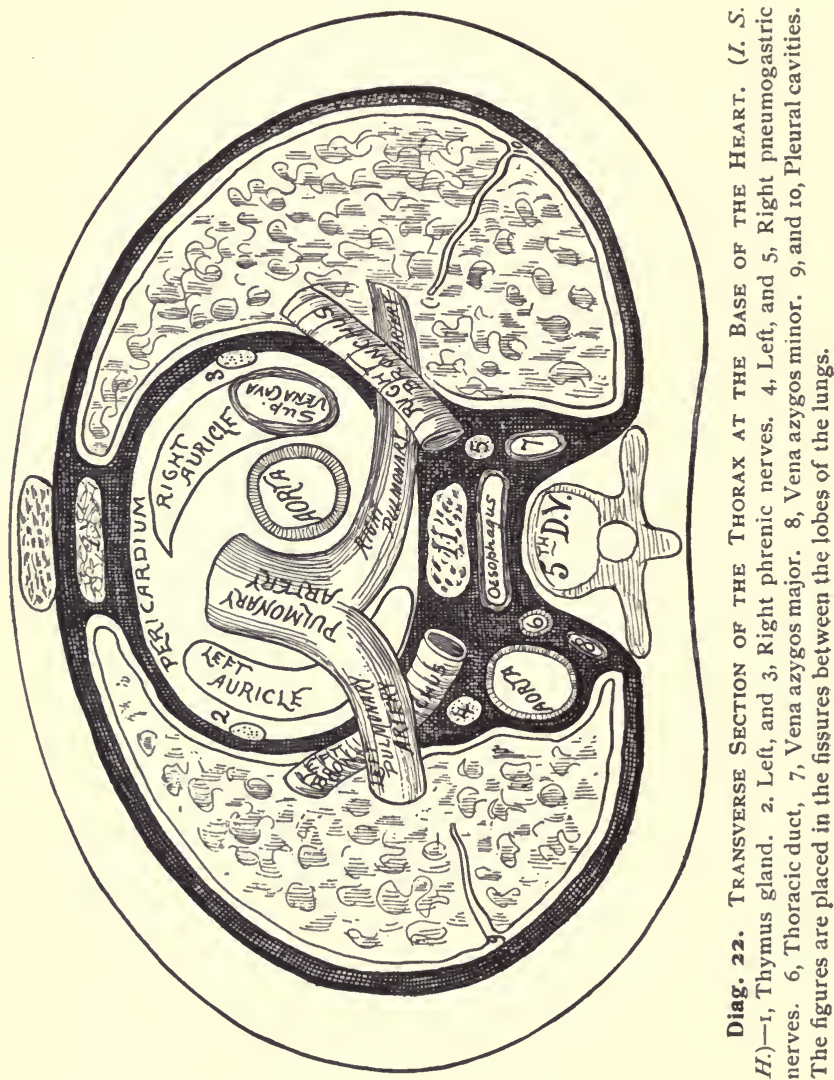

ward and to the left from the level of the second right cartilage (or the first intercostal space) to the body of the fourth (or third) dorsal vertebra on the left side. The highest por- 


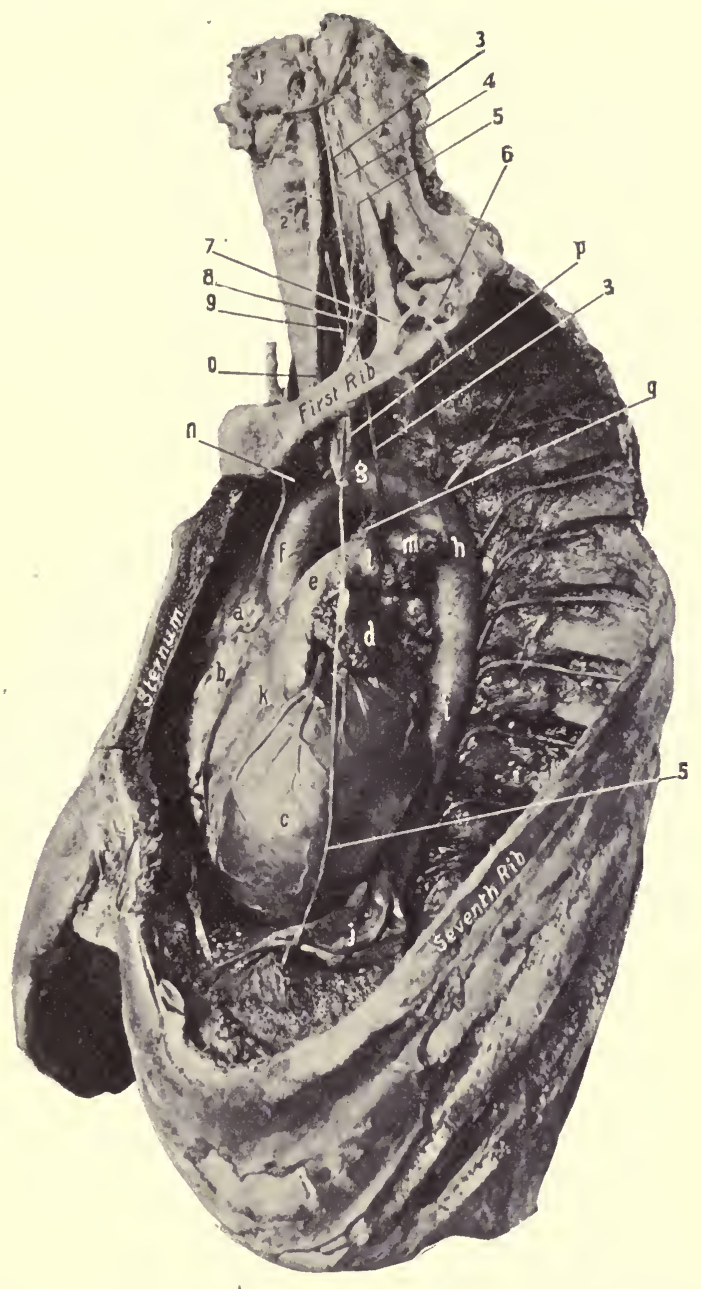

From the Second Prize Dissection at the University Medical College, for 1895 , bv P. D. Shultz.

Fig. 72. Dissection of the Thorax. $-a$, Right auricle. $b$, Right ventricle. $c$, Left ventricle. $d$, Left auricle. $e$, Pulmonary artery. $f$, Ascending aorta. $g$, Transverse aorta. $h$, Descending aorta. $i$, Thoracic aorta. From it the left intercostal arteries are shown. $j$, Pericardium. $k$, Left coronary artery. $l$, Left pulmonary artery. $m$, Left bronchus. $n$, Innominate artery. $o$, Left common carotid. p, Left subclavian artery. $q$, Ductus arteriosus. I, Thyroid cartilage. 2, Trachea. 3, 3. Left pneumogastric nerve. 4, Left sympathetic cord. 5, 5, Left phrenic nerve. 6, Left subclavian artery. 7, Scalenus anticus muscle. 8, Internal mammary artery, left. 9, Left recurrent laryngeal. 
tion of the under surface of the arch is opposite the articulation between the first and second portions of the sternum. From its upper surface it gives off the innominate, left common carotid, and subclavian arteries.

Relations.-In front, pleuræ and lungs (in inspiration), left phrenic and pneumogastric and cardiac nerves, and the left superior intercostal vein; behind, trachea, osophagus, thoracic duct, deep cardiac plexus, left recurrent laryngeal nerve; above, its three branches and the left innominate vein; below, left bronchus, bifurcation of pulmonary artery, left recurrent laryngeal nerve, remains of ductus arteriosus, some lymphatic glands.

\section{The Descending Aorta. Diag. 22.}

This continues the arterial vessel from the ending of the transverse aorta (see above) to the level of the lower border of the fifth dorsal vertebra, where it becomes the thoracic aorta.

Relations.-In front, left lung and pleura covering it; behind, left side of the (third), fourth, and fifth vertebræ; at the right, œsophagus, thoracic duct, and the above vertebræ; at the left, lung covered by its pleura.

The Thoracic Aorta. Diags. 22, 23. Figs. 72, 74.

Is the continuation of the aorta from its descending portion (see above) along the front of the dorsal vertebræ to the twelfth, where it leaves the thorax by passing through the aortic opening in the diaphragm into the abdomen. At its beginning it is a little to the left of the middle line, but at its ending is over the middle line. It is one of the constituents of the posterior mediastinum.

Relations.-In front, root of left lung, œsophagus, diaphragm; behind, the dorsal vertebræ from the fifth to the twelfth inclusive, vena azygos minor (at the seventh or 
eighth), superior intercostal vein (at the fifth or sixth vertebræ); at the left, left lung and pleura, the œsophagus; at the right, the thoracic duct. For relations of œsophagus to artery, see page 343 .

The Innominate Artery. Fig. 7 I. Diag. 20.

It arises from the transverse aorta near its beginning, passes upward and outward to behind the right sterno-

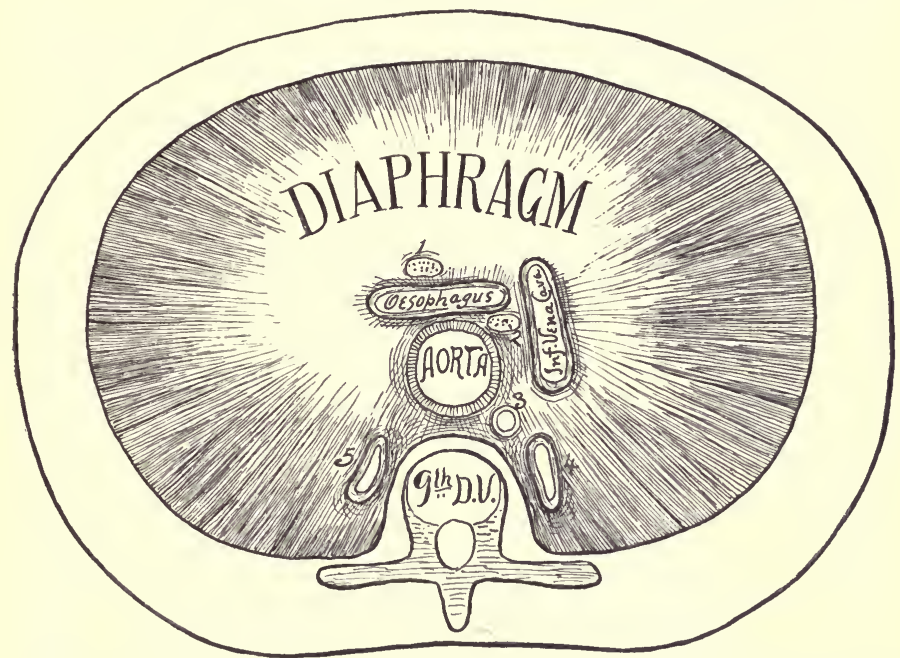

Diag. 23. Transverse Section of the Thorax just Above the Diaphragm. (I.S. H.) I, Left pneumogastric nerve. 2, Right pneumogastric nerve. 3, Thoracic duct. 4, Azygos major, and 5, Minor veins.

clavicular articulation, where it divides into the right common carotid and subclavian arteries. Its length is from one and one-half to two inches.

Relations.-In front, first piece of the sternum, origins of the right sternohyoid and sternothyroid muscles, right sternoclavicular joint, remains of thymus gland, left innominate and inferior thyroid veins, right cardiac nerves; 
behind, trachea, right lung, and pleura; at the right, right innominate vein, right pneumogastric nerve, right lung and pleura; at the left, left common carotid artery: at the ending of the artery, the trachea.

Thoracic portions of the left common carotid and subclavian arteries :-

The Left Common Carotid. Fig. 7 I. Diag. 20.

Arises from the middle of the upper surface of the transverse aorta, passes upward and outward into the neck. For rest of course see page IOI.

Relations (within thorax).-In front, manubrium, origins of the left sternohyoid and sternothyroid muscles, remains of thymus gland, left innominate vein; behind (and from below upward), trachea, œesophagus, thoracic duct, recurrent laryngeal nerve; at the right, innominate artery, trachea, inferior thyroid veins; at the left, left subclavian artery (behind), left pneumogastric nerve, left lung and pleura.

The Left Subclavian Artery. Figs. 70, 71. Diag. 20.

Is given off the transverse aorta at its terminus, extends almost vertically upward into the neck, passing behind the left sternoclavicular articulation.

Relations (within the thorax). In front : First left costal cartilage and adjacent portions of manubrium, left sternoclavicular joint, origins of the left sternohyoid and sternothyroid muscles, left lung and pleura, left innominate vein, left pneumogastric and cardiac nerves. Behind: Thoracic duct, longus colli muscle, vertebræ. At the right: Left common carotid (in front), trachea, left recurrent laryngeal nerve, œsophagus (behind).

For the continuation of the artery see page I08. 


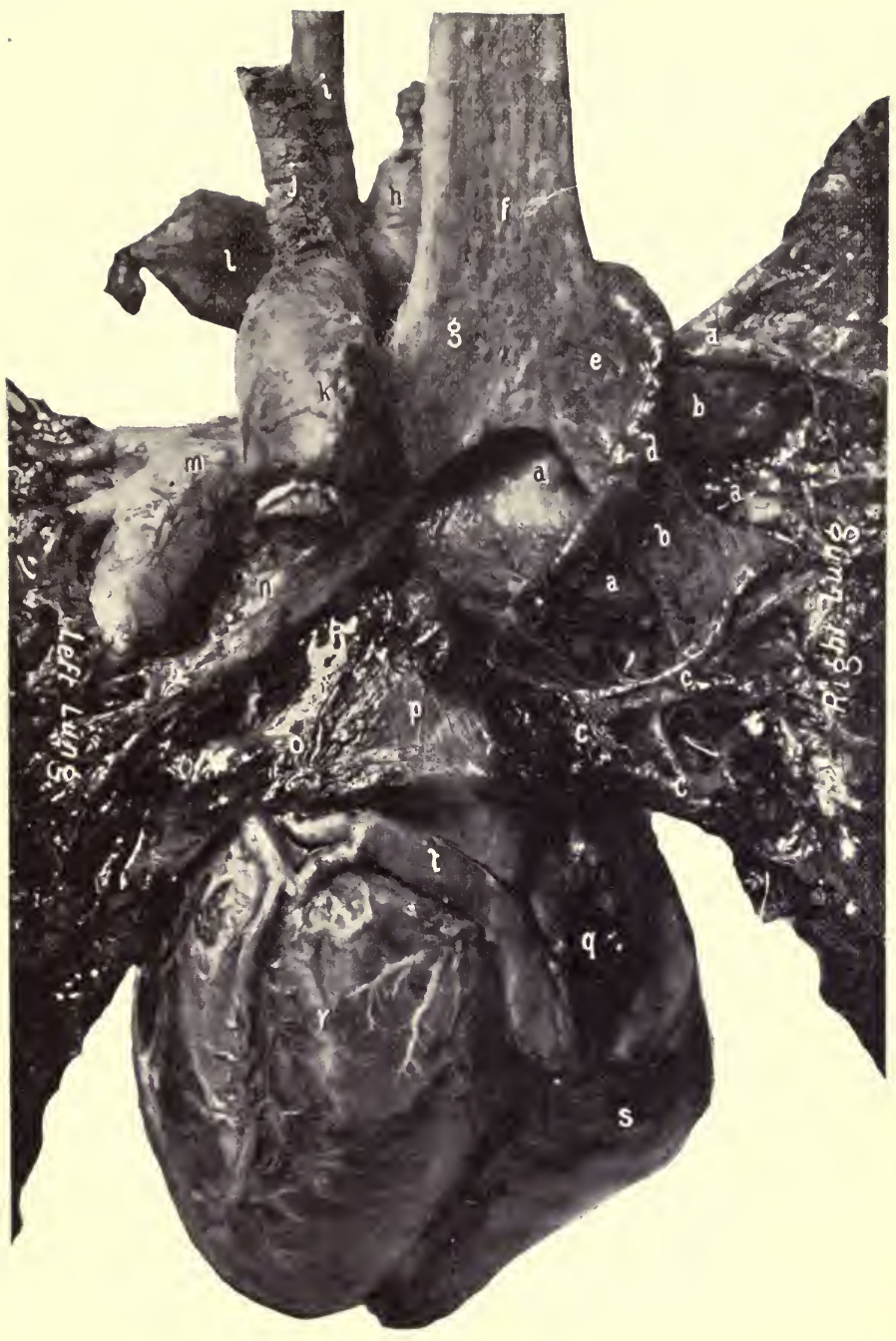

Fig. 73. Heart and Root of Lungs from Behind.- $a, a, a, a$, Right pulmonary artery and its branches. $b, b$, Upper and lower divisions of right bronchus. $c, c, c$, Right pulmonary veins. $d$, Vena azygos major. $e$, Right bronchus. $f$, Trachea. $g$, Left bronchus. $h$, Innominate artery. $i$, Left common carotid artery. $j$, Left subclavian artery. $k$, Descending aorta. l, Left innominate vein. $m$, Left pulmonary artery. $n$, Left bronchus. $o$, Left pulmonary vein. $p$, Posterior surface of left auricle. $q$, Orifice of inferior vena cava. $r$, Left ventricle. $s$, Right ventricle. $t$, Coronary sinus. 
The Pneumogastric Nerves. Figs. I 8, 7 1, 72.

Are found in the neck between the internal jugular vein and the common carotid artery, and behind both. See page 103 .

The right nerve comes forward between the vein and artery, crosses over the front of the subclavian artery and behind the right innominate vein (gives off the recurrent laryngeal nerve opposite the lower part of the artery), and descends backward and inward to the side of the osophagus and behind the root of the lung, where it forms the posterior pulmonary plexus. From the plexus it is continued by two branches along the posterior surface of the osophagus (forming the œsophageal plexus); finally these two cords unite, pass through the diaphragm, and are distributed to the posterior wall of the stomach.

The left pneumogastric at the base of the neck is between the left common carotid and subclavian arteries, descends behind the left innominate vein, over the front of the transverse aorta (at the lower border of which the left recurrent laryngeal nerve is given off), to the posterior part of the root of the left lung, where it forms a posterior pulmonary plexus. It then continues along the front of the œesophagus by two branches (forming an œesophageal plexus and communicating with the posterior plexus by branches), then these two branches unite and pass through the diaphragm to the anterior wall of the stomach.

The Right Recurrent Laryngeal Nerve. See Neck, page IO4.

\section{The Left Recurrent Laryngeal.}

Can be traced curving around the transverse aorta, external to the ductus arteriosus and above the pulmonary artery, to pass upward and inward behind the left common 
carotid, and ascend in the interval between the trachea and the œsophagus into the neck, $q$. $v$, page I04.

\section{DISSECTION.}

If the innominate veins have been tied off, proceed with the following (if they have not, then tie them off close to the superior vena cava) :-

Divide the branches of the aorta (or if they have been cut away in the neck, remove the attached portions with the aortic arch), divide the trachea at the opening of the chest, the phrenic, pneumogastric, and the recurrent (left) laryngeal nerves. Lift everything forward and cut the aorta across at the beginning of its downward curve. Separate the trachea from the œosophagus, divide the azygos major close to the superior cava, sever the attachments of the pericardium to the diaphragm, divide the inferior vena cava, and remove the heart, great vessels, adherent portions of nerves and veins, and the trachea and lungs from the thoracic cavity.

Place these parts into a common salt solution for later dissection.

Turn to the interior of the thorax.

The Esophagus. See page 1 24. Diags. 20 to 23.

Begins in front of the fifth cervical vertebra and behind the cricoid cartilage, nearly in the median line. It enters the upper opening of the chest a little to the left of the middle line and in front of the bodies of the vertebra, descends to the œesophageal opening in the diaphragm, and terminates in the stomach. In its course the thoracic duct intervenes between it and the bodies of the vertebræ above and the thoracic aorta below. Its length is from nine to eleven inches.

Relations.-In the neck: In front, trachea. Laterally, the lobes of the thyroid gland, the recurrent laryngeal nerves, common carotid and inferior thyroid arteries, thoracic duct (at the left). Behind, prevertebral fascia, longus colli muscle, lower cervical vertebræ (below the fifth).

In the thorax: In front, trachea, left common carotid and subclavian arteries, left bronchus, transverse aorta, pericardium, left pneumogastric nerve. Laterally, pneu- 
mogastric nerves, pleural sacs, and lungs. Aorta at left and vena azygos major at right. Behind, longus colli muscle, dorsal vertebræ, thoracic duct, third, fourth, and fifth right intercostal arteries, left lower azygos vein, aorta, right pneumogastric nerve.

DISSECTION.

Remove the œsophagus, tying a cord around its lower end as it passes through the diaphragm.

The Thoracic Duct. Diags. 20 to 23.

This begins in the abdomen as the duct of the receptaculum chyli in front of the first and second lumbar vertebræ and behind the abdominal aorta.

The duct passes into the thorax through the aortic opening at the right of the artery, and then along the front of the spine, between the thoracic aorta on the left and the vena azygos major on the right, until at the third dorsal vertebra it turns to the left behind the aortic arch and œsophagus. It passes upward at the left of the latter into the neck, arches over the left subclavian artery and the apex of the left lung to empty into the junction of the left jugular and subclavian as they are forming the left innominate vein.

At its opening into the vein there are two valves found. Other valves similar to those found in the veins are distributed throughout its length. It is about fifteen or eighteen inches long, and in diameter varies from one-fourth to one-sixth of an inch.

The thoracic duct receives the drainage from the lower extremities, abdominal organs (excepting the upper surface of the liver and abdominal walls), left lung, left side of the heart, left upper extremity, and left side of the head and neck. It empties all this collection into the beginning of the left innominate vein. 


\section{The Thoracic Sympathetic Nervous System. Fig. 74.}

This consists of a nerve cord which extends through the chest alongside of the dorsal vertebræ (continuous above with the cervical and below with the lumbar cord), and a series of enlargements or ganglia, twelve in number, which rest upon the heads of the ribs (excepting the last two, which lie upon the side of the body of the corresponding vertebræ).

The sympathetic branches are (external) to the intercostal nerves and (internal) to the thoracic viscera.

From the lower ganglia are given off the important splanchnic nerves, which, formed in the thorax, pass to the abdomen.

(I) The great splanchnic nerve is formed by filaments from the fifth to the ninth thoracic ganglia inclusive, passes through the crus of the diaphragm, and terminates in the semilunar ganglion of that side. (2) The lesser splanchnic nerve is composed of filaments from the eleventh and twelfth ganglia, takes the same course through the diaphragm as the great, and ends in the solar or renal plexus. (3) The smallest splanchnic nerve is a small branch from the last thoracic ganglia to the renal plexus.

The Branches of the Thoracic Aorta. (For the artery see page 338.) Figs. 72, 74.

(I) Pericardial, a few small arteries to the posterior surface of the pericardium. (2) Bronchial, three in number. The right usually rises from the first right (aortic) intercostal artery or from the front of the thoracic aorta by a trunk common to it and the left upper bronchial artery. It runs outward to the right lung on the back of the bronchus. The left bronchial are two in number, arising from the thoracic aorta one below the other, and both pass to the left lung along the back of the bronchus. The left upper may arise 
by the common trunk from the first right intercostal artery. The bronchial arteries supply the lungs, lymphatic glands (bronchial), œsophagus, and pericardium. (3) The œesophageal, four or five to the osophagus and mediastinum. (4) The intercostal arteries. There are nine or ten of these distributed to the parietes of the chest. The first and second spaces are supplied by the superior intercostal branch of the subclavian (see page I I 3). The right intercostals are slightly longer than the left, owing to the displacement of the aorta to the left of the median line. Their course is alike in all. Each artery runs outward, with the intercostal vein above and the nerve below, upon the external intercostal muscle, then between the external and internal intercostal muscles, and in the intercostal groove of the rib above; passing around the chest to the front, it finally anastomoses with the upper of the pair of anterior intercostal arteries from the internal mammary. The lower intercostal arteries anastomose with the musculophrenic branch of the internal mammary and the upper lumbar arteries. Each intercostal artery gives off a dorsal artery to the parts posterior to the chest, and a branch to the spine and cord. Also, a small collateral intercostal artery opposite the angle of the ribs. This artery runs forward along the top of the rib below and between the external and internal intercostal muscles to anastomose in front with the lower of the pair of anterior intercostal arteries from the internal mammary. (5) The pleural, muscular, and mammary (from the third, fourth, and fifth) are small branches from the intercostals.

The Intercostal Muscles.

The External.

Origin.-From the lower outer border of the upper eleven ribs external to the tubercles. 
Insertion.-Into the upper border of the eleven lower ribs a little external to the tubercles. Three or four of the lower muscles reach into the cartilages for their insertion. The direction of the fibres is from above downward and forward.

\section{The Internal.}

Origin.-From the inner border of the intercostal groove of the eleven upper ribs and cartilages; from the sternum around to the angles of the ribs.

Insertion.-The fibres pass downward and backward to the upper surface of the rib and cartilage below.

Nerve Supply.-The intercostal nerves.

Action.-The intercostal muscles act to bring together the ribs between which they are placed. The external intercostals act upon the ribs alone, the internal upon the ribs and cartilages. Taken together, the action of the intercostal muscles is to elevate the ribs and cartilages. From this action two results follow.

The chest is enlarged in two directions, transversely and anteroposteriorly. But this is not secured by the movement of the ribs upon two axes, as stated by most authorities; such an explanation is not warranted by the construction of the joints which unite the ribs to the spinal column. The reasons for the enlargement are these: First, the ribs move upon an axis passing through their heads and tubercles. This allows the rib to move as if hinged at these points, and the anterior end moves upward and downward. Second, the ribs increase in size from above downward (until the seventh rib is met with). Third, the ribs are not placed horizontally, but slope from behind downward and forward. Fourth, when the intercostal muscles act, they raise the ribs and cartilages into a more nearly horizontal 
position. Now this action substitutes a rib of greater for one of less dimensions, and the result is to increase the dimensions of the chest transversely, and anteroposteriorly by an amount equal to the difference between the areas of the sections of the thorax at the level of these two ribs.

The Intercostal Veins. Fig. 74.

These veins accompany the intercostal arteries and return the blood from the area which they supply. They terminate by emptying into the veins described below.

The Azygos Veins. Figs. 74, 73.

These present numerous variations in formation, course, and termination, but the following arrangement is the one usually found.

(I) The azygos major begins in the abdomen as a continuation of the ascending lumbar vein, or by a branch from the right renal, or vena cava inferior. It enters the chest through the aortic opening, lies to the right of the aorta upon the intercostal arteries and vertebræ, and at the level of the third or fourth dorsal vertebra arches over the right bronchus and empties into the superior vena cava (now removed) close to its pericardial attachment. It receives the nine or ten lower right intercostal veins, the azygos minor (opposite the sixth or seventh dorsal vertebra), the right superior intercostal vein, the left azygos superior (at times), the right mediastinal, bronchial, spinal, and œsophageal veins.

(2) The azygos minor also begins in the abdomen from the left renal or ascending lumbar veins, enters the chest through the left crus of the diaphragm, ascends at the left of the vertebræ to the level of the seventh or eighth, then turns to the right under the aorta to empty into the major azygos at the level of the sixth or seventh vertebra. The minor 


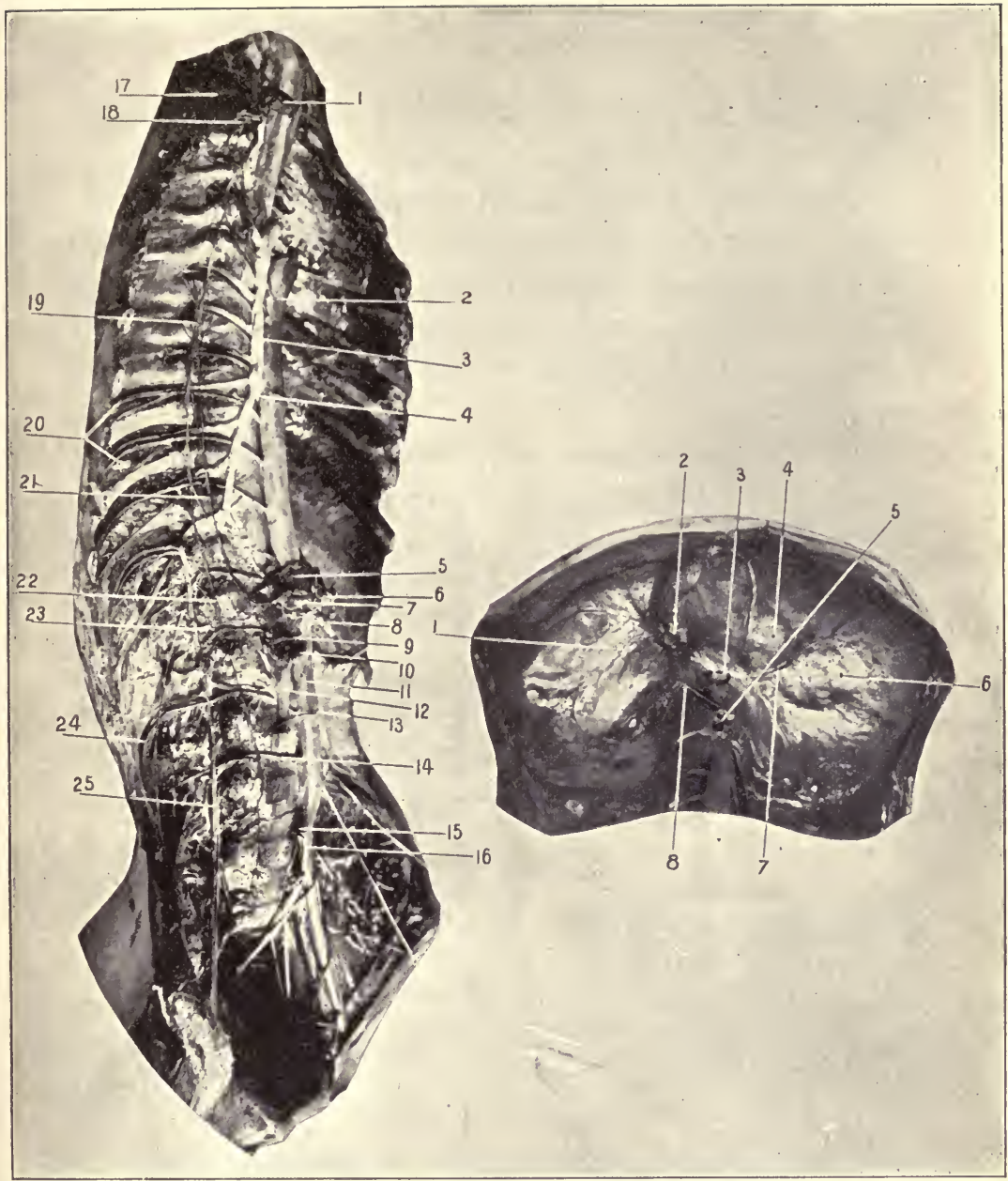

Fig. 74. Dissection of Thorax (and AbDomen).-I, Right subclavian artery. 2, Thoracic aorta and its intercostal branches on the right side. 3, Vena azygos major and its tributaries. 4, Point where the vena azygos minor empties into the major. 5, Remains of diaphragm. 6, Cœliac axis. 7, The right semilunar ganglion. This, with the left, forms a plexus (the solar) about the cœliac axis. 8, Superior mesenteric artery. 9, Right suprarenal artery. Io, Right renal artery. II, Abdominal aorta I2, Right crus of diaphragm. I3. Right spermatic artery. I4, One of the right lumbar arteries. The others are also shown, but not named. I5, Right, and 16. Left common iliac arteries. I7, Right axillary artery. I8, Right superior intercostal artery. 19, Thoracic sympathetic cord and ganglia. 20, Two sets of intercostal vessels and nerves. Their position with reference to each other is from above downward vein, artery, and 11erve. 2I, Great splanchnic nerve. 23, Vertical lumbar vein and its tributaries. Notice that it is continued directly into the azygos major. 24, Another lumbar artery. 25, Lumbar plexus.

Fig. 75. The Diaphragm From Below.-I, Right leaflet of tendon of diaphragm. 2, Opening of the inferior vena cava. 3, Esophageal opening. (Containing the œsophagus.) 4, Middle leaflet of the tendon of the diaphragm. 5, The aortic opening, with the aorta in position. 6, Left leaflet of tendon of diaphragm. 7, Left inferior phrenic artery. 8, Right inferior phrenic artery. 
receives the six left, lower intercostal veins, the left mediastinal, and œsophageal veins (lower).

(3) The left superior azygos-azygos tertius (often absent). When present, receives the fourth to the seventh or eighth intercostal veins inclusive, some mediastinal, and the left bronchial veins. It communicates with the left superior intercostal vein above, and empties into the azygos major below.

(4) The right superior intercostal vein is formed by the second, third, and sometimes fourth right intercostal veins, and empties into the azygos major as it arches to terminate in the vena cava superior.

The first right intercostal vein empties into the innominate or vertebral vein.

(5) The left superior intercostal, formed by the first two or three left intercostal veins, opens into the innominate (left). It communicates below with the left superior azygos.

The Intercostal Nerves. Fig. 74.

These are the anterior branches of the twelve dorsal nerves which supply the walls of the chest and abdomen. The first intercostal nerve sends four-fifths of its fibres to join the brachial plexus and the remaining fifth takes a course similar to the other intercostal nerves.

The intercostal nerves from the second to the sixth inclusive terminate anteriorly as the anterior cutaneous nerves of the thorax. See page 266. The next nerves below, from the seventh to the eleventh inclusive, course between the transversalis and internal oblique muscles, and terminate in front as the anterior cutaneous nerves of the abdomen. The course of the twelfth is given with the lumbar plexus. All these nerves give off lateral cutaneous 
branches (excepting the first), which pierce the overlying muscles and supply the side of the body from the axilla to the crest of the ilium. Each lateral cutaneous nerve divides into an anterior and posterior branch in passing to its distribution. In addition to this the lateral cutaneous branch of the second nerve passes to supply the posterior upper part of the arm, and is called the intercostohumeral nerve. See page 280 .

The Diaphragm. Figs. 7 I, 75.

Origin.-From the posterior surface of the ensiform appendix, from the inner surface of the cartilages of the six lower ribs, from the ligamentum arcuatum externum and internum, and by two crura from the anterior surface and intervertebral discs and anterior common ligament of the first to the fourth lumbar vertebræ (on the right side), and from the first to the second or third (on the left side).

Insertion.-Into the central tendon, which presents three portions or leaves, two lateral and one central.

Nerve Supply. - The phrenics, bringing filaments from the third, fourth, and fifth cervical nerves. These nerves pass through the muscle and are distributed to its under surface.

Action. - To produce inspiration by lengthening the vertical dimension of the thorax. To compress the viscera and aid in all expulsive efforts, as defæcation, micturition, parturition, and vomiting. In vomiting the diaphragm contracts to form a hard surface against which the stomach is compressed by the action of the abdominal muscles.

\section{Openings Through the Diaphragm.}

Aortic opening lies immediately in front of the spine. It gives passage to the aorta, vena azygos major, and thoracic duct.

The asophageal opening lies in front of the aortic to the 
left of the middle plane of the body. The œsophagus with the pneumogastric nerves pass through it. Also some small œesophageal arterial filaments.

The caval opening is in the right portion of the diaphragm at the posterior border of the central tendon where the right and middle leaflets join. Through it passes the inferior vena cava.

Through the crura pass the cord of the sympathetic nerve, the splanchnic nerves, and in addition, on the left side, the azygos minor vein.

Having removed the heart and great vessels (viz.: the ascending and transverse aorta and its branches, the pulmonary artery, the superior cava), the trachea and lungs, in one mass, they are to be dissected :-

(I) The heart, great vessels, coronary arteries, ventricles, and auricles are to be studied as they are seen from the front. (2) The structures and their relations which compose the root of the lungs are to be investigated. (3) Separate the heart (and vessels) from the lungs (and trachea). (4) Complete the dissection of the posterior of the heart (ventricles, auricles, and vessels), then open its cavities and learn their parts or contents. (5) Complete the study and comparison of the right and left lung, inflating them for this purpose. (6) Follow up the bronchi to the main subdivisions, three for the left and two for the right lung.

The Right Coronary Artery. Figs. 70, 7 I.

Comes off from the anterior sinus of Valsalva of the aorta, appears between the right auricle and pulmonary artery, follows the auriculoventricular groove to the right border of the heart, here divides into two terminal arteries. One continues the course around in the auriculoventricular groove to the back of the heart to anastomose with a 
similar one from the left coronary artery. The other, the interventricular, descends in the posterior (right) interventricular groove to the apex of the heart to enter into an anastomosis with the left artery. The right artery also gives off several other branches to the front and side of the heart (right auricle).

The Left Coronary Artery. Figs. 70, 71, 72.

Is given off from the left (posterior) sinus of Valsalva, issues between the left auricle and the pulmonary artery, curves downward in the left interventricular groove to the apex of the heart to form a free anastomosis with the right artery. It gives off a branch which turns backward in the auriculoventricular groove to anastomose with the similar branch of the right. Both arteries supply the auricles and beginning of the great vessels.

The Cardiac Veins. Figs. 70, 71, 72, 73 .

They take up the blood from the area of the heart supplied by the above arteries, which they accompany.

The coronary sinus extends from the anterior interventricular vein (of which it is a continuation) around the left margin of the heart to the right auricle. Near the auricle it receives the right (posterior) interventricular vein.

The anterior interventricular (great cardiac) vein follows upward in the sulcus with the left artery, it reaches the auriculoventricular groove, turns backward in it to the right auricle, into which it empties. The portion of the vein in the auriculoventricular groove is called the coronary sinus ; it is about an inch long, and receives the oblique vein of Marshall, posterior cardiac veins, and the posterior interventricular vein (accompanies the right coronary artery), the last just before it enters the auricle. 


\section{The Cardiac Nerves.}

The cardiac nerves are supplied from the pneumogastric, recurrent laryngeal nerves, and the three cervical ganglia.

The minute filaments from these sources interlace in the concavity of the aortic arch, in front of the right pulmonary artery (forming the superficial cardiac plexus), and behind the transverse aorta, between the trachea and the transverse aorta (forming the deep cardiac plexus).

The deep plexus receives branches from all the sources above, except from the left superior sympathetic cardiac and the left inferior cardiac of the pneumogastric. The superficial plexus is formed by the two exceptions above and by the branches from the deep plexus itself.

From these plexuses branches descend to the heart and lungs.

\section{Inferior Vena Cava.}

For formation and course see Abdomen.

It enters the thorax from the abdomen through the caval opening in the diaphragm to the right of the thoracic aorta, and in front of the dorsal vertebræ, and empties into the right auricle of the heart. It is covered by pericardium for about one-half inch.

The Heart. (See page 330.) Figs. 70 to 73.

Note its shape, size, position of parts, and their relations to each other. For the external relations see page 258 .

Open the ventricles by incisions from the pulmonary artery and the aorta to the apex through the right and left sides of the heart. Open the auricles by incisions parallel with the auriculoventricular groove.

The Right Auricle.-Openings, superior vena cava, inferior vena cava, auriculoventricular, coronary sinus, foramina Thebesii. 
The opening of the coronary sinus is found between that of the inferior vena cava and the auriculoventricular. It is guarded by the valve of Thebesius, formed by a reduplication of the endocardium.

The inferior caval opening is protected by the Eustachian valve.

The tubercle of Lower is found between the two caval openings and at their right.

The appendix and anterior surface of the auricle is thrown into ridges by the musculi pectinati.

The septum between the two auricles is depressed, forming the fossa ovalis (in the foetus it is an opening), surrounded by a border, the annulus ovalis.

The Left Auricle._Openings. Four pulmonary veins (two may open by one orifice), auriculoventricular, foramina Thebesii.

The musculi pectinati are found in the appendix.

The Right Ventricle.-Openings. The auriculoventricular from the auricle, the pulmonary artery. The former is guarded by the tricuspid valves, the latter by the semilunar.

The tricuspid valve is formed of three main and three secondary triangular flaps of membrane. These flaps or cusps are attached by their bases to the fibrous ring about the auriculoventricular orifice, and also to each other for a short distance from their bases.

The semilunar valves, three in number, situated at the beginning of the pulmonary artery (and aorta, also). They are reduplicated folds of the inner coat of the vessel plus some fibrous tissue. At the centre of the free margin is a small nodule, the corpus Arantii. By the peculiar arrangement of the fibrous tissue in the semilunar valves a lunated space is left on either side of each corpus Arantii, consisting 
only of the two layers of the endothelium of the vessel. These thin places admit of a closer apposition of the valves.

The sinuses of Valsalva are hollows behind each cusp of the semilunar valves (whether in pulmonary artery or the aorta).

The sinuses are arranged in the pulmonary artery with two in front (the left is slightly in front of the right) and one in the rear.

In the aorta these sinuses are arranged with one in front and two behind. See Coronary Arteries, pages 352 and 353.

The inner surface of the ventricle is corrugated by muscular ridges, the columnæ carneæ. These are found in three forms : one, attached by their whole lengths ; a second, by both ends; a third, by one end (musculi papillares), the other end being free and affording attachment to the chordæ tendineæ. The chordæ tendineæ are fibrous strings passing from the musculi papillares to the under surface and free borders of the primary and secondary portions of the tricuspid (and bicuspid) valve.

The Left Ventricle.-This ventricle is about three times thicker than the right one. From it extends the aorta, and into it empties the left auricle through the auriculoventricular opening. The aortic opening is provided with semilunar valves and the auriculoventricular with the bicuspid (mitral) valve, similar in every way to the semilunar and tricuspid of the right ventricle.

The auriculoventricular or bicuspid valve is composed of two main and two secondary flaps. Behind the semilunar valves the aortic wall is pouched; these pouches are called the sinuses of Valsalva, as above described.

The Trachea. Figs. I8, 70, 7I, 72. Diags. 20, 2 I.

The trachea is a fibromuscular tube that is prevented 
from collapsing like the œsophagus by the introduction of incomplete rings of cartilage.

These cartilaginous arches are incorporated within the structure of the tube. Their opening is placed behind, and the gap filled in with muscular tissue. The trachea is the continuation of the air tube below the larynx, or the cricoid cartilage. It terminates at its bifurcation into the two bronchi at the fourth or fifth thoracic vertebra. Its length is about five inches and its diameter nearly an inch.

Relations.-In the neck: In front, skin, fasciæ, anterior jugular veins, their communicating and the inferior thyroid veins, the isthmus of the thyroid gland, branches of the superior thyroid artery. Laterally (and in front), sternohyoid, sternothyroid muscles, lobes of the thyroid gland. Laterally, lobes of the thyroid gland carotid sheath and its contents. Behind, œsophagus (and at sides, recurrent laryngeal nerves and the inferior thyroid arteries).

In the chest: In front, origin of muscles as above. Remains of the thymus gland. Left innominate vein, transverse aorta, and the diverging innominate and left common carotid arteries, the deep cardiac plexus. Laterally, the lungs and their pleuræ, the pneumogastric nerves, and on the left side the recurrent laryngeal nerve. Behind, the œsophagus.

The blood supply is from the inferior thyroid arteries, the nerve supply from the pneumogastrics, recurrent laryngeals, and sympathetic nerves.

\section{The Bronchi. Fig. 73. Diag. 22.}

These are constructed the same as the trachea, of which they are the terminal branches.

The bifurcation of the trachea is not at the middle plane of the trachea, but more to the left side, consequently the 
right bronchus presents a more conspicuous opening than the left, and therefore is more liable to receive any object inspired into the trachea.

The right bronchus gives off a large branch to the upper lobe of the lung and then bifurcates to supply the middle and lower lobes.

The left bronchus divides into two branches, one for each lobe.

The right bronchus is shorter, wider, and more horizontal than the left. Its length is about an inch and its width a little more than half as much. The right pulmonary artery is below and then in front of the bronchus. The azygos major vein arches upward over the bronchus from behind to empty into the vena cava superior.

The left bronchus is longer, narrower, and more oblique than the right. Its length is about two inches. In front of it is the aortic arch, the left pulmonary artery is above and then in front of it. Behind the bronchus is the œsophagus, thoracic duct, and descending aorta.

The Lungs. Figs. 59, 68.

The lungs are two in number, the right and left. The right consists of three lobes and the left of two.

When the chest is intact they fill the pleural cavities, with the exception of the extreme lower angle between the chest and diaphragm. See page 263. The lung is described as having an apex, projecting into the root of the neck through the upper opening of the chest, and rising above the first rib about one and one-half inches (threefourths of an inch above the clavicle); a base, concave, to fit over the convexity of the diaphragm, upon which it rests; an outer convex surface opposed to the interior of the chest ; an inner surface, concave, fitting over the peri- 
cardium and heart and mediastinum; a root, at which point the visceral and parietal pleuræ become continuous, and where the bronchial tube, vessels, nerves, and lymphatics pass to (or from) the lungs; an anterior border, thin, notched on the left side for the heart (for its relation to the anterior of the chest see page 263); a posterior border, thick, rounded, lying alongside of the spinal column; and a lower border, which resembles the anterior. For its relations to the chest wall, see page 262 .

The main fissure which divides each lung into two lobes passes from the posterior border, three inches from the apex, downward around the outer surface of the lung to end at the lower portion of the anterior border.

Besides this main fissure the right lung has a secondary one that passes from the centre of the main fissure to the anterior border, dividing the upper lobe into two portions.

The structures constituting the root of the lung (Fig. 73), are the bronchus, pulmonary artery and vein, the bronchial vessels, nerves, lymphatic glands, the connecting tissue, and all surrounded by the pleura.

The relations of the pulmonary artery, vein, and bronchus are from before backward, bronchus, artery, and vein. On the right side, from above downward, the relation is the same, but on the left side it is artery, bronchus, and vein.

The lungs weigh about forty-two ounces, the right being a little heavier than the left.

Relations of the Roots of the Lungs.-Figs. 7 I, 72. Diag. 22. The right: In front, the phrenic nerve, anterior pulmonary plexus, superior vena cava, the right auricle of the heart; above, the azygos major; below, broad ligament of the lung; and behind, the pneumogastric nerve and the posterior pulmonary plexus. 
The left: In front, phrenic nerve and anterior pulmonary plexus ; above, the arch of the aorta ; below, same as right ; behind, descending aorta, pneumogastric nerve, and posterior pulmonary plexus.

\section{UPPER EXTREMITY AND THORAX, Posterior.}

\section{DISSECTION.}

Incisions.-(I) From the base of the skull (made by the one on the head and neck) in the median line along the back of the thorax (made by the one on the upper extremity), to terminate at the tip of the coccyx (extended by the one dissecting the lower extremity).

(2) From the median incision out along the spine of the scapula (same for head and neck, page 128) to meet the similar incision in front over the point of the shoulder.

(3) Carry a transverse cut from twelfth dorsal spine around the body to meet the similar one from the front.

This incision answers for both upper and lower extremities. Reflect the integument from the back of the neck, thorax, and trunk; from the arm to below the elbow. To expose the last no farther incisions in the skin are necessary; simply reflect it, beginning at the point where its anterior dissection stopped, and working from the outer to the inner side of the limb.

This method will also hold for the removal of the integument from the back of the forearm, hand, and fingers.

\section{The Superficial Fascia.}

The superficial fascia covering the back of the body from the base of the skull to the end of the spine is a dense, brawny layer composed of connective and granular fatty tissue. In the subject it is often found infiltrated by gravitated fluid.

The superficial fascia is continuous with the similar layer of other adjacent parts of the body. It should be removed in the same manner as the integument. In its removal an outlook should be kept for the superficial nerves and vessels. The cutaneous nerves are the posterior divisions 


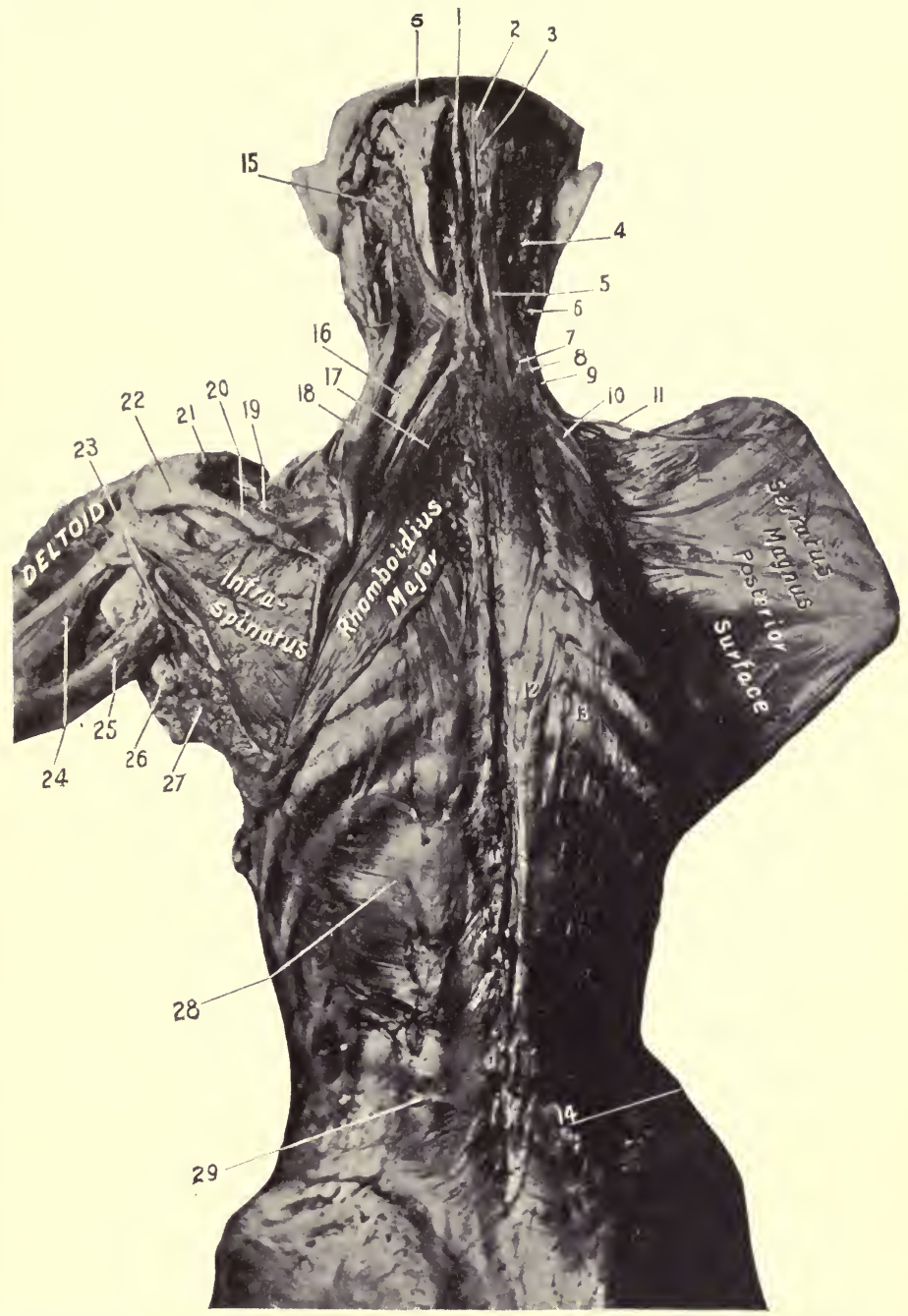

Fig. 76. Dissection of THE BACK.-1, Ligamentum nuchæ. 2, Third occipital nerve. (Internal cutaneous branch of the third cervical nerve.) 3, Great occipital nerve. 4, Trachelomastoid muscle. 5, 5, Complexus. 6, Posterior part of sternomastoid. 7, Transversalis cervicis. 8, Cervicalis ascendens. 9, Scalenus posticus. Io, Serratus posticus superior. II, Posterior scapular artery. I2, Longissimus dorsi. I3, Accessorius ad iliocostalem or ad sacrolumbalem. I4, Erector spinæ. I5, Splenius capitis. I6, Rhomboideus minor. 17, Rhomboideus major. 18, Levator anguli scapulæ. I9, Supraspinatus. 20, Spine of scapula. 2I, Clavicle. 22, Acromion process. 23, Teres minor. 24. Outer head of triceps. 25, Long head of triceps. 26, Tendon of latissimus dorsi. 27, Teres major. 28, Serratus posticus inferior. 29. The vertebral aponeurosis. 
of the lateral cutaneous branches of the intercostal nerves at the side, and the dorsal branches from the same source along the spine. The arteries are minute branches from the intercostals and the other vessels which will appear later.

\section{The Deep Fascia.}

This is a well-marked layer of dense connective tissue which covers in all the structures of the back. Above, it is attached to the base of the occipital bone along the superior curved line, and to the mastoid process of the temporal bone; laterally, in the neck, it becomes the outer layer of the deep cervical fascia, and is attached to the transverse processes of the cervical vertebræ; in the middle line it is attached to the spinous process of all the vertebræ from the skull to the coccyx, in the neck forming the ligamentum nuchæ; back of the shoulder it is attached to the spine and acromion process of the scapula, and becomes continuous with the fascia covering the deltoid; lower down it covers in the latissimus dorsi muscle, and at its anterior border becomes continuous with the axillary fascia (see page 268); below it is attached to the crest of the ilium, and above the bone reaches around the body, covering in the external oblique muscle.

\section{DISSECTION.}

Remove the deep fascia, exposing the first layer of muscles.

The Trapezius. Fig. 77 .

Origin.-From the inner third of the superior curved line of the occipital bone and occipital protuberance, from all the spines of the cervical vertebræ by means of the ligamentum nuchæ, from the spines of all the dorsal vertebræ and the supraspinous ligament. 
Insertion.-Into the posterior portion of the upper surface and border of the outer third of the clavicle; into the upper border of the spine and acromion process and the slight tubercle near the base of the spine of the scapula.

Nerve Supply.-The spinal accessory and deep branches of the third and fourth cervical nerves. They are distributed to the anterior surface of the muscle.

Action.-The spine and head being the fixed points and both muscles acting, the shoulders will be drawn backward and the points of the shoulders slightly elevated. The muscles acting singly and in parts from the fixed spine : if the upper portion acts, the clavicle and acromion will be raised (shoulder elevated); if the middle portion of the muscle acts, the scapula will be drawn toward the spine, a slight rotation taking place at the same time; if the lower portion acts, the vertebral border of the spine will be drawn downward (the shoulder carried downward and backward), at the same time the point of the shoulder is very slightly elevated. Acting from the shoulder as the fixed point, both muscles will retract the head and neck and elevate the chin ; one muscle acting, will carry the chin to the opposite side and flex the neck laterally.

The muscle fixing the shoulders gives the accessory muscles of inspiration a better chance for acting.

The Deltoid. Fig. 60.

Origin.-From the anterior border of the outer third of the clavicle, from the outer margin of the acromion, lower lip of the spine of the scapula, and the infraspinatus fascia.

Insertion.-For a distance of two or three inches into the outer surface of the humerus just above its middle, at a rough triangular surface. 
Nerve Supply.-The circumflex, which furnishes fibres from the fifth and sixth cervical nerves.

Action.-To abduct the arm to a right angle with the body. The anterior portion of the muscle will flex and adduct the arm to (less than) a right angle, while the posterior portion will adduct and extend the arm to half a right angle.

The elevation of the arm beyond a right angle is accomplished through the action of the trapezius and serratus magnus muscles by producing a rotation of the scapula and elevation of the outer end. The clavicle moves upward and at the same time rotates upon its long axis.

The deltoid really is a continuation of the direction of the fibres of the trapezius and so extends its action.

\section{DISSECTION.}

Cut the trapezius away from its insertion, divide the nerves passing to it, and roll the muscle up to the spine.

Complete the cleaning of the latissimus dorsi muscle.

\section{The Latissimus Dorsi.}

Origin.-From the spines and supraspinous ligaments of the lower six dorsal and all the lumbar vertebræ, and through the lumbar aponeurosis, from the spines of the sacral vertebræ and the outer lip of the posterior third of the crest of the ilium, from the outer surface of the three or four lower ribs external to their angles, and from the outer surface of the lower angle of the scapula.

Insertion.-Into the bottom of the bicipital groove of the humerus.

Nerve Supply.-The long or second subscapular nerve. The filaments coming from the fifth cervical nerve.

Action.-The origin being the fixed point, the muscle acts to draw the humerus (and shoulder) downward, backward, 
and inward, and to rotate the humerus inward (adduction, extension, and internal rotation of humerus).

The insertion being the fixed point, the muscle will raise the body and swing it forward (if both muscles are acting) or to one side (if only one muscle acts). Acting upon the ribs, it will raise them and act as an accessory muscle of inspiration.

\section{DISSECTION.}

Cut the deltoid away from its origin and turn it downward and forward; in doing so save its nerve and arterial supply.

Disarticulate the clavicle from its acromial attachment; divide the coracoclavicular ligaments and draw the clavicle out of the way.

Remove the supra- and infraclavicular fasciæ and clean the muscles covered by them. Also, clean the levator anguli scapulæ, rhomboid, and teres muscles. Remove the fascia covering the triceps.

The superficial fascia of the arm has been already described (see page 282).

The cutaneous nerves of the back of the shoulder and arm.

The shoulder receives filaments through the acromial nerves from the third and fourth cervical nerves. See page 9I.

The deltoid region of the arm and a small surface of the outer part of the arm from the deltoid half-way to the elbow is supplied by the circumflex nerve.

The rest of the outer portion of the arm is supplied by the superior external cutaneous branch of the musculospiral nerve.

The inner half of the back of the arm is supplied from above downward by the internal cutaneous branch of the musculospiral, the intercostohumeral, and the lesser internal cutaneous nerves. 
The Levator Anguli Scapulæ. Fig. 76.

Origin.-From the posterior tubercles of the transverse processes of the three or four upper cervical vertebræ.

Insertion.-Into the upper fourth of the vertebral border of the scapula.

Nerve Supply. - The third and fourth cervical nerves.

Action. - To raise the inner portion of the scapula, at the same time depressing the shoulder.

The Rhomboideus Major and Minor. Fig. 76.

These muscles will be described together, as they are really one.

Origin.-From the lower portion of the ligamentum nuchæ, the spinous processes of the last cervical and the four or five upper dorsal vertebræ, and the supraspinous ligament.

Insertion.-Into the lower three-fourths of the vertebral border of the scapula.

Nerve Supply._Branches from the fifth cervical nerve.

Action.-To draw the scapula to the spine and at the same time elevate the inner border; this also depresses the outer portion of the bone (and the shoulder).

The Supraspinatus. Fig. 76 .

Origin.-From the inner two-thirds of the supraspinous fossa and the inner surface of the supraspinous fascia.

Insertion.-Into the upper facet upon the greater tuberosity of the humerus. Its under fibres are also continued into the capsular ligament of the shoulder-joint.

Nerve Supply._-From the fifth cervical nerve through the suprascapular.

Action.-To abduct the humerus, but more especially to hold the head of the humerus against the glenoid cavity of the scapula. 


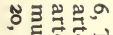

를

要过

ㄷ.ड. 0

.

두웅

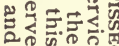

2 $3=2$

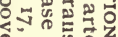
. $-\omega^{2}<0$ 둥

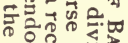
$\overrightarrow{0}=\sum_{1}=$

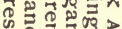

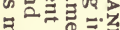
. 픔이 舟 ज5유

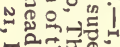

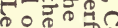
¿응

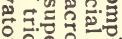
तก응

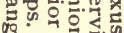
둥. $\exists \infty D_{N}$

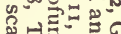
象 \%

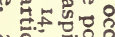

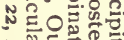

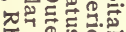
둔 可

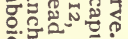
으을

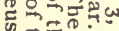

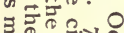
는 으을 일 ة E. 둘 을룰. N

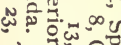
$\rightarrow-\infty$ 20ㄴㄷㅡ. एँ 눙ㅇํㅇ पर

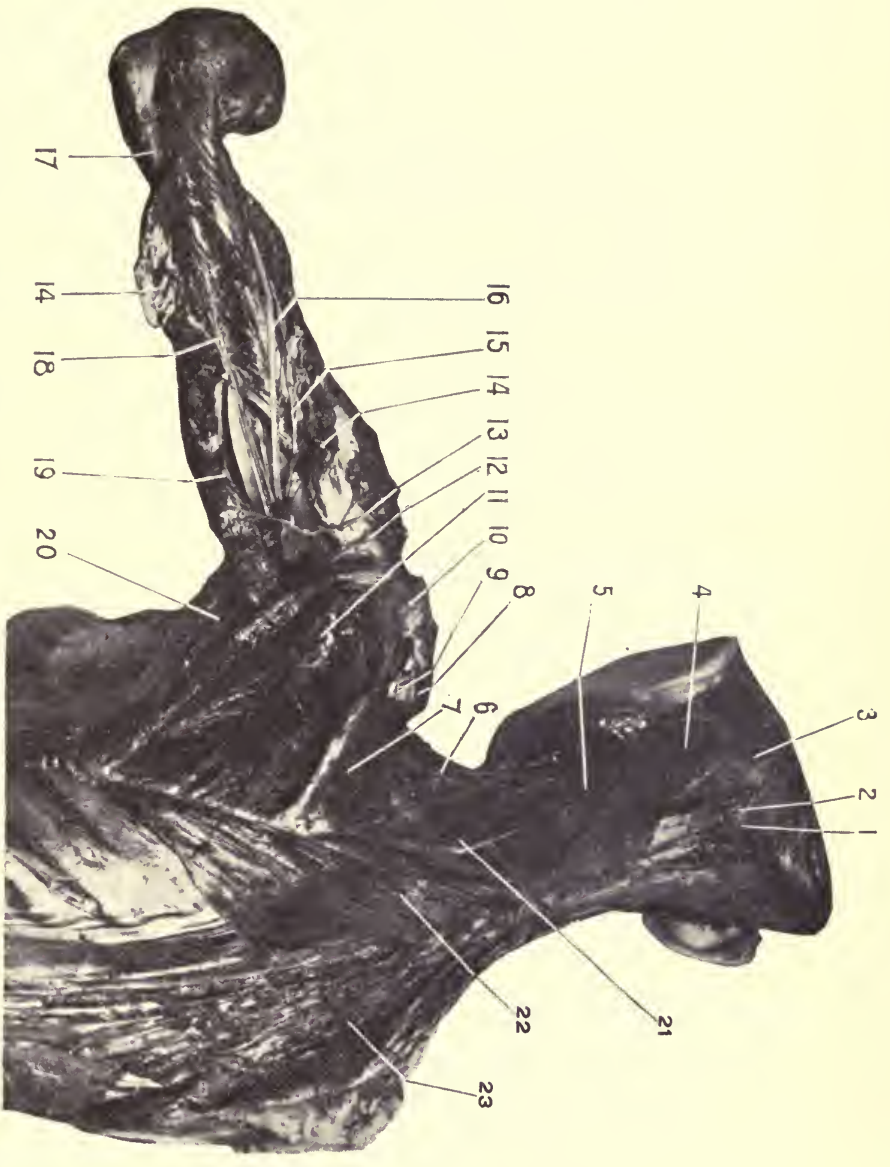
ㄱ. 글 구 F役

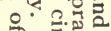
दूลे ㄱ. ก불ㄹㅇㅢ

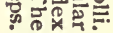


The Infraspinatus. Fig. 76.

Origin.-From the under surface of the spine of the scapula, from the inner two-thirds of the posterior surface of the scapula reaching from the spine to the origin of the teres muscles, and from the inner surface of the infraspinous fascia.

Insertion.-Into the middle facet upon the greater tuberosity of the humerus, and into the capsular ligament of the shoulder-joint.

Nerve Supply.-The same as the supraspinatus. See above.

Action.-Primarily it is an external rotator of the arm; if the arm is extended and drawn forward the muscle will adduct and extend it slightly.

The Teres Minor. Figs. 76, 77.

Origin.-From the upper two-thirds of the posterior surface of the axillary border of the scapula, and from the septa between it, the teres major, and infraspinatus muscles.

Insertion. - Into the lowest facet upon the greater tuberosity, and into the shaft of the bone for an inch below.

Nerve Supply. - The circumflex. The filaments are furnished by the fifth cervical nerve.

Action.-An external rotator and adductor of the arm.

The Teres Major. Figs. 76, 77.

Origin.-From the lower third of the posterior surface of the axillary border, and from the posterior surface of the inferior angle of the scapula, and from the septa which intervene between it and the teres minor and the infraspinatus muscles.

Insertion. - By a tendon nearly two inches broad into the inner bicipital ridge of the humerus. 
Nerve Supply. - The lowest or third subscapular, bringing filaments from the sixth and seventh cervical nerves.

Action.-To rotate the arm inward and adduct it, also to draw the arm backward when it has been carried forward.

The Triceps. Fig. 77.

Origin.-The long or middle head rises from the axillary border of the scapula just below the glenoid cavity (and from the lower portion of the capsule of the shoulder-joint).

The external head comes from the posterior surface of the humerus above, and external to the musculospiral groove (reaching up as high as the insertion of the teres minor).

The internal head from the surface of the humerus below and internal to the same grocve (reaching up to the insertion of the teres major).

The external and internal heads also arise from the posterior surface of the external and internal intermuscular septa opposite their bony origins.

Insertion.-Into the tip of the olecranon process of the ulna, and into the deep fascia of the forearm.

Nerve Supply.-The musculospiral. The source of the filaments is from the seventh and eighth cervical nerves.

Action.-Primarily, upon the forearm to extend it; secondarily, upon the arm to adduct and support it.

The Subanconeus.-This is not a separate muscle, merely some fibres of the triceps which are inserted into the posterior part of the ligament of the elbow-joint. Its function is to raise the ligament in extension of the joint and keep it out of the way of the bones.

\section{DISSECTION.}

Find the suprascapular artery and nerve, trace them over the upper border of the scapula. Divide the supraspinatus muscle and follow the artery and 
nerve through the scapular notch into the infraspinous fossa, to terminate in the muscle there.

Find the dorsalis scapulæ artery and trace its course upon the back of the scapula, cutting as much of the overlying muscle away as is necessary to follow it.

In the same way dissect out the circumflex nerve and the posterior circumflex artery to their distribution to the teres minor and deltoid muscles.

Divide the rhomboid muscles vertically midway between their attachments and reflect them.

At the upper part of the vertebral border of the shoulder find the posterior scapular artery and trace its branches as far as possible.

Cut the latissimus dorsi transversely at its middle and reflect its central portion, severing it from its costal attachments.

\section{The Serratus Posticus Superior. Fig. 76 .}

Origin.-From the lower portion of the ligamentum nuchæ, the spines of the last cervical and first three dorsal vertebræ, and the supraspinous ligaments.

Insertion.-Into the posterior surface of the second to the fifth ribs inclusive, just external to their angles.

Nerve Supply. - The second and third intercostal nerves. Action.-To raise the ribs and aid in inspiration.

The Serratus Posticus Inferior. Fig. 76.

Origin.-From the spines of the last two dorsal and first two lumbar vertebræ.

Insertion.-Into the lower borders of the last four ribs.

Nerve Supply. - The tenth and eleventh intercostals.

Action.-To draw the ribs downward, thus affording a fixed point for the action of the diaphragm and so aiding inspiration.

The Posterior Scapular Artery. Fig. 76.

Usually arises from the third portion of the subclavian, but may come off as the lower branch of the transverse cervical.

For its course from the subclavian see page i I 3 . When 
found at the back it is covered by the trapezius, levator anguli scapuli, rhomboid muscles. Its course is around the upper angle and along the vertebral border of the scapula to its lower angle.

It anastomoses, $(a)$ in the supraspinous fossa, with the suprascapular; $(b)$ in the infraspinous fossa, with the dorsalis scapulæ, and $(c)$ at its terminus with the subscapular.

Its muscular branches supply the adjacent muscles.

\section{The Suprascapular Artery and Nerve. Fig. 77.}

The artery is one of the branches of the thyroid axis. See page II 2. Origin of nerve. See page 315.

In this part of the dissection it is found passing into the supraspinous fossa over the transverse ligament of the scapula, while the suprascapular nerve passes under it, through the foramen which it forms out of the notch in the scapula.

The nerve and artery take the same course through the supraspinous fossa and great scapular notch, and terminate in the infraspinous fossa. Both supply the supra- and infraspinatus muscles and the shoulder joint.

The artery anastomoses with the posterior scapular in the supraspinous fossa, the dorsalis scapular in the infraspinous fossa, and the acromial branch of the acromiothoracic over the spine and acromial process of the scapula.

The Dorsalis Scapulæ. Fig. 77 .

This large branch from the subscapular (really the continuation of the artery) appears at the back in the triangular interval between the subscapularis above, the teres major below, and the long tendon of the triceps at the outer side, and under cover of the teres minor, between which and the bone it passes into the infraspinous fossa to anastomose with the suprascapular and posterior scapular arteries. 


\section{The Posterior Circumflex Artery and Circumflex} Nerve. Fig. 77.

The artery is a branch from the third portion of the axillary, the nerve comes from the posterior cord of the brachial plexus. See pages 279 and 3 I 8 . They are found coming through a quadrilateral space bounded above (and in front) by the subscapularis (behind by the teres minor), below by the teres major, internally by the long head of the triceps muscle, and externally by the humerus.

The nerve supplies the teres minor and deltoid muscles and sends branches to the shoulder-joint and the skin over the deltoid and middle third of outer surface of the arm. The artery supplies these muscles, the joint of the shoulder, and anastomoses with the anterior circumflex, and by a large branch with the superior profunda arteries.

\section{The Superior Profunda Artery and the Musculospiral Nerve. Fig. 77.}

These are found together as they course through the musculospiral groove, from the inner to the outer side of the arm, where the nerve terminates by dividing into its radial and posterior interosseous branches (see page 3i 8), and the artery by anastomosing with the radial recurrent. The artery also sends a branch upward to anastomose with the posterior circumflex and one downward-the articular-which, descending in the substance of the triceps, supplies that muscle, and terminates in the anastomotic circle about the elbow-joint (for which see page 387 ).

\section{DISSECTION.}

Divide the levator anguli scapula and rhomboid muscles. Clean the inner surface of the serratus magnus, noting its attachments.

Remove the serrati muscles and clean the fascia covering the extensor muscles of the spine and note its attachments. 
The Vertebral Aponeurosis. Fig. 76.

This aponeurosis starts in the cervical region as a very thin layer, but gradually becomes thicker as it descends, until in the lower dorsal and lumbar regions it is somewhat thicker. In the last region it receives the tendons of the latissimus dorsi and serratus posticus inferior muscles. It is attached in the median line to the spines and supraspinous ligaments of the vertebræ extending from the sacrum to the base of the skull; laterally it passes in the cervical region to the tips of the transverse processes, in the dorsal region to the angles of the ribs, and in the lumbar region to the crest of the ilium, and becomes continuous with the lumbar fascia. From the lumbar portion of the aponeurosis arises the latissimus dorsi and the serratus posticus inferior muscles.

\section{DISSECTION.}

Remove the fascia and expose the muscles beneath. Separate them carefully and make out the limits of each.

NotE.-The person dissecting the lower extremity removes the fascia from the lumbar region, the one on the thorax the portion there, and the one on the head and neck the cervical portion.

\section{The Extensor Muscles of the Spine. Fig. 76.}

The spinal gutter alongside of the spinous processes is filled up by a muscular mass extending from the sacrum to the base of the skull.

This muscular mass, though really continuous and constituting one muscle, in action is divided into separate portions for convenience of reference.

Below, the mass is found blended together to form the erector spinæ, which fills in the lumbar portion of the spinal gutter. Above, the continuation of the erector spinæ is represented by three columns. The inner column, represented by the spinalis dorsi; the middle column, by the longissimus dorsi, transversalis cervicis, and trachelomas- 
toid; the external column, by the iliocostalis, accessorius, and cervicalis ascendens.

\section{The Erector Spinæ.}

Origin.-From the lowest two or three dorsal and all the lumbar and sacral spines, from the back of the sacrum, from the posterior one-fifth of the crest of the ilium and the posterior iliac spines.

Insertion.-Its fibres pass into the spinalis dorsi (inner), longissimus dorsi (middle), and iliocostalis, or sacrolumbalis (external) muscles.

Nerve Supply.-The posterior branches of the lumbar nerves.

Action.-See below.

The Spinalis Dorsi. (Inner column.)

Origin.-The inner portion of the erector spinæ.

Insertion.-The spinous processes of the upper dorsal vertebræ (four to nine).

Nerve Supply.-The posterior branches of the dorsal nerves.

Action.-To extend the thoracic portion of the spine.

The Longissimus Dorsi. (Middle column.)

Origin. - The middle portion of the erector spinæ.

Insertion.-(Inner tendons.) The transverse processes of the dorsal, and the accessory tubercles of the lumbar vertebræ.

(Outer set.) To the transverse processes of the lumbar vertebræ, to the lower ten ribs external to their tubercles.

Nerve Supply and Action.-See below.

Transversalis Cervicis. (Middle column.)

Origin.-From the transverse processes of the upper four or five dorsal vertebræ. 
Insertion.-Into the posterior tubercles of the transverse processes of the cervical vertebræ from the second to the sixth inclusive.

Nerve Supply.-External posterior branches of the dorsal nerves.

Action.-See below.

Trachelomastoid. (Middle column.)

Origin.-From the four or five upper transverse processes of the dorsal vertebræ, from the articular processes of the lower four cervical vertebræ.

Insertion.-Into the posterior border of the mastoid process.

Nerve Supply.-The posterior branches of the cervical nerves.

Action of the Middle Column.-To extend the spine in the cervical, dorsal, and lumbar regions; to flex the spine laterally; to extend and flex the head laterally and rotate the chin to the same side.

Iliocostalis (Sacrolumbalis). (External column.)

Origin.-The outer portion of the erector spinæ.

Insertion.-Into the angles of the six lower ribs.

Nerve Supply.-External posterior branches of the lumbar nerves.

Action.-See below.

Accessorius. (External column.)

Origin.-From the angles of the lower six ribs.

Insertion.-Into the angles of the six upper ribs and the transverse process of the seventh cervical vertebra.

Nerve Supply.-The posterior division of the dorsal nerves.

Action.-See below. 
Cervicalis Ascendens. (External column.)

Origin.-From the angles of the five upper ribs.

Insertion.-Into the posterior tubercles of the transverse processes of the fourth, fifth, and sixth cervical vertebræ.

Nerve Supply.-The external posterior branches of the cervical nerves.

Action of External Column.-To extend and flex laterally the cervical, dorsal, and lumbar regions of the spine. To draw the ribs downward and act to aid expiration.

\section{DISSECTION.}

Remove the above muscles to expose the deeper layers which occupy the space between the spines and tips of the vertebræ and transverse processes of the vertebræ.

The muscles comprising the two last layers will not be given here, as their study and dissection is more theoretical than practical.

The sixth layer is composed of the complexus (see page I32), semispinalis dorsi, semispinalis colli, multifidus spinæ, and rotatores spinæ. The seventh layer is formed of the interspinales and the intertransversales.

For the suboccipital muscles see Head and Neck, page I 34.

\section{The Posterior Branches of the Spinal Nerves.}

For the cervical nerves see page 129.

The dorsal, lumbar, and sacral : These are found coming through the muscles filling the spinal gutter in two sets, an external and internal set. They supply the overlying muscles and terminate in the integument.

Not much time need be spent in dissecting out these nerves; they can be examined as the dissection proceeds.

\section{DISSECTION.}

Continue to remove the integument from the forearm, hand, and fingers, as before directed. See page 360 .

The arrangement of the superficial veins should be noted. There is a great amount of variation in their formation. The usual plan is given below. 


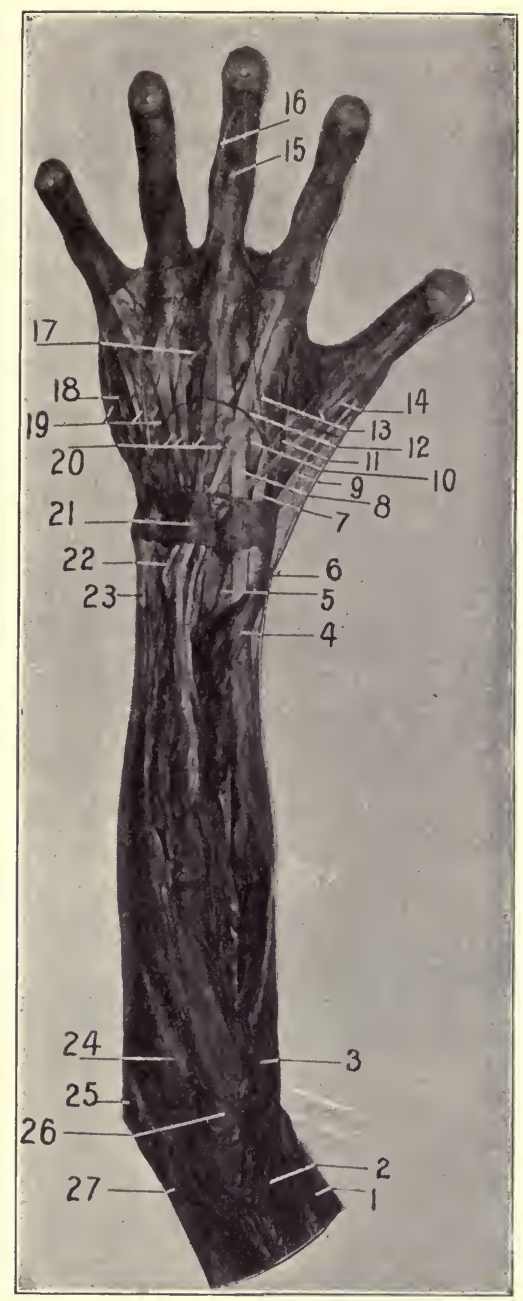

Fig. 78. Dissection of the Forearm, Posterior. [This specimen presents the "clubbed" fingers typical of tubercular diathesis.] - I, Brachialis anticus. 2, Supinator longus (brachioradialis). 3, Extensor carpi radialis longior. 4, The extensor ossis metacarpi pollicis. 5, The anterior interosseous artery, having passed to the posterior of the forearm. 6 , Radial nerve. 7, Tendon of the extensor carpi radialis longior. 8, Tendon of the extensor carpi radialis brevior. 9, Tendon of the extensor brevis pollicis (extensor primi internodii pollicis). Io, Posterior radial carpal arterv. II, Dorsalis pollicis (which also gives off the dorsalis indicis). 12, Communicating branch between the radial and the dorsal cutaneous branch of the ulnar nerves. I3, Radial digital branches. I4, Extensor longus pollicis (extensor secundi internodii pollicis). 15. The middle portion of a tendon of the extensor communis digitorum. 16, The lateral portion of the same tendon. 17, Slip connecting the tendon of the extensor communis digitorum of the ring finger with that of the middle. 18, Tendon of the extensor minimi digiti. 19, Digital branches of the dorsal cutaneous nerve (posterior carpal of ulnar). 20, Tendons of the extensor communis digitorum. 21, The posterior annular ligament. 22, The extensor indicis. 23, Extensor carpi ulnaris. 24, The anconeus. 25, The olecranon process. 26, The external condyle of humerus. 27, Triceps. 


\section{The Superficial Veins of the Forearm.}

There are usually two main trunks, the posterior ulnar and posterior radial veins. These begin at the back of the hand in an irregular plexus and proceed up the back of the forearm, communicating with each other by branches. The posterior ulnar curves forward just below the internal condyle of the humerus to unite with the anterior ulnar to form the common ulnar. See page 282. The posterior radial empties into the radial at the outer side of the upper part of the forearm.

\section{The Superficial Nerves of the Forearm and Hand-}

These are-for the forearm the external cutaneous branch of the musculospiral and a posterior branch of the cutaneous portion of the musculocutaneous nerve, for the outer half; and the posterior division of the internal cutaneous, for the inner half of the forearm.

In the hand the radial nerve supplies the outer half of the dorsum and the outer two and one-half fingers, while the dorsal cutaneous branch of the ulnar nerve completes the inner half of the back of the hand and two and onehalf fingers.

\section{DISSECTION.}

Remove the deep fascia from the back of the forearm, hand, and fingers. At the wrist leave a band of the deep fascia about an inch wide, the posterior annular ligament of the wrist.

Clean all the muscles, vessels, and nerves presenting.

The Posterior Annular Ligament. Fig. 78.

This will be found to consist of the fibres of the deep fascia which have been reinforced by additional transverse fibres. This thickened band is about an inch wide, and is attached from the outer border of the radius around the back of the wrist to the inner border of the pisiform and 
cuneiform bones. It is not attached to the ulna, but passes over it, helping to hold it in place.

By extensions from the inner surface of the ligament to the ridges of the radius, the grooves upon the back of that bone are converted into canals, through which the long tendons of the muscles from the posterior of the forearm to the hand pass. These canals are arranged as follows :-

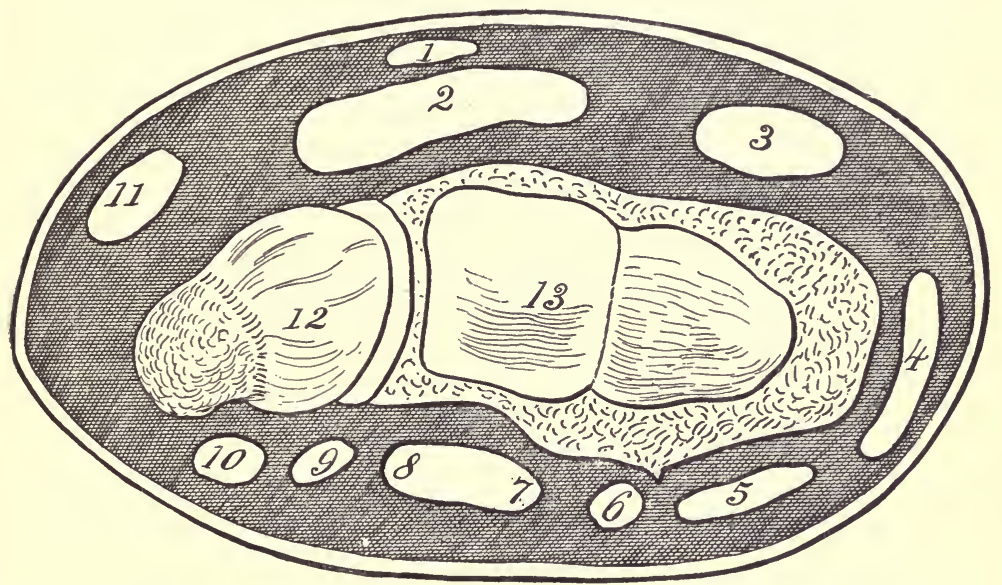

Diag. 24. The Annular Ligament of the Wrist and the Approximate Relations of the Various Tendons. (I. S. H.) - I, Palmaris longus. 2, Middle compartment of annular ligament, anterior, contains the tendons the flexor sublimis and profundus digitorum, the flexor longus pollicis, and the median nerve. 3, Flexor carpi radialis. 4, Extensor ossis metacarpi pollicis, extensor brevis pollicis. 5, Extensor carpi radialis longior and brevior. 6, Extensor longus pollicis. 7, Extensor indicis, and 8, Extensor communis digitorum in one compartment. 9, Extensor minimi digiti. Io, Extensor carpi ulnaris. I1, Flexor carpi ulnaris. 12, Ulna. 13, Radius.

Upon the outer side of the radius are the tendons of the extensor metacarpi pollicis and the extensor brevis pollicis; upon the back of the radius at the outer side are the tendons of the extensor carpi radialis longior and brevior, separated, however, by the synovial membrane from each 
other; next is the extensor longus pollicis in a narrow canal; then in a broad one lies the tendons of the extensor communis digitorum and the extensor indicis ; in a compartment behind the radio-ulnar articulation is the slender tendon of the extensor minimi digiti, and lastly, lying in the groove at the outer side of the styloid process of the ulna, is the tendon of the extensor carpi ulnaris.

Extensor Carpi Radialis Longior. Fig. 78.

Origin.-From the lower third of the external condyloid ridge of the humerus and the intermuscular septa.

Insertion. - Into the radial side of the base of the second metacarpal bone.

Nerve Supply. - The musculospiral (fibres from the sixth cervical nerve).

Action.-Extend the wrist, feeble flexor of forearm, slight abductor of wrist.

Extensor Carpi Radialis Brevior. Fig. 78.

Origin.-By the common tendon from the external (epi) condyle of the humerus, and from the intermuscular septa.

Insertion. - Into the radial side of the base of the third metacarpal bone.

Nerve Supply. - The posterior interosseous branch of the musculospiral. The fibres coming from the sixth and seventh cervical nerves.

Action.-To extend the wrist, slight extensor of the forearm.

Extensor Communis Digitorum. Fig. 78 .

Origin.-By the common tendon from the external (epi) condyle of the humerus, and from the intermuscular septa, and from the inner surface of the deep fascia of the forearm.

Insertion. - The muscle is provided with four tendons which pass through the fourth compartment of the pos- 
terior annular ligament in a bunch. Lower down they separate to pass to their respective fingers. Across the knuckles the tendons of the three outer fingers are united by fibrous bands which diverge from the tendon of the ring finger to the tendons of the middle and little finger on either side. The insertion into the fingers is as follows :From the side of each tendon a fibrous extension passes to the lateral ligaments of the metacarpophalangeal articulations. Beyond this each tendon divides into three slips. The middle slip is inserted into the base of the second phalanx, the two lateral slips pass forward to be inserted into the base of the last phalanx.

Each tendon of the extensor communis digitorum receives laterally the insertions of the lumbricales (see page 307) and the dorsal and palmar interossei muscles (palmar, see page 309 ; dorsal, page 388).

Nerve Supply. - The posterior interosseous (the filaments issue from the seventh cervical nerve).

Action.-To extend the fingers, hand, and wrist, to slightly extend the forearm.

Extensor Minimi Digiti. Fig. 78.

Origin.-The same as the preceding, with which it unites for the upper third of its length.

Insertion.-Into the tendon of the common extensor going to the little finger.

Nerve Supply.-Same as the preceding.

Action.-To extend the little finger, the hand, and the wrist, and a very feeble extensor of the elbow.

Anconeus. Fig. 78 .

Origin.-From the back of the external (epi) condyle of the humerus, and from the posterior ligament of the elbowjoint. 
Insertion.-Into the outer surface of the olecranon, and the upper fourth of the ulna.

Nerve Supply. - The musculospiral (through the seventh and eighth cervical nerves).

Action.-To extend the forearm.

\section{Extensor Carpi Ulnaris. Fig. 78.}

Origin.-From the external (epi) condyle of the humerus by the common tendon, from the upper two-thirds of the posterior ridge of the ulna in common with the flexor carpi ulnaris and the flexor profundus digitorum, and from the inner surface of the deep fascia of the forearm and the adjacent intermuscular septa.

Insertion.-Into the back of the base of the metacarpal bone of the little finger.

Nerve Supply. - The posterior interosseous (the filaments from the eight cervical).

Action.-To extend the wrist, to adduct the wrist, to feebly extend the forearm.

\section{DISSECTION.}

Separate the superficial muscles from each other clear to their origins; do not remove the annular ligament yet until the deeper muscles have been studied, after which it can be cut away as far as necessary to expose the relations of the tendons to each other and to the back of the wrist.

Trace the recurrent interosseous artery upward to the elbow, cutting through the anconeus muscle. Follow downward from the supinator brevis, the posterior interosseous artery and nerve, the former to its anastomosis with the termination of the anterior interosseous, and the latter to the ganglion upon the back of the wrist.

Complete the cleaning of the radial artery and its branches, noting the formation of the posterior carpal anastomoses.

Remove the fascia from the interossei muscles and demonstrate their insertion.

Extensor Ossis Metacarpi Pollicis. Fig. 79.

Origin.-From the outer surface of the ulna for two 


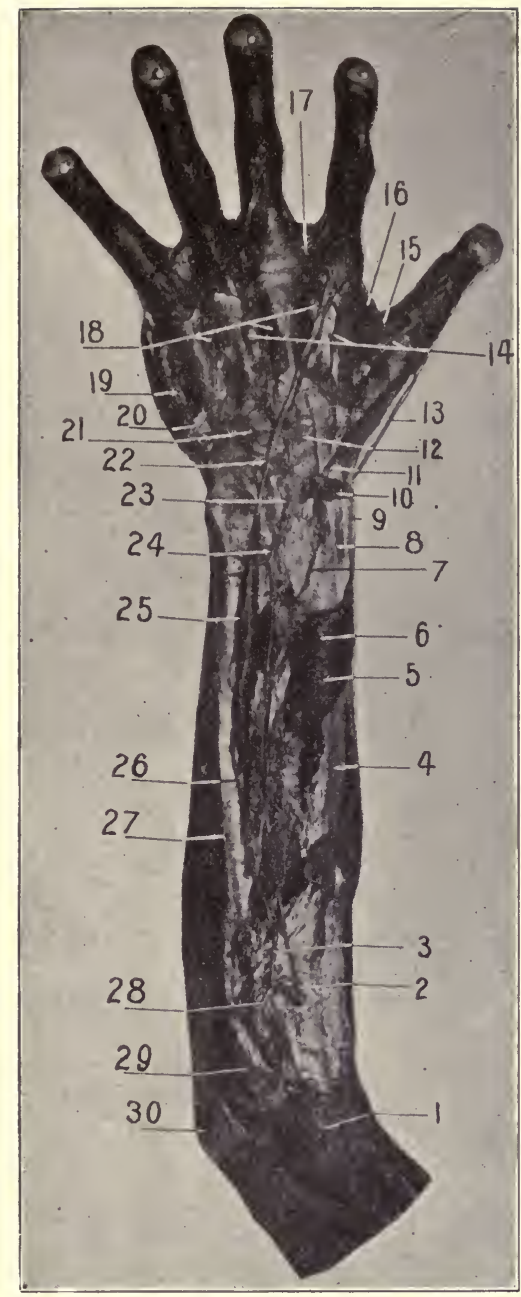

Fig. 79. Dissection of the Forgarm, Posterior.-i, External condyle. 2, Posterior interosseous nerve coming through the supinator brevis muscle. 3, The posterior interosseous artery. 4, Radius. 5, Extensor ossis metacarpi pollicis. 6, Extensor brevis pollicis (extensor primi internodii pollicis). 7, Outer branch of the anterior interosseous. 8, Tendon of the extensor carpi radialis brevior. 9, Styloid process of radius. 10, Small portion of the posterior annular ligament. 11, Tendon of the (divided) extensor longus pollicis (extensor secundi internodii pollicis). 12, Posterior carpal arch, radial side. 13, Radial nerve, cutaneous branch to thumb. I4, Digital arteries. 16, Abductor indicis, first dorsal interosseous muscle. 17, Anastomosis of dorsal with palmar digital arteries. 18, The three inner interossei. 19, Abductor minimi digiti. 20, Tendon of the extensor carpi ulnaris. 21, Posterior carpal arch, ulnar side. 22, The extensor indicis. 23, Ganglionic enlargement of the posterior interosseous nerve at the back of the wrist-joint. 24, Inner division of the anterior interosseous artery. 25, Posterior interosseous artery. 26, The same. 27, The ulna. 28. Recurrent interosseous artery. 29, Anconeus. 30, Olecranon process. 
inches above the middle, from the middle third of the posterior surface of the radius, and from the intervening portion of the interosseous membrane between its bony origins; from the intermuscular septa.

Insertion. - Into the outer aspect of the base of the first metacarpal bone, and into the deep fascia of the ball of the thumb.

Nerve Supply.-The posterior interosseous (from the seventh cervical nerve).

Action.-Extensor and abductor of thumb, abductor of hand, slight supinator of forearm.

Extensor Brevis Pollicis. (Extensor primi internodii pollicis.) Fig. 79.

Origin.-From the posterior surface of the interosseous membrane and the radius below the above muscle, with which it is continuous.

Insertion.-Into the outer surface of the base of the first phalanx of the thumb.

Nerve Supply.-Same as the above.

Action.-To extend and abduct the thumb, to abduct the hand.

Extensor Longus Pollicis. (Extensor secundi internodii pollicis.) Fig. 79 .

Origin.-From the posterior surface of the ulna for its middle third, from the adjoining portion of the interosseous membrane.

Insertion.-Into the base of the second phalanx of the thumb.

Nerve Supply._-Same as the above.

Action.-To extend the thumb and hand, to supinate the forearm. 
Extensor Indicis. Fig. 79.

Origin.-From the posterior surface of the ulna and the interosseous membrane just below the extensor longus pollicis.

Insertion.-Into the tendon of the common extensor for the index finger.

Nerve Supply.-Same as the above.

Action.-To extend the index finger and hand.

Supinator Brevis. Figs. 67, 79 .

Origin.-By the common tendon from the external (epi) condyle of the humerus, from the external lateral ligament of the elbow-joint, from the orbicular ligament, from the ridge and triangular surface of bone below the lesser sigmoid cavity of the ulna, from the intermuscular septa.

Insertion.-Into the upper third of the radius from its neck to the insertion of the pronator radii teres below, and limited by the oblique line in front.

Nerve Supply. - The posterior interosseous, which passes through the muscle from the front to the back of the forearm. (The fibres come from the sixth cervical nerve.)

Action.-To supinate the forearm.

\section{The Posterior Interosseous Artery. (See page 297.)} Fig. 79.

This is a branch from the common interosseous in the anterior part of the forearm. It turns backward, passing between the radius and ulna on either side, and the interosseous membrane below, and the oblique ligament above, and appears in the posterior part of the forearm between the supinator brevis and the extensor ossis metacarpi pollicis muscles. It descends between the deep and superficial layers of muscles until just above the wrist it terminates in branches which anastomose with the anterior iriterosseous 
and the posterior carpal branch of the radial. Its branches are distributed to the surrounding muscles and to the wristjoint.

The Posterior Interosseous Nerve. Fig. 79.

Is a branch from the musculospiral just above the external (epi)condyle in the interval between the supinator longus and the brachialis anticus. See page 3i 8 . It descends to the anterior surface of the supinator brevis, through which it passes to the back of the forearm, where it is found under the superficial layer of muscles. As it descends it passes under the deep muscles to the interosseous membrane, upon which it lies, and ends just above the wrist-joint in a small ganglion.

The nerve gives off branches to all the muscles at the back of the forearm and the ganglion, to the wrist and carpal joints.

The Termination of the Anterior Interosseous Artery. Fig. 79 .

This is found coming through the interosseous membrane about an inch and a half above the wrist. It descends to the back of the wrist, where it enters into an anastomosis with the posterior carpal of the radial. In its course it forms an anastomosis with the posterior interosseous above the wrist.

\section{The Recurrent Interosseous Artery. Fig. 79.}

Is a branch of the common or the posterior interosseous. It is found on the back of the forearm as it curves upward over the supinator brevis. It runs upward between this muscle and the anconeus to the back of the elbow-joint between the external (epi) condyle and the olecranon process, where it enters into the elbow anastomosis. 
The arteries which form the anastomosis about the elbow-joint are the following:- In front of the elbow: On the outer side there is the termination of the superior profunda above and the recurrent radial below; on the inner side the anterior branch of the anastomotica magna above and the anterior ulnar recurrent below.

Behind the elbow: On the outer side the articular branch of the superior profunda above, and the recurrent interosseous below; on the inner side the posterior branch of the anastomotica magna and the inferior profunda above, with the posterior ulnar recurrent below.

On the back of the elbow all these arteries inosculate to form a free plexus and communication with each other.

\section{The Radial Artery at the Wrist. Fig. 79.}

Continuing the description of the artery from the lower end of the radius where it was left off, see page 293.

The artery is seen to run backward around the lower end of the radius, between the tendons of the extensor ossis metacarpi and brevis pollicis and the external lateral ligament of the wrist-joint, to the interval between the bases of the first and second metacarpal bones, where it lies under the tendon of the extensor longus pollicis muscle. It now passes forward and inward between the first and second metacarpal bones and the two heads of the abductor indicis muscle to the palmar side of the hand, where it is continued as the deep arch. See page 3 IO.

The Branches of the Radial at the Back of the Wrist.(I) The posterior carpal arises as the radial issues from under the tendon of the extensor brevis pollicis, runs inward across the back of the wrist, and terminates by anastomosing with the posterior ulnar carpal and the anterior and posterior interosseous arteries. From this posterior 
carpal arch the second and third dorsal interosseous arteries are distributed to the third and fourth interosseous spaces. (2) The first dorsal interosseous, or the metacarpal : a small branch from the radial under the tendon of the extensor longus pollicis, to the space between the second and third metacarpal bones. (3) The dorsalis pollicis and (4) the dorsalis indicis are small arteries distributed to the dorsal surface of the thumb and index fingers. The dorsalis pollicis divides into two branches to either side of the thumb, while the dorsalis indicis supplies the outer side of the index finger.

\section{The Collateral Digital Arteries.}

The dorsal digital arteries divide at the web of the fingers into small branches to the adjacent sides of the dorsal surface of the fingers.

The posterior carpal branch of the ulnar is found issuing from the front of the forearm under the tendon of the flexor carpi ulnaris muscle. It passes to the back of the wrist and enters into the posterior carpal anastomoses. See above.

The Dorsal Interossei. Fig. 79.

These are four in number, one on either side of the middle finger, one to the radial side of the index, and one to the ulnar side of the ring finger respectively.

Origin.-By two heads from the contiguous sides of the metacarpal bones between which they lie. The origin is more extensive from that metacarpal bone of the finger into which they are inserted.

Insertion.-Into the base of the first phalanx, and into the lateral margin of the tendon of the extensor communis digitorum. 
Nerve Supply.-The ulnar through the deep palmar branch (the filaments coming from the eighth cervical nerve).

Action.-To extend the second and third phalanges, to flex the first phalanges of the four fingers to which they

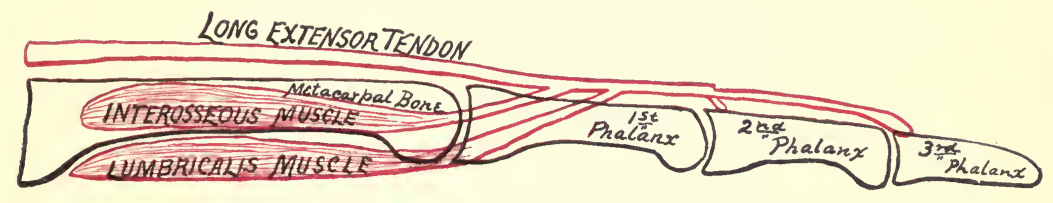

Diag. 25. DiAgRAM OF THE INSERTION OF THE LUMBRICALES AND INTEROSSEI Muscles into the Aponeurotic Tendon of the Extensor Communis Digitorum, to Illustrate their Double Action as Flexors of the First Phalanges and Extensors of the Last Two Sets of Phalanges. ( $I$. $S$. $H$,)

are attached. This action is in common with the palmar set of interossei and the lumbricales. The distinctive action of the dorsal set of muscles is abduction of the fingers from a line through the middle finger.

\section{THE SPINAL CORD.}

\section{DISSECTION.}

The dissection of the spinal cord is not practicable in the usual dissectingroom subject, for the cord is so soft that its gross structure cannot be determined.

A perfectly fresh subject that has been injected as soon as received, or a " pickled subject" that has been preserved with a zinc injection and a hardening preservative solution, is to be selected.

If the brain has been removed as previously described, the dissection can be continued by cleaning out all the mass of muscles which fill in the spinal gutters from the base of the skull to the end of the sacrum. Clean the spinous processes, laminæ, and articular processes, noting the ligaments of these parts of the spine. 
Beginning above with a pair of curved bone cutters (having a blunt-pointed lower blade), carefully sever all the laminæ on both sides from above downward, keeping as close to their outer extremities as possible. Remove the laminæ, the spinous processes, and connecting ligaments. Clean the posterior surface of the dura. Carefully trace outward the nerve trunks as they pass from the dura to enter the intervertebral foramina.

Open the dura carefully from above downward.

\section{The Spinal Dura. Figs. 80, 8 I.}

This membrane is similar in structure to the cranial dura, with which it is continuous at the foramen magnum. It differs in this point that it does not in the spine form the inner periosteum for the vertebræ as it does for the cranial bones, though it is connected to the periosteum and ligaments of the former by a loose connective tissue. It extends from the foramen magnum to about the second or third sacral vertebra, beyond which it is prolonged as a cord-like sheath for the filum terminale of the cord, and is attached to the base of the coccyx.

The dura is pierced by the spinal nerve, around which it is prolonged in the shape of funnel-like sheaths for a short distance, until it blends with the sheaths of the nerves.

The inner surface of the dura is smooth, as in the brain, and is separated in a similar manner from the arachnoid membrane by the subdural space. The spinal dura does not send processes into the fissures of the cord.

\section{The Spinal Arachnoid.}

This reaches as low as the dura, to the inner surface of which it is closely applied (but separated from it by the subdural space, which in the normal condition is very narrow). It surrounds the nerves within the tubular sheaths of the dura and terminates like that membrane by becoming continuous with the sheaths of the nerves. 


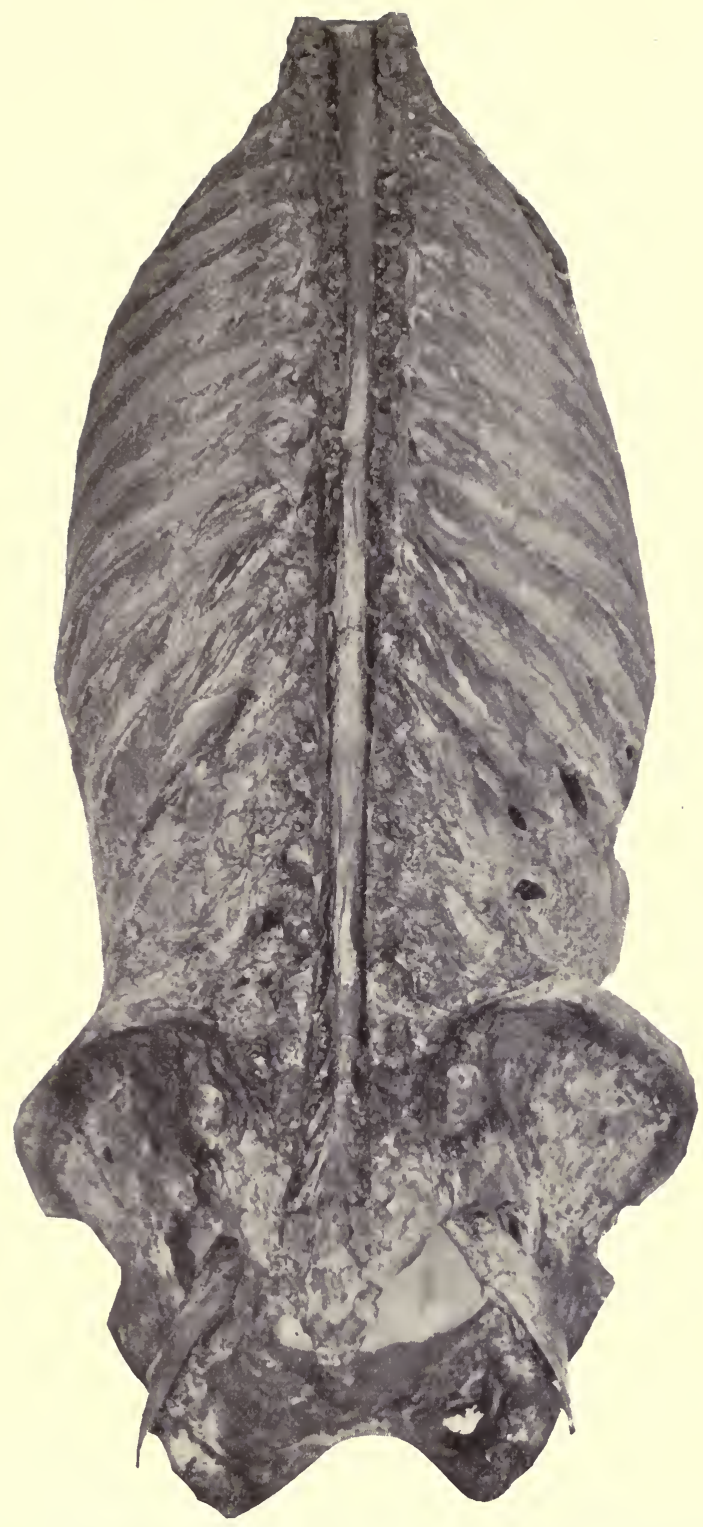

Fig. 80. Dissection of the Spinal Cord. [Photograph of the first prize dissection at the University Medical College, I895, by J. J. Moorhead.]-The dura not opened. The tubular processes which pass outward enclosing each pair of the spinal nerves are well shown. 
The Spinal Pia. Fig. $8 \mathrm{I}$.

This, like in the brain, is the vascular membrane which encloses the cord from the foramen magnum to the end of the cord proper at the second lumbar vertebra, beyond which, under the name of the filum terminale (mostly pia but little cord), it extends to the base of the sacrum.

From the lateral margins of the cord (pia) saw-teeth-like processes pass outward to be attached by the apices of the teeth to the inner surface of the dura (and thus pin the arachnoid to the dura at these points). There are $2 \mathrm{I}$ of these teeth, and they are termed the ligamentum denticulatum. They serve to hold the cord steadily in its proper position within the centre of the spinal canal.

The space between the arachnoid and the pia is the spinal subarachnoidean space and communicates with the similar spaces of the brain. It contains the cerebrospinal fluid, which can thus flow freely from the spinal canal into the subarachnoid spaces at the base of the brain, and thence by way of the foramen of Majendie into the ventricular cavities of the same.

The pia dips into the anterior and posterior fissures of the cord.

The pia and arachnoid are connected by filaments of connective tissue constituting the subarachnoid tissue. It becomes thickened around the vessels which pass to (and from) the cord at its posterior fissure and here forms an incomplete partition called the septum.

The Spinal Cord. Fig. 8I.

The spinal cord is 16 to 18 inches in length, extending from the margin of the foramen magnum to the first or second lumbar vertebra. Its diameter is from twoto three-fifths of an inch. It weighs about one ounce. 


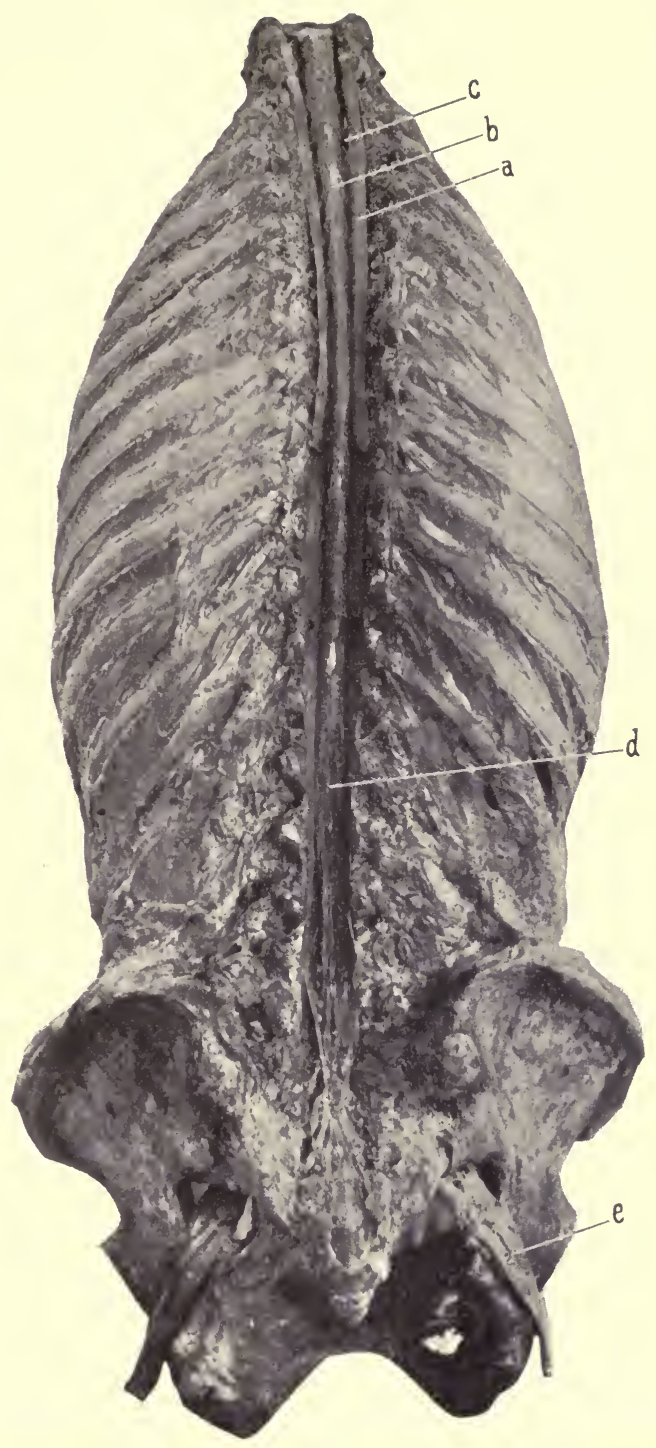

Fig. 8I. Dissection of the Spinal Cord. [Photograph of the first prize dissection at the University Medical College, 1895, by J. J. Moorhead.]- $a$, Dura of cord reflected. $b$, Arachnoid and pia of cord still enveloping it. The former membrane is very thin and cannot be demonstrated in such a dissection. The pia and its vascular construction is apparent. $c$, One of the spinal nerve roots. Many of the others are shown. $d$, The cauda equina. $e$, The great sciatic nerve. 
Its sections are generally cylindrical, except in the cervical region, where they become slightly flattened from before backward. It is divided into three regions, cervical (four inches long), the dorsal (ten and one-half), and the lumbar (the rest of the cord). In the first and third regions, the cord is slightly enlarged and forms the cervical and lumbar enlargements.

The cervical enlargement extends from the third cervical to the second dorsal vertebra. The lumbar from the ninth to the last dorsal or first lumbar vertebra.

The cord is divided by two incomplete fissures into two symmetrical halves. The central substance which connects the halves of the cord is the commissure, and within it is a minute canal, the central canal of the cord, which opens into the fourth ventricle at the calamus scriptorius. It is the remains of the central canal which extended through the primitive cerebrospinal axis.

The anterior fissure of the cord is a wider but shallower fissure than the posterior, which is narrow and deep. The first is one-third, the second one-half of the anteroposterior diameter of the cord. These fissures are co-extensive with the anterior and posterior fissures of the medulla.

The surface of the cord is further divided by two shallow grooves-the anterolateral and posterolateral,--from which issue the anterior and posterior nerve roots, into three tracts, the anterior, lateral, and posterior. Here it is apparent that the anterior tract is continuous with the pyramidal tract of the medulla, the lateral with the lateral, and the posterior into the funiculi gracilis and cuneatus of the medulla.

On section, the characteristic arrangement of the white and gray matter of the cord becomes apparent. The gray 
matter is enclosed within the white, and exists in the shape of two large " commas" ('), turned with the head anterior, the convex surfaces turned toward each other, and connected at their middle by the gray commissure.

Here we must pause. The subject of the internal arrangement of the cord and brain is most fascinating, but it does not properly enter into the scope of such a manual of anatomy as this. However, the student should not be content to let the matter rest here, but should pass from this hasty and necessarily incomplete description of the brain and cord to the microscopic anatomy of the same, then to their functions, and lastly to the application of all this knowledge to the workings of medical and surgical practice.

\section{The Spinal Nerves. Fig. $8 \mathrm{I}$.}

They arise from the side of the cord in 32 pairs by two sets of roots as already indicated, the anterior (motor) and posterior (sensory), the former being smaller than the latter. Inasmuch as the spinal cord does not reach to the lower extremity of the spinal canal, it follows that, as the spinal nerves leave the cord they have to descend in order to leave by their proper foramen, the downward inclination of the nerves increases from above downward, until at the lower end of the cord the canal is filled with the nerves from below the last dorsal vertebra, which run parallel with each other and then slightly outward to reach their foramen of exit ; this arrangement produces a tasseled appearance named the cauda equina.

The point at which the spinal nerves leave the side of the cord and their points of emergence are given in the following table copied from Gowers :- 

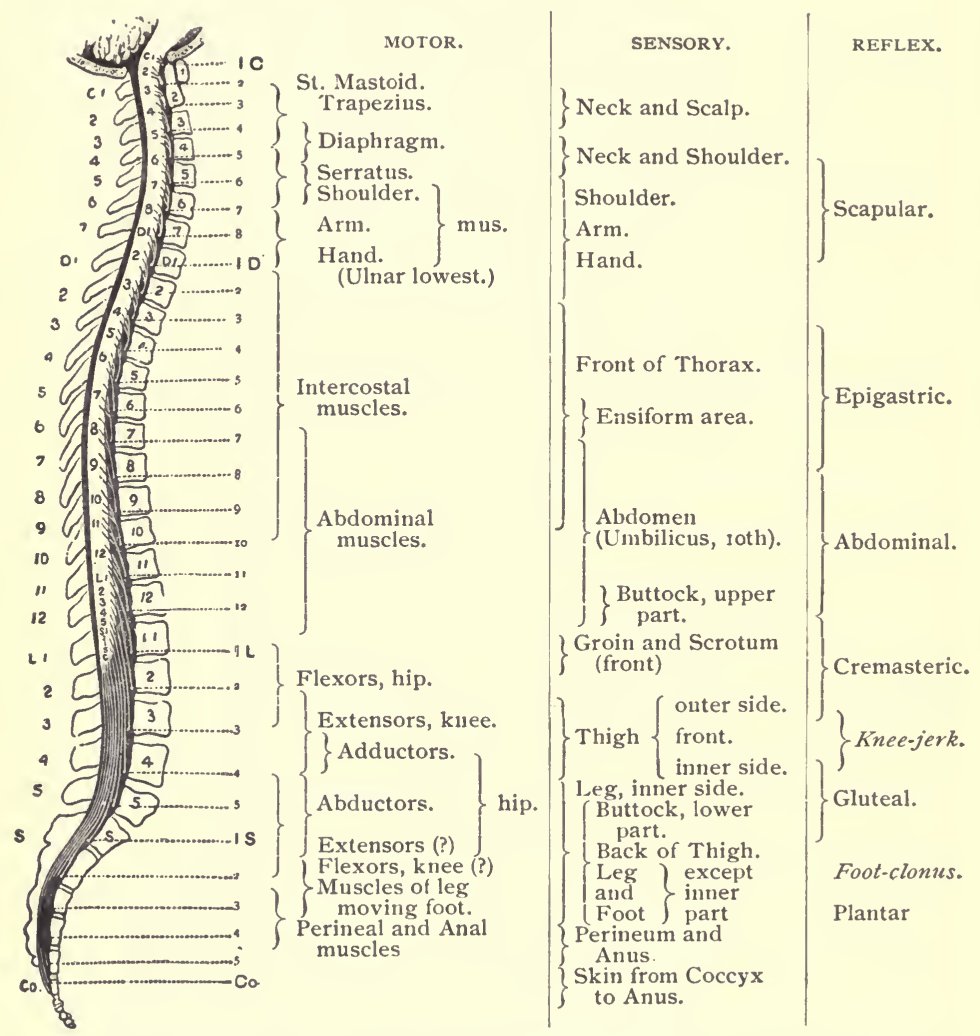

Diagram and Table showing the Approximate Relation to the Spinal Nerves of the Various Motor, Sensory, and Reflex Functions of the Spinal Cord (from anatomical and pathological data). 


\section{THE PERINEUM, Male.}

The perineum is a diamond-shaped space corresponding to the boundaries of the pelvic outlet.

The pelvic outlet is formed by the lower borders of the ossa innominata joined in front at the symphysis and behind by the wedge-shaped sacrum.

\section{LANDMARKS.}

The borders of the perineal space can be felt, and are formed by the under surface of the pubic arch, in front; the rami of the pubes and ischium, and the tuberosities of the latter, at the side anteriorly; the lower border of the gluteus maximus, beneath which can be outlined the great sacrosciatic ligaments, at the side posteriorly; and the tip of the coccyx in the posterior median line.

In the median line, in front of the coccyx, is the anus, and in front of the anus reaching up to the base of the scrotum is the bulb of the penis.

Laterally, in the anterior part of the perineum, the crura of the penis will be felt covering the ischiopubic rami; and posteriorly, at the side of the anus, is a depression filled with fat-the ischiorectal fossa.

The perineum is divided by a transverse line from the tuberosities of the ischium into the anterior compartment, or the perineum proper, and the posterior division, or the ischiorectal fossæ.

In order to understand the construction of the pelvic floor it will be well to first study the arrangement of the fasciæ which assist in closing the pelvic outlet and support the pelvic viscera in position. Start from the interior of the pelvis. Attached to the inner surface of the obturator membrane and the margin of the bone adjacent thereto, 
especially posteriorly and below, is the obturator internus (see Fig. I I4); and covering over the front of the sacrum is the pyriformis, which disappears through the greater sacrosciatic foramen. These muscles are covered by the obturator and pyriformis fascia respectively.

The Obturator Fascia. Fig. I I 3. Diags. 26, 27.

It covers the obturator internus and is attached to the inner surface of the pelvis around the origin of the muscle;

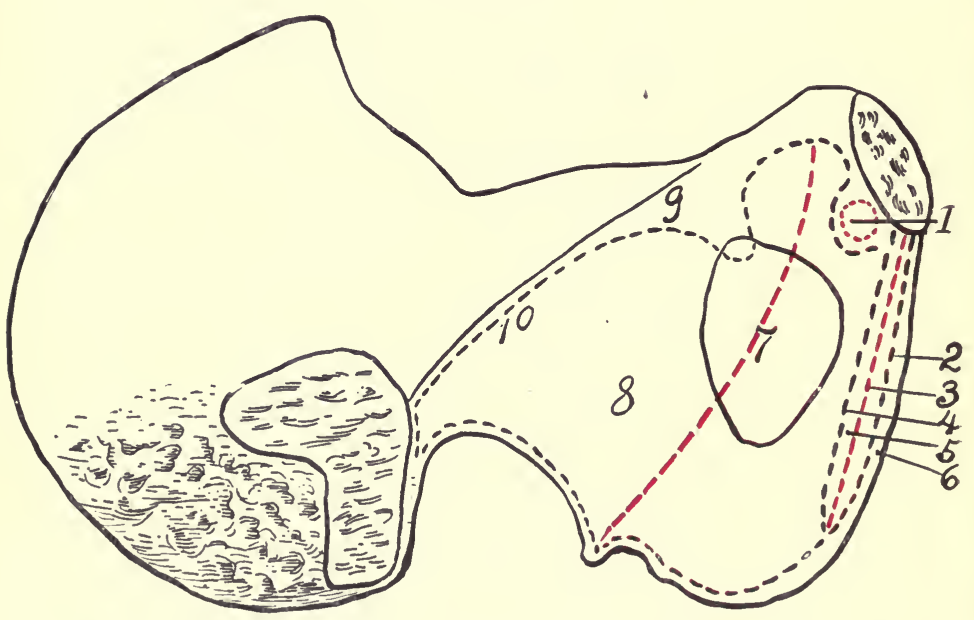

Diag. 26. The Attachments of the Pelvic Fascia. (Modified from Morris, I. S. H.)-I, Levator ani muscle. 2, Perineal fascia. 3, Anterior layer of triangular ligament. 4, Posterior layer of triangular ligament. 5, Compressor urethræ muscle. (Deep transversus perineæ.) 6, Erector penis (Ischiocavernosus). 7, The "white line." 8 , Origin of the obturator internus. 9, Iliopectineal line. Io, Dotted line shows attachment of the obturator fascia.

above, to the iliopectineal line ; in front, to the inner surface of the pubes a little external to the symphysis, to the inner lip of the rami of the pubes and ischium; below, to the inner 
lip of the tuberosity of the ischium, blending with the falciform extension of the great sacrosciatic ligament; behind, about the margin of the great sacrosciatic notch and to the front of the articular surface for the sacrum, and continuous above with the posterior end of the iliopectineal line. By its attachment to the great sacrosciatic ligament the fascia is carried over the lesser sacrosciatic foramen; underneath the ligament an extension of the fascia is prolonged outward, with the obturator internus muscle, into the gluteal region.

The obturator fascia is continuous behind with the thin membrane covering the pyriformis muscle; at its anterior part it is pierced by the obturator nerve and vessels. It is crossed obliquely from above downward by the line of attachment of the rectovesical fascia, the so-called "white line," which curves downward and backward from the inner and upper surface of the pubes across the middle of the obturator foramen and ends at the base of the spine of the ischium. The obturator fascia above the "white line" is within the pelvic portion of the abdominal cavity, and is covered by peritoneum; the portion below is extrapelvic and belongs to the perineum. This perineal or anal portion furnishes a canal (of Alcock) through which the pudic vessels pass.

\section{The Pyriformis Fascia. Fig. I I 3.}

This is a thin membrane covering the muscle and sciatic plexus of nerves. It is adherent about the origin of the pyriformis and blends in front with the obturator and rectovesical fasciæ.

The Rectovesical Fascia. Fig. I I 3. Diags. 26, 27, 28.

This may be said to come off the inner surface of the obturator fascia along a line reaching from the inner, 
upper surface of the body of the pubes to the spine of the ischium. This fascial line is slightly concave upward, and from its appearance is called the "white line."

Posteriorly the rectovesical fascia blends with the pyriformis fascia. In the angular interval between the recto-

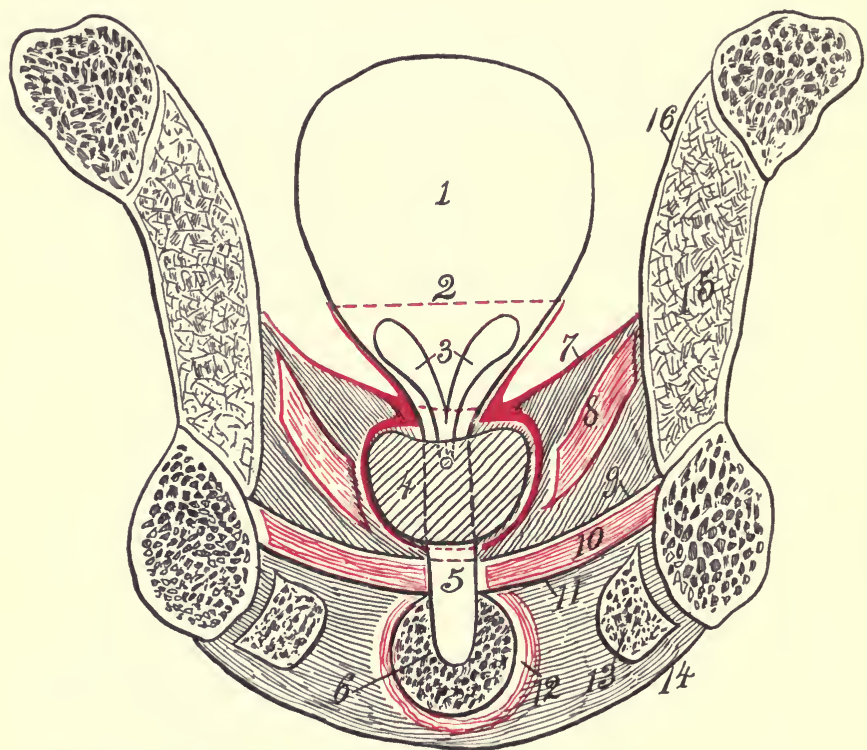

Diag. 27. Transverse Section of Pelvis through Anterior Portion of Perineum. (Modified from Quain, I. S. H.)-I, Bladder. 2, Dotted line to show the extent to which the vesical reflection of the rectovesical fascia reaches. 3, Base of bladder and seminal vesicles. 4, Prostate enclosed in its capsule of rectovesical fascia. 5. Urethra. 6, Bulb of corpus spongiosum. 7, Rectovesical fascia. 8, Levator ani muscle. 9, Deep layer of triangular ligament. 10, Compressor urethra muscle. II, Superficial layer of triangular ligament. I2, Accelerator urinæ. 13, Erector and crus penis. 14, Perineal fascia. 15, Obturator internus muscle. 16, Obturator fascia.

vesical and obturator fasciæ the levator ani muscle is attached (along the so-called "white line"). The rectovesical fascia drops downward and inward, covering the upper surface of the levator ani (and coccygeus muscles), 
reaching to the bladder, prostate, and rectum. Beneath these structures the fascia is continuous with the similar layer from the opposite side. The fascia thus completely closes the pelvic outlet and supports the pelvic viscera.

At the side of the rectum, bladder, and prostate the fascia splits into two layers,- - an upper layer that is reflected upward on to the rectum for a distance of two inches or so and becomes lost ; on to the bladder it reaches upward above the seminal vesicles (thus shutting them out from the pelvic cavity proper), then merges into the wall of the bladder. The lower layer is continued inward to join the opposite layer beneath the bladder, and also sends a lamina downward to enclose the prostate, form its capsule, and become continuous with the deep layer of the triangular ligament. Between the fascia, bladder, and prostate lies a collection of large veins known as the vesicoprostatic plexus. The rectum lies in close contact with the prostate and the base of the bladder, separated from them only by a layer of the rectovesical fascia, which is reflected upward and downward upon it. The lower layer is lost in the muscular tissue of the rectum above the anus; the upper reflection can be traced about two inches, when it, too, is lost in the substance of the bowel.

The portions of the fascia reaching to the rectum are called the true ligaments of the rectum, and the portions reaching to the sides of the bladder are likewise called the lateral true ligaments of the bladder. Besides these ligaments (all parts of the same fascial plane) the fascia which extends from both sides of the inner surface of the pubes to the front of the neck of the bladder and prostate are called the anterior true ligaments of the bladder and prostate. These are the best marked, and contain involuntary muscu- 
lar fibres. They are also called the puboprostatic ligaments.

In the female the rectovesical fascia encloses the vagina and forms a sheath for it similar to the prostate in the male.

The Anal (Ischiorectal) Fascia. Diags. 27, 28. Fig. 82.

This is a thin layer which covers the lower surface of the levator ani muscle and coccygeus muscles. Laterally, it is attached to the obturator fascia close under the origin of the levator ani muscle from the "white line;" behind, it blends with the obturator fascia ; in front, it becomes continuous with the triangular ligament; centrally, it passes into the fibrous tissue about the anus.

The prismatic-shaped cavity which is left between the lower part of the obturator fascia and the anal fascia is called the ischiorectal fossa.

The Deep Layer of the Triangular Ligament. Diags. 26, 27, 28.

Posteriorly, this ligament becomes continuous with the anal fascia (also the superficial layer of the triangular ligament and the perineal fascia, as will appear later). Laterally, it is attached all along the inner margin of the rami of the ischium and pubes; anteriorly, to the subpubic ligament, leaving here an opening for the single dorsal vein of the penis. The upper surface of the deep layer of the triangular ligament is continuous at the anterior margin of the levator ani muscle with the rectovesical fascia, which here forms the capsule for the prostate gland. The prostate gland rests upon this portion of the triangular ligament as on a shelf.

The triangular ligament (deep portion) is perforated at its apex by the dorsal vein of the penis ; at its base centrally, by the membranous urethra; at its base laterally, by the pudic artery, vein, and dorsal nerve of the penis. 
In the female this fascia is split for the passage of the vagina.

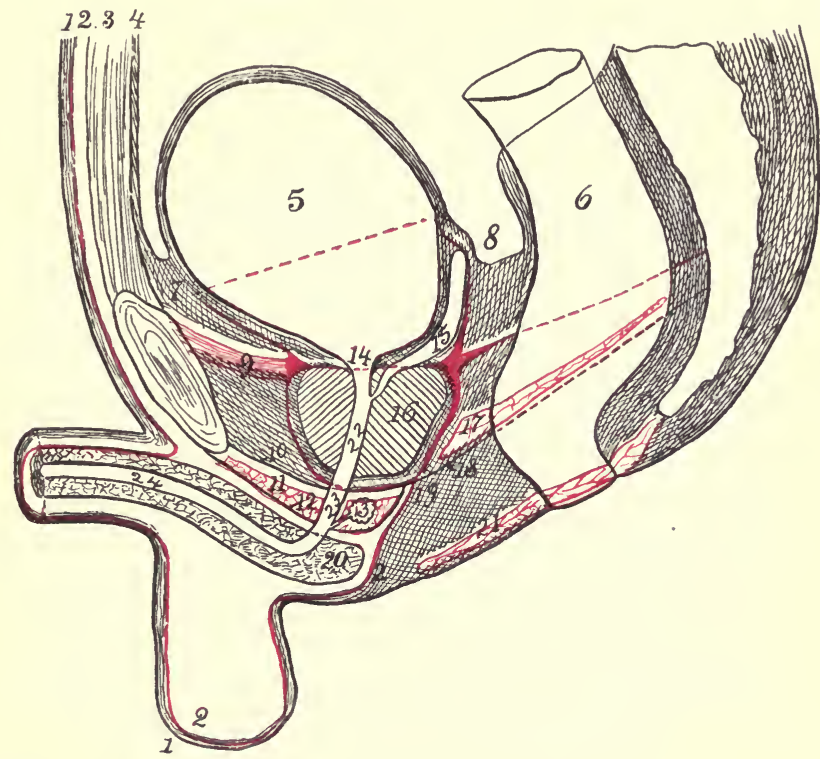

Diag. 28. Median, Vertical Anteroposterior Section of Male Pelvis.

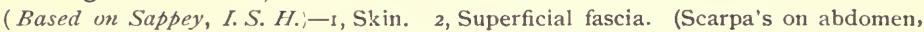
dartos on penis and scrotum, Colles' or perineal in the perineum.) 3, Muscle wall of abdomen. 4, Peritoneum, reflected over bladder and rectum. 5, Bladder. 6, Rectum. 7, Retzius' space. 8, Rectovesical pouch. 9, 9, Rectovesical fascia. The anterior thickened portion forms the puboprostate ligament. Io, Deep layer of triangular ligament. II, Compressor urethræ (deep transversus perineæ). I2, Superficial layer of the triangular ligament. 13, Cowper's gland and duct. I4, Internal meatus of urethra. 15, Seminal vesicle, shut out from general abdominal cavity by upward reflection of rectovesical fascia. 16, Prostate gland enclosed within a capsule formed by the downward extension of the rectovesical fascia. 17, Levator ani muscle. 18, Anal fascia. 19, Meeting point (really line) of two layers of triangular ligament, the perineal and anal fasciæ. 20, Bulb of corpus spongiosum. 2I, Sphincter ani. Figs. 18 and 19 are placed within the ischiorectal fossa. 22, Prostate. 23, Membranous and 24, Spongy parts of urethra.

\section{The Superficial Layer of the Triangular Ligament.} Diags. 26, $27,28$.

This is attached along the inner margin of the ramus of 
the ischium and pubes external to the line of the deep fascia. Behind, it is continuous with the deep layer; in front, it extends to the subpubic ligament and is pierced here for the dorsal vein, arteries, and nerves of the penis.

It is separated from the deep layer by the compressor urethræ muscle, imbedded in which are the dorsal nerve and vein of the penis, the internal pudic artery and its branches to the bulb, the crus, and the dorsal artery of the penis, as well as the Cowper's glands and the membranous urethra. It is pierced by the membranous urethra, the ducts of Cowper's glands, the artery of the bulb, the dorsal artery, vein, and nerve of the penis.

\section{DISSECTION.}

Fill the rectum with oakum and take two or three stitches in the anus.

Incisions. - (I) In the median line from the perineoscrotal junction (base of the scrotum) to the tip of the coccyx, cutting close to the anus upon either side.

(2) Make an incision at the anterior and posterior end of No. I, about three inches transversely outward toward the right and left. Do not cut across the base of these flaps, nor make any other transverse incisions.

Reflect the integument in the form of two large flaps. In doing so be careful not to cut away the external sphincter of the anus, which lies close to the skin. Notice that while the skin is thick in the other parts of the perineum, about the anus it is very thin, and puckered into radiating lines by the contraction of the external sphincter.

\section{The Superficial Fascia. Diag. 28.}

This consists of two layers:-

(a) A subcutaneous layer which varies in thickness according to the amount of adipose tissue present. When this is carefully scraped away a well-marked but thin layer of fascia is seen covering the deeper parts. This is the second layer of the superficial fascia, and is called the perineal fascia, or fascia of Colles.

(b) The perineal fascia, fascia of Colles, the deep 


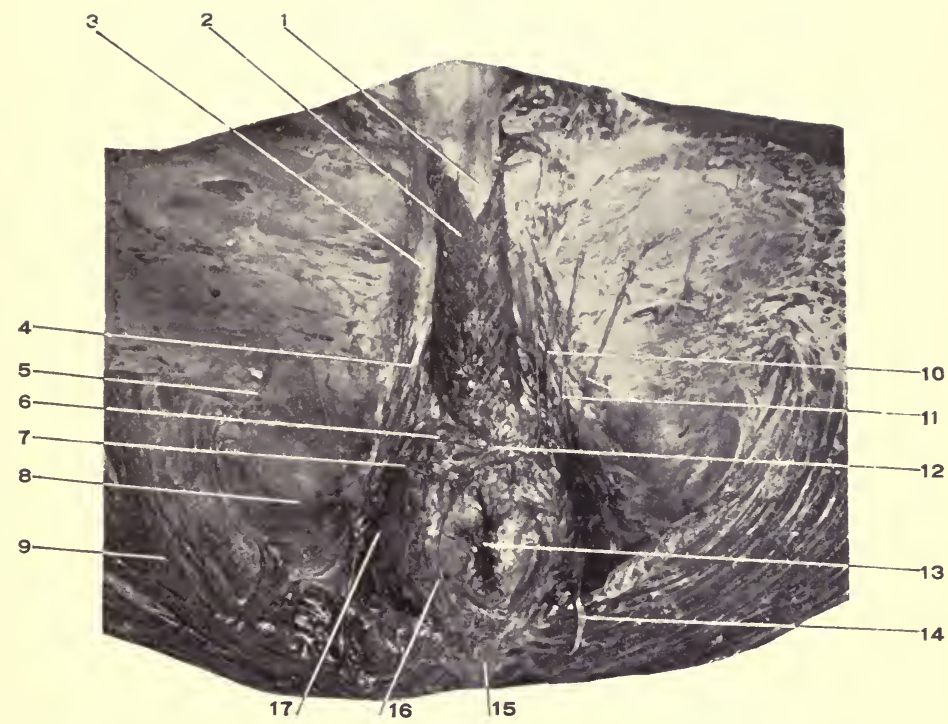

Fig. 82. Dissection of the Male Perineum. Perineal triangle and contents.-I, Corpus spongiosum. 2, Accelerator urinæ (bulbocavernosus). 3, Crus penis (origin of the corpus cavernosus). 4, Erector penis (ischiocavernosus). 5, Inferior pudendal nerve. 6, Transversus perineæ muscle (superficial). 7, Trausverse perineal artery. 8, Tuberosity of the ischium. 9, Gluteus maximus. Io, Superficial perineal artery. I1, Superficial perineal nerves. 12, Tendinous centre of perineum. 13, Anus. 14, Fourth sacral nerve. 15, Tip of coccyx. 16, Sphincter ani. 17, Ischiorectal fossa and anal fascia. 
layer of the superficial fascia. All these terms are applied to the membranous lamina of the superficial fascia, which covers over the structures forming, and contained within, the perineal triangle. It is attached behind to the base of the triangular ligament, curving backward over the transverse perinei muscle to do so ; laterally, to the inner margin of the rami of the ischium and pubes external to the attachment of the superficial layer of the triangular ligament. Forward, it passes over the bulb of the penis into the scrotum, where it receives a reinforcement of involuntary muscular fibres, and is called the dartos of the scrotum (it is also continuous with the dartos of the penis or labium majus). After leaving the scrotum (and penis or labium majus) it passes upward to become continuous with the deep layer of the superficial (Scarpa's) fascia of the abdomen over the spermatic cord (round ligament) at the external abdominal ring.

The perineal fascia, after surrounding the scrotum and penis (as the dartos), becomes attached to the front of the symphysis pubis as explained on page 438 , forming the suspensory ligament of the penis, or clitoris.

The Anus. Figs. 82, 85 .

The anus is the opening of the bowel externally. The lower part of the rectum and the anus is surrounded by circular muscle fibres, the external sphincter, that wrinkles the skin into radiating lines. For this muscle see below.

The Inferior Pudendal Nerve, or Nerve of Sœmmering. Fig. 82 .

This nerve is a branch from the small sciatic under the gluteus maximus; it winds forward around the tuberosity of the ischium, perforates the deep layer of the superficial fascia about an inch external to the tuberosity, passes for- 
ward and inward to the scrotum, which it supplies. It also supplies the integument of the inner and upper part of the thigh.

In the female it is distributed to the labium majus.

Sphincter Ani Externus. Figs. 82, 83.

Origin.-From the tip of the coccyx.

Insertion.-Into the tendinous centre of the perineum.

It is a broad, though thin, muscle which encircles the anus, and by its contraction firmly closes it.

Nerve Supply. - The inferior hemorrhoidal, from pudic, and the fourth sacral.

Action.-To close the anus.

\section{DISSECTION.}

Carefully pick out the fat filling the posterior half of the perineum, demonstrate the boundaries of the fossa exposed and the vessels and nerves crossing it.

The Ischiorectal Fossa. Fig. 82.

The posterior portion of the perineum.

This fossa is of an irregular prismatic shape. Its outer wall is (approximately) vertical and is formed by the lower portion of the obturator fascia. Its inner wall is formed by the anal fascia backed by the levator ani and coccygeus muscles and the external sphincter of the anus, with the tendinous centre of the perineum in front and the tip of the coccyx behind. In front, the fossa is limited by the junction of the perineal fascia and the superficial and deep layers of the triangular ligament and the anal fascia; behind, by the margin of the gluteus maximus muscle, the great sacrosciatic ligament, and the junction of the obturator and anal fasciæ. The contents of the fossa: Adipose tissue, inferior hemorrhoidal arteries, vessels, and nerves. 
The Hemorrhoidal Plexus.

This venous plexus begins around the anus and extends upward upon the rectum. It empties the blood by the inferior hemorrhoidal vein into the internal pudic vein; by the middle, into the internal iliac and superior hemorrhoidal; and by the superior, into the inferior mesenteric, which opens finally into the portal vein. This establishes a free venous communication from the anus to the portal vein, and so to the liver. There are no valves in these veins, so the blood can be dammed back from the liver or from the heart.

The Inferior Hemorrhoidal Artery. See page 4I 2.

The Inferior Hemorrhoidal Nerve. See page 4I 3 .

\section{DISSECTION.}

Carefully divide the perineal fascia along the median line and across its posterior attachment and reflect the two portions. If the fascia has been partially destroyed in the dissection remove any parts left and complete the cleaning of the structures forming and contained within the perineal triangle.

\section{The Perineal Triangle}

Is formed by the erector penis, accelerator urinæ, and transversus perinea muscles.

It contains the superficial and transverse perineal vessels and nerves.

Erector Penis. (Ischiocavernosus.) Fig. 82.

Origin.-From the inner surface of the tuberosity and ramus of the ischium.

Insertion.-Into the outer and under surface of the crus penis, and by a small slip around the crus penis, to pass over the dorsal vein of the penis (situated posterior to the anterior slip of the accelerator urinæ). This small slip is 


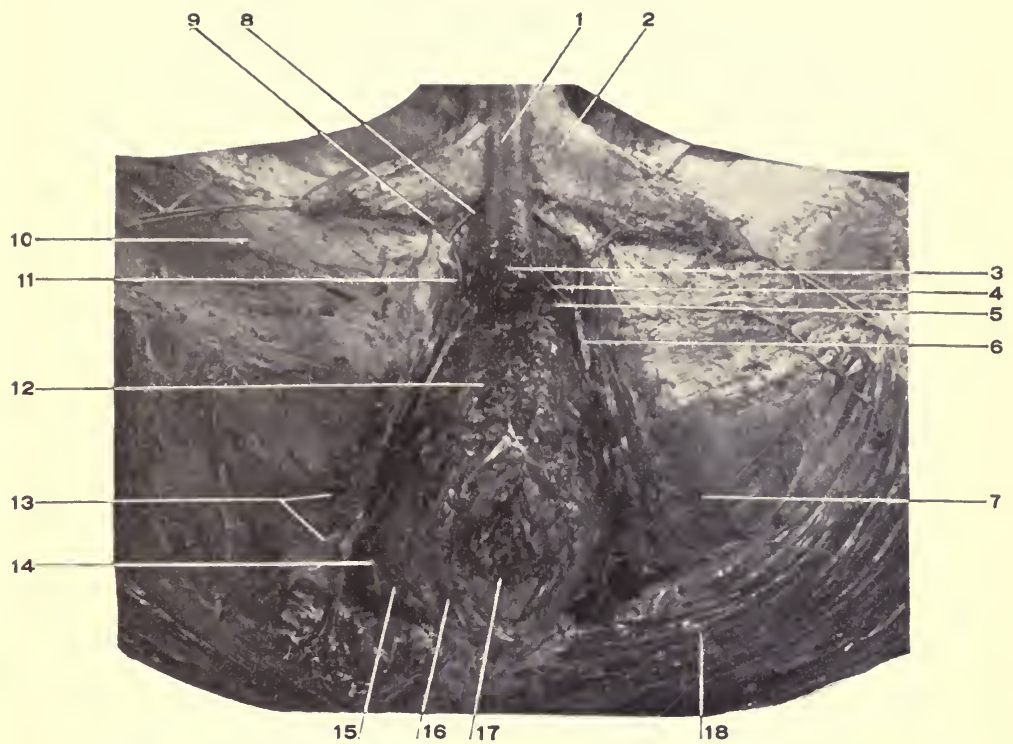

Fig. 83. Dissection of the Perineum, Muscles of the Triangle Removed and the Crura Turned to One Side,-I, Corpus spongiosum. 2, Corpus cavernosum. The crus is cut away from its attachment and turned to the side. 3 , Bulb of the corpus spongiosum. 4, Arterial branch to the corpus spongiosum. 5, Artery of the bulb. 6, Internal pudic artery. 7, Tuberosity of the ischium. 8, Dorsal artery of the penis. 9, Artery to the corpus cavernosum. Io, Fascia lata. II, Internal pudic artery. 12, Compressor urethræ muscle (the deep transversus perineæ). This muscle is thin and not easily demonstrated in a photograph. 13, The superficial perineal nerves turned outward. I4, Inferior hemorrhoidal vessels and nerve. I5, Levator ani muscle. 16, Sphincter ani. 17, Anus. 18, Gluteus maximus. 
not constant in man; it is named, from its action, the compressor venæ dorsalis penis.

Nerve Supply.-Perineal.

Action.-To compress the dorsal vein of the penis (by the small slip mentioned above) and so produce erection of the penis. The main part of the muscle compresses the crus penis, and so aids erection and maintains the penis erect after it becomes engorged.

Transversus Perinæi. (Superficial transversus perinai.) Fig. 82.

Origin.-From the inner surface of the tuberosity of the ischium.

Insertion.-Into the tendinous centre of the perineum.

Nerve Supply.-Superficial perineal.

Action.-To fix the tendinous centre, aid in supporting the pelvic floor.

Accelerator Urinæ. (Bulbocavernosus.) Fig. 82.

Origin.-From the median raphe, reaching from the tendinous centre of the perineum forward upon the bulb of the penis to opposite the lower border of the symphysis.

Insertion.-In three portions: ( I) A small set of fibres anteriorly, which surround the corpora cavernosa (and, of course, the corpus spongiosum) to be inserted upon the dorsum of the penis above (or over) the dorsal vein of the penis. (2) A middle set, the most of the muscle, which passes forward around the corpus spongiosum (and so between it and the corpora cavernosa) to the dorsum of the corpus spongiosum. (3) A third set posteriorly, also small, which surrounds the bulb of the penis behind the membranous urethra, and is inserted into the dorsum of the bulb and the superficial layer of the triangular ligament close to the exit of the urethra. 


\section{Nerve Supply.-Perineal.}

Action.-The anterior portion compresses the dorsal vein of the penis and so produces erection of the organ. The middle portion compresses the bulb and its vessels and so aids the action of the anterior portion. The posterior part compresses the membranous urethra and expels its contents (urine or semen). This last action is continued by the two other portions of the muscle, acting peristaltically from behind forward.

The Internal Pudic Artery. Figs. 82, 83, I I 2, I 4.

This is the smaller branch of the bifurcation of the anterior trunk of the internal iliac artery, the larger being the sciatic. It leaves the pelvic cavity with the sciatic between the coccygeus and pyriformis muscles, through the greater sacrosciatic foramen, curves around the base of the spine of the ischium, having the internal pudic nerve on its internal and the nerve to the obturator internus muscle on its external side; then re-enters the pelvic cavity (ischiorectal fossa) through the lesser sacrosciatic foramen; the artery now runs forward along the outer surface of the ischiorectal fossa, being contained in a canal (Alcock's) formed by the separation of the obturator fascia. In this part of its course the artery is situated about an inch above the lower margin of the tuberosity of the ischium, and is accompanied by the pudic nerve, which divides soon after entering the ischiorectal fossa into the superficial perineal nerve and the dorsal nerve of the penis ; the former nerve is below and the latter above the artery (subject standing).

The artery continues forward, perforates the posterior layer of the triangular ligament close to the ramus of the ischium, along which it extends between the two layers of 
the triangular ligament and within the substance of the compressor urinæ muscle to the anterior part of the space, where it divides into the artery of the crus and the dorsal artery of the penis. The latter continues the course of the internal pudic forward and upward, perforating the superficial layer of the triangular ligament close to the opening for the dorsal vein of the penis, then passes between the crura and symphysis pubis and through the suspensory ligament of the penis to the dorsal surface of that organ, where it lies external to the dorsal vein, which is centrally placed. It continues along the dorsum of the penis to the glans, where it ends by breaking up into a coronary anastomosis. It also sends branches to the prepuce. The dorsal artery is accompanied in its course by the nerve of the same name which is placed outside of the artery.

Branches of the Internal Pudic.

(I) The inferior hemorrhoidal branch (or branches, I to 3 ) is given off as the pudic enters the rectovesical fossa. It passes through the fat, filling the fossa, to the rectum and anus. Above, it anastomoses with the middle hemorrhoidal of the anterior division of the internal iliac. (Consult the intestinal anastomoses.)

(2) The superficial perineal artery is given off at the base of the triangular ligament, perforates the perineal fascia, crosses over or behind the transverse perineal muscle, extends forward between the erector and crus penis and the accelerator urinæ muscles, to the base of the scrotum, where it ends in supplying the superficial parts of that structure. It gives off the transverse perineal artery to the muscle of that name, besides supplying the adjacent parts.

(3) The artery to the bulb. This is a considerable artery which arises from the internal pudic within the space between the layers of the triangular ligament. Its course 
is forward and inward through the compressor urethræ muscle. When near the middle line it penetrates the superficial layer of the triangular ligament and enters the bulb. It supplies Cowper's glands, the membranous urethra, the bulb, and the corpus spongiosum.

(4) The artery to the crus. The larger of the terminal branches of the internal pudic. This perforates the anterior layer of the triangular ligament, enters the crus penis (corpus cavernosum), through the centre of which it passes to its anterior extremity, giving off numerous branches in its course to this body.

(5) The dorsal artery of the penis. The smaller branch of the bifurcation of the internal pudic. For its course, see above.

\section{The Internal Pudic Vein.}

This begins as a branch from the corpus cavernosum, receives a communicating branch from the dorsal vein of the penis, retraces the course of the internal pudic artery, receiving in its course branches corresponding to the branches of the artery, and finally empties into the beginning of the internal iliac vein.

The Internal Pudic Nerve. Figs. 82, 83, I I 2, I I4.

This is found in the dissection of the perineum, as it enters the ischiorectal fossa through the lesser sacrosciatic foramen along with the internal pudic artery. It courses along the side of this fossa with the artery, being contained in a canal in the obturator fascia, and divides into the perineal and dorsal nerve of the penis (clitoris). Before this division the nerve gives off the (I) inferior hemorrhoidal branch, which passes inward to the external sphincter of the anus and the integument over it. 
(2) The perineal nerve. This - lies below the artery (subject standing). It penetrates the perineal fascia close to the base of the triangular ligament, divides into two branches, the posterior or external and the internal or anterior. These branches pass through the perineal triangle and terminate in the scrotum (female, the labium majus). From this nerve muscular branches pass to the transversus perinæi, accelerator urinæ (sphincter vaginæ), erector penis (clitoris), and filaments also pass to the compressor urethra, bulb, and mucous membrane of the urethra.

(3) The dorsal nerve of the penis lies first above the internal pudic artery, continues with the artery into the triangular space, crossing the artery to get below it. It leaves the triangular space with the dorsal artery of the penis, being situated at the outer side of the artery, and terminates in branches to the glans. For the course of the artery see above.

The nerve supplies in its course branches to the corpus cavernosum, glans, and integument of penis.

In the female the nerve is smaller, but takes a similar course and distribution.

\section{DISSECTION.}

Remove the erector penis, accelerator urinæ, and transversus perineæ muscles. Clean the bulb (corpus spongiosum), crura (corpora cavernosa), and the anterior layer of the triangular ligament.

The Corpus Spongiosum. (Is described here, but see page 440 for dissection of penis.) Fig. 83 .

This is a cylindrical tube of fascia containing erectile tissue, and traversed through its centre by the urethra. It is placed below and between the two crura, to which it is firmly bound by the sheath of the penis.

It is dilated at each end; the anterior dilatation is called 
the glans. (See dissection, page 440.) It is a heartshaped enlargement, which spreads out laterally and backward beyond the crura, which terminate within it. The margin of the glans is called the corona glandis, and the constricted portion behind it the neck of the penis. The glans shows a vertical slit at its extreme end, the external urinary meatus. The posterior dilatation is named the bulb. It is about one and one-half inches long, and projects a little beyond the membranous urethra, which enters this portion of the corpus spongiosum. The bulb lies upon (really below) the anterior triangular ligament, being surrounded by the accelerator urinæ muscle and covered by the perineal fascia.

The portion of the corpus spongiosum between the glans and bulb is called the body. Through its centre the urethra passes, the space between the urethra and sheath of the corpus being filled with erectile tissue. The corpora cavernosa and the corpus spongiosum are bound together by the elastic fascial sheath of the penis, which extends from the root of the penis to the glans. Within this elastic sheath are found the deep dorsal nerves, arteries, and vein of the penis, while external to it are the superficial dorsal vessels and nerves. The former arteries and nerves are from the internal pudic artery and nerve; the vein empties into the prostatic sinus plexus. The latter vessels are from the external superficial and deep pudic arteries; the veins open into their venæ comites, and the nerves are branches of the iliohypogastric. See page 440.

Beneath the skin and external to the fibrous sheath lies a membranous layer composed of fibrous and muscular tissue-the dartos-which is continuous with the similar layer of the scrotum and the fascia of Scarpa. See pages 404,438 . 
The Corpora Cavernosa. Figs. 82, 83.

Each corpus consists of a cylindrical tube of dense fascia (tunica albuginea) containing erectile tissue. It is pointed at both ends, the anterior being short and imbedded in the substance of the glans, the posterior longer, more slender, and tapering, and being attached firmly to the inner margin of the ramus of the pubes and ischium. This portion of the corpus cavernosum is called the crus penis.

The artery of the crus enters it close to its bony attachment, and runs through its centre to the anterior extremity, giving off in its course numerous branches to the erectile tissue. The vein of the crus emerges where the artery enters, and is continued posteriorly under the name of the Internal Pudic Vein, q. v., page 4I3.

The two crura are separated below, where they are fastened to the pubic arch, and at their anterior extremity, where they terminate in the glans, but the intervening portion is firmly united by an envelope of fascia in addition to their own proper sheaths.

When the crura are cut apart the line of division shows numerous transverse striations, called the septum pectiniforme.

Anterior Portion of the Triangular Ligament. See page 403.

\section{DISSECTION.}

Remove what is left of the anterior layer of the triangular ligament. Expose the compressor urethræ, Cowper's glands, the deeper branches of the internal pudic artery and nerve.

Compressor Urethræ. (Deep Transverse Perinci.) Fig. 83.

Origin.-From the inner margin of the rami of the ischium and pubes.

Insertion.-Into a median raphé. 
Nerve Supply.-Dorsal nerve of the penis.

Action.-To compress the membranous urethra, which it surrounds, expelling its contents (urine or semen). To aid erection by compressing the emergent veins of the penis. To compress Cowper's glands and so force out their secretion. This may be independent of the emission of semen or in connection with that act.

Cowper's Glands. Diag. 28.

These are two glands about as large as small peas, situated between the two layers of the triangular ligament, close to and behind the membranous urethra, and under (above) the bulb of the penis.

The duct of a gland is about an inch long. It pierces the anterior layer of the triangular ligament, the corpus spongiosum, and opens into the floor of the urethra (bulbous portion).

\section{DISSECTION.}

Carefully remove the compressor urethræ muscle and the nerve and artery and their branches.

Remove the hemorrhoidal vessels and nerves.

Section a portion of the gluteus maximus.

The Posterior Layer of the Triangular Ligament. See page 402.

The Anal Fascia. See page 402.

\section{DISSECTION.}

Remove the posterior layer of the triangular ligament and the anal fascia (which are continuous, as explained).

Levator Ani. Fig. 83.

Origin.-From the inner surface of the body of the pubis, from the angle between the obturator and rectovesical fascix-the so-called "white line"-and from the inner surface of the spine of the ischium. 
Insertion.-Into the tip of the coccyx, into the median raphé extending from the coccyx to the anus, into the lateral surfaces of the rectum, also into the median raphé as far forward as the tendinous centre of the perineum. In the female the anterior fibres of the muscle are attached to the side of the vagina.

Nerve Supply.-Inferior hemorrhoidal, fourth and fifth sacral.

Action.-To elevate the pelvic floor and rectum, prostate (vagina) and bladder. To diminish the pelvic cavity, thus compressing the abdominal viscera, and aid in all expulsive efforts. The levator ani and the coccygeus have been called the pelvic diaphragm, as their combined action resembles the action of the diaphragm.

\section{Coccygeus.}

Origin.-From the inner surface of the spine of the ischium.

Insertion.-Into the side of the coccyx and the last two pieces of the sacrum and the lateral ligaments connecting the two bones.

Nerve Supply._Fourth and fifth sacral and the coccygeal.

Action.-Draws the coccyx forward and aids the levator ani.

\section{DISSECTION.}

Remove the levator ani, cutting it away at its origin and insertion.

Consult the description of the rectovesical fascia, page 399.

Draw the rectum backward, and keeping close to it cut the fascia between it and the prostate and bladder until the rectovesical fold of peritoneum is reached. Clean the prostate and base of bladder.

\section{The Prostatic Plexus.}

This is a plexus situated within the capsule of the prostate and between the rectovesical fascia and the neck of the bladder. 


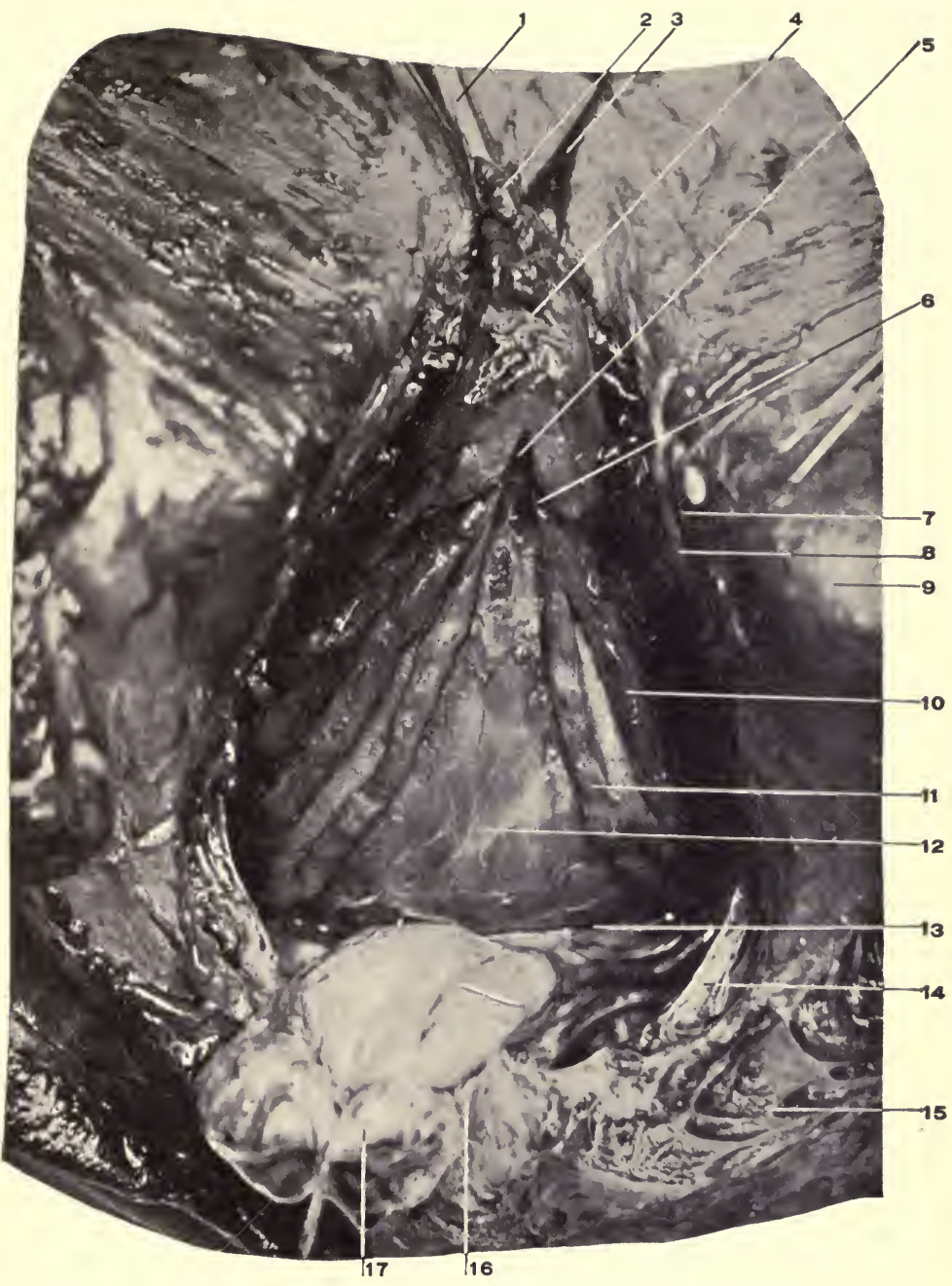

Fig. 84. Dissection of the Perineum. The Prostate ann Base of the BLADDER. - I, Tube in the urethra. 2, The urethra. 3, Dorsal vein of the penis. 4, Prostate gland. 5, Notch in the prostate where the 6 , Ejaculatory duct enters. 7, Dorsal nerve of the penis. 8, Internal pudic artery. 9, Tuberosity of the ischium. Io, A seminal vesicle. II, The vas deferens. 12, The external trigone of the bladder. 13. Point where the peritoneum is reflected from the bladder to the rectum, rectovesical pouch. 14, Great sacrosciatic ligament. 15, Gluteus maximus muscle. 16, Coccyx. 17, Rectum. 
It receives the two veins of divisions of the dorsal vein of the penis, the veins of the prostate and bladder, communicates with the hemorrhoidal plexus, and empties by a right and left branch into the corresponding internal iliac vein.

\section{The Vesical Plexus}

Is formed by the veins which surround the bladder and at its base empty into the prostatic plexus.

In the female, the vaginal plexus surrounds the vagina and opens into the hemorrhoidal and vesical plexuses. The uterine plexus terminates in the ovarian veins.

Base of the Bladder. See page 537.

The external trigone (Fig. 84) is the surface of the bladder included between the diverging vasa deferentia and the peritoneal reflection from the bladder to the rectum, which is from one to one and one-half inches from the base of the prostate.

The Seminal Vesicles. Fig. 84.

These are two in number, two inches long, one-half inch wide, one-quarter inch thick, situated outside the vas deferens, one and one-quarter inches above, and, like the vas, inclining downward and inward to the prostatic notch to empty into the common duct. Their function is the storage of the seminal fluid.

Relations.-Behind is the rectum; in front, the bladder and termination of the ureters ; internally, the vas deferens; below, the prostate. They are enclosed in a capsule from the rectovesical fascia. When unraveled each vesicle is found to consist of a single tube about four inches long, with numerous blind outgrowths.

Vas Deferens. See page 542.

The Prostate. Fig. 84. Diags. 27, 28.

This is a fibromuscular gland surrounding the neck of the bladder and the beginning of the urethra. It is heart- 
shaped, one and one-half inches across the base and one and one-quarter inches in vertical extent, placed with its base uppermost and the apex below. There is a notch at the base dividing the gland into two lobes. Into this notch the vasa deferentia and seminal vesicles converge to unite into the common ejaculatory ducts which traverse the prostate downward and forward to open into the floor of the prostatic urethra. The gland is enclosed in a capsule derived from the rectovesical fascia, upon which it rests. At the apex of the gland, at the place where the urethra issues, the fibres of the levator ani muscle are wanting (the muscle being behind the gland), and at this point the rectovesical fascia becomes continuous with the deep layer of the triangular ligament.

Relations.-Above : The bladder, vasa deferentia, seminal vesicles. Behind: Rectum separated only by a thin layer of rectovesical fascia. Below: The rectovesical fascia and deep layer of the triangular ligament. In front: Puboprostatic ligaments. Laterally: Capsule derived from the rectovesical fascia, and separating it from the levator ani muscle. The prostatic venous plexus encloses the gland, being between it and its capsule. Into the front of the plexus the deep vein of the penis empties.

The Lower Portion of the Rectum. For the upper part, see page 504. Fig. 84. Diag. 28.

The lower portion of the rectum extends from the tip of the coccyx to the anus, a distance of one and one-half inches. This part of the rectum curves downward and backward ; consequently, in introducing a tube into the rectum it should first be pointed forward and upward or toward the umbilicus, then it may be carried backward toward the sacrum. In children this precaution should be followed. 
The second part of the rectum is enclosed by the internal sphincter, which is only an increase in the circular muscular fibres, and has the levator ani supporting it at its sides. Fig. 82.

In front, is the lower part of the prostate, the base of the triangular ligaments, the transverse peroneal muscles.

\section{THE PERINEUM, Female.}

\section{The Landmarks.}

Consult the male perineum for the boundaries of the pelvic outlet, page 397. Notice the various differences between the male and female pelvic outlet. The pubic arch in the female is wider than in the male, and the tuberosities of the ischium are farther apart.

Comparative measurements of the male and female pelvis (internal) :-

The female pelvis (Grandin and Jarman, "Obstetric Surgery").

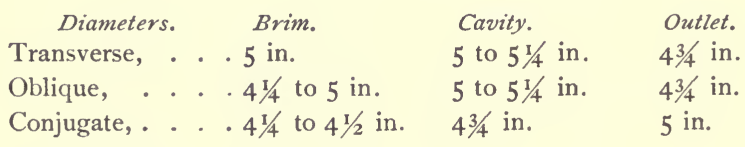

The male pelvis.

Diameters. Brim.

Transverse, . $4 \frac{1}{2}$ in.

Oblique, .. $4 \frac{1}{4} \mathrm{in}$.

Conjugate, . . . 4 in.
Cavity.

$4^{1 / 2}$ in.

$4 \frac{1}{2}$ in.

$41 / 2$ in.
Outlet.

$31 / 2$ in.

4 in.

$3 \%$ in.

The External Genitals. Fig. 85 .

These should be carefully identified. In comparing the following descriptions with other text-books many smaller 
differences will be marked; these differences are believed to be justified by a careful examination of the subject (living as well as dead) and from the conclusions of other observers.

The Vulva.- - The external genitals of the female, excepting the mons veneris" ("Foster's Dictionary").

The Pudendum.- "The external genitals (especially those of the female, including the vulva and mons veneris ") (same). Quain uses the terms synonymously to include the mons veneris, labia majora and minora, the hymen or its remains, the clitoris, and meatus urinarius.

(I) The Mons Veneris.-The fatty elevation covering the pubes.

(2) The Labia Majora.-The homologue of the scrotum, remaining permanently cleft along the middle line (this cleft is the rima pudendi, or genitalis). The labia majora form two elevations reaching from the mons veneris in front toward the tendinous centre of the perineum. The posterior junction of these labia is said to form the posterior commissure, but an examination of the subject (living and dead) will convince any one that they do not unite in this manner, but flatten out into the smooth covering of the perineum.

(3) The Labia Minora.--Related morphologically to the integument and foreskin of the penis. They are two folds of integument (the word is used advisedly) smaller than the labia majora, located internal to the labia majora, by which they are usually concealed from sight. They are joined in front over the clitoris, forming its prepuce, and behind by the fourchette, a thin fold of membrane.

(4) The Clitoris.-Akin to the penis of the male. Its blunt extremity is called the glans clitoridis, and is covered by the prepuce, formed by the labia minora. 
(5) The Fourchette.-The narrow fold or band of membrane (integument) which joins the labia minora posteriorly (usually torn in childbirth).

(6) The Vestibule.-This is the median gutter which extends from the clitoris in front to the hymen or its remains behind, and is bounded laterally by the labia minora. Into it opens the meatus urinarius.

(7) The Hymen.-An irregular, ring-like fold of membrane (probably integument), which surrounds the entrance to the vagina. It is really external to and below the vagina (D. S. Lamb). Torn in childbirth always. The numerous small nodules remaining to mark the site of the hymen in women who have had children are called the carunculæ myrtiformes.

(8) The Meatus Urinarius.-The external opening of the female urethra is in the median line of the vestibule and about three-fourths to one inch from the clitoris.

(9) The Fossa Navicularis is the shallow depression between the hymen (internally) and the fourchette (externally.)

(I0) The Orifice of the Vagina lies in the middle line. It is narrowed in the virgin by the hymen, and surrounded in the matron by the carunculæ myrtiformes.

(I I) Opening of the Ducts of the Glands of Bartholin is on the inner side of the labia minora, external to the hymen, and on a level with the middle of the vaginal orifice.

The Anus. See page 406.

\section{DISSECTION.}

Distend the vagina and rectum with oakum, and take several interrupted sutures in the labia majora and the anus. 


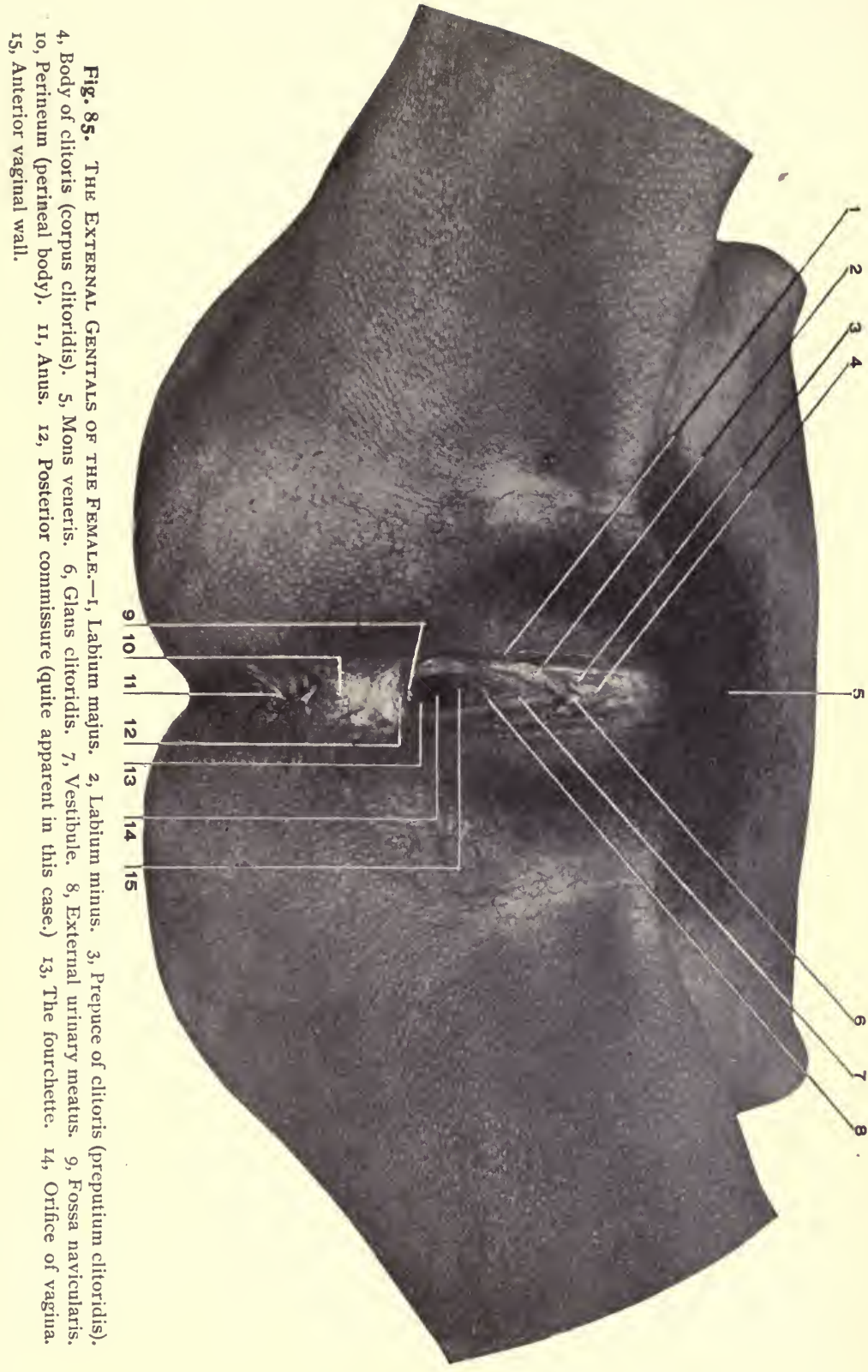


Incisions.-(I) Incise the labia majora close to their inner margin from the mons veneris to the median line of the perineum; carry the incision around the anus to the coccyx behind.

(2) Make the transverse incisions as indicated on page 404.

Carefully remove the integument, especially about the labial margin and around the anus.

The descriptions of the various structures given under the male perineum will apply with a slight modification to the female parts.

The Superficial Fascia. See page 404.

This consists of the two layers, these layers being pierced by the vaginal opening. The deep layer forms in the female an imperfect dartos of the labia majora as it does the dartos of the scrotum in the male.

Sphincter Ani Externus. See page 407.

The Ischiorectal Fossa, situation, formation, boundaries, and contents. See page 407. Fig. 86.

The Internal Pudic Artery. See page 4I I. Fig. 86.

In the female there are these variations: the superficial perineal artery is larger than in the male and is distributed to the labia majora and minora. The artery of the bulb ends in the bulb of the vestibule, and is smaller than in the male. The artery of the crus is a small branch to the crus clitoridis.

The dorsal artery of the clitoris is much smaller than the corresponding artery in the male.

The Internal Pudic Nerve. See page 4I3. Fig. 86.

In the female the superficial perineal nerves supply the labia, the erector clitoridis, the sphincter vaginæ, and the compressor vaginæ. The dorsal nerve of the clitoris is a small branch which supplies that organ. 
DISSECTION.

Remove the perineal fascia and dissect out the perineal triangle. This will be more difficult to do than in the male, as the parts are smaller, the sphincter vaginæ (corresponding to the accelerator urinæ) imperfectly developed and perforated by the vaginal orifice.

After careful dissection, consult the male perineal triangle, page 408 .

Transversus Perinæi. Same as in the male. See page 4 IO.

The Erector Clitoridis. Smaller, but similar to the erector penis in the male. See page 408.

\section{The Sphincter Vaginæ.}

In the male the accelerator urinæ or the bulbocavernosus, page 4IO. Originates from the tendinous centre of the perineum, passes forward to the parts about the clitoris, surrounding the vagina. It is an indistinct, imperfectly developed plane of muscular fibres.

\section{DISSECTION.}

Divide the transversus perineæ and sphincter vaginæ muscles and reflect them. Cut away the erector clitoridis.

Draw the clitoris forward and remove the mucous membrane between it and the urethral orifice.

The orifice of the urethra presents in the middle line nearly midway between the pubic arch and the vaginal orifice.

The Bulb of the Vagina, or Bulbi Vestibuli.

This is the female representative of the corpus spongiosum in the male. The bulb of the vagina consists of two portions, each about an inch long and lying upon the front and sides of the vaginal opening and just under the sphincter vaginæ muscle. The bulbs are connected in front over the meatus urinarius by a narrow neck (pars intermedialis of Köbelt).

The Clitoris. Figs. 85, 86.

Morphologically identical with the male penis. It is 
composed of two small corpora cavernosa attached similarly to the corpora cavernosa in the male. See page 4 I6. The corpora cavernosa converge from the pubic arch, unite to form the body of the clitoris, and terminate in a blunt extremity (different from the male) which is called the glans clitoridis.

The clitoris is covered by a modified integument, and is suspended from the symphysis pubis by a small ligament similar to the suspensory ligament of the penis.

The Artery and Nerve to the Bulb. See pages 4I2, 4 I4.

The Artery and Nerve to the Corpus Cavernosum and the Dorsal Artery and Nerve of the Clitoris. See pages 4I3, 4I4. Fig. 86.

The Anterior Layer of the Triangular Ligament.

The description on page 403 will answer for this structure in the female, in addition noting that it is perforated by the vagina.

\section{DISSECTION.}

Remove the anterior layer of the triangular ligament.

Compressor Urethræ. Constrictor Vaginæ. (Deep Transversus Perinai.)

The compressor urethræ muscle occupies the same position in the female that it does in the male. Besides its urethral function it also constricts the vagina. It is not so well developed as in the male. See page 4 I6.

Various small muscles are described in this plane by various dissectors. It is sufficient if the student now recognizes the above muscle.

The Glands of Bartholin. (Couper's Glands in the male.)

These are two small glands lying opposite to the posterior half of the vaginal orifice, just behind the bulbs of the vagina, imbedded within the constrictor vaginæ muscle. 


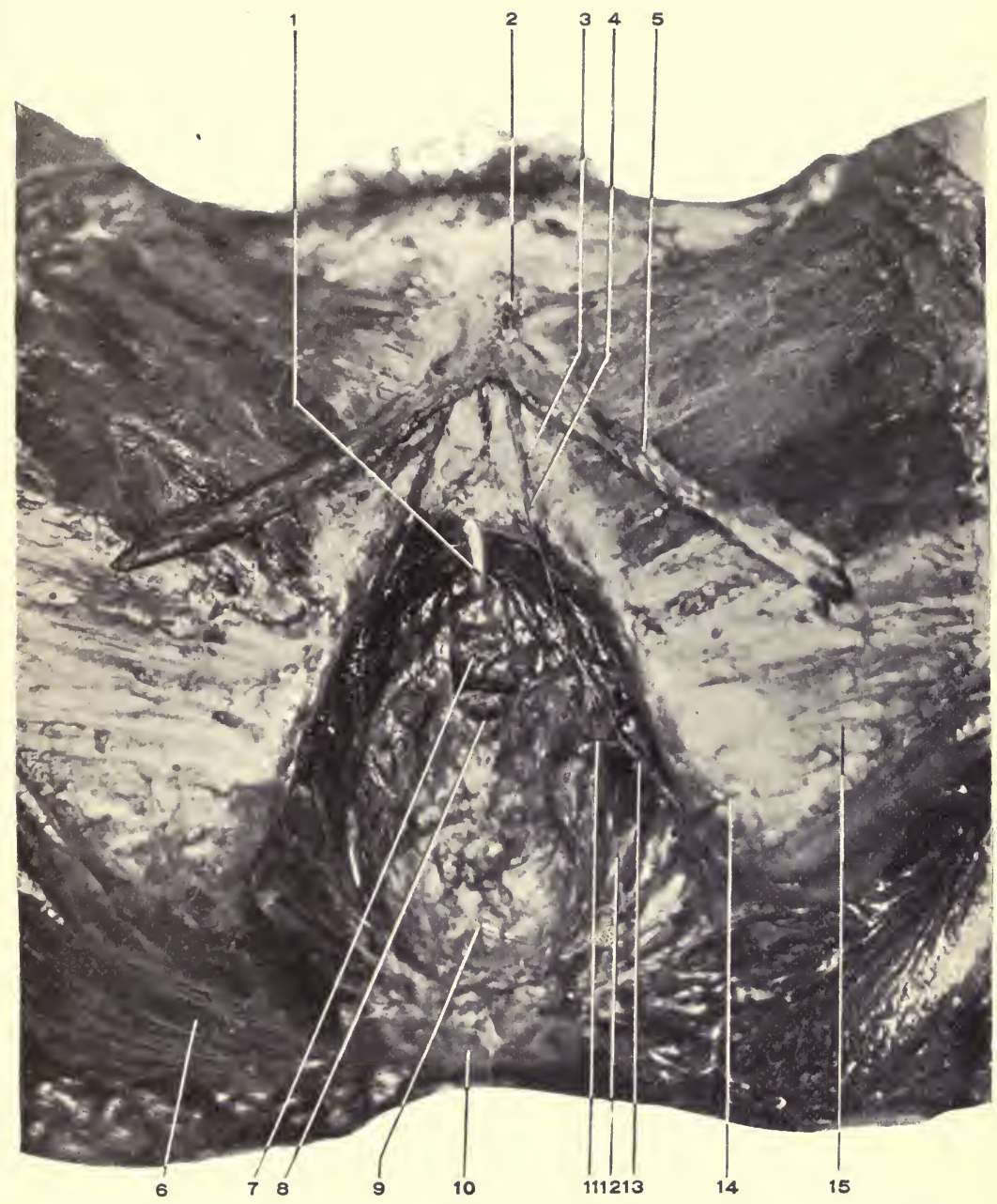

Fig. 86. Dissection of the Female Perineum.- I, Tube in the urethra. 2, Glans clitoridis. 3, Dorsal artery of the clitoris. 4, Dorsal nerve of the clitoris. 5, Corpus cavernosum. It terminates in the crus clitoridis. 6, Gluteus maximus. 7. Anterior vaginal wall. 8, Vaginal orifice. 9, Anus (rectum). Io, Coccyx. II, Superficial perineal artery. 12, Inferior hemorrhoidal artery and the ischiorectal fossa. 13, The internal pudic artery. 14, Tuberosity of the ischium. 15, Fascia lata. 
They open by a duct, about three-fourths of an inch long, opposite the middle of the vaginal opening.

The Posterior Layer of the Triangular Ligament

Is the same as in the male (see page 402), besides being pierced by the vagina. It is continuous with the anal fascia covering the levator ani muscle.

Levator Ani and Coccygeus Muscles. See pages 4I7, $4 \mathrm{I} 8$.

\section{DISSECTION.}

Draw the rectum backward and separate it from the vagina until the rectovesical fold of peritoneum passing between them is reached. Notice how far this fold reaches downward into the rectum and vagina.

In like manner separate the vagina from the urethra and base of the bladder. Expose the ureters, and carefully note their position with reference to the uterus, vagina, and bladder.

The Rectum. See page $42 \mathrm{I}$.

For the description of the rectovesical fascia, see page 399. The Vagina.

This membranous canal is usually described as one of the parts of the internal organs of generation of the female, but it properly belongs to the external genitals. It extends from the hymen upward and backward to enclose the lower portion of the uterus. The angle of reflection from the uterus is called the fornix. Its anterior wall is about three inches long, its posterior, about four. In the natural state the two walls are in contact and the fissure between them resembles the letter " $\mathrm{H}$," a long transverse limb and two short vertical limbs.

Relations.-In front: The urethra, base of bladder, and ureters, which enter the bladder in front of the vagina one and one-half inches below the level of the cervix. Behind: The rectum, from which it is separated; above, by the rectovesical fold of peritoneum which descends between the uterus and upper inch of the vagina in front and the rectum 
behind, forming the pouch of Douglas; below, the vagina is separated from the rectum for its last inch by the fibrous structures which, taken together, form the " perineal body ;" in the middle portion the vagina lies in contact with the rectum. At the sides : Above, the vagina is crossed by the ureters from above downward and forward; below, it is embraced by the levatores ani muscles.

The mucous membrane (probably modified integument) is thrown into longitudinal ridges upon the anterior and posterior walls of the vagina. These ridges are called the columne rugarum. They extend upward from the vaginal orifice.

The veins form a plexus about the vagina and communicate with the hemorrhoidal and vesical plexuses.

The arteries are from the anterior division of the internal iliac, viz. : vaginal, internal pudic, vesical, and uterine.

The nerves are from the internal pudic, the fourth sacral, and the hypogastric sympathetic plexus.

The Perineal Body. Fig. 85 .

This is the mass of elastic, fibrous, and connective tissue, with the adjacent portions of muscles, which fills in the space between the lower end of the vagina and vaginal orifice in front and the rectum and anus behind. It is triangular in anteroposterior section, the base at the integument, the apex at the junction of the vaginal and rectal walls. The sides of the triangle measure about an inch.

The Uterus. See Pelvic Viscera.

The Female Urethra. Figs. 85, 86.

Is about one and one-half inches long and one-fourth inch in diameter. It lies along the anterior wall of the vagina. It opens into the bladder three-fourths of an inch behind the middle of the pubic symphysis, and externally in the middle line just in front of the orifice of the vagina. 


\section{THE ABDOMEN, Exterior.}

Boundaries.

The cavity of the abdomen is limited above by the thorax and diaphragm, below by the pelvis and levator ani, behind by the bodies and intervertebral substances of the lumbar vertebræ and adjacent muscles, laterally and in front by the abdominal muscles and their aponeuroses.

LANDMARKs. Figs. 87, 98.

Above and in the middle line is the ensiform appendix; extending outward and downward from it are the cartilaginous portions of the seventh, eighth, and ninth ribs, forming a continuous line, then the tips of the tenth, eleventh, and twelfth ribs. The tip of the tenth rib is to be noticed especially, as it is the point at which the upper horizontal line is drawn in mapping out the abdominal regions. It can easily be distinguished from the others, as it is the first freely movable rib met with in passing from the ensiform outward.

Below, there is in the middle line the junction of the two pubic bones, or the symphysis pubis. At an inch externally is the spine of the same. If the subject is very fleshy the spine of the pubes is concealed and difficult to locate in the usual way, but by invaginating the scrotum (or labium majus) on the finger and abducting the thigh so as to render tense the tendon of the adductor longus muscle, then carrying the finger up along the tendon of this muscle to its origin, the spine will be found immediately above it. The finger can also be entered into the external abdominal ring, as it is situated just over and to the outside of the spine. The spine is important in differentiating inguinal 


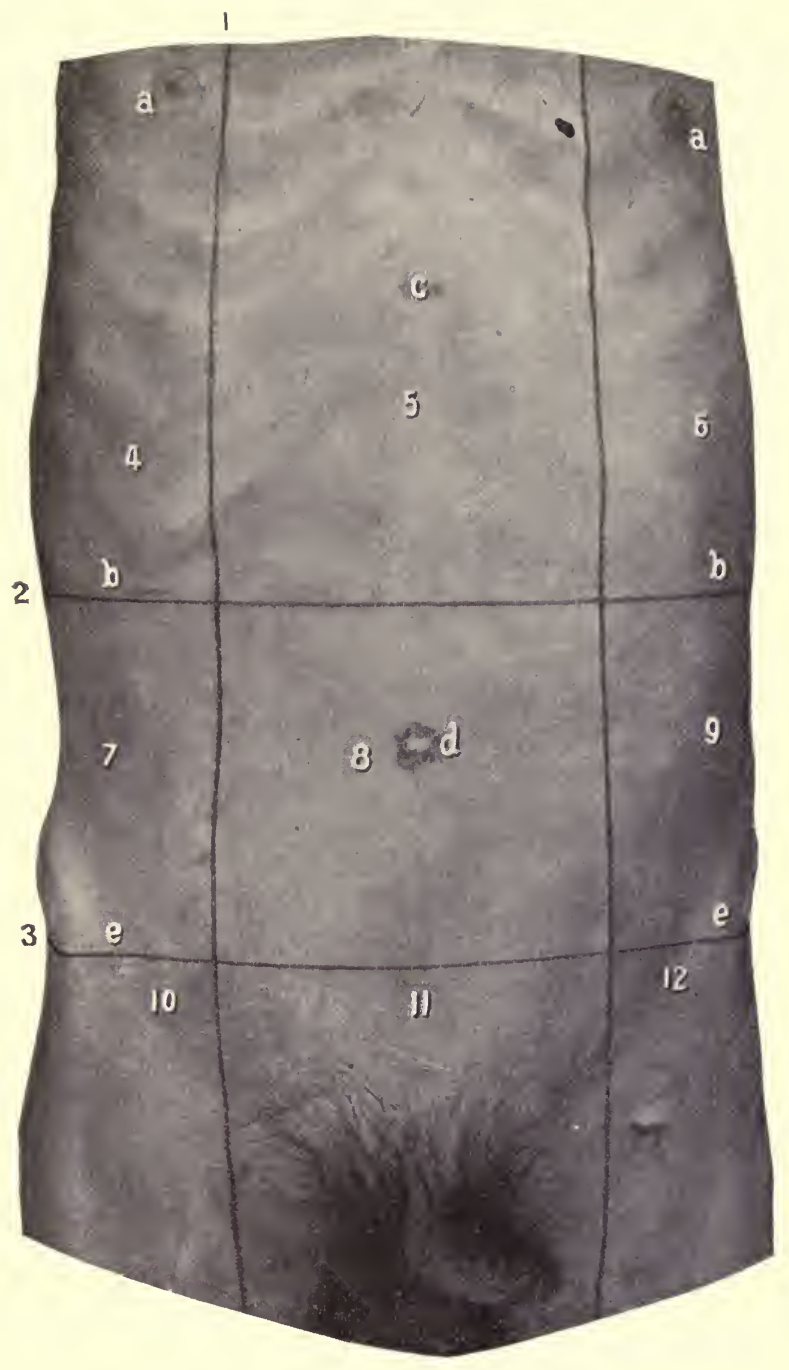

Fig. 87. The Abdominal Regions.- I, A vertical line drawn from the middle of Poupart's ligament. 2, The upper transverse line drawn at the level of the tip of the tenth rib. 3, The lower transverse line drawn at the level of the anterior superior iliac spines. $a, a$, Nipples. $b, b$, Tip of tenth ribs. $c$, Ensiform appendix. $d, \mathrm{Um}-$ bilicus. $e, e$, Anterior superior iliac spines. 4, Right hypochondriac, 5, Epigastric, 6, Left hypochondriac, 7, Right lumbar, 8, Umbilical, 9, Left lumbar, Io, Right inguinal, II, Hypogastric, I2, Left inguinal, regions. 
from femoral hernia, the former being to the inside and the latter to the outside of it. More externally, and always located with ease, is the anterior superior spine of the ilium. The crest of this bone terminates in this point, below which is a notch. In making measurements from this point, the thumb, over which is stretched the measuring tape, should be carried up from below until arrested in the notch below the spine. When the measurement is so made, the spine is truly a "fixed point," but as usually performed, by trying to hold the thumb or finger upon the spine, it is not a fixed point, as the measuring finger will slide all around the place over an area of an inch.

The dense band of fibres stretching between the anterior spine of the ilium and the spine of the pubes, formed by the aponeurosis of the external oblique muscle, is Poupart's ligament. It is a very important landmark. The umbilicus is always present, always distinct, hence always easily located. Its position in the middle line a little below the midpoint between the ensiform and symphysis is very constant, the variations not amounting to enough to render it unreliable as a most important reference point. Directly behind it is the body of the third lumbar vertebra.

Just below the ensiform the abdominal wall is depressed forming the "pit of the stomach" or scrobiculus cordis. A furrow passes from this depression to a little below the umbilicus, marking the linea alba. Below this the abdominal wall is not depressed.

The masses of the recti muscles and the shallow grooves outside of them are usually seen. The grooves, when present, lie over the linea semilunares. The masses of the recti may be cut up transversely by shallow depres- 
sions at the ensiform, umbilicus, and midway between the two. These transverse markings correspond to the tendinous intersections in the recti muscles.

\section{Abdominal Regions. Figs. 87, 98.}

The abdomen is arbitrarily divided into regions by four lines, two horizontal and two vertical. The horizontal lines are drawn, one connecting the anterior superior spines of the ilium, the other between the tips of the cartilages of the tenth ribs. The vertical lines are formed by erecting a perpendicular at the middle of Poupart's ligament on each side.

The regions thus formed are named as follows, from right to left. The first tier: The right hypochondriac, the epigastric, the left hypochondriac. The second tier: The right lumbar, the umbilical, and the left lumbar. The third tier: The right inguinal, the hypogastric, the left inguinal.

The portions of the viscera that lie in the above regions may be determined approximately by consulting Figs. 96 to I06. It is to be noted that these relations are not constant for the same subject at different times, or for any two subjects at the same time. At best they are only relative.

The surface area of the various viscera will be given in connection with the description of those viscera.

\section{DISSECTION.}

Shave or cut the hair from the pubes.

Incisions.-(I) From the ensiform to the symphysis, then along the dorsum of the penis.

(2) Transversely around the thorax at the level of the ensiform.

(3) From the symphysis outward, following the direction of Poupart's ligament and the crest of the ilium.

Remove the integument only, from this area. 
Superficial Fascia. Fig. 88.

This consists of two layers, the external or the subcutaneous, the internal, deep, or fascia of Scarpa.

(I) The external layer is composed of connective and adipose tissue, the varying quantities of the latter determining the thickness of the subcutaneous tissue. Above it is continuous with the superficial fascia of the thorax, and below with that of the thigh.

In this layer lie the subcutaneous vessels and nerves.

The more important of the vessels are (a) The superficial (superior) external pudic, which forms with the artery of the other side an anastomotic arch over the pubes, and at the side of the root of the penis gives off the superficial dorsal artery to that organ. (b) The superficial epigastric. This passes upward toward the umbilicus, having crossed the middle of Poupart's ligament. These arteries are branches from the femoral; for their origin, see Dissection of Thigh. They are usually divided in operations upon the inguinal lymphatics and herniotomies. (c) Numerous cutaneous arterioles from the intercostals, lumbars, deep epigastrics, and superficial circumflex iliacs.

The veins take the names of, and accompany, the arteries.

The cutaneous nerves are unimportant, and need not detain the student for their dissection. They come from the lower intercostals and the first lumbar nerves.

\section{The Lymphatics. Fig. 88.}

The only lymphatic glands of any importance are those grouped around the superficial epigastric artery, over and above the middle of Poupart's ligament. They drain the superficial region of the lower zone of the abdomen, the 


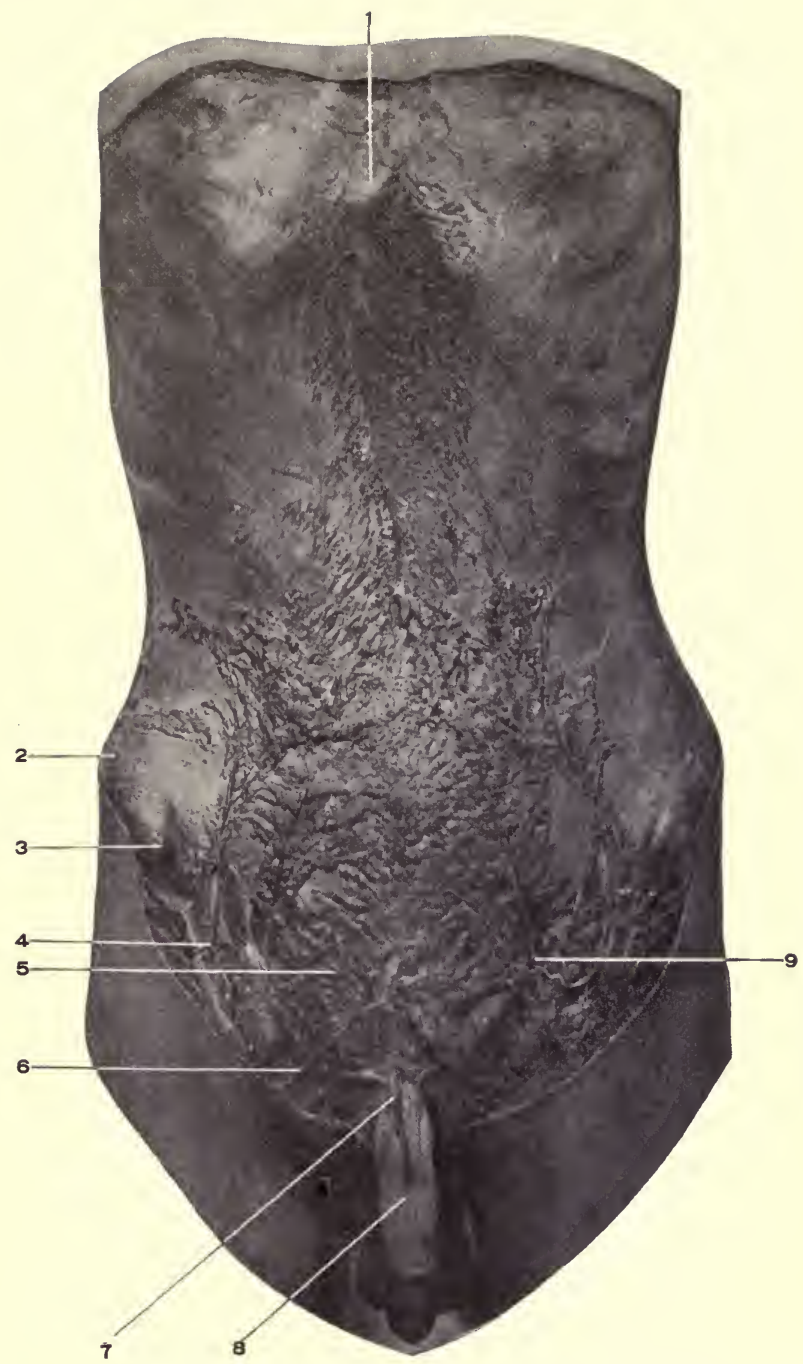

Fig. 88. Dissection of the ABdomen.-I, Ensiform appendix. 2, Crest of ilium. 3, Anterior superior spine of ilium. 4, Superficial epigastric artery. 5, Superficial fascia. 6, Superficial external pudic artery It forms an anastomotic arch over the pubes with the opposite artery. 7. The superficial dorsal artery of the penis. A branch of the above. 8 , The dartos of the penis. Continuous with the superficial fascia of the abdomen. 9, Superficial epigastric vein. The inguinal glands were small and do not show plainly. 
upper part of the thigh, the perineum, the scrotum, the anterior two-thirds of the urethra and superficial parts of the penis (external genitals in the female).

The connection of the inguinal lymphatics with the genitals of the male and female explains why they become enlarged in inflammatory conditions of those parts. Such enlargement following venereal disease is termed a bubo.

The removal of the inguinal glands is a simple operation on the cadaver, but in the living, if they are matted together by inflammatory exudations, it may become quite an undertaking.

(2) The deep layer of the superficial fascia. Fascia of Scarpa. Fig. 90. This is a thin layer of tissue in the upper part of the abdomen, where it passes into the deep fascia of the thorax; below the umbilicus it forms a dense plane that backs the external layer, and is separated from the muscle beneath by a little areolar tissue. It is attached in the middle line along the linea alba, below to the external part of the crest of the ilium, the deep fascia of the thigh close to Poupart's ligament until the spermatic cord is reached, over which it is reflected, then to the front of the pubes and symphysis. Over the cord and from the front of the symphysis, the two layers of the superficial fascia become firmly united, lose the adipose tissue, acquire elastic and muscular fibres, and thus altered in structure pass over the penis and into the scrotum as the dartos sheath, then into the perineum to become continuous with the superficial perineal or Colles' fascia. See page 404. The band of fibres from the lower part of the linea alba and the front of the symphysis which passes on to the penis, forms the suspensory ligament for that organ. The extension of the fascia through the scro- 


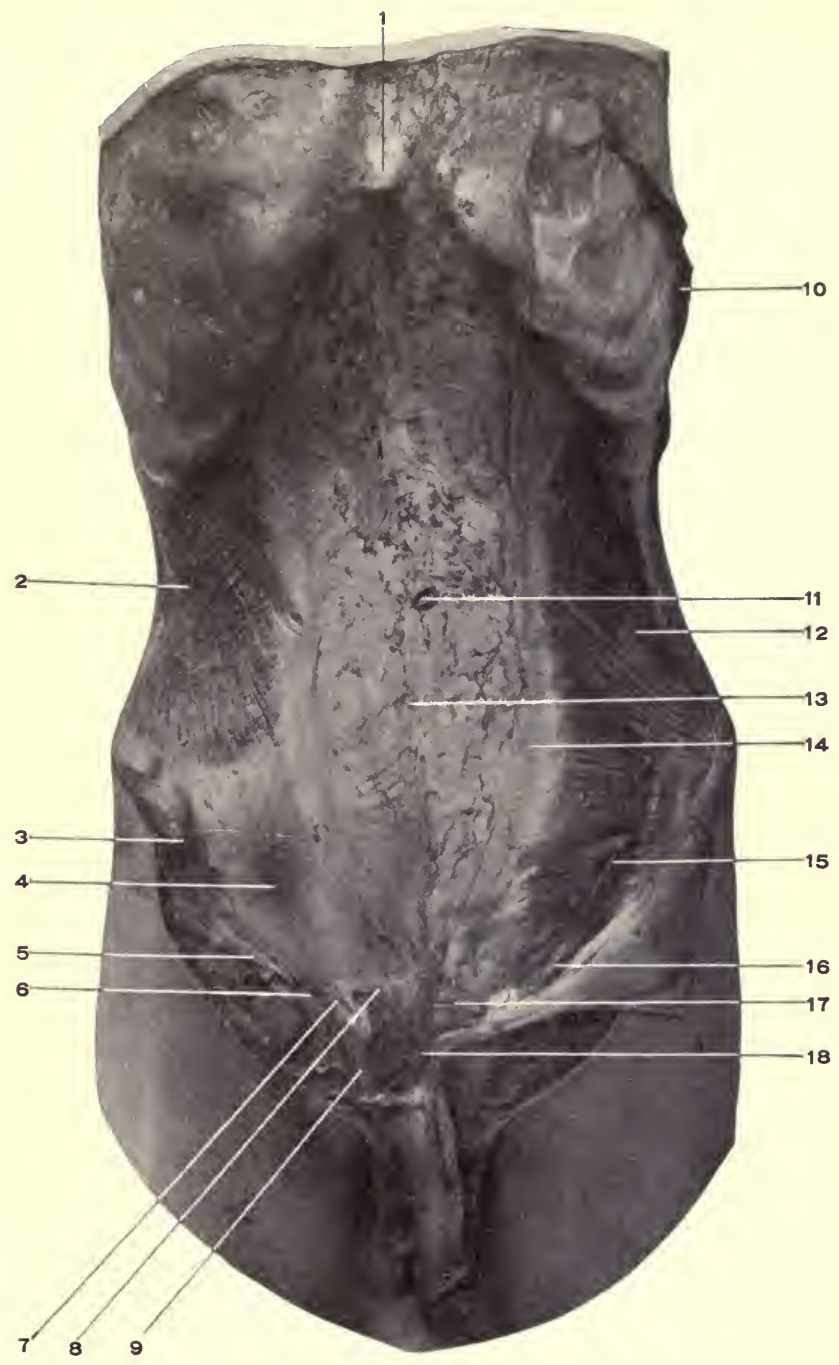

Fig. 89. Dissection of the AbDomen.-1, The ensiform appendix. 2, Muscle portion of the external oblique. 3, Anterior superior iliac spine. 4, Aponeurotic portion of the external oblique. 5, Poupart's ligament. 6, Outer pillar of the external abdominal ring, 7, The external abdominal ring closed by the external spermatic fascia. 8, The internal pillar of the external ring. 9, The spermatic cord. 10, Origin of external oblique. 11, Umbilicus. 12, Internal oblique. 13, The linea alba. 14, The linea semilunaris. 15, The iliohypogastric nerve. 16, The inguinal canal. 17, Conjoined tendon. is, The suspensory ligament of the penis. 
tum to the perineum, and the gap left over the cord, explains how extravasated urine may find its way from the perineum into the scrotum, and even to the lower abdominal region.

The Penis. Figs. 88 to 93 .

Is divided into the root or base, at the symphysis; the glans or head; the neck, just back of the glans; and the body, between the neck and root.

The penis is slung from the symphysis pubis by the suspensory ligament, formed from the deep layer of the superficial fascia (Scarpa's), of the abdomen. See above.

The integument covering the glans is called the prepuce or the foreskin. The angle of the penis is the bend which the flaccid organ makes at the symphysis pubis.

The Superficial Vessels and Nerves of the Penis.The arteries are derived from the superficial external pudic, the nerves from the terminal branch of the iliohypogastric. The veins are venæ comites of the arteries.

The Sheaths of the Penis are described in connection with the Corpus Spongiosum on page 4I 4.

\section{DISSECTION.}

Incise the superficial fascia transversely at the level of the umbilicus and vertically in the middle line. Reflect the several portions, leaving the lower segment fastened by its lower attachments. Notice, $(a)$ the iliohypogastric nerve, which appears through the aponeurosis of the external oblique above the spine of the pubes and passes to the dorsum of the penis. (Superficial dorsal nerve of the penis.) (b) The gap in the superficial fascia over the spermatic cord. (c) The formation of the suspensory ligament of the penis. Incise the dartos sheath along the dorsum of the penis, reflect it laterally. It will be found continuous with the superficial fascia of the abdomen and dartos of the scrotum. 


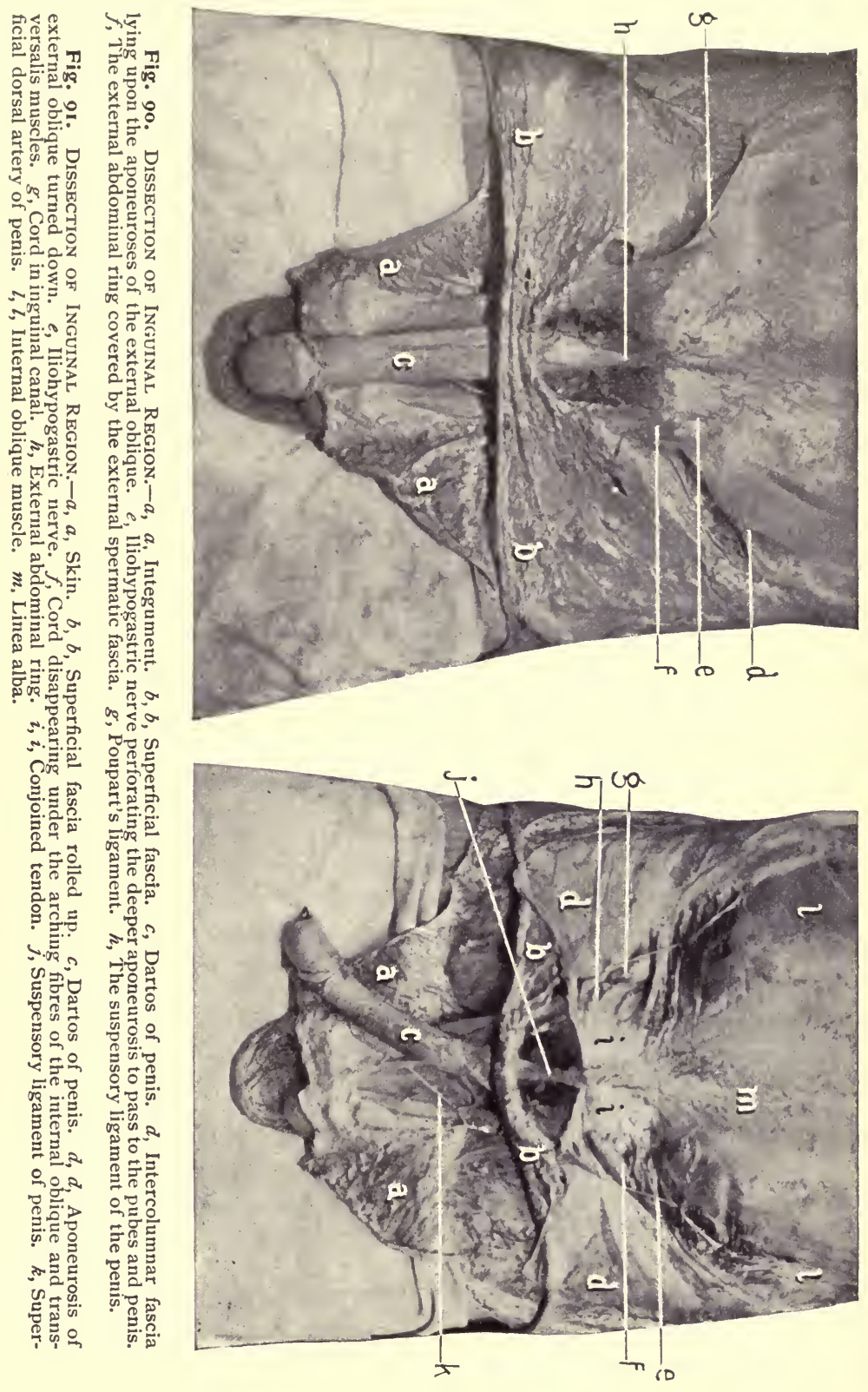


The Deep Vessels and Nerves of the Penis.-The arteries are from the internal pudic, page 4I2. Nerves from the internal pudic nerve, page 4I4.

The Dorsal Vein of the Penis. (Usually single, may be double.)

This emerges from a venous plexus which surrounds the glans penis, passes backward along the dorsum of the penis between the two dorsal arteries, receives in this part of its course large branches from the body of the penis which emerge from between the corpus spongiosum and the corpora cavernosa and wind around the side of the penis to enter the dorsal vein.

Continuing backward, the vein penetrates the suspensory ligament of the penis, then passes through the opening in the triangular ligaments close to the under surface of the pubic arch, divides into two branches which terminate in the prostatic plexus.

Before passing through the triangular ligaments the dorsal vein sends communicating branches to the internal pudic veins. See page 4r3. Within the pelvis it communicates with the obturator vein.

\section{DISSECTION.}

Divide the elastic sheath of the penis along the median line, separating the corpora cavernosa from each other, and from the corpus spongiosum. Consult the descriptions of these structures as already given on pages $4 \mathbf{1} 4,416$.

Leave the corpus spongiosum attached by the membranous urethra to the prostate and bladder, keep them covered with a damp cloth until such time as the bladder has been dissected and the urethra can be opened from end to end.

Incise the skin of the scrotum along the median raphé, and reflect it. Identify the dartos and its connections with the superficial abdominal fascia, then reflect it like the integument. Remove the testicle from the scrotum.

The various coverings of the cord and testicle (Fig. 93) are those that are derived from the several layers of the 
abdominal wall. How these coverings are secured is explained later; only their names can be given now. First is the dartos, as already described. Second, the external spermatic fascia. Third, the cremasteric fascia. Fourth, the infundibuliform fascia. Fifth, the extraperitoneal tissue. In addition to these the testicle has a partial envelope formed of peritoneum, called the tunica vaginalis.

Of these various layers only the first, third, and last can be demonstrated apart from their sources. For the first, see page 406 ; for the second, page 460 ; the last, the tunica vaginalis, is a covering the testicle has acquired from the peritoneum, see page 460 . It consists of two layers; the visceral, enveloping the testicle, except at its posterior part ; and the parietal, forming the walls of the sac containing the gland.

\section{DISSECTION.}

Open the tunica vaginalis and recognize the following parts; The testicle proper and the epididymis.

The tunica albuginea (Fig. 93) is the dense white fibrous covering of the testicle. At the posterior margin of the testicle there is an extension from this capsule into the body of the gland of a fibrous septum called the mediastinum, from which radiate secondary septa dividing the organ into lobes.

The epididymis lies at the back of the testicle and is divided into the head or globus major, the body, and the tail or globus minor, from which the vas deferens extends. See page 542 .

Obliquus Externus Abdominis. Fig. 89.

Origin.-From the outer surface and lower margin of the eight lower ribs, by muscular slips that interdigitate, the three lower ones with the latissimus dorsi, the rest with the serratus magnus. 
Insertion.-By muscular fibres into the anterior half of the external lip of the iliac crest. By its aponeurosis, which unites in the middle line with the opposite muscle (as well as all the other lateral abdominal muscles), to form the linea alba, extending from the ensiform to the symphysis. By a dense band of the aponeurosis, which extends from the anterior superior spine of the ilium over the femoral vessels to the spine, iliopectineal line, crest and front of the pubes. The portion of this band attached to the spine of the pubes is Poupart's ligament, the reflection to the iliopectineal line is Gimbernat's ligament.

The Linea Alba. Fig. 89. Diag. 29.

This is the fascial junction of all the lateral abdominal muscles in the middle line from the ensiform appendix to the symphysis pubis.

A gap is left between the fibres of the external oblique over the spine of the pubes, through which passes the spermatic cord in the male and the round ligament in the female. This is the external abdominal ring or opening. Figs. 89 to 93 .

The outer border of the ring is formed by Poupart's ligament, the inner, by the fibres of the aponeurosis passing to the front of the pubes; these borders are called columns. In order to strengthen this weak spot in the abdominal wall these columns are bound together by fibres passing at right angles to them, and extending outward to near the anterior superior iliac spine. These fibres constitute the intercolumnar fascia. From the lower part of the intercolumnar fascia and the margins of the ring a thin membrane descends upon the cord; this is the external spermatic fascia. It is these structures that close in the gap between the separated fibres of the external oblique and effectually 


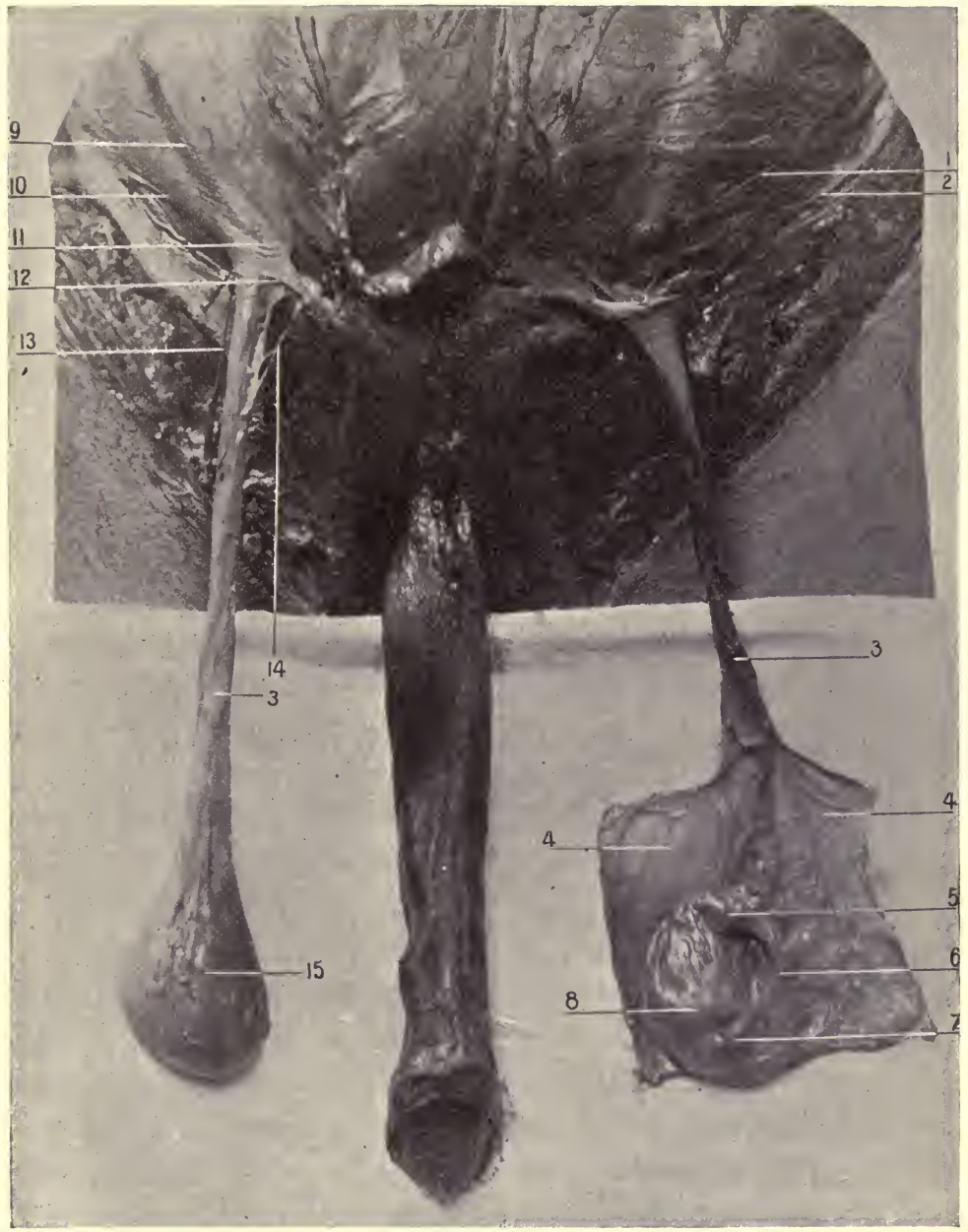

Fig. 92. Dissection of Testicles.-I, Iliohypogastric nerve. 2, Poupart's ligament. 3, 3, Spermatic cord. 4, 4, Tunica vaginalis laid open. 5, Head, 6, Body, 7, Tail of the epididymis. The head is also called the globus major and the tail the globus minor. 8, Testicle enclosed within the tunica albuginea. 9, Intercolumnar fascia. Io, Outer pillar (Poupart's ligament). II, Inner pillar of the external abdominal ring. 12, The external abdominal ring. 13, Ilio-inguinal nerve. 14, Cremasteric,branch of deep epigastric artery. 15, Testicle enclosed within the tunica vaginalis. 
obliterate any ring. In health and normally there is no "ring." In this connection I wish to caution the student in reference to the usual anatomical descriptions of " rings," and "canals." In the living subject there are no abdominal rings except during the descent of the testicle in fotal life or of a hernia at a later period. While in the subject a ring exists only if the above conditions appertain, or as a result of the dissector's labors. These so-called abdominal rings are then only places where openings can be made, or are "potential" rings. The usual habit of giving measurements to these potential rings is to be deprecated, as normally there are no rings, and if made by the dissector they may have any dimensions, according to his fancy or preconceived ideas. By applying measurements to openings that are normal only at a certain time in the individual's life an erroneous opinion is formed of the true relations of the parts, that will interfere, not only with a correct anatomical knowledge of the subject, but also with surgical work upon such regions.

\section{The Triangular Fascia. Fig. 89.}

Just above the symphysis some fibres of the external oblique pass across the median line to be inserted in the opposite ilicpectineal line and pubic crest, forming a small triangular ligament or fascia. The triangular ligament lies behind the internal column of the external abdominal ring, and in front of the conjoined tendon. It will not be seen until the external oblique is reflected. In the male the spermatic cord escapes from the external ring and passes into the scrotum ; in the female the round ligament appears, to quickly become lost in the tissues in front of the pubes. Actions of the External Oblique Muscle.-To protect, also to compress the abdominal viscera, and thus aid defæcation, 
micturition, vomiting, parturition, expiration. If one acts it rotates the thorax to the same side, or the pelvis to the opposite side, and flexes the spine laterally. Both acting will flex the thorax upon the pelvis or the reverse.

Nerve Supply. Page 453.

\section{DISSECTION.}

At the level of the anterior superior spine of the ilium make a small transverse cut through the aponeurosis of the external oblique, until the muscle beneath is seen. Insert a knife-handle or finger in this opening, and, raising the external oblique, divide it transversely toward the medial line, until the linea semilunaris is reached. Reflect the upper part of the muscle, first raising it carefully from the one beneath, and dividing its aponeurosis over the linea semilunaris ; cut away the two or three upper costal attachments of the muscle and turn it backward as far as it will go. The lower portion is to be turned back in a similar manner, dividing the aponeurosis as near the linea alba as convenient, and down to the pubis. This leaves a triangular flap attached by Poupart's ligament, and showing the external ring.

Obliquus Internus Abdominis. Fig. 89.

Origin.-From the posterior layer of the lumbar fascia, from the anterior two-thirds of the middle lip of the iliac crest, and from the outer half of Poupart's ligament.

Insertion.-By the conjoined tendon into the iliopectineal line and crest of the pubes, by its aponeurosis into the whole length of the linea alba from ensiform to symphysis, and by muscular fibres into the lower borders of the last three or four ribs.

The conjoined tendon is formed by the union of the fibres of the internal oblique and transversalis muscles, internal to the middle of Poupart's ligament, into a common tendon, that is inserted into the iliopectineal line and crest of the pubes. The conjoined tendon lies directly back of the external abdominal ring, and strengthens this naturally weak spot. The fibres of the muscles passing into the con- 
joined tendon arch over the spermatic cord (or round ligament).

In the angle between the external and internal oblique muscles is seen the cord (or round ligament) resting upon Poupart's ligament, and disappearing under the arching fibres above mentioned. The space occupied by the cord or round ligament is the inguinal canal. (Fig. 9I.) The same remarks apply to this canal that were made in reference to the abdominal rings - it is not a canal except when made such by a hernia or by the dissector. It is a "potential" canal. It extends from the internal to the external abdominal rings, a distance of one and one-half inches. The floor is formed by Poupart's ligament, upon which the cord is seen to rest. In front is the aponeurosis of the external oblique; behind, the conjoined tendon, internal oblique, and fascia of the transversalis (will be seen later). Above, at outer part, the muscular arch of the two internal muscles.

As the cord is examined, it will be found covered with detached loops of muscular fibres, which are reflected upon it in gradually lengthened festoons as low as the testicle. These muscular loops constitute the cremaster muscle, and with the connective tissue binding the loops together is known as the cremasteric fascia. The cremaster muscle is usually described as detached portions of the internal oblique. Some fibres no doubt come from this source, and some from the fotal structure called the gubernaculum. See page 460 .

The cremaster is supplied by the genitocrural nerve. There is neither cremaster muscle nor fascia in the female.

The ilio-inguinal nerve (Fig. 92) can be traced from where it passed through the external ring, upward and outward until it disappears through the internal oblique and transversalis. It lies close to Poupart's ligament. 


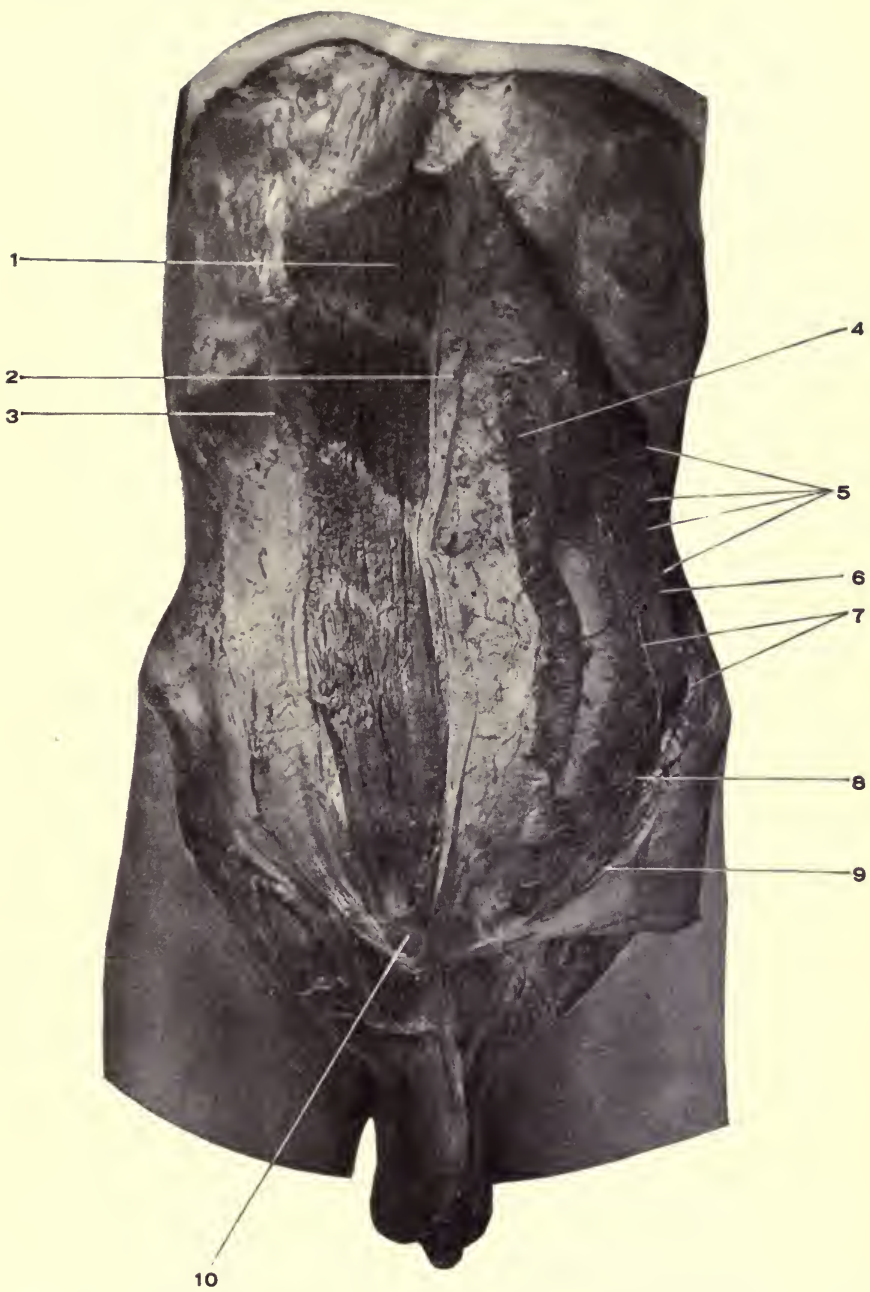

Fig. 93. Dissection of the Abdomen.-I, The rectus muscle with three tendinous intersections. 2, Inner, 3, Outer portion of the anterior layer of the sheath of the rectus. 4, Internal oblique reflected inward. 5, Intercostal nerves. 6, Transversalis muscle. 7 , Muscular branches of the deep circumflex iliac artery. 8, lliohypogastric nerve. 9, Poupart's ligament. Io, Pyramidalis muscle. 
The iliohypogastric nerve (Fig. 89) appears through the internal oblique muscle just above, and an inch internal to the iliac spine, and takes a course downward and forward upon the internal oblique.

The linea semilunaris (Fig. 89) will be seen at the outer border of the rectus muscle. It is formed by the junction of the aponeuroses of the internal oblique and transversalis muscles. The aponeurosis of the internal oblique divides into two layers at the outer border of the rectus muscle for its upper three-fourths. Half of the aponeurosis passes behind the rectus with the whole of the transversalis for this distance, and half in front with the whole of the external oblique for the same extent. In the lower fourth the blended aponeuroses of all three muscles pass in front of the rectus.

Its sheath behind in the lower fourth is then only composed of the Fascia of the Transversalis, see page 454.

The semilunar fold of Douglas (Fig. 94) is the free margin of the posterior sheath of the rectus muscle. It is about midway between the umbilicus and the pubes (and of course behind the rectus).

The aponeurosis of the external oblique joins the internal oblique inside of the linea semilunaris.

Actions of Internal Oblique.-Same as for External, q. $v$. Nerve supply. - See page 453.

\section{DISSECTION.}

Incise the internal oblique muscle transversely inward from the anterior superior iliac spine to the linea semilunaris, and vertically from the same point to the lower border of the ribs, then along their margin until the outer edge of the rectus is reached. Reflect this quadrilateral flap of internal oblique, being careful to leave the nerves and arteries resting upon the transversalis.

Do not attempt to separate the lower triangular portions of the internal oblique from the transversalis, as they are so intimately blended together that the separation cannot be done properly.

The posterior portion : of the internal oblique can be cut away from the ribs and crest of the ilium and left hanging by the lumbar fascia. 
Transversalis Abdominis. Figs. 91, 94.

Origin.-By fleshy slips from the inner surfaces of the six lower costal cartilages, interdigitating with the origins of the diaphragm. From the lumbar fascia, and the anterior three-fourths of the inner lip of the crest of the ilium, and from the outer third of Poupart's ligament.

Insertion.-By the conjoined tendon as given. See page 447. Into the whole length of the linea alba, which it helps to form.

\section{The Lumbar Fascia. Fig. 76. Diag. 29.}

Consists of three layers. The anterior, from the bases of the transverse processes of the lumbar vertebræ. The middle, from the tips of the same processes. The posterior, by the broad sheath of the erector spinæ muscle from the spinous processes and the supraspinous ligaments of the lumbar and adjoining dorsal and sacral vertebræ. Between the first and second layers is the quadratus lumborum; between the second and third, the erector spinæ. That portion of the anterior layer extending from the first lumbar vertebra to the tip of the last rib is called the ligamentum arcuatum externum.

Lying upon the tranversalis muscle are the nerves which are passing forward to supply the abdominal muscles. They are the lower six intercostal and the iliohypogastric and ilio-inguinal from the first lumbar.

Along with the nerves are found branches from the intercostal and lumbar arteries. The deep circumflex iliac artery gives off several large branches which pass upward between the internal oblique and the transversalis muscles. In the dissection of the preceding muscles it has been noticed that the fibres of the three layers run obliquely or at right angles to each other. The fibres of the trans- 
versalis pass horizontally, of the internal oblique upward and forward, of the external oblique downward and forward.

This arrangement insures protection against ventral her-

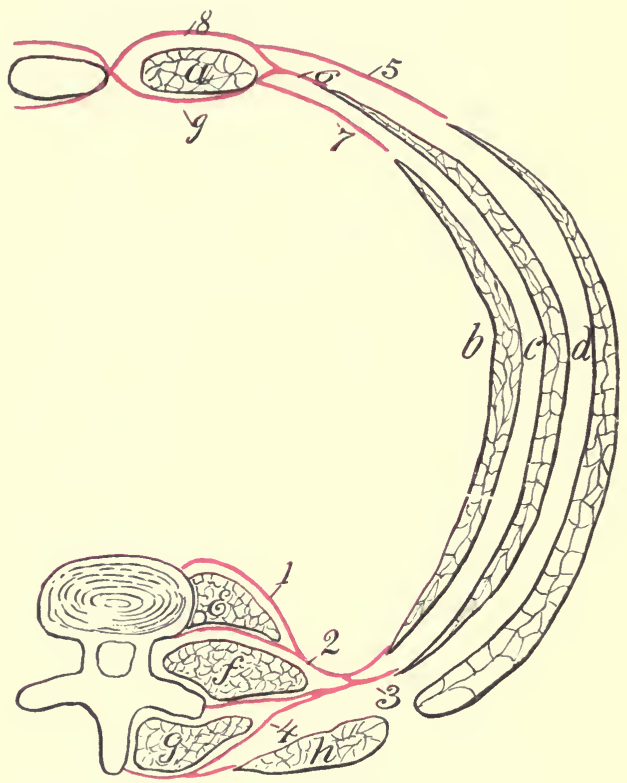

Diag. 29. The Arrangement of the Abdominal Muscles and Lumbar Fascia. (From Holden.)-1, Iliopsoas fascia. 2, Anterior layer of lumbar fascia. 3. Middle layer of lumbar fascia. 4, Posterior layer of lumbar fascia. 5, Aponeurosis of external oblique. 6, Aponeurosis of internal oblique. 7, Aponeurosis of transversalis. 8, Anterior portion of sheath of rectus. 9, Posterior portion of sheath of rectus. $a$, Rectus abdominis. $b$, Transversalis. $c$, Internal oblique. $d, E x-$ ternal oblique. $e$, Psoas magnus. $f$, Quadratus lumborum. $g$, Erector spinæ mass. $h$, Latissimus dorsi.

nia, and is made use of in some operations upon the abdominal viscera where the incision has to be carried through the fleshy part of the muscles, as for removal 
of the appendix vermiformis, or for the formation of an artificial anus in either iliac fossa, the criss-cross arrangement of the fibres forming a reliable sphincter in the latter case.

\section{DISSECTION.}

Incise the sheath of the rectus muscle from the ensiform to the symphysis, three-fourths of an inch from the linea alba. Reflect the two portions, being careful to avoid cutting into the muscle at the tendinous intersections, which are attached to the anterior portion of the sheath, but not to the posterior.

The Pyramidalis. Fig. 9I.

This small triangular muscle is found lying upon the rectus at its lower part.

Origin.-From the crest, anterior surface of body and symphysis of the pubes.

Insertion.-By a slender tendon into the linea alba, halfway between the umbilicus and symphysis.

Action.-To make the linea alba taut, to assist the rectus.

Rectus Abdominis. Fig. 91.

Origin.-By a direct tendon from the crest of the pubes, by a reflected tendon from the structures in front of the symphysis across the middle line.

Insertion.-Into the front of the cartilages of the fifth, sixth and seventh ribs, and outer margin of the ensiform.

Action.-To compress the viscera and aid in all expulsive efforts, as defæcation, micturition, vomiting, expiration, parturition. To flex the spine anteriorly, bringing the thorax and pelvis nearer together.

Nerve Supply for all the Abdominal Muscles.-The six lower intercostal nerves and the iliohypogastric and ilioinguinal branches of the first lumbar nerve. 


\section{DISSECTION.}

Raise the rectus from its sheath, beginning at its inner margin. The deep epigastric artery will be seen entering the posterior part of the muscle from below and the superior epigastric from above. The deep artery is a branch of the external iliac, and the latter, of the internal mammary. They anastomose in the substance of the rectus and form the longest arterial anastomosis in the body

Divide the deep epigastric as it enters the muscle, cut the nerves at the outer border of the muscle, and the muscle itself at its middle. Reflect the two portions.

The posterior part of the sheath of the rectus can now be seen. The upper three-fourths is formed by the aponeurosis of the transversalis with one-half of the internal oblique, and ends below in a free border, called the semilunar fold of Douglas, midway between the umbilicus and the symphysis, or at the junction of the upper three-fourths with the lower one-fourth of the rectus. The lower onefourth is formed by the transversalis fascia.

\section{DISSECTION.}

Cut through the transversalis from the fold of Douglas to the anterior superior iliac spine, saving the iliohypogastric and inguinal nerves. This gives below a triangular flap consisting of the fibres of the transversalis and internal oblique attached by the conjoined tendon and Poupart's ligament.

The posterior part of the rectus sheath is to be divided along the linea alba to the ensiform, then the transversalis and overlying muscles removed entirely from their attachments to the ribs, ilium, and lumbar fascia. The peritoneum nust not be perforated in this separation. To succeed in this will take some time and patience.

In reflecting the lower triangular flap do not take up the fascia transversalis, which is closely united to the muscle, but is distinct from it.

The Fascia Transversalis. Fig. 94.

This is a layer of condensed connective tissue interposed between the transversalis muscle and the extraperitoneal tissue. It is thin in the upper region of the abdomen where it becomes continuous with the fascia covering the 


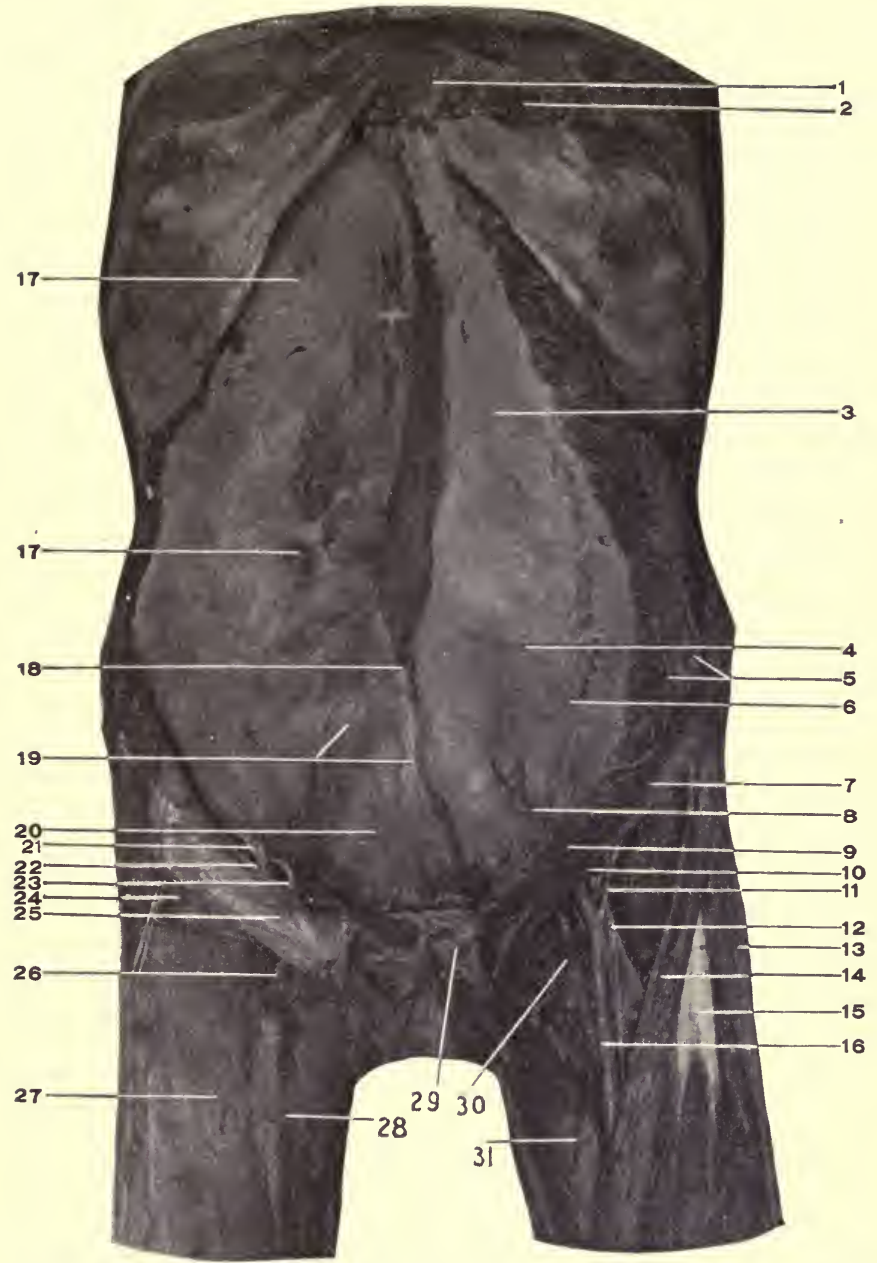

Fig. 94. Dissection of the Abdomen.-1, Superior epigastric artery. 2, Rectus abdominis, reflected. 3, Posterior portion of sheath of the rectus abdominis. 4, Semilunar fold of Douglas. 5, Muscular branches of deep circumflex iliac artery resting upon the transversalis nuscle. 6, Iliohypogastric nerve. 7, Ilio-inguinal nerve. 8, Deep epigastric artery covered by transversalis fascia. 9, Site of the internal abdominal ring. 10, Poupart's ligament. Ii, Deep circumflex iliac artery, having a low origin below Poupart's ligament. 12, Anterior crural nerve. Lines 8 to 12 cross the iliacus muscle. 13, Tensor vaginæ femoris. 14, Sartorius. I5, Rectus femoris. 16, Femoral artery lying in Scarpa's space or triangle. Notice its boundaries, floor, and contents. 17, 17, Peritoneum. The parietal layer. 18, Inner margin of Douglas's fold. 19, Obliterated hypogastric arteries. 20, Urachus. 21, Spermatic cord. 22, The opening (infundibular) of the internal abdominal ring in the transversalis fascia. 23, Deep epigastric artery and relation to internal abdominal ring. 24, Transversalis muscle, reflected. 25, Transversalis fascia. 26, Saphenous opening. 27. Fascia lata. 28, Long saphenous vein. 29, Pyriformis muscle. 30, Femoral vein. 31. Adductor longus muscle. 
diaphragm, but becomes thicker as the inguinal region is approached, and when Poupart's ligament is reached is a membrane of considerable density.

Attachments.-Below, to the crest of the ilium, to the whole of Poupart's ligament (excepting over the femoral vessels, where it passes under Poupart's ligament to form the anterior sheath of the same), to the outer part of Gimbernat's ligament, to the iliopectineal line and inner surface of the pubes and symphysis. In the middle line it is continuous from side to side. Laterally, it passes into the loose tissue in those parts. Above, it becomes continuous with the fascia lining the diaphragm. The portion of the fascia beneath Poupart's ligament and arching over the femoral vessels is called the crural arch.

The internal abdominal ring (Fig. 94) is a potential opening through the fascia transversalis, formed by the passage through it of the spermatic cord in the male and the round ligament in the female. It is situated just above the middle of Poupart's ligament. From the margins of the internal ring there is a thin layer of membrane projected upon the cord (or round ligament); this is the infundibuliform process of the fascia transversalis. For its formation, see page 460 .

\section{DISSECTION.}

Incise the transversalis fascia transversely at the level of the umbilicus and along the middle line, providing it has been demonstrated as a continuous layer; reflect its lower portion, which is thicker than the upper and lies in front of the deep epigastric artery, and turn this portion downward. Trace the deep epigastric artery to its source, raising the peritoneum and contents from the iliac fossa. Examine the relations about the internal ring.

The remaining portion of the deep epigastric artery has been exposed by this dissection. It is seen to arise from the front of the external iliac close to Poupart's ligament, 
having the cord or round ligament curving around its outer side, and passing upward and inward to enter the sheath of the rectus muscle, over the semilunar fold of Douglas, to finally inosculate with the superior epigastric in the substance of the muscle.

The position of the artery is between the two abdominal rings, or to the inner side of the internal, and to the outer side of the external ring. It takes part in the formation of Hesselbach's triangle, constituting its outer side, the other boundaries being the outer margin of the rectus to the inside and Poupart's ligament for the base. Through this triangle the direct inguinal hernia must pass.

The Formation of the Abdominal Rings, Coverings of the Testicle and Cord.-In order to make the subject clear we must turn to the development of the parts.

Take the time in the development of the fœtus when the testicle is at the back part of the abdomen, below the kidney and behind the peritoneum, which encloses its front and sides, forming the mesorchium. The testicle has the gubernaculum, a fibromuscular cord passing from its lower border through the abdomen to be attached in three main divisions, a middle band passing to the bottom of the scrotum, an anterior one to the structures in front of the pubes, a posterior one to the tissues over the ischiatic region.

The testicle descends to reach the inner surface of the abdominal wall and the gubernaculum shortens; whether the testicle descends because of the contraction of the gubernaculum, or its shortening is only an incident in the descent of the testicle, are disputed points with no way of solution. The testicle is preceded in its descent by a process of the peritoneum which is drawn downward as it were by the gubernaculum. It also takes with it the peritoneum by which it is nearly enclosed. Arriving against the inner 
surface of the abdominal wall the testicle makes no hole, ring, or opening through its layers in the sense of a division or separation of fibres, not this, but it bulges in front of itself first the transversalis fascia, then the transversalis

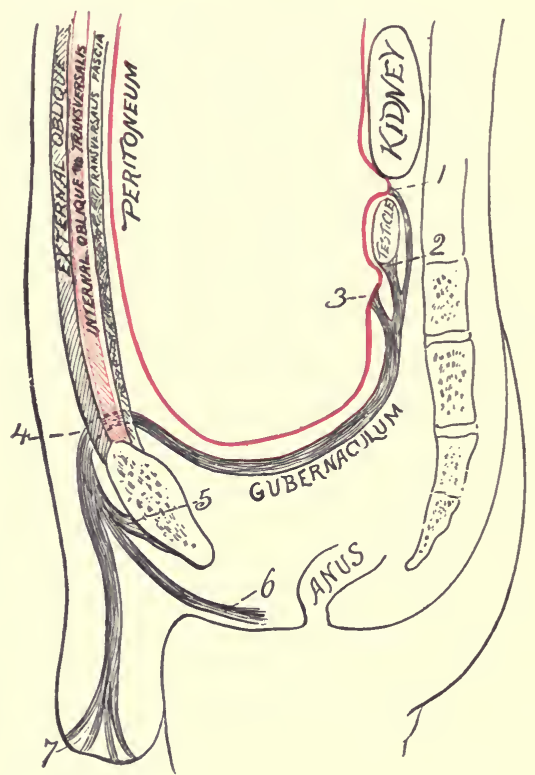

Diag. 30. Diagrammatic Representation of the Descent of the Testicle And Formation of Abdominal Rings and Coverings for the Testicle and CORD. (I.S. H.)-I, The attachment of the gubernaculum to the peritoneum above the testicle. 2, Its attachment to the testicle. 3, Its attachment to the peritoneum below the testicle. 4, Passage of gubernaculum through the abdominal wall. 5, Anterior band attached over pubic region. 6, Posterior band attached over ischiatic region. 7 , Middle band attached to base of scrotum.

muscle, and internal oblique, and finally the external oblique ; stretching and thinning these several layers in front of itself it carries them all into the scrotum to form the coverings for itself, the cord and for any hernia following this 
course. The testicle may be arrested in its course and never leave the abdominal cavity, it may remain in the inguinal canal, or it may be directed to the front of the pubes or into the perineum by the slips of gubernaculum outside of the scrotum. After the passage of the testicle these

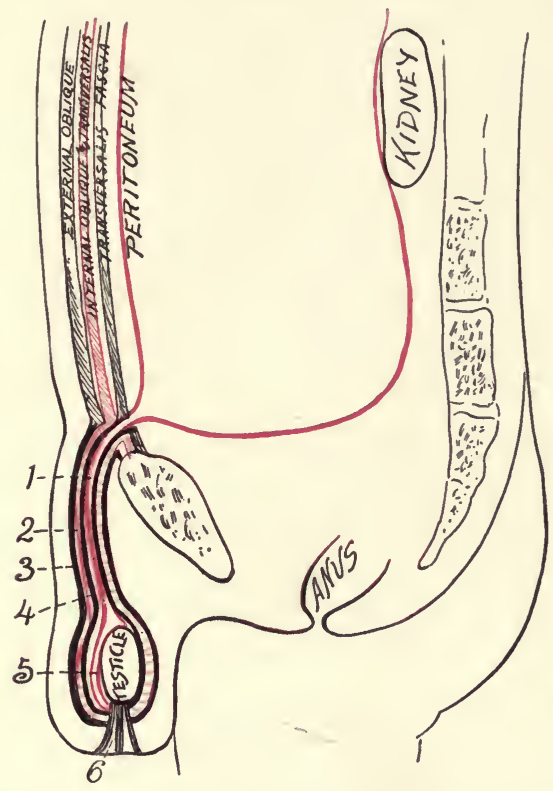

Diag. 31. (I.S. H.) See Diag. 30.-I, Vaginal process of peritoneum. 2, Infundibuliform fascia. 3, External spermatic fascia. 4, Cremasteric fascia. 5, Tunica vaginalis. 6, Remains of middle band of gubernaculum.

several layers of the abdominal wall contract upon the cord where it passes through them, and the funnel-shaped processes derived from them become so thinned that in the adult they cannot be demonstrated except close to their points of origin (at the rings). In this connection consult a surgery for the various forms of inguinal hernia. 
The covering derived from the transversalis fascia receives the name of the internal spermatic or infundibuliform fascia ; that from the internal oblique muscle, partially composed of separate muscle loops and supporting connective tissue, forms the cremaster muscle (and fascia); that from the external oblique constitutes the external spermatie fascia. By the passage of the testicle into the scrotum the internal and external portions of the gubernaculum become inverted, while the middle remains as a band joining the gland to the bottom of the scrotum. The inverted portions of the gubernaculum help to form the cremasteric muscle in part. The internal and external bands of the gubernaculum sometimes draw the testicle to the region of their attachment ; this explains the abnormal position of this organ in front of the pubes or in the region of the ischium or perineum. If the natural process is arrested the testicle may never leave the interior of the abdomen or it may be left in any part of its course through the abdominal wall (at any point in the inguinal canal).

The places where the testicle enters and leaves the abdominal wall are called rings, and its course between these points the inguinal canal.

As the testicle descends it takes with it a tubular process of peritoneum called the funicular process. Naturally, this tube becomes closed at the internal ring and close to the testicle, and the intervening portion entirely obliterated. The portion attached to the testicle forms its tunica vaginalis.

If the process is arrested any of these conditions may prevail: If the tube remains open above, fluid may pass into it, forming a congenital hydrocele, or the intestine may become prolapsed into the tube, giving rise to a congenital hernia. If it becomes closed at its extremities but open between, a hernia may still descend behind the funicular 
process, forming the infantile variety of rupture ; in this case three layers of peritoneum intervene between the bowel and skin; or fluid may distend the tube, producing an encysted hydrocele of the cord.

Even if nature's processes are completed a hernia may develop, pass out through the internal ring, inguinal canal, and external ring. This is the indirect form of inguinal hernia. If the hernial protrusion leaves through Hesselbach's triangle (see page 457) and appears at the external ring, it becomes the direct form of inguinal hernia.

The coverings of the indirect inguinal hernia are these: The peritoneum, extraperitoneal tissue, the infundibuliform fascia, the cremasteric fascia, the external spermatic fascia, the superficial and deep layers of the superficial fascia, and the integument.

The direct inguinal hernia takes a course through Hesselbach's triangle and directly out through the external ring. Its coverings are the peritoneum, extraperitoneal tissue, fascia transversalis, conjoined tendon, external spermatic fascia, two layers of the superficial fascia and the skin.

\section{DISSECTION.}

Raise the peritoneum from the iliac fossæ, exposing the iliac vessels. This shows how they may be reached and ligated without opening the peritoneum.

Clean the extraperitoneal tissue from the surface of the peritoneum ; it will be found thicker in the lower than the upper part.

Find three fibrous cords passing from the umbilicus downward to the region of the bladder. The two outside ones are the obliterated hypogastric arteries, the middle one the urachus. The arteries return the blood in the fotus to the placenta, the urachus connects the bladder with the allantois. By inflating the bladder these cords can be easier dissected out, at the same time it will be seen that the peritoneum does not cover the anterior wall of the bladder for a distance of nearly two inches above the pubes. This area of bladder uncovered by peritoneum and separated from the inside of the abdominal wall by extraperitoneal tissue is the space of Retzius. Through this space suprapubic cystotomy is performed. 


\section{THE ABDOMEN, Interior.}

The Peritoneum. Fig. 94.

The peritoneum may be called a closed sac (in the female it is not strictly such, as the Fallopian tubes open into it) lining the abdominal cavity. Imagine the abdominal cavity empty, line this throughout with a thin membrane. From the back push in the alimentary canal and the organs connected with it, from below the urinary viscera. The thin membrane is pushed forward by the various organs which receive a covering from the membrane, either complete or partial according to the extent that they extend into the general cavity. This membrane is the peritoneum, the layer lining the abdominal walls is the parietal, that covering the abdominal organs is the visceral.

Leaving the adult peritoneum for the time, it will be necessary to follow the development of this membrane as well as that of the abdominal viscera in order to gain a correct idea of the relations as they are found in the adult. The primitive alimentary canal is simply a straight tube extending along near the posterior part of the embryo and held to this position by a fold of peritoneum. This fold of peritoneum forms only an incomplete, vertical septum through the greater portion of the abdominal cavity (Diag. 32, Nos. 3, 4, 5), but in the upper portion this fold extends clear forward to the anterior abdominal wall (Diag, 32, No. 7). This portion of the septum is also joined to the under surface of the diaphragm.

The alimentary canal soon becomes differentiated by growth into stomach, duodenum, small intestine, and large intestine.

The peritoneum receives different names according to the 
part of the alimentary canal to which it is attached. That to the stomach is the mesogaster, to the duodenum is mesoduodenum, to the small intestine is mesentery, to the

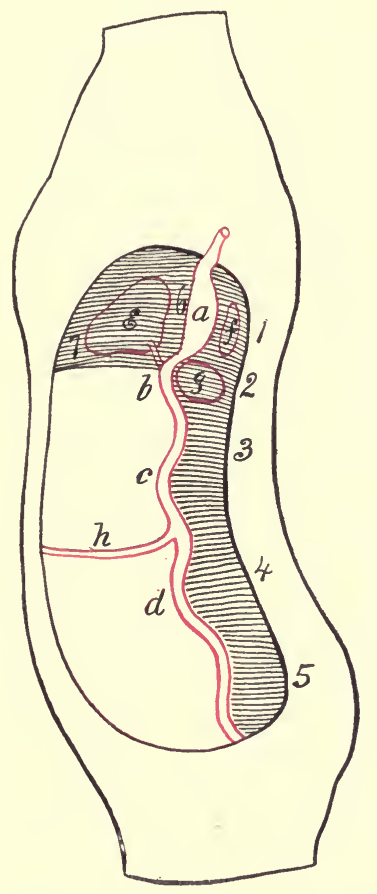

Diag. 32. To Illustrate the Development of the Alimentary Canal and Peritoneum. The development is traced within the outlines of an adult abdominal cavity in order to make the subject clearer. (I. S. H.) - a, Stomach. $b$, Duodenum. $c$, Small intestine. $d$, Large intestine. $e$, Liver. $f$, Spleen. $g$, Pancreas. $h$, Vitello-intestinal duct. I, Mesogaster. 2, Mesoduodenum. 3, Mesentery. 4, Mesocolon. 5, Mesorectum. 6, Gastrohepatic omentum. 7, Suspensory ligament of liver.

large intestine mesocolon, then mesosigmoid, and mesorectum.

There are arteries developing during this stage. Along the back of the fotus is the aorta, lying between the two 
folds of the peritoneum that attaches the alimentary canal to the back of the foetus. From the aorta branches pass to the various parts of the intestinal tube : To the stomach passes the gastric; to the

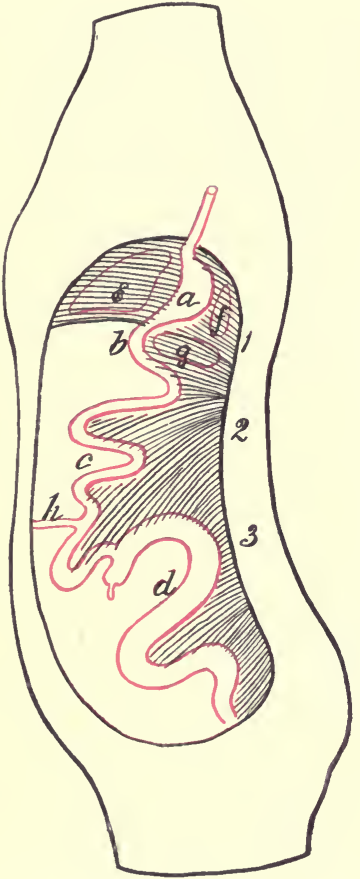

Diag. 33. A Second Stage in the Process of Development. ( $I$. $S$. H.)-Increase in size of stomach, length of small intestine, and size and length of large intestine. Decrease in size of vitello-intestinal duct. small and large intestine the superior mesenteric; and to the large intestine the inferior mesenteric. All these arteries run to their distribution between the folds of the peritoneum which encloses the alimentary canal.

The growth of the bowel is mostly below the stomach and duodenum, and takes place faster than the growth of the corresponding part of the peritoneum, consequently the small intestine is thrown into numerous folds.

One of these folds is in the duodenum and another in the large intestine (Diag. 33, b). As development proceeds the bend in the large intestine is brought nearer to the duodenal curve and the mesentery acquires a distinct $\mathrm{neck}$. (Diag. 34.)

There now occurs a most interesting change. This change is the rotation of the intestinal tract below the duodenum on the superior mesenteric artery as an axis, the large intestine, or the colic part of it, passing from the left 
up over the duodenum to the right to a position at the right side of the abdominal cavity, while the small intestine passes to the left and lies within the arch formed by the large in-

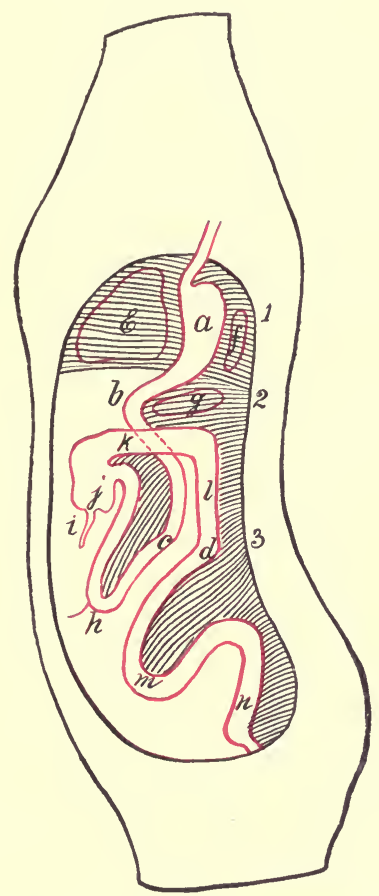

Diag. 34. A Third Stage in the Process of Development. (I. S. H.) The large intestine having passed upward and crossed from left to right in front of the duodenum. $-a$, Stomach. $b$, Duodenum. $c$, Small intestine. $d$, Large intestine now differentiated into several portions-as $i$, Vermiform appendix. $j$, Cæcum. $k$, Transverse colon (ascending colon not yet developed). $l$, Descending colon. $m$. Omega loop. $n$, Rectum. e, Liver. $f$, Spleen. $g$, Pancreas. I, Mesogaster. 2, Mesentery, 3, Mesocolon.

testine. (Diags. 34 and 35.) This rotation forms a twist in the mesenteric stalk and a fossa that remains in the adult as the duodenojejunal fossa. 
At the beginning there is no separation between the abdominal and thoracic cavities; such a division is formed later by the development of the diaphragm. The mesogaster does not stop with the stomach, but passes forward

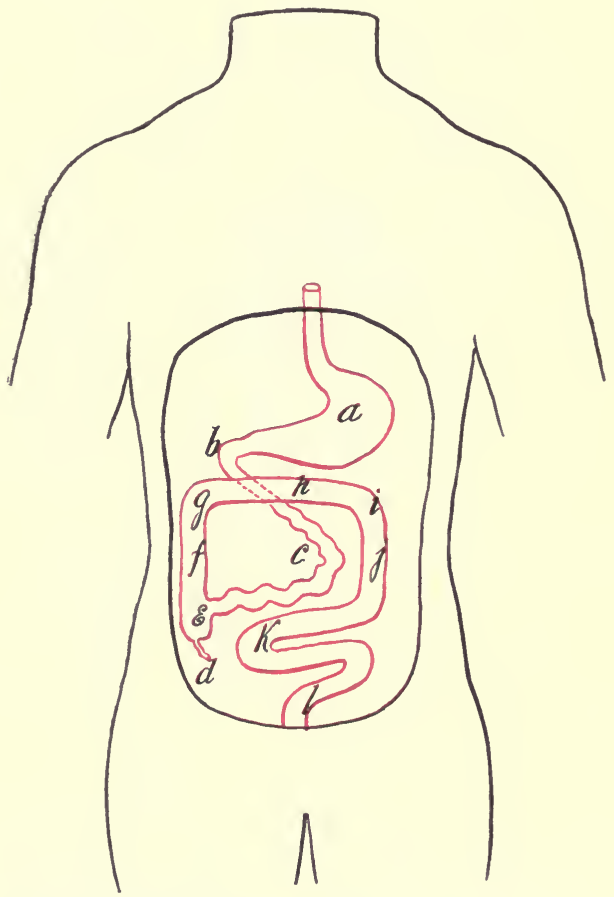

Diag. 35. The final Stage in the Process of Development. (I. $S$. $H$.) The alimentary canal in its adult position. $-a$, Stomach. $b$, Duodenum. $c$, Small intestine. $d$, Vermiform appendix. $e$, Cæcum. $f$, Ascending colon. $g$, Hepatic flexure. $h$, Transverse colon. $i$, Splenic flexure. $j$, Descending colon. $k$. Omega loop. $\zeta$, Rectum.

to the anterior abdominal wall and diaphragm, on to which it is reflected. Within the two layers of the anterior mesogaster (in front of the stomach), and springing from the 
duodenum, to which it is connected by a duct, is a mass of budding cells that subsequently forms the liver. The fotal liver, then, is in front of the stomach, between the layers of the anterior mesogaster (future hepatic ligaments and gastrohepatic omentum), by which it is connected to the diaphragm the stomach, and duodenum. Into the last opens the duct of the liver. Behind the stomach another mass of cells springs up from whose growth the spleen is formed; this also develops between the two layers of the mesogaster. In the same way the pancreas springs from a mass of cells in the mesoduodenum (posterior to the gut). (Diags. 32, 33, 34, e.f.g.)

Coincident with the rotation of the small and first parts of the large intestine, the stomach, first part of duodenum, and liver turn over toward the right side of the abdominal cavity (Diag. 35), while the spleen is crowded over to left side of the abdomen.

In order to understand these new relations and how they come to be formed, we must consider how the peritoneal attachments can be shifted.

There is more surface to be covered than there is peritoneum to cover it. Some parts must be only partially covered by peritoneum in this case; this is what really occurs. In the beginning there is enough peritoneum to cover the primitive gut, but the gut, liver, spleen, etc., growing faster than the peritoneum, some of them become deprived of their peritoneal covering in part. The attachments of the peritoneum to the abdominal wall are also shifted by the peritoneum being drawn up in one direction more than in another, until the base of the original attachment is completely altered. For instance, the colon rotates to the right side of the abdomen, the right layer of the mesocolon is taken up by the growth of the other abdomi- 
nal organs (chiefly the liver), until the colon may be left covered only in front and at the sides (the usual condition), or, less peritoneum being drawn off from the colon, there will be left a distinct mesocolon of varying length. In the same manner the entire attachment of the gut below the stomach is moved to the left.

As a part of this process of peritoneal alterations the Formation of the Omentum must next be explained.

It is formed by the pouching to the left and forward of the mesogaster below the spleen and as far as the duodenum.

This bag will be composed of two layers of the peritoneum originally forming the mesogaster. It comes to hang down over the intestines covering them in. In a vertical section of the fœetus from before backward, the dilated bag of mesogaster (omentum) is seen to come off from the lower part of the stomach, and after making the downward loop, to return to the back of the abdomen, where it is attached: the transverse mesocolon and the mesentery are attached separately and in this order below the posterior omental attachment. (Diags. 36, 37.)

As growth goes on, the under layer of omentum is drawn away from the spine and then from the upper part of the colon, while the inner layer comes to rest upon the colon and to form the upper layer of its mesocolon. (Diag. 38.) This gives the typical formation seen in the adult vertical anteroposterior sections. If the process is interrupted, the foetal condition prevails and the omentum is separate from and above the transverse mesocolon at their posterior attachments. (Diag. 37.) This failure of development is a predisposing condition to volvulus of the bowel.

The omentum passing to the colon and becoming attached 
to it in the above manner is called the gastrocolic or the great omentum, to distinguish it from the peritoneal bands

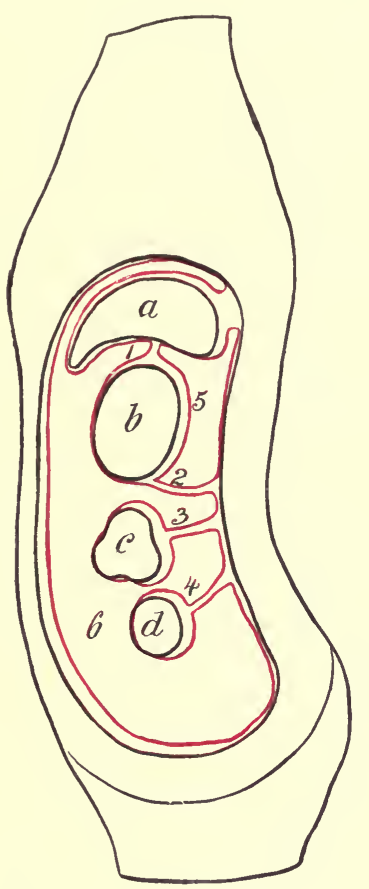

Diag. 36. The Development OF THE GREAT OMEntum. (Modified from Minot.) The abdominal viscera are supposed to be in their final stage of development while the peritoneum is drawn in its fotal state. $-a$, Liver. $b$, Stomach. $c$, Transverse colon. $d$, Small intestine. I, Gastrohepatic omentum. 2, Mesogaster from the stretching of which the great omentum is formed. 3, Mesocolon. 4, Mesentery. 5, Cavity of great omentum. 6, General peritoneal cavity. 5, and 6, communicate at right of gastrohepatic omentum (foramen of Winslow).

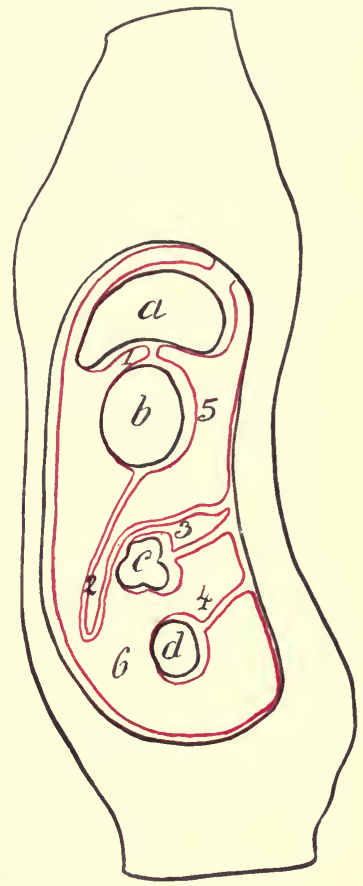

Diag. 37. A Second Stage in the Development of the Great OMENTUM. (Modified from Minot.) The condition here represented, viz. of the great omentum passing for a separate attachment to the back of the abdominal cavity above the transverse mesocolon sometimes is found to prevail in the adult. Letters and numbers as in Diag. 36.

joining the stomach to other abdominal viscera, as the 
gastrohepatic and gastrosplenic omenta to the liver and spleen, respectively.

The cavity of the great omentum is also called the cavity of the lesser peritoneum. It communicates with the general peritoneal cavity by means of the opening to the right and behind the gastrohepatic omentum, which in the fotus is a very wide opening, but in the adult the primitive relations have become so altered that this opening will only admit two fingers, and receives the name of the foramen of Winslow.

The posterior attachment of the peritoneum in the fotus is, as already stated, in a straight line ; in the adult it is in several lines, none of which are straight. (Diag. 39.) The course in the adult that represents the primitive peritoneal attachment is above, beginning with the falciform ligament of the liver, through the gastrophrenic ligament of the stomach, the gastrosplenic omentum (all that is left of the original mesogaster), then to the attachment of the descending mesocolon, the sigmoid mesentery, and the mesorectum, the last coming back to the middle line in the lower part of its course, all the rest lying to the left of the middle line. The other adult attachments are acquired in the manner already explained. They are for the small intestines and the ascending and transverse colon. The mesenteric attachment starts above to the left of the second lumbar vertebra and passes downward to the right iliac fossa. From the last point the mesentery for the ascending colon extends upward to the lower border of the thorax, then turns to the right, forming the transverse mesocolon. At the upper end of the ascending colon a band of peritoneum passes off horizontally to be attached to the lateral abdominal wall between the crest of the ilium and the lower border of the thorax. Upon this shelf the right extremity of the liver 
rests, hence it is called the sustentaculum hepatis. Similarly, at the point where the transverse colon bends downward to form the descending colon, a peritoneal process passes outward to the tenth rib; this band is the costocolic ligament, or, as the spleen rests upon it, the sustentaculum splenis.

To return to the fotal condition once more, from the front of the small intestine a duct passes off to the yolk sac; this is the vitello-intestinal duct. Usually this duct becomes entirely obliterated, but sometimes it may persist in the adult as Meckel's diverticulum, usually as a very short, blind tube, from one to three feet from the cæcum; less often does it reach any considerable length, but when it does it may become a menace to the individual from forming adhesions and a portion of the bowel being strangulated by it.

Peritoneal reflections as seen

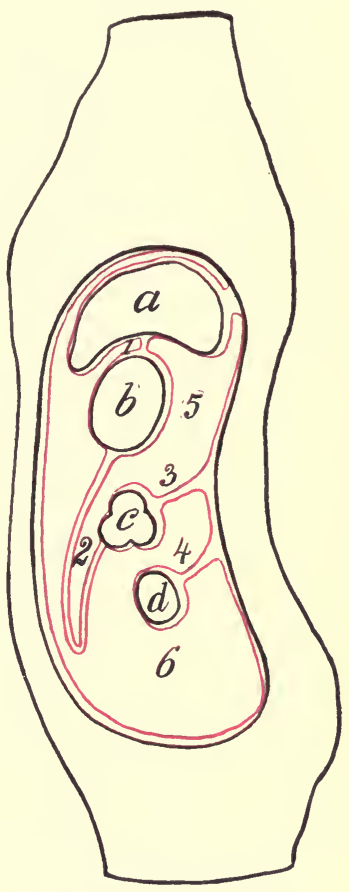

Diag. 38. The Final Stage in the Development of the Great OMENTUM. Letters and numbers as in Diag. 36. when the abdomen is opened in the adult. The peritoneum is divided, for purposes of description, into various parts, depending upon the location. The portion of the membrane lining the interior of the abdominal cavity is called the parietal layer, that covering the abdominal organs, the visceral. The peritoneum 
passing between various parts of the viscera, or viscera and abdominal wall, is further classified as omentum, that is, peritoneum attaching the stomach to adjacent organs, as gastrohepatic, gastrocolic, and gastrosplenic omenta ; mesentery,

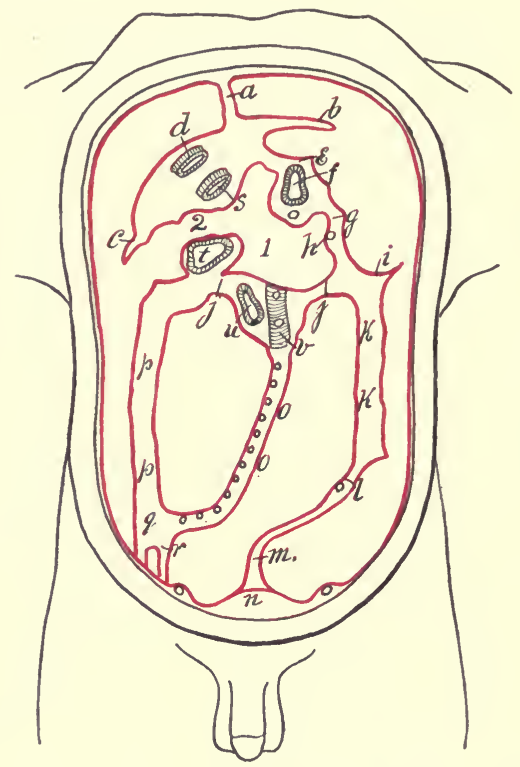

Diag. 39. The Parietal, Peritoneal Attachments of the Abdominal VISCERA. (From Cunningham, in Morris's Anatomy.)-a, Falciform ligament of liver. $b$, Left lateral. $c$, Right lateral ligaments of liver. $d$, Inferior vena cava. $e$, Gastrophrenic ligament. $f$, Esophagus. $g$, Gastrosplenic omentum. $h$, Splenic artery. $i$, Costocolic ligament. $j, j$, Transverse mesocolon. $k, k$, Descending colon. $l$, Sigmoid mesocolon. $m$, Mesorectum. $n$, Bladder. $o, o$, Mesentery. $p, p$, Ascending colon. $q$, Cæcum. $r$, Vermiform appendix. $s$, Inferior vena cava. $t$, Duodenum. $u$, Duodenum. $v$, Abdominal aorta. 1 , Cavity of great omentum. 2, Foramen of Winslow.

the peritoneal attachment of the intestine below the stomach to the posterior abdominal wall, the part of the bowel being indicated by a combination of the term meso- and the portion of the bowel, as mesoduodenum, mesocolon, sigmoid- 
mesentery, mesorectum ; and ligament, the process of peritoneum that connects and supports organs not parts of the alimentary canal, as the ligaments of the liver, uterus, etc. ; exceptions are the gastrophrenic and costocolic ligaments.

The hollows formed by the reflections of the peritoneum are called fossæ, the position being indicated by the names of the parts between which the fossa is formed, as rectovesical fossa:

The anterior parietal layer of peritoneum has been exposed. It is seen to cover the abdominal organs and reach from the pelvic cavity to the under side of the diaphragm. Attention has already been called to the fact that the peritoneum can be raised from the iliac fossæ, exposing the vessels there. The membrane is now to be opened by an incision from the ensiform to the symphysis at the left of the middle line, and one horizontally across just below the umbilicus.

Reflect the four segments. Attached to the right upper one are two ligaments of the liver. Save these. Diag. 40.

To follow the peritoneum, the membrane is seen lining the under surface of the diaphragm to the posterior portion ; it then is reflected on to the liver and the osophageal end of the stomach, forming the anterior layer of the coronary and lateral ligaments of the liver and the gastrophrenic ligament of the stomach ; then, covering the upper surface of the liver, the peritoneum turns over its anterior border to line its under surface as far backward as the point of entrance of the hepatic vessels; at this place the membrane descends to the lesser curvature of the stomach, forming the anterior layer of the gastrohepatic omentum.

The peritoneum covers the entire anterior surface of the stomach and first portion of the duodenum, and from the greater curvature of the former and front of the latter this 
anterior layer (having been joined by the peritoneum covering the posterior wall of the stomach, vide infra) falls.

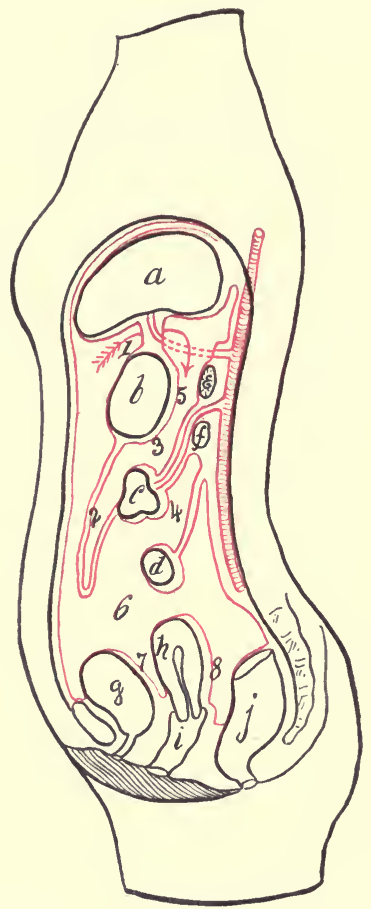

Diag. 40. A Diagrammatic Median anteroposterior Section of the Abdominal Cavity, to Illustrate the Reflections of the Peritoneum. (Modified from Quain.)-a, Liver. $b$, Stomach. $c$, Transverse colon. $d$, Small intestine. $e$, Spleen. $f$, Pancreas. $g$, Bladder. $h$, Uterus. $i$, Vagina. $j$, Rectum. I, Gastrohepatic omentum. The arrow passes around its right (free) margin from 6 , the general peritoneal cavity, into 5 the cavity of the great omentum through the foramen of Winslow. 2, Great omentum. 3, Transverse mesocolon within which is the superior mesenteric artery. 4, Mesentery. 5, Cavity of great omentum. 6, General peritoneal cavity. 7, Uterovesical pouch. 8, Pouch of Douglas.

downward in a broad fold over the small and large intestines and returns behind this first layer to be attached to 


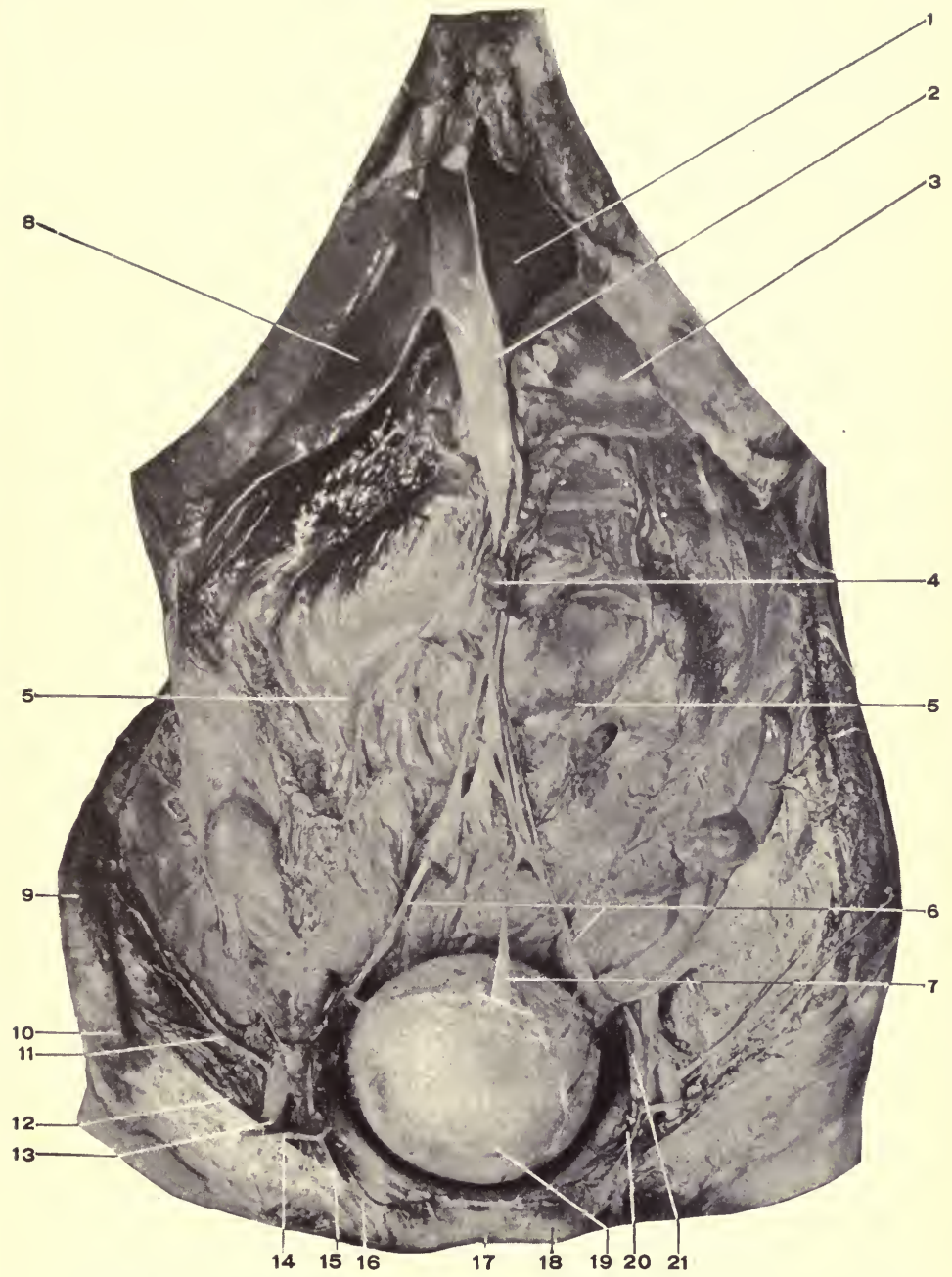

Fig. 95. Abdominal Viscera (Male). [Subject horizontal.]-I, Left lobe liver. 2, Falciform ligament of the liver. 3, Stomach. 4, Umbilicus. 5, 5, Great omentum covering intestines. 6, Obliterated hypogastric arteries. 7, Urachus. 8, Right lobe liver. 9, Crest. Io, Anterior superior spine of ilium. II, Deep circumiflex iliac artery. 12, Poupart's ligament. I3, External iliac artery. 14, Deep epigastric artery. 15, Spermatic cord. Notice the relations of 14 and 15. 16, Pubic branch of I4. I7, Symphysis pubis. 18, Pubic spine. 19, Bladder moderately distended. 20, Site of femoral ring. 21, External iliac vein. 
the transverse colon. This is the great, or gastrocolic omentum.

Raise the great omentum and with it the transverse colon. The peritoneum passing from this part of the colon to the posterior abdominal wall is the lower layer of the transverse mesocolon.

From the posterior attachment the peritoneum extends down upon the small intestines; enclosing them, it returns to the abdominal wall, these two surfaces forming the right upper and left lower layers of the mesentery. It will be seen that the mesentery has a short base which is attached to the abdomen in an oblique line extending from the left of the second lumbar vertebra downward to end in the right iliac fossa, that its intestinal end equals the length of the small intestine, that its width is about eight inches.

From the lower end of the mesentery the peritoneum is seen to cover the beginning of the large intestine-the cæcum-and the little process-the vermiform appendix - that extends off from it. The appendix has a narrow mesentery, the cæcum has none; both are free in the peritoneal cavity. Upward from the cæcum passes the ascending colon, which is found covered with peritoneum in front and at the sides, that at the right being continued as the lining or parietal layer of the abdomen, that to the left being continued into the right surface of the mesentery. Sometimes the colon has a distinct mesentery.

The bend formed by the junction of the ascending and transverse colon is the hepatic flexure ; from its outer border an extension of the transverse mesocolon is found which is attached to the adjacent abdominal wall. This is the sustentaculum hepatis, because the right extremity of the liver rests upon it.

At the left the transverse colon takes a sudden bend 
downward to become the descending colon. This bend is the splenic flexure. At this point the transverse mesocolon is seen to be continued to the under surface of the tenth rib, forming the costocolic ligament or the sustentaculum splenis, as it supports the spleer.

The descending colon is covered like the ascending, front and sides, the peritoneum at the right passing into the left lower surface of the mesentery, to the left, to line the lateral abdominal wall. In both the posterior surface is usually bare; this allows the bowel to be entered from the back without opening into the general peritoneal cavity. The large loop of intestine following the descending colon is the omega loop, or the sigmoid flexure. It has a distinct mesentery about three inches long and the same width. This allows the gut to hang free in the pelvic cavity. When the hollow of the sacrum is reached the peritoneum passes around to cover in the front of the rectum, descending to a point about an inch above the level of the tip of the coccyx, then reaches upward, covering in the back and sides of the bladder until its top is attained, from which point it is continued on to the anterior abdominal wall. The cavity between the rectum and bladder is the rectovesical fossa. The peritoneal folds formed around the rectum and bladder are termed the ligaments of those organs.

In the female the condition is altered by the interposition of the uterus and vagina between the bladder and the rectum.

Posteriorly the peritoneum reaches as low as the upper part of the vagina, anteriorly only to the lower part of the uterus. The hollow behind the uterus is the rectovaginal fossa, Douglas's pouch, the one in front of it the uterovesical. Laterally from the uterus the peritoneal folds 
called the broad ligaments pass to the sides of the pelvic cavity.

Between the front wall of the bladder and the inner surface of the pubes and lower abdominal wall is a space, not covered by peritoneum, called the space of Retzius.

The circuit thus traversed outlines the cavity of the greater peritoneum, to distinguish it from the cavity of the lesser peritoneum or the great omentum. Remember that these two cavities are continuous with each other, but through a constricted neck which is called the foramen of Winslow. This opening lies behind the gastrohepatic omentum, opening at the right into the greater cavity just outlined, and continued at the left into the cavity to be examined. A finger can be introduced from right to left, behind the gastrohepatic omentum, into this foramen.

If a transverse circuit be made at the upper part of the abdominal cavity the peritoneum will be seen to have the following arrangement (Diag. 4I):-

It will be easily recognized that the peritoneum lines the anterior and lateral walls of the abdomen. It will also be seen to cover the front of the liver (interrupted by the falciform ligament) and the stomach, and that it connects these organs forming the anterior layer of the gastrohepatic omentum. Continued to the left, the peritoneum turns around the fundus of the stomach and passes on to the spleen. The portion of the peritoneum between the stomach and the spleen is the left layer of the gastrosplenic omentum. From the spleen the peritoneum extends to the back of the abdominal cavity, forming the left layer of the phrenosplenic ligament which holds the spleen in place. The fold forming the last ligament turns outward and covers the abdominal wall.

Passing to the right, from the upper surface of the liver, 


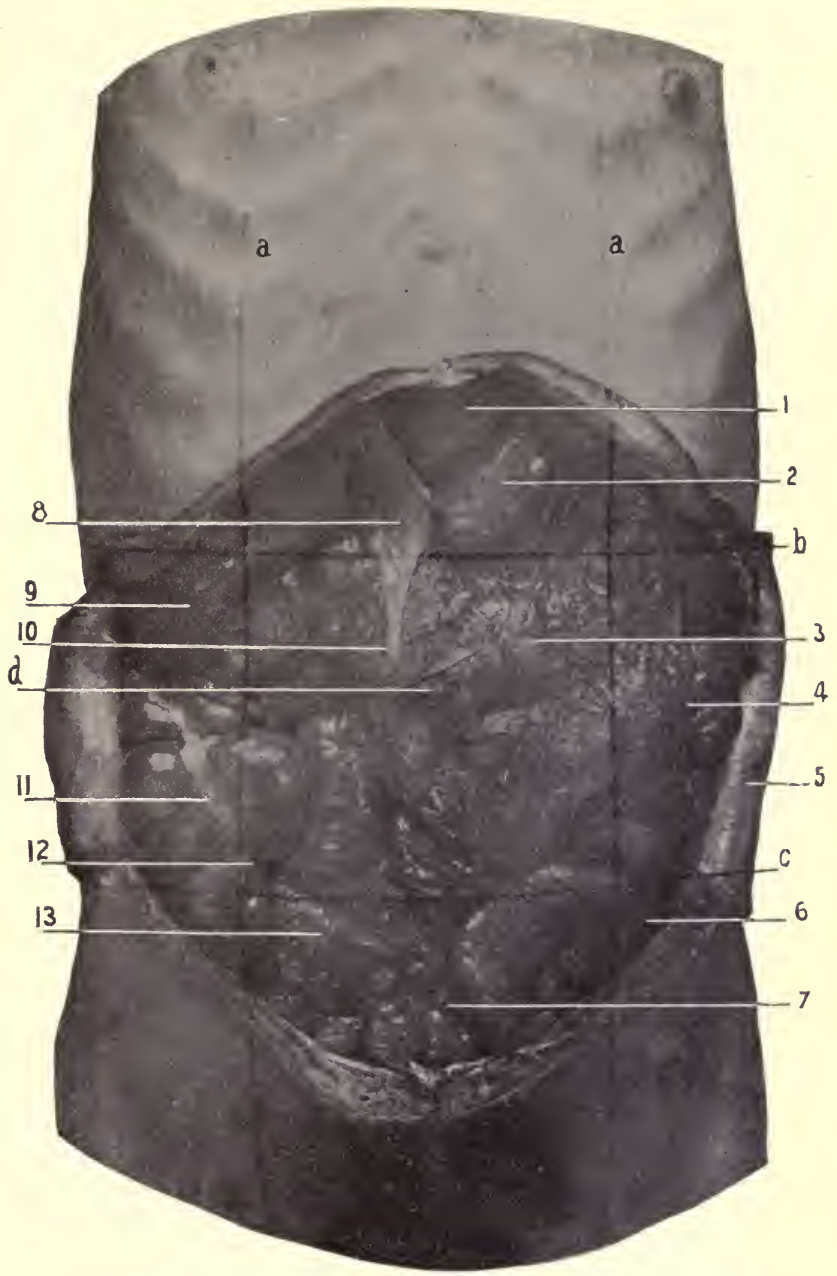

Fig. 96. A Composite Photograph of the Abdominal Wall and Viscera (MALE). [Subject horizontal.]-A photograph of the exterior of the abdomen was taken; then, upon the same plate, a photograph of the abdominal viscera as exposed without moving the camera or subject. Consult in this connection Figs. 87 (taken before) and 97 (taken after Fig. 96) in order to show the parts more sharply. $-a, a$, Vertical lines. $b$, Upper horizontal line. $c$, Lower horizontal line. $d$, Umbilicus. I, Left lobe of liver. 2, Stomach. 3. Transverse colon showing through the great omentum. In this subject the great omentum was found as shown in the figure. 4, Coils of small intestine. 5, Abdominal wall. 6, Inguinal region-left and right. Notice the small amount of viscera in this region. 7, The hypogastric region. 8, Falciform-suspensory-ligament of the liver. 9, Right lobe of liver. Io, Round ligament in free margin of 9. II, Ascending colon showing one of the longitudinal muscular bands. 12, Junction of the cæcum and vermiform appendix. 13, Ileum close to the crecum. 
the peritoneum is seen to turn around to its under surface, which it covers. When the small opening of the foramen of Winslow is reached this layer becomes continuous with the anterior layer already described, and then is said to " pass through the foramen," forming the posterior covering for the liver and stomach and also of the gastrohepatic

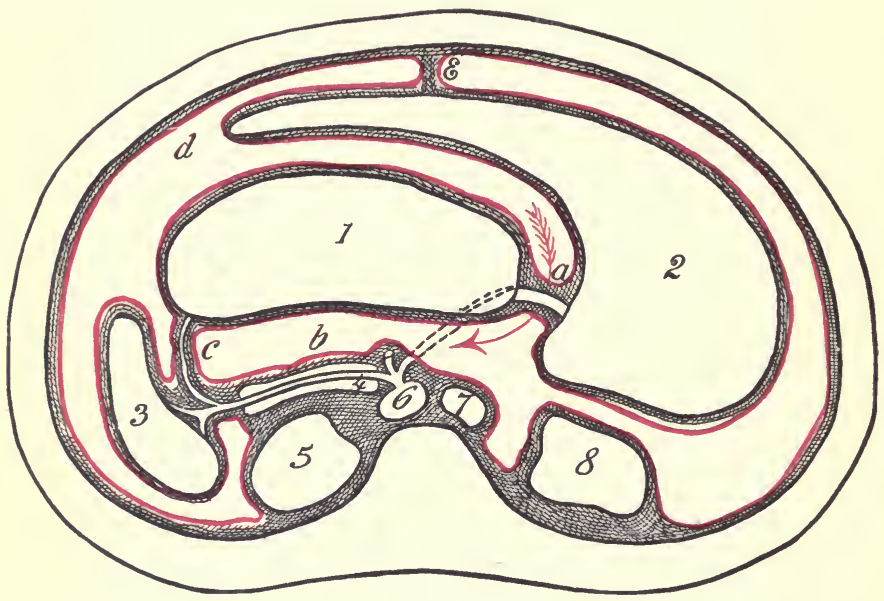

Diag. 41. A Transverse Section in the Upper Region of the Abdomen, (Modified from Heath's Anatomy.)-I, Stomach. 2, Liver. 3, Spleen. 4, Pancreas. 5, Left kidney. 6, Aorta at the cœliac axis and its three branches. 7, Inferior vena cava. 8, Right kidney. $a$, Gastrohepatic omentum. $b$, Cavity of great omentum. $c$, Gastrosplenic omentum. $d$, General peritoneal cavity. Arrow indicates its communication through foramen of Winslow with $b$.

omentum. (Remember, we are now within the cavity of the great omentum or lesser peritoneum.) Passing onward to the left, this layer covers in the posterior wall of the stomach and turns to reach the spleen (forming the right side of the gastrosplenic omentum), then from the spleen to the back part of the abdomen, and turning to the right covers the structures at the back of the abdomen, viz.: 


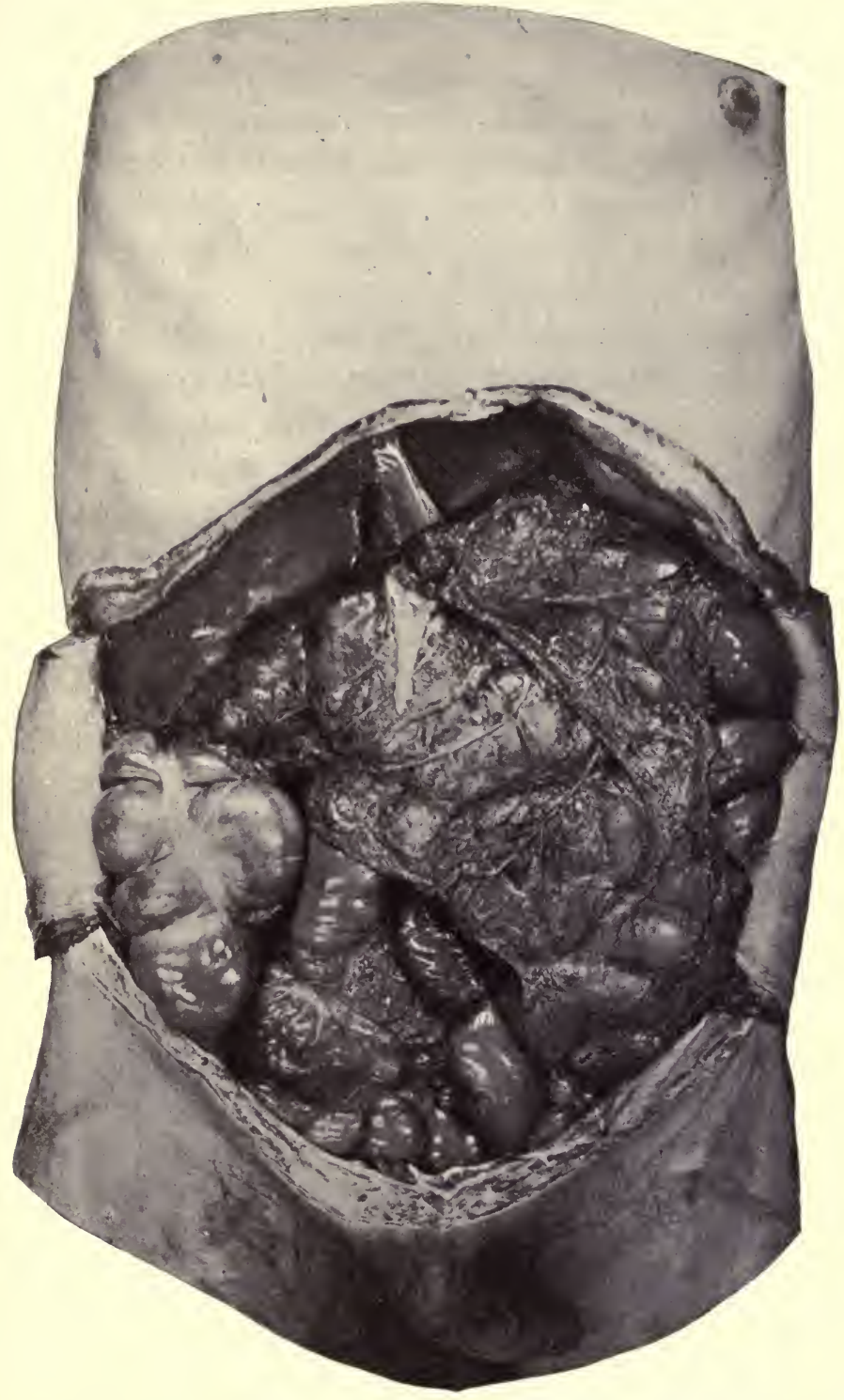

Fig. 97. Abdominal Viscera (Male). [Subject horizontal.]-For the names of the exposed parts consult the preceding photograph. 
the pancreas, aorta, vena cava (forming the posterior wall for the cavity of the great omentum), and extending still to the right, may be said to "pass through the foramen of Winslow" (forming its posterior wall) and become continuous with the parietal peritoneum behind the right lobe of the liver.

A transverse tracing at a lower level is very much simpler and can be easily followed from the diagram 42 .

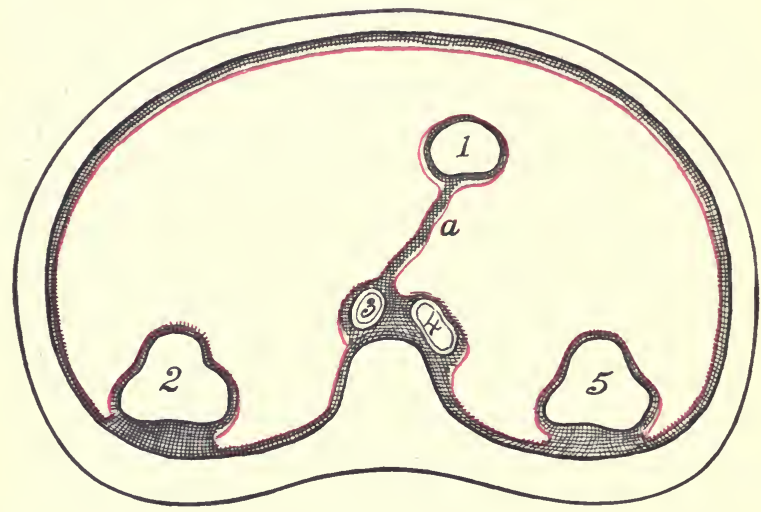

Diag. 42. A Transverse Section in the Middle Region of the rbdomen. (After Morris's Anatomy.)-I, Small intestine. 2, Descending colon. 3, Aorta. 4, Inferior vena cava. 5, Ascending colon. a, Mesentery.

\section{The Abdominal Viscera Covered by Peritoneum.}

The stomach, liver, spleen, first portion of the duodenum, all of the small intestine, cæcum, vermiform appendix, transverse colon, omega loop, first part of the rectum (described in two parts), the ovaries, Fallopian tubes, and the uterus.

The Peritoneal Fossæ.

In the lower part of the abdomen is found the fossa between 
the rectum and bladder (male) called the rectovesical. In the female there are two fossæ here, one between the rectum and the uterus and vagina,- - the rectovaginal or the pouch of Douglas-and a second one between the uterus and the bladder, the uterovesical.

The Intersigmoid Fossa.-This is a small pouch-may be large enough to admit a ball having a radius of two inches-located in the middle of the base of the sigmoid mesentery on its left surface. The opening of the fossa is to the left and downward.

Fossa Duodenojejunalis.-This pouch will admit the "first joint of the thumb." It is found at the left of the vertical or last portion of the duodenum. The opening is upward. Into these last two pouches a portion of the intestine might work its way, thus forming a retroperitoneal hernia.

\section{DISSECTION.}

Incise the anterior layer of the gastrocolic omentum parallel with and just below the greater curvature of the stomach. This will open into the cavity of the great omentum. The hand introduced into this cavity will find the liver above, the stomach in front, the pancreas and colon behind, and the finger can be carried to the right behind the stomach to appear through the foramen of Winslow behind the gastrohepatic omentum. The peritoneum lining the cavity of the greater omentum then covers the posterior surface of the stomach and first part of the duodenum, becomes blended with the anterior covering of the stomach at its lower border as already stated, then loops downward to form the inner layer of the greater omentum, and on the return becomes attached to the upper (anterior) surface of the transverse colon, which it covers, then, passing backward to the abdominal wall, forms the upper layer of the transverse mesocolon. The base of the transverse mesocolon covers in the duodenum and pancreas. From this point the peritoneum extends upward to the under surface of the diaphragm, then on to the liver, forming the posterior layer of the coronary and lateral ligaments of that organ, also of the gastrophrenic ligament. From the posterior margin of the stomach the anterior and posterior layers of peritoneum pass backward to enclose the spleen, gastrosplenic omentum, then on to be attached to the posterior abdominal wall, forming the splenophrenal ligament. 
Summary of the Development of the Viscera and Peritoneum.

(I) The alimentary canal is first undifferentiated and a straight tube.

(2) It is attached to the backbone by a thin membrane - the primitive peritoneum. Also for its upper portion to the anterior abdominal wall. Diag. 32.

(3) As development proceeds the various parts of the alimentary canal appear, as stomach, duodenum, small intestine, large intestine (colon, omega loop, rectum).

(4) The peritoneum attached to these various parts of the intestinal canal receives special names according to the part to which it is attached, as mesogaster, mesoduodenum, mesentery, mesocolon, sigmoid mesentery, mesorectum.

(5) As the alimentary canal grows it becomes thrown into loops, the greatest lengthening taking place in the part known as the small intestine. Diag. 33.

(6) The primitive relations are destroyed by the large intestine crossing over to the right side of the abdominal cavity in front of the small intestine (duodenum), this brings the small intestines to the left side of the abdominal cavity.

(7) This also brings out new attachments for the mesentery of the large intestine (colon, ascending and transverse).

(8) The lower (pyloric) end of the stomach and the liver share in the change about, by falling over to the right side of the abdominal cavity, while the spleen and the upper (cardiac) end of the stomach turn to the left. Similarly, the head of the pancreas turns to the right, while its tail points to the left. Diags. 34,35 .

(9) The liver is developed between the folds of the anterior mesogaster, passing from the anterior border of the stomach to the diaphragm. It is connected with the 
duodenum by its duct, which opens into this part of the bowel.

The pancreas arises from budding cells posterior to the duodenum and between the two layers of the mesoduodenum ; its duct opens into that of the bile duct.

The spleen arises posterior to the stomach, and also between the two layers of the mesogaster.

(I0) After the rotation of the intestines and the change to the oblique position by the stomach, the great omentum is formed by the pouching of the mesogaster, below the spleen and as far as the first part of the duodenum, to the left and forward, to form a bag that hangs down in front of the rest of the intestines. Diags. 36, 37 .

(I I) In the formation of the great omentum, the attachments of the primary mesogaster and transverse mesocolon are altered, until the great omentum seems, in the adult, to come from the lower border of the stomach to the anterior border of the colon, having looped down to form the apron. Consequently there is only one process of peritoneum which forms the posterior attachment of the great omentum, and it is between the folds of this the great omentum that the colon is situated, and the part of the great omentum passing to the backbone is called the transverse mesocolon. Diags. 38 and 40.

(I2) If the foetal form remains throughout life the posterior fold of the omentum returns to the backbone for a separate attachment above the attachment of the mesocolon. Diag. 37 .

The description of the abdominal viscera will be brief and only include the gross anatomy. The descriptions will also be given consecutively, and their dissection later. This is to avoid unnecessary repetition. 
The dissector is expected to first read the descriptions and then to expose the organs according to the plan as given later, identifying as he goes the main points regarding each organ.

Too much stress cannot be given to the study of the intimate and projected surface relations of the various organs of the abdominal cavity. Every year sees surgeons successfully invading new fields, hitherto forbidden ground; in the last few years no part of surgery has yielded more brilliant results than in the domain of the abdomen.

The student is urged to do more than to casually identify the main abdominal relations; he is to study for himself the position of each and every organ without reference to what may be written about it. Read what has been said, then study and think for himself. He will find there are many surprises in store for him if he studies in this way.

The Liver. Figs. 96, 97, 104, I05, 108, 109.

Position and Size.-The liver occupies the dome-shaped space under the diaphragm on the right side, reaching across the middle line a distance of two and one-half inches. It is the largest gland in the body, weighing about three and one-half pounds. It measures twelve inches from side to side, six and one-half from before backward, and three and one-half vertically in its thickest part (in the right lobe).

The Ligaments.- The liver is held in position by five ligaments, all, except the round, derived from the peritoneum which encloses almost the entire gland, and all (with the same exception) attached to the diaphragm.

(I) The suspensory, falciform, or broad ligament is the first one seen on opening the abdomen. It is attached to the right of the middle line from the umbilicus backward to the posterior part of the diaphragm, and below to the liver, 


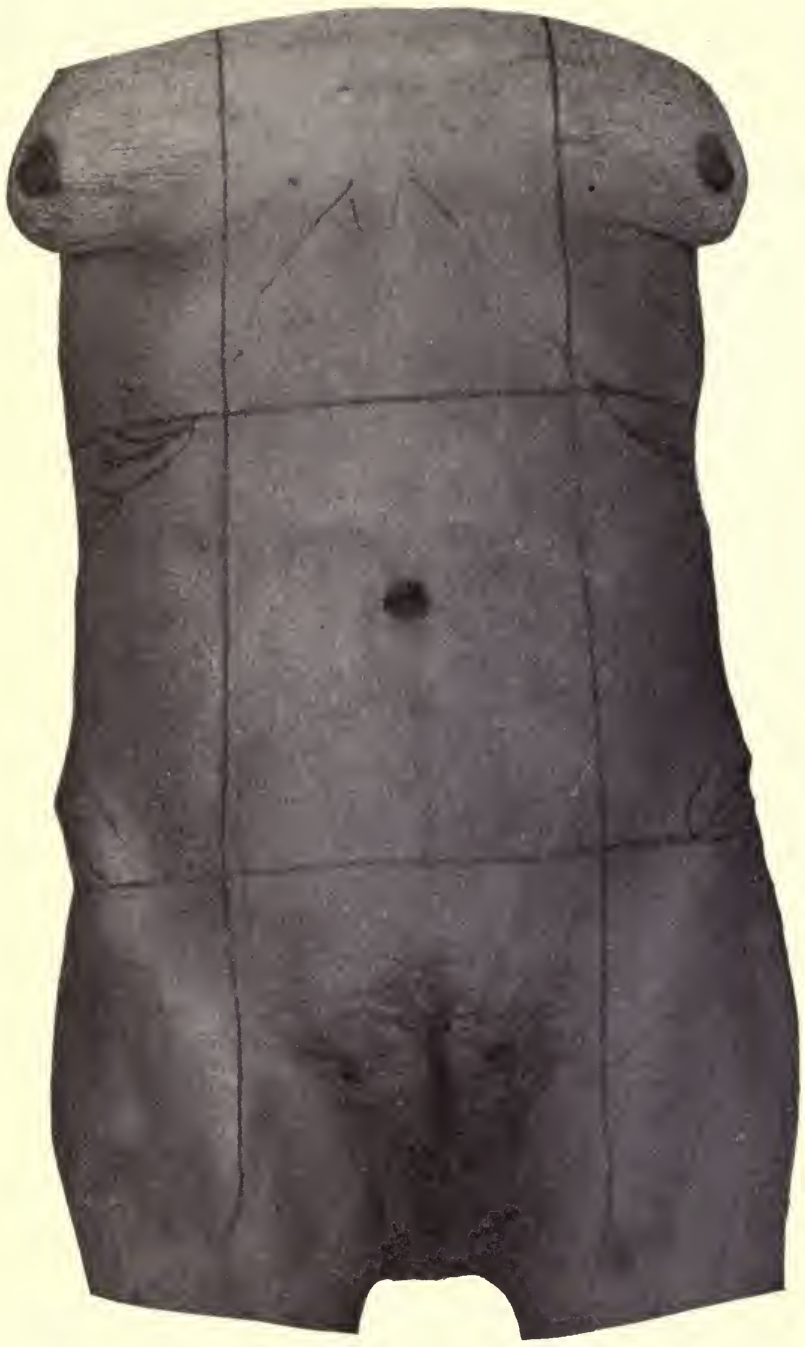

Fig. 98. Abdominal Regions (Female). [Subject horizontal.]-For the names of the various regions consult Fig. 87 . This photograph should be used in connection with the following photographs to determine the visceral surface relations. 
dividing the gland into the right (larger) and left (smaller) lobes,

(2) In the free margin of the suspensory, is the round ligament, a fibrous cord representing the umbilical vein. It extends from the umbilicus to the under surface of the liver, and disappears in the umbilical fissure.

(3) Extending from side to side, at right angles to, and continuous with, the suspensory ligament, is the coronary ligament.

$(4,5)$ The extreme ends of the coronary ligament are called the right and left lateral ligaments.

Relations.-The liver will be seen to be in relation with the following organs. (Not all will appear at this stage, but as before remarked, they are given here consecutively, and later may be confirmed or disproved) :-

Above, the upper surface lies against the diaphragm. Below, the lower surface comes in contact with the hepatic flexure of the colon, gall bladder (part of the liver), stomach (cardiac and pyloric ends and anterior surface), the first and second parts of the duodenum, the right kidney, and the gastrohepatic omentum. Behind, the diaphragm and its crura, the tenth and eleventh dorsal vertebræ, the œesophagus, the aorta (separated from the liver by the diaphragm), vena cava, and the right suprarenal body.

Surfaces, Lobes, and Fissures.-(I) The upper surface is convex to fit in the concavity of the diaphragm, and is divided by the suspensory ligament into the right and left lobes.

(2) The lower surface is generally concave, and is separated from the posterior by the transverse fissure. Into the transverse fissure pass the portal vein, hepatic artery, and hepatic nerves, coming out of the hepatic duct.

The right lobe is divided from the left, by the longi- 


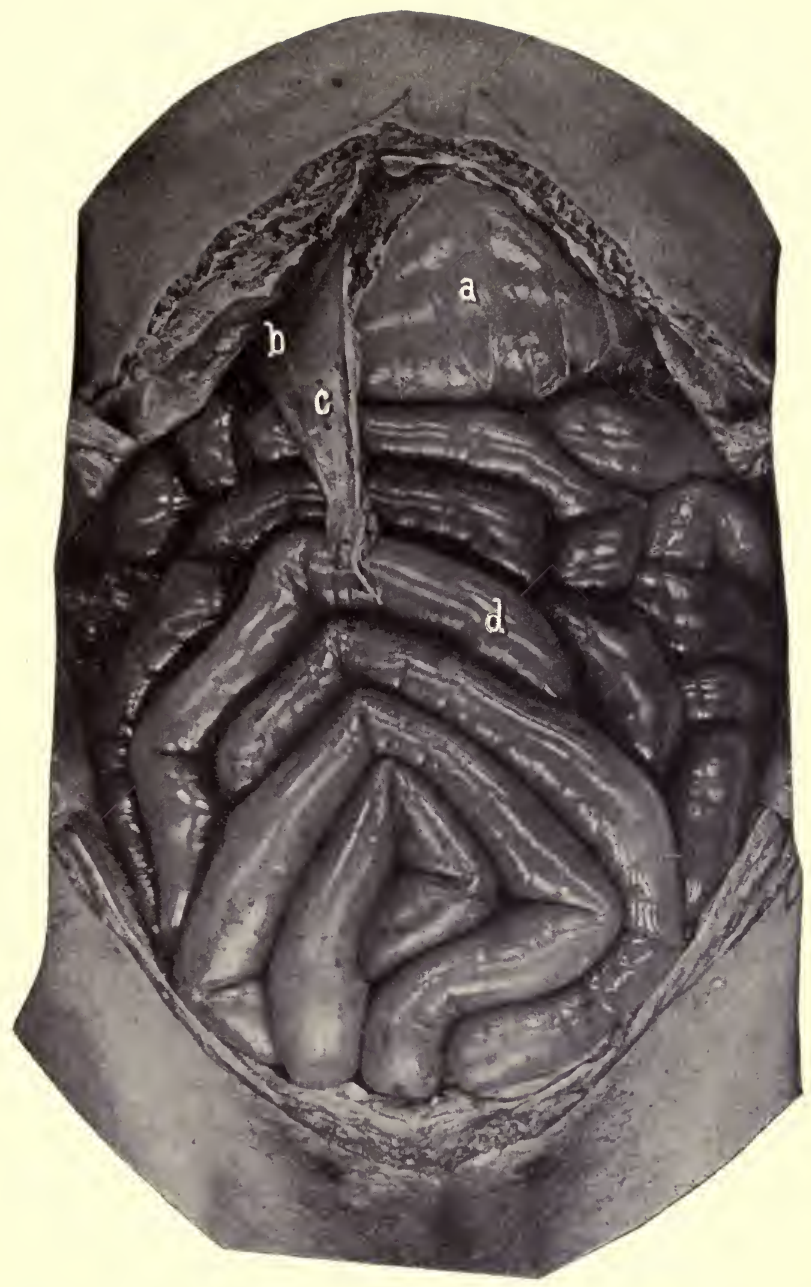

Fig. 99. Abdominal Viscera (Female). [Subject horizontal.]- $a$, Stomach. $b$, Liver. $c$, Falciform ligament of the liver, in the free (right) margin of which is the round ligament. $d$, The coils of small intestines. The photograph shows the exact condition that was found on opening the abdomen. The great omentum was out of sight, being rolled up under the stomach. 
tudinal flssure. This fissure consists of two parts; the anterior (the umbilical), in which the umbilical vein lies; and the posterior for the ductus venosus, a venous trunk which joins the umbilical vein to the vena cava (by way of a hepatic vein). These veins are fotal structures. The gall bladder occupies a shallow hollow-the fissure of the gall bladder. Between the fissures for the umbilical vein and the gall bladder, is the quadrate lobe, bounded behind by the transverse fissure and in front by the anterior border of the liver. The quadrate lobe is often connected to the left over the umbilical fissure forming the pons hepatis.

The lower surface of the liver shows several slight depressions produced by contact with the various abdominal organs beneath it; these are called impressions. They are for the stomach, hepatic flexure, duodenum, and right kidney.

(3) The posterior surface is poorly marked. It may be defined as that part of the liver behind the transverse fissure. It is deeply concave from side to side to accommodate the spine and crura of diaphragm. It contains a vertical gutter in which lies the vena cava inferior, and called the fissure for the vena cava. At the left of the vena cava is the Spigelian lobe, separated from the left lobe by the fissure for the ductus venosus.

The Spigelian lobe is connected with the right by a slender tongue or ridge of liver substance known as the caudate lobe.

It will be seen that the fissures of the liver can be arranged like the letter "H." The right leg is made up of the fissures for the gall bladder and vena cava, the left by the umbilical and fissure of the ductus venosus, the cross bar is the transverse fissure. 
The anterior border is thin and sharp, and is notched at the umbilical fissure. To the right of this notch is the fundus of the gall bladder. If distended it will project beyond the liver border.

The gall bladder (Figs. I04, I05) is a membranous sac situated in the fissure of that name, used as a reservoir for the bile. Its duct is the cystic duct which unites with the hepatic to form the common bile duct. (For the course of the latter see Gastrohepatic Omentum.)

It must be remembered that in living, healthy people the relations of the abdominal organs to each other and to the surface of the body varies from time to time with respiration, position of the body and fullness of the stomach, and that in disease the variations are innumerable. Consequently, all measurements are only relative, and true for the subject and at that particular time. However, if our measurements are not exactly correct they serve to give us standards from which to note variations. Hence, the need that every student should investigate for himself the relations of the subject he is at work upon, without reference to the text-book figures ; later he should make the comparison between his data and that in the text-books.

The Surface Area of Liver Projection. Figs. 96, 98, I08, I09.-The upper border of the liver is represented by a line, beginning two and one-half inches at the left of the median line on a level with the sixth left sternochondral articulation, and drawn slightly downward to cross the sternum at the sternoxiphoid articulation, then toward the right, curved slightly upward, to reach a point one inch below the right nipple; from here the line is carried directly around the chest to end at the eighth dorsal vertebra.

The lower liver border is traced by an oblique line starting at the left over the eighth costal cartilage (a very indefi- 
nite point), and drawn to the right, crossing the middle line of the body midway between the ensiform and the umbilicus, reaching the right ninth costal cartilage (another blind point), then running parallel with the lower margin of the thorax to the eleventh rib, then along the lower border of this rib to the spine.

The interlobular notch is half-way between the right vertical line (see page 435) and linea alba. The fundus of the gall bladder lies just below and behind the tip of the cartilage of the tenth right rib, or in the notch between the right rectus muscle and the thoracic margin. The cartilages given above are "areas" not " points," hence considerable latitude is allowed.

The Gastrohepatic Omentum. Diag. 36, No. I. Fig. 103.

The double layer of peritoneum which extends from the transverse fissure and under surface of the liver to the upper border of the stomach and first portion of the duodenum is the gastrohepatic omentum. It consists of three distinct parts : (I) A superior, triangular portion of a dense white ligamentous structure, - the tendinous portion. (2) A middle, oval portion opposite the lesser curvature of the stomach. This segment is so thin that it is easily torn and through it can be seen the Spigelian lobe of the liver,- the flaccid part. (3) An inferior part which is thick, massive, and at the right ends in a rounded border which forms the anterior margin of the foramen of Winslow. In this free margin are the hepatic vessels and nerves. The vessels are the portal vein, hepatic artery and duct. The duct is to the right, the artery to the left, and the vena porta behind the two. (For further information consult a careful article by Byron Robinson, on the Gastrohepatic Omentum, in the (N. Y.) Medical Record for Aug. IO, I 895.) 


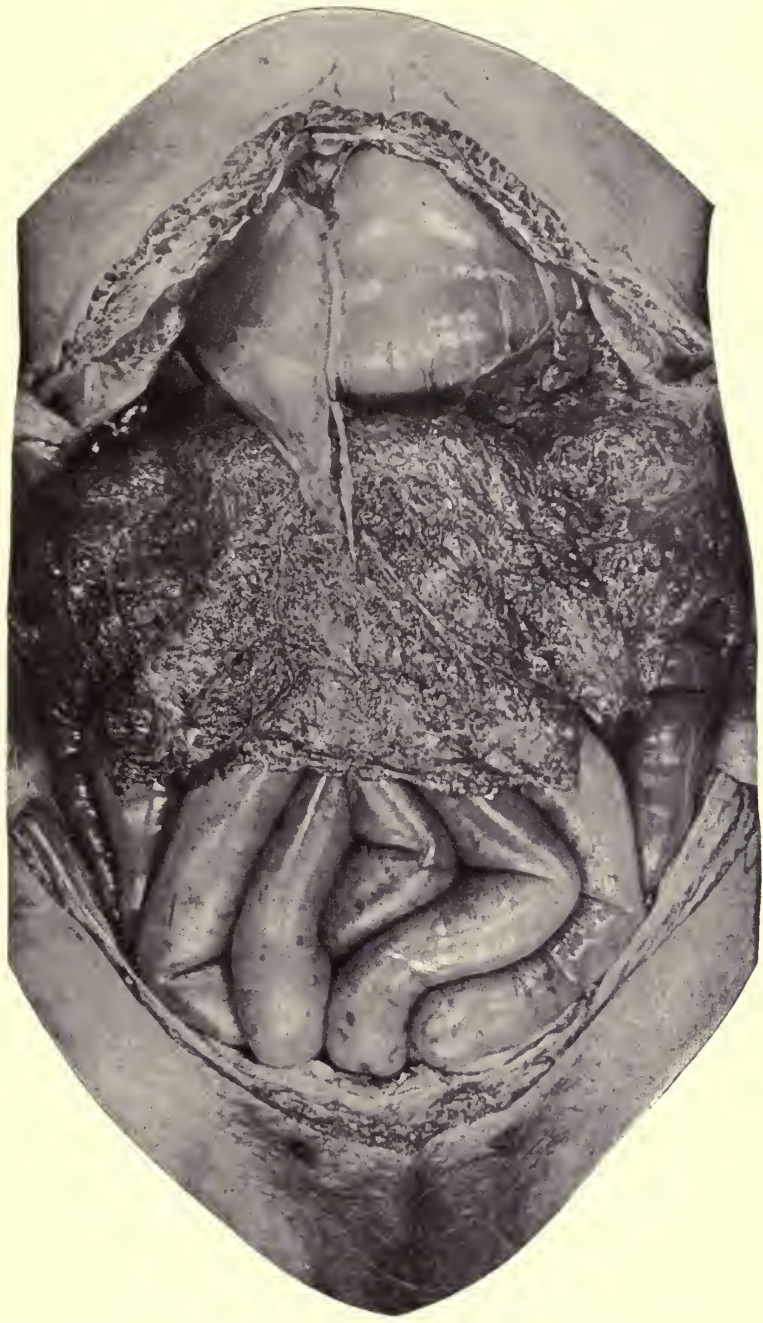

Fig. 100. Abdominal Viscera (Female). [Subject horizontal.]-This photograph shows the great omentum unrolled and spread out over the intestines. 
The Stomach. Figs. 96 to IOO, 103, IO4, 105.

The stomach lies on the left side of the abdomen, below the diaphragm and left lobe of the liver. It is about twelve inches long, four inches wide, and weighs four and one-half ounces. It is entirely covered by peritoneum. See $D e-$ velopment, page 462. Its long axis is placed obliquely from above, behind, and at the left, to downward, forward, and to the right.

It has two openings, the cardiac, the opening of the œsophagus into the stomach, and the pyloric, the beginning of the intestinal canal. There is no constriction about the cardia, but around the pylorus is a muscular collar forming a sphincter.

The short border, or the lesser curvature, is three and one-half inches long and concave. From it passes the gastrohepatic omentum to the liver. See above. From the longer border, or the greater curvature, hangs down the broad peritoneal apron, the greater or gastrocolic omentum.

The fundus is the dilated end of the stomach to the left of the cardia. At the osophagus the anterior and posterior layers of peritoneum come together and pass on to the diaphragm, forming the gastrophrenic ligament.

To the right along the lesser curvature these layers after joining, form the gastrohepatic omentum and continue to the liver, while below from the greater curvature these two layers unite to form the great omentum, and returning again to the colon may also be called the gastrocolic omentum.

Relations. - The anterior surface is in relation with the liver, diaphragm, and anterior abdominal wall. The area of stomach opposed directly to the abdominal wall is represented by a triangle formed on the right by the anterior 
margin of the liver, on the left by the margin of the costal cartilages, and below by the upper horizontal line. See page 435 .

Behind, the posterior surface of the stomach helps to form part of the anterior wall of the cavity of the great omentum (lesser peritoneum). It lies in front of the pancreas, great vessels, crura of diaphragm, solar plexus, from all of which it is separated by the anterior layer of the transverse mesocolon, also in front of the spleen and the gastrosplenic omentum, the splenic artery, left kidney and suprarenal body. Above, are the liver, gastrohepatic omentum, diaphragm, and œsophagus. Below, the gastrocolic or greater omentum, transverse colon and its mesocolon.

The Surface Area of the Stomach Projection.-The stomach being one of the most movable organs by reason of its loose peritoneal connections and from the varying states of distention or emptiness, it follows that to attempt to give any fixed outlines of its position is almost useless. The cardiac end is the only point that can be fixed with any degree of exactness. This lies behind the seventh costal cartilage, one inch to the left of the sternum. The fundus rises up behind and above the location of the apex of the heart, to the level of the sixth costochondral articulation.

The pylorus is the most uncertain portion; usually it is found one inch to the right of the median line and two inches below the ensiform.

The upper border will be indicated by a short curve, with concavity upward, connecting the cardiac and pyloric orifices. The greater curvature is drawn upward from the cardia to the point reached by the fundus, then with a wide curve is drawn outward, to the left and downward, then to the right and after crossing the middle line midway between 
the ensiform and umbilicus the border is curved upward to the lower part of the pylorus.

If the lower border of the stomach reaches below the umbilicus the case is one of pathological distention.

The stomach is entered through the triangle above given. The incision may be parallel with the costal margin or through the semilunar line.

\section{The Intestine.}

The intestine is the alimentary canal below the stomach. It is divided into the small and large intestine. The small intestine consists of the duodenum, the jejunum, and the ileum. The large intestine is divided into the cæcum, ascending, transverse, and descending colon, the omega loop (sigmoid flexure), and rectum.

The Duodenum. Figs. 103, 104, 105.-Though this portion of the intestine is one of the last to be dissected, it will be described now along with the rest of the intestine. The duodenum comprises the first ten inches of gut below the pylorus, and is about two inches in diameter. It is divided into four portions, the proximal ascending part, two inches long; the descending portion, two and threefourths inches long; the transverse portion four to five inches long; and the distal ascending portion, one inch in length. The duodenum is the only fixed portion of the small intestine. Its adult position is secured through the rotation of the gut in the foetus (see page 462), by which the duodenum is crowded to the back of the abdominal cavity by the transverse colon which passes in front of it.

(I) The proximal ascending part. This is two inches long, passes upward to the right and ends opposite the neck of the gall bladder. It is enclosed by peritoneum prolonged from the stomach, and is freely movable. 


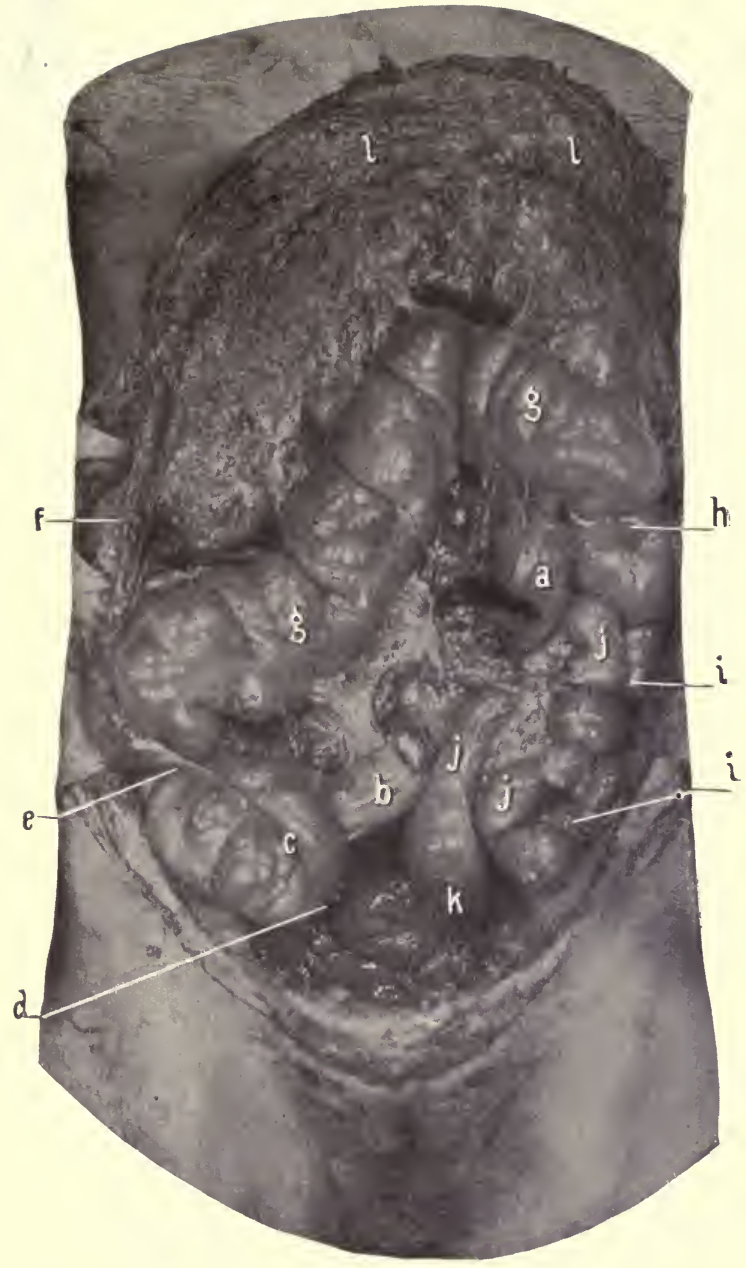

Fig. 101. Abdominal Viscera (FEmale). [Subject horizontal.]-The small intestines have been removed, the large intestine inflated, and the great omentum turned up over the thorax. $a$, End of duodenum and beginning of jejunum. $b$, End of ileum. The intervening portion of jejunum and ileum has been removed. $c$, Cæcum. The point of junction of ileum and colon is well shown and should be referred to the surface by consulting Fig. 98. $d$, The point of junction of the vermiform appendix with the cacum. In this case the process hung loosely down into the pelvic cavity. $e$, Ascending colon passing up behind a flexure of the transverse colon. $f$, The hepatic flexure. $g, g$, Transverse colon. $h$, Splenic flexure. $i, i$, Descending colon. $j, j, j$, Omega loop. $k$, Rectum. $l, l$, Great omentum. 
Relations.-Above and in front, liver, gall bladder, foramen of Winslow. Below, pancreas. Behind, the common bile and pancreatic duct, and vessels passing to the liver.

(2) The descending portion is two and three-fourths inches long, and extends from the neck of the gall bladder to the level of the second or third lumbar vertebra.

Relations.-Front, transverse colon and mesocolon. To the left, pancreas. To the left and posterior, the common bile duct. Behind, the right kidney, renal vessels, and vena cava.

This portion of the duodenum is covered only in front by the peritoneum. Into this part of the duodenum the common bile and pancreatic duct opens, at a point four inches from the pylorus.

(3) The third or transverse portion of the duodenum, four or five inches long, is the most fixed portion of the alimentary canal. It crosses the spine over the second or third lumbar vertebra, in a slightly oblique direction, the right end being a little lower than the left.

Relations.-In front, it is covered by the peritoneum, transverse colon and mesocolon, and the superior mesenteric artery. Above, the pancreas, inferior pancreaticoduodenalis artery, the superior mesenteric artery and vein. Behind, the crura of the diaphragm, vena cava, aorta.

(4) The fourth or distal ascending portion, usually described as a part of the third portion. This part is only one inch long, curves vertically upward, is nearly covered by peritoneum. It is held in position by a process of peritoneum containing some muscular fibres and called the musculus suspensorius duodeni (of Treitz), which extends from the left crus of the diaphragm and tissues about the cœliac axis. Behind this band is the small fossa duodenojejunalis, opening upward, and into which a retroperitoneal hernia might possibly find its way. 
The Small Intestine. Figs. 96 to 100.

The first two-fifths of the intestine below the duodenum is called the jejunum, the remaining three-fifths, the ileum. The coils of the small intestine fill in the space left by the arching colon at the sides and above, and the pelvic viscera below. They are attached to the posterior abdominal wall by the mesentery, a double layer of peritoneum, which reaches from the left side of the second lumbar vertebra to the right iliac fossa, a distance of four to six inches. The mesentery is about eight or nine inches in width, and in length along its intestinal border equals the jejunum and ileum. Between the layers of the mesentery the superior mesenteric artery ramifies. Sometimes a remnant of the vitello-intestinal duct is found on the outer border of the ileum at a distance of from one to three feet from the ileocæcal valve; if present it is called Meckel's diverticulum. When existing it may be a menace to the individual on account of forming adhesions with some other part of the abdomen, thus forming a band or bridge over or under which a portion of the intestine might become constricted. In the normal subject it will be seen that the mesentery is just short enough to keep the margin of the intestine from engaging in the abdominal rings or femoral opening, consequently in hernia the mesentery must be abnormally elongated congenitally or by acquisition.

The ileum joins the large intestine in the right inguinal region, at right angles to the larger gut, and from two to three inches from its lower blind extremity.

The small intestine opens by a valvular orifice, the ileocæcal valve, into the larger. This opening is behind a line joining the anterior superior iliac spine and the umbilicus, and at a distance of three inches from the first point. 
The Large Intestine. Figs. 96, 97, 98, I о , 102.

Reaches from the right inguinal region, upward and across the abdomen, then descends along the left side, to pass into the pelvic cavity.

It has a length of five and one-half feet, and a diameter of two inches.

The large intestine is not only larger than the small, but is distinguished from it by pendent masses of fat covered by peritoneum-appendices epiploice, and by having three bands of muscular tissue longitudinally disposed upon its exterior, one posteriorly at the mesenteric attachment, one anteriorly, and one internally. These bands being shorter than the bowel give it a sacculated appearance.

Its blind extremity, below the junction of the ileum, is the cæcum.

The cæcum lies in the right lumbar or inguinal region. It is about two and one half inches long and about three inches wide. From its lower, posterior surface the vermiform appendix comes off, and lies usually in a direction upward and to the left, but often the appendix may hang down into the pelvic cavity, or take any other position about the lower end of the cæcum. The cæcum and appendix are usually entirely enclosed by peritoneum (the appendix having a distinct, though small mesentery), and are free within the abdominal cavity, consequently any pus coming from the interior of either would be discharged into the general abdominal cavity and start up a (usually) fatal peritonitis, unless the exudation is shut off by inflammatory adhesions agglutinating the adjacent intestines together, to form a local pus cavity.

The point at which the appendix joins the cæcum is on the ilio-umbilical line (from anterior superior spine of ilium to umbilicus) at a distance from the iliac spine of two and 
one-half inches. As it is at this point that the pain is usually greatest in appendicitis, it is a good point to keep in mind. As it was first mentioned by McBurney it takes the name of that surgeon and is called McBurney's point.

From the cæcum to the liver the large intestine is called the ascending colon. Here taking a sudden bend to the left the bowel becomes the transverse colon, and the bend is the hepatic flexure.

On the left side of the body, under the spleen, the gut turns downward to form the descending colon while the bend is called the splenic flexure. The continuation of the large intestine is the omega loop and rectum.

The ascending colon (Figs. 96, 97, 98, IOI, I02) is usually only enveloped by peritoneum in front and at the sides, the posterior part of the bowel resting against the back of the abdomen. It may, however, be completely covered by peritoneum, in which case it is provided with a distinct mesentery.

Relations. - In front and at the right is the abdominal wall, at the left the coils of small intestine, below the cæcum, and behind the quadratus lumborum, and lower portion of the right kidney.

The hepatic flexure lies up under the overhanging edge of the liver, in close contact with the gall bladder. From its outer border, there is often a continuation of the transverse mesocolon to the side of the abdomen; upon this little shelf the right extremity of the liver rests (sustentaculum hepatis).

The transverse colon (Figs. 96, 97, 98, I I ) crosses the abdomen from right to left, and from the hepatic to the splenic flexure. It rises higher at its two extremities than in the middle, where it crosses the middle line just above the umbilicus.

Relations.-Above, gall bladder, liver, stomach. Behind, 
the third portion of the duodenum. Below, the small intestine. In front it is covered by the great omentum. Its mesentery is attached to the posterior abdominal wall over the duodenum and pancreas, it passes to enclose the colon then drops downward to form the greater omentum, then rises to the lower border of the stomach, and first portion of the duodenum. See Diag. 40.

The splenic flexure is in contact with the spleen above. From its outer margin the transverse mesocolon is continued to the under surface of the abdomen over the tenth rib. This band is the costocolic ligament, and its function is to support the spleen (sustentaculum splenis).

The descending colon (Figs. 98, IOI, IO2) extends from the splenic flexure to the left iliac fossa. It is covered in front and at the sides by peritoneum, its posterior surface being usually in contact with the abdominal wall. But, as with the ascending colon, this part of the colon may have a distinct mesentery. In this case the peritoneal cavity would be opened in performing lumbar colotomy ; if there is no mesentery the gut can be cut into without invading the general peritoneal cavity.

Relations.-In front and at the sides, the small intestine. Behind, the diaphragm, left kidney, quadratus lumborum muscle.

The omega loop, or sigmoid flexure (Figs. 96, 97, 98, IOI, IO2), is the next part of the bowel below the descending colon. It lies in the left iliac fossa, reaching into the pelvic cavity as low as the third portion of the sacrum, including in its extent the so-called first portion of the rectum, when that part of the bowel is described in three portions. Its length is 18 inches. When distended the apex of the loop may reach over to the right so far as to come in contact with the cæcum. At its lower end it is provided 


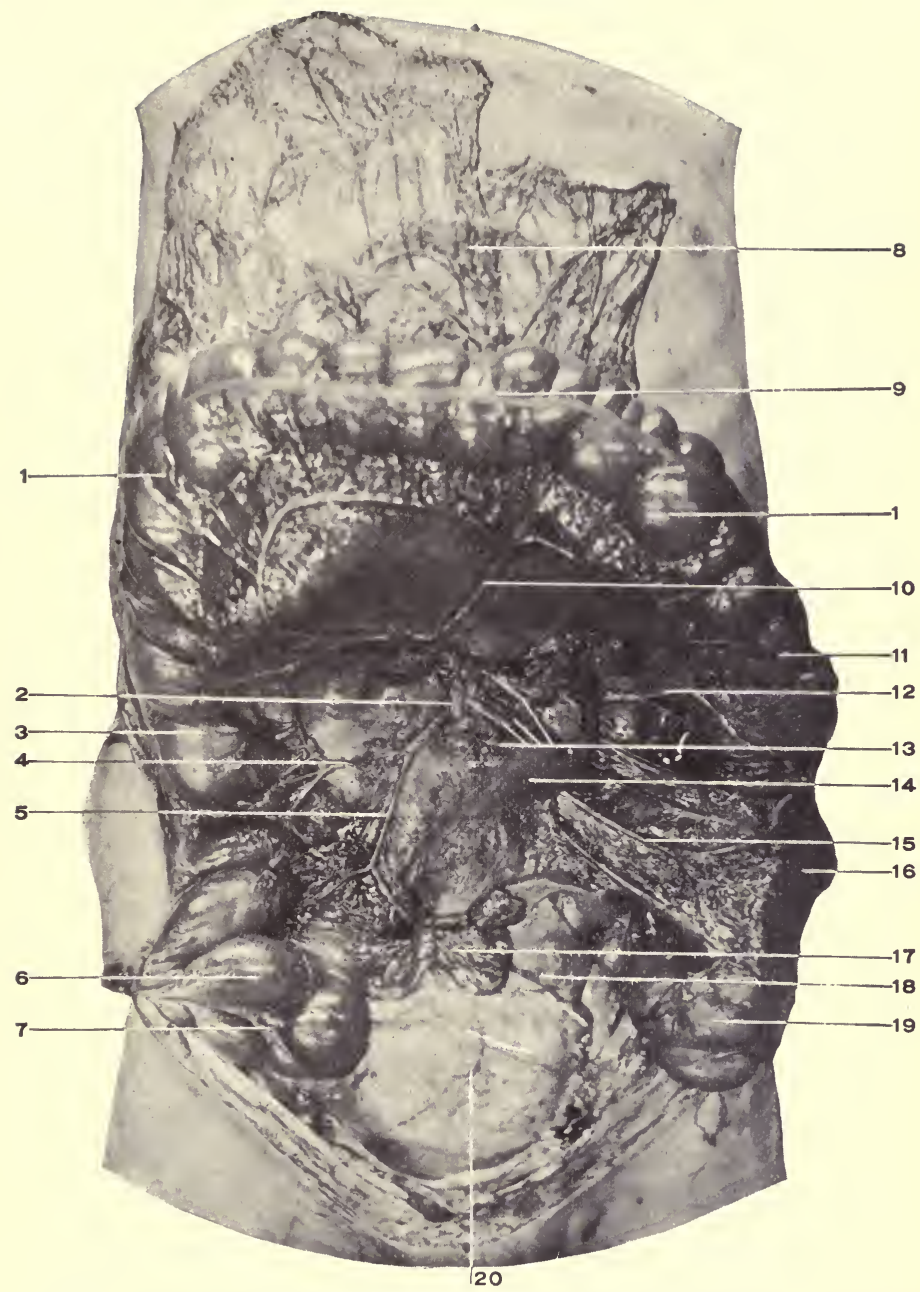

Fig. 102. Blood Supply to the Intestines. [Subject horizontal.]-I, I, Transverse colon. 2, Superior mesenteric artery. 3, Hepatic flexure of colon. 4, Right colic artery. 5, Ileocolic artery. 6, Crecum. 7. Junction of the vermiform process with cæcum. The process extends downward and inward behind the cæcum. 8, Great omentum. 9, Longitudinal muscular band on the large intestine. Io, Middle colic artery. I I, Splenic flexure of colon. 12, Left colic artery. I3, Vasa intestini tenuis. 14, Duodenum, transverse portion. 15, Sigmoid artery. 16, Descending colon. I7, Ileum divided close to the cacum. I8, Rectım. I9, Omega loop. 20, Bladder. The chain of arterial anastomoses upon the large intestine can be readily followed. Also the secondary, and in places the tertiary arterial loops. 
with a sort of sphincter, formed by an increase in the circular fibres, and a narrowing in the calibre of the bowel. The omega loop lies normally within the pelvic cavity. The loop has a distinct mesentery and is freely movable, being surrounded almost entirely by peritoneum.

The attachment of the omega mesentery crosses the psoas at right angles, the iliac vessels at their bifurcation, and curves downward into the pelvis toward the right so as to reach the median line. This line of attachment measures about three and one-half inches, and the mesentery is about the same in width.

Relations.-With the small intestines in front and to the inner side. With the cæcum and vermiform appendix (if distended) on the right. With the iliacus and psoas, genitocrural nerve, iliac vessels, first portion of sacrum behind. Below it rests upon the bladder and rectum and in the female, upon the uterus.

The rectum (Figs. I IO, I I I), is the last portion of the large intestine from the end of the omega loop at the third portion of the sacrum to the anus, and measures about five inches. It may conveniently be described in two portions. The first portion three and one-half inches, from the third portion of the sacrum to the tip of the coccyx. This part of the rectum is covered by peritoneum only upon its front and at the very beginning. In front of this portion are the bladder, seminal vesicle, trigone, posterior surface of the prostate. In the female, with the posterior surface of the cervix of the uterus and upper part of the vagina. Behind, it rests upon the lower part of the sacrum and the coccyx conforming to the sacral curve.

The peritoneum is reflected from the rectum to the bladder or the vagina, and in the bottom of this reflection is the rectovesical or rectovaginal fossa. 
In rectal operations it is important to know how far the peritoneum is from the seat of operation. Posteriorly the peritoneum is at a distance of five inches, anteriorly of three inches from the anus. The second portion, see page $42 \mathrm{I}$.

The Surface Area of the Intestines. - The abdomen is divided into regions as indicated, page 435 .

The cæcum occupies the right lumbar region; it is usually located in the text-books in the inguinal region, but this places it too low ; it may reach to, but not often below, the lower horizontal line (see page 435), and lying in the upper portion of the right iliac fossa.

The appendix joins the cæcum at a point on the ilioumbilical line two and one-half inches from the iliac end, or where this line is crossed by the right vertical.

The ileocæcal valve is three inches from the iliac spine on the ilio-umbilical line.

The ascending colon reaches upward through the right lumbar region to a little above the anterior liver border.

The hepatic and splenic flexures are situated in the right and left lumbar and hypochondriac regions; when the upper horizontal line is drawn between the tips of the tenth ribs, these flexures may not reach above this line, if so they lie within the lumbar regions. Between these flexures the transverse colon extends, looping downward to just above the umbilicus.

The descending colon occupies the left lumbar region, while the omega loop describes almost a circle from the left iliac fossa through the lower left portion of the umbilical region into the pelvis, to terminate in the rectum behind the middle line.

The operation of introducing a high rectal tube is sometimes described. If the rectum and omega loop is examined it will be seen that it is impossible to pass any tube 
beyond the middle of the loop; if the tube is introduced still further the bowel is carried on in front of it, and if too much force is used the bowel may be perforated, as has been done, and death follows.

The Spleen. Fig. 105.

The spleen is an oval gland, six by four by one and onehalf inches, which lies behind the fundus of the stomach. Its long axis is parallel with the tenth rib, its upper border with the upper edge of the ninth rib, and its lower border with the lower edge of the eleventh rib.

Its inner end is two inches from the left of the middle line behind, and its outer end at the mid-axillary line. It is covered by peritoneum except at the hilum where the vessels and nerves are situated.

The spleen is developed in the mesogaster. See page 467. The portion of the primary mesogaster between the stomach and spleen is now called the gastrosplenic omentum, and the portion of the mesogaster extending between the spleen and diaphragm is now called the phrenosplenic ligament. Above, the latter process is continuous with the gastrophrenic ligament, and below, the former passes into the left portion of the greater omentum.

The splenic artery, vein, lymphatics, and nerves enter or leave the spleen at the hilum of the spleen.

Relations.-In front, stomach and splenic flexure of the colon. To the outside, the diaphragm, inclusive of the ninth, tenth, and eleventh ribs. To the inside, the stomach, pancreas, left kidney. Below the spleen rests upon the costocolic ligament, and has the splenic flexure of the colon below and in front.

The blood supply for the spleen comes from the splenic branch of the cœliac axis; the splenic vein passes to unite 


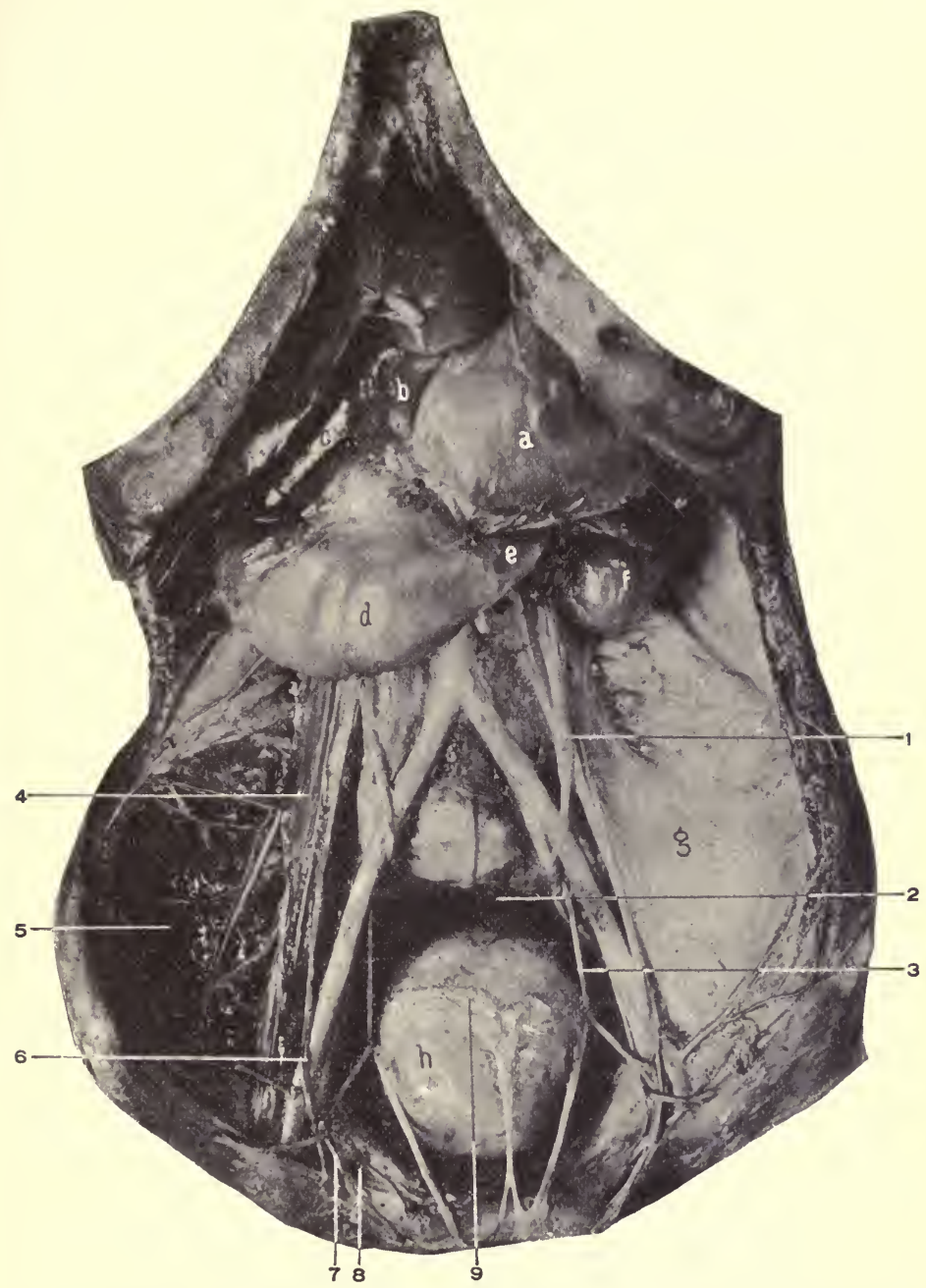

Fig. 103. Dissection of Abdominal Viscera (Male).- $a$, Stomach. $b$, Pylorus. $c$, Descending Portion of duodenum. $d$, Transverse portion of duodellum. $e$, Beginning of vertical portion of duodellum. $f$, Beginning of jejunum. $g$, Iliopsoas fascia. h, Bladder. 1, Left ureter. 2, Cut end of rectum. 3, Left superior vesical artery. 4, Psoas magnus. 5, lliacus. 6, Right spermatic vein. 7, Vas deferens. 8, Femoral ring. 9, A transverse line formed by dividing the peritoneum along its attachment to the bladder. In front of this line the bladder is uncovered by the peritonenm. 
with the superior mesenteric vein behind the pancreas and form the portal vein.

The nerve supply is from the solar plexus.

The Pancreas. Fig. 105.

The pancreas is an elongated gland with its larger extremity (the head), within the duodenal arch, its body (the middle portion) crosses the spine over the first lumbar vertebra, and the smaller extremity (or the tail) is in contact with the spleen.

Relations.-The gland lies behind the peritoneum, which forms the anterior layer of the transverse mesocolon, by which the gland is separated from the stomach. Behind, crura of the diaphragm, vena cava, aorta, superior mesenteric artery and vein, spine, left kidney and suprarenal body. Above, the upper border rests against the cœliac axis and has the splenic artery and vein running behind it. Below, the third part of the duodenum.

The duct of the gland opens into the common bile duct close to the duodenum; it is called the duct of Wirsung.

The blood-supply: From the splenic, hepatic, and superior mesenteric arteries. The return flow is through the splenic and superior mesenteric veins.

The lymphatics empty into the superior mesenteric glands.

The nerves are derived from the solar plexus and accompany the arteries to the gland.

\section{DISSECTION.}

Raise the liver and ribs as far upward as possible with chain hooks. Draw the stomach downward. Dissect off the peritoneum covering the hepatic artery, duct, and portal vein, trace the artery to the cœliac axis, and the gastric and splenic branches of the same. 
The Cœliac Axis. Figs. I04, I05, 106.

This is the second branch of the abdominal aorta. It is a large trunk coming off the front of the abdominal aorta just after it comes through the diaphragm.

The Solar Plexus. Figs. 74, 106, 107.

About the cœliac axis will be found the semilunar ganglia, the right and left, joined together by numerous nerve filaments composing the solar plexus (abdominal brain). From the solar plexus nerves pass to the various abdominal organs with the blood vessels. They form secondary plexuses upon, and take the names of, the arteries they accompany.

The Semilunar Ganglia. Figs. 74, I07.

Are irregularly shaped masses, one on either side of the cœliac axis and superior mesenteric arteries.

The greater and lesser splanchnic nerves are formed within the thorax (see page 345) ; after piercing the crus of the diaphragm they enter the upper part of the semilunar ganglion to take part in the formation of the solar plexus.

Branches of Coliac Axis.-The coliac axis divides into the gastric, hepatic, and splenic arteries.

The Gastric Artery. Figs. I04, 105.

This is the smallest of the three branches. It passes upward to the left to reach the stomach at the junction of the œesophagus, then turns downward, following the lesser curvature, until it anastomoses with the pyloric. Its branches are $(a)$ œsophageal, running upward to the œesophagus, to anastomose with the thoracic œesophageal, (b) the branches over the fundus, to unite with splenic branches, (c) terminal, anastomosing with the pyloric. 


\section{The Hepatic Artery,}

Next in size, comes off from the right side of the coliac axis. It takes a direction to the right and upward to the liver.

Branches.-(a) The pyloric. This is given off opposite the pylorus, passes to this end of the stomach, turns to the left and joins the terminal branches of the gastric. (b) The gastroduodenalis, really one of the branches of bifurcation of the hepatic, descends behind the pylorus. See its further course below. (c) The cystic. This supplies the gall bladder. (d) The terminal branches, right and left, to supply their respective lobes of the liver.

The relations of the hepatic artery, duct, and portal vein have been given. See Gastrohepatic Omentum, page 492. (e) The hepatic gives off several small pancreatic branches to that gland.

\section{DISSECTION.}

Divide the anterior layer of the greater omentum just below the greater curvature of the stomach. Turn the stomach up over the chest and fasten it there with chain hooks. Complete the dissection of the splenic and gastroduodenal arteries. Trace the common bile duct and portal vein as far as possible. Clean the anterior surfaces of the pancreas, duodenum, and the beginning of the superior mesenteric artery.

The Splenic Artery. Fig. I05-This is the largest of the cœliac branches. It passes behind the stomach toward the left to supply the spleen. It gives off $(a)$ numerous small branches to the pancreas as it courses along its upper border, called the pancreaticæ parvæ. (b) A large artery, the pancreatica magna, to the same gland. (c) Then the left gastro-epiploic which follows the greater curvature of the stomach between the layers of the great omentum, from left to right to inosculate with the right artery of this name. (d) The vasa brevia, several branches to the fundus of the stomach, which anastomose with the gastric. (e) The terminal branches to the spleen itself. 
The gastroduodenalis will be seen to divide into two branches : the right gastro-epiploic, which courses along the greater curvature of the stomach to unite with the left gastro-epiploic; and the superior pancreaticoduodenalis, which, descending in the interval between the duodenum and the head of the pancreas, supplies both and finally anastomoses with the inferior pancreaticoduodenalis from the superior mesenteric. Both gastro-epiploic arteries supply the stomach and send off long slender branches to the omentum, which follow backward to meet similar branches from the colic arteries.

These branches are the epiploic.

The Superior Mesenteric Artery. Figs. 102, 105.

Will be found coming out between the pancreas and third portion of the duodenum, arising from the front of the aorta. It gives off the inferior pancreaticoduodenalis, which turns to the left between the pancreas and the duodenum, supplying both, and terminating by anastomosing with the superior artery of the same name.

\section{DISSECTION.}

Let the stomach and liver drop back into the abdomen. Raise the great omentum and transverse colon upward on to the chest and fasten them there. Dissect off the presenting layer of the peritoneum from the superior mesenteric artery and its branches. It will not be necessary to clean all the intestinal branches to their distribution; clean their origin, and trace two or three, with their anastomoses, to the smaH intestine. It will be necessary to follow all the other branches coming off the right side of the mesenteric. They are the ileocolic, the right, and middle colic.

The superior mesenteric artery arises from the front of the aorta (as previously discovered), above the transverse duodenum and below the pancreas. It takes a curved direction downward and to the right iliac fossa.

(a) Its first branch is the inferior pancreaticoduodenalis, 
q. $\%$ above. (b) From the convex side the vasa intestini tenuis, I 2 to I 6 branches, are given off to supply the small intestine from the last portion of the duodenum to within a short distance from the cæcum. These intestinal branches form from three to five systems of arterial anastomoses between their origin and distribution. (c) The ileocolic supplies the last portion of the small intestine and the beginning (cæcum) of the large. It anastomoses on one side with the last intestinal branch and on the other with the $(d)$ colica dextra. This artery is distributed to the ascending colon. (e) The colica media supplies the transverse colon. These last three arteries form long arterial arches from one to the other, and from the main arches other secondary ones are formed before the intestine is finally reached. Atong with the artery runs the vein, only in the contrary direction, and at the right side of the artery. The vein disappears under the pancreas, where it helps to form the portal vein. See page 514. Its tributaries are the right gastro-epiploic, pancreaticoduodenal (superior and inferior), besides the veins corresponding to the branches of the superior mesenteric artery.

\section{DISSECTION.}

Tie two strong strings around the small intestine six inches from the duo. denum and the same distance from the cæcum, divide the intestine between the cords at both points, cut it away from its mesentery, and remove it entirely. Clean the inferior mesenteric artery, tracing its branches to the descending colon, omega loop, and rectum.

The Inferior Mesenteric Artery. Figs. I02, 105.

Is given off from the left side of the abdominal aorta one and one-half inches above its bifurcation.

Its Branches.

(a) The colica sinistra supplies the descending colon. (b) The sigmoid is distributed to the sigmoid, or omega 


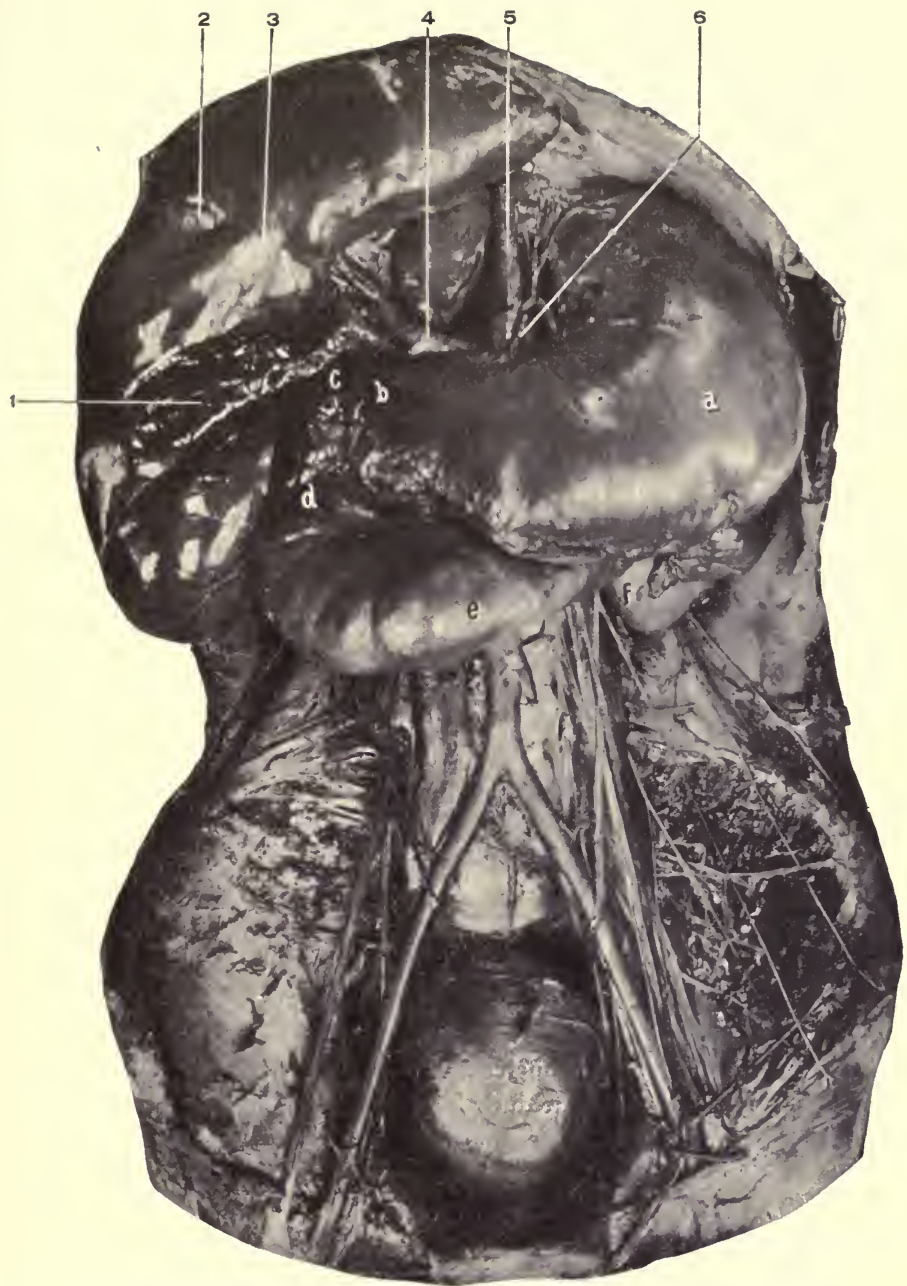

Fig. 104. Dissection of Abdominal Viscera (MAle), $-a$, Stomach. 3, Pylorus. $c$, Ascending duodenum. $d$, Descending duodenum. $e$, Transverse duodenum. $f$, Left kidney. $\mathrm{r}$, Gall bladder. 2, Round ligament of liver in the umbilical portion of the longitudinal fissure of liver. Between 1 and 2 is the quadrate lobe. 3, Pons hepatis. 4, Hepatic artery at the point where it is giving off the gastroduodenal. 5, Abdominal aorta. 6, Gastric artery. 
loop. (c) The superior hemorrhoidal to the upper part of the rectum. These arteries form a chain of anastomoses with one after the other, uniting on the right with the colica media, and below, in the pelvis, with the middle hemorrhoidal from the internal iliac.

The longest arterial arches will be found on either side of the colica media.

This completes the arterial supply of the alimentary canal from the œesophagus to the middle of the rectum.

\section{The Inferior Mesenteric Vein}

Drains the blood from the area supplied by the artery, passes upward at the left of the artery and behind the perineum to empty into the splenic vein just before it unites with the superior mesenteric vein to form the portal vein.

\section{DISSECTION.}

Double ligature the rectum, and divide it. Remove the large intestine entirely, carefully cutting through its peritoneal attachments close to the bowel.

Tie a cord about the œsophagus as close to the stomach as possible, and divide the former above it. Tie two cords around the first portion of the duodenum and divide between them. Remove the stomach and spleen, severing their ligaments of attachment to the diaphragm and the arteries and veins passing to and from them.

Raise the duodenum and pancreas, and complete the dissection of the common bile duct, the pancreatic duct, from where it joins the preceding to a distance of two or three inches into the gland, the portal vein, and the tributaries that form it.

\section{The Portal Vein. Fig. I05.}

This is formed behind the pancreas by the junction of the superior mesenteric and splenic veins. It passes upward toward the right behind the first part of the duodenum to the transverse fissure of the liver. It is about three inches long, and lies between the two layers of the gastrohepatic omentum. It receives the pyloric, gastric, cystic, the supe- 
rior mesenteric, and splenic. See Gastrohepatic Omentum, page 492 .

The Splenic Vein. Fig. I05.

Runs in company with the artery from the spleen to its junction with the superior mesenteric to form the portal vein. Its tributaries are the vasa brevia veins, left gastroepiploic, pancreatic, and inferior mesenteric.

\section{DISSECTION.}

Draw down the liver, carefully sever its ligaments until the vena cava is reached, secure room enough between the diaphragm and the liver to pass a ligature around the vein, tie it, and divide the vein below the ligature.

Find the vena cava below the liver and ligate it there, cutting above the ligature. The liver, duodenum, and pancreas can now be removed entirely, dividing the vessels that are found passing to them and the peritoneal processes which bind them to the posterior abdominal wall, remembering to keep close to the viscera that are being removed.

Clean the under surface of the diaphragm, the abdominal aorta and its remaining branches, the inferior vena cava and the veins emptying into it, the suprarenal bodies, kidneys and their blood supply, the ureters, the spermatic or ovarian arteries. For the present leave the pelvic viscera.

The Abdominal Aorta. Figs. 74, I06, 107.

Extends from the aortic opening in the diaphragm, over the lower margin of the twelfth dorsal vertebra, to the lower border of the fourth lumbar vertebra, where it divides into the two common iliac arteries. The point at which the aorta bifurcates is a little below and to the left of the umbilicus. The aorta lies at first in front of the vertebra, but below is a little to the left.

Relations.-In front, from above downward, liver, solar plexus, gastrohepatic omentum, stomach (at the osophagus), anterior layer of transverse mesocolon, vena porta (or ending of the splenic vein), the pancreas, left renal vein, third portion of the duodenum, mesentery, small intes- 
tines, lymphatic glands, and sympathetic plexus. Behind, the aorta rests upon the lumbar vertebræ and intervertebral discs, the anterior common ligament of the spine, left crus of the diaphragm, and left lumbar vein. At the right, right crus of diaphragm, right splanchnic nerves, Spigelian lobe of the liver, receptaculum chyli, vena cava inferior. At the left, the left crus of the diaphragm, left splanchnic nerves.

The Branches.

(I) The inferior phrenics. These arteries may arise separately or by a common trunk from the front of the aorta immediately after the aorta appears through the diaphragm, or they may arise from the cœliac axis. They are the right and left, and supply the right and left sides of the diaphragm. The right also sends small branches to the liver and right suprarenal body, the left to the œsophagus, spleen, and left suprarenal gland. The right artery passes behind the vena cava.

(2) The Cœliac Axis, see page 509.

(3) The right and left suprarenal. These are small arteries that come off the side of the aorta and supply the suprarenal bodies. Besides these, the suprarenal glands receive blood from the inferior phrenics (superior suprarenal blood supply), and from the renals (the inferior blood supply, the suprarenals themselves being the middle arteries).

(4) The right and left first lumbar. See below.

(5) Superior mesenteric. See page 5 I I.

(6) Right and left renal. Each renal artery arises from the side of the aorta, on a level with the first lumbar vertebra, and passes outward to the kidney, breaking up into several branches to enter at the hilum of the gland. The artery is in front of the beginning of the ureter and behind the renal vein. 


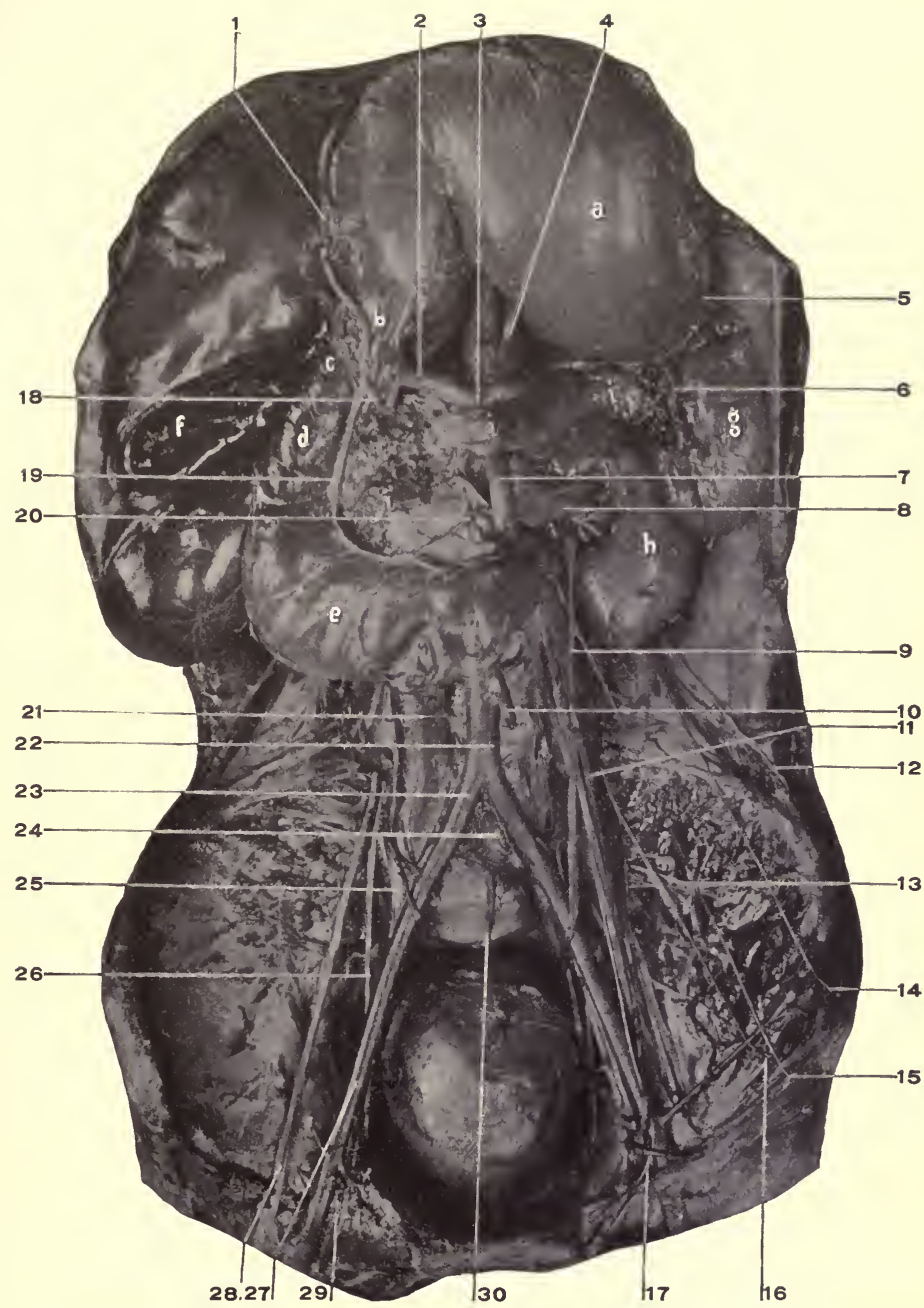

Fig. 105. Dissection of Abdominal Viscera (MAle), - $a$, Stomach. $b$, Pylorus. $c$, Ascending duodenum (when stomach is in natural position). $d$, Descending duodenum. $e$, Transverse duodenum. $f$, Gall bladder. $g$, Spleen. $h$, Kidney. $i, i, i$, Pancreas. I, Right gastro-epiploic artery. 2, Hepatic artery. 3, Coliac axis. 4, Gastric artery. 5, Left gastro-epiploic. 6, Splenic artery. 7, Superior mesenteric artery. 8, Renal vein. 9, Ureter. Io, Inferior mesenteric artery. II, Left spermatic vein. Trace it both ways. I2, Last dorsal nerve. I3, Iliolumbar artery. I4, Iliohypogastric nerve. 15, Ilio-inguinal nerve. I6, External cutaneous nerve. 17, Genitocrural nerve. 18, Gastroduodenal artery. 19, Superior pancreaticoduodenal artery. 20, Inferior pancreaticoduodenal artery. 2I, Inferior vena cava. 22, Aorta. 23, Common iliac artery. 24, Left and 25, Right common iliac veins. Notice their relations to their corresponding arteries. 26, Obturator nerve. 27, External iliac artery. 28, Anterior crural nerve. 29, External iliac vein. 30, Middle sacral artery. 
The right artery passes behind the vena cava, and is a little longer than the left.

(7) The right and left spermatic. Each is a long, slender artery that passes with the spermatic cord through the internal abdominal ring, the inguinal canal, and external ring to the scrotum as far as the testicle, which it supplies. The artery lies behind the peritoneum, and joins the cord (vas deferens) just before the internal abdominal ring is reached.

In the female the artery is the ovarian, and supplies the ovary and uterus. It has the same origin, and course until it enters the broad ligament and passes to supply the ovary and uterus.

(8) The right and left second lumbar arteries. See below.

(9) The inferior mesenteric. See page 5 I 2.

(IO) The right and left third lumbar. See below.

(I I) The right and left fourth lumbar.

The lumbar arteries arise from the posterior surface of the aorta, opposite the bodies of the lumbar vertebræ, pass outward beneath the origin of the psoas, then behind the quadratus lumborum, finally between the transversalis and internal oblique, and are distributed to the abdominal wall as far forward as the rectus muscle, anastomosing with the other abdominal arteries.

(I 2) The right and left common iliacs. See below.

(I 3) The sacra media. This small artery arises from the posterior surface of the aorta just above its bifurcation, passes down in front of the middle of the sacrum to the coccyx. It anastomoses with the sacral branches from internal iliac arteries.

The Common Iliac Arteries. Figs. 103 to 107

They extend from the bifurcation of the aorta, at the 
lower border of the fourth lumbar vertebra, to the junction of the sacrum and ilium, where they divide into the external and internal iliacs.

The right common iliac is slightly longer than the left (right, two inches, left one and three-quarters). They are crossed by the ureters near their bifurcation, and in the female, by the ovarian arteries.

For the relation to the corresponding veins, see below.

The External Iliac Arteries. Figs. I03 to I07.

Continue the direction of the common iliac arteries from their termination, outward beneath Poupart's ligament, where they become the femoral.

Under Poupart's ligament the external iliac artery is at a point midway between the anterior superior iliac spine and the symphysis pubis. A line drawn from this point to onehalf of an inch below and at the left of the umbilicus, will indicate the course of the common and external iliac arteries.

The external iliac is crossed by the vas deferens in the male, the round ligament in the female, and by the deep circumflex iliac vein in both.

For relations to vein, see below.

Branches.

(I) The deep epigastric. See page 456. Figs. 94, IO3 to 107.

(2) The deep circumflex iliac. Figs. 106, 107. Arises from the outside of the external iliac and takes a course outward, following the direction of Poupart's ligament and the crest of the ilium, to anastomose by its terminal branches with the iliolumbar artery.

Its muscular branches perforate the transversalis muscle and ascend between this and the internal oblique muscles, supplying the abdominal wall and anastomosing with the 
lower intercostals, lumbar, and lateral branches of the deep epigastric arteries.

The External Iliac Veins. Figs. I03, I O4, 105.

They begin under Poupart's ligament, as the continuation of the femoral veins, at the inside of the external iliac arteries.

Each passes upward along the inside of the artery to terminate where the artery begins by joining with the internal iliac vein to form a common iliac vein.

The left vein is at the inner side of the artery for its entire course, and posterior to the artery. The right vein starts at the inner side of the artery and at its termination has begun to pass behind it.

Tributaries. - The deep epigastric, and circumflex iliac veins, which collect the blood from the area supplied by the arteries of the same names, also a pubic vein which joins the obturator and external iliac veins.

The Internal Iliac Veins will be given later.

The Common Iliac Vein. Figs. I03 to I06.

Is formed by the junction of the external and internal iliac veins. It extends from the sacro-iliac articulation to the right of the aortic bifurcation, where they unite to form the vena cava inferior. The left vein is behind and at the inside of the left artery, and ends behind the right artery. The right vein is first behind, then behind and at the outside of its artery.

Tributaries.-The iliolumbar, and middle sacral veins.

The Vena Cava Inferior. Fig. I06.

Is formed at the right of the abdominal aorta, and behind the right iliac artery over the fifth lumbar vertebra, by the union of the two common iliac veins, and passes upward 
to leave the abdominal cavity through the caval opening in the diaphragm. It lies at the right side of the aorta, in contact with it below, but separated from it above by the right crus of the diaphragm and Spigelian lobe of the liver.

The vena cava is crossed by the right spermatic artery, transverse colon, mesentery, duodenum, pancreas, portal vein, and liver, and lies in a deep groove in the substance of the liver (the caval fissure).

Tributaries.-(I) The common iliac veins.

(2) The lumbar, four on each side, the left being a little longer than the right.

The lumbar veins are joined together by a vertical vein which runs upward in front of the transverse processes of the vertebræ to communicate above with the azygos major (on the right side), and azygos minor (on the left side). See page 348 .

(3) The spermatic veins (ovarian in the female). Return the blood from the testicle; on the cord they form a plexus of dilated and convoluted veins called the pampiniform plexus. In the female this plexus is formed about the ovary. The left spermatic (or ovarian) vein empties into the left renal vein.

(4) The renal veins. Return the blood from the kidneys.

The left is a little longer than the right, and receives the left spermatic (or ovarian) vein. The renal artery lies behind the vein.

(5) The suprarenal veins. These return the blood from the bodies of the same name. The left suprarenal usually empties into the left renal, the right into the vena cava.

(6) The hepatic veins. Two or three large veins. Return all the blood from the liver and empty into the vena cava as it lies in the caval fissure of the liver.

(7) The inferior phrenic veins. Collect the blood from 
the diaphragm, and accompany the arteries of the same name. The left usually empties into the left suprarenal, or renal.

The Kidneys. Figs. 106, 107.

These organs are situated at the back of the abdominal cavity, on either side of the spinal column, supported by an investment of adipose and connective tissue. The long axis of the kidney is vertical, its length 4 inches, corresponding to the last two dorsal and first two lumbar vertebræ, width $2 \frac{1}{2}$ inches, and thickness $1 \frac{1}{4}$ inches. The right is usually a little lower than the left, owing to the liver above it crowding it downward.

Relations.-Posteriorly, the kidney rests upon the diaphragm in front of the eleventh and twelfth ribs, the quadratus lumborum, and psoas muscles, from which it is separated by the diaphragmatic fascia from the first, the anterior layer of the lumbar fascia from the second, and by the iliopsoas fascia from the third. Behind it pass the twelfth dorsal, iliohypogastric, and ilio-inguinal nerves.

The relations of the pleura are important though not intimate. Its parietal reflection descends into the angle between the diaphragm and the thorax, to the level of the lower border of the twelfth dorsal vertebra, and is represented by a line crossing the neck of the twelfth rib and the outer end of the eleventh. The incisions for reaching the kidney may open into the pleural cavity if carried too high.

Anterior surface of the kidney. The right is crossed in its upper half by the liver, in the lower half by the ascending colon and duodenum (latter covers inner one-fourth of lower half). The left has the stomach in front of its upper third, the splenic artery and pancreas in front of the middle third, and the colon in front of the lower third. 


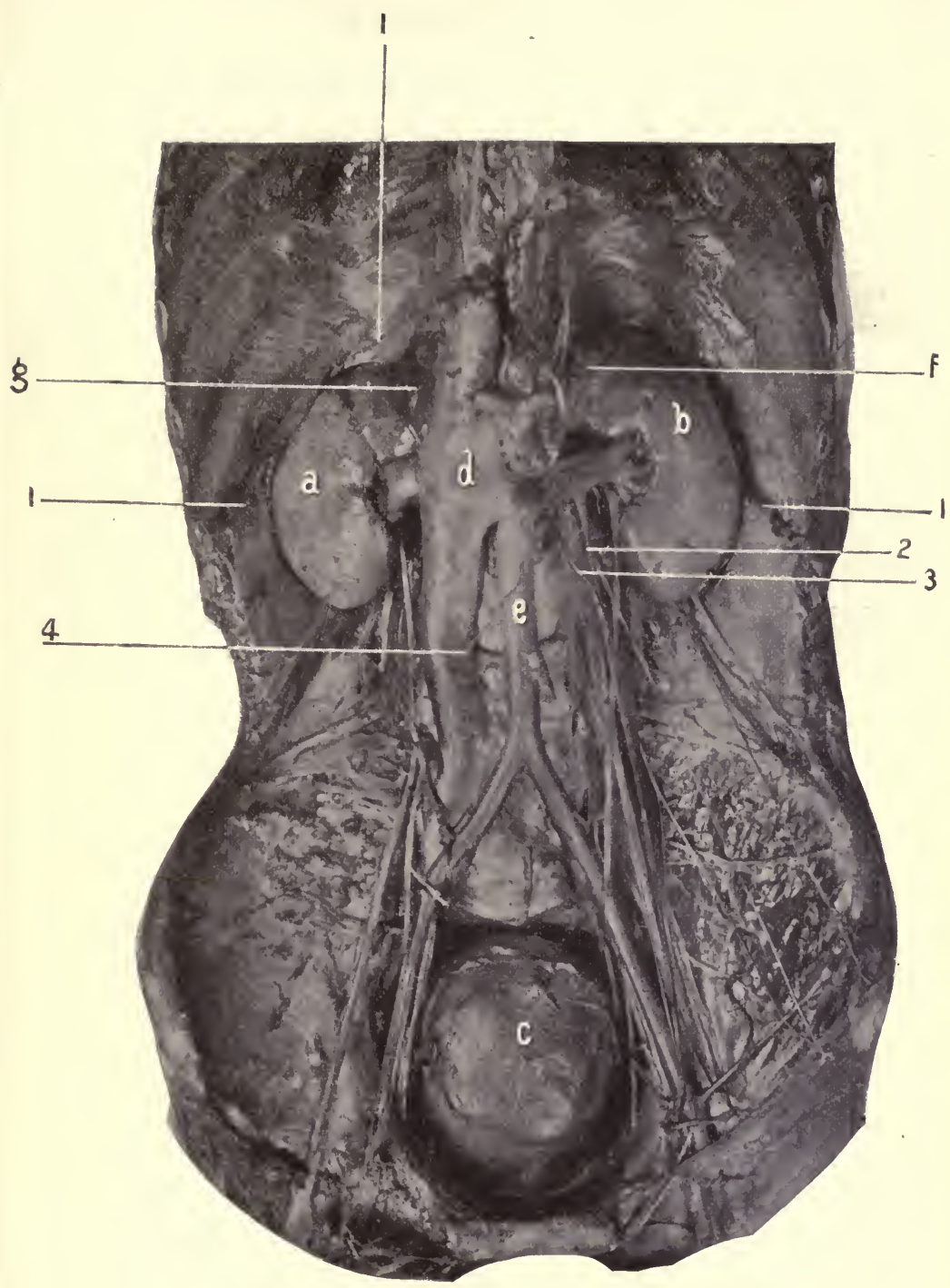

Fig. io6. Dissection of Abdominal Viscera (MAle), $-a$, Right, and $b$, Left kidney. $c$, Bladder. $d$, Inferior vena cava. $e$, Abdominal aorta. $f$, Left, and $g$, Right suprarenal bodies. I, I, I, Origin of diaphragm. 2, Left spermatic vein emptying into renal vein. 3, Left spermatic artery. 4, Right spermatic artery. 
On the outside, the right lies against the liver for its upper three-fourths, the left against the spleen for about the same extent. Above, the suprarenal bodies rest upon the upper and inner margin of the kidneys. On the inner side are the arteries, veins, and exit of the ureters.

The arteries are derived from the Aorta. The veins empty into the Vena Cava. The nerves are derived from the solar and aortic plexuses. The lymphatics empty into the receptaculum chyli.

The Suprarenal Bodies. Figs. 106, I07.

These small triangular glands are found resting upon the upper and inner borders of the kidneys. Their base is about one and three-fourths inches, their height about one and one-fourth inches.

The right suprarenal body is behind the liver, duodenum, and in contact with the vena cava, the left behind the stomach, pancreas, splenic artery, and has the spleen to the outer side.

The arteries are from the Aorta, Inferior Phrenic, and Renal. The veins empty into the vena cava, or renal. The nerves are derived from the same source as the kidney. The lymphatics pass to the renal glands. The kidneys and suprarenal bodies are behind the peritoneum.

The Ureters. Figs. 105 to 107, I I 2.

Are the excretory ducts of the kidneys, and pass downward to empty into the base of the bladder. They are from twelve to sixteen inches long, and below the kidney one-sixth of an inch in diameter. At the kidney they become expanded to form the sinus, into which the calices empty. In its course to the bladder the ureter crosses the psoas muscle, genitocrural nerve, common iliac artery and vein. It lies behind the peritoneum, and is crossed by the 
spermatic vessels (in female, ovarian), and at the bladder by the vas deferens (in the female by the round ligament).

The Ilio-Psoas Fascia. Fig. I03.

Is attached to the crest of the ilium, the outer part of Poupart's ligament blending with the transversalis fascia; when the femoral vessels are reached it passes behind them to form the posterior layer of their sheath (as the transversalis does the anterior layer); it is then attached to the iliopectineal line, brim of the true pelvis, to the bodies and intervertebral substances, and bases of the transverse processes of the lumbar vertebræ, as well as to the tendinous arches which bridge over the lumbar arteries; when the diaphragm is reached this fascia becomes thickened to form the ligamentum arcuatum internum, extending from the body of the first to the transverse process of the second lumbar vertebra, arching over the psoas muscle; along the outer margin of the psoas the fascia is attached to the anterior lamella of the lumbar fascia.

It is the existence of this fascia that determines the course of purulent collections beneath it to point beneath Poupart's ligament at the outer side of the femoral artery, forming a psoas abscess. Such abscesses are, in the great majority of cases, due to tubercular disease of the lumbar vertebræ (Pott's disease).

The anterior layer of the lumbar fascia is seen covering the quadratus lumborum muscle. Its attachments have already been given (see page 45I). Below, it becomes attached to the crest of the ilium and the iliolumbar ligament ; above, it forms the ligamentum arcuatum externum, $q . v$. (page $45 \mathrm{I}$ ).

The Femoral Opening. Figs. I03, 106.

To the inner side of the external iliac vein, just under 
Poupart's ligament, is seen a dimple that is usually occupied by a small lymphatic gland. This dimple or small fossa is due to a slight depression in the transversalis fascia, as it pouches into a small gap that is left between the vein and Gimbernat's ligament. The gap (as seen after dissection or after being made by a hernia) is the femoral ring, and the transversalis fascia that covers it over is the septum crurale. A femoral hernia bulges the septum crurale before it in its descent to leave the abdominal cavity by the femoral opening. As the dissection progresses the femoral opening will be seen to have these boundaries: To the inside, Gimbernat's ligament, conjoined tendon, and triangular ligament ; in front, Poupart's ligament ; to the outside, the external iliac vein covered by the sheath (formed by the union of the iliac fascia beneath, with the transversalis fascia above); behind, the pubic portion of the fascia lata covering the pectineus muscle and the horizontal ramus of the pubic bone.

The description of the canal will be given later.

\section{DISSECTION.}

Ligate the vena cava at its beginning, divide and remove it, with all the veins emptying into it.

Carefully remove the iliopsoas and anterior layer of the lumbar fasciæ. Dissect out the receptaculum chyli, splanchnic nerves, sympathetic cord and ganglia, the beginning of the lumbar arteries, the branches of the lumbar plexus external to the psoas muscle, the psoas and iliacus muscles. Follow the vas deferens to the bladder, or the round ligament to the uterus.

Trace the ureters to the bladder.

The Lumbar Sympathetic Ganglia and Cord. Figs. 74, I06, I07, I I 3 .

Consist of four ganglia connected together by the sympathetic cord, which above comes from the thorax, and below passes to the sacral ganglia. These ganglia lie in 
front of the vertebræ, the right behind the vena cava, the left behind the outer border of the aorta, both close to the anterior border of the psoas muscle. The cord passing to the sacral ganglia goes behind the iliac vessels, internal to the psoas muscle.

\section{The Receptaculum Chyli.}

This is the dilated pouch from which the thoracic duct arises. It receives the lymph and chyle from the abdominal walls and organs. See page 344 .

It lies to the right and behind the abdominal aorta, between it and the right crus of the diaphragm, and upon the body of the second lumbar vertebræ. It is one and onehalf inches long and one-quarter of an inch in diameter.

Psoas Magnus. Figs. 103, 107.

Origin.-By five muscular slips from the anterior surfaces and lower borders of the transverse processes of the lumbar vertebræ, from the sides and intervertebral discs of the last dorsal and lumbar vertebræ. Over the centre of the bodies of the vertebræ small gaps are left in the muscular attachment for the passage of the lumbar vessels and lumbar branches of the sympathetic nerves.

Insertion.-By a strong tendon into the lesser trochanter of the femur.

Nerve Supply.-Muscular branches from the anterior division of the second and third lumbar nerves.

Action.-For action on femur see under Iliacus. On the spine it flexes the last dorsal and lumbar vertebræ upon the pelvis.

Iliacus. Figs. 103, 107.

Origin.-From the base of the sacrum, the sacro-iliac and iliolumbar ligaments, the iliac fossa, the iliac crest, 
spinous processes and bone between them, from the capsule of the hip joint.

Insertion.-Into the outer border of the tendon of the psoas, also into an inch of the line from the lesser trochanter to the linea aspera.

Nerve Supply._By branches from the anterior crural, derived from the second and third lumbar nerves.

Actions. - The psoas magnus and iliacus act as one muscle upon the femur. They are the direct and most powerful flexors of the thigh, upon the abdomen, or the reverse. From the insertion of their tendon into the lesser trochanter, this insertion being outside of the centre of motion of the head of the femur in the acetabulum. It follows that flexion being arrested, the great trochanter will be raised (carried forward) and internal rotation will result.

\section{Psoas Parvus. Fig. 107.}

When present this muscle will be found arising from the intervertebral disc between the bodies of the last dorsal and first lumbar vertebræ, and from the adjacent portions of those vertebræ.

Insertion.-Into the iliopectineal line.

Nerve Supply-From the first lumbar nerve.

Action.-To flex the pelvis on the spine or the reverse.

Quadratus Lumborum. Figs. I06, 107.

Origin.-From the iliolumbar ligament, from the inner lip of the crest of the ilium for two inches posterior to the iliolumbar ligament, from the tips of the transverse processes of the three lower lumbar vertebræ.

Insertion.-Into the inner half of the lower border of the last rib, and the transverse processes of the three upper lumbar vertebræ. 
Nerve Supply.-Filaments from the last dorsal and upper lumbar nerves.

Action.-A lateral flexor of the spine. By fixing the last rib it gives the diaphragm a firm point for contraction and so aids inspiration.

\section{DISSECTION.}

Trace the branches of the lumbar nerves through the psoas muscle.

The Last Dorsal Nerve. Figs. 105, 106, I07.

Courses outward and downward just below the lower border of the last rib, crossing the quadratus lumborum, to disappear through the lumbar fascia, and enter between the transversalis and internal oblique muscles, where it divides into two branches.

The anterior branch continues to the rectus and pyramidalis muscles, the lateral or iliac pierces the internal and external oblique muscles about three inches above the crest of the ilium and turns downward to supply the integument over the crest of the ilium in front of and as low down as the great trochanter.

The Lumbar Plexus. Figs. 103 to I07.

The lumbar plexus is formed by the anterior divisions of the first four lumbar nerves and a branch from the last dorsal.

From the fourth lumbar nerve a branch descends to join the fifth (anterior) division, and the trunk thus formed passes to the upper margin of the sacral plexus as the lumbosacral cord.

The lumbosacral cord lies between the inner border of the psoas muscle and the base of the sacrum, and behind the iliac vessels. The lumbar nerves are connected to the sympathetic ganglia by slender filaments. 
The Branches of the lumbar plexus are as follows:-

The first nerve having received the branch from the last dorsal, divides into the iliohypogastric and ilio-inguinal. From the first and second is formed the genitocrural. From the second and third, the external cutaneous. From the second, third, and fourth, the anterior crural and obturator. From the third and fourth, the accessory obturator. And from the fourth the branch passes to enter into the lumbosacral cord.

All these branches are formed within the substance of the psoas magnus muscle.

\section{The Nerves.}

(I) The iliohypogastric branch. From the first lumbar. Issues from the upper border of the psoas, crosses the quadratus lumborum and iliacus obliquely to enter the transversalis muscle over the middle of the crest of the ilium. It divides into two branches; the iliac, which pierces the abdominal muscles and supplies the integument over the outer and posterior surface of the crest as far as the great trochanter, being posterior to the iliac branch of the last dorsal nerve.

The hypogastric branch, which passes through the internal oblique about one inch in front of the anterior superior iliac spine, extends downward and forward to appear through the external oblique about two inches above the spine of the pubes, supplying the integument in front of the pubes and dorsum of the penis.

(2) The ilio-inguinal. From the first lumbar. It is found at the outer border of the psoas just below the iliohypogastric, takes a course parallel with it but a little lower, to follow close to the crest of the ilium and Poupart's ligament, and appear through the external 


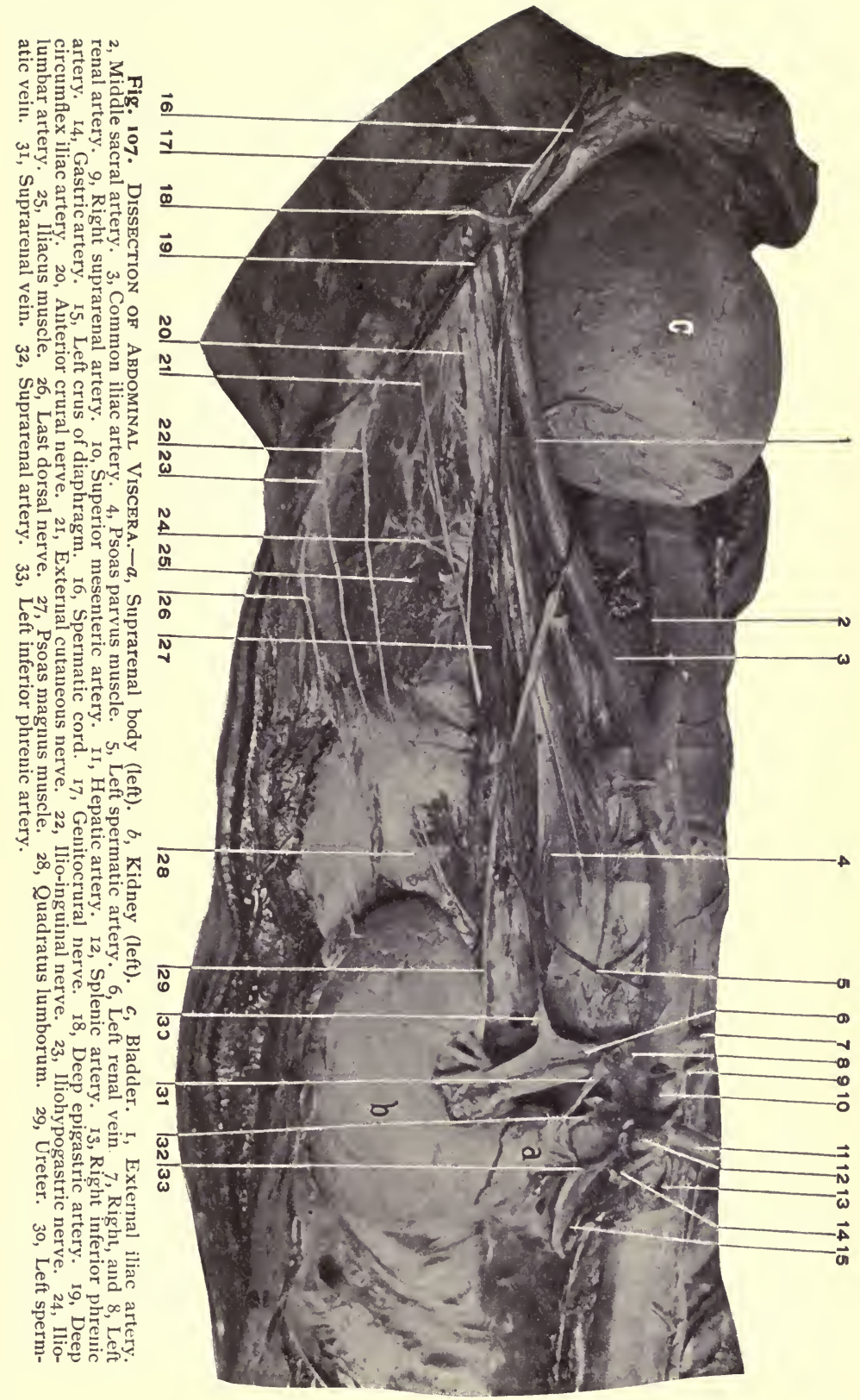


abdominal ring behind the cord (or round ligament), to be distributed to the integument over the upper and inner part of the thigh and outer surface of the scrotum or labium majus.

(3) The genitocrural, from the first and second lumbar nerves.

This nerve appears at the inner side of the psoas, runs downward upon its anterior surface, and divides just above Poupart's ligament into the genital and crural branches. The genital branch turns outward to join the cord and leave the abdomen through the internal abdominal ring to supply the cremaster muscle (in the female the round ligament). The crural branch continues downward under Poupart's ligament at the outer side of and in front of the external iliac and femoral arteries, pierces the fascia lata at the lower border of the saphenous opening (two inches below Poupart's ligament), and supplies the central and upper region of the front of the thigh.

(4) The external cutaneous. From the second and third nerves. Emerges from the outer border of the psoas muscle, below the crest of the ilium, extends outward and downward across the iliacus muscle, passes under Poupart's ligament close to the anterior superior iliac spine, in front of the origin of the sartorius, descends vertically in a canal in the fascia lata for four or five inches, then becomes superficial and supplies the integument over the anterior and outer surfaces of the thigh, nearly to the knee. Just after coming out from under Poupart's ligament, a branch is given off that turns backward to supply the skin over the great trochanter.

(5) The anterior crural nerve. Formed from the second, third, and fourth lumbar nerves. Descends in the interval between the psoas and iliacus muscles, being covered by 


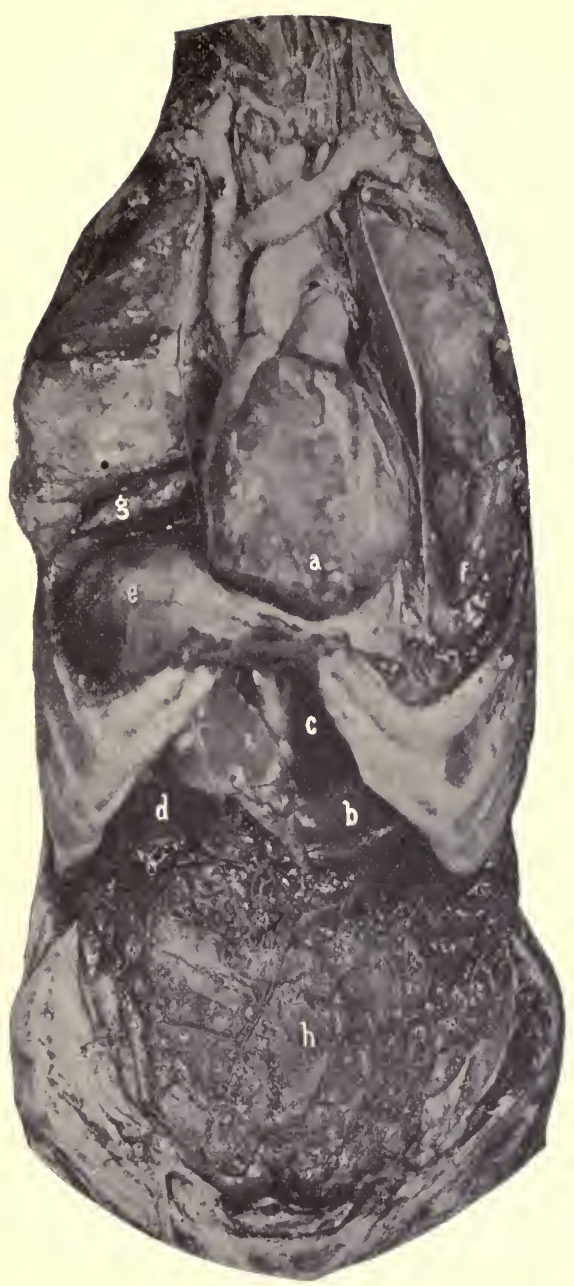

Fig. 108. The Mutual Relations of the Thoracic and Abdominal VisCERA. [Figs. 108 and rog were made after long transfixion pins had been passed through the lower border of the thorax and driven firmly into the plank upon which the subject rested. These pins were inserted previous to any dissection and the photographs were taken with the subject horizontal.] $-a$, Heart in its natural position. $b$, Stomach. $c$, Left lobe of liver. $d$, Right lobe of liver. $e$, Diaphragm covering liver. $f$, Left and $g$, Right lung. $h$, The great omentum covering the intestines. 
the former ; under Poupart's ligament it lies external to the femoral artery ; a little lower it breaks up into its branches, muscular and cutaneous. See Thigh, anterior.

The muscular branches supply the pectineus (by a branch which passes behind the femoral vessel), sartorius, rectus femoris, vasti, and crureus. The hip joint receives a small branch from the nerve to the rectus, and the knee, from the nerves to the vasti and crureus.

The cutaneous branches. The middle pierces the fascia lata three or four inches below Poupart's ligament and supplies the anterior region to the knee. The internal is distributed to the antero-internal lower two-thirds of the thigh and knee.

The long or internal saphenous nerve. Follows the femoral artery through Hunter's canal until the artery turns backward through the opening in the adductor magnus. In this canal the nerve lies first to the outside of the artery, then crosses it in front to reach the inside.

From Hunter's canal, the nerve continues downward, passes between the sartorius and gracilis (behind the former as usually given), and appears superficial at the inner side of the knee, and supplies the antero-internal surfaces of knee, leg, and ankle. In its course from Hunter's canal to the knee the nerve is accompanied by the superficial branch of the anastomotica magna artery. In the leg, the nerve follows the internal saphenous vein. Just above the knee the nerve gives off the branch which supplies the anterointernal surface of that joint.

(6) The obturator nerve. From the second, third, and fourth lumbar nerves. Descends between the psoas and base of the sacrum, behind the iliac vessels, passes forward and inward just below the level of the brim of the pelvis and between the peritoneum and pelvic fascia, to leave the pelvis 


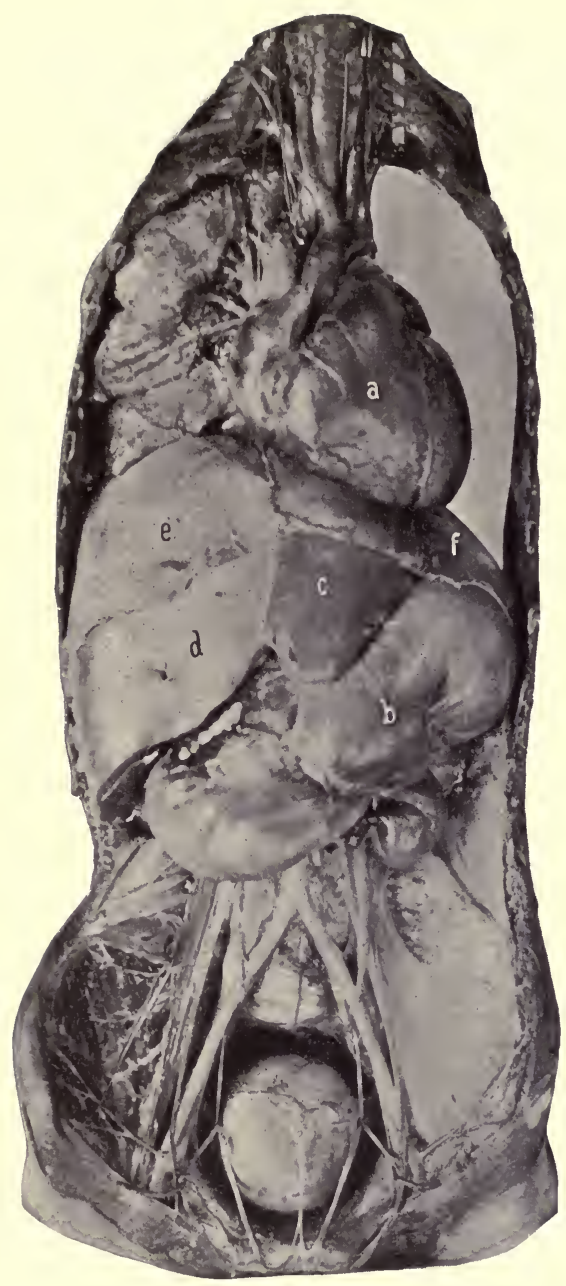

Fig. 109. Mutual Relations of Thoracic and Abdominal Viscera. [The ribs have been entirely removed. Subject horizontal.] $-a$, Heart turned more to the left than normal, owing to the left lung having been removed. $b$, Stomach. Its fundus pushes upward behind apex of heart. $c$ and $d$, Left and right lobes of the liver. $e$ and $f$, Right and left halves of the diaphragm. The close anatomical (as well as clinical) relations between the heart, lungs, liver, and stomach are shown in this and the preceding Figure. 
by the gap in the upper margin of the obturator membrane. It is accompanied in its course by the artery of the same name if it is a branch from the internal iliac. In the thigh the nerve issues from the obturator canal above or through the upper part of the obturator externus muscle, where it divides into two branches. The anterior branch descends between the pectineus and adductor longus in front and the adductor brevis behind, supplying these muscles and terminating in the gracilis. It also sends a cutaneous branch to the lower and inner third of thigh. The posterior branch goes behind the adductor brevis and supplies the adductor magnus, continues downward, pierces the adductor magnus just above the femoral opening, joins the popliteal vessels, and ends in the posterior part of the kneejoint.

The obturator externus receives its nerve supply from the posterior division of the nerve. A filament is also sent to the hip-joint.

(7) The obturator accessory. When present arises from the third and fourth lumbar nerves. Descends between the psoas and internal iliac artery (behind both) over the anterior border of the pubes to the pectineus, which it supplies, then communicates with the obturator nerve and continues to the hip-joint.

\section{DISSECTION.}

Inflate the bladder. Dissect the peritoneum from it and the rectum. Clean the internal iliac vessels and their branches, also the sacral plexus. In doing this notice the pelvic fascia, the so-called "white line," and the rectovesical fascia.

To dissect the pelvic cavity and viscera requires that the lateral wall shall be removed from one side as represented in the plates; as this destroys the side removed for dissection of the gluteal region, it is not practicable, and the dissector must do the best possible with the pelvis intact.

Trace the ureters, vasa deferentia, and expose the seminal vesicles. In 
the female carefully note the relations, position of bladder, uterus, uterine ligaments, ovaries, tubes, and rectum. Trace the round ligaments, ureters, ovarian and uterine arteries. See the Dissection of Perineum, page 397 male, and 422 female.

The Bladder. Figs. 84, 95, IO3 to I I 3.

The bladder is situated in the pelvic cavity. In the living, when empty it is probably round; when distended, oval. When empty it lies entirely behind the symphysis; if moderately distended, reaches to the top; if fully distended, may reach two inches above the symphysis, and in pathological distention may extend to or even above the umbilicus. When distended the long axis of the bladder is parallel with a line connecting the umbilicus and the anus.

Peritoneal Covering.-From the anterior abdominal wall the peritoneum extends on to the top, sides, and down the back of the bladder to within an inch or an inch and a half of the prostate, where it is reflected on to the rectum, forming the rectovesical pouch in the male; (in the female the peritoneum extends backward on to the uterus, and the fossa is called the uterovesical).

The reflections of the peritoneum from the bladder to the adjacent walls of the abdomen or viscera are termed "false" ligaments, and they are well named on account of the false impression that they convey of their function and appearance. The student needs simply to remember that the bladder is underneath the pelvic peritoneum and raises it upward as it fills (the loose covering of peritoneum allowing this action, and steadying the bladder when full).

Retzius' space is the interval between the anterior wall of the bladder and the inner surface of the symphysis pubes, and lower abdominal wall. The extent of space varies with the distention of the bladder. When the bladder is empty the space is obliterated; when the bladder is very full the 
space may extend two inches above the top of the symphysis. As the bladder rises it takes with it the peritoneum; consequently, the viscus can be opened in this condition without entering the peritoneal cavity. The extent of space is further increased by inflating the rectum, thus crowding the bladder upward and forward. This manœuvre is utilized in suprapubic cystotomies.

\section{The True Ligaments of the Bladder.}

(a) The urachus. This is the fibrous cord that extends from the top of the bladder to the umbilicus, and represents the canal which connected the bladder with the allantois in the fotus. See Fig. 95. (b) and (c) The obliterated hypogastric arteries. These fibrous cords extend from the sides of the bladder, where they are continuous with the superior vesical arteries, to the umbilicus. In the fotus they were pervious and carried the fotal blood to the mother. (d) The rectovesical fascia. Parts of this sheet of fascia are designated as the anterior and lateral true ligaments of the bladder and the ligament of the rectum. See page 399.

Relations of the Bladder (when moderately distended). -Anteriorly, the inner surface of the pubic bones and anterior portions of the obturator internus muscles. Laterally, ureters, obliterated hypogastric arteries, vas deferens (in the male, round ligament in the female), anterior branches of the internal iliac artery, levator ani and obturator internus muscles. Posteriorly; the upper, peritoneal surface, with the omega loop; the lower, nonperitoneal, with the rectum, seminal vesicles, terminations of ureters, and vasa deferentia. In the female the posterior surface is in relation to the uterus and upper part of the vagina.

Superiorly, the small intestines and omega loop. Infe- 


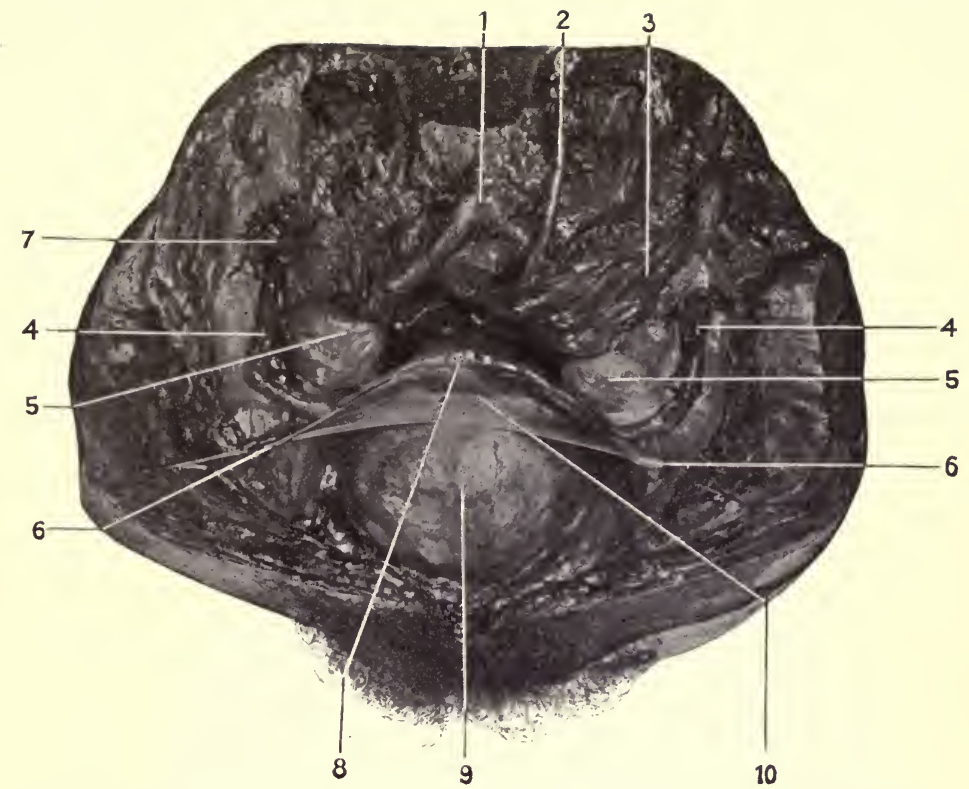

Fig. 110. Female Pelvic Viscera.-1, Aorta at bifurcation. 2, Inferior mesenteric artery. 3, Rectum. 4, 4, Fallopian tube. 5, 5, Ovaries. 6, 6, Round ligaments. 7, Fimbriated extremity of Fallopian tube. 8, Uterus. 9, Bladder moderately distended. Io, Uterovesical pouch. 4, 5, 6, Are enclosed within a fold of peritoneum which constitutes the broad ligament of the uterus.

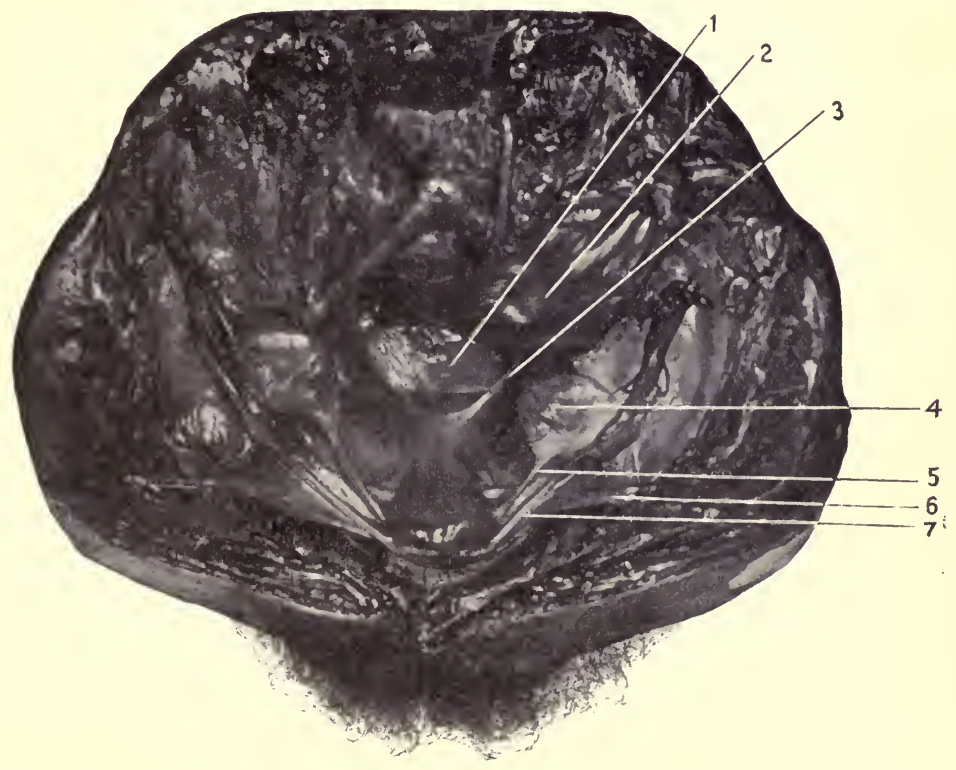

Fig. I11. Female Pelvic Viscera. (Bladder empty.)-I, Douglas' pouch. 2, Rectum. 3, Recto-uterine ligament. 4, Ovary. 5, Ovarian (utero-ovarian) ligament. 6, Round ligament. 7, Fallopian tube. Its fimbriated extremity is very plain. 
riorly, prostate, seminal vessels, urethra, rectovesical fascia, venous plexuses.

The External Trigone. Fig. 84. This is a triangular surface on the posterior wall of the bladder bounded at the apex by the prostate, on the sides by the diverging seminal vesicles and the vas deferens, and above by the reflection of the peritoneum from the posterior wall of the bladder to the front of the rectum. This fold is from one to one and onehalf inches from the prostate and three and one-half inches from the anus.

This surface of the bladder is in close relation to the rectum and only separated from it by some rectovesical fascia. Through this space a trocar might be introduced from the rectum into the bladder, though it is not practiced now.

The Ureter. Figs. 103 to 107, I 12.

This is a small canal (one-sixteenth of an inch in diameter), which extends from the kidney to the bladder, carrying the urine from the former to the latter. Its average length is twelve to sixteen inches. It lies behind the peritoneum and is enclosed in the extraperitoneal tissue.

After crossing the psoas muscle and the iliac vessels it passes to the lateroposterior wall of the bladder, curves downward and inward to enter the substance of the bladder in front of the upper end of the seminal vessels ; after traversing the bladder wall they open upon its inner surface about three-fourths of an inch apart and the same distance posterior to the internal opening of the urethra. In the male the vas deferens passes between the ureter and bladder. In the female, the ureter runs in front of the cervix uteri and upper part of the vagina (between these and the bladder). At the base of the bladder, where the ureters are entering 


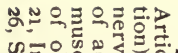

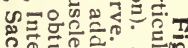

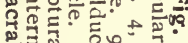

드영유은

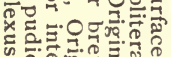

की

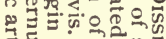

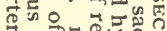

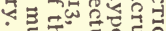

ถึ.

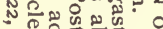

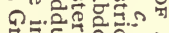

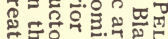

क

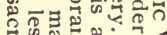

边

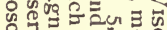

的

ก.

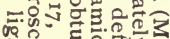

绎

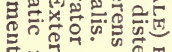

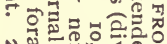

记

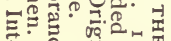

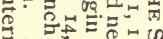

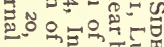

두유

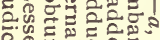

3 억

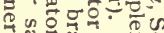

¿

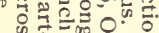

ก

๑두을

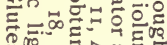

क्ष

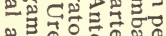

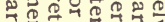

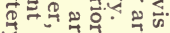

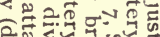

을 3 .

<.르

此出

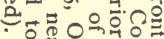

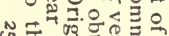

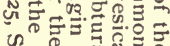
นn

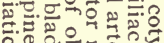
รั

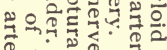

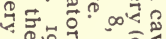
C눈 : 을

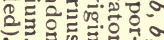

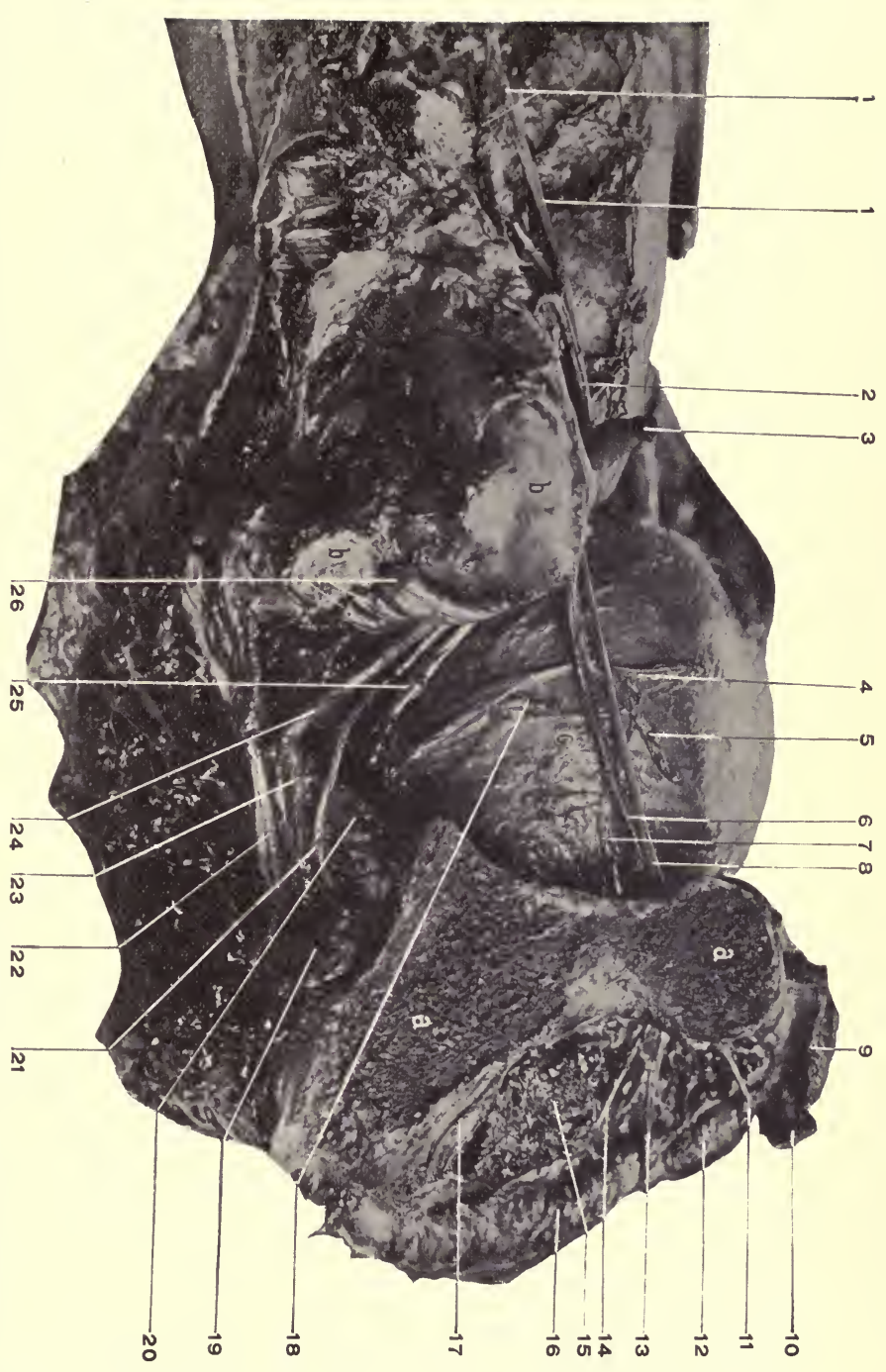


its wall, they lie one and one-half inches posterior to the prostate and two inches apart. Their course through the bladder wall is very oblique, so that they issue only threefourths of an inch posterior to the internal opening of the urethra and the same distance apart.

The Vas Deferens. Figs. 92, 94, I03, I I $2,84$.

One on each side. Conveys the seminal fluid from the testicle to the seminal vesicles. It is about twenty-four inches long and one-sixteenth of an inch in diameter.

Starting from the globus minor of the testicle, it ascends with the structures forming the cord through the external abdominal ring, the inguinal canal, the internal ring, around the deep epigastric artery. It now leaves the other structures of the cord and runs inward across the psoas muscle and external iliac vessels to the side of the bladder. In the abdomen it lies behind the peritoneum for its entire course to the bladder. Descending along the posterior wall of the bladder, it passes in front of the obliterated hypogastric arteries and the ureter (between them and the bladder) to the notch at the base of the prostate. In the last part of its course it is between the bladder and the rectum. In the prostatic notch the vas unites with the seminal vesicle to form a single tube, the ejaculatory duct, which continues through the prostate to open upon the floor of the urethra. The length of the duct is about three-fourths of an inch.

The one and one-half inch of the vas adjacent to the prostate is dilated and receives the name of ampulla. See page 420.

The Uterus. Figs. I IO, I I I.

The uterus is the reproductive organ of the female. It varies in size with the age and condition of the individual. In 
the normal adult it measures about three inches in length, two inches in width at the upper part and one at the lower, and one inch in thickness. It is placed, with its widest dimension transverse, between the bladder and rectum and at the upper end of the vagina, into which it projects. The organ is divided into the body, the upper portion; the isthmus, the middle narrow portion; and the cervix, the lower portion. The cervix is about an inch long; the upper part outside of the vagina is the extravaginal part, the lower half-inch within the vagina, intravaginal. The intravaginal portion of the cervix presents the opening of the cavity of the uterus, or the os uteri.

Peritoneal Reflections. - The peritoneum is reflected from the bladder over the uterus to the rectum. It covers the body of the uterus in front, and behind descends on to the vagina about an inch before it is reflected on to the rectum. The anterior depression between the bladder and the uterus is the uterovesical fossa; the posterior, between the rectum and the vagina and uterus, is the recto-vaginal, or the pouch of Douglas.

The Ligaments of the Uterus. - The peritoneum forms semilunar folds between the sides of the uterus and the bladder in front and the rectum behind; these are the anterior and posterior ligaments of the organ. These folds constitute the lateral boundaries of the anterior and posterior, or uterovesical and rectovaginal pouches.

From the sides of the uterus the peritoneum extends in a broad double fold to the lateral pelvic wall, forming the broad ligaments of the uterus.

In the free margin of each ligament is the Fallopian tube; on the posterior surface, the ovary connected to the uterus by a band about one and one-half inches long (the ligament of the ovary); at about the middle of the 
broad ligament is the ovarian artery and round ligament; at its base the ureter, uterine artery, veins, and nerves.

\section{The Round Ligament. Figs. I IO, I I I.}

Is a fibromuscular flattened cord about five inches long, extending from the upper angle of the uterus outward and forward behind the peritoneum, to cross the iliac vessels, curve around the deep epigastric artery, and leave the abdomen by the internal abdominal ring. After passing through the inguinal canal the round ligament issues from the external ring and becomes lost in the tissues in front of the pubes. It carries with itself into the inguinal canal a tubular process of peritoneum, called the canal of Nuck; this sometimes persists into adult life and forms a sac for an inguinal hernia. The cord receives blood from the ovarian, superior vesical, and deep epigastric arteries.

The Fallopian Tubes. Figs. I IO, I I I.

One on each side, about five inches long, extend outward from the superior angle of the uterus in the free border of the broad ligament. Near the uterus they are very small, gradually enlarge as they pass outward, finally terminate in a tasseled, or fimbriated extremity. It is attached by one of these fimbriæ to the ovary. The opening of the tube into the peritoneum is the only communication that the peritoneal sac has with the exterior of the body.

\section{The Ovary. Figs. I IO, I I I.}

The ovary is a small body one and one-half by threefourths by half an inch, oval in outline, its long axis nearly vertical, situated upon the posterior surface of the broad ligament of the uterus, and connected to the uterus by a band one and one-half inches long that is called the ligament 


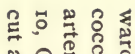

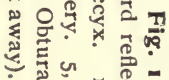

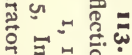

륭

马્ّ

르눈

콜 苟 号

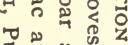

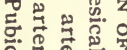

द

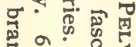

हैं

요손

을 0 혹

茞罚

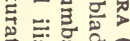

क्ष

\#

贾焉国

द प

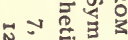

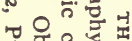

물 焉

o

०ै ๘

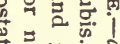

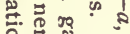

क्ष०

象 $\infty$

邑。

- Oु है

ผ

००ड क

ฏ

要

क द

ㄴ..눈

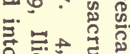

웡

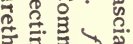

要的?

可要
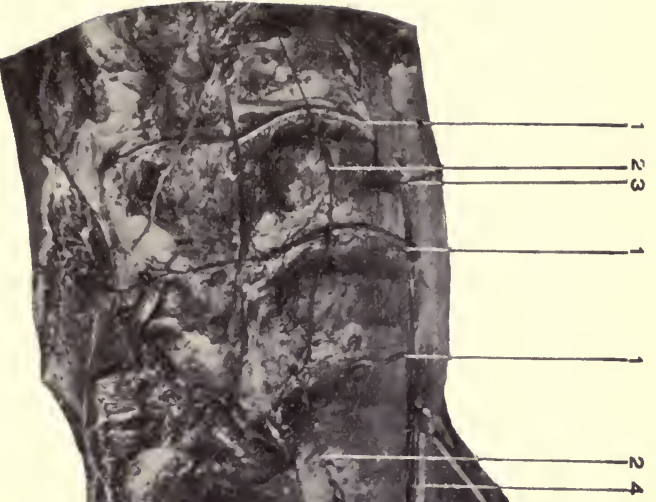
of the ovary, and to the Fallopian tube by one of the fimbrix.

The Veins form a Plexus about the ovary and in the meshes of the broad ligament (the pampiniform plexus). The right ovarian vein opens into the vena cava, the left into the left renal vein.

The Lymphatics.-The distribution of the lymph drained from the female genitals is important as giving a clue to the site of a possible infection. The vulvar lymphatics empty into the inguinal glands. Inflammation of the vulva may cause an enlargement of the inguinal glands constituting, as in the male, a bubo.

The vaginal and uterine lymphatics pass to the pelvic glands, from the round ligament to the inguinal glands, and from the broad ligament to the lumbar glands.

The Nerves are derived from the third and fourth lumbar, hypogastric, and renal sympathetic plexuses.

The Internal Iliac Artery and its Branches. Figs. I I2, I I 3, I I 4 .

The internal iliac is the posterior branch of the common iliac, as the external is the anterior branch. The terms external and internal as applied to these arteries are misnomers, for both arteries are internal, within the abdominal cavity, the proper designation would be as indicated, anterior and posterior.

The internal iliac descends along the pelvic wall to the upper margin of the great sacrosciatic foramen, where it divides into the anterior and posterior trunk.

The anterior trunk gives off the vesical, hemorrhoidal, uterine, vaginal, obturator, sciatic, and pudic branches.

(a) The vesical are three in number, the superior, middle, 
and inferior, which are distributed to the bladder. The superior gives off the deferential artery to the vas deferens (in the female a similar one to the round ligament), and at the bladder the obliterated hypogastric, which in the fotus was pervious. (b) The middle hemorrhoidal. This supplies the middle section of the rectum, anastomosing above with the superior hemorrhoidal from the inferior mesenteric and below with the inferior hemorrhoidal from the internal pudic. (c) Uterine. Supplies the lower section of the uterus; the two arteries form an anastomosis around the neck of the cervix by the circular artery. (d) The vaginal. Four or five small arteries to the side of the vagina. The obturator. It runs forward just below the nerve which it accompanies through the obturator canal. For the terminal distribution, see page 583 . The pelvic branches are small, only one of them being of any size, viz. : the pubic, which anastomoses upon the inner surface of the pubes with the pubic branch of the deep epigastric. Frequently this anastomosis is by larger trunks, or the obturator may rise (one in three and a half cases) from the deep epigastric artery and pass to the obturator canal at the outer side of the femoral opening, or in a few cases (one in ten) the artery may encircle the femoral opening (likewise any protrusion through it, as femoral hernia) to gain the obturator canal around the inner side of the femoral opening. The sciatic artery is the larger terminal branch of the anterior trunk of the internal iliac. It passes downward to leave the pelvis through the great sacrosciatic foramen, below the pyriformis muscle. For its continuation, see page 6r 3. $(g)$ The internal pudic, the smaller terminal branch, passes through the same opening with the sciatic, but above and internal to it. For the rest of the artery, see page 4II. 
The Posterior Division of the Internal Iliac Artery.

(a) The iliolumbar artery. This passes outward and upward beneath the common iliac vessels and psoas magnus muscle, where it divides into two main trunks, the upper and lower, which supply the adjoining parts and anastomose with the last lumbar, gluteal, and deep circumflex iliac arteries. (b) The two lateral sacral pass inward across tine sacrum to anastomose with the sacra media, to supply the surrounding parts, and, penetrating the sacral foramina, to be distributed to the posterior sacral region. (c) The gluteal. This is the direct continuation of the posterior trunk, It leaves the pelvic cavity with the superior gluteal nerve through the great sacrosciatic foramen above the pyriformis muscle. For its distribution, see page 6 2 .

\section{The Internal Iliac Vein}

Is formed at the great sacrosciatic foramen by the junction of the gluteal, sciatic, and internal pudic veins. The venous trunk then ascends and joins the external iliac vein at the sacro-iliac articulation to form the common iliac vein. Besides the above veins the internal iliac receives the lateral sacral, iliolumbar, obturator, prostatic, vesical, hemorrhoidal (vaginal and uterine in the female) veins.

\section{DISSECTION.}

Close to the bladder divide the remaining ligaments, vessels, nerves, ureters, and vasa deferentia passing to it.

Remove the bladder and open it from its apex along its anterior wall to the urethra, and this also for its entire length along the upper surface.

\section{The Openings of the Ureters.}

These openings are three-fourths of an inch behind the internal urinary meatus and the same distance apart.

The beginning of the urethra is called the internal meatus. This opening lies in the median line, three-fourths 


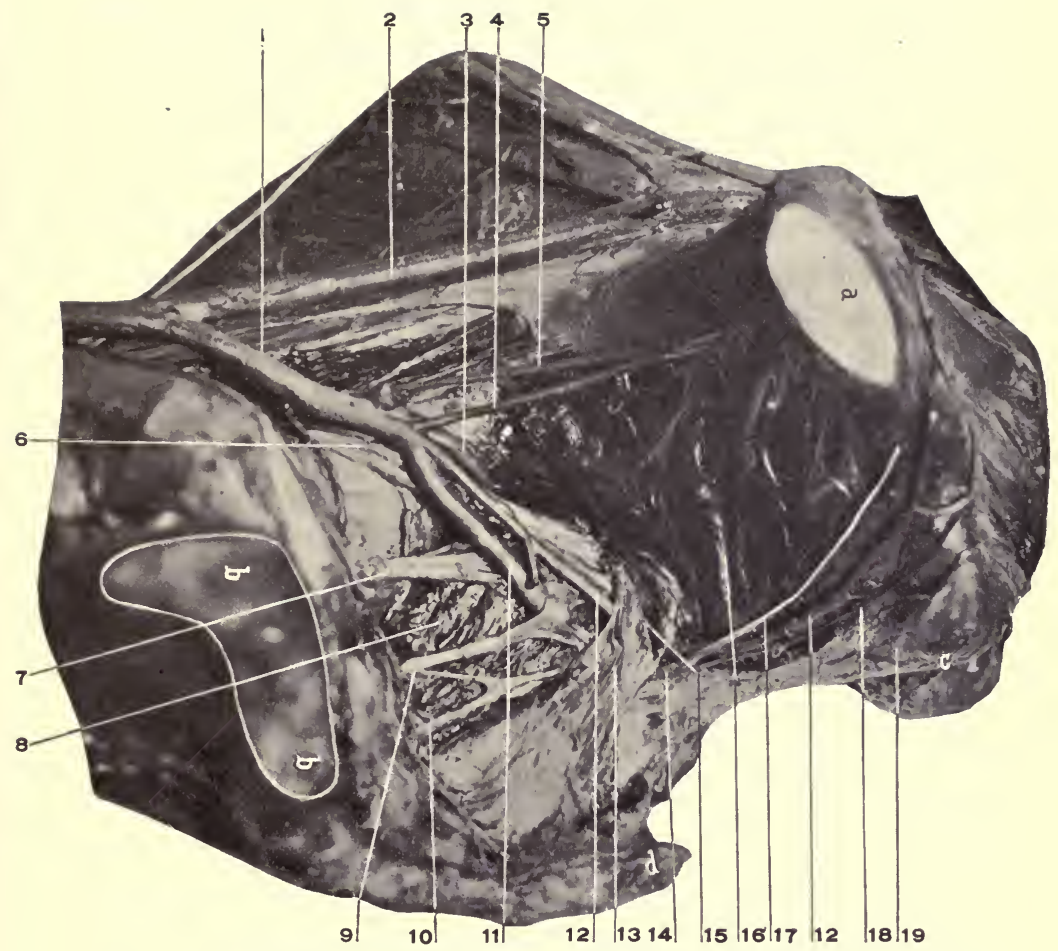

Fig. 114. Dissection of Pelvic Wall (Male) from the Side.- $a$, Symphysis pubis. $b, b$, Articular surface of sacrum. $c$, Tuberosity of ischium. $d$, Tip of coccyx. I, Internal, 2, External iliac artery. 3, Gluteal artery. 4, Obturator artery. 5, Obturator nerve. 6, Lumbosacral cord. 7, First sacral nerve. 8, Pyriformis muscle. 9, Second, 10, Third sacral nerves. 11, Sciatic artery. 12, 12, Internal pudic artery. 13, Coccygeus muscle and small sacrosciatic ligament. 14, Internal pudic nerve. 15, Spine of ischium. 16, Obturator internus muscle. 17, (Deep) dorsal nerve of penis. 18, (Superficial) perineal nerve. 19, Attachment of great sacrosciatic ligament to tuberosity of the ischium. 
of an inch behind the lower margin of the symphysis and two inches above the level of the perineum.

Internal Trigone.-This is the triangular area mapped out by connecting the orifices of the ureters and internal meatus of the urethra by imaginary lines.

\section{The Urethra.}

This is the membranous tube which forms the outlet to the bladder and seminal vesicles. Its length is approximately six inches when the penis is relaxed. Its diameter to the size of the penis is as four to nine (Otis).

It is divided into the prostatic portion (one and onequarter inches long), the membranous portion (one-half to three-fourths of an inch long), and the spongy portion (the remainder of the canal).

The prostatic portion pierces the prostate from base to apex, lying nearer the anterior than the posterior surface of the gland.

On section the canal is $U$-shaped, with the convexity forward and the "legs" backward.

Within the prostatic portion the following structures are found:-

(I) The verumontanum, or colliculus seminalis. This is a vertical elevation along the posterior wall of the urethra, about half an inch long. At its centre is the opening of a blind canal (2), the sinus pocularis, or the uterus masculinus. Embryologically related to the female uterus. Below the opening of the sinus pocularis appear (3) the orifices of the ejaculatory ducts, one on each side of the median line. The depressions along the base of the verumontanum are called (4) the prostatic sinuses, and into them open (5) the ducts of the prostatic glands.

The membranous portion of the urethra is the only fixed 
part of the canal. It is held in place by the two layers of the triangular ligament, between which it extends. It is surrounded by the compressor urethræ muscle, and has the glands of Cowper in close relation to it.

The spongy portion of the urethra. This presents two dilatations: one at its beginning, the pars bulbosa, and one at its termination, the fossa navicularis.

The external meatus is the vertical opening of the urethra in the glans. Along the spongy portion of the tube are several small blind depressions opening forward, called lacunæ. One of these, the lacuna magna, is situated about an inch from the external meatus, and may catch the point of a sound or catheter. The urethra shows on cross section as a transverse slit in the membranous and spongy portions of the urethra, and at the external meatus as a vertical slit.

\section{The Sacral Plexus. Fig. I I4.}

This is formed by the union of the Lumbosacral Cord, (see page 529), and the anterior divisions of the first three sacral nerves to form a broad ribbon at the entrance of the great sacrosciatic foramen. It rests upon the origin of the pyriformis muscle. The fourth sacral nerve enters into the plexus indirectly by sending a branch to join the internal pudic nerve. The fifth sacral nerve has no part in the sacral, but enters into the formation of the coccygeal plexus. The fourth sacral nerve has most of its filaments distributed independently of the sacral plexus, to the skin over the lower internal portion of the gluteus maximus muscle, to the integument of the anus, besides furnishing branches to the external sphincter ani, coccygeus, and levator ani muscles.

The Branches of the Sacral Plexus.

(a) The superior gluteal. This is formed by two 
branches, one from the lumbosacral cord and the other from the first sacral nerve. It passes out of the pelvis with the gluteal artery, through the great sacrosciatic foramen and above the pyriformis muscle, supplies the gluteus medius and minimus, between which it runs forward to terminate in the tensor vaginæ femoris. (b) The inferior gluteal, formed by filaments from the lumbosacral cord, the first and second sacral nerves, leaves by the same foramen as the above, only below the pyriformis muscle (where it is usually contained in the same sheath with the small sciatic nerve, giving rise to the statement that it is a branch of the small sciatic), and terminates in the gluteus maximus muscle. The branch to the pyriformis muscle is from the second and third sacral nerves. (d) The nerve to the quadratus femoris arises from the third sacral nerve, passes through the great sacrosciatic foramen below the pyriformis muscle, descends in front (anterior to) the obturator internus and gemelli muscles to be distributed to the anterior surface of the quadratus femoris muscle. In its passage over the hipjoint it sends a branch to it. (e) The small sciatic nerve comes from the posterior surface of the second and third sacral nerves, makes its exit from the pelvis through the great sacrosciatic foramen below the pyriformis muscle. For the rest of its course, see page 604. ( $f$ ) The nerve to the obturator internus arises from the second and third sacral nerves, goes out through the great sacrosciatic foramen under the pyriformis, re-enters the pelvis through the smaller sacrosciatic foramen, and is distributed to the muscle from which it is named. $(g)$ The internal pudic arises by three roots from the second, third, and fourth sacral nerves, takes the same course as the nerve above until the pelvis is re-entered, it then follows along with the internal pudic artery through the canal (Alcock's) beneath the 
obturator fascia, to supply the structures of the perineum, see page 4I3. (h) The great sciatic nerve. This is the termination of the sacral plexus. It is the largest nerve in the body. From its origin it passes outward through the great sacrosciatic foramen below the pyriformis muscle into the gluteal region; for further course, see page 6ro.

The sacral gangliated cord is the continuation of the lumbar cord from behind the iliac vessels to the central ganglion (impar) over the front of the coccyx; there are four small ganglia usually in the course of the cord, but may be fewer. 


\section{THE LOWER EXTREMITY, Anterior.}

\section{LANDMARKs. Fig. 87, 98, i I 5 .}

The landmarks at the lower part of the abdomen belong also to the lower extremity. They are the symphysis, body, and spine of the pubes, Poupart's ligament, the anterior superior spine and crest of the ilium. Below the crest of the ilium to the outer part of the thigh is the great trochanter. Its upper border is on a level with the centre of the hip-joint.

Nélaton's line is drawn from the anterior superior spine of the ilium to the tuberosity of the ischium, and it crosses normally to the top of the great trochanter.

About the knee the prominent condyles of the femur, the tuberosities of the tibia, the head of the fibula, and the patella should all be examined. Especially should the varying positions of the patella in flexion and extension of the leg be appreciated. The ridge of the tibia along the front of the leg produces the shin, and as the bone is covered by skin and subcutaneous fascia alone, injuries here are liable to produce wounds through them to the bone.

At the ankle the prominent malleoli are recognized. The internal is in front of the external and does not reach so low down. Measurements for difference in the limbs are usually from the anterior superior spines of the ilium to the internal malleoli. See page 434.

At the foot the most prominent bony parts are the head of the astragalus (if the foot is fully extended).

Behind the foot is the os calcis, which can be felt on both sides. On the inside is the prominent tubercle of the scaphoid and head of the first metatarsal bone. The sustentaculum tali of the os calcis lies in front of and one inch below 


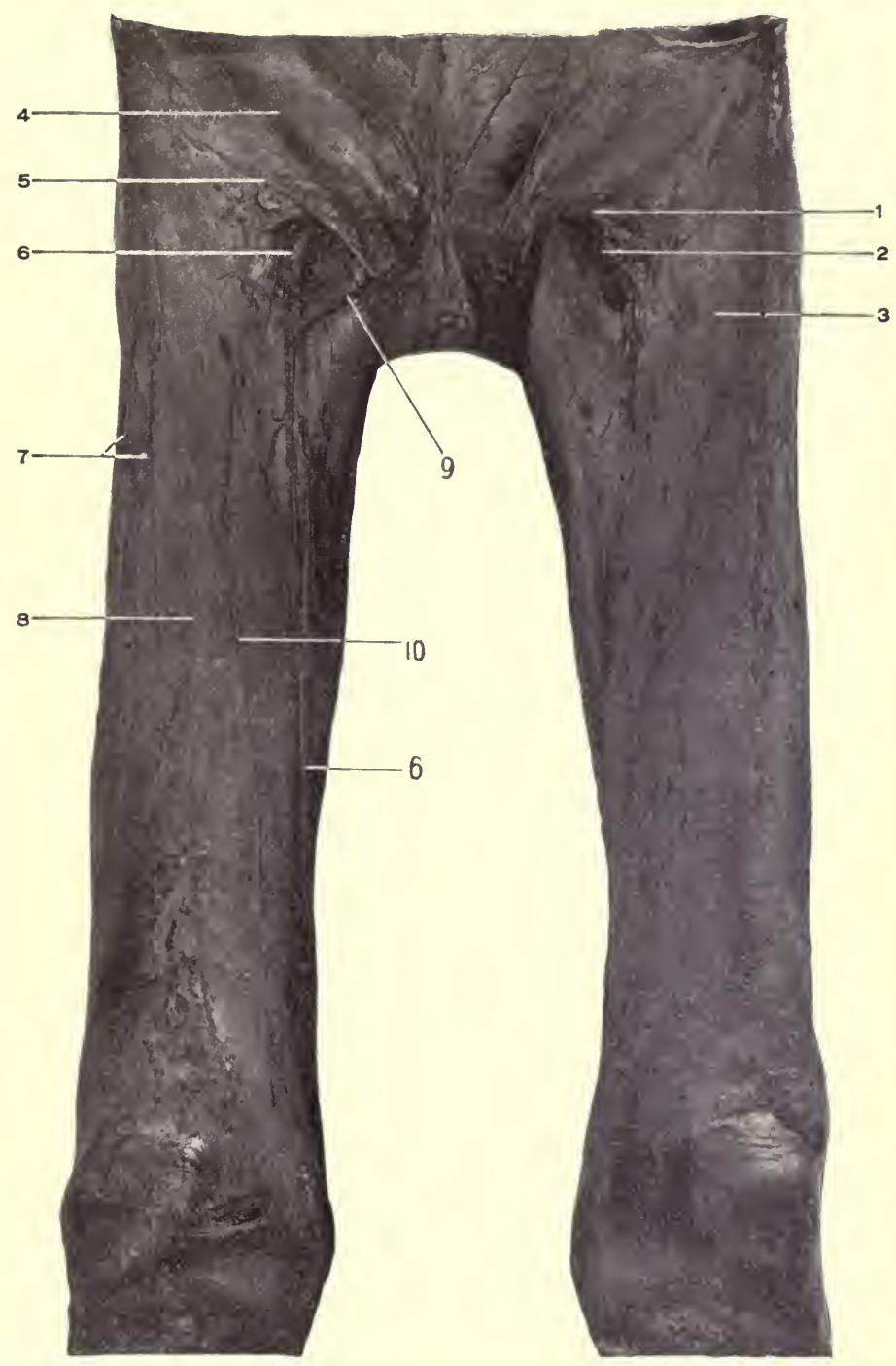

Fig. 115. Dissection of Thigh, ANTERIOR.-I, Falciform process of fascia lata. 2, Saphenous opening. 3. Fascia lata. 4, Aponeurosis of external oblique. 5, Poupart's ligament. 6,6 , Long, or internal, saphenous vein. 7,8 , Cutaneous branches of external cutaneous nerve. 9, Superficial external pudic vein. 10, Middle cutaneous nerve. 
the internal malleolus. The tubercle of the scaphoid is one and one-fourth of an inch in front of the same point. On the outer side of the foot the os calcis, cuboid, and base of the fifth metatarsal bones can be felt.

\section{DISSECTION.}

Incision.-(I) From the middle of Poupart's ligament down the front of the thigh to below the patella.

(2) Two short transverse cuts at the lower end of (I).

Remove the skin only, turning it off laterally as far as possible.

\section{The Superficial Fascia. Fig. I I 5.}

This fascia varies in thickness with the amount of adipose tissue present. It is described as consisting of two layers, the superficial and deep, but this distinction can be readily made only in the upper third of the thigh. Within the superficial fascia are contained the superficial vessels, nerves, and lymphatics.

The superficial layer of the fascia passes up over Poupart's ligament to become continuous with a similar layer of the abdomen, behind with the fascia of the buttocks, and below passes into that covering the leg.

The deep layer is only demonstrated with ease in the upper third of the thigh. It consists of a thickening of the under surface of the superficial fascia and is attached above to the crest of the ilium, Poupart's ligament, spine, crest, and front of the pubes; internally and externally it blends with the fascia lata, centrally it covers over the saphenous opening and is strongly attached to its outer and lower margins and loosely to its inner margin, forming the cribriform fascia.

\section{DISSECTION.}

Trace the superficial arteries, veins, and nerves. Locate the femoral lym. phatics. 
The Superficial Arteries. Figs. 88, 1 I 5.

Are the superficial external pudic. The superficial epigastric. See page 436. The superficial circumflex iliac. See page 572 .

Superficial twigs of the muscular branches of the femoral are seen after penetrating the fascia lata along the front of the thigh.

The superficial branch of the anastomotica magna will be found on the inner side of the knee with the internal saphenous nerve.

The Superficial Veins. Fig. I I 5.

The internal saphenous vein is found at the inner side of the knee as it ascends from the leg. It passes upward to enter the saphenous opening (passing through the cribriform fascia), and empty into the femoral vein. In its course up the thigh it receives the external and internal cutaneous femoral veins, and at the saphenous opening the superficial external pudic, epigastric, and circumflex iliac veins.

\section{The Superficial Nerves. Fig. I I 5.}

These will be demonstrated as the fascia lata is cleaned, though filaments of the main branches will be found at this stage. It is not advisable to delay the dissection to look especially for them. They are branches from the last dorsal, between the crest of the ilium and great trochanter; External Cutaneous; the middle and internal cutaneous from the Anterior Crural; the Genitocrural (see page 532); and the Ilio-inguinal (see page 530).

\section{The Femoral Lymphatics. Fig. I I 5.}

These will be found below the middle of Poupart's ligament and grouped about the saphenous opening. They receive the drainage of the superficial area of the lower 
extremity represented by the distribution of the internal saphenous vein (internal, anterior, and posterior area of lower extremity).

\section{The Prepatellar Bursa}

Will be found over the patella, between it and the deep fascia. A vertical incision will open into the bursa.

\section{DISSECTION.}

Remove all of the superficial fascia, leaving the vessels and nerves lying upon the fascia lata.

Recognize the deep layer of the superficial fascia and its attachments, then remove it carefully and expose the saphenous opening, and vein passing through it. Save the denser, curved outer band and the thinner inner portion of the fascia bounding the saphenous opening.

The Fascia Lata, Anterior Portion. Figs. I I 5, i I6.

This is a strong tube of membrane enclosing the thigh. Attached below to the bony points about the knee-condyles of femur, patella, tuberosities of tibia, head of the fibula. Above, anteriorly, Poupart's ligament, spine, crest, and front of the pubes (iliac portion). The pubic portion is under the iliac portion and is attached to the iliopectineal line, and externally blends with the iliac fascia from the pelvis. (See page 525.) Internally, to the rami of the pubes and ischium. Posteriorly, see page 600.

(a) Intermuscular Septa.-A strong one is found externally between the vastus and the biceps muscles attached to the external lip of the linea aspera. A less distinct septum is found in front of the adductor muscles, and a third one behind them. From the under surface of the fascia lata there also extend off processes to enclose all the muscles of the thigh in separate compartments.

(b) The Iliotibial Band.-This is a reinforcement of the transverse fibres of the fascia lata along the outer side of 


$$
1
$$


the thigh, by vertical fibres extending from the anterior portion of the crest of the ilium to the head of the fibula and external tuberosity of the tibia. Into the upper fourth of this band the tensor vaginæ femoris is inserted.

(c) Internal Process.-An internal process of the fascia lata extends from the notch and inferior anterior iliac spine, along the inner surface of the tensor vaginæ femoris muscle, to join the iliotibial band just below the insertion of the muscle.

(d) The Saphenous Opening.-The confusion in the descriptions of the saphenous opening all arise from the fact that the opening does not exist in the normal condition. It is a product of a femoral hernia or of the dissector. Different dissectors manufacture different sorts of openings, according to their preconceived ideas derived from the descriptions they have read, or as a result of careless dissection, or from the varying conditions of the fascia itself, (for not all fasciæ have the margins of the opening equally plain).

Stated briefly, the saphenous opening is simply a gap produced by the separation of the iliac and pubic portions of the fascia lata, the iliac portion having the external and more superficial attachment (Poupart's ligament, spine of pubes), the pubic portion having the more internal and deep attachment (to the iliopectineal line and iliac fascia behind the femoral vessels). Through this gap passes the internal saphenous vein, superficial external pudic and superficial epigastric arteries, and the genitocrural nerve.

The opening is about one inch below the inner portion of Poupart's ligament, its vertical diameter one and onefourth or one-half inch, its transverse, one-half to threefourths of an inch. These measurements are after dissection. The outer or iliac portion, bounding the opening, 
is a somewhat thickened band extending from the spine of the pubes and the inner part of Poupart's ligament, outward and downward under the saphenous vein to blend with the pubic portion on the inner side of the opening. This curved free border of the iliac portion of the fascia lata is the falciform ligament or process.

There is no band on the inner side of the opening as on the outer, because the sheet of fascia (pubic portion) extends upward and outward, under, behind the femoral vessels, forming an almost level plane of fascia with which the falciform process blends at its lower portion. This pubic portion of the fascia lata helps to form the posterior covering for the femoral vessels. The opening is covered over by the deep layer of the superficial fascia, which is firmly attached to the falciform process (outer and lower margin of the opening) and but slightly to the pubic or inner margin of the fascia lata.

This firm attachment of the cribriform fascia to the outer and lower margin of the opening and its loose attachment to the pubic portion explains (at least partially) why a femoral hernia takes an upward and inward course as soon as it leaves the saphenous opening.

This covering to the saphenous opening is called the cribriform fascia, because it is perforated by the internal saphenous vein, superficial external pudic and superficial epigastric arteries, and (sometimes) the genitocrural nerve.

\section{The External Cutaneous Nerve. Fig. I I 5.}

This will be found as it issues from under Poupart's ligament close to the anterior superior spine of the ilium. Its course is downward along the outer side of the thigh, in a canal formed by the fascia lata, for four or five inches, it then becomes superficial and supplies the integument of the 
anterior and outer surfaces of the thigh nearly to the knee. Just below the spine of the ilium it gives off a branch which turns backward to supply the integument over the great trochanter.

The Internal Cutaneous Nerve. Figs. I I 5, I I 7.

It descends across the femoral artery and divides into two branches: an anterior, which pierces the fascia lata at the middle and lower thirds of the thigh and supplies the integument of the inner anterior surface of the lower third of the thigh.

It descends across the vessels and gives off branches which perforate the fascia lata at various levels to supply the integument of the inner surface of the thigh. The two terminal branches are described as the anterior and posterior branches. The anterior is distributed to the front of the lower third of the thigh and knee, while the posterior branch extends downward to the upper third of the back of the leg.

The Middle Cutaneous Nerve. Figs. I I 5, I I 7.

May exist as a single or double trunk. It (or one of the divisions) usually pierces the sartorius muscle, then the fascia lata at the upper and middle thirds of the thigh, and supplies the integument of the front and inner sides of the thigh to the knee.

\section{DISSECTION.}

Carefully remove the fascia lata, with the superficial vessels and nerves, from the front of the thigh; leaving Poupart's ligament intact above; the iliotibial band on the outside; and exposing the gracilis muscle on the inside.

Sartorius. Fig. I I 7 .

Origin.-From the anterior superior spine of the ilium and the upper part of the notch below. 


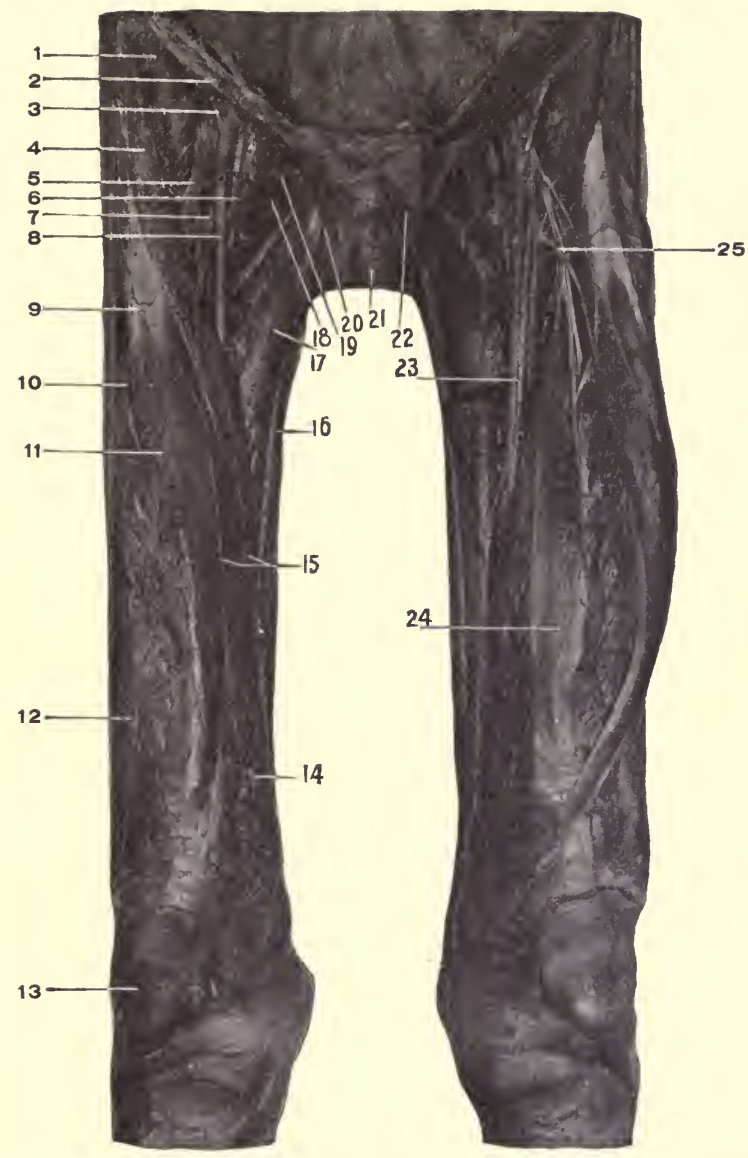

Fig. 117. Dissection of Thigh, Anterior.-I, Iliacus muscle. 2, Poupart's ligament. 3, Deep circumflex iliac artery (femoral artery has been pulled lower than natural). 4, Sartorius muscle. 5, Anterior crural nerve. 6, Femoral vein. 7, The profunda artery. 8, Femoral artery. 9, Rectus femoris muscle. ro, The external cutaneous nerve. II, Middle cutaneous nerve. I2, Vastus externus muscle. I3, Patella. 14, Vastus internus muscle. 15, Internal cutaneous nerves. 16, Gracilis muscle. 17, Adductor longus muscle. 18, Pectineus muscle. 19, Deep external pudic artery. 20, Spermatic cord. 21, Penis. 22, Pyramidalis muscle turned downward. 23, Long, or internal, saphenous nerve crossing the femoral artery. 24, Crureus muscle. 25 , The external circumflex artery. 
Insertion.-Into the front of the tibia just internal to the tubercle by an inverted $\mathrm{J}$-shaped tendon, the curve of the $\mathrm{J}$ hooking over the tendons of the gracilis and semitendinosus muscles. Also, into the deep fascia over the lower part of the knee-joint and upper part of the leg.

Nerve Supply.-The anterior crural, through its middle cutaneous branch, which pierces the muscle at its upper and middle thirds.

Actions.-To flex the thigh on the abdomen, to flex the leg on the thigh, to rotate the thigh outward, to rotate the leg (when semiflexed) inward. It has a slight action of abduction, but no action tending to cross (adduct) the thighs. Acting from below it will flex the pelvis on the femur, and slightly rotate it toward the opposite side.

\section{Tensor Vaginæ Femoris. Figs. I17, II 8 .}

Origin.-From the anterior one inch of the outer lip of the crest of the ilium, the upper portion of the notch below, and from the fascia covering the gluteus medius (a portion of the fascia lata).

Insertion.-Into the inner surface of the fascia lata at its upper fourth, and by the continuation of the fascia, under the name of the iliotibial band, into the head of the fibula and external tuberosity of the tibia. The muscle at its insertion is found between two laminæ of the fascia lata. An external part of the fascial covering of the thigh, which reaches up to the crest of the ilium ; and an internal layer, which passes up to the anterior inferior spine of the ilium and the tendons of the rectus muscle.

Nerve Supply.-The superior gluteal, which comes forward between the gluteus medius and minimus, and enters the posterior surface of the muscle.

Action.-To tense the fascia lata, to flex the thigh, to 
abduct the thigh, to rotate the thigh inward; through the iliotibial band, to flex the leg, to rotate the leg outward, when flexed.

Acting from below it preserves the horizontal position of the pelvis upon the femur (in this connection acting with the gluteus maximus), and rotates it to the same side.

Rectus Femoris. Fig. II 7 .

Origin.-By the straight (anterior) tendon from the anterior inferior spine of the ilium, by the reflected (posterior) tendon from the outer surface of the ilium just above the acetabulum.

Insertion.-Into the upper border of the patella.

Nerve Supply.-The anterior crural.

Action.-To extend the leg on the thigh, or vice versa; to flex the thigh on the abdomen or the reverse.

Pectineus. Figs. I I 7, I 20.

Origin.-From the iliopectineal line and the triangular surface of bone in front of it, between the iliopectineal (pubal) eminence and the spine of the pubes. From the under inner surface of the fascia lata covering the muscle.

Insertion.-Into a line extending from the back of the lesser trochanter to the linea aspera of the femur.

Nerve Supply.-The obturator, anterior crural (constant), and the accessory obturator (when present).

Action.-To flex the thigh, to adduct the thigh, to rotate the thigh slightly externally when it is partially flexed, but if the thigh is kept extended it will rotate it internally in the same way that the iliopsoas muscle does. The explanation is this : the centre of motion is at the hip-joint ; the muscular attachments are to the shaft of the femur two inches external to the centre of motion; flexion being resisted the 
great trochanter will be raised; this produces internal rotation of the shaft of the femur. The iliopsoas and pectineus have this action.

Adductor Longus. Figs. II 7 , II 9.

Origin.-From the front of the body of the pubes just under the crest.

Insertion.-At the middle third of the femur, into the inner lip of the linea aspera.

Nerve Supply.-The obturator nerve through its anterior division.

Action.-To adduct, flex, and rotate the femur outward. If the femur is the fixed point, it will flex the pelvis and rotate it to the same side.

Gracilis. Figs. I I 7, I 9 .

Origin.-From the inner margin of the rami of the pubes and ischium, and from the lower half of the symphysis pubis.

Insertion.-By a long slender tendon into the inner surface of the tibia in the angle formed by the tendon of the sartorius.

Nerve Supply. - A branch from the anterior division of the obturator nerve.

Action.-To adduct the thigh, to flex the leg; when the leg is flexed to rotate it internally.

The Internal Saphenous Nerve. Figs. I I 7, I 19.

The longest branch of the anterior crural nerve. Beginning just below Poupart's ligament where the anterior crural nerve breaks up into its terminal branches, the internal or long saphenous nerve descends along the outer side of the femoral artery through Scarpa's triangle, then enters Hunter's canal with the femoral vessels. In the canal the 


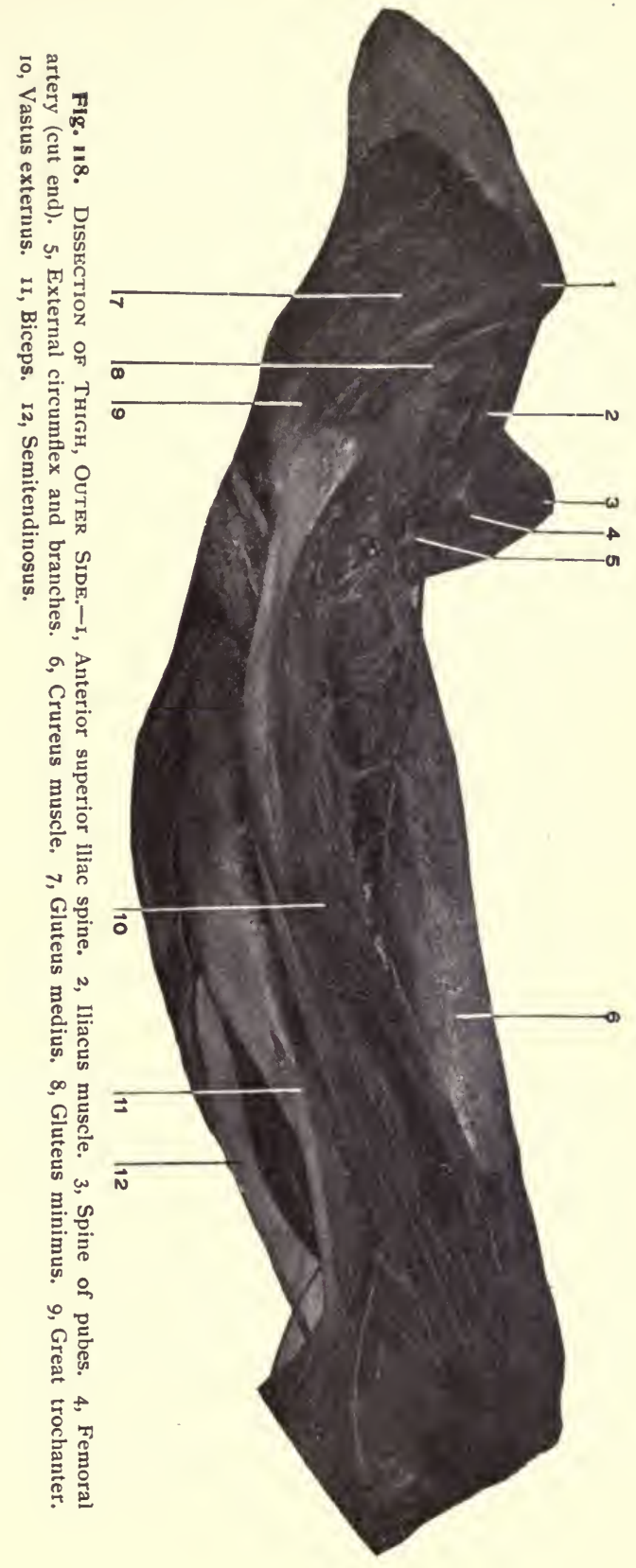


nerve crosses over the front of the artery from its outer to its inner side. Leaving the femoral artery, when it turns backward through the opening in the adductor magnus, the nerve continues downward between the tendons of the sartorius (in front) and the gracilis (behind) and becomes superficial just below the inner side of the knee joint. Here it joins the internal saphenous vein, which it accompanies along the inner side of the leg to the ankle, and terminates a short distance below the internal malleolus.

While under cover of the sartorius muscle the internal saphenous nerve gives off the patellar branch which pierces the sartorius and runs downward and forward to the front of the knee. It supplies the integument over the inner side of the knee, leg, and ankle.

The Nerve to the Pectineus Muscle.

This is a small but constant branch given off from the anterior crural just under Poupart's ligament. It passes downward and inward behind the sheath of the femoral vessels, with which it is closely united to the front of the pectineus muscle.

The Muscular Branches of the Anterior Crural. Figs. I I 7, I I9, I 20.

They are distributed to the sartorius (usually from the middle cutaneous), rectus femoris, vastus externus and internus, crureus, and pectineus muscles. The hip joint receives a branch from the nerve to the rectus. The articular branches to the knee joint are filaments from the branches to the rectus, vasti, and crureus muscles, which passing onward through the muscles terminate in the upper part of the capsule and synovial membrane of the knee joint.

Scarpa's Triangle. Fig. I I7.

This is a triangular space located upon the front of the 
upper part of the thigh. It is formed by the sartorius muscle upon the outside, the adductor longus on the inside, and Poupart's ligament above, which forms the base of the triangle.

The "floor" of the space is formed from without inward by the lower portion of the iliacus, the tendon of the psoas magnus, the pectineus, the adductor longus, and (in thin people) the adductor brevis muscles. The triangle is crossed from the middle of the base to the apex at the junction of the sartorius and adductor longus by the femoral vessels and the anterior crural nerve and its branches.

The relations of these structures under Poupart's ligament is nerve, artery, and vein from without inward.

Internal to the vein is the femoral opening or canal. See below, also page 525 .

At the apex of the triangle the vein turns backward so as to lie nearly behind the artery, while the branches of the nerve are to the outside of and in front of the vessels.

The triangle is covered by the fascia lata, the two layers' of the superficial fascia, and the integument.

\section{The Femoral Canal}

Is the space left within the femoral sheath (at the inside of the femoral vein, between the vein and Gimbernat's ligament and the conjoined tendon). There is no canal unless made by the descent of a femoral hernia or by the dissector. When a femoral hernia develops it leaves the abdomen through the femoral opening at the inner side of the terminus of the external iliac vein, descends along the inside of the femoral vein, and makes its appearance through the saphenous opening. 
The Femoral Artery. Figs. I 7 to I 20.

This is that portion of the great arterial trunk to the lower extremity which extends from beneath Poupart's ligament to the opening in the adductor magnus muscle. It is a continuation of the external iliac above, and is continued as the popliteal below.

The course of the artery is indicated by a line drawn from the mid-point between the anterior superior iliac spine and the symphysis pubis to the adductor tubercle, the thigh being slightly abducted, flexed, and rotated outward.

The femoral artery passes through Scarpa's triangle from the middle of its base to its apex, and through Hunter's canal.

Its relations will be considered in these localities.

Relations of the Femoral Artery.-Scarpa's triangle. See above. Here the artery is centrally placed between the femoral vein on the inside and the anterior crural nerve and its branches on the outside.

The vein as it descends passes to a deeper level than the artery, so that at the apex of the triangle it comes to occupy a position behind and internal to the artery.

The internal cutaneous branch of the anterior crural nerve crosses over the front of the artery in the lower part of the triangle, while the internal saphenous nerve continues along the outer side of the vessel.

In this part of its course the artery rests at its upper part upon the tendon of the psoas magnus (which separates it from the pelvis and the capsule of the hip-joint) the nerve to the pectineus and that muscle itself. At its lower portion, upon the profunda artery and vein (which intervene between the femoral and the pectineus muscle), and the adductor longus. In thin people a small portion of the 


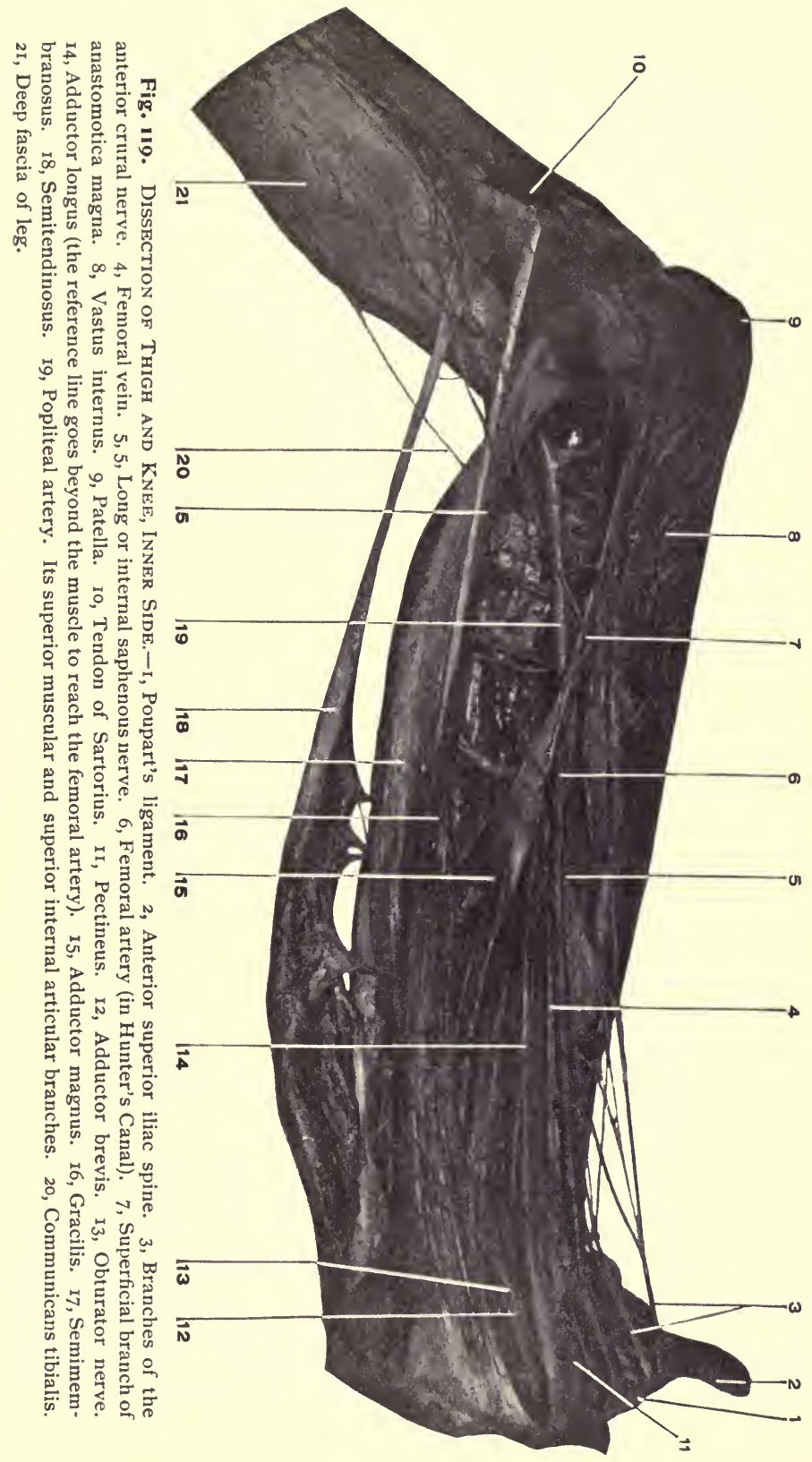


adductor brevis muscle appears between the pectineus and adductor longus.

The artery is covered by the integument, superficial fascia, fascia lata, and is crossed by some small cutaneous nerve filaments and veins.

Hunter's Canal.-See page 575. In its passage through this triangular shaped canal the femoral artery lies between the vastus internus on the outside, the adductor longus and magnus on the inside, and beneath the aponeurotic membrane which unites these muscles. The femoral vein accompanies the artery, being placed behind and internal to the artery at the upper part of the canal, and behind and slightly external to the artery at the opening in the adductor magnus.

The internal saphenous nerve also passes through the canal with the artery and crosses it from the outside to the inside; it leaves the artery at the lower part of the canal by passing in front of the tendon of the adductor magnus muscle, while the artery passes through the opening in the same. In front of the artery (in addition to the aponeurotic covering and the internal saphenous nerve) are the sartorius muscle, the fasciæ, and integument.

The Branches of the Femoral Artery.

(1) The superficial epigastric. See page 436. This is a small branch from the front of the femoral just below Poupart's ligament. It pierces the fascia lata, then turns upward and inward on to the abdomen to ramify in the subcutaneous tissue as high as the umbilicus.

(2) The superficial circumflex iliac.-This is another small branch from the superficial epigastric, or from the femoral close to it. It passes through the fascia lata, runs outward just below Poupart's ligament and breaks up into branches which supply the superficial tissues about the 
crest of the ilium. In its course it gives off twigs to the iliacus, sartorius muscles, and the inguinal lymphatic glands.

(3) The superficial (superior) external pudic arises from the inner side of the femoral, passes through the fascia lata or the saphenous opening, extends upward and inward to the lower part of the abdomen, where it meets its fellow from the other side. As it is opposite the side of the root of the penis it gives off the superficial dorsal artery to that organ.

(4) The deep (inferior) external pudic.-A branch in common with the above, or just below it, from the inner side of the femoral. It takes a course inward across the pectineus and adductor longus muscles (to which it gives muscular branches) passes through the fascia lata at the inner border of the adductor longus, breaks up into branches which supply the scrotum (labium in the female) and the inner side of the thigh.

(5) The deep femoral. See page 579.

(6) The anastomotica magna.-This artery is given off from the femoral just before it turns backward through the femoral opening, at the lower end of Hunter's canal.

It divides into two branches, the superficial and deep. The former takes a superficial course along with the long or internal saphenous nerve to the inner side of the knee, while the latter, the deep branch, takes a deeper course through the fibres of the vastus internus muscle in front of the tendon of the adductor magnus to the deeper parts about the knee. Both of these arteries enter into an anastomosis with the internal articular branches from the popliteal; the superficial with the inferior, and the deep with the superior, internal articular branches.

(7) The muscular branches.-These are small branches, 
varying in number, to the muscles in relation to the femoral artery in its course.

\section{The Femoral Vein.}

The femoral vein begins at the opening in the adductor magnus, where it is the continuation of the popliteal vein, and ends under Poupart's ligament, beyond which point the venous trunk is continued as the external iliac vein. The relations of the femoral vein have been sufficiently given with those of the artery, q. $\%$, page 570.

The Tributaries.-(I) The venæ comites of the anastomotica magna artery. (2) The venæ comites of the muscular branches. (3) The deep femoral vein. The deep femoral vein retraces the course of the profunda artery, receiving as it does so the venæ comites corresponding to the branches of the profunda femoris artery, and finally empties into the femoral vein about an inch and a half below Poupart's ligament. In its course the vein lies at the inside and in front of the profunda artery. (4) The long or internal saphenous vein. This large subcutaneous vein empties into the femoral opposite the saphenous opening. See page 557. The femoral vein contains several valves.

The anterior crural nerve. See Lumbar Plexus, page 532.

\section{DISSECTION.}

Divide the iliotibial band just below the tensor muscle and turn the latter upward; at the same time look for its nerve supply.

Divide the sartorius, gracilis, and rectus at their middle and reflect their ends, saving their nerves.

Incise the fascia stretching across the front of Hunter's canal, and expose the femoral artery, vein, and internal saphenous nerve.

Divide the adductor longus at its middle and lower thirds (below where the nerve enters it). Draw the pectineus upward, dividing it through the middle if necessary. Cut through the iliacus under Poupart's ligament and turn the lower part downward. Divide the femoral artery below the origin of the profunda. 
Hunter's Canal. Figs. I I9, I 20.

This so-called canal is no "canal " at all until its contents are removed. It may be described as the angular space between the insertions of the vastus internus on the outside and the adductor longus and magnus muscles on the inside, and reaching from the opening of the adductor magnus upward to the apex of Scarpa's triangle. This angular interval is "roofed" over by an aponeurotic membrane extending between the vastus internus and the adductor longus and magnus muscles, and through it passes the femoral artery, vein, and long saphenous nerve, the situation of these structures being from before backward, nerve, artery, and vein.

Vastus Externus. Figs. I I 8, I 2 I.

Origin.-From the upper half of the anterior intertrochanteric line, from the front of the great trochanter, from the horizontal line which marks the base of the great trochanter, from the outer part of the gluteal ridge (insertion of the gluteus maximus), from the whole length of the outer lip of the linea aspera.

Insertion.-Into the upper and outer margins of the patella, and by a strong aponeurosis into the external tuberosity of the tibia and the deep fascia of the leg.

Nerve Supply. - The anterior crural.

Action.-To extend the leg, the outward pull of the muscle owing to the obliquity of its lower fibres, resists a corresponding internal traction of the vastus internus.

Vastus Internus and Crureus. Figs. I I 7, I I9, I 20.

Origin.-From the front and sides of the femur for its upper two-thirds (crureus portion), from the lower half of the anterior intertrochanteric line, and its continuation (spiral line) to the linea aspera, from the whole length of 
the inner up of the linea aspera, from the internal intermuscular septum and the tendon of the adductor magnus (vastus portion).

Insertion.-Into the upper border of the patella behind the rectus femoris tendon (the crureus portion), into the inner margin of the patella, and by an aponeurotic expansion into the internal tuberosity of the tibia and the deep fascia of the leg (vastus portion).

Nerve Supply.-The anterior crural.

Action.-To extend the leg; the vastus internus through its lower fibres resists the outward pull of the vastus externus.

\section{The Ligamentum Patellæ.}

This is the continuation of the tendons of the four muscles described as attached to the patella, namely the rectus femoris, the vastus externus, the vastus internus and crureus, which having, in common, the action of extension of the leg are known as the quadriceps extensor femoris. The ligamentum patellæ extends from the apex and lower margins of the patella to the lower portion of the tibial tubercle. It is about an inch wide above, and three-fourths. of an inch below, one-fourth of an inch thick, and from two to three inches in length.

The patella should be regarded as a sesamoid bone developed within the tendon of the quadriceps extensor; its presence renders the action of those muscles much more effective than they would be without it.

Adductor Brevis. Figs. I I9, I 20.

Origin.-From the front of the body and descending ramus of the pubes below the adductor longus and between the gracilis and the obturator externus. 


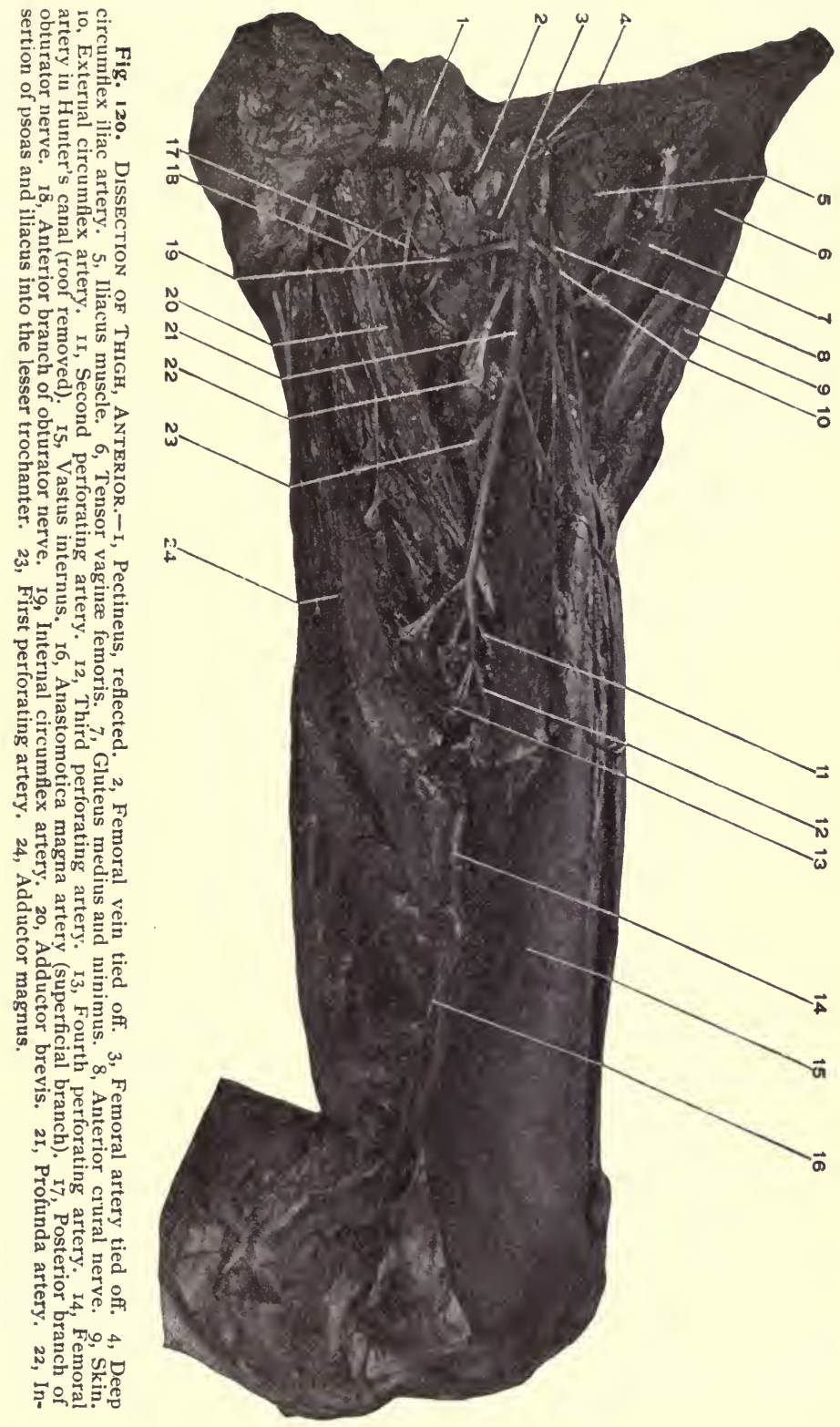


Insertion.-At the upper third of the shaft of the femur, into the inner lip of the linea aspera and the line extending upward to the lesser trochanter.

Nerve Supply.-From the anterior division of the obturator nerve.

Action.-To adduct and rotate the thigh outward; also, a slight flexor of the thigh.

Adductor Magnus. Figs. I 19, I 20.

Origin.-From the front of the rami of the pubes and ischium, and from the lower part of the tuberosity of the ischium.

Insertion.-Into the back of the femur internal to the insertion of the gluteus maximus, along the whole length of the linea aspera between the adductor brevis, adductor longus, and vastus internus on the inside, and the short head of the biceps on the outside, into the internal condyloid ridge and ending in a strong tendon, which is attached to the adductor tubercle on the internal condyle of the femur.

Nerve Supply.-The obturator through its posterior division, and the great sciatic which gives a branch to the posterior surface of the muscle.

Action.-To adduct the thigh, to rotate it outward (the upper part of the muscle); and to extend the thigh (through the fibres arising from the ischial tuberosity).

The femoral opening in the adductor magnus muscle.This is a gap that is left along the insertion of the muscle at the middle and lower thirds of the thigh through which the femoral artery and vein pass to enter the popliteal space.

Besides the opening for the femoral artery and vein the adductor magnus muscle is perforated along its insertion close to the bone by the branches (three) of the profunda which pass to the back of the thigh. 
The Deep Femoral-Profunda Femoris. Figs. I I 7, I 8 , I I9, I 20.

This is the largest and most important branch of the femoral. It arises from the outer and back part of the femoral at a point varying from one to two inches from Poupart's ligament, the average distance being about one and one-half inches. Its course is downward and inward behind the femoral artery and vein, then behind the adductor longus muscle, behind which it continues to just above the femoral opening in the adductor magnus, when it perforates the latter close to its insertion and enters the popliteal region as the last perforating artery.

In its course it lies upon the iliacus, pectineus, adductor brevis and magnus muscles. The vastus internus is at the outer side of the artery.

The Branches of the Profunda.

(I) The External Circumflex.-It arises from the outer side of the profunda near the latter's origin. It passes outward under the sartorius and rectus femoris muscles, interlacing with the branches of the anterior crural nerve, and at the outer side of the thigh divides into the ascending, transverse, and descending branches. (a) The ascending branch (or branches) takes an upward course in the interval between the tensor vaginæ femoris, sartorius, rectus femoris, gluteus medius and minimus, and iliacus muscles. It supplies all these muscles, also, the hip joint, and anastomoses with branches from the superior gluteal and deep circumflex iliac arteries. (b) The transverse branch (one to three) winds outward around the femur, under the vastus externus, to anastomose at the back of the thigh with the first perforating of the profunda, the sciatic, and internal circumflex arteries. (See page 614.) (c) Descending branches: These are large ones which turn 
downward to supply the front and outer side of the muscles of the thigh. A long branch reaches to the outer side of the knee and anastomoses with the superior external articular branch from the popliteal. Other branches in front extend to the knee joint and at the inner side anastomose with the deep branch of the anastomotica magna.

(2) The Internal Circumflex arises from the inner and back part of the profunda artery near its origin. It passes backward between the tendon of the psoas and pectineus, then between the adductor brevis and the obturator externus, and continues between the quadratus femoris and the adductor magnus to the back of the thigh, where it anastomoses with the sciatic, external cutaneous, and the first perforating artery of the profunda, thus forming the "crucial anastomosis." In its course the internal circumflex supplies the muscles adjacent to it, the hip joint, anastomoses with the obturator artery, and gives off a branch of considerable size which follows the tendon of the obturator externus muscle to the back of the hip joint (above and behind the quadratus femoris) to anastomose with the sciatic, and gluteal arteries.

(3) The Perforating Arteries.-There are three of these given off from the profunda, and counting the termination of that vessel itself, makes four. The three perforating are branches from the profunda as it lies behind the adductor longus muscle. They all pass through the adductor magnus (the two upper ones in addition piercing the adductor brevis) by means of aponeurotic openings close to the bone, and appear upon the back of the adductor magnus where they form a chain of anastomoses with each other. In addition the first artery anastomoses with the sciatic, internal circumflex and the external circumflex, the third perforating with the termination of the profunda, 


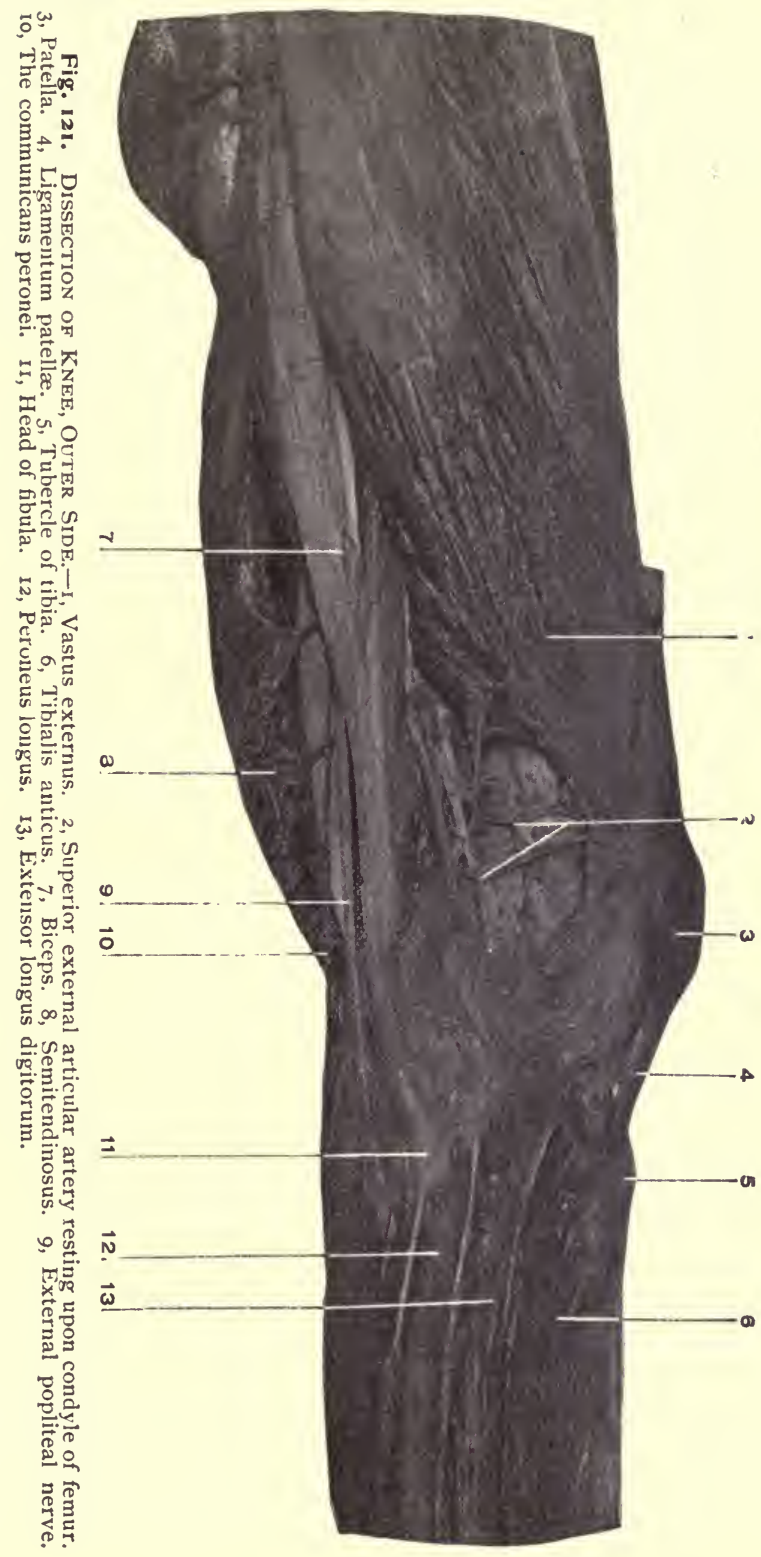


which is called the fourth perforating artery. It passes through a similar gap in the adductor magnus close to its insertion and just above the opening for the femoral artery, to the back of the adductor magnus, where it forms an anastomosis with the third perforating, and the superior muscular and articular branches of the popliteal. All the perforating arteries supply the muscles near them.

\section{Subcrureus.}

The innermost fibres of the crureus which pass to the synovial membrane of the knee joint. Its function is to keep the synovial membrane raised up out of the way of the patella.

Obturator Externus. Fig. I 20.

Origin.-From the inner portion of the external surface of the obturator membrane, and from the adjacent portions of the rami of the pubes and ischium.

Insertion.-Into the digital fossa of the great trochanter.

Nerve Supply.-Posterior division of the obturator nerve.

Action.-To adduct the thigh, to rotate the thigh outward, to flex the thigh. This is the muscle which should have been called the "sartorius" as it is the muscle which crosses the legs.

The Obturator Nerve. Figs. I 19, I 20.

For its formation, and course through the pelvis, see page 534 .

The nerve emerges into the thigh through the obturator gap in the obturator membrane along with the obturator artery. It divides into two branches, an anterior and a posterior. The anterior branch passes over the upper margin of the obturator externus, over the adductor brevis, and under the pectineus and adductor longus muscles. 
The anterior branch supplies the pectineus, adductor brevis and longus, and the gracilis muscles, the hip joint, and the integument at the middle of the inner side of the thigh.

The posterior branch descends through the obturator externus muscle, then between the adductor brevis and magnus, and is continued as a long, slender filament through the lower part of the last muscle into the popliteal space, through which it descends on the anterior surface of the popliteal vessels to the posterior ligament of the knee joint.

The posterior branch supplies the obturator externus, and adductor magnus muscles, the hip and knee joints.

\section{The Accessory Obturator Nerve. Fig. I07.}

For the formation, course, and distribution of this nerve, see page 536 .

The Obturator Artery. Figs. I 12, I I4, I 20.

This is a branch from the anterior division of the internal iliac artery. For its course and branches within the pelvis, see page 547 .

The artery appears with the nerve at the upper margin of the obturator externus muscle, which it perforates, and divides into an internal and an external branch. These course along the internal and external margins of the obturator foramen respectively, anastomose with each other at the lower border of the foramen and supply branches to the adjacent muscles. In addition the internal branch anastomoses with the internal circumflex, and the external branch passes outward below the hip (which it supplies) and terminates in small branches which anastomose with the terminal branches of the sciatic artery. 


\section{DISSECTION.}

Incisions. - (I) Continue the incision down the front of the leg and foot to toes.

(2) Make liberating tıansverse cuts at the ankle and one across the base of the toes.

(3) Carry incisions along the top of the toes.

Remove the integument from the leg, foot, and toes.

Leave the superficial veins and nerves lying upon the deep fascia. In removing the skin from the toes look out for and save the digital nerves.

\section{The Superficial Fascia.}

The superficial fascia of the leg and foot is continuous with that of the thigh. It varies in thickness but is always thin over the antero-internal surface of the tibia, the malleoli, and the dorsum of the foot.

\section{The Deep Fascia. Fig. I 22.}

The deep fascia continues the fascia lata from the thigh, being directly continuous with it in the popliteal space and indirectly about the front of the knee where the deep plane of fascia is attached to the tuberosities of the tibia and the head of the fibula.

In front, the fascia is continuous with the periosteum along the margins of the antero-internal surface of the tibia. At the outer side of the leg, the fascia is fastened to the fibula by two intermuscular septa which enclose the peroneus longus and brevis. At the ankle, the deep fascia is firmly attached to the bony points about the joint and passes into the deep fascia covering the dorsum and sole of the foot. In the popliteal space and about the ankle, the fascia is reinforced by transverse fibres to which, in the latter instance, the names of annular ligaments are given.

The Anterior Annular Ligament. Fig. I 23.

(I) The upper portion: This is a band about two inches 
wide that passes from the front of the tibia to the front of the fibula just above the ankle joint. It contains one synovial sheath for the tendon of the tibialis anticus. The tendons of the other muscles pass under it without any synovial investment.

(2) The lower portion. This band lies just below the level of the joint, and is arranged like a "horizontal " $<$. The single limb being external and attached to the upper anterior surface of the calcis.

The single limb is split for the passage of the tendons of the extensor longus digitorum and the peroneus tertius, and is lined by synovial membrane common for these tendons.

The upper "leg" of the $<$ is attached to the internal malleolus, passing over the tendon of the extensor proprius hallucis and mostly under the tendon of the tibialis anticus, a thin investment covering the tendon of the latter muscle.

The tendon of the former muscle has a separate synovial sheath under this portion of the ligament. The lower "leg" of the $<$ passes over the tendons of the extensor proprius hallucis and tibialis anticus to the inner side of the foot where it blends with the plantar fascia.

The synovial sheath of the tibialis anticus is prolonged from the upper portion, down under this part of the annular ligament.

The External Annular Ligament. Fig. 123.

This is that portion of the deep fascia of the leg and foot, which extends from the external malleolus to the outer posterior and lower borders of the os calcis. It retains the tendons of the peroneus longus and brevis in place behind the external malleolus. The tendons are surrounded by a common synovial sheath. 
The Internal Annular Ligament. Fig. I3 I.

This is the portion of the deep fascia reaching from the internal malleolus to the inner lower and posterior borders of the os calcis.

For the relations of the important structures which pass under this ligament, see page 632 .

\section{The External (Short) Saphenous Vein.}

This begins at the outer side of the venous plexus found upon the dorsum of the foot. It passes backward along the outer margin of the foot, below the external malleolus, then upward over the back of the leg, perforates the deep fascia of the leg at the lower part of the popliteal space and empties into the popliteal vein.

(For the dissection of the vein at the back of the leg, see page 6i 8 .)

The vein receives its tributaries from the outer side of the foot, outer and back parts of the leg, and a large vein from the lower part of the thigh just before it perforates the deep fascia.

\section{The Internal (Long) Saphenous Vein.}

This emerges from the inner side of the dorsal venous arch of the foot. Its course is upward in front of the internal malleolus, along the antero-internal border of the leg to the inner side of the knee, where it lies behind the internal condyle of the femur. Its further course and tributaries are given on page 557 .

The internal saphenous receives the subcutaneous veins from the inner side of the foot and leg.

Both saphenous veins communicate with the venæ comites of the deep parts of the leg by veins which perforate the deep fascia at irregular intervals. They also communi- 


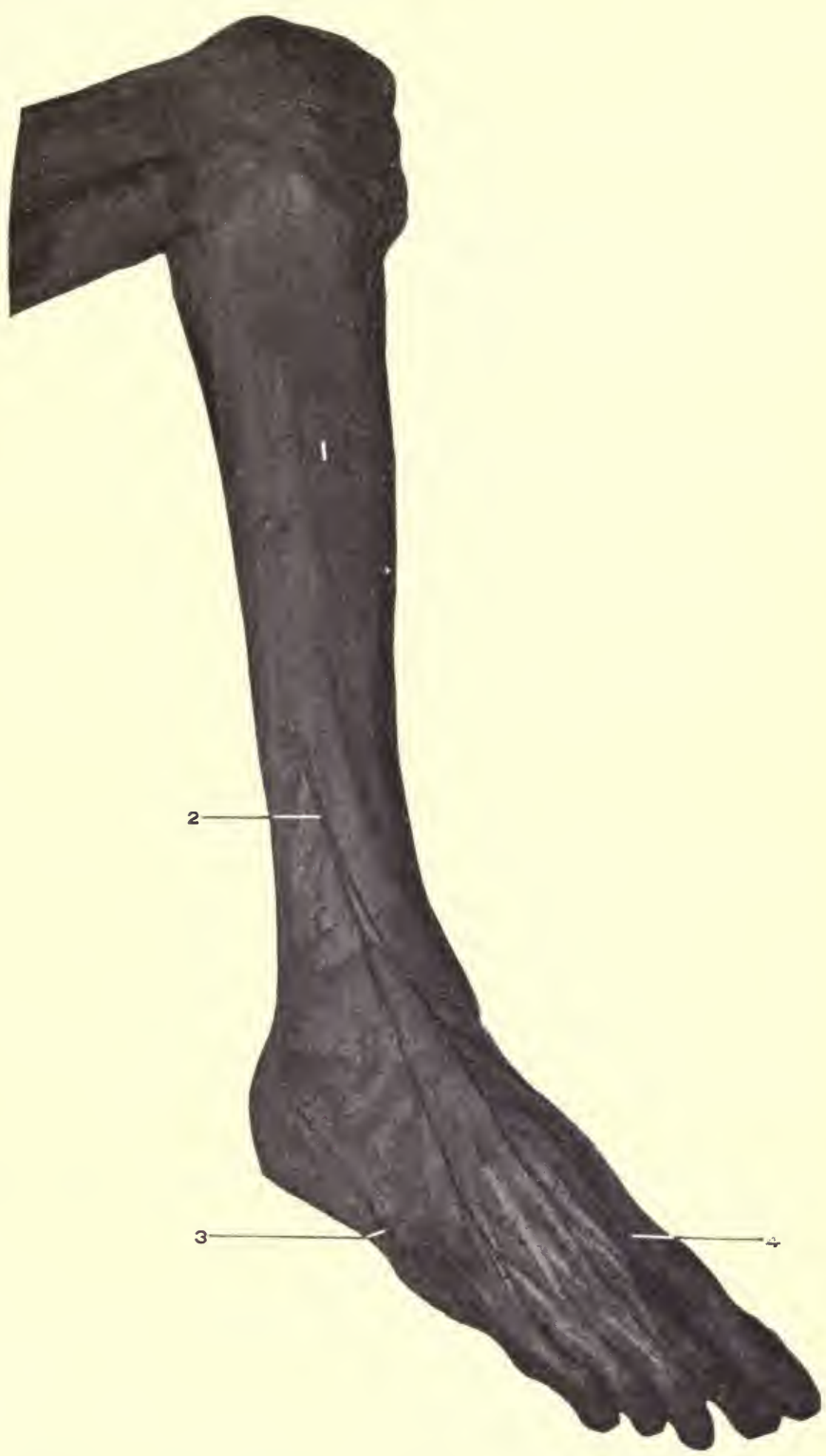

Fig. 122. Dissection of Leg, Anterolateral Region.-i, Deep fascia. 2, Musculocutaneous nerve. 3, Short or external saphenous nerve. 4, Internal branch of anterior tibial. 
cate with each other at variable points. They both have numerous valves.

The Internal (Long) Saphenous Nerve. See page 566.

The cutaneous branches of the external popliteal nerve are two small nerves which supply the superficial region of the upper and outer thirds of the leg.

The Musculocutaneous Nerve. Fig. I 22.

This is one of the terminal branches of the external popliteal nerve just after it has turned around the neck of the fibula, the other being the anterior tibial.

The musculocutaneous nerve descends between the peroneus longus and brevis, which it supplies, pierces the deep fascia at the outer side of the leg at its lower and middle thirds, and is distributed to the integument covering the outer and front of the lower third of the leg, the dorsum of the foot, the front and inner side of the great toe, the contiguous sides of the second and third, third and fourth, and fourth and fifth toes. The opposite sides of the first and second toes are supplied by the termination of the anterior tibial nerve, and the outer side of the little toe and foot receives its innervation from the external saphenous nerve. Both of these nerves receive a filament from the musculocutaneous.

The External or Short Saphenous Nerve.-See page 6 I6.

DISSECTION.

Remove the deep fascia, leaving the annular ligaments in place.

Clean all the muscles, vessels, and nerves upon the front of the leg and foot as far as possible without dividing any.

At the last, after the relations of parts are fully seen, then remove the anterior annular ligaments, and the extensor brevis digitorum, and complete the foot. 
Tibialis Anticus. Figs. I 23, I 24.

Origin.-From the under surface of the external tuberosity of the tibia, from the upper half or two-thirds of the outer surface of the tibia, from the adjacent portion of the interosseous membrane, from the upper third of the inner surface of the deep fascia covering the muscle, and from the intermuscular septum between the extensor longus digitorum and itself.

Insertion.-Into the adjoining portions of the inner and lower surfaces of the internal cuneiform and first metatarsal bones.

Nerve Supply. - The anterior tibial branch of the external popliteal nerve.

Action.-To flex the foot upon the leg, to raise (invert) the inner border of the foot, to adduct the anterior part of the foot.

Extensor Longus Digitorum. Figs. I 23, I 24,

Origin.-From the external tuberosity of the tibia, from the front of the head of the fibula, from the upper threefourths of the anterior surface of the fibula, from the upper third of the interosseous membrane, and for the same extent from the inner surface of the deep fascia covering the leg, and from the intermuscular septa between it and the bordering muscles.

Insertion. - Into the four outer toes by separate tendons which divide into three slips. The middle slip in each case being inserted into the base of the second phalanx, the other two tendons passing around the middle one to be inserted into the bases of the third phalanx.

From the margins of each tendon slips pass to the lateral ligaments of the metacarpophalangeal articulations.

The tendons of the interossei, lumbricales, and extensor 
brevis digitorum muscles blend with the margins of the long extensor tendons along the first phalanges.

Nerve Supply.-The anterior tibial.

Action.-To extend the toes, to flex the foot upon the leg. The action upon the toes is most marked upon the first phalanges, the extension of the second and third phalanges being effected through the interossei and lumbricales.

Extensor Proprius Hallucis. Figs. I 23, I 24.

Origin.-From the middle two-fourths of the anterior surface (antero-internal) of the fibula and from the interosseous membrane adjacent to this origin.

Insertion.-Into the dorsal surface of the base of the last phalanx of the great toe. At the metatarsophalangeal articulation a fibrous expansion from the tendon passes to the lateral ligaments of the articulation.

Nerve Supply.-The anterior tibial.

Action.-To extend the great toe, to flex the foot, to adduct the fore part of the foot, to raise (invert) the inner border of the foot. Due to the tendon being crossed by the lower leg of the $<$ ligament.

See Attachments of Annular Ligament, page 585.

Peroneus Tertius. Figs. I23, I 24.

Origin.-From the lower fourth of the anterior surface of the fibula, being continuous with the extensor longus digitorum, from the adjacent interosseous membrane, and intermuscular fascia.

Insertion.-Into the upper surface of the base of the fifth metatarsal bone.

Nerve Supply.-The anterior tibial.

Action.-To flex the ankle-joint, to raise the outer border of the foot (eversion), to abduct the fore part of the foot. 


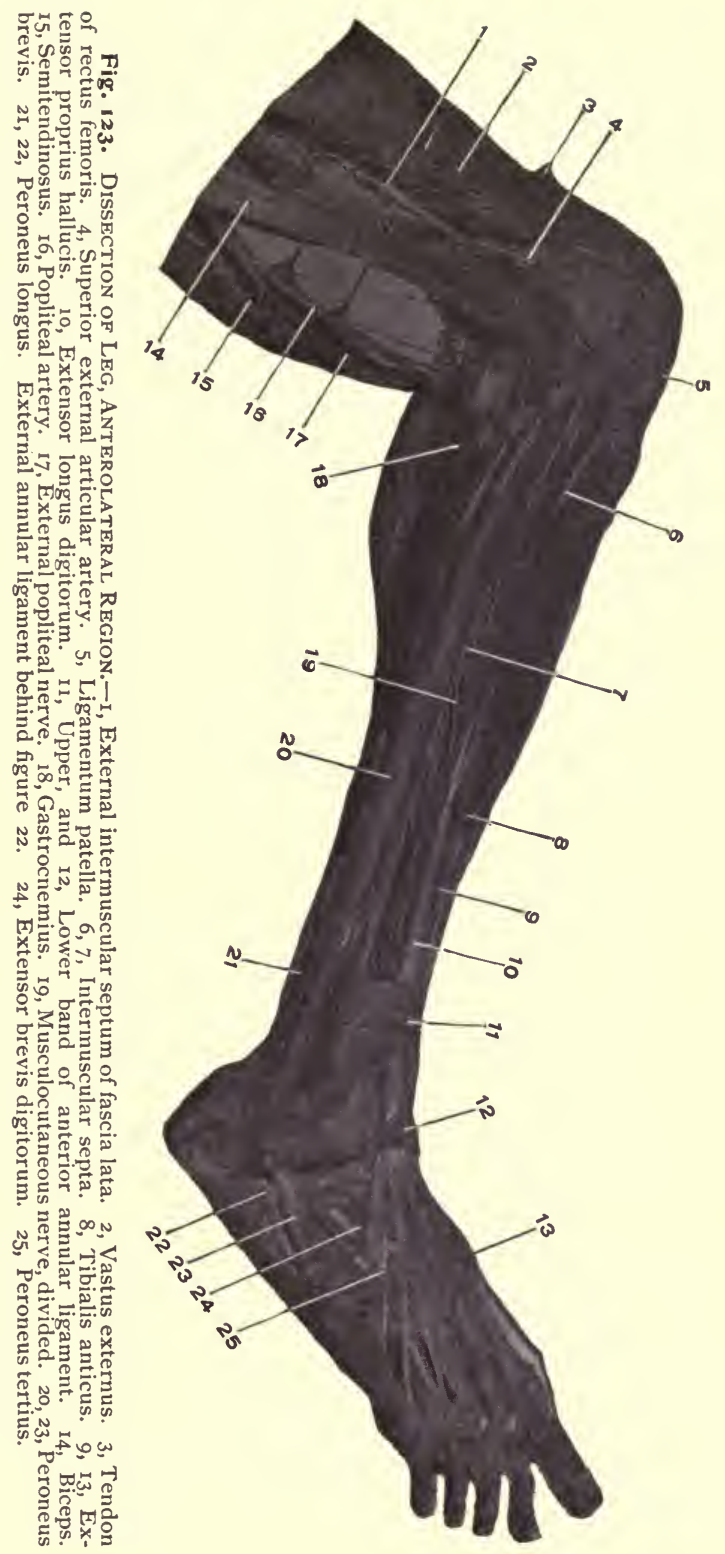


Extensor Brevis Digitorum. Fig. I24.

Origin.-From the outer and upper surface of the fore part of the os calcis, from the external calcaneo-astragaloid ligament, and from the lower band of the anterior annular ligament.

Insertion.-By four tendons. The first tendon is inserted into the outer surface of the base of the first phalanx of the great toe; the rest of the tendons merge into the outer margins of the tendons of the extensor longus digitorum (just over the bases of the first phalanges), and by them are attached to the second and third phalanges.

Verve Supply. - The anterior tibial.

Action.-To extend and adduct (toward the middle line of the second toe) the first phalanx of the great toe, to extend the third, second, and first phalanges of the next three toes. The action of the muscle upon the toes is more upon the distal than the proximal segments of them.

\section{Dorsal Interossei. Fig. I 25.}

There are four dorsal interossei muscles. There is one on either side of the second toe; then the third and fourth toes have each a muscle upon their outer (fibular) side.

Origin.-From the adjacent sides of the metatarsal bones between which they lie. Between the two heads pass the posterior perforating arteries. (In the case of the first muscle it is the communicating artery.)

Insertion.-Into the bases of the first phalanges, and into the lateral margins of the tendons of the extensor longus digitorum; upon the inner side of the second toe for the first muscle, and upon the outer side of the second, third, and fourth toes for the remaining muscles.

Nerve Supply. - The external plantar branch of the posterior tibial nerve. 
Action.-Same as in the hand ( $q . v$., page 389 ), to flex the first set of phalanges, to extend the second and third sets of phalanges, to abduct the toes from the middle line drawn through the second toe.

\section{The Anterior Tibial Artery. Figs. I 24, 225.}

This artery is the anterior branch of bifurcation of the popliteal, just at the lower border of the popliteus muscle. See page 623. The course of the anterior tibial artery is forward between the two heads of the tibialis posticus muscle, then between the tibia and fibula, where the artery crosses over the top of the interosseous membrane to gain the front of the leg.

It turns downward resting upon the interosseous membrane and between the tibialis anticus and the extensor longus digitorum for the upper third of its course, then between the tibialis anticus and the extensor proprius hallucis for the middle third, then finally between the tendon of the extensor proprius hallucis and the tibia for the lower third. In front of the ankle joint the name of the artery is changed to the dorsalis pedis. The artery has on either side an accompanying vein which frequently communicate with each other. The anterior tibial nerve lies at the outer side of the artery for its upper third, over the front of the artery for its middle third, and then again at the outer side of the artery for the lower third.

The course of the artery is indicated upon the outer surface of the leg by a line drawn from the inner margin of the head of the fibula to the mid-point in front of the ankle joint between the malleoli. The deeper guide to the artery is the intermuscular space at the outer margin of the tibialis anticus muscle, for the upper twothirds; and between the tendons of the extensor proprius 
hallucis and the tibialis anticus for the lower third of the leg.

\section{The Branches of the Anterior Tibial.}

(I) The posterior tibial recurrent. This is found when dissecting the back of the leg. It runs upward between the popliteus muscle and the posterior ligament of the knee joint. It is a small branch and often wanting.

(2) The anterior tibial recurrent. This is a branch of considerable size that runs upward through the origin of the tibialis anticus close to the bone, and supplies the outer side of the front of the knee, anastomosing with the external inferior and superior articular from the popliteal and the long articular branch of the external circumflex to the outer side of the knee.

(3) The muscular branches are numerous and supply the muscles bordering upon the artery.

(4) The malleolar. The external is slightly larger than the internal. They pass to the external and internal malleolar regions, which they supply ; in their course they run beneath the tendons of the muscles at either side of the anterior tibial artery. The internal malleolar forms an anastomosis with the posterior tibial, internal plantar, and the internal calcanean; the external, with the anterior peroneal, the external plantar, and the tarsal branch of the dorsalis pedis.

The Anterior Peroneal. See page 633. Fig. I 25.

It is found as it appears through the interosseous membrane, between the tibia and the fibula, about two or three inches above the ankle joint. It passes downward between the two bones of the leg to the front of the outer side of the ankle, which it supplies, and where it enters into an anastomosis with the external malleolar artery. 
The Dorsalis Pedis Artery. Fig. I 25.

This is the continuation of the anterior tibial from the front of the ankle, along the front of the foot, to the space between the first and second metatarsal bones, where it terminates by dividing into the dorsalis hallucis and the deep communicating artery.

In its course it passes between the tendons of the extensor proprius hallucis and the extensor longus digitorum, and is crossed near its termination by the inner tendon of the extensor brevis digitorum.

The venæ comites lie upon either side of the artery, and the anterior tibial nerve at its outer side.

The course of the dorsalis pedis is indicated by a line drawn from the mid-point between the malleoli to the interval between the first and second metatarsal bones.

The Branches of the Dorsalis Pedis.

(I) The internal tarsal. Several small branches to the inner side of the foot.

(2) The external tarsal turns outward beneath the extensor brevis digitorum muscle to the outer side of the foot, where it enters into an anastomosis with the external malleolar, anterior peroneal, external plantar, and the metatarsal arteries.

(3) The metatarsal artery passes outward under the tendons of the extensor brevis digitorum muscle and across the heads of the four outer metatarsal bones to the outer border of the foot.

Above, it anastomoses with the external tarsal, and at the outer side of the foot with the external plantar. From the front part of the artery the three interosseous branches are given off.

The interosseous arteries pass forward in the second, 
third, and fourth spaces, resting upon the interosseous muscles. At their beginning they communicate with the plantar arch by means of the posterior perforating branches, and at the metatarsophalangeal articulations they communicate with the corresponding plantar digital artery, through the anterior perforating branches. The interosseous arteries then divide into the dorsal collateral digital vessels which supply adjacent sides of the dorsal surface of the four outer toes. The outer side of the fifth toe being supplied by a small branch from the fourth dorsal interosseous artery just before it bifurcates.

The Dorsalis Hallucis artery is the continuation of the dorsalis pedis to the metatarsophalangeal joint where it divides into two collateral digital branches to the adjacent sides of the first and second toes. The dorsalis hallucis communicates just before its division with the corresponding plantar digital artery, and near this point gives off the dorsal collateral digital branch to the inner side of the great toe. This last branch is inconstant.

\section{The Communicating Artery. Fig. I 25.}

This is a branch of bifurcation of the dorsalis pedis, and corresponding to the first posterior perforating artery, passes backward through the interval between the first and second metatarsal bones to the sole of the foot, where it completes the plantar arch by anastomosing with the external plantar. It then continues forward as the fifth plantar digital artery, between the first and second toes, receives the anterior perforating from the dorsalis hallucis, sends a branch to the plantar surface of the inner side of the great toe, and divides into the collateral digital (plantar) branches for the contiguous sides of the first and second toes. 


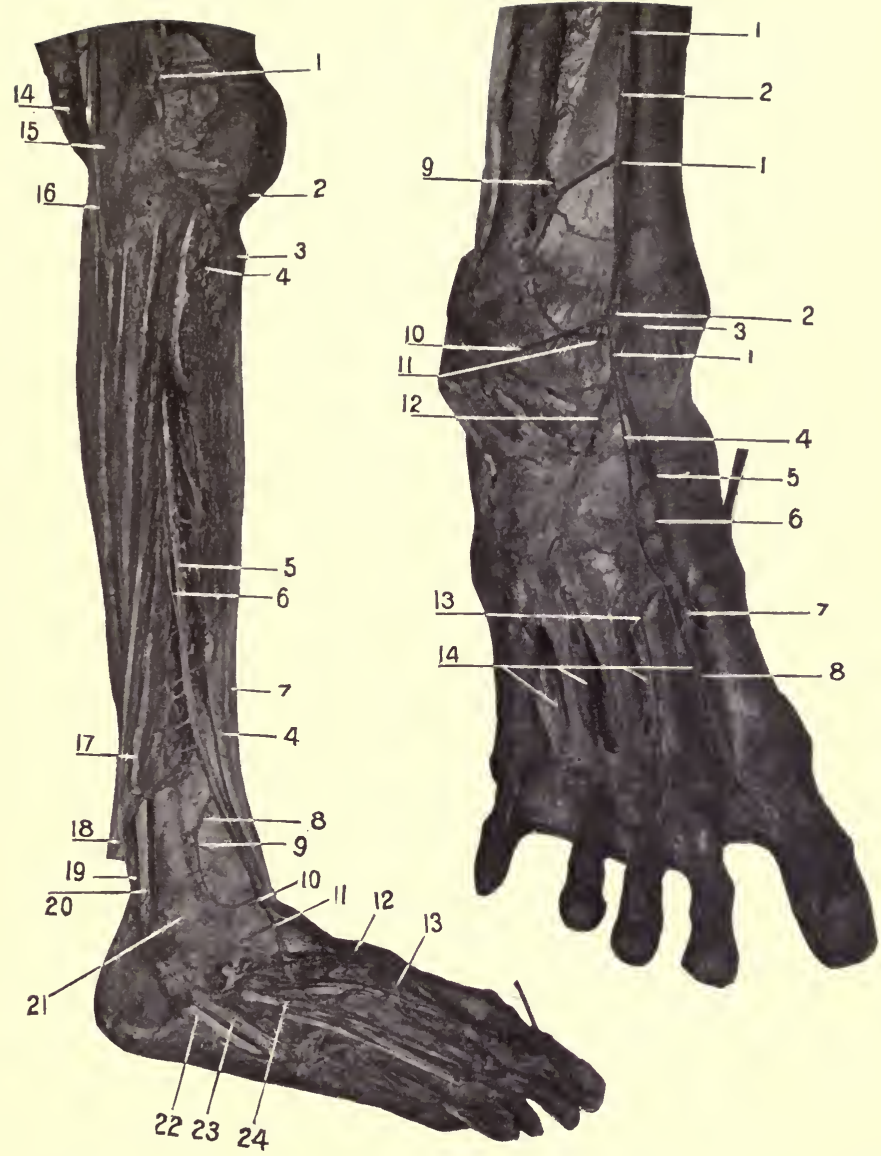

Fig. 124. Dissection of Leg, Anterolateral Region.-I, Superior external articular artery. 2, Ligamentum patella. 3, Tubercle of tibia. 4, 4, Tibialis anticus. 5. Anterior tibial artery and muscular branches. 6, Anterior tibial nerve. 7, Tibia. 8, Articular branch, anastomosing with 9, Anterior peroneal and Io, The external malleolar. II, Tarsal artery. I2, Dorsalis pedis I3, Metatarsal artery. I4, Semitendinosus. 15, Tendon of biceps. 16, External popliteal nerve. 17. Extensor proprius hallucis. 18, Extensor longus digitorum. 19, 22, Peroneus longus. 20, 23, Peroneus brevis. 21, External malleolus. 24, Extensor brevis digitorum.

Fig. 125. Dissection of Foot, Dorsal Surface.-I, I, I, Anterior tibial nerve. 2, 2, Anterior tibial artery. 3, Internal malleolar artery. 4, Internal branch of anterior tibial nerve. 5, Internal tarsal artery. 6, Dorsalis pedis artery. 7, Communicating artery. 8, Dorsalis hallucis artery. 9, Anterior peroneal artery. Io, External branch of anterior tibial nerve traced to extensor brevis digitorum which has been reflected. II, External malleolar artery. 12, External tarsal artery. 13, Metatarsal artery. I4, The four dorsal interossei. 
The Anterior Tibial Nerve. Figs. I 24, I 25.

The remaining branch of division of the external popliteal nerve, the other being the musculocutaneous.

The anterior tibial nerve descends through the substance of the extensor longus digitorum muscle, keeps at the outer side of the anterior tibial artery for its upper third, then runs upon the anterior surface of the artery in its middle third, leaves the artery in its lower third, remaining at its outer side, passes with the long tendons under the two bands of the anterior annular ligament, and terminates here by dividing into two branches, an internal and external.

The internal branch continues forward along the outer side of the dorsalis pedis artery to the first interosseous space, receives a branch from the musculocutaneous and divides into collateral digital nerves to the adjacent sides of the great and second toes. The external branch turns outward under the extensor brevis digitorum, becomes slightly enlarged, and from this enlargement filaments pass to the muscles and tarsal articulations.

In its course the anterior tibial supplies muscular branches to the extensor longus digitorum, tibialis anticus, extensor proprius hallucis, peroneus tertius, and the extensor brevis digitorum; articular branches to the ankle and the tarsal joints. 


\section{THE LOWER EXTREMITY, Posterior.}

LANDMARKs. Figs. 76, I 26.

The landmarks as given on page 554 should be consulted.

The crest of the ilium curves backward to terminate in the posterior superior spinous process of the ilium. The spines of the sacrum and the termination of the spinal column in the coccyx are easily felt.

The tuberosity of the ischium is an important reference point to which the posterior part of Nelaton's line is carried.

The difficulty in determining what is the most prominent part of the tuberosity of the ischium is the difficulty belonging to all so-called anatomical " points," which are in reality "broad surfaces." This difficulty of exactly locating these various "points" becomes increased in the living, especially if there is a thick overlying pad of fatty tissue. The student must be cautioned in regard to such "points" as the costal cartilages, tuberosity of the ischium, condyles and tuberosities of the various long bones. Such landmarks are called "points" by common consent, but in reality they are very considerable areas.

The bony parts about the popliteal region demand attention, and the ham-strings, which form the lateral boundaries of the same. They are rendered prominent by extending the leg. The tendo-Achillis is readily appreciated, especially if the foot is flexed.

\section{DISSECTION.}

Incisions.-(I) Continue the median incision of the trunk to the tip of the $\operatorname{coccyx}$.

(2) Make a transverse cut from the last dorsal vertebra outward (same as 3 , page 360 ).

(3) From the middle of the sacrum carry a curved incision outward over the crest of the ilium to join the similar anterior one. 
Remove the integument from the lumbar, gluteal, and posterior crural regions.

To expose the back of the thigh (and for that matter the leg) it will not be necessary to make median and transverse incisions. But reflect the integument from the outer to the inner side of the limb, and from above downward, at this time as far downward as the calf of the leg. Do not cut this large skin flap away but use it for covering the part.

\section{The Superficial Fascia, Posterior Portion.}

This layer is continuous with the fascia described upon the anterior aspect of the thigh. See page 556 .

Upon the small of the back and over the buttocks, the superficial fascia is usually very thick and consists of very coarse granular adipose tissue. See page 360.

\section{DISSECTION.}

Remove the superficial fascia from the entire area corresponding to the integument, saving the cutaneous nerves and veins.

\section{The Fascia Lata, Posterior Portion.}

The posterior portion of the fascia is continuous with the anterior at the sides of the thigh, with the deep fascia of the leg in the popliteal space, and is attached above and below and at the sides, as given on page 558 .

Above, the fascia divides into two laminæ between which the gluteus maximus lies, and into the angle of division at the outer side of the thigh it is inserted similar to the tensor vaginæ femoris. The external lamina is the stronger, and is attached to the $\operatorname{coc} c y x$ and the sacrum. The internal layer blends with the outer margin of the great sacrosciatic ligament. The two layers become united at the margins of the gluteus maximus, the external portion then passing over the gluteus medius and being fastened to the crest of the ilium, the internal to the tuberosity of the ischium and the pubic arch.

The portion of the fascia lata into which the gluteus 


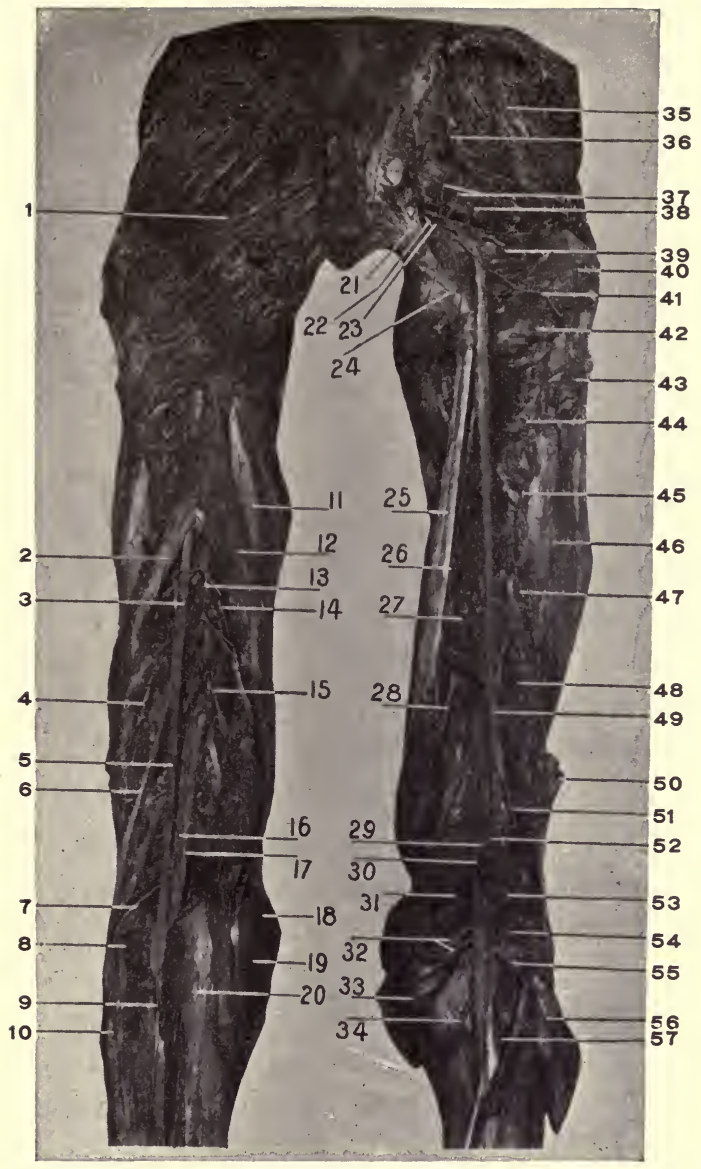

Fig. 126. Dissection of Gluteal Region, Back of Thigh and KNeE.-I, Gluteal maximus. 2, Long head of biceps. 3, Great sciatic nerve. 4, Short head of biceps. 5, Internal popliteal nerve. 6, External popliteal nerve. 7, Posterior saphenous artery (cutaneous). 8, Outer head of gastrocnemius. 9, Communicans tibialis nerve. 10, Communicans fibularis (peronei). 11, Semimembranosus. 12, Senitendinosus. 13, Perforating artery. I4, Muscular branches of great sciatic to II and 12. 15. Fourth perforating artery. 16, Popliteal vein. 17, Popliteal artery. 18, Tendon of gracilis. I9, Tendon (divided) of Sartorius. 20, Inner head of gastrocnemius. 21, Great sacrosciatic ligament. 22, Sciatic artery. 23, Small sciatic nerve and branches. 24, Tuberosity of ischium 25, Seminembranosus. 26, Nerve to 25. 27, Adductor magnus, 28, Perforating artery, 29, Popliteal vein. 30, Popliteal artery. 31, Superior internal articular artery. 32, Inferior muscular (sural) arteries. 33, Inner head of gastrocnemius. 34, Internal inferior articular artery. 35, Gluteus medius. 36, Gluteal artery. 37, Muscular branches (divided) to the gluteus maximus (removed). 38, Pyriformis. 39, Obturator internus and gemelli. 40, Great trochanter. 4I, Anastomotic branch of sciatic artery. 42, Quadratus femoris. 43, Insertion of gluteus maximus. 44, First perforating artery. 45, Second perforating artery. 46, Vastus externus. 47, Third perforating artery. 48 , Short head of biceps. 49, Great sciatic nerve. 50, Cut end of long head of biceps. 5I, External poplitea! nerve. 52, Internal popliteal nerve. 53, Superior external articular artery. 54 , Nerve to outer head (56) of gastrocnemius. 55, Inferior muscular (sural) arteries. 56 , Outer head of gastrocnemius. 57, Plantaris. 
maximus is inserted is that thickened layer already described as the iliotibial band, see page 558 .

In the popliteal space the fascia lata is much increased in strength by the addition of transverse fibres which bind together the sides of the space. The external or short saphenous vein pierces the fascia at this place.

Upon the inner portion of the thigh the fascia is thinner than elsewhere.

\section{The Cutaneous Nerves.}

These are the terminal filaments of the last dorsal and the posterior branch of the iliohypogastric nerves over the upper part of the gluteal region and the great trochanter; the cutaneous branches of the sacral nerves over the inner portion of the gluteal region; the recurrent branches of the small sciatic over the lower portion of the gluteus maximus ; branches from the small sciatic down the back of the thigh, and popliteal space; and the posterior branches of the external cutaneous (see page 56r) along the outer side of the thigh.

\section{DISSECTION.}

Remove the fascia lata from the gluteus maximus muscle, cutting in the direction of the fibres of the muscle. Expose the muscle entirely.

Incise the fascia in the middle line from the gluteus maximus to below the knee and remove it from the deeper parts, noting the compartments for the muscles. Save the small sciatic nerve.

Clean the muscles of the buttocks, thigh, and the upper part of the popliteal space.

Gluteus Maximus. Fig. I 26.

Origin.-From the crest and the outer surface of the ilium posterior to the superior curved line thereof, from the lower half of the posterior and lateral part of the sacrum, from the side of the coccyx, from the lumbar aponeurosis between the ilium and sacrum, and from the posterior surface of the great sacrosciatic ligament. 
Insertion.-Into the iliotibial band over the great trochanter of the femur. Into the gluteal (vertical) ridge which extends from the posterior surface of the great trochanter to the outer bifurcation of the linea aspera. A distance of about two inches.

Nerve Supply.-The inferior gluteal branch of the sacral plexus. This nerve is usually included within the sheath of the small sciatic and consequently is said to be a branch of that nerve. See page 552 .

Action.-The attachments of all the posterior muscles of the thigh should be very carefully determined, then their action when the thigh is in an extended position, and finally when it is flexed at various angles. It will only be by such careful study that the complex actions of these gluteal and external rotator muscles will be understood.

To attempt to commit to memory the action of muscles from the book is all wrong, unless the demonstration is carried out upon the subject (living or dead) and by the use of the skeleton.

Action of the gluteus maximus.

(I) When the thigh is extended, it acts as an external rotator; its upper fibres will assist abduction, and its lower fibres will assist adduction; the action of the whole muscle neutralizes these movements.

(2) When the thigh is flexed. The muscle is a powerful extensor of the thigh, it is also a strong abductor (in flexion); abduction diminishes as flexion decreases and extension increases (in other words, as the individual assumes an upright position).

(3) Upon the leg, it will assist flexion when once started, and external rotation, through the iliotibial band, along with the tensor vaginæ femoris.

In full extension of the leg the muscle helps to main- 
tain the knee in that position and to resist flexion, yet after flexion is once started its action (if any) is as indicated above.

(4) Upon the trunk. Taking its fixed point from below the muscle will extend the trunk upon the thighs as in assuming an upright position after stooping forward.

The action of the gluteus maximus and tensor vaginæ femoris, through the attachment of the iliotibial band is very necessary in the maintenance of the trunk erect when standing upon one leg. They act here like the guy rope to a derrick in lifting a weight, the femur being the upright, the pelvis the cross beam, and the body the weight; the centre of motion being at the hip joint.

The Small Sciatic Nerve. Fig. I 26.

The small sciatic nerve is formed by branches from the second and third sacral nerves, passes through the great sacrosciatic foramen under the pyriformis muscle, then beneath the gluteus maximus and behind the fascia lata through the middle of the thigh into the popliteal space, where it pierces the deep fascia and supplies the integument over the popliteal space and the upper third of the calf of the leg.

Its Branches are :-

(I) Recurrent cutaneous branches to the integument over the gluteus maximus.

(2) The inferior pudendal. This branch turns inward under the gluteus maximus, pierces the deep fascia about an inch external to the tuberosity of the ischium (see page 406,) and runs forward to supply the inner side of the thigh and the scrotum (or the labium majus).

(3) The femoral cutaneous branches supply the skin over the back of the thigh and popliteal space. 


\section{DISSECTION.}

Divide the gluteus maximus at its outer and middle thirds and reflect the two portions.

The outer portion is to be carefully dissected to demonstrate the double insertion of the muscle and the bursa between it and the great trochanter, and the inner portion is to be lifted up and turned back on to the sacrum to show its extensive origin and the bursa which intervenes between the muscle and the tuberosity of the ischium.

Divide the nerve and arterial supply close to the muscle.

Remove entirely the inner portion of the muscle by cutting it away from its origins.

A large multilocular bursa will be found between the tendon of the gluteus maximus and the great trochanter of the femur. A second one intervenes between the muscle and the tuberosity of the ischium.

Gluteus Medius. Figs. I I 8, I 26, I 27.

Origin.-From the external surface of the ilium between the middle curved line below and the superior curved line and the anterior four-fifths of the external lip of the iliac crest above, from the deep surface of the fascia lata covering it.

Insertion.-Into the oblique line upon the outer surface of the great trochanter. The direction of this oblique line is from above and behind, downward and forward.

Nerve Supply.-The superior gluteal (fourth and fifth lumbar, and first sacral nerves).

Action.-At all times the muscle acts as an abductor of the thigh, and serves to maintain the pelvis level when standing upon one foot, as in walking, etc.

The anterior portion of the muscle acts as an internal (forward), the posterior, as an external (backward) rotator of the thigh.

Pyriformis. Figs. I I 4, I 26, I 27.

Origin.-From the front of the lateral margin of the 
second, third, and fourth pieces of the sacrum, and from the ridges of bone between the first, second, third, and fourth sacral foramina.

From the deep surface of the great sacrosciatic ligament, and the upper margin of the great sacrosciatic notch.

Insertion. - Into the upper border of the great trochanter near the front. The tendon of the pyriformis muscle is closely joined, near the trochanter, to the common tendon of the obturator internus and the gemelli muscles. If the tendons are carefully separated it will be found that the tendon of the latter muscles passes beneath and in front of that of the former muscle; the posterior muscles have the anterior insertion into the great trochanter.

Nerve Supply.-Muscular branches from the second sacral nerve of the sacral plexus.

Action.-If the thigh is extended the muscles act solely as an external rotator. When the thigh is flexed it acts as an abductor. Acting from a fixed point upon the trochanter the pyriformis will carry the other side of the pelvis backward, whether the femur be extended or flexed.

Obturator Internus and Gemelli. Figs. I I 4, I 26, I 27.

(I) Obturator internus. From the inner surface of the true pelvis (including the obturator membrane), below a line drawn from the upper margin of the obturator foramen to the junction of the iliopectineal line near its terminus, and above a line drawn about the base of the spine of the ischium. Behind the muscle reaches to the anterior margin of the great sacrosciatic foramen, and in front, it extends on to the rami of the pubes and ischium. It also arises from the obturator fascia which covers it. See page 398. (2) Gemellus superior. From the external surface of the spine of the ischium. (3) Gemellus inferior. 
From the upper and back part of the tuberosity of the ischium, along the lower border of the groove for the tendon of the obturator externus. The tendon of the obturator internus leaves the pelvis through the small sacrosciatic foramen; after coming through the foramen it receives the two gemelli muscles, the superior above and the inferior below, on either side. These small muscles are really portions of the obturator internus arising external to the pelvic cavity.

Insertion.-The common tendon of the three muscles is attached to the front of the upper margin of the great trochanter (in front of the pyriformis as explained. See Pyriformis).

Between the tendon of the obturator internus and the margin of the small sacrosciatic foramen is a large bursa; another one may exist between the tendon and the capsule of the hip joint.

Nerve Supply.-By muscular branches from the sacral plexus. The nerve to the obturator internus gives a branch to the gemellus superior, while the gemellus inferior receives its supply from the nerve to the quadratus femoris. See pages 610, 6r 2.

Action.-To rotate the thigh outward (external rotators) when the limb is extended. If the thigh be flexed the muscles act as abductors of it.

The femur being the fixed point, the opposite side of the pelvis will be carried backward in extension or flexion.

Quadratus Femoris. Figs. I26, I27.

Origin.-From the outer lip of the tuberosity of the ischium.

Insertion.-Into the linea quadrati, which extends vertically downward from the middle of the posterior intertrochanteric line for about one and one-half inches. 
Nerve Supply.-A branch from the sacral plexus, see page $6 \mathrm{I} 2$.

Action.-To rotate the thigh outward and adduct it when the limb is extended. If the thigh is flexed it acts as an external rotator and abductor of it.

Acting from the femur, it will depress the opposite side of the pelvis and carry it backward.

Obturator Externus. See page 582.

Biceps Flexor Cruris (Femoris) (Biceps Femoris).

Origin.-(1) The long head, in common with the semitendinosus from the (anterior) lower and internal facet upon the tuberosity of the ischium. (2) The short head, from the whole length of the external lip of the linea aspera and the upper two-thirds of the external condyloid ridge, and from the external intermuscular septum.

Insertion. - Into the head of the fibula at the base of the styloid process, by a prolongation of some of the anterior fibres of the tendon into the external tuberosity of the tibia, and by others from the back of the tendon into the deep fascia of the leg.

Nerve Supply. - The great sciatic (from the first, second, and third sacral nerves).

Action.-(I) Upon the leg (both heads) the biceps is a flexor of the leg, and in a flexed position becomes an external rotator (supinator). (2) Upon the thigh (the long head) the muscle is an extensor for all the ordinary purposes of locomotion and the like, the gluteus maximus being only called into action by some heavier work to be done. It also has a slight action as an external rotator of the thigh. (3) Upon the body, it is used in raising it from a stooping position (an extensor of the pelvis). 
Semitendinosus. Fig. I 26.

Origin.-By a tendon common to it and the long head of the biceps from the lower (anterior) and internal facet upon the tuberosity of the ischium.

Insertion.-By a long slender tendon into the inner surface of the tibia opposite its tubercle, where it lies behind the sartorius and below the gracilis. Also, into the deep fascia of the leg.

A bursa intervenes between the three tendons and the internal lateral ligament of the knee.

Nerve Supply.-Great sciatic. See biceps, above.

Action.-(I) Upon the leg, to flex the leg and in a flexed position to rotate it inward (pronation). (2) Upon the thigh, it is an extensor, and used for the lesser muscular efforts. (3) Upon the pelvis it acts as an extensor, as in raising the body from a stooping position.

Semimembranosus. Fig. I 26.

Origin.-From the external and upper (posterior) facet upon the tuberosity of the ischium. To get to this position the tendon of the semimembranosus passes forward of, then external to, the common tendon of the long head of the biceps, and the semitendinosus muscles.

Insertion.-Into the transverse groove upon the back of the internal tuberosity of the tibia; by a strong recurrent bundle of fibres, which crosses the knee joint obliquely, into the outer posterior surface of the external condyle of the femur; and by a continuation of the direct fibres into the fascia covering the popliteus muscle, and so into the oblique line upon the posterior surface of the tibia.

Nerve Supply. - The great sciatic, the filaments coming from the first, second, and third sacral nerves.

Action.-(I) Upon the leg, the muscle acts as a flexor, 
and in flexion to rotate the leg inward (pronation). Upon the thigh, it has the same action as the semitendinosus. (3) Upon the body, the muscle acts as the semitendinosus.

The Great Sciatic Nerve. Figs. I I 2, I I 4, I 26, I 27.

This is the largest nerve in the body. It is formed within the pelvis but close to the great sacrosciatic foramen, by the convergence of the lumbosacral cord, the first, second, and third sacral nerves (anterior branches, see page 553).

It emerges through the great sacrosciatic foramen being below the pyriformis muscle, and descends into the thigh until at its lower third, when it divides into the external and internal popliteal nerves. In its course the great sciatic nerve lies between the tuberosity of the ischium and the great trochanter, and crosses the gemellus superior, tendon of the obturator internus, gemellus inferior (tendon of the obturator externus), the quadratus femoris, and adductor magnus muscles.

It lies under the gluteus maximus, and long head of the biceps muscles, and is covered by the fascia lata, superficial fascia and the integument.

In the upper part of its course it is accompanied by the arteria comes nervi ischiadici.

Branches.-(I) Muscular, to the biceps, semitendinosus, semimembranosus, and adductor magnus. (2) Articular, to the hip and knee joints. The latter through the internal and external popliteal branches.

The Internal Pudic Nerve. See page 4I3.

The Nerve to the Obturator Internus.

This branch of the sacral plexus arises from the second 


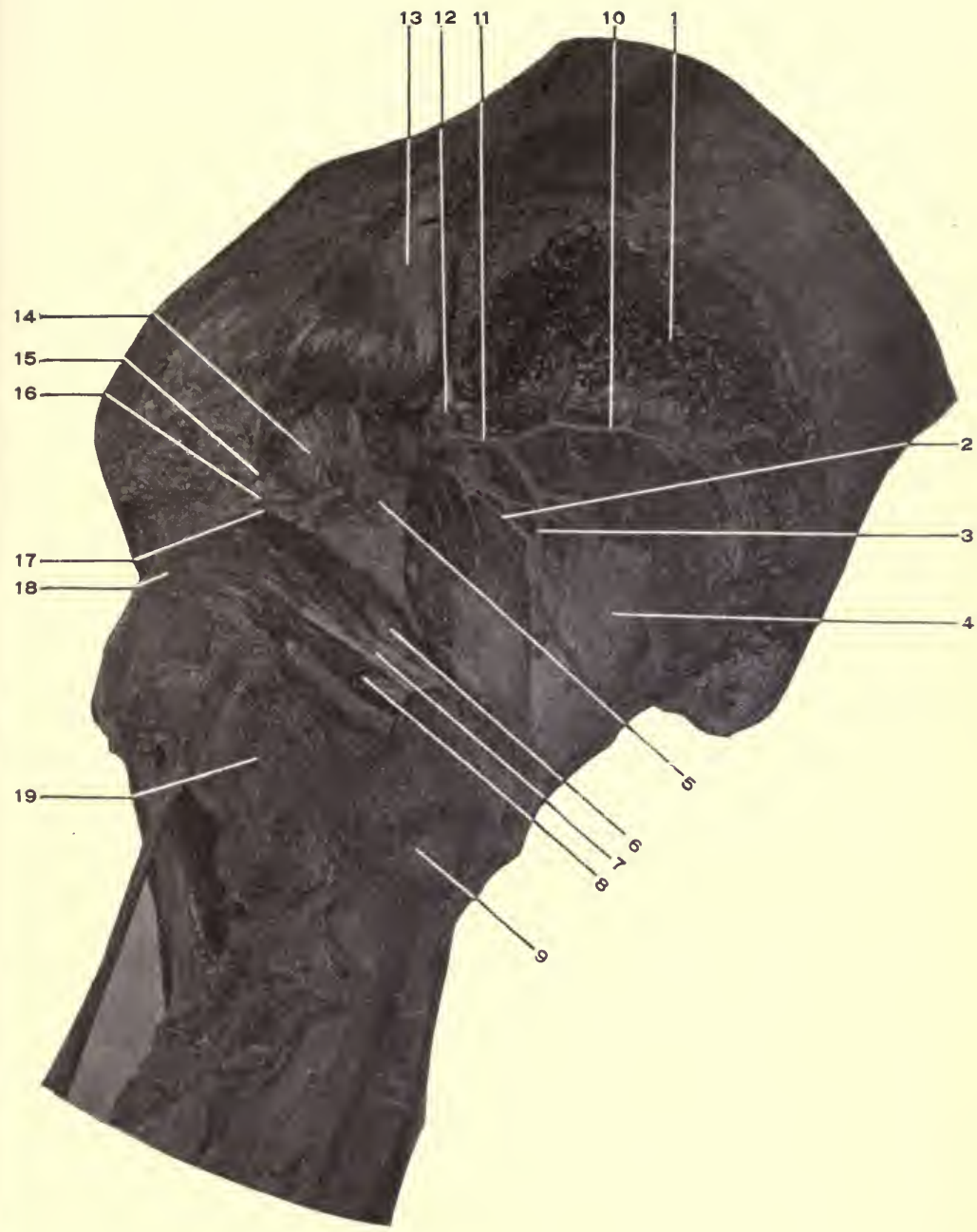

Fig. 127. Dissection of Gluteal Region.-1, Origin of gluteus medius. 2, Superior gluteal nerve. 3, Inferior branch of deep division (II) of gluteal artery. 4, Gluteus minimus. 5, Stub of great sciatic nerve. 6, Gemellus superior. 7, Obturator internus. 8, Gemellus inferior. Look for the obturator externus tendon between 8 and 19. 9, Great trochanter. Io, Superior branch of deep division of gluteal artery. II, Deep division of gluteal artery. 12, Superficial division of gluteal artery. 13, Pyriformis reflected. 14, Great sacrosciatic foramen (filled with the structures it transmits). 15, Internal pudic nerve. 16, Internal pudic artery. 17, Nerve to the obturator internus muscle. 18, Great sacrosciatic ligament. 19, Quadratus femoris. 
and third sacral nerves, issues from the great sacrosciatic foramen below the pyriformis, curves around the base of the ischial spine and re-enters the pelvic cavity and is distributed to the obturator internus muscle.

As the nerve crosses the spine it gives a branch to the gemellus superior. Here it lies external to the internal pudic artery. (The artery lies between the internal pudic nerve (internal) and the nerve to the obturator internus (which is external).)

\section{The Nerve to the Quadratus Femoris.}

This muscular branch arises from the third sacral nerve, leaves the pelvic cavity through the great sacrosciatic foramen, close to the bone, then passes under (anterior to) the gemellus superior, tendon of obturator internus, and gemellus inferior muscles, then over (posterior to) the tendon of the obturator externus to the anterior surface of the quadratus femoris to which it is supplied.

The gemellus inferior is supplied by a branch from this nerve.

The Gluteal Artery. Figs. I I 2, I I 4, I 26, 127.

This is the largest of the three arteries which leave the pelvis through the great sacrosciatic foramen. It is really the continuation of the posterior division of the internal iliac and passes out through the great sacrosciatic foramen above the pyriformis muscle, close to the bone. A halfinch from the margin of the foramen it divides into a superficial and deep branch.

(I) The superficial branch. This breaks up into branches which ramify beneath and within the gluteus maximus muscle and supply the surrounding parts. (2) The deep branch. Gives a nutrient artery to the ilium and then divides into a superior and an inferior branch, which pass 
outward and forward between the gluteus medius and minimus muscles; the superior keeping close to the bone along the origins of the muscles, the inferior keeping company with the superior gluteal nerve. (3) Besides these branches, the gluteal artery gives off within the pelvic cavity small vessels to the obturator internus, pyriformis, levator ani, and coccygeus muscles and the pelvis itself.

The gluteal artery anastomoses with the lateral sacral, sciatic, deep circumflex iliac, and the external circumflex (of the profunda).

The Internal Pudic Artery. See page 4I I.

The Sciatic Artery. Figs. I 1 2, I 14, I 26, I 27.

This is the larger branch of bifurcation of the anterior division of the internal iliac. It comes through the great sacrosciatic foramen below the pyriformis muscle and passes downward under the gluteus maximus to terminate in the upper part of the thigh.

It supplies numerous branches to the surrounding parts, a few of which are named.

(I) The coccygeal branch; this pierces the great sacrosciatic ligament, and supplies the gluteus maximus and the superficial parts over the coccyx and sacrum. (2) The anastomotic, a long slender branch to the great trochanter, around which it anastomoses with the gluteal, external circumflex, ascending branch of the internal circumflex, and the first perforating of the profunda. It supplies the external rotators of the thigh and the hip joint. (3) Comes nervi ischiadici, a small branch which supplies the great sciatic nerve. (4) Numerous cutaneous branches below the gluteus maximus. The sciatic artery through its lower branches anastomoses with the first perforating of the pro- 
funda (below), the external circumflex, and the internal circumflex upon the outer and inner sides of the femur. This arrangement of communicating branches constitutes the "crucial anastomosis." The student must not expect to find the inosculating branches forming any well defined "cross." However, as one artery (the sciatic) is above, another (first perforating) is below, and two others upon either side (the external and internal circumflex) a somewhat "cross-shaped" anastomosis is formed. (5) Within the pelvis the sciatic gives twigs to the adjacent muscles, nerves, and pelvic viscera.

The Great Sacrosciatic Ligament. Figs. I 26, I I4.

This is a very strong band of fibres extending from the posterior, superior, and inferior iliac spines, and from the side and posterior surface of the sacrum and coccyx to the inner margin of the tuberosity of the ischium, along which it is prolonged as a long curved band (the falciform process) for an inch and a half.

A part of the fibres of the great sacrosciatic ligament pass into the tendon of the long head of the biceps (flexor biceps femoris).

This muscle then may be said to take an origin from the surface from which this ligament rises.

The great sacrosciatic ligament converts the small sacrosciatic notch into a foramen (assisted by the small ligament of the same name).

From the posterior surface of the ligament the gluteus maximus arises; from the anterior surface some fibres of the pyriformis.

To the outer margin the deeper lamina of the fascia lata is attached; at its inner border it becomes continuous with the obturator fascia. 
It is usually pierced by the coccygeal branch of the sciatic artery.

The Small Sacrosciatic Ligament. Fig. II4.

This arises from the side of the sacrum and coccyx, anterior to the great sacrosciatic ligament, with which it blends.

It is inserted into the spine of the ischium. It converts: the great sacrosciatic notch into a foramen (assisted by the great sacrosciatic ligament to a slight extent). From it arise some fibres of the coccygeus muscle.

The Sacrosciatic Foramina. Figs. I 14, I 27.

These are formed by the great and small sacrosciatic ligaments bridging over the great and small sacrosciatic notches as mentioned above.

The greater foramen transmits the pyriformis muscle; above the muscle the gluteal artery and vein and the superior gluteal nerve. Below the muscle the sciatic artery and vein, the great and small sciatic nerves, the internal pudic artery, vein, and nerve, and the nerves to the obturator internus and quadratus femoris muscles.

The smaller foramen transmits the obturator internus muscle (its tendon), the internal pudic artery, vein, and nerve, and the nerve to the obturator internus muscle.

\section{DISSECTION.}

Cut through the gluteus medius at its outer and middle thirds. Reflect the two parts.

Gluteus Minimus. Figs. I 18, 127.

Origin.-From the external surface of the ilium between the middle and inferior curved lines, extending from the margin of the great sacrosciatic notch behind, to the notch between the anterior, superior, and inferior spines of the ilium. 
Insertion.-Into a rough area upon the front of the great trochanter.

A small bursa is found between the tendon and the trochanter.

Nerve Supply.-The superior gluteal; see Gluteus medius, page 605 .

Action.-The same as the gluteus medius, only in a less degree.

It is an abductor of the thigh; the anterior portion of the muscle rotates the thigh forward (inward), and the posterior portion backward (outward). It acts upon the pelvis to keep it level or bring the crest toward the great trochanter, also to swing the other side forward, as in walking.

The Superior Gluteal Nerve. Fig. 127.

This is formed by a branch from the lumbosacral cord and the first sacral nerve. Its course is through the great sacrosciatic foramen above the pyriformis muscle, forward between the gluteus medius and minimus muscles, which it supplies, and terminates in the deep surface of the tensor vaginæ femoris muscle (see page $55 \mathrm{I}$ ).

\section{DISSECTION.}

Remove the integument from the back of the leg, following the general directions on page 600 for exposing the thigh.

When the heel is reached, cut the skin flap away entirely.

The External or Short Saphenous Nerve. Figs. I22, I 28 .

This is formed at the middle of the back of the leg, superficial to the deep fascia (the branches which form it having pierced the deep fascia already), by the tibial and fibular communicating cutaneous nerves.

It descends along the outer surface of the leg, curves forward below the external malleolus, and runs along the 

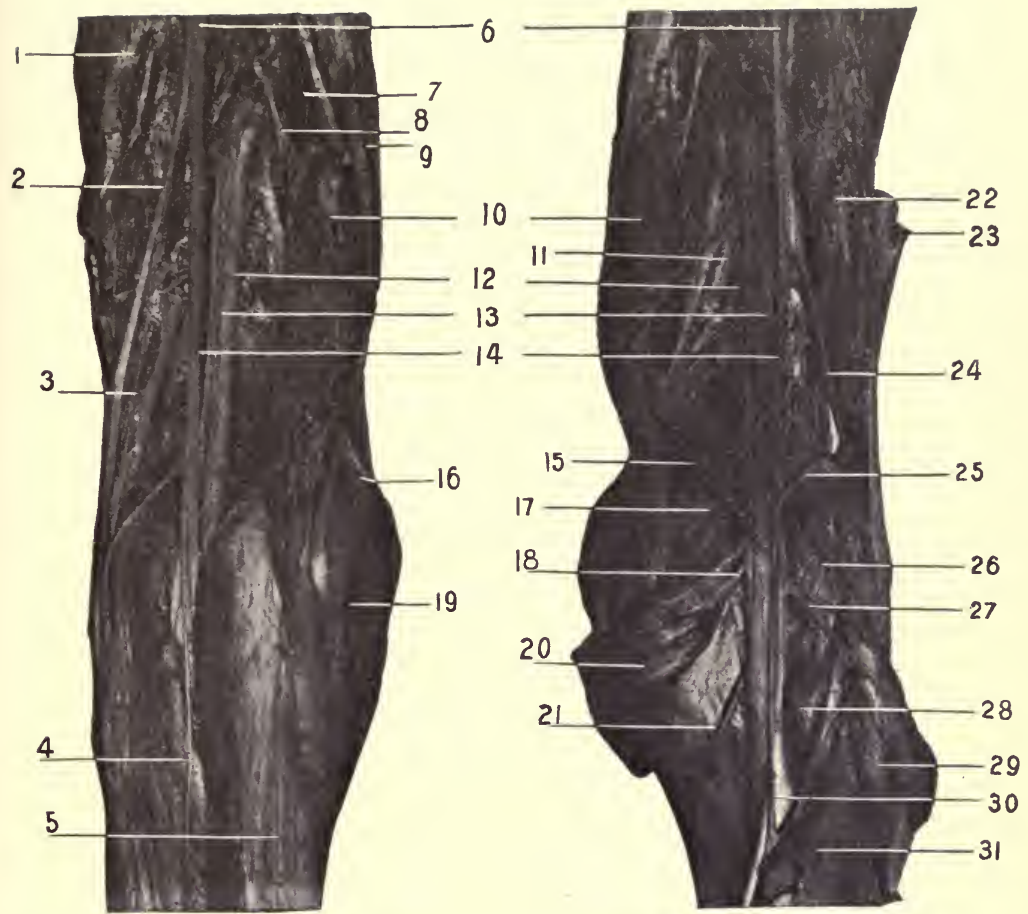

Fig. 128. Dissection of the Popliteal Space.-1, Short head of biceps. 2, External popliteal nerve. 3, Communicans peronei. 4, Communicans tibialis. 5, Gastrocnemius. 6, Great sciatic nerves. 7, Muscular branch to semimembranosus. 8 , Last perforating artery. 9, Semitendinosus. 10, Semimembranosus. 11, Tendon of adductor magnus. 12, Popliteal arteries. 13, Popliteal veins. I4, Internal popliteal nerves. 15, Internal superior articular artery. 16, Gracilis. 17, Nerve to inner head gastrocnemius. 18, Inferior muscular (sural) arteries. 19, Tendon of Sartorius. 20, Inner head gastrocnemius. 21, Inferior, internal articular artery. 22, Short head of biceps. 23, Long head of biceps (divided). 24, External popliteal nerve. 25, Superior, external articular artery. 26, Nerve to outer head of gastrocnemius. 27, Inferior muscular (sural) arteries. 28, Plantaris. 29, Outer head gastrocnemius. 30, Nerve to soleus. 3r, Soleus. 
outer border of the foot and little toe, where it terminates. The nerve communicates with the musculocutaneous on the dorsum of the foot, supplies the integument along its course, and is accompanied by the external saphenous vein.

\section{The External or Short Saphenous Vein.}

For its formation, see page 586 .

The vein follows upward along the outer part, then the back of the leg to the middle of the popliteal space, where it pierces the deep fascia and empties into the popliteal vein. Its tributaries are numerous cutaneous veins from the outer and back parts of the foot and leg, and a large descending one from the back of the thigh. It communicates with the deep veins through the deep fascia, and with the internal saphenous near its termination.

The Deep Fascia. See page 584 .

\section{DISSECTION.}

After cutting through the fascia in the middle line remove it together with the external saphenous vein, but leave the nerve behind.

Clean out the popliteal space.

The External Popliteal Nerve. Fig. I 28.

This is the outer branch of bifurcation of the great sciatic nerve, and runs from the upper part of the popliteal space along the inner border of the tendon of the biceps muscle to the neck of the fibula, around which it turns, lying close to the bone and covered by the peroneus longus muscle. Here it divides into its two terminal branches, musculocutaneous and the anterior tibial, for which see pages 588 and 598 .

\section{Branches of the External Popliteal.}

(I) Articular, to the knee joint, which they reach by following the superior and inferior external articular branches. 
of the popliteal artery.

(2) Cutaneous. Two in number, one to the upper and outer part of the leg. The other (the fibular communicating, peroneal, or nervus communicans peronei) passes to the back of the calf, where at the middle of the leg it joins with the tibial communicating (nervus communicans tibialis) to form the short or external saphenous nerve. (3) The recurrent articular nerve, a small branch at the point of bifurcation of the external popliteal; it follows the anterior tibial recurrent artery to the front of the knee.

\section{The Internal Popliteal Nerve. Fig. I 28.}

This is larger than the external popliteal and is the direct continuation of the great sciatic. It extends from the bifurcation of the great sciatic vertically through the middle of the popliteal space and becomes the posterior tibial at the lower border of the popliteus muscle.

The nerve is the most superficial of the important contents of the popliteal space. In the upper third it lies external to the popliteal vessels, in the middle directly behind them, and in the lower third to their inside. In front of the nerve lies the popliteal vein and in front of the vein the artery. The order then is, from behind forward, nerve, vein, and artery.

\section{Branches.}

(I) Articular, to the inner side and back of the knee joint being found with the superior and inferior internal and azygos articular branches of the popliteal. (2) Cutaneous, this is the tibial communicating (nervus communicans tibialis). It is given off the internal popliteal nerve at the middle of the popliteal space, descends in the interval between the two heads of the gastrocnemius, pierces the deep fascia of the leg, is joined by the fibular communicating, 
and the trunk becomes the external saphenous. (3) The muscular branches. These supply the gastrocnemius, plantaris, popliteus, and soleus, muscles.

Gastrocnemius. Figs. I 28, I 29, I 3 I.

Origin.-By two heads from the posterior surface of the femur above each condyle and from the condyloid ridges of the same.

Insertion.-By the tendo-Achillis, common to it and the soleus, into the lower part of the posterior surface of the os calcis.

A bursa is found under the inner head of the muscle and another between the tendo-Achillis and the upper part of the os calcis.

Nerve Supply. - The internal popliteal.

Action.-To flex the leg upon the thigh, to extend the foot upon the ankle.

In the first case the muscle acts with the flexors of the knee, and in the second with the extensors of the foot and ankle.

The Popliteal space. Fig. I 28.

This is the hollow behind the knee joint. It is diamondshaped.

Its boundaries are formed as follows :-

Above, the biceps externally, the semitendinosus, semimembranosus, the gracilis, and the sartorius, internally. Below, by the diverging heads of the gastrocnemius and in addition the plantaris at the outer side. On either side of the middle of the space are the condyles of the femur.

The "floor" of this space is formed by the back of the lower end of the femur, the posterior ligament of the knee joint, the posterior part of the articular rim of the tibia (covered by the above ligament) and the popliteus muscle 
(covered by the aponeurosis derived from the tendon of the semimembranosus muscle). The contents of this space, are : The popliteal artery, vein, nerves, and their branches; the small sciatic and terminal branch of the obturator nerves; the external or short saphenous vein; lymphatic glands, and adipose tissue.

The relations of the artery, vein and nerves.-The popliteal nerves are the most superficial, the external running along the inner edge of the tendon of the biceps muscle, the internal descending vertically through the space. The vein is deeper than the nerve and slightly internal to it (in the upper part of the space). The artery is still deeper than the vein (in close union with it) and a little to its inner side (in the upper half of the space).

\section{DISSECTION.}

Divide the two branches that go to form the external saphenous nerve, and the inner and outer head of the gastrocnemius just above their junction.

Turn the lower part of the muscle downward and separate the upper heads, saving their nerve supply. Clean the parts presenting

\section{Plantaris. Figs. I 28, I 29, I3 I.}

Origin.-From the lower part of the external bifurcation of the linea aspera, the posterior surface of the femur adjacent thereto, and from the posterior ligament of the knee joint.

Insertion.-By a long slender tendon which descends between the gastrocnemius and the soleus to be attached into the os calcis at the inner side of the tendo-Achillis.

Nerve Supply.-The internal popliteal.

Action.-A feeble flexor of the leg upon the thigh, and extensor of the foot upon the leg.

Popliteus. Fig. I 30.

Origin.-From the anterior portion of the transverse 
groove near the lower margin of the external condyle of the femur, from the posterior ligament of the knee joint.

Insertion.-Into the triangular surface of the tibia above the oblique line, and into the deep surface of the aponeurosis which covers the muscle.

Nerve Supply. - The internal popliteal, which distributes a branch to the anterior surface of the muscle.

Action.-To flex the leg upon the thigh; in flexion it will rotate the leg internally (pronation).

When the leg is extended the tendon of the muscle crosses the transverse groove upon the external condyle; in flexion the tendon lies in the groove.

Soleus. Figs. I 29, I 3 I.

Origin.-From the posterior surface of the head of the fibula and the upper third of the same, from the external intermuscular septum, from the oblique line and from the inner border of the tibia as low as the middle of the bone, from a tendinous arch which joins the back of the tibia and fibula, and covers the popliteal vessels and nerves.

Insertion.-By the tendo-Achillis, which it helps form, into the lower part of the posterior surface of the os calcis.

Nerve Supply._-The internal popliteal.

Action.-To extend the foot, as in raising the body in walking, etc.

The Tendo-Achillis. Figs. I 29, I 3 I.

This is the strongest tendon in the body. It is formed by the junction of the tendons of the gastrocnemius and soleus muscles, and is attached to the lower part of the posterior surface of the os calcis. Its dimensions are length; from the middle of the leg to the heel, three to five inches, five-eighths of an inch wide, three-eighths of an inch thick. 
The Popliteal Vein. Fig. I 28.

The popliteal vein is formed by the junction of the venæ comites of the anterior and posterior tibial arteries, at the lower border of the popliteus muscle, and passing upward becomes the femoral vein at the opening in the adductor magnus muscle. Its relation to the popliteal artery. It lies behind the artery and crosses it slightly obliquely from below upward, and from the inner to the outer side.

The Popliteal Artery. Figs. I 28, I 29, I 30.

The popliteal artery is a continuation of the femoral from the opening in the adductor magnus muscle to the lower border of the popliteus muscle, where it terminates by dividing into the anterior and posterior tibial arteries opposite the lower margin of the tibial tubercle. The course of the artery is indicated by a line drawn vertically through the popliteal space.

Relations. - The artery rests upon the floor of the popliteal space, which is formed by the posterior surface of the lower end of the femur, the posterior ligament of the knee joint, the posterior margin of the tibia (covered by the posterior ligament), and the popliteus muscle, from which it is separated by the fascia covering the muscle.

The popliteal vein lies close behind the artery, being a little external to it above, and slightly internal to it below.

The internal popliteal nerve is behind (or superficial to) both artery and vein, and crosses them about the middle of the space, from the outer to the inner side (above downward).

The semimembranosus muscle covers the upper part of the artery (vein and nerve), the inner head of the gastrocnemius, the lower part of it, the fascia (two layers) and integument for its entire course. 
The artery lies between the semimembranosus and biceps above, and between the two heads of the gastrocnemius below, the plantaris being at the outer side.

Branches of the Popliteal.

(I) Muscular or sural. These are divided into two groups, superior and inferior, from the upper and lower portions of the popliteal.

The superior are three or four in number, supply the structures of the upper half of the popliteal space and anastomose with the lower perforating branches of the profunda, and the superior articular branches of the popliteal.

The inferior sural, two in number, supply the muscles at the lower part of the popliteal space and calf of the leg.

From the inferior sural cutaneous branches are given off to the superficial structures upon the back of the leg.

A larger branch may accompany the external saphenous vein; if present it is called the external saphenous artery.

(2) Articular. Five in number. Figs. I I 9, I 2 I, I 28, I 29, I30. (a) The superior external articular. Passes transversely outward above the external head of the gastrocnemius and under (in front of) the biceps to the front of the outer side of the upper part of the knee, where it anastomoses with the descending branch of the external circumflex, above; the deep branch from the anastomotica magna and the superior internal articular, across the front of the knee; and the inferior external articular, below. (b) The inferior external articular. This takes a course outward under (in front of) the external head of the gastrocnemius, then under the external lateral ligament of the knee and tendon of the biceps muscle (just above the head of the fibula) to the front of the lower part of the knee, where it anastomoses with the external superior external articular, above ; the anterior tibial recur- 


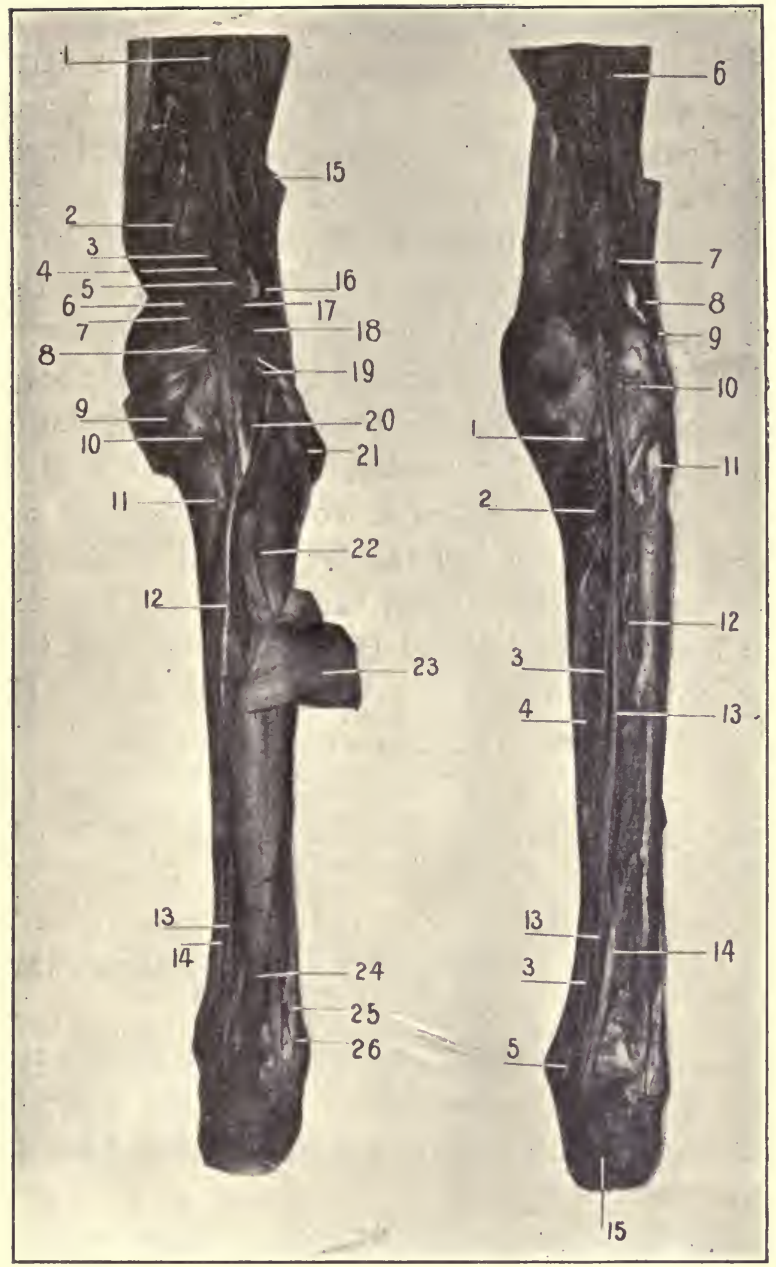

Fig. 129. Dissection of I,eg, Posterior.- I, Great sciatic nerve. 2, Tendon of adductor magnus. 3, Popliteal artery. 4, Popliteal vein. 5, Popliteal nerve. 6 , 7, 8, 9, 10, see I5, I7, I8, 20, 21, Fig. I28. I 1, Popliteus. I2, Tendon of plantaris. 13. Posterior tibial nerve. 14, Posterior tibial artery. 15, I6, 17, 18, 19, 20, 21, see 23, $24,25,26,27,28,29$, Fig. 128. 22, Soleus. 23, Lower part of gastrocnemius. 24, Tendo-Achillis. 25, 26, Tendons of peroneus longus and brevis.

Fig. 130.-1, Internal inferior articular artery. 2, Popliteus muscle. 3, 3, Posterior tibial artery. 4, Flexor longus digitorum. 5, Tendon of tibialis posticus. 6, Great sciatic nerve. 7, Internal and 8, External popliteal nerves. 9, Biceps tendon. 10, External inferior articular artery. 11, Head of fibula. 12, Peroneal artery. 13, 13, Posterior tibial nerve. I4, Flexor longus hallucis. 15, Os calcis. 
rent, below; and the internal inferior articular across the front of the knee. (c) The superior internal articular. Arises from the popliteal opposite the external articular. It runs inward along the insertion of the inner head of the gastrocnemius, then under (in front of) the tendons of the inner ham-string and adductor magnus muscles to the front of the upper part of the knee and here anastomoses with the deep branch of the anastomotica magna, above; the superior external articular, across the front of the knee; and the inferior internal articular, below. (d) The inferior internal articular. Takes a course downward and inward under (in front of) the inner head of the gastrocnemius muscle, lying upon the popliteus muscle, then passes between the internal lateral ligament and the tibia, to the front of the lower part of the joint. Here it ends in branches which anastomose with the inferior external articular, over the front of the tibia; and the superior internal articular, and superficial branch of the anastomotica magna above. (e) The azygos articular. This comes off the front of the popliteal, at the middle of the back of the knee and passes directly forward into the joint.

The arterial anastomosis about the knee joint is arranged in a superficial and deep system. The former lying between the integument and fascia and the latter close to the bones.

\section{DISSECTION.}

Divide the tendon of the plantaris and tendo-Achillis near their insertion and cut the soleus away from its tibial origin.

Turn the muscles outward. Clean the deep layer of muscles, and the vessels and nerves.

Inasmuch as the deep muscles of the leg pass to their insertion into the foot this part of them has not been dissected out at present. 
They will be described at this time however, and the student can review them after the foot has been dissected.

Flexor Longus Digitorum. Figs. I 30, I 3 I, I 33.

Origin.-From the posterior surface of the tibia, extending from the oblique line to the junction of the middle and lower thirds of the bone, from the deep fascia which covers the muscle, and from the intermuscular septum.

Insertion (see Dissection of Foot). - Into the bases of the last phalanges of the four outer toes.

The tendon of the muscle occupies a special fibrous canal lined with synovial membrane behind the internal malleolus (being posterior to the tendon for the tibialis posticus). Turning forward into the foot the tendon crosses below that for the flexor longus hallucis, to which it is connected by a fibrous band, then receives the insertion of the flexor accessorius and divides into the four terminal tendons; they then pass through a button-hole slit in the tendons of the short flexor and on to their insertion. (See the Lumbricales.

Nerve Supply.-The posterior tibial.

Action.-To flex the toes, beginning with the last phalanges and passing backward, to flex the joints of the foot (mediotarsal), to extend the foot.

Flexor Longus Hallucis (Pollicis). Figs. I30, I 3 I, I 33.

Origin.-From the posterior (and internal) surface of the fibula for its lower two-thirds, from the deep surface of the fascia which covers the muscle, from the intermuscular septa on either side.

Insertion (see Dissection of Foot). - Into the base of the last phalanx of the great toe.

The tendon of this muscle slightly grooves the back of the tibia and astragalus, passes forward under the sustenta- 
culum tali; for this distance the tendon is contained in a fibrous canal lined with synovial membrane. It continues forward above the tendon of the flexor longus digitorum, to which it gives a slip, and passes to its insertion.

Nerve Supply. - The posterior tibial.

Action.-To flex the great toe, to flex the mediotarsal joints of the foot, to extend the foot upon the leg.

The two above muscles are intimately concerned in walking and all allied acts; by their contraction the integrity of the arch of the foot is preserved.

Tibialis Posticus. Figs. I 30, I 3 I.

Origin.-From the posterior surface of the tibia external to the origin of the flexor longus digitorum, and extending from the oblique line to the middle and lower thirds of the shaft ; from the postero-internal surface of the fibula internal to the origins of the soleus and the flexor longus hallucis, and extending from the neck to the lower fourth of the shaft; from the posterior surface of the interosseous membrane for its upper three-fourths; from the intermuscular septa on either side and from the surface of fascia covering the three deep muscles of the leg.

Insertion (see Foot).- Into the tubercle of the scaphoid, the os calcis (anterior part of the sustentaculum tali), the under surface of the base of the first metatarsal, three cuneiform and cuboid bones, by a broad expansion of the tendon of the muscle which passes into the ligaments which bind the bones of the foot together. The tendon of the muscle passes behind the internal malleolus, being the most anterior of the structures at this place. In this course it is contained in a stout fibrous canal lined with synovial membrane.

Nerve Supply. - The posterior tibial. 


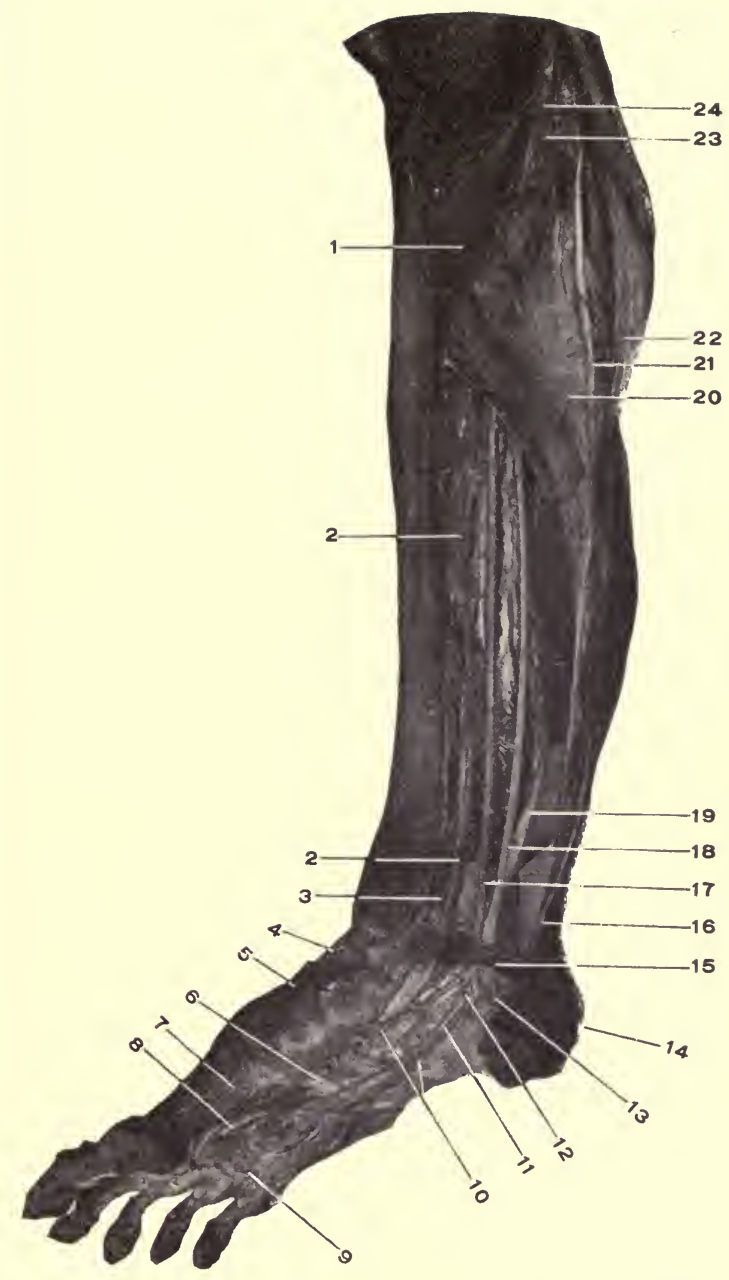

Fig. 131. Dissection of Leg and Foot, Posterolateral. Region.-i, Popliteus. 2, Flexor longus digitorum. 3, Tibialis posticus. 4, Dorsalis pedis artery. 5 , Tendon (divided) tibialis anticus. 6, Tendon of peroneus longus. 7, Flexor brevis hallucis. 8, Adductor hallucis. 9, Transverse pedis. Io, Internal and external plantar arteries. II, External plantar nerve. 12, Cut end of internal plantar nerve. I3, Internal calcanean nerve. 14, Os calcis. 15, Internal annular ligament. 16, TendoAchillis and tendon of plantaris. I7, Posterior tibial artery. 18, Posterior tibial nerve. 19, Flexor longus hallucis. 20, Soleus. 21, Plantaris. 22, Gastrocnemius. 23, Internal popliteal nerve. 24, Popliteal artery. 
Action.-To extend the foot upon the ankle, to adduct the foot, to raise the inner border of the sole (inversion). The action of this muscle tends to prevent the production of flat foot by holding the tarsal bones firmly together, and inverting the sole.

Peroneus Longus. Figs. I23, I24, I 34.

Origin.-From the external tuberosity of the tibia, from the outer surface of the head and upper two-thirds of the shaft of the fibula (lying posterior to the peroneus brevis), from the intermuscular septa on either side, and from the inner surface of the deep fascia in its upper third.

Insertion (see Foot).- The tendon of the muscle runs behind the external malleolus (having the tendon of the peroneus brevis in front), in front of the peroneal tubercle on the os calcis, through the groove in the cuboid, across the foot to the base of the first metatarsal bone and the internal cuneiform.

Behind the malleolus the two tendons have a common synovial sheath, in the sole the long tendon has a special one. A sesamoid bone is often found in the tendon as it crosses the cuboid bone.

Nerve Supply.-The musculocutaneous which passes through the muscle.

Action.-To extend the foot on the leg, to abduct the fore part of the foot, to depress the ball of the great toe (inner border of the foot) and raise the outer border of the foot (producing eversion of the sole). It is an important factor in maintaining the anteroposterior and transverse arches of the foot and in walking.

Peroneus Brevis. Figs. I23, I 24.

Origin.-From the lower two-thirds of the outer surface of the fibula, overlapping the peroneus longus for its lower 
third. From the intermuscular septa and deep fascia of the leg.

Insertion (see Dorsum of Foot).-Into the prominent base of the fifth metatarsal bone. The tendon of the muscle lies in front of that for the peroneus longus; as the two pass behind the external malleolus, one synovial sheath here encloses them both.

Nerve Supply.-The musculocutaneous.

Action.-To extend the foot, to abduct the foot, to raise the outer border of the foot. In all its actions it is much inferior to the peroneus longus.

The Posterior Tibial Nerve. Figs. I28, I30, I3I.

This is the continuation of the internal popliteal nerve from the lower border of the popliteus muscle to the midpoint between the internal malleolus and the point of the heel, where it terminates by dividing into the external and internal plantar nerves.

The posterior tibial nerve lies first at the inner side of the corresponding artery, but crosses behind the artery (just after the peroneal branch is given off) to the outer side, which position it keeps for the rest of its course.

Branches.-(I) Muscular to the tibialis posticus, flexor longus digitorum, and flexor longus hallucis. (2) Articular, one or more filaments to the ankle joint. (3) Cutaneous, internal calcanean (calcaneoplantar). Pierces the internal annular ligament and supplies the integument over the inner side of the heel, and back part of the sole.

The Anterior Tibial Artery. See page 593.

The Posterior Tibial Artery. Figs. I30, I3I.

This is the continuation of the popliteal, of which it is the larger terminal branch, from the lower border of the 
popliteus muscle to the point mid-way between the internal malleolus and the point of the heel, at which place it divides into the internal and external plantar vessels.

The artery is covered by the skin, superficial and deep fascia, the gastrocnemius and soleus muscles, and the deep intermuscular fascia of the leg. The muscular covering is wanting in the lower third of its course.

The artery crosses the posterior surface of the tibialis posticus, flexor longus digitorum, tibia, and the internal lateral ligament of the ankle joint. At the ankle the artery is covered by the annular ligament and origin of the abductor hallucis muscle.

The artery has an accompanying vein upon either side which frequently communicate by cross branches. The posterior tibial nerve is at its beginning at the inner side of the artery, but crosses it where the peroneal artery arises, and continues at the outer side of the artery for the rest of its course.

Behind the internal malleolus the posterior tibial artery is the central structure of five others.

From before backward the order is, tendon of the tibialis posticus, tendon of the flexor longus digitorum, artery, posterior tibial nerve, and (at a little distance, and on a deeper plane) the tendon of the flexor longus hallucis. Besides these the venæ comites lie, one in front the other behind the artery and next to it.

Branches.-(I) Muscular, numerous to the surrounding muscles. (2) The mutricnt artery of the tibia, largest in the body, arises near the beginning of the posterior tibial, and enters the nutrient foramen at the upper and middle thirds of the outer border of the tibia.

(3) Cutaneous (two or three), which reach the inner side of the leg between the superficial and deep sets of muscles.

(4) A communicating 
branch to the peroneal under the tendon of the flexor longus hallucis, and about an inch above the ankle joint. (5) One or more small internal malleolar branches to the inner side of the ankle. (6) The artery below, which is its largest branch.

\section{The Peroneal Artery. Figs. I 25, I 30.}

This is a branch from the posterior tibial, about an inch below the lower border of the popliteus muscle; it turns outward and downward along the fibular side of the leg, lying deeply placed between the tibialis posticus and flexor longus hallucis, or else under the latter. About three inches above the ankle joint it gives off the anterior peroneal and then continues under the name of the posterior peroneal to behind the external malleolus, where it breaks up into several terminal branches to the outer and back part of the os calcis. There are two accompanying veins. Branches of the Peroneal Artery.

(I) Muscular branches to the adjacent muscles. mutrient artery to the fibula, in the middle and lower thirds of the leg. (3) The anterior peroneal. This artery is given off about three inches above the ankle joint, passes forward between the tibia and fibula and through the interosseous membrane to the front of the leg, then turns downward to supply the outer part of the front of the ankle. See page 594. (4) The communicating branch (or branches), anastomoses with the posterior tibial across the lower end of the tibia. See Posterior Tibial. (5) The cutaneous branches to the integument upon the outer side of the leg. (6) The external calcanean, supplies the parts at the outer side of the os calcis. This with the small terminal branches of the peroneal anastomose about the ankle with the external malleolar, anterior peroneal, tarsal 
(on outer side) and with the posterior tibial and internal calcanean branch of the external plantar (on the inner side).

\section{DISSECTION.}

No incision through or around the sole is necessary.

Working from the heel forward, dissect the skin off in one piece to the sides of the sole and toes (where its removal was stopped when on the dorsum of the foot).

Cut away the entire flap.

The superficial fascia will be found as a thick pad of fatty and connective tissue covering the sole.

It is to be entirely removed so as to thoroughly expose the deep fascia. For this purpose the curved scissors will be found to be the most useful.

Be careful to save the digital nerves which lie imbedded in the fat between the processes of the plantar fascia.

The Superficial Fascia, or the subcutaneous tissue.

This layer is much thickened in the foot to form pads at the bearing points of the sole for the protection of the deeper parts. These pads are found at the heel, ball of the foot and toes, and are seen to be composed of a coarse meshed framework of connective tissue filled with fat.

The Plantar Fascia. Fig. I 32.

This is the specialized portion of the deep fascia of the foot which preserves its shape and protects its deeper parts. It covers the entire sole of the foot, but the central portion is much stronger and thicker than the lateral portions.

The middle portion of the plantar fascia is narrow behind where it is attached to the under surface of the back part of the os calcis, and widens out as it passes forward to the fore part of the foot where at the base of the toes it divides into five slips, one for each toe. Each slip passes forward, splits to enclose the tendons of the long and short flexor muscles of the toes, and is inserted into the lateral ligaments of the metatarsophalangeal articulation, and the deep transverse ligament of the heads of the tarsal bones. 


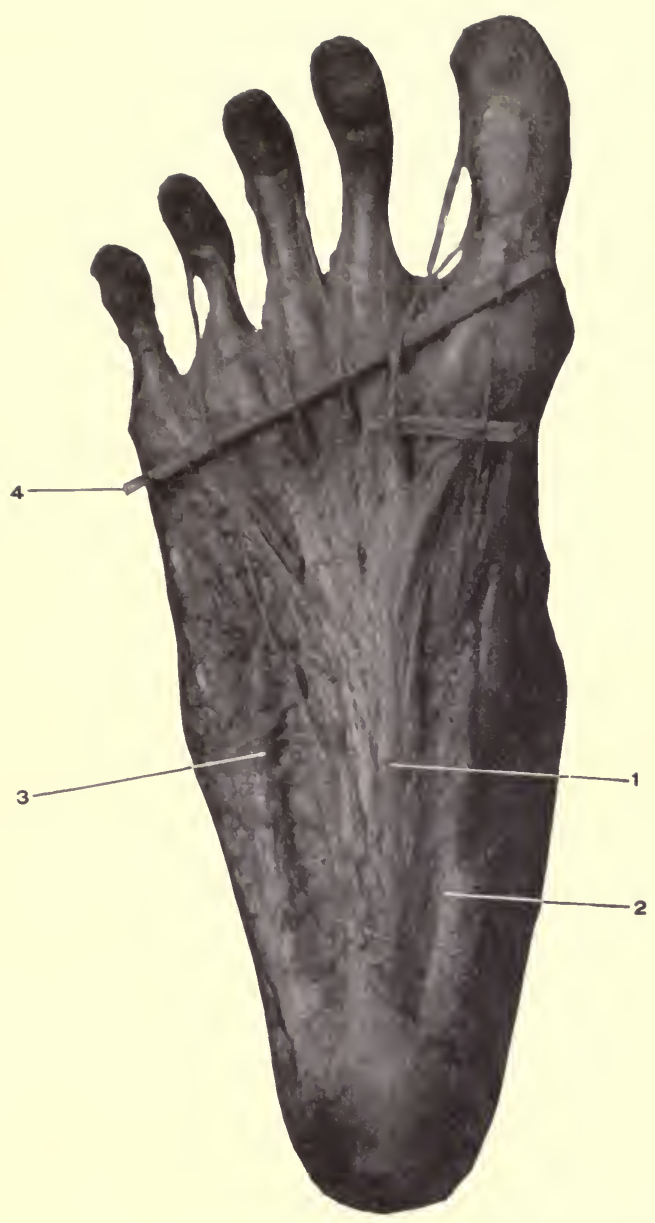

Fig. 132. Dissection of the Foot, Plantar Surface.-i, Middle or central portion of plantar fascia. 2, Internal and 3, External portions. 4, The digital nerves (raised on a small rod). 
Between these fasciculi pass the lumbricales and interossei muscles and the digital vessels and nerves.

The central portion of the plantar fascia is connected at its margins to the ligaments which cover the under surface of the bones of the foot, by fibrous septa, the external and internal intermuscular septa; these divide the foot into three compartments.

The lateral portions of the fascia are continuous with the margins of the central portion and pass into the deep fascia covering the dorsum of the foot and the lateral annular ligaments of the ankle. In addition the external portion has a strong band which extends from the outer surface of the os calcis and the external annular ligament, and is attached to the base of the fifth metatarsal bone, and the internal portion passes from the inner side of the os calcis and internal annular ligament forward, to be inserted into the base of the first phalanx of the great toe.

\section{DISSECTION.}

Remove the plantar fascia by cutting away altogether the outer and inner portions. Incise the central part transversely about three inches from the heel, raise the anterior portion from the muscle beneath and carefully dissect it forward, dividing the strong intermuscular septum on each side of the muscle, until the insertions of the fasciculi are reached. Stop here to examine how the flexor tendons pass through the divided fasciculi while the digital nerves, vessels, and lumbrical muscles lie between the fasciculi themselves, then remove the fascia entirely. Leave the posterior portion attached to the muscle.

\section{The First Layer.}

The Flexor Brevis Digitorum. Fig. I 33.

Origin.-From the anterior part of the greater (inner) tubercle of the os calcis, from the deep surface of the middle portion of the plantar fascia for its posterior third, and from the sides of the intermuscular septa. 
Insertion.-By four tendons into the bases of the second phalanges of the four smaller toes. The tendons of the brevis divide for the passage of the tendons of the flexor longus digitorum, then reunite, forming a buttonhole for the passage of the tendons of the flexor longus digitorum, they then again divide and pass to their insertion into the bases of the second phalanges.

Nerve Supply.-The internal plantar.

Action.-To flex the four outer toes, beginning at the second phalanges, to flex the bones of the foot and preserve the arch.

Abductor Hallucis (Pollicis). Fig. I 33.

Origin.-From the inner margin of the greater tubercle of the os calcis, the deep part of the internal annular ligament, the side of the intermuscular septum, and from the deep surface of the plantar fascia covering it.

Insertion.-Into the inner margin of the base of the first phalanx of the great toe.

The tendon of this muscle is joined before its insertion by the inner half of the tendon of the flexor brevis hallucis.

Beneath the origin of the muscle is a fibrous arch which reaches from the internal intermuscular septum to the deep ligamentous structures at the inner border of the foot, and through which pass the plantar vessels and nerves.

Nerve Supply.-The internal plantar.

Action.-To abduct the great toe from the middle line of the foot (passes through the middle of the second toe), to flex the first phalanx of the great toe, to preserve the arch of the foot.

Abductor Minimi Digiti. Fig. I33.

Origin.-From the under surface of the os calcis in front of both tuberosities, from the side of the external intermus- 
cular septum, and from the deep surface of the band reaching from the outer side of the os calcis (the lesser tuberosity) to the base of the fifth metatarsal bone.

Insertion. - The outer side of the base of the first phalanx of the little toe, the outer side of the base of the fifth metatarsal bone.

Nerve Supply.-The external plantar.

Action.-To abduct the little toe from the middle line of the foot, to flex the first phalanx of the little toe. (Abduction of the first phalanx carries with it the rest of the phalanges, but flexion is only of the first phalanx).

\section{The Internal Plantar Nerve. Figs. I 32, I 33.}

This is larger than the external plantar. It is accompanied by the corresponding artery for a part of its course.

It runs forward between the abductor hallucis and the flexor brevis digitorum muscles and ends at the middle of the foot by dividing into three (or four) digital branches. These are named from within outward.

(I) The first digital branch supplies a muscular branch to the flexor brevis hallucis and cutaneous branches to the inner side of the great toe. (2) The second sends a muscular branch to the first lumbricalis, then bifurcates to supply the adjacent sides of the first and second toes. (3) The third supplies the second lumbricalis, and the contiguous sides of the second and third toes. (4) The fourth; this communicates with the superficial division of the external plantar nerve, and supplies the opposite sides of the third and fourth toes. The digital nerves supply the joints of the toes which they pass over. Their branches to the toes after their bifurcation are called collateral digital nerves. There are six digital and ten collateral digital nerves, the internal plantar furnishing four of the digital and seven 


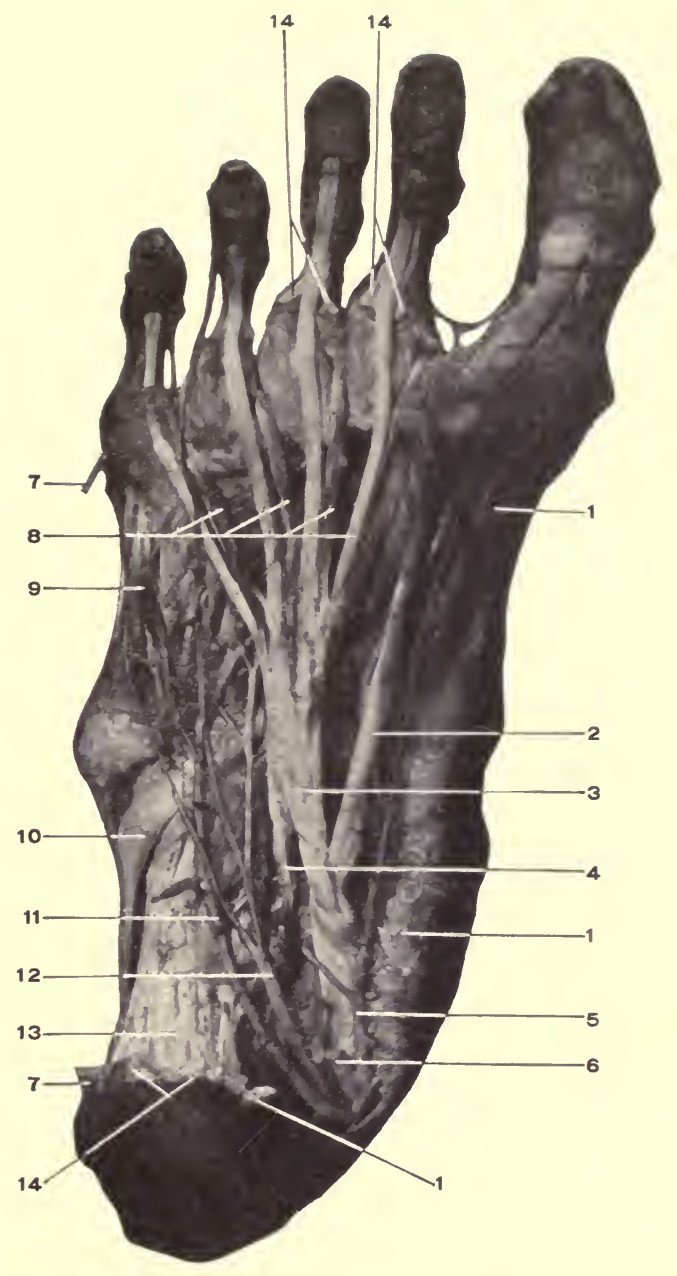

Fig. 133. Dissection of Foot, Plantar Surface.-I, I, I, Abductor hallucis removed. 2, Tendon of flexor longus hallucis. 3, Tendon of flexor longus digitorum. 4, Flexor accessorius. 5, Internal plantar artery. 6, Cut end of internal plantar nerve. 7, 7, Abductor minimi digiti removed. 8, Lumbricales. 9. Flexor brevis minimi digiti. Io, Tendon of peroneus longus. II, External plantar artery. 12, External plantar nerve. 13, Long inferior calcaneocuboid ligament. 14, 14, 14, Remains of flexor brevis digitorum. 
collateral digital branches; the external plantar furnishes the remainder, two digital and three collateral digital nerves. These are numbered from within outward.

The Internal Plantar Artery. Figs. I 33, I 34.

The smaller branch of the posterior tibial behind the internal malleolus. Its course is forward and slightly outward, under cover of the abductor hallucis muscle, then between this muscle and the flexor brevis digitorum, to the ball of the great toe where it terminates in branches which anastomose with the fifth and sixth plantar digital arteries.

\section{The Branches of the Internal Plantar Artery.}

(I) Muscular, to the adjacent muscles. (2) Cutaneous, to the integument of the inner segment of the sole. Anastomotic, beneath the abductor hallucis to the inner side of the foot to anastomose with the internal malleolar and internal tarsal arteries of the dorsum of the foot. Superficial digital, small branches which pass forward in the inner three interosseous spaces with the digital branches of the internal plantar nerve, and anastomose with the digital arteries.

\section{DISSECTION,}

Section the internal plantar nerve where it appears in the sole. Divide the abductor muscles about one inch in front of their origin, and the flexor brevis digitorum transversely across at its middle.

Reflect the parts.

\section{The Second Layer.}

Flexor Accessorius. Fig. I 33.

Origin.-The internal, larger, head, from the inner surface of the os calcis. The external, smaller, head from the outer margin of the os calcis in front of the external tuberosity, and from the long plantar ligament. 
Insertion.-Into the outer margin and upper surface of the tendon, of the flexor longus digitorum.

Nerve Supply.-The external plantar.

Action.-To convert the oblique pull of the flexor longus digitorum into a direct one, and thus assist in flexing the four outer toes. Acting alone, or when the flexor longus digitorum is blocked by the full extension of the ankle, it will still flex the toes.

The Lumbricales. Four in number. Fig. I33.

Origin.-Arise from the clefts between the tendons of the flexor longus digitorum (being attached to two adjacent tendons excepting the most internal, which arises from the inner margin of the internal tendon).

Insertion.-Into the aponeurotic expansion of the tendon of the extensor longus digitorum at the inner side of the four outer toes.

Nerve Supply. - The two (sometimes only one) inner muscles are supplied by the internal plantar, the two (sometimes three) outer are supplied by the external plantar.

Action.-These muscles will flex the first set of phalanges (as their tendons pass below the centre of motion of the metatarsophalangeal articulations), and extend the last two sets of phalanges (because their tendons are inserted into the tendons of the long extensor muscle above the centre of motion of these articulations). Besides this common action, the internal one will abduct the second toe, and the rest adduct the three outer toes with reference to the middle line of the foot (which passes through the centre of the second toe).

For the tendons of the flexor longus hallucis and the flexor longus digitorum, see these muscles, page 627 . 
The External Plantar Nerve. Figs. I 33, I 34.

This is the outer branch into which the posterior tibial divides behind the internal malleolus. The nerve (with the external plantar artery) runs forward and outward between the flexor brevis digitorum and the flexor accessorius, and divides into two branches at the base of the fifth metatarsal bone, the superficial and deep.

Before this division the nerve supplies muscular branches to the flexor accessorius and the abductor minimi digiti, and cutaneous twigs to the outer border of the sole of the foot.

The superficial branch. This supplies muscular branches to the interossei in the space between the fourth and fifth toes, and the flexor brevis minimi digiti, a communicating branch to the internal plantar, and digital branches to the adjacent one and one-half outer toes (adjacent side of the fourth and fifth, and outer side of the latter).

The deep branch. This accompanies the external plantar artery into the deep parts of the foot. It supplies the two (sometimes three) outer lumbricales, adductor hallucis, transversus pedis, and the rest of the interossei (those in the three inner spaces).

The External Plantar Artery. Figs. I33, I34.

This is the larger branch into which the posterior tibial divides behind the internal malleolus. The artery passes forward and outward, being covered by the abductor hallucis and the flexor brevis digitorum, to the outer segment of the foot until it reaches the base of the fifth metatarsal bone, where it turns short inward, passing deeply between the interossei muscles and the lumbricales and flexor tendons, and terminates at the posterior part of the space between the first and second metatarsal bones where it forms 
a junction with the communicating branch of the dorsal pedis and so completes the plantar arch. The artery is accompanied by two venæ comites and the external plantar nerve.

Branches of the External Plantar Artery.

(I) Muscular, numerous small branches to the near-by muscles.

(2) Calcanean (internal), two or three branches to the inner side of the os calcis.

(3) Cutaneous, to the integument along the outer border of the sole.

(4) Anastomotic, pass over the outer border of the foot to anastomose with the external malleolar, tarsal and metatarsal branches upon the dorsum of the foot.

All these branches come off the artery before it turns inward to form the plantar arch.

From the plantar arch arise the following :

(5) Articular, pass backward to the joints of the tarsus.

(6) Posterior perforating, three, which ascend through the back part of the three outer interosseous spaces and anastomose on the dorsum of the foot with the dorsal interosseous arteries (branches of the metatarsal artery. See page 596).

(7) Digital branches. Four in number; arise from the front of the arch, (a) The first digital, from the beginning of the arch at the base of the fifth metatarsal bone, to the outer side of the little toe, crossing in its course the flexor brevis, and the adductor minimi digiti muscles. and $(d)$ The second, third, and fourth digital arteries pass forward upon the interossei muscles through the middle of the fourth, third and second interosseous spaces to the web of the toes where each divides into two collateral digital branches to the adjacent sides of the toes. 
The fifth and sixth digital arteries are both branches of the communicating artery from the dorsum of the foot. The sixth supplies the inner side of the great toe and anastomoses backward with the internal plantar artery; the fifth takes a forward course like the other digital arteries, divides into two collateral digital branches at the web of the toes and supplies the opposing sides of the great and second toes.

There are then six digital, and ten collateral digital arteries, numbered from without inward.

\section{DISSECTION.}

Divide the tendon of the flexor longus digitorum just in front of the accessorius and reflect it, with the lumbricales, forward.

Clean all the remaining muscles and tendons.

\section{The Third Layer.}

Flexor Brevis Hallucis (Pollicis). Fig. I 34 .

Origin.-From the inner border of the cuboid bone, from the under surface of the tendon of the tibialis posticus, over the cuneiform bones.

Inscrtion.-By a divided tendon, one part with the abductor hallucis into the inner border of the base of the first phalanx, the other with the tendon of the adductor hallucis into the outer border of the same bone. A sesamoid bone is found in each head.

Nerve Supply.-The internal plantar.

Action.-To flex the first phalanx of the great toe.

The Adductor Hallucis. Fig. I 34.

Origin.-From the plantar surface of the bases of the second, third, and fourth metatarsal bones, and from the sheath of the tendon of the peroneus longus.

Insertion.-Along with the outer head of the flexor 


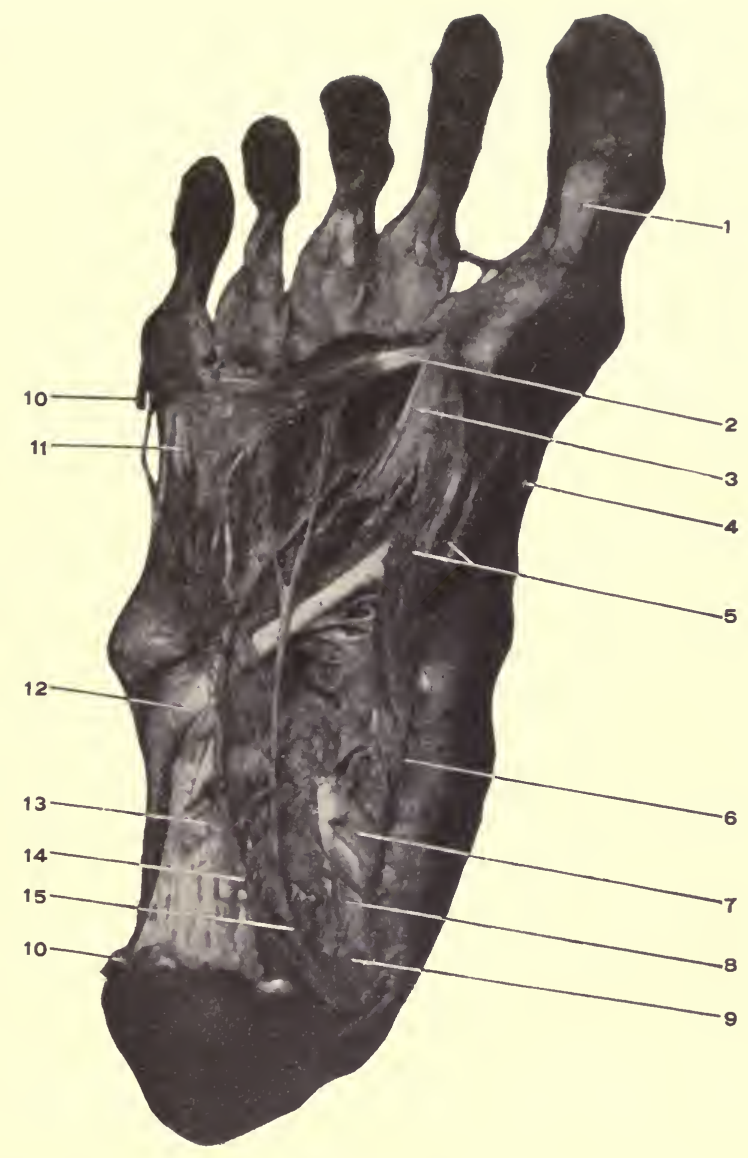

Fig. 134. Dissection of Foot, Plantar Surface.-1, Cut tendon of flexor longus hallucis. 2, Transversus pedis. 3, Adductor hallucis. 4, Abductor hallucis (end of tendon). 5, Flexor brevis hallucis. 6, Internal plantar artery. 7, Cut tendon of flexor longus digitorum. 8, Cut tendon of flexor longus hallucis. 9, Cut end of internal plantar nerve. ro, ro, Cut ends of abductor minimi digiti. II, Flexor brevis minimi digiti. 12, Tendon of peroneus longus. 13, Long inferior calcaneocuboid ligament. 14, External plantar artery, plantar arch and branches. 15, External plantar nerve. 
brevis hallucis into the outer surface of the base of the first phalanx of the great toe.

Nerve Supply. - The external plantar nerve.

Action.-To adduct and flex the first phalanx of the great toe.

Transversus Pedis. Fig. I 34.

Origin.-From the under surface of the three outer metatarsophalangeal articulations, and from the deep transverse metatarsal ligaments.

Insertion.-In common with the adductor hallucis, see above.

Nerve Supply.-The external plantar.

Action.-To adduct the first phalanx of the great toe, to draw the metatarsal bones together.

Flexor Brevis Minimi Digiti. Fig. I 33 .

Origin.-From the under surface of the base of the fifth metatarsal bone and from the sheath of the tendon of the peroneus longus muscle. Into the outer part of the base of the first phalanx of the little toe.

Nerve Supply.-The external plantar.

Action.-To flex and abduct (feebly) the first phalanx of the little toe.

The Fourth Layer.

Plantar Interossei.-Three in number. (Dorsal, four, see page 592.) Fig. I 34.

Origin.-From the under and inner surface of the three outer metatarsal bones.

Insertion.-Slightly into the inner portion of the base of the first phalanges of the third, fourth, and fifth toes, and more especially into the inner margin of the aponeurotic expansion of the tendon of the extensor longus digitorum at the back of the first phalanges of the above toes. 
Nerve Supply.-The external plantar.

Action.-These muscles have the action common to the dorsal interossei and the lumbricales, of flexing the first set. of phalanges and extending the rest; their special action is to adduct the first phalanges of the three outer toes to the middle line running through the second toe.

\section{DISSECTION.}

The tendons of the peroneus longus, and tibialis posticus remain to be dissected. For these muscles see pages 630 and 628 . 


\section{ADDENDUM.}

\section{Pronator Quadratis.}

Origin.-From the antero-internal surface of the ulna for its lower one-fourth.

Insertion.-Into the anterior surface and outer border of the radius for its lower one-fourth.

Nerve Supply. - The anterior interosseous (median). Action.-To pronate the forearm.

The Axillary Space.-This is an irregularly-shaped pyramid, with the following boundaries :-

Anteriorly, the clavicle; the subclavius, pectoralis major and minor muscles; the costocoracoid membrane; the pectoral and clavipectoral fascix (the latter an extension of the costocoracoid membrane).

Posteriorly, the subscapularis, teres major, and latissimus dorsi muscles.

Internally, the serratus magnus muscle; portions of the first to the fourth ribs inclusive, with the intervening muscles and fascia.

Externally, the upper portion of the humerus; long head of the biceps and the coracobrachialis.

The apex is between the clavicle and first rib, and is occupied by the brachial plexus and large blood vessels.

The base is formed by the axillary fascia.

The average weight of the adult human brain is, for males, $49 \frac{1}{2}$ ounces; for females, 44 ounces. 


\section{INDEX.}

ABDOMEN, boundaries of, 432

dissection of, 435

exterior of, 432

interior of, 462

dissection of, 473

landmarks of, 432

subcutaneous nerves of, 436

superficial fascia of, 436

lymphatics of (inguinal), 436 veins of, 436

Abdominal aorta, 5 I5

dissection of, 515

cavity, 432

muscles, nerve supply for, 453

regions, 435

ring, external, 444

internal, 456

rings, formation of, 457

viscera, covered by peritoneum, 482 seen on opening the abdomen, 479, $48 \mathrm{I}, 489$

Abducens nerve (sixth cranial), 57, 201

Abductor hallucis, 637

minimi digiti, 306, 637

pollicis, 306

Abscess, cervical, I4I psoas, 525

retropharyngeal, I4I

Accelerator urinæ, 410

Accessorius, 375

Achillis, tendo-, 622

Acromial artery, 272, 278

nerves, 76,91

Acromioclavicular joint, 256

Acromion process, 256

Acromiothoracic artery and branches, 272,278

Action of muscles on vocal cords, I47

Adam's apple, 69

Adductor brevis, 576

hallucis, 644

longus, 566

magnus, 578

(obliquus) pollicis, 308

(transversus) pollicis, 308

Afterbrain, 169, 171, I75

Ala cinerea, 252

Alcock's canal, 399, 4II

Alimentary canal, development, 462

Ampulla of vas deferens, 542

Amygdaloid lobe of cerebellum, 242 nucleus, 236

Anal fascia, 402

Anastomosis about the elbow, 387 about the knee, 624,626

"crucial," 580, 6I4
Anastomotica magna (brachial), 287

(femoral), deep branch, 573

superficial branch, 557

Anatomical " points," 599

"canals," 446, 599

" rings," 446, 599

Anconeus, 38I

Angle of penis, 440

Angular artery, 65

convolution (or gyrus), 187

process of frontal bone, external, I8 internal, 18

Ankle, annular ligaments of, anterior, 584 external, 585 internal, 586

Annular ligament of wrist, anterior, 302 posterior, 378

Annulus ovalis, 355

Ansa hypoglossi, 88

Anterior crural nerve, $532,557,568$ muscular branches of, 568

temporal artery, 26

tibial artery and branches, 593, 594

triangles of neck, 82

Anterolateral ganglionic arterial group, I62

Anteromedian ganglionic arterial group, 162

Antrum of Highmore, opening of, 148

Anus, 406

Aorta, 334

abdominal, 515

arch of, 334

ascending, 334

descending, 338

thoracic, 338

branches of, 345

transverse, 336

Aperture of Eustachian tube, I4I, 145

of larynx, I 45

of mouth, I4I

of nares, $14 \mathrm{I}$

of thorax (superior), 323

Apex of heart, 260

of lung, 262

Aponeurosis of external oblique (abdo-

lumbar, 451 men), 444

of occipitofrontalis, 25

pharyngeal, I 24

transversalis, 454

vertebral, 373

Appendices epiploicæ, 500

Appendix, auricular, 354, 355

ensiform, 255, 432

vermiform, 476,500 
Aqueduct of Sylvius, 171, 240 development of, 175

Arachnoid, lymphatics of, 156 membrane, 154 nerves of, 156 removal of, 156 septum, 392 spinal, 390

Arachnoidal villi, 155

Arantius, corpus of, nodules, 355

Arbor vitæ (cerebellum), 244

Arch of aorta, 334 palniar, deep, 310 , and superficial, 303 plantar, 643 supra-orbital, I8 zygomatic, 18

Arches of fauces, anterior and posterior, 142

palatine, anterior and posterior, 142

Arciform fibers or tract, external, 248, 254

Areola, nipple (mammary gland), 266

Arm and forearm, deep fascia of, 283 dissection of, anterior, 282 posterior, 360

landmarks of, 255 to $25^{8}$ superficial, 264, 282

nerves of, $283,365,378$ veins of, 282,378

Arrangement of peritoneum, 473

Artery or arteries :-

abdominal aorta, 515

acromial, 272, 278

acromiothoracic and branches, 272, 278

alar thoracic, 278

anastomosis about the elbow, 387 knee, 626

crucial, 580, 614

anastomotica magna (brachial), 287

(femoral), deep. 573; and superficial branch, 557

angular, 65

anterolateral ganglionic group (brain), 162

anteromedian ganglionic group (brain), 162

aorta, 334

articular, anterior circumflex, 279

azygos, 624

of external plantar, 643

of internal circumflex, 580

of interosseous recurrent, 386

of popliteal, 624

of posterior circumflex, 372

of sciatic, 6ri3

of superior profunda, 287, 372

of suprascapular, 371

of temporal, 62

ascending aorta, 334

cervical, 111

palatine, 95

pharyngeal, 96

auditory, 164

auricular, anterior, 62

deep, 120

posterior, 96

axillary and branches, 275, 276

azygos articular, 624

basilar, 158,164

branches, 164

bicipital, 279
Artery or arteries :-

brachial and branches, 286, 287

bronchial, 345

buccal, 104

(facial), 64

(internal maxillary), 12 I

to bulb, 412

calcanean, external, 633

internal, 643

carotid, common, IOI

left, 340

external, 92

internal, 98,158

carpal, anterior, of radial, 294

of ulnar, 297

carpal, posterior of radial, 387

of ulnar, 297,388

central, of retina, 53

cerebellar, inferior and superior, I64

cerebral, anterior, $15^{8}, 163$

middle (artery of cerebral hemorrhage), 160, 163

posterior, 160,164

cervical, ascending, III

of cervix of uterus, 547

deep (profunda cervicis), II 3, I35

superficial, I12, I30

transverse, I12

choroid, anterior and posterior, 163

ciliary, 53

circle of Willis, 158, 162

circumflex (axillary) anterior, 278

posterior, 279,372

femoral, external, 579 internal, $5^{80}$

iliac deep, 510 superficial, 557,572

clavicular, 272

coccygeal, 613

cœliac axis, 509

colica, dextra, media, sinistra (left, middle, and right), 512

collateral digital of fingers, 305,388 of toes, 596,643

comes nervi ischiadici, 613 phrenici, 328

communicating cerebral, anterior, 160 posterior, 160

dorsalis pedis, 596

of palm, 304

coronary, inferior (face), 64

left (heart), 353

right (heart), $35^{2}$

superior (face), 65

cortical of cerebral, anterior, 163 middle, 163 posterior, 164

cricothyroid, 93

crucial anastomosis, 580, 614

of crus clitoridis, 426

penis, 413

cystic (gall bladder), 510

deferential to vas, 547

dental, 121, 122

descending aorta, 338

digital foot, 643 collateral, dorsal, 596 plantar, 643

hand, 304, 388 collateral, dorsal, 388 
Artery or arteries :-

digital hand collateral, palmar, 305

dorsal, of clitoris, 426 of penis, deep, 412,413 superficial, 436,573

of tongue (lingual), 94

dorsalis hallucis, 596

indicis, 388

linguæ, 94

pedis, 595

pollicis, 388

scapulæ, 278,371

epigastric, deep, 454,456

superior, 321, 454

superficial, $436,557,572$

epiploic, 511

ethmoidal, anterior and branches, 54,149

posterior, 53, 149

facial, in face and branches, 64

in neck and branches, 94

transverse, ó 2

femoral, 570

branches, 572

deep (profunda femoris), 579

frontal, 26,54

ganglionic arterial groups (brain), anterolateral, anteromedian, posterolateral, 162, and pos-

gastric, 509 teromedian, I63

gastroduodenalis, 5 Io, 5 Ir

gastro-epiploic, left, 5 Io

right, 51 i

gluteal, 548, 612

hemorrlioidal, inferior, 412

middle, 547

superior, $5^{14}$

hepatic, 510

humeral, 272,278

hyoid (lingual), 94

(superior thrroid), 93

hypogastric (obliterated), 538, 547

ileocolic, 512

iliac, common, 518

circumflex, deep, 519

superficial, 557,572

external, 519

internal and branches, 546

iliolumbar, 548

infra-orbital, 122

innominate, 339

intercostal, anterior (internal mamaortic, 346

$$
\text { mary), } 321
$$

superior, 346

interossei (hand), dorsal, 388 palmar, 310

(foot), dorsal, 595

interosseous, anterior, 296, 386

common, 296

posterior, 297,385

recurrent, 386

labial, inferior, 64

lachrymal, 50,53

laryngeal, inferior, 112 superior, 93

lateral nasal, 65 sacral, 548

lenticulostriate (cerebral hemorrhage), 162
Artery or arteries :-

lingual, 94

lumbar, 518

malleolar branches of anterior tibial, 594

of posterior tibial, internal, 633

mammary, external, 279

internal, II $3,320,346$

masseteric branches of facial, 64

of internal maxillary, I2I

maxillary, internal, 120

median, 297

meningeal anterior, small, and posterior, 44

of anterior ethmoid, 44

of ascending pharyngeal, 44

of occipital, 44

of posterior ethmoid, 44

middle, I2I

posterior, 96

small, I2I

of vertebral, 44

mental of facial, 95

mesenteric, inferior, 512

superior, 51 I

metacarpal, 388

metatarsal, 595

musculophrenic, $32 \mathrm{I}$

mylohyoid, 12I

nasal, of infra-orbital, 122

of anterior ethmoidal, 54

ophthalmic, 54

of sphenopalatine, 148

lateral, 65

nasopalatine, 122,148

nutrient of brachial, 287

interosseous, 297

of femur, third perforating, 580

of fibula, 633

of humerus, 287

of ilium, 612

of radius, 297

of ulna, 297

of tibia, 632

obliterated hypogastrics, 538,547

obturator, 547,583

occipital, 95, 130

cesophageal, 346

of bulb, 412

of cervix. circular, 547

of crus clitoridis, 426

of crus penis, $4 \mathrm{I} 3$

of gastric, 509

of inferior thyroid, II2

of larynx, 156

of phrenic, 516

ophthalmic, 53

branches of, 26,53

orbital, 62

ovarian, $5^{18}$

palatine, ascending or inferior, 95

superior, 122

palmar arch, deep, 310

superficial, 303

interossei, 310

palpebral, 54

pancreatic, from hepatic, 5 ro

pancreatica magna, 510

pancreaticæ parvæ, 510

pancreaticoduodenalis, inferior, 51I 
Artery or arteries :-

pancreaticoduodenalis, superior, $5 \mathrm{II}$

parotid, 62

perforating of deep femoral, 580 foot, anterior and posterior, 596,643

hand, anterior, 310

internal mammary, $32 \mathrm{I}$

profunda femurs, 580

pericardial, 345

perineal, superficial, 4I2

transverse, 412

peroneal, 633

anterior, 594

posterior, 633

pharyngeal, ascending, 96

phrenic, inferior, 516

plantar arch, 643

digital, 640,643

collateral, 643

external, 642

internal, 640

pleural, 346

popliteal and branches, 623,624

princeps cervicis, 130

pollicis, 3 to

profunda cervicis, I1 $13,130,135$ inferior (brachial), 287

superior (brachial), 287, 372

femoris, 579

pterygoid, 121

pterygopalatine, 122

pubic, 547

pudic internal, 4II, 547

in female, 426

deep external, 573

superficial external, 557, 573

pulmonary, 332

left, 334

right, 332

pyloric, 5 so

radial, carpal, anterior, 294 posterior, 387

at wrist, 387

in forearm, 293

in hand, 310

recurrent, 294

volar, 294

radialis indicis, 310

ranine of lingual, 94

recurrent, arch, deep palmar, 3 Io interosseous, 386

radial, 294

tibial, anterior and posterior, 594

ulnar, anterior and posterior, 296

renal, 516

sacra media, 518

sacral, lateral, 548

middle, 518

scapular, posterior, I12, I13, 370

dorsal, 278, 371

sciatic, $547,6 \mathrm{I}_{3}$

septal, 148, 149

sigmoid, 512

spermatic, 518 .

sphenopalatine, 148

splenic, 510

sternal (internal mammary), 32I

sternomastoid, of occipital, 96

of superior thyroid, 93

stylomastoid, 96
Artery or arteries :-

subclavian, 107

left, $34^{\circ}$

sublingual, of lingual, 94

submaxillary of facial, 95

submental of facial, 95

subscapular, 278, 371

superficial volar, 294

suprahyoid, 94

supra-orbital, 18,26, 53

suprarenal, si6

suprascapular, I12, 371

sural, inferior and superior, 624

system of brain, cortical, 163 ganglionic, 162

tarsal, external and internal, 595

temporal, 26, 62

anterior, 26

deep, I 21

middle, 62

posterior, 26

thoracic, 278

of acromiothoracic, 272

alar, 278

aorta, 338

branches of, 345

long, 278

superior, 276

thyroid axis, III

inferior, 112

superior, 93

thyroidea ima, II5

tibial, anterior, 593

branches, 594

posterior, 631

branches, 632

tibial recurrent, anterior and posterior,

tonsillar,

traclueal, 112

transverse, aorta, 336

of basilar, 164

cervical, II 2

of external circumflex, 579

facial, 62

perineal, 412

tympanic, 120

ulnar, in forearm, 295

in hand, 304

carpal, anterior and posterior, 297, 388

recurrent, anterior and posterior, 296

uterine, 547

vaginal, 547

vasa brevia, 510

intestini tenuis, 513

vas deferens, 547

vertebral, $110,15^{8}$

vesical, inferior, middle, superior, 546

vidian, 122

volar, superficial, 294

Articular branches of circumflex, posterior, 372

of circumflex, internal, 580

of interosseous recurrent, 386

of plantar, external, 643

of popliteal, 624

of profunda, superior (brachial), 287 , 372

of sciatic, $61_{3}$

of suprascapular, 37 I 
Articular branches of temporal, 62 nerves of auriculotemporal, 28 of circumflex, 372

of crural, anterior, 568

of interosseous, posterior, 386

of median, 288,317

of obturator, 536,583

of popliteal, external, 618

internal, 6ig

of tibial, anterior, 598

posterior, 631

of ulnar, 316

Arytenoideus, I46

Aryteno-epiglottic folds, 143

Aryteno-epiglottidis, 146

Ascending aorta, 334

cervical artery, III

colon, 476,501

convolution, frontal, 22, 185

parietal, 24,186

or orbital branches of Meckel's ganglion, I5I

palatine artery, 95

pharyngeal artery, 96

Astragalus, head, 554

Auditory artery, 164

meatus, externus 18

nerve (eighth cranial), 204

Auricle, right, 354

left, 355

Auricular appendix, 354, 355

artery, deep, 120

anterior, 62

posterior, 96

muscles, 25

nerve, great, 75,90

Auricularis magnus nerve, 75,90

Auriculotemporal nerve, 26, 100

Auriculoventricular openings, 354; 355 valves, 355,356

grooves, 330

Axilla, boundaries of, 257, 268, 270, 275, 648

Axillary artery, 275, 276

fascia, 268

lymphatics, 274

space, 648

vein, 275

Axis, thyroid, II I

coliac, 509

Azygos artery, 624

vein, left, superior, 350 major and minor, 348

uvula, 144

BACK, dissection of, 372

fascia of, 362,373

landmarks of, 128, 255, 599

muscles of, 373

nerves of, 376

Band, iliotibial, $55^{8}$ vocal, 145

Bartholin, glands of, 428

Basal, ganglia of brain, 226, 230, 234, 236 gray commissure, 238

Base of brain, 192

of bladder, 420,537

of heart, 260,330

of lungs, $26335^{8}$
Base of skull, interior, 38

Basilar artery, I58, 164

Basilic vein, 283

median, 283

Bell, nerves of, external respiratory (long thoracic), 28o, 315

internal respiratory (phrenic), I00, 325

superior respiratory (spinal accessory), 76, 91, 206

Bend of elbow, $29^{2}$

Biceps, 285

flexor cruris, 608

Bicipital artery, 279

groove, 256

of humerus, 256

Bicuspid valve, 356

Bile duct, common, 49I, 492 cystic, 49

Biventer cervicis, 132

Bladder, gall, 49I

urinary, 537 dissection of interior, 548

interior of, 548

ligaments of, lymphatics of, relations of, vessels and nerves of, 537

peritoneal covering of, 537

relations of, 538

Retzius' space, 478 , 537

trigone, external and internal, 420, 540,550

ligaments, false, 477,537

true, 401,538

Blood supply of brain, 158

Bodies, suprarenal, 524

Body of caudate nucleus, 228

of corpus callosum, 208

of fornix, 216

geniculate, external, 226, 230

internal, 224

of lateral ventricle, 2ro

of Luy's, 240

olivary, 248

of penis, $44^{\circ}$

perineal, $43 \mathrm{I}$

of pubes, 554

of uterus, 543

Brachia, or peduncles, corpora quadrigemina, 224

corpus callosum, 197, 210

Brachial artery, 286, 287

plexus, 312

veins, 286

Brachialis anticus, 285

Brachioradialis, 292

Brain, 166

base of, 192

blood supply of, 158

development of, I68

fissures of, 176,180

ganglia of, $226,230,234,236$

hardening of, 36

hemispheres of, 178

membranes of, 152

relations to cranium, 18

removal of, 32,36

topography of, 18

weight of, 648

Breast, female, 266

Bregma, I7 
Broad ligament of liver, 486

of uterus, 478,543

Bronchi, 357

Bronchial arteries, 345

veins, 359

Bronchus, left and right, $35^{8}$

Buccal artery (facial), 64 ; (internal maxillary), I2

nerve (facial), 6I; (fifth), II7

Buccinator, 67

Bulb, artery of, 412

olfactory, 199

of penis, $4 \mathrm{I} 5$

of posterior horn of lateral ventricle, $2 \mathrm{I}_{2}$ of vagina, 427

Bulbi vestibuli, 427

Bulbocavernosus (accelerator urinæ), 410

Bundle of Vic d'Azyr, 238

Burns' space, I39

Bursa in hand, 311

prepatellar, $55^{8}$

subgluteal (gluteus maximus), 605

Buttocks, landmarks of, 599

CAECUM, 476, 500

Calamus scriptorius, 252

Calcanean artery, external, 633 internal, 643

nerves, 631

Calcar (hippocampus minor), 190, 212

Calcarine fissure or sulcus, I90, I94

Callosal sulcus, 191, 192

Callosomarginal fissure or sulcus,, 190

Canal, Alcock's, 399, 4I I

alimentary, development of, 462

central, of spinal cord, 394

femoral, 569

of Hunter, 572, 575

inguinal, 448

of Nuck, 544

" Canals," anatomical, 446, 599

Capsule, external, 228, 234

internal, 234

of Tenon, 49

Cardiac arteries, left, 353 right, 352

nerves, 104,354

cervical, I04

inferior, 107

middle, 107

superior, 106

orifice of stomach, 494

plexus, deep and superficial, 354

veins, 353

Carotid artery, common, Ior left, 340

external, 92

internal, 98,158

plexus, 38 , I06

triangle, inferior and superior, 83

Carpal artery, radial anterior, 294 posterior, 387

ulnar anterior, 297 posterior, 297,388

"Carrying angle" or function, 258

Carunculæ myrtiformes, 424

Cauda equina, 395

Caudate body, head, tail of same, 228 lobe of liver, 490

\section{Caudate nucleus, 226}

Cava inferior, 354,520

superior, 327

Cavernous plexus, 106 sinus, 43

Cavities of heart, 354

Cavity of great omentum, 470

Central artery of retina, 53 canal of spinal cord, 394

lobe of the cerebrum (island of Reil), 187

Cephalic vein, 256, 269, 283 median, 283

Center of hearing, 204 of sight, 200

of smell, 200

Centrum ovale majus and minor, 207

Cerebellar arteries, inferior and superior I 64

peduncles, 244

Cerebellum, 242

development of, 17 r, 175

lobes of, 242

peduncles, inferior and superior, 244 middle, 195,244

Cerebral arteries, anterior, 158 middle (artery of cerebral hemorrhage), 160, 163 posterior, 160, 164

blood supply, $15^{8}$

crura, 196

development, 175

flexures, 174

hemispheres, 178

relations to exterior of cranium, I8

hemorrhages, 173

veins, 165,220

deep, 220

superficial, I65

ventricles, lateral, 2 II

fifth, 216

fourth, 248

third, 222

vesicles, 169

Cerebrum, I78

development of, I70, I7 I

fissures of, 180

inner surface of, 188

under surface of, 192

Cerebrospinal Huid, 155,156 function of, 156

Cervical abscess, 141

artery, ascending, III

deep, II3, I35

princeps cervicis, I30

superficial, 112, 130

transverse, II2

enlargement of spinal cord, 394

fascia, deep, I 36

first layer, 138

fourth layer, I4O

second layer, 138

third layer, 139

ganglion, inferior, 107

middle, ro6

superior, 106

nerve to trapezius, 89 , 9 I

nerves, 89

plexus, 89

superficial nerve $75,89,90$

sympathetic cord and ganglia, 106 
Cervical vein, deep, III, I36 transverse, 74,79

Cervicalis ascendens, 135,376

Cervicofacial nerve, $6 \mathrm{I}$

Cervix, circular artery of, 547 of uterus, 543

Chiasm or commissure, optic, 197, 200

Chorda tympani, II8, 203

Chordæ tendineæ, 356

Willisii, 34, 35

Choroid artery, anterior and posterior, I63

plexuses, development, 177, I78, 218

vein, 222

Choroideus sulcus, 230

Ciliary arteries, 53

nerves, 56

Circle of Willis, 158,162 function of, 161

Circular sinus, 44

Circumflex artery, anterior, 278 posterior, 279,372

femoral, external, 579 internal, 580

iliac, deep, 520 superficial, $346,557,572$

nerve, $318,319,365,372$

Circumvallate papiliße, I43

Claustrum, 234

Clava, 254

Clavicle, 255

Clavicular artery, 272

nerves, $76,89,91,266$

Claviculo-acromial joint, 256

Clavipectoral fascia, 272

Clitoris, 423,427

dorsál artery of, 426

glans of, 428

nerves of, 428

suspensory ligament of, 406, 428

Coccygeal artery, 613

Coccygeus, 418

Coliac axis and branches, 509

Colica dextra, media, sinistra, 512

Collateral fissure or sulcus, 190, 194

digital arteries of foot, 396, 643 of hand, 305,388

eminence, 190,212

fissure, 190, 194

Colles' fascia, 404

fracture, 258

Colliculus seminalis, 5.50

Colon, ascending, 476, $50 \mathrm{I}$

blood supply of, 5 I1, 512

descending, $477,501,502$

omega loop of, or sigmoid flexure, 477 , 502

transverse, 476,501

Columnæ carneæ, 356

rugarum of vagina, 431

Comes nervi ischiadici, 613 phrenici, 328

Commissure basal (gray), 238

(cerebral), anterior, 236 development of, 170,174

middle, posterior, 232

optic (nerve), 197, 200

Common bile duct, 491, 492

carotid artery, IOI left, 340
Common iliac arteries, 518

Communicans hypoglossi, 88,89

peronei nerve (fibular), 616, 6 I9

phrenici, 3 I4

tibialis nerve, 619

Communicating arteries, anterior and of foot, 596 posterior, 160

of ulnar, 304

Comparative measurements of male and female pelvis, 422

Complexus (and biventer cervicis), 132

Compressor of dorsal veins of penis, 410

urethræ, 416

vagina, 428

Condyles of femur, 554

of humerus, 257

Confluence of sinuses(torcular Herophili), 35,42

Conjoined tendon, 447

Constrictors of pharynx, inferior, middle, and superior, 125

Constrictor vaginæ, 428

Convolution or convolutions :-

angular, 187

dentate, 192

fornicate (gyrus fornicatus), 192

frontal lobe, indicated on exterior of cranium, 22

anterior, internal, posterior, 194

ascending, inferior, middle, and superior, 185

hippocampal (gyrus hippocampi), 192

lingulate, 192, 194

marginal, 191

occipital lobe indicated on exterior of cranium, 24

inferior, 194

middle and superior, 188

occipitotemporal, inferior, 194

superior, 192, 194

operculum, i86

orbital (frontal), anterior, internal, and external, or posterior, 194

paracentral, 186, 191

parietal lobe indicated on exterior of cranium, 22

ascending, inferior and superior, 186

supramarginal, 186

temporal lobe indicated on exterior of cranium, 24

inferior, 194

inferior, middle, and superior, 187

uncinate, I9I, I92, 194

Coracobrachialis, 284

Coracoid process, 256

Cord (nerve), lumbosacral, 529, $55^{1}$

spermatic, coverings for, 442

spinal, 392

sympathetic, cervical, 106

lumbar, 526

sacral, 553

thoracic, 345

Cords, vocal, true, and false, 145

Cornua of lateral ventricle, 2 II

Corona glandis of penis, 415,440

of clitoris, 428

radiata, 234 
Coronary artery, inferior, 64 left, 353

right, 352

superior, 65

ligament of liver, 473,488

sinus, 353

opening of, 354

vein, 353

Corpora albicantia, seu mammillaria, 197 development of, 175

cavernosa, 416

quadrigemina, 224

brachia of, 224

development of, 171, 175

striata, 226

Corpus Arantii, 355

callosum, 207

body, 208

development, 170,173

forceps major, 212 , and minor, 208

genu, 207

peduncles of, 197, 210

raphé, 210

rostrum, 196, 208

splenium, 207

tæniæ tectæ and striæ longitudinales, 210

tapetum, 208

dentatum, 246

fimbriatum, 218

geniculatum, externum, 226, 230

internum, 224

spongiosum, 414

striatum, 226

development, 170, 173, 232

vein of, 220

Corrugator supercilii, 30

Cortical arterial system of brain, 163 hemispheres, 178

veins of brain, superior and inferior, 165

Costocolic ligament, 47 I, 477

Costocoracoid ligament, 270 membrane, 139,270

Cotunnius, nerve of, 152

Counting the ribs, 257

Course of extravasated urine, $404,438,440$

Coverings for cord and testicle, $44^{2}$ how acquired, 457

Cowper's glands, 417

Cranial nerves, I98

eighth, 204

eleventh, 76, 9I, 206

fift h, 46, 201

first, 198

fourth, 50, 201

ninth, 98, 204

second, 57,200

seventh, 6r, 203

sixth, 57, 201

tenth, 103, 206

third, 57,200

twelfth, 88, 206

sinuses, 34

Craniocerebral topography, I7

Cremaster, 448

fascia, 448

formation of muscle and fascia, 460

Crest of ilium, 434, 557, 599

of occipital bone, external, 18
Crest of tibia (spine) (shin), 554

Cribriform fascia, 556

Cricoarytenoid, 146

lateralis, 147

Cricothyroid, 145

artery, 93

membrane, 146

Crista galli, 34

Crucial anastomosis, 580, 614

sulcus, 224

Crura cerebri, 195, 238

development of, 175, 175

of diaphragm, 351

Crural arch, 456

nerve, anterior, 532, 557, 568

septum, 526

Crureus, 575

Crus, artery of, 413

clitoridis, 426

penis, 416

Crusta, 240

Cruveilheir, plexus of, 112

Cuboid bone, 556

Cuneate lobe, I9I, 242

Curvature of stomach greater and lesser, 494

Cutaneous nerve, dorsal, 298

external (of thigh), 532, 557, 561, 602 (musculospiral), 378 of popliteal, $5^{88}$

intercostal, anterior, $35^{\circ}$

internal arm, 288, 316, 319, 378 (thigh), 557, 562

lateral, $35^{\circ}$ inferior, superior (musculospiral), 318,365

lesser, internal, 316, 319, 365

middle, 557,562

palmar, 298

nerves of abdomen, $436,35^{\circ}$

of arm, anterior, 283 posterior, 365,378

of back, 360

of dorsum of foot, 588,589 sole of foot, 638,642

of face, $46,6 \mathrm{r}$

of forearm, anterior, 283 posterior, 378

of gluteal region, 602

of hand, palm, 298 dorsum, 378

of neck, anterior, 75,76 posterior, 129

of shoulder, anterior, 264 posterior, 365

of thigh, anterior, 557 posterior, 602

of thorax, anterior, 36, 264 posterior, 350

Cystic artery, 510

duct, 491

vein, 514

Dartos, labium majus, 406

penis, 406,415

scrotum, 406

Decussation of anterior pyramids of medulla, 248

Deferential artery, 547 
Deformity, "gun-stock," $25^{8}$

Deltoid, 363

tubercle, 256

Dental arteries, I21, 122

nerves, anterior, middle, posterior, 150 plexus, superior, I5O

Dentate convolution, 192

fissure, 190, 194

ligament, 392

nucleus, 246

Depressor anguli oris, 62

labii inferioris, 63

Descendens hypoglossi, 88,90

Descending aorta, 338

colon, $477,501,503$

Descent of testicle, 457

Development of abdominal (inguinal) rings and coverings for the cord and testicle, 457

of alimentary canal, 462

of aqueduct of Sylvius, I7 I, I75

of auditory nerve, I7I

of brain, 168

of cerebellum, I7 I, I75.

of cerebrum, 170, 171

of choroid plexuses, 177,178

of commissure, anterior, 170, 174

of corpora albicantia, 175

of corpora quadrigemina, I7 I, I 75

of corpus callosum, 170, 173

of corpus striatum, 170, 173, 232

of crura cerebri, I71, 175

of fissures of brain, 176

of Rolando, 176, 177

of Sylvius, 176

transverse (Bichat), 176

of foramina of Monro, 171

of fornix, 170, 173

of infundibulum, I7 I, I75

of internal capsule, 232

of lamina cinerea, 175

terminalis, 170, 173

of liver, 467

of medulla, 171, 175

of olfactory lobe, 170, 172

of omentum, greater, 468

of optic thalamus, I7 I, I73, 232 nerve, $17 \mathrm{I}$

of pancreas, 467

of peritoneum, 462

of pineal gland, $17 \mathrm{I}$

of pituitary body, 17 I, I75

of pons, 171,175

of septum lucidum, 170, 173

of spleen, 467

of tuber cinereum, I75

of valve of Vieussens, i7 7

of velum interpositum, 177

of ventricles of brain, fifth, 173

$$
\text { fourth, } 171,176
$$

lateral, 170

third, 17 I

viscera (abdominal) and peritoneum,

Diaphragm, 351 summary of, 484

openings in, 35 I

Digastric, 78

Digital arteries collateral of hand, 305 , 388 of foot, 596,643
Digital arteries of hand, 304, 388

of foot, 643

nerves collateral of hand, fingers, 305 of toes, 640

of fingers, 305

of toes, 638

Dissection, abdomen exterior, 435

interior, 473

arm and forearm, anterior, 264, 282 posterior, 360,369

axilla, 274

back, 360, 372

bladder and urethra, interior, 548 exterior, 536

brain, 165,207

corpus callosum, 207

face, deep, II5 superficial, $5^{8}$

fascia lata, 558, 602

foot, dorsum, 588 sole, 634

forearm, 282, 290, 294 posterior, 376

fossa, ischiorectal, 407

gluteal region, 599

hand, anterior, 300 posterior, 376

head, 24 posterior, 128

heart, 354

larynx, I45

leg anterior, 584 posterior, 616

nasal fossæ, 148

neck, anterior, 69 posterior, 128

omentum, gastrocolic, $468,470,476,483$, 494

gastrohepatic, 473, 492, 494

gastrosplenic, 506

orbit, 48

palm of hand, 300

pelvic viscera, 536

penis, 435,440

perineum, female, 424

male, 404

peritoneum, 473

pharynx, 141

pia, 156, 165

removal of arachnoid, 156

of brain, 36

of deep fascia of leg, 588, 618

of dura, 34

of fascia lata, 562, 602

of heart and lungs from thorax, 343

of palmar fascia, 302

of pia, 165

of plantar fascia, 636

shoulder, anterior, 272 posterior, 360

skull, interior of base, 44

spinal cord, 389

testicle, 443

thigh, anterior, 556

posterior, 599

thorax and shoulder, anterior, 264

interior of, 319

posterior, 360

ventricles, lateral (brain), 214

third, 222 
Dissection, ventricles of heart, 355 wrist and hand, anterior, 300 posterior, 376

Diverticulum, Meckel's, 47 I, 499

Dorsal artery of clitoris, 426 of penis, superficial, 436,573 deep, 412, 413

of tongue, 94

digital arteries of hand, 388 of toes, 596

nerves of hand, 305

of foot, 638

enlargement of spinal cord, 394

nerve, last, $529,557,602$

of clitoris, 414,426

of penis, $4 \mathrm{I} 4$

nerves of, intercostal, 376

veins of hand, 378

of foot, 586

of penis, 440,442

Dorsalis hallucis, artery, 596

indicis artery, 388

lingual artery, 94

pedis artery, 595

pollicis artery, 388

scapulæ artery, 278, 371

Douglas, fold of, 450

pouch of, or cul-de-sac, 43 I, 477, 483, 543

semilunar fold of, $45^{\circ}$

Duct or ducts :-

Bartholin glands, 424

bile, common, $49 \mathrm{r}, 492$

cystic, $49 \mathrm{I}$

ejaculatory, $542,55^{\circ}$

hepatic, 491

lymphatic right, 107

nasal, opening of, 148

of pancreas (Wirsung), 508

of parotid gland (Stenson's), 66

of prostatic glands, 550

of Rivinus (sublingual gland), 120

of Steuson (parotid gland), 66

thoracic, 344

vitello-intestinal, 47 I

Wharton's, 86

of Wirsung, 508

Ductus arteriosus, 332

vellosus, 490

fissure of, 490

Duodenojejunal fossa, 483,498

$$
\text { formation of, } 465
$$

Duodenum, 496

impression on liver, 490

ligament of (Treitz), 498

Dura of the brain, 32 processes of, 34

of spinal cord, 390

EIGHTH cranial nerve, 204

Ejaculatory duct, 542 orifices of, 550

Elbow, arterial anastomosis about, 387 landmarks, 257

space anterior, 292

Eleventh cranial nerve, 76, 91, 206

Eminence, parietal, 18

tubal, of Eustachian tube, 142

Eminentia collateralis, 212 formation of, 190
Enlargement of spinal cord, cervical, dorsal, lumbar, 394

Ensiform appendix, 255, 432

Epididymis, 443

Epigastric artery, deep, 454, 456 superficial, $436,557,572$ superior, 321,454

vein, deep, 520 superficial, $436,5.57$

Epiglottis, 143

Epiploic arteries, 51 I

Erector clitoridis 427

penis, 408

spinæ, 374

Ethmoidal artery, anterior and branches, 54,149 posterior, 53,149

Eustachian tube, 141, 145

valve, 355

Extensor brevis digitorum, 592 pollicis, 384

carpi radialis brevior and longior, 380 ulnaris, 382

communis digitorum, 380

indicis, 385

longus digitorum, 589 pollicis, 384

minimi digiti, 38 I

muscles of spine, 373

ossis metacarpi pollicis, 382

primi internodii pollicis, 384

proprius hallucis, 596

secundi internodii pollicis, 384

External cutaneous nerve (arm), 283, 378 thigh, 532, 557, 561, 602

meatus, urinary, male, $415,55^{1}$ female, 424

of ear, 18

trigone of bladder, 420,540

Extremity, lower, landmarks of anterior, 554

landmarks of posterior, 599

FACE, dissection of, deep, II5 superficial, $5^{8}$

landmarks of region of, 24

Facial artery, in face, 64

in neck, 94

transverse, 62

nerve, $6 \mathrm{r}, 203$

cervicoiacial division, 6 I

temporofacial division, 6 I

vein, 62

in neck, 74

Falciform ligament or process of fascia lata, 56I of liver, 486

or limbic lobe of cerebrum, 192

Fallopian tube, 543, 544

False ligameuts of bladder, 477,537

vocal cords, 145

Fal $x$ cerebelli, 40 cerebri, 34

Fascia, anal or ischiorectal, 402

abdominal, superficial, 436

deep (Scarpa's), 438

axillary, 268

arm and forearm, deep, 283 superficial, 264, 282 
Fascia, back, deep, $3^{62}$

superficial, 360

cervical, deep, 136 first layer, 138 fourth (prevertebral) layer, I40 second layer, 138 third layer, 139

clavipectoral, 272

Colles' (perineal), 404

costocoracoid, 270

cremasteric, 448

formation of, 460

cribriform, 556

foot, superficial of sole, 634

dorsum, 584

forearm, 282,283

gluteal region, superficial, 600

hand, 282,360

iliac, of fascia lata, 560

iliopsoas, 525

infundibuliform, 456, 460

intercolumnar, 444

ischiorectal, 402

lata, dissection of, $55^{8}, 602$

iliac portion, pubic portion, 560

anterior portion, intermuscular septa, iliotibial band, $55^{8}$

internal process, saphenous opening, 560

falciform process, $56 \mathrm{I}$

posterior portion, 600

leg, deep, 584 removal of, 562,602

superficial, 584

lumbar, 45

masseteric, 59,138

neck, 360

obturator, 398

orbital, 49

palmar, 3 or

palpebral, 49

parotid, $59,{ }_{13} 8$

pectoral, 268

pelvic, 398 , et seq.

perineal, superficial or Colles', 404

deep or triangular ligaments of, 402,403

pharyngeal, 124

plantar, 634

removal of, 636

prevertebral, 140

pubic, of fascia lata, 560

pyriformis, 399

rectovesical, 399,538

Scarpa's, 438

shoulder, 264, 268, 360

spermatic, external, 444

internal or infundibuliform, 456, 460

temporal, 30

thigh, deep (fascia lata), 560, 600

superficial, 556, 600

thorax anterior, 264

posterior (thorax and back), 360

transversalis, 454

triangular, 446

(ligament), 402, 403

vertebral, 373

Fascial sheath of penis, 415

Fasciculus teres, 252

Fauces, anterior and posterior arches of, I42
Fauces, isthmus of, 142

pillars of, 142

Female genitals, external, 422

mamma, 266

pelvis, measurements of, 422

perineum, 422

urethra, 43 I

Femoral artery, 570

course of, $57^{\circ}$

deep (profunda femoris), 579

muscular branches of, 573

canal, 569

hernia, 526, 569

lymphatics, 557

opening (under Poupart's ligament), 525

(in adductor magnus), 578

ring, 525

vein and tributaries, 574

veins, cutaneous, external and internal, 557

deep (profunda femoris), and tributaries, 574

Femur, condyles of, 554

nutrient artery of, third perforating, $5^{80}$

Fibres, arciform, external, 248, 254 of Gerdy, 302

Fibula, head of, 554 nutrient artery of, 633

Fifth cranial nerve trunks of, 46,201 ventricle of brain, 216 development of, 173

Filum terminale, 390

Fimbria, 218

Fimbriated extremity of Fallopian tube, 544

First cervical nerve (suboccipital), I36 cranial nerve (olfactory), I98

Fissure, of brain, I76, I80

calcarine, I90, 194

callosal, 191, I92

callosomarginal, 190

of cerebrum, iso development of, 176

collateral, I90, 194

dentate or hippocampal, 190, 194

ductus venosus, 490

for gall bladder, 490

frontal, inferior, superior, and precentral, 22, 185

great longitudinal, j80, 194, I96

development, 176

hippocampal or dentate, I90, I94

intraparietal, 22, 186

of liver, ductus venosus, 490

longitudinal, 488

transverse, 488

umbilical, 490

vena cava inferior, 490

longitudinal of liver, 488

of lungs, outlining on chest, 263,359

of medulla anterior, 248

posterior, 252

occipital, inferior, middle, and supe-

olfactory, 194 rior, 24,188

orbital, 192

parieto-occipital, 22, 184, I90, I94

of Rolando, 2I, 182 
Fissure of Rolando, development of,

of spinal cord, anterior and posterior, sphenoidal, 46

of Sylvius, 21, 180, 194, 197

development of, 176

temporal, middle, superior, 24, 187

inferior, 194

transverse (Bichat), 180

development of, I7 6

of liver, 488

umbilical of liver, 490

vena cava of liver, 490

Flexor accessorius, 640

brevis digitorum, 636

hallucis, 644

minini digiti, 307

(in foot), 646

pollicis (inner head), 308

outer head, 306

carpi radialis, 291

ulnaris, 291

longus digitorum, 627

hallucis, 627

pollicis, 298

profundus digitorum, 300

sublimis digitorum, 294

tendons of profundus and sublimis in hand, 311

Flexure, hepatic, 476,501

sigmoid, $477,501,502$

splenic, $477,501,502$

Flexures of cerebral vesicles, 174

Flocculus, 242

Floor of anterior elbow space, 292

of lateral ventricles, 212

of popliteal space, 620

of Scarpa's triangle, 569

of third ventricles, 198

of ventricles of brain, fourth, 252

lateral, 212

third, 198

Fluid, cerebrospinal, 155, I56

Fold aryteno-epiglottic, 143

of Douglas, 450

glosso-epiglottic, 143

reduplicated, of corpus callosum, 208

Foot, arteries of, dorsalis pedis and

dissection of dorsum, 588 sole, 634

landmarks of, 554

veins of, $5^{86}$

Foramen cæcum, of medulla, 248

of skull, 35

of tongue, 142

dental inferior, 118

infra-orbital, 68,150

jugular, 40, 204

Key and Retzius, 155, 250

of Majendie, 155,250

mastoid, 42

mental, 68, I2I

of Monro, 218

development of, I7I

optic, 57,200

ovale, 48

rotundum, 48,149

sacrosciatic, greater and smaller, 6I5
Foramen, supra-orbital, 68

suprascapular, 112

of Winslow, 470,478

Foramina of Monro, 218

development of, 171

of exit for cranial nerves from skull, I98, et seq.

of Key and Retzius, I55, 250

of Scarpa, 152

of Stenso11, 149

Thebesii, 354

Forceps, major and minor of corpus callosum, 208, 212

Forearm, dissection of, 282, 290, 294 posterior, 376

fascia of deep, $2 \mathrm{~S}_{3}$ superficial, 264, 282

landmarks of, 255

subcutaneous nerves of, 283,378

veins of, 282,378

Forebrain, 169, 170, 171

Formation of abdominal (inguinal) rings and coverings for spermatic cord and testicle, 457

Fornicate convolution (gyrus fornicatus), 192

Fornix, body, genua, pillars, anterior and posterior, 216

development of, 170, 173

Fossa duodenojejunalis, 483,498

formation of, 465

intersigmoid, 483

ischiorectal, 402,407

Mohrenheim's, 84

nasal, 148

navicularis, 424

of urethra, 55

ovalis, 355

peritoneal, 473,482

rectovesical, rectovaginal, uterovaginal, $477,483,543$

of Rosenmüller, 142

of skull, anterior, middle, posterior, 38

uterovesical, $477,483,543$

Fourchette, 424

Fourth cranial nerve, 50, 201

ventricle, 250

Fovea, inferior and superior of fourth ventricle, $25^{2}$

Fracture, Colles', $25^{8}$

Frenum of epiglottis, 143

veli, 244

Frontal artery, 26, 54

bone, angular process, external and internal, 18

convolutions, ascending, inferior, middle, superior, 185

marginal, 191

orbital (anterior, internal, posterior), 194

lobe, 184

convolutions referred to exterior of cranium, 22

nerve, 49,56

sulcus inferior, 22, 185

orbital, 192

precentral, 22,185

superior, 22, 185

Fundus of stomach, 494

of uterus (body), 543 
Funicular process, 460

Funiculus cuneatus, 254 gracilis, 254

Galea capitis, 25

Galen, vein of, common, 40, 222 left and right, 222

Gall bladder, 491 fissure for, 490 relations immediate and superficial or external, 491, 492

Ganglia, basal (gray), of brain, 226, 230, 234,236

cervical, inferior, 107

middle, 106

superior, 106

coccygeal, 553

lumbar, 326

sacral, 553

semilunar, 509

thoracic, 345

Ganglion, Gasserian, 46

geniculate (facial), 202

impar, 553

jugular (ninth cranial nerve), 204

Meckel's, sphenopalatine or nasal, 15I

ophthalmic, lenticular, or ciliary, 56

otic, II9

petrous (ninth), 204

of pneumogastric (root and trunk), ro3 submaxillary, i 19

Ganglionic arterial system of brain, 162 anterolateral group, 162 anteromedian group, 162 posterolateral group, I62 posteromedian group, 163

veins of brain, 220

Gasserian ganglion, 46

Gastric artery, 509

vein, 514

Gastrocnemius, 620

Gastrocolic omentum, 468, 470, 476, 483, 494

Gastroduodenalis artery, 510, $5 \mathrm{II}$

Gastro-epiploic artery, left, 5IO right, 5 II

veins, $5 \mathrm{I} 2,5 \mathrm{I} 4$

Gastrohepatic omentum, 473, 492, 494

Gastrophrenic ligament, 473, 494

Gastrosplenic omentum, 506

Gemelli, inferior and superior, 606

Geniculate body, external, 226, 230 internal, 224 ganglion (facial), 202

Geniohyoglossus, 97

Geniohyoid, 96

Genitals, external, female, 422 male, 440

Genitocrural nerve, 532, 557

Genua of fissure of Rolando, 182

Genu, corpus callosum, 207

fornix, 216 of internal capsule, 234

Gerdy, fibres of, 302

Gimbernat's ligament, 445

Glabella, I7

Gland or glands :-

Bartholin, 428

Cowper's, 417
Gland or glands :-

lachrymal, 50

mammary, 266

Pacchionian, I55

parotid, 66

pineal, 224

pituitary, I7 I, 175, 197

prostatic, $55^{\circ}$

sublingual, I 19

submaxillary, 85

thymus, 324

thyroid, I 14

Glans clitoridis, 423,428

penis, 415,440

Globus pallidus (lenticular nucleus), 230

Glosso-epiglottic folds, 143

pouch, 143

Glossopharyngeal nerve, 98, 204

Glottis (larynx), I45

rima, 145

Gluteal artery, 548, 612

nerve, inferior, 552

superior, $55 \mathrm{I}, 6 \mathrm{I} 6$

region, dissection of, 599

landmarks of, 599

vein, 548

Gluteus maximus, 602

bursa under the muscle, 605

medius, 605

minimus, 6I5

Gracilis, 566

Great a uricular nerve, $75,89,90$

deep petrosal nerve, 46,151

longitudinal fissure of cerebrum, 180 , 196 development, 176

occipital nerve, I 29

omentum, $468,470,476,483,494$

cavity of, 470

sacrosciatic foramen, 6I5

ligament, 614

sciatic nerve, 553,6 ro

splanchnic nerve, 345

superficial petrosal nerve, I5I, 203

synovial bursa of palm of hand, $31 \mathrm{I}$

transverse fissure of cerebrum, 180 development, 176

trochanter, 554

tuberosity of humerus, 256

Groin, see inguinal region, 435

land marks of, 432

lymphatics of, 430 .

Groove, anterolateral of medulla, 248

auriculoventricular, 330

bicipital, 256

interventricular, 330

lateral of crus cerebri, 240

oculomotor, 240

Gubernaculum, 457

Gun-stock deformity, $25^{8}$

Gustatory nerve, II 8

Gyrus, angular, 187

fornicatus, 192

hippocampi, 192

uncinate, 191, 192, 194

HAM-STRINGS, 599

Hand, dissection of, 300

posterior, 376 
Hand, synovial membrane of, 311

Hardening of brain, 36

Head, anterior, I7

of astragalus, 554

of caudate nucleus, 228

dissection of, 24,128

of fibula, 554

of humerus, 257

landmarks of anterior, 17

of posterior, 128

of pancreas, 508

posterior, dissection of, 128

of radius, 257,258

Heart, 330

apex, 260

arteries of (coronary right and left), 352,353

auricles, 354,355

cavities of, 330

dissection of, 354

dullness, 260

openings of, $354,355,356$

outlining on chest wall, 260

position of, 259, 33I

relation to chest wall, 260

size and weight, 330

valves of, 355,356

veins of, 353

ventricles, left, 356

right, 355

Hemispheres of cerebellum, 242

of cerebrum, 178

Hemorrhoidal artery, inferior, 412

middle, 547

superior, $5 \mathrm{I} 4$

nerve, inferior, 413

plexus, 408

veins, 408

Hepatic artery, 5 Io

duct, 491

flexure of colon, 476,501 vein, 521 impression on liver, 490

Hernia, course of direct and indirect, inguinal, 460,46

coverings for direct and indirect inguinal, $46 \mathrm{I}$

femoral, 526,569

nature's protection against ventral hernia, 452

varieties of direct and indirect inguinal, $460,46 \mathrm{I}$

Hesselbach's triangle, 457

Highmore, antrum of, opening of, 148

Hindbrain, 169, 171, 175

Hippocampal convolution, 192

or dentate fissure, 190, 194

Hippocampus major, 214 formation of, 190

minor, 212

formation of, 190

Horns of lateral, ventricle, anterior, middle or descending, posterior, 210

Humeral artery, 272, 278

Humerus, nutrient artery of, 287

Hunter's canal, 572, 575

Hydrocele, 460, 46I

Hymen, 424

Hyoglossus, 97
Hyoid artery of lingual, 94

of superior thyroid, 93

Hypogastric artery, obliterated, 538, 547

Hypoglossal (cranial) nerve, 88, 206

loop, 88

Hypoglossi ansa, 88

communicantes, $88,89,90$

descendens, 88,90

Hypophysis, 175, 197

ILEOCOLIC artery, 512

valve, 499

Ileum, 499

Iliac artery, circumflex deep, 519

superficial, 557,572

common, 518

external, 519

internal, 546

portion of fascia lata, 560

vein, common, 520

circumflex deep, 520

superficial, 557

external, 520

internal, 548

lliacus, 527

Iliocostalis, 375

Iliohypogastric nerve, 440, 450, 451, 530, 602

Ilio-inguinal nerve, $448,451,530,557$

Iliolumbar artery, 548

Iliopsoas fascia, 525

Iliotibial band, $55^{8}$

Impar ganglion, 553

Impressions on the liver for duodenum, 490

for hepatic flexure, 490

for right kidney, 490

for stomach, 490

Inferior longitudinal sinus, 36

maxillary nerve, 48,117

petrosal sinus, 42

pudendal nerve, 406,604

vena cava, 575

fissure in liver for, 490

Inframaxillary nerve, 61,74

Infra-orbital artery, 122

canal, 150

foramen, 68,150

nerve, $6 \mathrm{I}$

plexus, 6r, I5

Infraspinatus, 368

Infratrochlear nerve, 56

Infundibuliform fascia (internal spermatic), 456,460

Infundibulum, 197

development of, 17 I, 175

frontal sinus, 148 opening of into nasal fossa, 148

Inguinal canal, 448

hernia, course of, 460,46 I

coverings for, $46 \mathrm{I}$

varieties of, $460,46 \mathrm{I}$

lymphatics, 436

rings, external, 444

internal, 456

formation of, 457

Inion, 18

Innominate artery, 339 veins, 92,326 
Interbrain, I69, I7 I, I75

Interclavicular notch, 255

Intercolumnar fascia, 444

Intercostal arteries, 346 anterior, 32I aortic, 346

artery, superior, 346

muscles, external, 346

internal, 347

action of, 347

nerves, $350,376,45 \mathrm{I}$ cutaneous branches, 266, 282, 45 I veins, 348

left and right superior, $35^{\circ}$

Intercostohumeral nerve, 266, 280, 282, 3I9, $35 \mathrm{I}, 365$

Internal capsule, 234 development of, 232

Interossei of foot, dorsal, 592 of hand, dorsal, 388 palmar, 309

sole of foot, 646

Interosseous artery, anterior, 296, 386 common, 296 of foot, dorsal, 595

of hand, dorsal, 388 palmar, 310

posterior, 297,385

recurrent, 386

nerve, anterior, 317 posterior, 293, 318, 386

Interpeduncular space, 198

Intersigmoid fossa, 483

Interventricular (coronary) artery, left, 353

right, 35

groove, 330

vein, anterior, 353

posterior, 353

Intestinal blood supply, 509, et seq.

Intestine, 496

development of, 462

large, 500

appendices epiploicæ, 500

muscular bands of, 500

sacculated formation, 500

divisions of,

ascending colon, 476 , 501

cæcum, 500

descending colon, $477,501,502$

omega loop or sigmoid flexure, 477 501,502

rectum, 504

splenic flexure, 501,502

transverse colon, 476 , 5 이

vermiform appendix, 500

relation to surface of body, 505

rotation of, 464

small, 499

divisions of

duodenum, 496
ileum, 499

jejunum, 499

Intraparietal sulcus, 22, 186

Ischiocavernosus (erector penis) 408

Ischiorectal fascia, 402

fossa, 402,407

dissection of, 407

region, 397

Ischium, tuberosity of, 599
Island of Reil, 187

Isthmus, of fauces, I42

of gyrus fornicatus, 192

of thyroid gland, II4

of uterus, 543

JACOBSON's nerve, 206

Jejunum, 499

Jugular ganglion (ninth nerve), 204

vein, anterior, 74,99

external, 74, 99

internal, 92

posterior external, 74

KEY and Retzius, foramina of, $155,25^{\circ}$

Kidney, 522

arteries of, 524

dissection of, 5 I 5

impression of right, on liver, 490

lymphatics of, 524

nerves, 524

relations of, 522

situation of, 522

size of, 522

veins of, 524

Knee of corpus callosum, 207

of fornix, 216

of internal capsule, 234

Knee-joint, arterial anastomosis about, 624,626

nerves to, $618,6 \mathrm{Ig}$

Knees of fissure of Rolando, 182

LABBÉ, vein of, 165

Labia majora and minora, 423

Labial artery, inferior, 64 nerves (superior maxillary), I5I

Labium majus, dartos of, 406

Lachrymal artery, 50, 53 gland, 50 nerve, 50,56

Lacuna magna, 55!

Lacunæ of urethra, 55

Lambda, I7

Lamina cinerea, 196 development of, 175

quadrigemina, 224

terminalis, 170, I73

Landmarks of abdomen, 432

of $\operatorname{arm}, 257$

of axillary space, 257

of back, 128, 255, 599

of cranium, 17

of elbow, 257

of face, 24

of foot, 554

of forearm, 255

of gluteal region, 599

of hand, 255

of head anterior, I7 posterior, I 28

of $\operatorname{leg}, 554$

of neck, anterior, 68 posterior, 128

of perineum, female, 422 male, 397

of popliteal space, 599

of shoulder, 256 
Landmarks of thigh, anterior, 432, 554 posterior, 599

of thorax, anterior, 255, 432

posterior, 319

of wrist, 258 ,

Large intestine, 500

Laryngeal artery (inferior thyroid), I12

(superior thyroid), 93

nerve, inferior or recurrent, I04 left, 34I

superior, 103

Larynx, arteries of, 146

dissection of, 145

fossa innominata, 146

interior of, 145

nerves of, 146

nerve supply to muscles, I47

ventricles of, 146

Last dorsal nerve, $529,557,602$

Lateral area of medulla, 248, 252

columns of medulla, 248,252

cutaneous branches of intercostal nerves, $35^{\circ}$

groove in crura cerebri, 240

ligaments of liver, 473,488

of uterus, 478,543

nasal artery, 65

sacral arteries, 548

sinuses of skull, 21, 40, 92

ventricle, 210

body, 210

bulb, 212

floor of, 212

horns, anterior, middle, posterior, 210

roof of, 210

Latissimus dorsi, 364

Leg, dissection of, anterior, $5^{84}$ posterior, 616

fascia of, deep, $584,5^{88}$

superficial, 584

landmarks of, 554

nerves of, cutaneous, $583,616,619$

veins of, cutaneous, 586,618

Lenticular ganglion (ophthalmic, ciliary), 56

nucleus, 228

Lenticulostriate artery, 162 in cerebral hemorrhage, 173

Lesser occipital nerve, $75,89,90,129$ omentum, 473, 492, 494

$\mathrm{sac}$ of peritoneum (sac of greater omentum), 470

splanchnic nerve, 345

superficial petrosal nerve, 203

Levator anguli oris, 60 scapulæ, 366

ani, 4I7

glandulæ thyroideæ, 98

labii inferioris, 63

superioris, 60

alæquæ nasi, 60

menti (labii inferioris), 63

palati, 144

palpebræ superioris, $5^{\circ}$

Ligament or ligaments :-

annular of ankle, anterior, 584

external, 585

internal, 586

of wrist, anterior, 302
Ligament or ligaments :-

annular of wrist, posterior, 378

of bladder, false, 477,537 true, 401,538

broad, of liver, 486

of uterus, 478,543

of clitoris, suspensory, 406, 428

coronary, of liver, 473,488

costocolic, 47 I, 477

costocoracoid, 270

duodenum (Treitz), 498

falciform of fascia lata, $56 \mathrm{I}$

of liver, 486

gastrophrenic, 473, 494

Gerdy, 302

Gimbernat's, 445

lateral of liver, 473,488

of uterus, 478,543

of liver, 486

musculus suspensorius duodeni

of ovary, 543,544

palm, superficial, transverse, 301

penis, suspensory, $406,438,440$

peritoneal, of bladder, 477,537

of colon (costocolic), 471,477

of liver, $473,486,488$

of rectum, 477

of spleen, phrenosplenic, 506

of stomach, gastrophrenic, 473, 494

of uterus, broad, 478,543

phrenocolic, 471, 477

phrenosplenic, 506

Poupart's, 434, 445, 554

prostate, 401

pterygomaxillary, I23

puboprostatic, 402

rectum, 401

round of liver, 488

of uterus, 544

sacrosciatic, great, $6 \mathrm{I} 4$

small, 615

stylohyoid, 123

stylomaxillary, 123,138

suspensory of clitoris, 406,428

of duodenum, 498

of liver, 473,486

of penis, 438,440

tarsal external and internal, 29

transverse superficial of palm, 301

Treitz, 498

triangular of perineum, deep, 402 superficial, 403

uterus, $477,543,544$

vaginal ligaments (fingers), 303

wrist, anterior, 302

posterior, 378

Zinn, 52

Ligamentum arcuatum externum, 45I, 525

internum, 525

denticulatum, 392

nuchæ, 362

patellæ, 576

splenis, 471,477

sustentaculum hepatis, 471,476

Limbic lobe, 192

Limbs internal capsule, anterior and posterior, 234

Line, nasolambdoidal, 2 I 
Line, Nelaton's, 554, 599 white, of obturator fascia, 399, 400

Linea alba, 444

semilunaris, 450

Lineæ transversæ, 435, 453

Lingual artery, 94

nerve II 8

tonsil, 143

triangle, 85

Lingula (cerebellum), 244

Lingulate convolution, 192, I94

Liver, 486

anterior border of, $49 \mathrm{I}$

development of, 467

fissures of, 488

impressions, 490

colic (hepatic flexure),

duodenal,

gastric,

renal (right kidney),

ligaments of, 486,488

lobes of, 488

notch, interlobular, 493

relations, 488

surface, 491

size, 486

surfaces, 488

weight, 486

Lobes of cerebellum, 242

amygdaloid, 242

cuneate, 242

flocculus, 242

postero-inferior, 242

posterosuperior, 242

quadrate, 242

slender, 242

tonsillar, 242

vermiform, 242

of cerebrum, central (insula), 187

cuneiform, 191

falciform or limbic, 192

frontal, 22, 184

island of Reil, 187

limbic, 192

occipital, 24,188

olfactory, 172, 198

parietal, 22, 186

temporal, 24, 187

quadrate, igr

of liver, caudate, 490

lateral, left and right, 488

quadrate, 490

Spigelian, 490

of lung, left, $35^{8}$

right, $35^{8}$

Locus cæruleus, 252

niger, 240

Long or middle subscapular nerve, 318

or internal saphenous nerve, $534,566,588$ vein, $557,574,586$

thoracic artery, 278

Longissimus dorsi, 374

Longitudinal fissure (great), brain, I8o, 194,196

of liver, 488

sinus, inferior, 36

superior, 20, 34

Longus colli, 127

Lower extremity, dissection of, anterior, 556
Lower extremity, dissection of, posterior, 599

landmarks of, anterior, 554 posterior, 599

Lower, tubercle of, 355

Lumbar arteries, 518

enlargement of spinal cord, 394

fascia, 451

ganglia of sympathetic cord, 526

nerves, 376

plexus, 529

sympathetic cord, 526

veins, 521

ascending, $348,52 \mathrm{I}$

Lumbosacral cord, 529, 55 t

Lumbricales, of foot, 641

of hand, 307

Lung, apex, base, surfaces, 262, 358

bordeis, fissures, root, 359

outlining on chest, 262 fissures of, 263,359

nerves, vessels of, 359

reaches into base of neck, 262, $35^{8}$

Lunated space in semilunar valves, 35.5

Luy's body or nucleus, 240

Lymphatics, of abdomen (inguinal), 436

of arachnoid, 156

of axilla, 274

femoral, 557

inguinal, 436

mammary, 266

ovarian, 546

uterine, 546

vaginal, 546

vulvar, 546

Lymphatic duct, right, 107

thoracic, 344

Lyre of the fornix, 218

MAJENDiE, foramen of, I55, 250

Malar nerve (superior maxillary), I50 (facial, temporofacial division), $6 \mathrm{I}$

Male genitals, 440

perineum, 397

Malleolar arteries, external and internal, 594 internal, 594, 633

Malleoli, external and internal, 5.54

Malleolus, internal, relations behind, 632

Mammary artery, external, 279 internal, $113,320,346$

gland, 266

lymphatics, 266

vessels and nerves, 266

Manubrium, 255

Marginal convolution, I9I

sinuses, 42

Marshall, oblique vein of, 353

Masseter, 67

Masseteric artery, branch of facial, 64 of internal maxillary, 121

fascia, 59,138

nerve, 117

Mastoid process, 18

Maxillary antrum (of Highmore), opening of, 148

artery, internal, 120

nerve, inferior division of fifth, 48, II7 superior division of fifth, 48 , 149 
Maxillary vein, internal, 72

"McBurney's point," 501

Measuring for shortening in lower extremity, 434, 554 upper extremity, 256

Meatus, auditory, external, 18 nasal, inferior, middle, superior, 148 urinary, female, external, 424 male, external, $415,55 \mathrm{I}$

(and female), internal, 548

Meckel's diverticulum, 471, 499

(or nasal or spheilopalatine) ganglion, 151

Median artery, 297

nerve, 288, 298, 317

vein, 282

basilic, 283

cephalic, 283

Mediastinal arteries, 321.

veins, 327

Mediastinum, 322

anterior, middle, superior, 322

posterior, 323

testis, 443

of thorax, 322

Medulla, 196, 246

development of, 17 I, 175

fissures of, anterior, 248 posterior, $25^{2}$

Medullary la minæ, 230

velum, inferior (tela choroidea, inferior), 220,250 superior (tela choroidea, superior), 220

Membrane, costocoracoid, 270

cricothyroid, 146

thyrohyoid, 146

Membranes of brain, arachnoid, 154 dura, 32 pia, 156

of spinal cord, arachnoid, 390

dura, 390 pia, 392

Meningeal artery, anterior, 44 of ascending pharyngeal, 44 middle, 44,121

posterior, 44,96

small, 121

nerves of glossopharyngeal, 206

of hypoglossal, 206

of inferior maxillary, 117

of pneumogastric, 103

of superior maxillary, 150

Meninges of brain and spinal cord, see membranes of same above

Mental artery (facial), 95

(inferior dental), 121

foramen, 68,121

nerve, 68,118

Mesenteric artery, inferior, 512 superior, $5 \mathrm{II}$

vein, inferior, 514,515

superior, 512,514

Mesentery, $463,472,476,499$

development of, 463

of sigmoid flexure or omega loop, 504

of testicle, 457

of vermiform appendix, 476

Mesocolon, transverse, 463,476

Mesoduodenum, 463
Mesogaster, 463

Mesorchium, 457

Mesorectum, 504

Metacarpal artery, 388

Metatarsal artery, 595

Midbrain, 169, 171, 175

Mitral valve, 356

Mohrenheim's fossa, 84

Monro, foramen of, 171,218

Mons veneris, 423

Morgagni, sinus of, 126

Motor oculi nerve (third cranial), 57, 200 Muscle:-

abdominal muscles, nerve supply for, 453

abductor hallucis, 637

minimi digiti, 306,637

pollicis, 306

accelerator urinæ (bulbocavernosus), 410

accessorius, 375

adductor brevis, 576

hallucis, 644

longus, 566

magnus, 578

pollicis (obliquus), 308

(transversus), 308

anconeus, $3^{8} \mathrm{I}$

aryteno-epiglottidis, 146

arytenoideus, 146

auricular, 25

azygos uvulæ, 144

biceps, 285

biceps flexor crureus, 608

biventer cervicis, 132

brachialis anticus, 285

brachioradialis, 292

buccinator, 67

bulbocavernosus (accelerator urinæ), 410

cervicalis ascendens, 135,376

coccygeus, 418

complexus (and biventer), 132

compressor urethræ, 416

vaginæ, 428

venæ dorsalis penis, 410

constrictor, inferior, middle, superior

vaginæ, 428 (of pharynx), 125

coracobrachialis, 284

corrugator supercilii, 30

cremaster, 448

formation of, 460

crico-arytenoid, 146

lateralis, 147

cricothyroid, 145

crureus, 575

deltoid, 363

depressor angulı oris, 62

labii inferioris, $6_{3}$

diaphragm, 35I

digastric, 78

erector clitoridis, 427

penis, 408

spinæ, 374

extensor brevis digitorum, 592

pollicis (primi internodii), 384

carpi radialis brevior and longior, 380

ulnaris, $3^{82}$

communis digitorum, 380 
Muscle, extensor indicis, 385 longus digitorum, 589 pollicis (secundi internodii), 384 minimi digiti, $3^{81}$

muscles of spine, 373

ossis metacarpi pollicis, 382

primi internodii pollicis, 384

proprius hallucis, 590

secundi internodii pollicis, 384

flexor, accessorius, 640

brevis digitorum, 636

hallucis, 644

minimi digiti, 307,646

pollicis (inner head), 308

(outer head), 306

carpi radialis, 291

ulnaris, 29I

longus digitorum, 627

hallucis, 627

pollicis, 298

profundus digitorum, 300

sublimis digitorum, 294

tendons in palm of hand, 311

foot, first layer, 636

fourth layer, 646

second layer, $64^{\circ}$

third layer, 644

gastrocnemius, 620

gemelli, inferior and superior, 606

geniohyoglossus, 97

geniohyoid, 96

gluteus maximus, 602

medius, 605

minimus, 615

gracilis, 566

hyoglossus, 97

iliacus, 527

iliocostalis (sacrolumbalis), 375

infraspinatus, 368

intercostals, external, 346

internal, 347

interossei of foot, dorsal, 592 plantar, 646

of hand, dorsal, 388 palmar, 309

ischiocavernosus (erector penis), 408

larynx, muscles of, nerve supply for, 147

latissimus dorsi, 364

levator anguli oris, 60 scapulæ, 366

ani, 417

glandulæ thyroideæ, II5

labii inferioris, 63 superioris, 60

levator labii superioris alæquæ nasi, 60 proprius, 60

menti (labii inferioris), 63

palati, I44

palpebræ superioris, 50

longissimus dorsi, 374

longus colli, 127

lumbricales of foot, $64 \mathrm{I}$

of hand, 307

masseter, 67

mylohyoid, 8 I

obliquus abdominis externus, 443 internus, 447

capitis, inferior and superior, 134

inferior, 58

superior, 50
Muscle, obturator externus, 582

internus, 606

occipitofrontalis, 25

omohyoid, 79

opponens minimi digiti, 309

pollicis, 309

orbicularis oris, 63

palpebrarum, 28

palatoglossus, 144

palatopharyngeus, 143

palmaris brevis, 300

longus, 291

pectineus, 565

pectoralis major, 268

minor, 274

peroneus brevis, 630

longus, 630

tertius, 590

plantaris, 621

platysma myoides, 70

popliteus, 62I

pronator radii quadratus, 648 teres, 290

psoas magnus, 527

parvus, 528

pterygoideus externus, I23

internus, 124

pyramidalis, 453

nasi, 29

pyriformis, 605

quadratus femoris, 607

lumborum, 528

menti (depressor labii inferioris), 63

quadriceps extensor femoris, 576

rectus abdominis, 453

sheath of, 454

capitis anticus, major and minor, 126 lateralis, 126

posticus, major and minor, I34

externus (eye), 52

femoris, 565

inferior, $5^{8}$

internus, 52

superior, 52

rhomboideus, major and minor, 366

risorius, 59

sacrolumbalis (iliocostalis), 375

salpingopharyngeus, 145

sartorius, 562

scalenus anticus, 100

medius, roo

posticus, ror

semimembranosus, 609

semitendinosus, 609

semispinalis colli, 135

serratus magnus, 279

posticus, inferior and superior, 370

soleus, 622

sphincter ani externus, 407 internus, 422

vaginæ, 427

spinalis dorsi, 374

splenius capitis et colli, I3I

sternocleidomastoid, 76

sternohyoid, 80

sternothyroid, 80

styloglossus, 97

stylohyoid, 79

stylopharyngeus, 97

subanconeus, 369 
Muscle, subclavius, 272

subcrureus, 582

subscapularis, 279

supinator brevis, 385

longus, 292

supraspinatus, 366

temporal, 30

tensor palati, 144

vaginæ femoris, 564

teres, major and minor, 368

thyro-arytenoid, 147

thyrohyoid, 80

tibialis anticus, 589

posticus, 628

trachelomastoid, I3I, 375

transversalis abdominis, 451

cervicis (colli), I35, 374

transversus pedis, 646

perinæi, deep, 416 superficial, 410

trapezius, 362

triangularis sterni, 320

triceps, 369

vastus externus and internus, 57.5

zygomaticus, major and minor, 59

Muscles moving the vocal cords, I47

Muscular bands of the large intestine, 500 fasciæ of orbit, 49

Musculi papillares, 356

pectinati, 355

Musculocutaneous nerve (arm), 288, 3 I5 (leg), 588 319,378

Musculophrenic artery, 321

Musculospiral nerve, 290, 318, 319, 365, 372,378

Musculus suspensorius duodeni (Ligament of 'Treitz), 498

Mylohyoid artery, $12 \mathrm{I}$

muscle, $8 \mathrm{I}$

nerve, 81,118

NARES, posterior, I4I

Nasal artery, 54

lateral, 65

duct, opening of, 148

fossæ, dissection of, 148

openings into, 148

(Meckel's) ganglion, I5I

nerves, 56, 149, 151, 152

or nasopalatine artery, 122

Nasion, 17

Nasolambdoidal line, 21

Nasopalatine artery, 148

nerve, 148,152

Nates of corpora quadrigemina, 224

Neck dissection, anterior, 69 posterior, 128

landmarks of anterior, 68

posterior, 128

of penis, 415,440

triangles of, $8 \mathrm{I}$

veins of, 74

Nélaton's line, 554, 599

Nerve or nerves:-

abducens (sixth cranial), 57, 201

acromial, 76, 89, 91, 266, 365

ansa hypoglossi, 88

anterior crural, 532, 557, 568
Nerve or nerves:-

of arachnoid membrane, 156

articular to knee, 618, 619

auditory, 204

auricular (facial), 6 I

auricularis magnus, $75,89,90$

auriculotemporal, 26, II 7

of back, 376

Bell, respiratory,

external (long thoracic), 280, 315

internal (phrenic), I00, 325

superior (spinal accessory), 76, 91, 206

buccal (facial), 6 I

(fifth), II7

calcanean, 631

calcaneoplantar cutaneous, 631

cardiac, 104, 354

cervical, 104

inferior, 107

middle, 107

superior, 106

carotid plexus, 48, 106

cavernous plexus, I06

cervical cardiac, 104, 106, 107

cord and ganglia, 106

nerves, 89

plexus, 89

superficial, $75,89,90$

cervicofacial, division of facial, $6 \mathrm{r}$

chorda tympani, II8, 203

ciliary, 56

circumflex, 318, 319, 365, 372

clavicular, $76,89,91,266$

communicans hypoglossi, 88,89

fibularis, 616, 619

peronei, 6I9

phrenici, 3 I4

tibialis, 616, 619

cranial, 198

eighth, 204

eleventh, 76, 9I, 206

fifth, 46, 201

first, 198

fourth, 50,201

ninth, 98, 204

second, 57,200

seventh, 6I, 203

sixth, 57, 201

tenth, 103, 206

third, 57,200

twelfth, 88, 206

crural anterior, 532, 5.57, 568

Cruveilheir, plexus of, I12

cutaneous nerves of abdomen, 436, $35^{\circ}$ of arm, anterior, 283 posterior, 365,378

of back, 360

of chest, 264, 350

of face, $46,6 \mathrm{I}$

of foot, dorsum, 588,598

sole, 638,642

of iorearm, anterior, 283

posterior, 378

of gluteal region, 602

of hand, dorsum, $37^{8}$ palm, 298

of head, anterior, $25,46,49,6 \mathrm{I}, 75$, 129

posterior, 129

of leg, anterior, 588 
Nerve or nerves:-

cutaneous, of leg, posterior, 616, 6I9 of neck, anterior, 75,76 posterior, 129

of perineum, 406,413

of shoulder, anterior, 264 posterior, 365

of thigh, anterior, 557 posterior, 602

of thorax, anterior, 264, 350 posterior, 360

dorsal of foot, 588,589 hand, 298,378

external (musculocutaneous), 283 (musculospiral), 378 (thigh), 532, 557, 561, 602

internal (arm), 288, 316, 319, 378 lesser, 316, 319, 365

(thigh), 557, 562

inferior (musculospiral), 318, 365

middle (thigh), 557, 562

palmar, 298

superior (musculospiral), 318, 365

dental, anterior, middle, posterior, superior, 150

inferior, 117

descendens hypoglossi, 88, 90

digital of foot, 638

of hand, 305

collateral of foot, 640

of hand, 305

dorsal or intercostal, 376

of clitoris, 414, 426

last, $529,557,602$

of pelis, 414,440

facial (seventh cranial), 6I, 203

femoral cutaneous, 604

fifth cranial, 46,201

first cranial, 198

fourth cranial, 50, 201

frontal, 49,56

genitocrural, 532,557

glossopharyngeal (ninth cranial), 98, 204

gluteal, inferior, $55^{2}$

superior, 55I, 616

great auricular, $75,89,90$

gustatory (lingual), 118

hemorrhoidal, inferior, 4I3

hypoglossal (twelfth cranial), 88, 206 loop (ansa hypoglossi), 88

hypoglossi communicantes, $88,89,90$ descendens, 88,90

iliohypogastric, $450,451,530$ cutaneous branches, 602 dorsal of penis, 440

ilio-inguinal, $44^{8}, 45^{\mathrm{I}}, 53 \circ, 557$

inferior dental, 117,150

maxillary, 48, II7

pudendal, 406

inframaxillary, $6 \mathrm{I}, 74$

infra-orbital (facial), $6 \mathrm{I}$ plexus, 6I, I5I

infratrochlear, 56

intercostal, $350,376,45 \mathrm{I}$

anterior cutaneous branches, 266,451 lateral cutaneous branches, 282, 45I

intercostohumeral, 266, 280, 282, 319 , 351,365

interosseous, anterior, 317

posterior, $293,318,386$
Nerve or nerves:-

Jacobson's, 206

labial (superior maxillary), I5I

lachrymal, 50, 56

laryngeal inferior or recurrent, Io4

left, 342

superior, 103

larynx, nerves of, 146

last dorsal, 529, 557, 602

lingual or gustatory, I18

lumbar, 376

plexus, 529

lumbosacral cord, 551, 529

malar (facial), 6I

(superior maxillary), I50

masseteric, II7

maxillary, inferior, $48, I_{7}$

superior, 48,149

median, 288, 298, 316, 317, 319

mental, 68, i1 8

meningeal (glossopharyngeal), 206

(hypoglossal), 206

(inferior maxillary), II7

(pneumogastric), $\mathrm{IO}_{3}$

(superior maxillary), 150

motor oculi (third cranial), 57, 200

musculocutaneous (arm), 288, 315, 319, (leg) $5^{88^{3}}$

musculospiral, 290, 318, 319, 365, 372, 378

mylohyoid, 81, i18

nasal, 56, 149

(superior maxillary), 15

superior (superior maxillary), 152

nasopalatine, 148,152

obturator, 534,582

accessory, $536,5^{8} 3$

occipital, great, 129

small, $75,89,90,129$

third, I 29

oculomotor (third cranial). 57, 200

œsophageal, I04, 342

olfactory (tract, bulb, nerves), I 98

optic (second cranial), 57, 197, 200

(commissure, tracts, nerves)

ophthalmic, 48,54

orbital or temporomalar, 150

(Meckel's ganglion), I5I

parotid, 66

palatine, anterior, $\mathrm{I} 5 \mathrm{I}$

external, $\mathbf{I}_{52}$

posterior, 152

palmar cutaneous, 298, 301

palpebral (superior maxillary), I5I

perineal, 414

penis, dorsal nerve, 4I4

petrosal, great or deep, 46,151

superficial, external, 203 great, I5I, 203 lesser, 203

pharyngeal, I03, 106 plexus, 106

phrenic, 89, 100, 324

plantar, external, 642 internal, 638

plexus, brachial, 312

cardiac, deep, 354

superficial, 354

carotid, 48, 106

cavernous, 106 


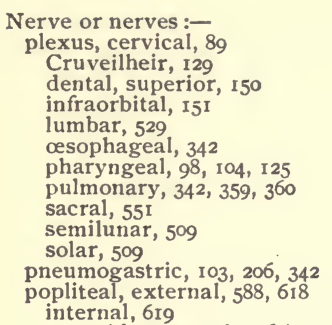

pterygoid, external and internal, ${ }_{117}$ pudendal, long or inferior, 406, 604 pudic, internal, 413,552

in female, 426

pulmonary plexus, posterior, $341,359,360$ radial, $293,318,378$

recurrent articular of popliteal, 619 of small sciatic, 604

laryngeal, 342

rhomboid, 314

sacral, 376, 551, 602

cutaneous filaments, 602

fourth, 55 I

plexus, 551

saphenous, external or short, 616

internal or long, $534,566,588$

sciatic, great, 553,610

small, 552, 602, 604

second cranial, 57,200

semilunar, 509

septal or nasopalatine, 148,152

or nerve of Cotunnius, 152

seventh cranial, 6I, 203

sixth cranial, 57,201

Sommering, 406

solar, 509

sphenopalatine, 149

spinal, 376, 395

accessory, 76, 91, 206

splanchıic, great, lesser, smallest, 345

sternal, 76, 89, 91, 266

stylohyoid, 6r

subclavian, 3I5, 3 I9

suboccipital, 136

subscapular, upper, middle, lower, 318 319

superficial cervical, $75,89,90$

supra-acromial, 76, 89, 91, 266, 365

supraclavicular, 76, 89, 91, 266

supramaxillary, 6I

supra-orbital, 25,49

suprascapular, 315, 3I9, 371

suprasternal, 76, 89, 9I, 266

supratrochlear, 25,49

supply for abdominal muscles, 453

sympathetic cord and ganglia, cervical, I06

lumbar, 526

sacral, 553

thoracic, 345

temporal (facial), 26, 6I

(inferior maxillary), 117

(superior maxillary), I50

temporofacial, division of facial, 61

temporomalar, 150
Nerve or nerves:-

tenth cranial (pneumogastric), 103, 206, 342

third cranial (motor oculi), 57, 200

thoracic, anterior, external, 272, 315, 319 internal, 316, 319

posterior or long, 280, 315

tibial, anterior, 598

posterior, 631

trigeminal (fifth cranial), 46, 201

trochlear (fourth cranial), 50, 201

tympanic, 206

to abdominal muscles, 453

arachnoid, 156

larynx, 146, 147

muscles of larynx, 147

obturator internus, 552,610

ovary, 546

pectineus, 568

penis, 414,440

pterygoideus, externus, 117

internus, 117

pyrifornis, 552

quadratus femoris, 612,552

trapezius, 89,91

ulnar, 288, 297, 298, 316, 319

Vidian, I5I

of Wrisberg, see lesser internal cutaneous, $316,319,365$

Ninth cranial nerve (glossopharyngeal), 98,204

Nipple, 255, 266

Nose, fossæ of, 148

meatuses of, 148

Notch, interclavicular or suprasternal, 255

sciatic, greater and smaller, $6 \mathrm{r}_{5}$

supra-orbital, 25

Nuck, canal of, 544

Nucleus, amygdaloid, 236

caudate, 226

dentate, 246

lenticular, 228

locus niger, 240

red, 240

subthalamic or Luy's, 240

Nutrient artery to clavicle, 113

artery, of femur (third perforating), 580

of fibula, 633

of humerus (brachial), 287

of ilium, $6 \mathrm{r}_{2}$

of radius, anterior interosseous, 297

of tibia, 632

of ulna, anterior interosseous, 297

Nymphæ (labia minora), 423

ObliquUs abdominis, externus, 443

internus, 447

capitis, inferior and superior, 134

inferior (eye), $5^{8}$

superior (eye), 50

Obliterated hypogastric arteries, 538, 547

Obturator artery, 547,583

accessory nerve, $536,5^{83}$

externus, 582

fascia, 398

internus, 606

nerve to, 6 ro 
Obturator nerve, 534,582 accessory, 536, 583

Occipital artery, 95, 130.

bone, external protuberance (crest), 18 superior curved line, 18

convolutions, inferior, middle, supeinferior, 194 rior, 188

lobe, 189

convolutions of, 189,194

referred to exterior of cranium, 24 sulci of, 24,188

nerves, great, 129

small, $75,89,90,129$

third, 129

protuberance, external, i8

sinus, 42

sulci, inferior and superior, 24, 188

triangle, 84

Occipitofrontalis, 25

Occipitotemporal convolution, inferior, 194

superior, I92, 194

Oculomotor groove, 140

nerve (third cranial), 57, 200

Esophageal arteries, i1 2, 346, 509, 516 nerves, 104, 342

Esophagus, 343

Olecranon, 257

Olfactory bulb, 198

lobe, development of, 170, 172

nerve, 198

sulcus, I94

tract, 198

Olivary body, 248

Omega loop, 477, 501, 502

Omental arteries (epiploic), 5 II

Omentum, 472

cavity of greater, 468,470

development of greater, 468

gastrocolic or greater, $468,470,476,483$, 494

gastrohepatic, lesser, $473,492,494$

gastrosplenic or third, 506

Omohyoid, 79

Opening, femoral, 525

in adductor magnus, 578

Fallopian tube, 544

saphenous, 560

superior of thorax, 323

of ureters into bladder, 548

Openings into auricle, left, 355 right, 354

in diaphragm, 35I

into nasal fossæ, 148

into stomach, cardiac and pyloric, 494

of the ducts of prostatic glands, 550 of glands of Bartholin, 424

Operculum, i 86

Ophthalmic artery, 26, 53

division of fifth cranial nerve, 48,54

ganglion, 56

nerve, 48,54

veins, inferior and superior, 54

Opponens minimi digiti, 309

pollicis, 309

Optic chiasm or commissure, 197

nerve, $57,197,200$

thalamus, 230

development of, 17 I, I73, 232
Optic thalamus, commissure, middle and posterior, 232

pulvinar, 230

sulcus choroideus, 230

tubercle, anterior, 230

tracts, 197

Orbicularis oris, 63

palpebrarum, 28

Orbit, dissection of, 48

Orbital artery, 62

convolutions of frontal lobe, 194

fascia, 49

sulcus, I92

or temporomalar nerve, 150

Orifice of ejaculatory ducts, 550

of stomach, cardiac, pyloric, 494

of vagina, 424

Os calcis, 554

uteri, 543

Otic ganglion, I19

Outlining on exterior of body the shape of the brain, 18

colon, 505

heart and great vessels, 259

intestines, 505

liver, 49I

lungs, and fissures, 262

spleen, 506

stomach, 495

Ovale centrum, majus and minus, 207

Ovarian artery, 518

ligament, 543,544

lymphatics, 546

plexus, 546

veins, 521

Ovary, 544

ligament of, 543,544

lymphatics of, 546

nerves of, 546

PACCHIONian glands, 155

Palate, soft, 142

Palatine arteries, 95, 122

arches, anterior and posterior, 142

nerves, 151, 152

Palatoglossus, I44

Palatopharyngeus, 143

Palm, dissection of, 300

Palmar arch, deep, 3 Io superficial, 303

fascia, 30 I

nerves, cutaneous, 298 , 301

synovial sac for long flexor tendons, 3 I

Palmaris brevis, 300

longus, 291

Palpebral arteries, 54

fascia, 49

nerves, I5I

Pampiniform plexus, female, 546 male, 521

Pancreas, 508

development, 467

duct (Wirsung), 508

position and relations, 508

Pancreatic arteries, 510

veins, 515

Pancreatica magna, 510

Pancreaticæ parvæ, 5 10 
Pancreaticoduodenalis artery, inferior, 5 II superior, 51 I

veins, 512

Paracentral lobe, or convolution, I86, I9I

Parietal convolution, ascending, inferior, eminence, 18 uperior, 186

lobe, 186

indicated on exterior of skull, 22

Parieto-occipital fissure, $184,190,194$ on exterior of skull, 22

Parotid, arterial branches to, 62 fascia, 59,138

gland, 66

duct of (Stenson's), 66

nerves, 66

veins, 66

Pars bulbosa of urethra, 55

Patella, 554

ligament of (ligamentum patellæ), 576

Pectineus, 565

Pectoral fascia, 268

Pectoralis major, 268

minor, 274

Peduncles of cerelvellum, I96, 244

of cerebrum, 196

of corpus callosum, 197, 210

of pineal gland, 224

Pelvic fascia, 398 et seq. viscera, 537 dissection of, 536

Pelvis, comparative measurement of male

Penis, 440 and female, 422

angle, 440

arteries of, dorsal, superficial, 436, 573

body, 440 deep, 412, 413

bulb, 415

coverings, 442

crura of, 416

dartos, 406, 415

dissection, 435,440

elastic sheath of, 415

glans, 415, 440

neck, 4I5

nerves, dorsal, deep, 414 superficial, 440

prepuce, 440

suspensory ligament, $406,438,440$

vein, dorsal, 442

Perforated space, anterior, 194, 196 posterior, I97

Perforating arteries, of foot, 596, 643 of hand, 3ro

of internal mammary, 321

of profunda femoris, 579

Pericardial arteries, 345

veins, 327

Pericardium, 328

Perineal arteries, superficial, 4I 2 transverse, 4I2

body, 43 I

fascia, deep layer, 402, 403 superficial, deep layer (Colles'), 404 nerves, 414

triangles, 408, 427

Perineum, female, 422 dissection, 424
Perineum, landmarks, 422

nuale, 397

dissection, 404

landmarks, 397

triangle of, 408

Peritoneal ligaments of the bladder, 477 colon (costocolic), 47 I, 477

liver, 486

rectum, 477

spleen, 506

stomach, 473, 494

uterus, 478

fossæ, 473,482

reflections, 471,483

over bladder, 537

uterus, 543

Peritoneum, 462

development of, 462

divisions, parietal, visceral, 462

ligaments, 473

mesentery, 472

omenta, 472

line of attachment to posterior abdominal wall, 470

traced in section, anteroposterior, 473 transverse sections, 478

viscera, covered by, 482

Peroneal artery, 633

anterior, 594

posterior, 633

nerve, from external popliteal, 619

vein, 633

Peroneus, brevis, б́зо

longus, 630

tertius, 590

Pes hippocampi, 214

Petrosal nerve, deep, great, 46, I5I

superficial, external, 203

great, 151, 203

lesser, 203

sinuses, inferior, superior, 42, 92

Petrous ganglion (glossopharyngeal), 204

Pharyngeal aponeurosis, 124

artery, ascending, 96

muscles, 125

nerves, 103,106

plexus, 106

tonsil, 142

Pharynx, 124

dissection of, I4I

interior of, $14 \mathrm{I}$

muscles of, 125, 143

Phrenic artery, 516

nerve, $89,100,324$

vein, inferior, 521

Phrenocolic ligament, 47I, 477

Phrenogastric ligament, 473, 494

Phrenosplenic ligament, 506

Pia (brain), 156

dissection of, 165

removal of, 165

(spinal), 392

Pillars (or columns) of the external abof the fauces, 142

of the fornix, anterior and posterior. 216

Pineal gland, 224

development of, 171

Pituitary gland, 197 
Pituitary gland, development of, I7 I, 175

Plantar arch, 643

artery, digital, 640,643

collateral, 643

external, 642

internal, 640

fascia, 634

nerve, extermal, 642

internal, 638

Plantaris, 621

Platysma myoides, 70

Pleuræ, 323

relation to the chest walls, 264

Pleural arteries, 346

Plexus, brachial, 3I2

cardiac, deep and superficial, 354

carotid, 48, 106

cavernous, 106

cervical, 89

Cruveilheir, 129

dental, superior, I50

infraorbital, 15I

lumbar, 529

œsophageal, 342

pharyngeal, 98, 104, 125

pulmonary posterior, $342,359,360$

sacral, $55^{\mathrm{I}}$

semilunar, 509

solar, 509

venous, choroid of lateral ventricle, 218 fourth ventricle, 220,250

third ventricle, 220

dorsal of foot, 586,638

of hand, 378

hemorrhoidal, 408

ovarian, 546

pampiniform, female, 546 male, 521

prostatic, 418

vesical, 420

vesicoprostatic, 401

Pneumogastric (tenth cranial), 103, 206, 342

Pomum Adami (Adam's apple), 69

"Points," anatomical, 599

"Point, McBurney's," 501

Sylvian, I8

Pons, I96, 246

development of, 171, 175

hepatis, 490

Popliteal artery, 623,624

nerve, external, 588, 618 internal, 619

space, 620

dissection, 62 I

landmarks of, 599

vein, 623

Popliteus, 621

Portal vein, 492, 514

Posterior auricular artery, 96

circumflex artery, 279, 372

nares, 141

scapular artery, I12, II3, 370

Pouch of Douglas, 431, 477, 483, 543

glosso-epiglottic, 143

rectovaginal, 483,543

rectovesical, 483

uterovesical, 483,543

Poupart's ligament, 434, 445, 554

Precentral sulcus, I95
Prepatellar bursa, $55^{8}$

Prepuce of clitoris, 423

of penis, 440

Princeps cervicis, artery, I30

pollicis, 310

Process, acromion, 256

angular, external (E. A. P.) and internal, 18

coracoid, 256

malar, 24

mastoid, 18

olecranon, 257

styloid of radius and ulna, $25^{8}$

vermiform of cerebellu m, 242

zygomatic, I 8

Processus vaginalis of peritoneum, 460

Profunda artery (brachial), inferior and superior, 287.372

cervicis (deep cervical), I1 $3,130,135$

cervicis vein, I I I, I36

femoris artery, 579

vein, 574

Pronator quadratus, 648

radii teres, 290

Prostate, 420 capsule of, 401,421

ligament, 401

Prostatic glands, opening of ducts, $55^{\circ}$ plexus, venous, 418

sinus, $55^{\circ}$

urethra, 550

Protuberance, external occipital, 18

Psoas abscess, 525

magnus, 527

parvus, 528

Pterygoid artery, 121

nerves, II 7

Pterygoideus externus, 123

internus, 124

Pterygomaxillary ligament, I23

Pterygopalatine artery, 122

Pubes, 554

body of, 554

spine of, 554

symphysis of, 554

Pubic artery, 547

portion of fascia lata, 560

spine, 554

Puboprostatic ligament, 402

Pudic artery, external, deep, 573 superficial or superior, 557,573

internal, 4I I, 547

in female, 426

nerve, internal, 413,552

in female, 426

vein, internal, 413

superficial, external, 436, 557

Pudendal nerve, inferior, 406,604

Pudendum, 423

Pulley for tendon of superior oblique, 50

Pulmonary artery, 332

left, 334

right, 332

plexus, posterior, $341,359,360$

sinuses (Valsalva), 356

valves (semilunar), 355

veins opening into left auricle, 359

Pulvinar (optic thalamus), 230

Putamen (lenticular nucleus), 230

Pyloric artery, 510 
Pyloric orifice of stomach, 494 vein, 514

Pylorus, 494

Pyramidalis abdominis, 453

nasi, 29

Pyramids of medulla, 248

Pyriformis, 605

fascia of, 399

QUADRATE lobe of cerebellum, 242 cerebrum, I9I of liver, 490

Quadratus femoris, 607

nerve to, 552,612

lumborum, 528

menti (depressor labii inferioris), 63

Quadriceps extensor femoris, 576

Quadrigeminal bodies, 224

nates, testes, crucial sulcus, 224

RADIAL artery, in forearm, 293

in the hand, 3 ro

at the wrist, 387

carpal, anterior, 294

posterior, 310

recurrent, 294

nerve, $293,318,378$

vein, 282,378

Radialis indicis artery, 310

Radius, nutrient artery to, 297

Ranine artery, 94

Raphe of corpus callosum, 210

Receptaculum chyli, 527

Recess, tonsilar, 142

Rectal artery, inferior, 412

middle, 547

superior, 514

tube, objection to, 506

Rectovaginal fossa, $477,483,543$

Rectovesical fascia, 399,538

fossa, 477,483

Rectum, lower portion, 42I

upper portion, 5OI, 504

ligaments of, 401,477

Rectus abdominis, 453 sheath of, 454

capitis anticus, major and minor, 126 lateralis, 126 posticus, major and minor, 134 femoris, 565

inferior (eye), 58

internus (eye), 52

superior (eye), 52

Recurrent arteries, interosseous, 386 palmar arch, deep, 310

radial, 294

tibial, anterior, posterior, 594

ulnar, anterior, posterior, 296

nerves, laryngeal, 342

of popliteal, 6I9

of small sciatic, 604

Red nucleus, 240

Reduplicated fold of corpus callosum, 208

Reflections of peritoneum, $47 \mathrm{I}, 483$

Regions of the abdomen, 435

of the face, 22

Reil, island of, 187

Relations at the elbow, 257
Relations behind the internal malleolus, 632

Removal of arachnoid, 156

of brain, 36

of dura, 34

of fascia lata, 562, 602

of deep fascia of leg, 588,618

of heart and lungs from thorax, 343

of palmar fascia, 302

of pia, 165

of plantar fascia, 636

Renal artery, 516

vein, 521

Restiform body (or tract) of medulla, 244 , 254

Retina, central artery of, 53

Retropharyngeal abscess, I4 I

Retzius, space of, 478,537

Rhomboid nerve, 314

Rhomboideus, major and minor, 366

Ribs, how to count, 257

Rima glottidis, I45

pudendi or genitalis, 423

Ring, abdominal (inguinal), external, 444 internal, 456 formation of, 457

" Rings," so-called anatomical, 446, 599

Risorius, 59

Rivinus, ducts of, 120

Rolando, fissure of, $2 \mathrm{I}, \mathrm{I} 82$ development of, 177

Root of lungs, 359

of penis, 440

Rosenmüller, fossa of, 142

Rostrum of corpus callosum, 196, 208

Rotation of intestines, 464

Round ligament of liver, 488

of uterus, 544

Rugæ of vagina, 43 I

SAc, pleural, 323

pericardial, 328

peritoneal (greater omentum), 470

Sacculations of large intestine, 500

Sacra media, 518

Sacral arteries, lateral, 548

middle, 518

nerves, $376,551,602$

plexus, $55^{1}$

sympathetic cord and ganglia, 553

Sacrolumbalis (iliocostalis), 375

Sacrosciatic foramina, greater, smaller, 615

ligament, great, 614 small, 615

Salivary glands, $66,85,119$

Salpingopharyngeus, 145

Saphenous lymphatic glands (femoral), 557

nerve, external or short, 616

internal or long, $534,566,588$

opening, 560

vein, external or short, 586,618 internal or long, $557,574,586$

Sartorius, 562

Scalenus anticus, medius, posticus, 100, IOI

Scaphoid bone, tubercle of, 554

Scapular artery, posterior, I12, I13, 370 
Scapular artery, dorsal, 278, 37 I

Scarpa, fascia of, $43^{8}$

foramina of, 152

triangle of, 568,570

Sciatic artery, 547, 6I3

nerve, great, 553,610

small, $552,602,604$

notch, greater and lesser, $6 \mathrm{I}_{5}$

Scrotum, dartos, 406

Second cranial nerve, 57,200

Semilunar fold of Douglas, 450

ganglion, 509

valves, 355

Semimembranosus, 609

Seminal vesicles, 420

Semispinalis colli, I35

Semitendinosus, 609

Septal artery, I48, 149

nerve (of Cotunnius), I48, I52

Septum, arachnoid, 392

crural, 526

lucidum, 214

development of, 173

pectiniforme, 416

Serratus magnus, 279

posticus, inferior and superior, 370

Seventh cranial nerve, 61,203

Sheaths of long flexor tendons in palm, 303

of rectus abdominis, 454

chin, 554

Shortening, measuring for, in lower extremity, 434, 554

in upper extremity, 256

Shoulder, cutaneous nerves of, anterior, 264

posterior, 365

dissection of, anterior, 272

posterior, 360

landmarks of, 256

Sight, center of, 200

Sigmoid artery, 512

flexure of colon, 477, 501, 502

sinus (lateral), 21, 40, 92

Sinus, cavernous, 43

circular, 44

confluence of (torcular Herophili), 35,

coronary, 353,354

lateral, 21, 40, 92

longitudinal, inferior, 36

superior, 20,34

marginal, 42

of Morgagni, 126

occipital, 42

petrosal inferior, 42, 92

superior, 42

prostatic (pocularis), 550

straight, 40

transverse, 44

of Valsalva, 356

Sixth cranial nerve, 57,201

Skull, interior of base, 38

dissection of interior, 44

fossæ of, anterior, middle, posterior, $3^{8}$

Small intestines, 499

occipital nerve, 75, 89, 90, I 29

sacrosciatic ligament, $6 \mathrm{I}_{5}$

sciatic nerve, $552,602,604$

center of, 200
Socia parotidis, 66

Sœmmering, nerve of, 406

Soft palate, 142

Solar plexus, 509

Soleus, 622

Space, anterior elbow, 292

axillary, 257, 268, 270, 275, 648

of Burns, 139

interpeduncular, 198

lunated in semilunar valves, 355

perforated, anterior, 194, 196

posterior, 197

popliteal, 620

of Retzius, 478,537

subarachnoid, anterior, I54

posterior, I55

spinal, 392

subdural, 32, 152

spinal, 391

Spermatic artery, 518

fascia, external, 444

formation, 460

internal, 456

plexus (pampiniform), 521

veins, 521

Sphenoidal fissure, 46

Sphenopalatine artery, 148

(Meckel's) ganglion, 15I

Sphincter ani, externus, 407 internus, 422

vaginæ, 427

Spigelian lobe of liver, 490

Spinal nerves, 376,395

Spinal accessory nerve, 76, 91, 206

arachnoid, 390

canal (central), 394

cord, 392

dissection of, 389

fissure, anterior and posterior, 394

dura, 390

muscles, 373

nerves, 395

posterior branches, 376

pia, 392

Spinalis dorsi, 374

Spine of ilium, anterior superior, 554 posterior superior, 599

of pubes, 554

of scapula, $25^{6}$

of tibia, 554

Splanchnic nerves, great, lesser, smallest,

Spleen, $506^{345}$

development, 467

ligaments, 506

position, 506

relations, 506

size, 506

Splenic artery, 510

flexure of colon, 477, 501, 503

vein, 515

Splenium of corpus callosum, 207

Splenius capitis et colli, $1_{3}$ I

Spongy portion of urethra, 550

Stenson's duct (parotid), 66 foramina, 149

Sternal artery (internal mammary), 321 nerve $76,89,91,266$

Sternocleidomastoid, 76

Sternohyoid, 80 
Sternomastoid artery (occipital), 96 (superior thyroid), 93

Sternothyroid, 80

Stomach, 494

fundus, 494

impression on liver, 490

openings, cardiac pyloric, 494

position, 494

relations to adjacent organs, 494

to surface of body, 495

Straight sinus, 40

Striæ acusticæ, 252

longitudinales, 210

Styloglossus, 97

Stylohyoid, 79

Stylohyoid ligament, I23

Styloid processes of radius and ulna, 258

Stylomastoid artery, 96

Stylomaxillary ligament, 123, 138

Stylopharyngeus, 97

Subanconeus, 369

Subarachnoid space, anterior, 154 posterior, 155 spinal, 392

tissue, 154,392

Subclavian artery, 107 left, 340

nerve, 315,319

triangle, 84

vein, 92,99

Subclavius, 272

Subcrureus, 58

Subcutaneous nerves of the abdomen, 436 of the arm, anterior, 283

posterior, 365,378

of the back, 360

of the chest, 264,350

of the face, $46,6 I$

of the foot, dorsum, 588,598

sole, 638,642

of the forearm, anterior, 283

posterior, 378

of the gluteal region, 602

of the hand, dorsum, 378

palm, 298

of the head, anterior, $25,46,49,61,75$, I 29

posterior, 129

of the inguinal region, 436

of the leg, anterior, 588

posterior, 616,619

of the neck, anterior, 75,76

posterior, 129

of the perineum, 406, 4I3

of the shoulder, anterior, 264

posterior, 365

of the thigh, anterior, 557

posterior, 602

of the thorax, anterior, 264,350

posterior, 360

Subdural space, 32, 152, 391

Sublingual artery, 94

duct (of Rivinus), 120

gland, 119

Submaxillary arteries, 95

duct (Wharton's), 86

ganglion, 119

gland, 85

triangle, 82

Submental artery, 95
Suboccipital nerve, 136

triangle, 132

Subparietal sulcus, I9I

Subscapular artery, 278, 37 I

nerves, lower, middle, upper, 318, 319

Subscapularis, 279

Subthalamic nucleus, 240

Sulcus, basilar, 246

calcarine, 190, 194

callosal, I9I, 192

central, 189

choroideus, 230

circular, 189

collateral, 190, 194

crucial, 224

frontal, inferior, precentral, superior, 22,185

hippocampal or dentate, 19c, 194

intraparietal, 22, 186

lateral (crus cerebri), 240

medulla, anterior, 248 ; anterolateral, 248

posterior, 252 ; posterolateral, 252

occipital, inferior, superior, 24, 188

olfactory, I 94

orbital, 192

parieto-occipital, 22, I84, I90, 194

posterior olivary, 248

precentral, I95

subparietal, Igr

temporal, inferior, 194; middle, superior, 24,187

Summary of the development of the abdominal viscera and peritoneum, 484

Superficial cervical nerve, 75, 89, 90

volar artery, 294

Superficial fascia, abdomen, 436, 438

arm, 264, 282, 283

back, 360,362

foot, dorsum, $5^{84}$

forearm, anterior, 282,283

gluteal region, 600

hand, 282,360

inguinal region, 436,438

leg, $5^{84}$

neck, 360

perineum, 404

shoulder, anterior, 264

posterior, 360

sole, 634

thigh, anterior, 556

posterior, 600

thorax, anterior, 264

posterior, 360

Superior curved line of the occipital bone, 18

longitudinal sinus, 20,34

oblique muscle (eye), 50

(capitis), 134

profunda artery, 372,387

Supinator brevis, 385

lollgus, 292

Supra-acromial nerves, $76,89,91,266$, 365

Supraclavicular nerves, 78, 89, 9I, 266

Suprahyoid artery, 94

Supramarginal convolution, I86

Supramaxillary nerve, 6 I

Supra-orbital arch, I8

artery, 18, 26, 53 
Supra-orbital foramen, 68

nerve, 25,49

notch, 25

Suprarenal arteries, 516

bodies, 524

veins, 521

Suprascapular artery, II2, 37 I

foramen, II 2

ligament, II2

nerve, 315, 319, $37 \mathrm{I}$

notch, II 2

vein, 74, 99

Supraspinatus, 366

Suprasternal nerves, 76, 89, 9I, 266 notch, 255

Supratrochlear nerve, 25, 49

Sural arteries, 624

Suspensory ligament, clitoris, 406,428 duodenum, 498 of liver, 473,486 of penis, $406,438,440$

Sustentaculum hepatis, $47 \mathrm{I}, 476$ splenis, 47I, 477 tali, 554

"Sylvian point" on skull, I8

Sylvius, aqueduct of, 240 development of, 176

fissure of, 21, 180, 194, I97

Sympathetic cords and ganglia, cervical, I06

lumbar, 526

sacral, 553

thoracic, 345

Symphysis pubis, 554

Synovial membrane of the palm and fingers, 3 I I

T ENIA semicircularis, 236

tectæ, 210

Tail of caudate nucleus, 228

of epididymis, 443

of pancreas, 508

Tapetum, 208

Tarsal arteries, external and internal, 595 ligaments, external and internal, 29

Tegmental (red) nucleus, 240

Tegmentum, 240

Tela choroidea, inferior, 220, 250 superior, 220

Temporal artery, 26, 62

anterior, 26,

deep, 12I

middle, 62

posterior, 26

convolutions, inferior, middle, superior,

fascia, 30 187, 194

lobe, 187

referred to exterior of cranium, 24

muscle, 30

nerves (facial), 26, 6I

(inferior maxillary), II 7

(superior maxillary), I50

auriculotemporal, 26, i 7

deep, II 7

sulci, inferior, 194

middle, superior, 24,187

vein, 72

Temporofacial division facial nerve, 6I
Temporomalar nerve, I50

Temporomaxillary sinus or vein, 72

Tendo-Achillis, 622

Tendo-oculi, 28, 29

Tendon, conjoined, 447

Tendons of long flexor muscles of fingers in palm, $31 \mathrm{I}$

Tenon's capsule, 49

Tensor palati, I 44

vaginæ femoris, 564

Tenth cranial nerve (pneumogastric), $103,206,342$

Tentorium cerebelli, 38

Teres major and minor, 368

Testes of corpora quadrigemina, 224

Testicle and cord, coverings of, 442 dissection of, 443 formation of same, 457

Testicle, 442

descent of, 457

dissection of, 443

gubernaculum of, 457

mediastinum of, 443

mesentery of (mesorchium), 457

tunica albuginea, 443

vaginalis, 443,460

Thebesius, foramina of, 354

valve of, 355

Thecæ of tendons of flexors of fingers, 303

Thigh, dissection, anterior, 556 posterior, 600

fascia lata, removal of, 562,602

landmarks, anterior, 432,554 posterior, 599

superficial arteries, veins, nerves, 557 , 602 fascia, 556

Third cranial nerve (motor oculi), 57, 200 ventricle, 222

Thoracic aorta, 338,345

artery, 278

alar, 278

long, 278

superior, 276

duct, 344

nerves, anterior, external, 272, 3I5, 3I9 internal, 316, 319

posterior or long, 280, 315

spinal (intercostal), 350, 376, 45

twelfth or last, $529,557,602$

opening, superior, 323

viscera, 334 et seq.

Thorax, 319

dissection of, 264

interior, 319

posterior, 360

fascia of anterior, 268

posterior, 360

landmarks of, anterior, 255, 432

posterior, 319

mediastina, 322

movements of, 347

opening in, superior, 323

viscera within, 334 et seq.

Thymus gland, 324

Thyro-arytenoid, 147

Thyrohyoid, 80

membrane, 146

Thyroid artery, inferior, III 
Thyroid artery, superior, 93 axis, III gland, II4 veins, IIs

Thyroidea ima, II5

Tibia, nutrient artery of, 632 malleolus of, 554

tuberosities of, 554

Tibial artery, anterior, 593, 594 posterior, 631,632 recurrent, 594

nerve, anterior, 598 posterior, 631

veins, 593,632

Tibialis anticus, $5^{89}$ posticus, 628

Tissue, subarachnoid, I54, 392

Tongue, 142 artery of, dorsal (lingual), 94

Tonsil, lingual, I43 pharyngeal, I42

Tonsillar artery (facial), 95 lobe of cerebellum, 242 recess, $\mathrm{I}_{42}$

Tonsils, I42

Torcular Herophili, 35, 42

Trachea, 356

Tracheal artery, II 2

Trachelomastoid, I3I, 375

Tracts, archiform, external, 248, 254 olfactory, 198 optic, 197, 200

Transversalis abdominis, $45^{\mathrm{I}}$ cervicis, 135, 374 artery, I12 vein, 74,99

fascia, 454

Transverse aorta, 336

of basilar, 164

cervical artery, II2 vein, 74,99

colon, 476 , 50 I of external circumflex, 579

facial artery, 62

fissure of brain, 180 development, i76 of liver, 488

mesocolon, 476

perineal artery, 412

sinus, 44

Transversus pedis, 646 perinæi, deep, 416 superficial, 410

Trapezius, 362

Treitz, ligament of, 498

Triangle, Hesselbach's, 457

of neck, 8r

anterior, 82

carotid, inferior, 83 superior, 83

lingual, 85

occipital, 84

posterior, 82

subclavian, 84

submaxillary, 82

suboccipital,132

perineal, 408

of Scarpa, 568, 570

Triangular fascia, 446 ligament, deep, 402
Triangular ligament, superficial, 403 space at base of bladder, external, 420 , 450

internal, $55^{\circ}$

Triangularis sterni, 320

Triceps, 369

Tricuspid valve, 355

Trigeminal nerve (fifth cranial), 46, 201

Trigone, external, $420,54^{\circ}$

internal, $55^{\circ}$

Trigonum habenulæ, 232

hypoglossi, 252

Trochanter, great, 554

Trochlear nerve (fourth cranial), 50, 201

Trolard, vein of, 165

Tubal eminence of Eustachian tube, 142

Tube, Eustachian, I41, 145

Fallopian, 543, 544

rectal, objection to, 506

Tuber cinereum, 196, 197 development of, 175

Tubercle of Lower, 355 deltoid, 256

of optic thalamus, anterior, 230 of scaphoid, 554

Tuberculum acusticum, 252

Tuberosity of humerus, 256 of ischium, 554,599

of tibia, 554

Tunica albuginea, of testicle, 443 vaginalis, of testicle, 443,460

Twelfth cranial nerve, 88,206 or last dorsal (intercostal), 529, 557, 602

Tympanic artery, 120 nerve (Jacobson's), 206

ULNAR artery, in the forearm, 295

in the hand, 304

carpal branches of, anterior, 297 posterior, 297, 388

recurrent, anterior and posterior, 296 nerve, $288,297,316,319$

vein, anterior, 282

common, 282 posterior, 282,378

Umbilical arteries (obliterated hypogastric), $53^{8}, 547$

fissure of liver, 490

ring, 434

vein, 490

Uncinate convolution, I9r, 192, 194

Upper extremity and thorax, landmarks of, 255

posterior, 360

measuring for shortening, 256

Urachus, 538

Ureter, 524, 540

calices, relations, sinus, 524

course, length, relations, size, $54^{\circ}$

openings of, 548

Urethra, dissection of interior, 548

female, 43 I

male, 550

fossa navicularis, $55 \mathrm{I}$

lacunæ, 55I

membranous portion, 550

pars bulbosa, $55 \mathrm{I}$

prostatic portion, $55^{\circ}$

spongy portion, $55^{\circ}$ 
Urethral triangle (perineal), 408

Urinary bladder, 537,548

meatus, external, female, 424 male, 4I 5, 55I

internal, 548

Urine, course of extravasated, 404,438 , 440

Uterine artery, 547

plexus (venous), 420

Utero-inguinal ligament (round), 544

Utero-ovarian ligament (ligament of ovary), 543,544

Uteropelvic ligament (broad), 473, 543

Uterovesical ligament (anterior), 543 pouch or fossa, $477,483,543$

Uterus, 543

body, cervix, isthmus, 543

ligaments, broad, 477

round, 544

peritoneal, 543

lymphatics, 546

nerves of, 546

position, relations, size, 543

Uterus masculinus, $55^{\circ}$

Uvula, 142

VAGINA, 430

bulb of, 427

orifice of, 424

relations of, 430

Vaginal arteries, 547

ligaments (fingers), 303

lymphatics, 546

plexus (venous), 420

Vagus (pneumogastric) nerve, 103, 206, 342

Vallecula of cerebellum, 242

Valsalva, sinuses of, .356

Valve, Eustachian, 355

ileocecal, 499

mitral (bicuspid), 356

semilunar, 35.5

Thebesius, 355

tricuspid, 355

of Vieussens, 244

Vas deferens, 542

ampulla, course, ejaculatory duct, artery of, 547 length, relations, size, 542

Vasa brevia, 5 10

intestini tenuis, 512

veins, $5^{1} 5$

Vastus externus, 575

internus, 575

Vein or veins :

acromiothoracic, 270

anastomotic of Trolard, 165

angular, 62

of arm and forearm, 282, 378

ascending (lumbar), 521

axillary, 99,275

azygos major and minor, 348

left superior, 350

basilic, 283

median, 283

brachial, 286

bronchial, 359

cardiac, 353

cephalic, 256, 269, 283
Vein or veins :-

cephalic, median, 283

cerebral, deep, 220

choroid, 222

Galen, 222

corpus striatum, 220

superficial or cortical, 165 anastomotic vein of Trolard, 165

basilar, 165

inferior, 165

middle, 165

posterior anastomosing vein of Labbé, 165

superior, 165

cervical, deep (profunda cervicis), I II I36

transverse (cervicis), 74, 99

choroid, 222

circumflex, iliac, deep, 520

superficial, $436,557,572$

coronary, 553

corpus striatum, 220

cystic, 5 I4

dorsal of penis, 442

superficial, 440

epigastric, deep, 520

superficial, 436,557

facial, 62

in neck, 74

femoral, 574

deep (profunda femoris), 574

subcutaneous or superficial, 557

of foot, anterior, 586

forearm, 378

of Galen, 222

common, 40, 222

ganglionic or deep cerebral or central 220

gastric, 514

gastro-epiploic, 512, $5^{14}$

gluteal, 548

of heart, 353

hemorrhoidal, 408

plexus, 408

hepatic, $52 \mathrm{I}$

iliac, common, 520

external, 520

internal, 548

deep circumflex, 520

superficial circumflex, 557

innominate, 92,326

intercostal, 348

left superior, $35^{\circ}$

right superior, 350

interventricular, anterior and posterior, 353

jugular, anterior, 74, 99

external, 74, 99

jugular, internal, 92

posterior, external, 74

of Labbé, 165

leg, 586,6 I 8

lumbar, $52 \mathrm{I}$

mammary, internal, 327

maxillary, internal, 72

median, 282

basilic, 283

cephalic, 283

mediastinal, 327

mesenteric, inferior, 514,515 
Vein or veins :-

mesenteric, superior, 512, 514

of neck, 74

oblique, of Marshall, 353

obturator, 548

ophthalmic, inferior and superior, 54

ovarian, 521

pampiniform (plexus), male, 521

female, 546

pancreatic, 515

pancreaticoduodenalis, inferior and

parotid, 66 superior, 512

of penis, 442

pericardial, 327

peroneal, 633

phrenic, inferior, 52

popliteal, 623

portal, 492, 514

profunda cervicis, III, 136

femoris, 574

prostatic (plexus), 418

pudic, internal, 413

external, superficial, 436, 557

pulmonary, 359

pyloric, 514

posterior, 378

radial, 282

renal, 521

saphenous, external or short, 586,618

internal or long, $557,574,586$

spermatic (pampiniform plexus), 52I

splenic, 515

subclavian1, 92, 99

superficial epigastric, 557

of $\mathrm{a} 1 \mathrm{~m}$ and forearm, 282

of forearm and hand, posterior, $37^{8}$ of thigh, 557

suprarenal, 521

suprascapular, 74,99

temporal, 72

temporomaxillary sinus, 72

thyroid gland, II

tibial, anterior, 593

posterior, 632

transverse, cervical, 74, 99

Trolard, vein of, 165

ulnar, anterior, 282

common, 282

posterior, 282,378

uterine, 420

vaginal plexus, 420

vasa brevia, 515

vertebral, III, I36

vertical (lumbar), 52 I

vesical (plexus), 420

vesicoprostatic (plexus), 40

Velum interpositum, 218 development, 177

Vena azygos, major and minor, 348 sinistra, superior, tertia, $35^{\circ}$

cava, inferior, 354,520

fissure in liver for, 490

superior, 327

Ventricle, fifth, 216

development, I73

fourth, 250
Ventricle, fourth, development, I7I, I76

lateral, 2 10

development, 170

dissection of, 214

third, 222

development, I7 1

dissectioll of, 222

floor of, 198

Ventricles of heart, 355,356

of larynx, 146

Vermiform appendix, 476, 500

process of cerebellum, 242

Vermis, inferior and superior (cerebel. lum), 242

Vertebral aponeurosis, 373

artery, IIO, $15^{8}$

muscles, 373

vein, III, I36

Vertical lumbar vein, 520

Verumontanum, 550

Vesical arteries, inferior, middle,superior, 546

plexus (venous), 420

Vesicles, cerebral, 169 seminal, 420

Vesicoprostatic plexus (venous), 401

Vesiculæ seminales, 420

Vestibule of vagina, 424

Vicq d'Azy r, bundle of, 238

Vidian artery, 122

canal, I5I

nerve, I5I

Vieussens, valve of, 244

Villi, arachnoidal, I55

Viscera, abdominal, 486 covered by peritoneum, 482

summary of development, 484 cranial, 166

pelvic, 537

thoracic, 321

Vitello-intestinal duct, 471

Vocal cords, true and false, 145

Volar artery, superficial, 294

Vulva, 423

WEIGHT of brain, 648

Wharton's duct, 86

"White line" of obturator fascia, 399, 400

Willis, circle of, 158,161

cords of, 34,35

Winslow, foramen of, 470,478

Wirsung, duct of, 508

Womb, or uterus, 543

Wrist, annular ligament of, anterior, 302 posterior, 378

dissection of anterior, 300

posterior, 376

landmarks of, 258

X1PHOID appendix, 432

$Z_{\text {INN, ligament of, } 52}$

Zygomatic arch, I8

Zygomaticus, major and minor, 59 






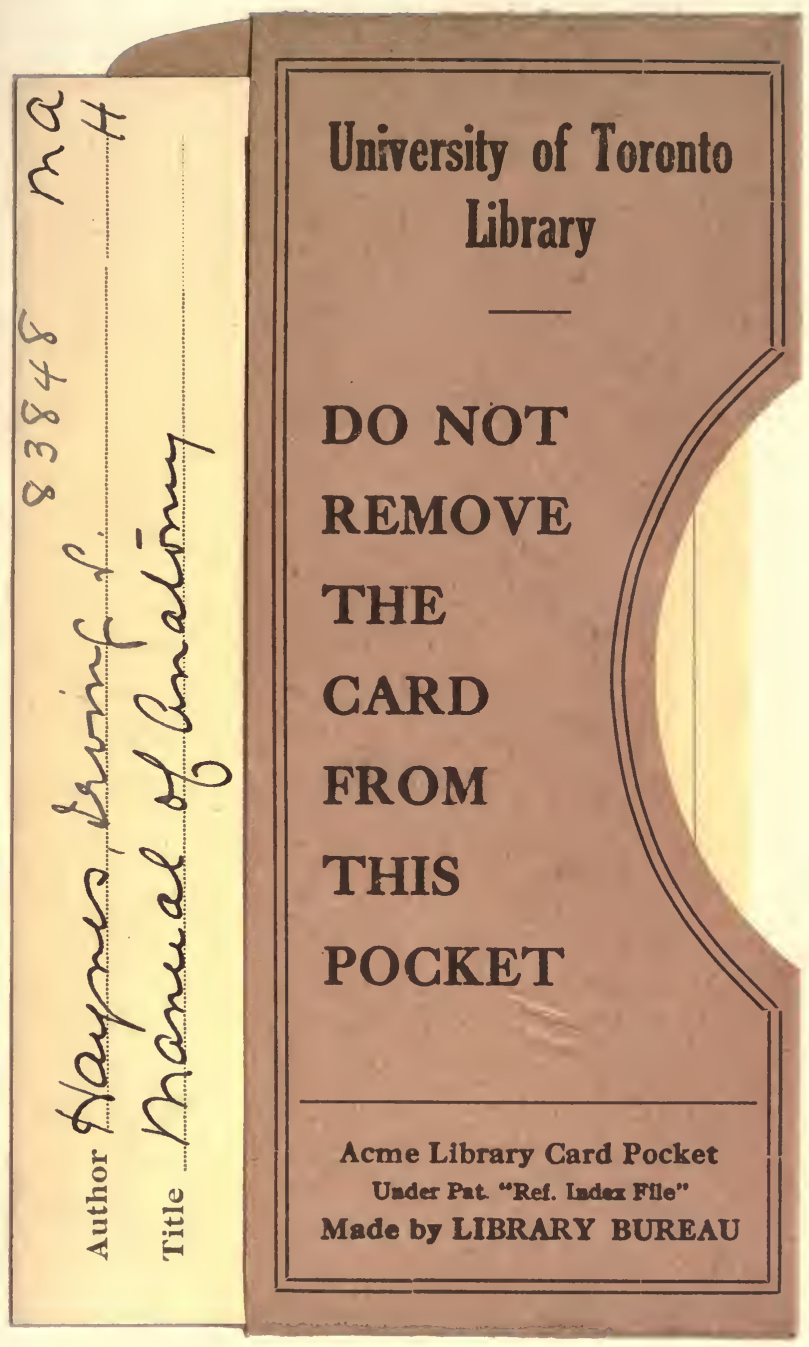


